\title{
DIFFICULT-TO-TREAT RHEUMATOID ARTHRITIS
}

Nadia M.T. Roodenrijs 


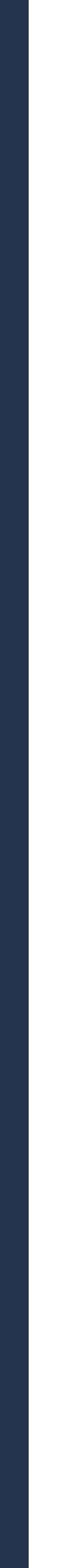






\section{DIFFICULT-TO-TREAT RHEUMATOID ARTHRITIS}

Nadia M.T. Roodenrijs 
ISBN 978-94-6416-572-2

Printed by Ridderprint

Printing of this thesis was kindly supported by Chipsoft, Fresenius Kabi Netherlands, Infection \& Immunity Utrecht, Pfizer and UCB Pharma.

(C) Nadia M.T. Roodenrijs, 2021

All rights reserved. No part of this publication may be reproduced or transmitted in any form or by any means, without permission from the author. The copyright of articles that have been published has been transferred to the respective journals. 


\section{DIFFICULT-TO-TREAT RHEUMATOID ARTHRITIS}

MOEILIJK TE BEHANDELEN REUMATOIDE ARTRITIS

(met een samenvatting in het Nederlands)

\section{Proefschrift}

ter verkrijging van de graad van doctor aan de Universiteit Utrecht op gezag van de rector magnificus, prof. dr. H.R.B.M. Kummeling, ingevolge het besluit van het college voor promoties in het openbaar te verdedigen op dinsdag 1 juni 2021 des middags te 12.15 uur

door

Nadia Maria Theresia Roodenrijs geboren op 3 mei 1990 te Wateringen 
Promotoren: $\quad$ Prof. dr. J.M. van Laar Prof. dr. F.P.J.G. Lafeber

Copromotoren: Dr. M.C. van der Goes Dr. P.M.J. Welsing 


\section{TABLE OF CONTENTS}

1 General introduction 9

2 Is prediction of clinical response to methotrexate in individual rheumatoid 23 arthritis patients possible? Results of a systematic literature review

3 The multi-biomarker disease activity score tracks response to rituximab treatment in rheumatoid arthritis patients: a post hoc analysis of three cohort studies

4 Characteristics of difficult-to-treat rheumatoid arthritis: results of an international survey

5 EULAR definition of difficult-to-treat rheumatoid arthritis

6 Immunological mechanisms underlying DMARD inefficacy in difficult-totreat rheumatoid arthritis: a narrative review with systematic literature search

7 Diagnostic issues in difficult-to-treat rheumatoid arthritis: a systematic literature review informing the EULAR recommendations for the management of difficult-to-treat rheumatoid arthritis

8 Pharmacological and non-pharmacological therapeutic strategies in difficult-to-treat rheumatoid arthritis: a systematic literature review informing the EULAR recommendations for the management of difficultto-treat rheumatoid arthritis

9 EULAR recommendations for the management of difficult-to-treat rheumatoid arthritis

10 Difficult-to-treat rheumatoid arthritis: contributing factors and burden of disease

11 Health care utilisation and economic burden of difficult-to-treat rheumatoid arthritis: a cost-of-illness study

12 Non-adherence in difficult-to-treat rheumatoid arthritis from the perspectives of patients and rheumatologists: a concept mapping study

237

263

291

13 General discussion

\section{A ADDENDUM}

Summary

Nederlandse samenvatting

Acknowledgements

List of publications

Contributing authors 



\section{CHAPTER 1}

\section{General introduction}

Parts of this chapter have been published in:

Ann Rheum Dis 2019;78:e105. 


\section{Rheumatoid arthritis}

Rheumatoid arthritis (RA) is the most common chronic inflammatory joint disease.' It is primarily characterised by pain and stiffness in joints caused by synovitis, which can result in irreversible cartilage damage and joint dysfunction if left untreated. Additionally, extra-articular manifestations can occur, such as rheumatoid nodules, and also more systemic manifestations like interstitial lung disease and vasculitis., ${ }^{2,3}$ On top of these direct consequences of RA, patients have an increased risk of comorbidities, such as cardiovascular diseases, ${ }^{4}$ osteoporosis ${ }^{5}$ and mental disorders. ${ }^{6}$

All these manifestations result in a significant patients' burden: reduced physical functioning, quality of life ${ }^{8}$ and premature mortality. ${ }^{9-11}$ With this, RA is accompanied by a substantial economic burden, not only derived by direct costs related to health care utilisation, but also as a result of functional and work disability. ${ }^{12-14}$

\section{Epidemiology and pathogenesis}

RA affects approximately $1 \%$ of the total adult population and is almost twice as common in women than in men.1.15-18 Global prevalence of RA is highest in Western Europe, North America and Australia, and lowest in Southeast Asia, North Africa and the Middle East. ${ }^{15}$ RA is a multifactorial disease, of which the exact cause is unknown.1,19 Several risk factors have been identified that may increase the risk of RA development. As environmental factors, smoking, ${ }^{20-23}$ obesity ${ }^{24,25}$ and an aberrant composition of the gut microbiome ${ }^{26-28}$ increase the risk of developing RA. Additionally, a positive family history for RA increases the risk, suggesting a role for genetic factors. ${ }^{29,30}$ Currently, HLA-DRBl shared epitope alleles are identified as the strongest genetic risk factor. ${ }^{31,32}$ This genetic risk factor is most prominent among patients who are positive for rheumatoid factor (RF) and anti-citrullinated protein antibody (ACPA). ${ }^{33,34}$ These autoantibodies can already be found before disease onset and can therefore also be considered as risk factors. ${ }^{35,36}$ Nevertheless, they are more likely to reflect the pathophysiological process, which already starts before onset of symptoms. ${ }^{35,36}$

Autoantibodies are thought to play a crucial role in the pathogenesis of RA and seropositivity for these autoantibodies is associated with more severe disease.19,37 However, autoantibodies do not occur in all patients, which underscores the heterogeneous pathogenesis of RA. Autoantibodies are thought to result from a decrease in self-tolerance triggered by environmental and genetic factors. ${ }^{38,39}$ Their interaction with antigens results in an activated immune system and an increased inflammatory state. Subsequently, this can result in synovitis, extra-articular manifestations and increased risk of certain comorbidities.

\section{Diagnostics}


Hitherto, no single clinical feature or specific test to diagnose RA exists. For research purposes, classification criteria have been developed containing the number of inflamed joints, seropositivity (RF and/or ACPA positivity), presence of acute phase reactants and the duration of symptoms. ${ }^{40}$ These classification criteria are frequently mistakenly used to diagnose RA. Classification criteria have primarily been developed to align study populations and are not meant to diagnose individual patients. ${ }^{41,42}$ Therefore, the diagnosis of RA should be made by the rheumatologist, weighting the combination of symptoms, signs and results of diagnostic tests (e.g. laboratory markers, radiography and pathology).,43

After diagnosing RA, medication should be started and disease activity should be followed over time. With the introduction of tight control and treat-to-target strategies, recommendations endorse regular assessment of disease activity and adaptation of treatment if remission or low disease activity is not reached. ${ }^{44-46}$ To be able to assess or monitor disease activity and treatment response, reliable diagnostic measures are needed.

Unfortunately, a perfect test assessing disease activity does not exist. Composite indices, which combine several measures regarded to reflect activity of the disease, are the most frequently used scores to assess disease activity. Especially the disease activity score assessing 28 joints (DAS28) is frequently used, which combines a swollen joint count (SJC) of 28 joints, a tender joint count (TJC) of these joints, patient's global assessment of disease activity and an acute phase reactant (erythrocyte sedimentation rate (ESR) or C-reactive protein (CRP)). ${ }^{47}$ Composite indices have several disadvantages possibly resulting in a suboptimal reflection of disease activity in individual patients: joints of the ankles and the feet are not included in the most frequently used scores (i.e. DAS28, clinical disease activity index (CDAI) and simplified disease activity index (SDAI)), while these joints are frequently involved in RA patients; and composite indices contain subjective components (i.e. patient's global assessment and TJC), which results in high variability between assessors and makes composite indices unreliable at patient level. ${ }^{48,49}$ Implementation of laboratory markers and imaging measures has been studied, although this has not yet shown sufficient benefit. ${ }^{50-52}$ Thus, whilst validated composite indices are not optimal for assessing disease activity at patient level, better diagnostic measures currently do not exist and therefore the use of composite indices is still endorsed in current RA management recommendations. ${ }^{44}$

\section{Therapeutic strategies}

Treatment of RA consists of a combination of pharmacological and nonpharmacological strategies.' Disease-modifying antirheumatic drugs (DMARDs) are the most important part of the therapeutic strategy, as these drugs do not only suppress symptoms, but additionally suppress inflammation and thereby prevent joint 
damage. ${ }^{53}$ DMARDs are frequently combined with glucocorticoids (GCs) as well as analgesics (e.g. paracetamol, opioids) and anti-inflammatory drugs (i.e. non-steroid anti-inflammatory drugs (NSAIDs)). Additionally, non-pharmacological interventions, such as physiotherapy, can be used. ${ }^{43}$ Treatment is aimed at reaching remission or at least low disease activity in order to prevent joint damage and to limit the patients' burden on the short and long term. ${ }^{44}$

The introduction of biological ( $b^{-}$, such as tumour necrosis factor inhibitors (TNFi)) and targeted synthetic (ts-, such as janus kinase inhibitors (JAKi)) DMARDs has improved patients' outcomes significantly. ${ }^{1,54}$ Due to cost constraints, conventional synthetic (cs) DMARDs - specifically methotrexate, if no contraindications are present - are still the first step in the pharmacological treatment of RA according to the European League Against Rheumatism (EULAR) recommendations. ${ }^{44}$ Until csDMARDs manifest their efficacy (typically within the first 2 to 3 months), csDMARDs may be combined with GCs to reduce symptoms in the initiation phase. If no improvement is achieved after 3 months or if the treatment goal is not achieved after 6 months, the generally more expensive b- or tsDMARDs may already be added if poor prognostic factors are present. Poor prognostic factors for RA severity, e.g. high SJCs and presence of early erosions, RF and/or ACPA, have been implemented in management recommendations to avoid valuable time loss. ${ }^{44,55-58}$ Despite implementation of these factors, full stratification of patients - and therewith personalised medicine - in order to obtain optimal treatment response is still not possible. ${ }^{59,60}$ With drugs being ineffective, valuable time in the management of disease may be lost: patients' disease remains active, which increases the risk of joint damage, loss of physical functioning and reduced quality of life.,61

\section{Difficult-to-treat rheumatoid arthritis}

Despite significant improvement in the management of RA over the past decades, around $5 \%$ to $20 \%$ of RA patients remain symptomatic after failing several lines of b/ tsDMARDs. ${ }^{62-64}$ These patients can be referred to as having 'difficult-to-treat (D2T)' RA. Suboptimal assessment of disease activity (and even of the diagnosis of the disease itself) and the absence of predictors of treatment response may all contribute to this disease state.

In the Netherlands, almost 260,000 patients had a diagnosis of RA in the year 2019 (1year prevalence).${ }^{65}$ If $5 \%$ to $20 \%$ of these patients can be classified as having D2T RA, this reflects around 13,000 to 52,000 patients in the Netherlands. Comparable numbers of patientswere found with other diagnoses, suchas Parkinson's disease (1-year prevalence 48,500), lung cancer (10-years prevalence 24,100), acquired immunodeficiency syndrome (AIDS) or human immunodeficiency virus (HIV) infection (1-year prevalence 23,000) and cardiac arrest (1-year incidence 16,800). ${ }^{66}$ This emphasises that the D2T RA population is still substantial. 
With the introduction of tight control and treat-to-target strategies in the management of RA, DMARD treatment can already be changed if no improvement is achieved within 3 months. Therewith, several lines of $b / t s D M A R D$ can already be failed within 1 year. After failure of $a b / t s D M A R D$, the only recommendation applicable to these patients is to switch to another b/tsDMARD with or without a different mechanism of action (Figure 1, adapted from Smolen et al).44 Further changes in the treatment of individual D2T RA patients are unfortunately generally based on trial-and-error, as comprehensive management recommendations for these patients are lacking.

Importantly, DMARD switches are only indicated in case of inflammation related to RA, whereas the symptoms and signs in D2T RA are often much more complex: in each individual D2T RA patient, one or more factors - of inflammatory and non-inflammatory origin - may potentially contribute to the D2T RA state (Figure 2, adapted from de Hair et al). ${ }^{62,67}$ The heterogeneity of D2T RA is exemplified in Table 1, in which three patient cases are presented who can be referred to as having D2T. This heterogeneity further complicates the management of these patients.

In daily practice, the recognition of factors contributing to D2T RA is suboptimal and the importance of these potentially contributing factors is unknown. This lack of knowledge and the absence of management recommendations for D2T RA hamper patient care. Suboptimal management of D2T RA patients may result in a significant burden: symptoms and signs are persisting, without clear expectation that the situation will improve in the (near) future. Additionally, the D2T RA state may affect social life, work disability and direct and indirect health care cost substantially. Several patient partners have underlined the major burden and one of them gave the following response: 'Reading about D2T RA is like reading the story of my life. I was also treated by trialand-error in the past and still suffer the consequences. That is why I feel that more

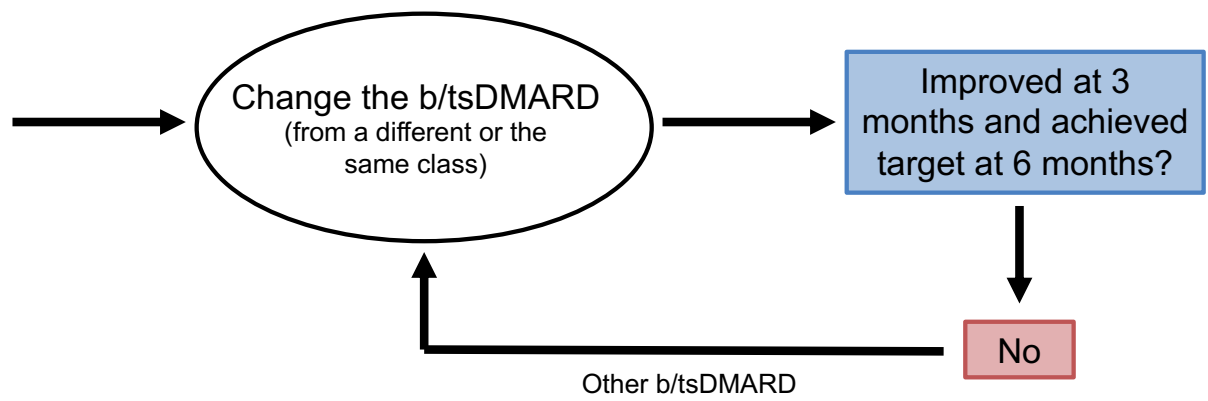

Figure 1. The loop of changing $\mathrm{b} /$ tsDMARDs in current EULAR RA recommendations ${ }^{44}$ In the current EULAR RA management recommendations, after failure of a first b/tsDMARD, patients enter the loop resulting in DMARD switching in case of insufficient clinical benefit.

DMARD: disease-modifying antirheumatic drug; EULAR: European League Against Rheumatism; RA: rheumatoid arthritis.

Adapted from Smolen et $\mathrm{al}_{{ }^{44}}$ with permission from BMJ Publishing Group LTD. 
attention for this topic and research in this area can be of great value to all patients like me.' This further emphasises that management, and therewith outcomes of D2T RA patients, should be improved.

Furthermore, the management of D2T RA patients is complicated by the lack of uniform terminology and a clear definition to describe and classify the D2T RA population. In addition to D2T, terms as 'persistent', 'treatment refractory' and even 'true refractory RA' (only applicable to patients in whom DMARDs are truly ineffective due to immunological mechanisms) are used in the current literature. ${ }^{63,67-69}$ Additionally, different definitions are used to classify this patient group, e.g. failure of different numbers of previous b/ tsDMARDs. ${ }^{70-72}$ Uniform terminology and a clear definition are therefore essential to unify and boost further research and to improve the management of D2T RA patients.

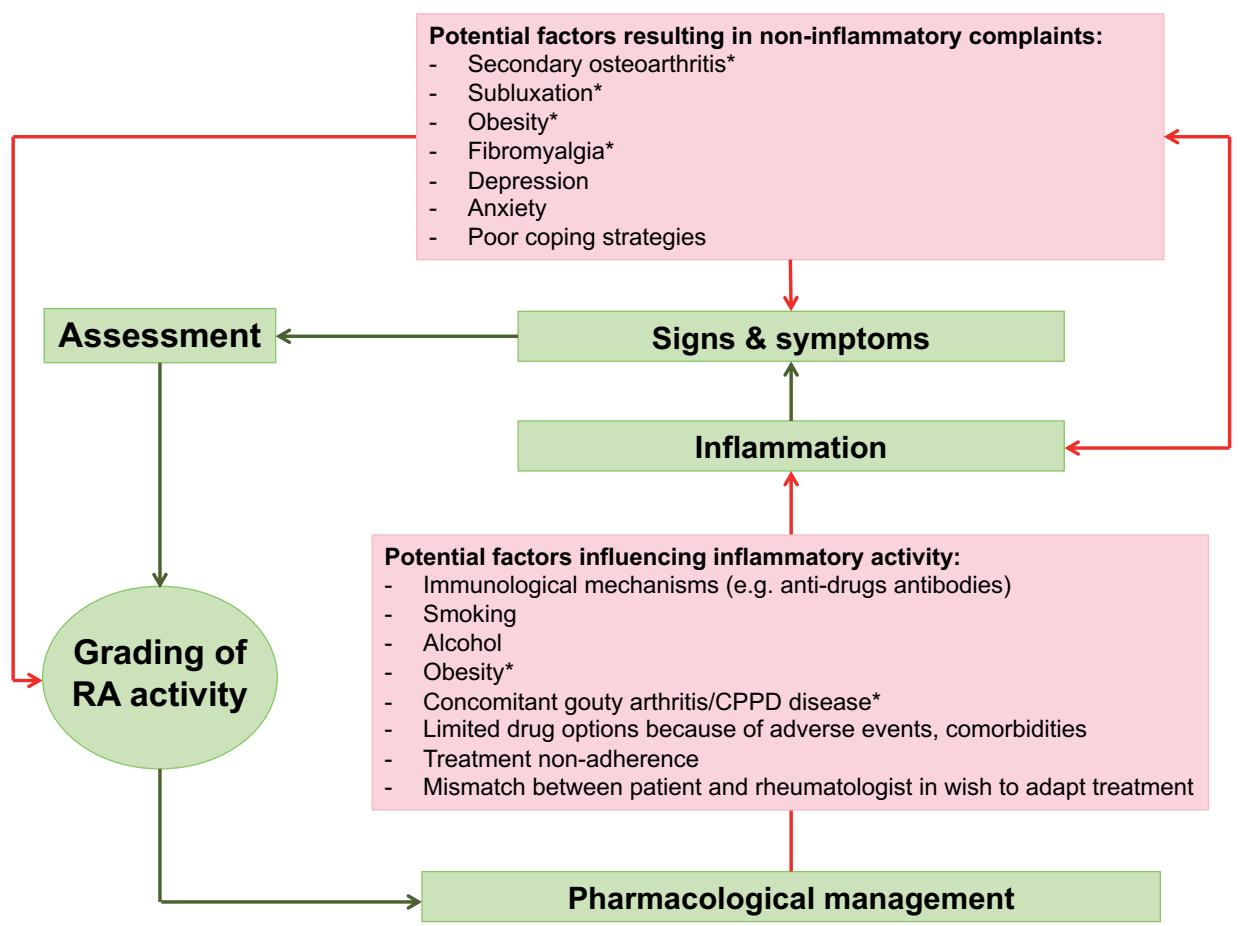

Figure 2. The management cycle of D2T RA, including factors potentially contributing to the disease state The drug management cycle is shown in green. Factors in pink, which influence (red arrows) the drug management cycle and lead to difficulty in grading RA activity or drug effectiveness. All these factors have been associated with established RA and/or persistent disease activity, and may in different grades contribute to D2T RA in individual patients.

CPPD: calcium pyrophosphate deposition disease; D2T: difficult-to-treat; RA: rheumatoid arthritis; *: Factors that might also hamper proper grading of disease activity.

Adapted from de Hair et $a_{1}^{62}$ with permission from Oxford University Press. 
Table 1. Typical D2T RA cases reflecting the heterogeneity of factors contributing to this disease state

\begin{tabular}{|c|c|c|c|c|}
\hline Case & $\begin{array}{l}\text { Patient } \\
\text { (sex, age) }\end{array}$ & Medical history & DMARD history & $\begin{array}{l}\text { Factor(s) potentially } \\
\text { contributing to D2T RA }\end{array}$ \\
\hline 1 & $\begin{array}{l}\text { Male, } \\
55 \mathrm{yr}\end{array}$ & $\begin{array}{l}\text { RA with active } \\
\text { inflammation }\end{array}$ & $\begin{array}{l}\text { Methotrexate, hydroxychloroquine, } \\
\text { sulfasalazine, azathioprine, leflunomide, } \\
\text { gold im, tocilizumab, abatacept, } \\
\text { adalimumab, etanercept, infliximab, } \\
\text { anakinra, rituximab, ustekinumab, } \\
\text { baricitinib }\end{array}$ & $\begin{array}{l}\text { Possibly 'true' refractory } \\
\text { RA, resulting from } \\
\text { immunological } \\
\text { mechanisms }\end{array}$ \\
\hline 2 & $\begin{array}{l}\text { Male, } \\
73 \mathrm{yr}\end{array}$ & $\begin{array}{l}\text { RA, aortic } \\
\text { dissection type } \\
\text { B - graft, graft } \\
\text { infection, chronic } \\
\text { kidney disease, } \\
\text { duodenal ulcer }\end{array}$ & $\begin{array}{l}\text { Methotrexate, hydroxychloroquine, } \\
\text { prednisolone, etanercept, rituximab }\end{array}$ & $\begin{array}{l}\text { Limited drug options due } \\
\text { to comorbidities }\end{array}$ \\
\hline 3 & $\begin{array}{l}\text { Female, } \\
47 \mathrm{yr}\end{array}$ & $\begin{array}{l}\text { RA, pain syndrome, } \\
\text { obesity }\end{array}$ & $\begin{array}{l}\text { Methotrexate, hydroxychloroquine, } \\
\text { sulfasalazine, leflunomide, tocilizumab, } \\
\text { abatacept, adalimumab, certolizumab } \\
\text { pegol }\end{array}$ & $\begin{array}{l}\text { Predominantly non- } \\
\text { inflammatory factors }\end{array}$ \\
\hline
\end{tabular}

Im: intramuscular; RA: rheumatoid arthritis; yr: years.

\section{Aims and outline of this thesis}

The aims of this thesis are to define and improve our understanding of D2T RA. This can contribute to the optimisation of the management of D2T RA patients and therewith to improve outcomes of this patient population. To this end, systematic literature reviews (SLRs) on the current state of knowledge have been performed and management recommendations have been developed. Additionally, studies have been performed to further characterise D2T RA to get more insights into this disease state.

In Chapter 2, a SLR is presented on the predictors of clinical response to methotrexate treatment, which is still the first step in the management of RA. Reliable predictors could help to avoid periods with unnecessary active RA and joint damage, and might diminish the risk of the development of the D2T RA state.

In Chapter 3, the ability of the multi-biomarker disease activity (MBDA) score to measure disease activity upon treatment with rituximab (a bDMARD used typically after multiple lines of bDMARDs failed) is assessed. The MBDA score combines twelve serum biomarkers and may therefore be a more objective alternative for the assessment of disease activity than traditional measures (clinical assessment and composite indices). In D2T RA patients specifically, the MBDA score may be useful to assess the presence or absence of inflammation, if doubt remains after assessment using traditional measures.

In Chapter 4, the concept of D2T RA is explored via an international survey among rheumatologists. Additionally, issues are explored that should be addressed in the 
management of D2T RA and that are not covered by current EULAR management recommendations.

In Chapter 5, the EULAR definition of D2T RA is presented. This definition is established by the EULAR Task Force on the development of management recommendations for D2T RA and is based on the international survey (Chapter 4), a scoping literature review and extensive discussions.

In Chapter 6, a narrative review is presented, which is based on a systematic literature search. In this review, it is explored whether 'true' refractory RA exists and, if so, how often it occurs. Additionally, underlying mechanisms of DMARD inefficacy as well as (unacceptable) side effects in D2T RA patients are explored and summarised.

In Chapter 7, a SLR is presented on diagnostic issues in D2T RA. This SLR is performed to inform the EULAR Task Force on the development of management recommendations for D2T RA. Here, it is firstly explored how to optimally confirm the diagnosis of RA in a D2T RA patient and how to optimally diagnose or rule out mimicking alternative or coexisting diseases. In addition, this SLR focusses on the assessment of the presence of inflammation in D2T RA and on the influence that comorbidities may have on this assessment.

In Chapter 8, a SLR is presented on pharmacological and non-pharmacological therapeutic strategies in D2T RA. This SLR is also performed to inform the EULAR Task Force on the development of management recommendations for D2T RA. First, pharmacological and non-pharmacological therapeutic strategies in D2T RA patients are explored that could be used to treat inflammatory activity and complaints not directly related to inflammation (non-inflammatory complaints). Second, this SLR focusses on the optimisation, and therefore also the identification, of a mismatch in goal setting between patients and health care professionals and of suboptimal selfmanagement.

In Chapter 9, the EULAR recommendations for the management of D2T RA are presented. The evidence identified in the SLRs (Chapter 7 and 8) is used as the scientific base of these recommendations.

In Chapter 10, D2T RA is further clinically characterised by identifying risk factors at RA onset and factors contributing to D2T RA (of inflammatory and non-inflammatory origin). Additionally, subgroups of D2T RA patients are explored and the clinical burden of D2T RA is determined.

In Chapter 11, the economic burden of D2T RA is assessed by identifying health care utilisation and cost-of-illness. Additionally, determinants of these costs are identified.

In Chapter 12, treatment non-adherence, which may substantially contribute to D2T RA, is further assessed. The perspectives of patients and rheumatologists on barriers and facilitators of optimal treatment adherence are thematically structured, prioritised and compared. 
In Chapter 13, the results of the studies are put in a broader perspective, including discussions on their strengths, limitations and clinical implications. Furthermore, future perspectives and research opportunities are discussed as well as concluding remarks. 


\section{References}

1. Smolen JS, Aletaha D, McInnes IB. Rheumatoid arthritis. Lancet 2016;388:2023-38.

2. Carmona L, González-Alvaro I, Balsa A, et al. Rheumatoid arthritis in Spain: occurrence of extra-articular manifestations and estimates of disease severity. Ann Rheum Dis 2003;62:897-900.

3. Turesson C, Jacobsson L, Bergström U. Extraarticular rheumatoid arthritis: Prevalence and mortality. Rheumatology 1999;38:668-74.

4. Avina-Zubieta JA, Thomas J, Sadatsafavi M, et al. Risk of incident cardiovascular events in patients with rheumatoid arthritis: A metaanalysis of observational studies. Ann Rheum Dis 2012;71:1524-9.

5. Xue AL, Wu SY, Jiang $L$, et al. Bone fracture risk in patients with rheumatoid arthritis. Med (United States) 2017;96.

6. Marrie RA, Hitchon CA, Walld R, et al. Increased Burden of Psychiatric Disorders in Rheumatoid Arthritis. Arthritis Care Res 2018;70:970-8.

7. Taylor PC, Moore A, Vasilescu R, et al. A structured literature review of the burden of illness and unmet needs in patients with rheumatoid arthritis: a current perspective. Rheumatol Int 2016;36:685-95.

8. Matcham F, Scott IC, Rayner L, et al. The impact of rheumatoid arthritis on quality-oflife assessed using the SF-36: A systematic review and meta-analysis. Semin Arthritis Rheum 2014;44:123-30.

9. England BR, Sayles H, Michaud K, et al. CauseSpecific Mortality in Male US Veterans with Rheumatoid Arthritis. Arthritis Care Res 2016;68:36-45.

10. Maassen JM, Goekoop-Ruiterman Y, Groenendael H Van, et al. OP0219: Mortality of Rheumatoid Arthritis Patients, Treated to Target at Low Disease Activity: 17-years Follow-up of the BeSt Cohort. Ann Rheum Dis 2020;79:136-7.

11. Sparks JA, Chang SC, Liao KP, et al. Rheumatoid Arthritis and Mortality among Women during 36 Years of Prospective Follow-Up: Results from the Nurses' Health Study. Arthritis Care Res 2016;68:753-62.

12. Furneri G, Mantovani LG, Belisari A, et al. Systematic literature review on economic implications and pharmacoeconomic issues of rheumatoid arthritis. Clin Exp Rheumatol;30:S72-84.

13. Hsieh P-H, Wu O, Geue $\mathrm{C}$, et al. Economic burden of rheumatoid arthritis: a systematic review of literature in biologic era. Ann Rheum Dis 2020;79:771-7.

14. Sokka $\mathrm{T}$, Kautiainen $\mathrm{H}$, Pincus $\mathrm{T}$, et al. Work disability remains a major problem in rheumatoid arthritis in the 2000s: Data from 32 countries in the QUEST-RA Study. Arthritis Res Ther 2010;12:R42.

15. Cross $M$, Smith $E$, Hoy $D$, et al. The global burden of rheumatoid arthritis: estimates from the global burden of disease 2010 study. Ann Rheum Dis 2014;73:1316-22.

16. Eriksson JK, Neovius M, Ernestam S, et al. Incidence of rheumatoid arthritis in Sweden: A Nationwide population-based assessment of incidence, its determinants, and treatment penetration. Arthritis Care Res 2013;65:870-8.

17. Hunter TM, Boytsov NN, Zhang $X$, et al. Prevalence of rheumatoid arthritis in the United States adult population in healthcare claims databases, 2004-2014. Rheumatol Int 2017;37:1551-7.

18. Myasoedova E, Crowson CS, Kremers HM, et al. Is the incidence of rheumatoid arthritis rising? Results from Olmsted County, Minnesota, 1955-2007. Arthritis Rheum 2010;62:1576-82.

19. Mclnnes IB, Schett $G$. The pathogenesis of rheumatoid arthritis. $N$ Engl $J$ Med 2011;365:2205-19.

20. Di Giuseppe D, Orsini N, Alfredsson L, et al. Cigarette smoking and smoking cessation in relation to risk of rheumatoid arthritis in women. Arthritis Res Ther 2013;15.

21. Liu X, Tedeschi SK, Barbhaiya M, et al. Impact and Timing of Smoking Cessation on Reducing Risk of Rheumatoid Arthritis Among Women in the Nurses' Health Studies. Arthritis Care Res 2019;71:914-24.

22. Stolt P, Bengtsson C, Nordmark B, et al. Quantification of the influence of cigarette smoking on rheumatoid arthritis: Results from a population based case-control study, using incident cases. Ann Rheum Dis 2003;62:83541.

23. Sugiyama D, Nishimura K, Tamaki K, et al. Impact of smoking as a risk factor for developing rheumatoid arthritis: A metaanalysis of observational studies. Ann Rheum Dis 2010;69:70-81.

24. Qin $B$, Yang $M$, Fu $\mathrm{H}$, et al. Body mass index and the risk of rheumatoid arthritis: A systematic review and dose-response meta-analysis. Arthritis Res Ther 2015;17:86.

25. Ljung L, Rantapää-Dahlqvist S. Abdominal obesity, gender and the risk of rheumatoid arthritis - a nested case-control study. 
Arthritis Res Ther 2016;18:277.

26. Manasson J, Blank RB, Scher JU. The microbiome in rheumatology: Where are we and where should we go? Ann Rheum Dis 2020;79:S727-33.

27. Radstake TRDJ. Extraintestinal translocation of microbes and tissue specificity in rheumatic musculoskeletal disease (RMD): its more than a gut feeling. Ann Rheum Dis 2018;77:1702-4.

28. Zhang $X$, Zhang $D$, Jia $H$, et al. The oral and gut microbiomes are perturbed in rheumatoid arthritis and partly normalized after treatment. Nat Med 2015;21:895-905.

29. Silman AJ, MacGregor AJ, Thomson W, et al. Twin concordance rates for rheumatoid arthritis: results from a nationwide study. Rheumatology 1993;32:903-7.

30. Frisell $T$, Holmqvist $M$, Källberg $H$, et al. Familial risks and heritability of rheumatoid arthritis: Role of rheumatoid factor/anti-citrullinated protein antibody status, number and type of affected relatives, sex, and age. Arthritis Rheum 2013;65:2773-82.

31. Gregersen PK, Silver J, Winchester RJ. The shared epitope hypothesis. an approach to understanding the molecular genetics of susceptibility to rheumatoid arthritis. Arthritis Rheum 1987;30:1205-13.

32. Dedmon LE. The genetics of rheumatoid arthritis. Rheumatology (Oxford) 2020;59:2661-70.

33. Raychaudhuri S, Sandor C, Stahl EA, et al. Five amino acids in three HLA proteins explain most of the association between MHC and seropositive rheumatoid arthritis. Nat Genet 2012;44:291-6.

34. Viatte $S$, Plant $D$, Han B, et al. Association of HLADRBl haplotypes with rheumatoid arthritis severity, mortality, and treatment response. JAMA - J Am Med Assoc 2015;313:1645-56.

35. Nielen MMJ, Van Schaardenburg D, Reesink HW, et al. Specific Autoantibodies Precede the Symptoms of Rheumatoid Arthritis: A Study of Serial Measurements in Blood Donors. Arthritis Rheum 2004;50:380-6.

36. Rantapää-Dahlqvist S, De Jong BAW, Berglin E, et al. Antibodies Against Cyclic Citrullinated Peptide and IgA Rheumatoid Factor Predict the Development of Rheumatoid Arthritis. Arthritis Rheum 2003;48:2741-9.

37. Van Gaalen FA, Van Aken J, Huizinga TWJ, et al. Association between HLA class II genes and autoantibodies to cyclic citrullinated peptides (CCPS) influences the severity of rheumatoid arthritis. Arthritis Rheum 2004;50:2113-21.
38. Alivernini S, Fedele AL, Cuoghi I, et al. Citrullination: The loss of tolerance and development of autoimmunity in rheumatoid arthritis. Reumatismo 2008;60:85-94.

39. Catrina Al, Deane KD, Scher JU. Gene, environment, microbiome and mucosal immune tolerance in rheumatoid arthritis. Rheumatology 2014;55:391-402.

40. Aletaha D, Neogi T, Silman AJ, et al. 2010 Rheumatoid arthritis classification criteria: An American College of Rheumatology/ European League Against Rheumatism collaborative initiative. Ann Rheum Dis 2010;69:1580-8.

41. Aggarwal R, Ringold S, Khanna D, et al. Distinctions between diagnostic and classification criteria? Arthritis Care Res (Hoboken) 2015;67:891-7.

42. Porter D, Basu N, Siebert S. Classification criteria: time for a rethink? Ann Rheum Dis 2020;79:1264-6.

43. Combe $\mathrm{B}$, Landewe $\mathrm{R}$, Daien $\mathrm{Cl}$, et al. 2016 update of the EULAR recommendations for the management of early arthritis. Ann Rheum Dis 2017;76:948-59.

44. Smolen JS, Landewé RBM, Bijlsma JWJ, et al. EULAR recommendations for the management of rheumatoid arthritis with synthetic and biological disease-modifying antirheumatic drugs: 2019 update. Ann Rheum Dis 2020;79:685-99.

45. Smolen JS, Breedveld FC, Burmester GR, et al. Treating rheumatoid arthritis to target: 2014 update of the recommendations of an international task force. Ann Rheum Dis 2016;75:3-15.

46. Stoffer MA, Schoels MM, Smolen JS, et al. Evidence for treating rheumatoid arthritis to target: Results of a systematic literature search update. Ann Rheum Dis 2016;75:16-22.

47. van der Heijde DM, van 't Hof M, van Riel PL, et al. Development of a disease activity score based on judgment in clinical practice by rheumatologists. J Rheumatol 1993;20:579-81.

48. Jacobs JWG, Ten Cate DF, van Laar JM. Monitoring of rheumatoid arthritis disease activity in individual patients: still a hurdle when implementing the treat-totarget principle in daily clinical practice. Rheumatology 2015;54:959-61.

49. Gaujoux-Viala C, Mouterde G, Baillet A, et al. Evaluating disease activity in rheumatoid arthritis: Which composite index is best? A systematic literature analysis of studies comparing the psychometric properties of 
the DAS, DAS28, SDAl and CDAl. Jt Bone Spine 2012;79:149-55.

50. Colebatch AN, Edwards CJ, Østergaard $M$, et al. EULAR recommendations for the use of imaging of the joints in the clinical management of rheumatoid arthritis. Ann Rheum Dis 2013;72:804-14.

51. Østergaard M, Pedersen SJ, Døhn UM. Imaging in rheumatoid arthritis - status and recent advances for magnetic resonance imaging, ultrasonography, computed tomography and conventional radiography. Best Pract Res Clin Rheumatol 2008;22:1019-44.

52. Caporali R, Smolen JS. Back to the future: Forget ultrasound and focus on clinical assessment in rheumatoid arthritis management. Ann Rheum Dis 2018;77:18-20.

53. Kerschbaumer A, Sepriano A, Smolen JS, et al. Efficacy of pharmacological treatment in rheumatoid arthritis: a systematic literature research informing the 2019 update of the EULAR recommendations for management of rheumatoid arthritis. Ann Rheum Dis 2020.

54. Kievit W, Fransen J, de Waal Malefijt MC, et al. Treatment changes and improved outcomes in RA: an overview of a large inception cohort from 1989 to 2009. Rheumatology 2013;52:1500-8.

55. Scott DL, Coulton BL, Symmons DPM, et al. Long-term outcome of treating rheumatoid arthritis: results after 20 years. Lancet 1987;329:1108-11.

56. van der Heijde DMFM, van Riel PLCM, van Leeuwen MA, et al. Prognostic factors for radiographic damage and physical disability in early rheumatoid arthritis. A prospective follow-up study of 147 patients. Rheumatology 1992;31:519-25.

57. van Leeuwen MA, van Rijswijk $M H$, Sluiter $W J$, et al. Individual relationship between progression of radiological damage and the acute phase response in early rheumatoid arthritis. Towards development of a decision support system. J Rheumatol 1997;24:20-7.

58. Smolen JS, Van Der Heijde DMFM, St.Clair EW, et al. Predictors of joint damage in patients with early rheumatoid arthritis treated with high-dose methotrexate with or without concomitant infliximab: Results from the ASPIRE trial. Arthritis Rheum 2006;54:702-10.

59. de Jong TD, Vosslamber S, Verweij CL. Moving towards personalized medicine in rheumatoid arthritis. Arthritis Res Ther 2014;16:110.

60. Isaacs JD, Ferraccioli G. The need for personalised medicine for rheumatoid arthritis. Ann Rheum Dis 2011;70:4-7.

61. Aletaha D, Smolen J, Ward MM. Measuring function in rheumatoid arthritis: Identifying reversible and irreversible components. Arthritis Rheum 2006;54:2784-92.

62. de Hair MJH, Jacobs JWG, Schoneveld JLM, et al. Difficult-to-treat rheumatoid arthritis: an area of unmet clinical need. Rheumatology (Oxford) 2017;57:1135-44.

63. Buch $\mathrm{MH}$. Defining refractory rheumatoid arthritis. Ann Rheum Dis 2018;77:966-9.

64. Kearsley-Fleet L, Davies R, De Cock D, et al. Biologic refractory disease in rheumatoid arthritis: Results from the British Society for Rheumatology Biologics Register for Rheumatoid Arthritis. Ann Rheum Dis 2018;77:1405-12.

65. Reumatoïde artritis (RA) | Cijfers \& Context | Huidige situatie | Volksgezondheidenzorg.info. https://www.volksgezondheidenzorg.info/ onderwerp/reumatoïde-artritis-ra/cijferscontext/huidige-situatie\#node-prevalentiereumatoïde-artritis-huisartsenpraktijk (accessed 23 Nov 2020).

66. Ranglijst aandoeningen op basis van vóórkomen | Volksgezondheidenzorg.info. 2019.https://www.volksgezondheidenzorg. info/ranglijst/ranglijst-aandoeningen-opbasis-van-vóórkomen (accessed 23 Nov 2020).

67. Roodenrijs NM, de Hair MJ, van der Goes MC, et al. Correspondence to viewpoint 'Defining refractory rheumatoid arthritis' by Buch. Ann Rheum Dis 2019;78:e105.

68. Bécède $M$, Alasti $F$, Gessl I, et al. Risk profiling for a refractory course of rheumatoid arthritis. Semin Arthritis Rheum 2019;49:211-7.

69. Buch MH. Response to 'Correspondence to viewpoint 'Defining refractory rheumatoid arthritis' by Buch' by Roodenrijs et al. Ann Rheum Dis 2019;78:e106.

70. Takanashi S, Kaneko Y, Takeuchi T. FRI0079 Characteristics of difficult-to-treat rheumatoid arthritis. Ann Rheum Dis 2020;79:612-3.

71. Batko B, UrbańskiK, Świerkot J, et al. Comorbidity burden and clinical characteristics of patients with difficult-to-control rheumatoid arthritis. Clin Rheumatol 2019;38:2473-81.

72. Richard L, Gonde H, Devauchelle A, et al. SAT0108 Characteristics of multiresistant patients to biological treatments in rheumatoid arthritis, and associated factors: an observational and retrospective study in 
385 patients. Ann Rheum Dis 2019;78:1120-1. 



\section{CHAPTER 2}

Is prediction of clinical response to methotrexate in individual rheumatoid arthritis patients possible? Results of a systematic literature review

Joint Bone Spine 2019;83;13-23.

Nadia. M.T. Roodenrijs, Marlies C. van der Goes, Paco M.J. Welsing, Janneke Tekstra, Jacob M. van Laar, Floris P.J.G. Lafeber, Johannes W.J. Bijlsma, Johannes W.G. Jacobs 


\section{Abstract}

\section{Objectives}

To identify, by a systematic literature review, predictors of clinical response to methotrexate treatment in rheumatoid arthritis patients, which would facilitate personalised treatment.

\section{Methods}

PubMed and Embase databases were searched for original articles. Additionally, congress abstracts of European League Against Rheumatism and American College of Rheumatology annual meetings of the past 2 years were screened. Articles describing predictors of clinical response to methotrexate after 3 to 6 months were included, since this reflects the time span used to determine treatment effectiveness and decide on treatment changes in treat-to-target recommendations.

\section{Results}

Thirty articles were included, containing 102 different predictors and 11 predictive models. Nineteen predictors and 2 predictive models were studied in multiple cohorts. Female gender was found to be a predictor of non-response in two studies (odds ratios 0.55 and 0.54 ), but these findings could not be replicated in two other studies. In two studies, smoking predicted non-response (adjusted odds ratios 0.35 and 0.60 ), although this was inconsistent over all response criteria assessed. Rheumatoid factor positivity predicted non-response in two studies (adjusted hazard ratio 0.6l, adjusted odds ratio 0.40), but this was not found in three other studies. Heterogeneity in studies prohibited further comparison of predictive values between studies. Additionally, a validated epigenetic model was found (area under the curve 0.90 and 0.91).

\section{Conclusions}

No predictors were identified reliably predicting clinical response to methotrexate after 3 to 6 months in the individual patient: clinical predictors were weak. However, a promising epigenetic model was found that needs further validation. 


\section{Introduction}

The European League Against Rheumatism (EULAR) recommendations and American College of Rheumatology (ACR) guidelines for treatment of rheumatoid arthritis (RA) state that methotrexate (MTX) should be part of the first treatment strategy unless patients have contraindications or early intolerance.,2 They also recommend treat-totarget strategies. EULAR recommendations endorse that treatment should be adjusted if there is no improvement at 3 months after start of treatment, or if the treatment target has not been reached at 6 months after start of treatment. Still, with this regimen, valuable time may be lost, increasing the risk of joint damage, loss of physical function and decreased quality of life, and unnecessary exposure to adverse events of a drug that is not sufficiently effective.

To avoid unnecessary time loss, prognostically unfavourable factors for RA at time of diagnosis, e.g. high swollen joint counts, and presence of rheumatoid factor (RF) and/or anti-citrullinated protein antibodies (ACPA), have been implemented in management recommendations. ${ }^{1-3}$ Presence of these factors allows for an earlier start of biological (b-) or targeted synthetic (ts-) disease-modifying antirheumatic drugs (DMARDs). Prediction of response to MTX, if possible, could be an additional factor to consider when contemplating an earlier start of b/tsDMARDs.

MTX is considered the first-choice treatment in RA based on its efficacy, safety and relatively low costs. MTX monotherapy has been reported to lead to an ACR70 response rate of $25 \%$ at 6 months. ${ }^{4}$ In these trials, MTX was not combined with glucocorticoids (GCs) or other DMARDs. In combination therapy, higher response rates have been reported. ${ }^{5-8}$ Nonetheless, a considerable proportion of early RA patients does not achieve low disease activity or remission with MTX-based treatment within the window of opportunity. Many studies have assessed predictors of response to treatment with MTX in RA patients, but few predictors have been validated and, if validated, conflicting results have been presented. Genome-wide association studies with data-driven methodology have led to a large number of new, especially genetic, predictors. These efforts are in line with the concept of personalised treatment, which is expected to become one of the key principles of future health care. This makes prediction of treatment response highly relevant. Therefore, the aim of this systematic review was to explore and summarise all known factors that predict clinical response to MTX.

\section{Methods}

\section{Search strategy and study inclusion}

The databases PubMed and Embase were searched until December 18, 2018. The core 
components of this search strategy contained the domain (patients with RA who started MTX) and outcome (clinical response at 3 to 6 months after start of MTX therapy, since this period reflects the time span used to determine treatment effectiveness and decide on treatment changes according to treat-to-target recommendations'). The determinant, i.e. predictors of treatment response, was not specified in the search strategy, as all possible types of predictors were of interest (see full search in Supplementary File 1). A limit was set to English or Dutch language. In Embase, only originals articles, systematic literature reviews and conference abstracts of EULAR and ACR annual meetings were selected. For conference abstracts, the search was limited to the past 2 years.

Eligible articles studied patients with RA of 18 years and older starting MTX for the first time. All doses and administration routes of MTX were allowed. Studies in which patients additionally started another DMARD or GC were excluded; concomitant DMARDs and/ or GCs, started at least 4 weeks before initiation of MTX and kept at a stable dose, were allowed. Eligible studies assessed clinical response with a validated composite score, e.g. EULAR/ACR response, disease activity score assessing 28 joints (DAS28), clinical disease activity index (CDAI), simplified disease activity index (SDAI), or health assessment questionnaire (HAQ). Furthermore, these studies had to contain quantitative data on one or more predictors for treatment response. All predictors measured at baseline were deemed of interest.

First, titles and abstracts obtained from the systematic search were screened according to the inclusion criteria by NMTR. Articles were also included for full-text screening if there was uncertainty about fulfilling the inclusion criteria. Thereafter, the full-text versions of the included abstracts were checked for eligibility. In case of doubt, eligibility was discussed with MCvdG until consensus was reached. In case of a missing full-text version, efforts were made to contact the authors to obtain the manuscript. If this did not succeed, the abstract was excluded if fulfilling the inclusion criteria was not sure. To check for articles missed in the search/screening process, the references of all included full-text articles and of reviews were screened.

\section{Data extraction and quality assessment}

Details on study type, patients baseline characteristics, type of predictor(s) and type and time point of outcome(s) were extracted from the included articles. Quantitative information on the prognostic value (relative risk (RR), odds ratio (OR), hazard ratio (HR), beta regression coefficient $(B)$, positive predictive value (PPV), negative predictive value (NPV), sensitivity, specificity, area under the receiver operating characteristic curve (AUC$\mathrm{ROC}$ ) or calculable) was extracted from text or tables, including information on whether univariate or multivariate analyses had been performed. If, in addition to quantitative information of one predictor, non-quantitative information of other predictors was given, this information was also extracted. A predictive value that was shown in a figure without 
providing exact values was extracted only for the direction of the predictive effect. Predictors were classified as patient characteristics, disease characteristics, genetic predictors or other laboratory markers.

The quality of included articles was assessed using the Quality in Prognostic Studies (QUIPS) tool for prognostic studies. ${ }^{9}$ Six domains of risk of bias were assessed: study participation; study attribution; prognostic factor measurement; outcome measurement; study confounding; and, statistical analysis and reporting. The risk of bias for each domain was summarised as low, moderate or high.

\section{Statistical analyses}

Predictive values of studies that shared details on type of analysis and definition and time point of clinical response were pooled to perform a meta-analysis. If this was not possible due to heterogeneity between studies, associations with different binary definitions of clinical response (EULAR good response category; ACR 70 response; DAS28 <2.6; stable low disease activity) and different time points of response with an OR (or a calculable OR) as predictive association measure were summarised to graphically present the data of predictors of the multiple cohorts. All predictors that were studied in multiple cohorts were summarised in tables showing the direction of predictive effects and, if possible, whether the effects were statistically significant.

In addition, all data was extracted to tables to show all reported/calculable quantitative association measures for predictors and predictive models.

\section{Results}

\section{Study characteristics}

The systematic literature search resulted in 845 unique articles; 27 of them finally fulfilled the selection criteria and additional references screening yielded 3 additional articles, resulting in 30 included articles for data extraction (Figure 1).10-39

Study characteristics of the included articles are shown in Table 1. Patients were predominantly female, had moderate to high disease activity and were predominantly RF and ACPA positive. Disease duration, dose and strategy of MTX treatment, being naïve for DMARDs and/or GCs or not and concomitant DMARD and/or GC treatment were heterogeneous between the included studies.

The risk of bias of the included articles is summarised in Table 2. None of the included articles or abstracts had a low risk of bias for all categories. Response outcome was well defined for the majority of articles, summarised as a low risk of bias.

A total of 102 different predictors were found in the included articles: 7 regarding patient characteristics, 22 regarding disease characteristics, 50 regarding genetic predictors, 


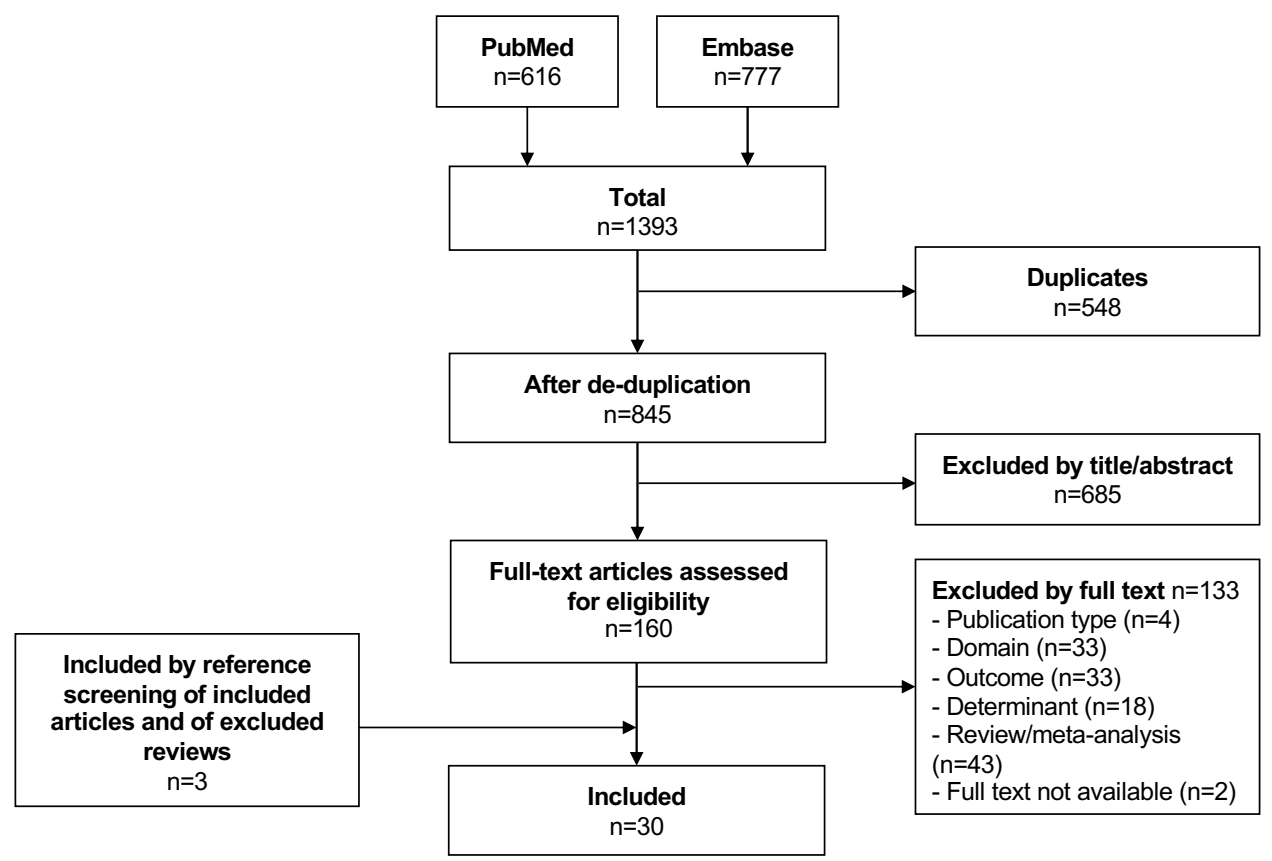

Figure 1. Flow chart of included articles n: number.

and 23 regarding other laboratory markers. In addition, 11 predictive models were found. Nineteen predictors and 2 predictive models were evaluated in multiple cohorts. The outcomes of clinical response were measured using different criteria (e.g. EULAR response categories, ACR categories, DAS28), and different cut-offs (e.g. DAS28<2.6,

Table 1. Study characteristics of included articles

\begin{tabular}{|c|c|c|c|}
\hline Study & Baseline characteristics & & \\
\hline $1^{\text {st }}$ Author, publication year & Which patients? & $\begin{array}{c}\text { Gender } \\
\text { (female), \% }\end{array}$ & $\begin{array}{c}\text { Age }(\mathrm{Y}) \\
\text { mean }(\mathrm{SD}) \$\end{array}$ \\
\hline Bugatti, $2017^{10}$ & RF and/or ACPA positive patients* & - & - \\
\hline \multirow[t]{4}{*}{ Carini, $2018^{11}$} & Cohort A: Responders* & 57 & $54(13.2)$ \\
\hline & Cohort A: Non-responders* & 67 & $58(14.5)$ \\
\hline & Cohort B: Responders* & 78 & 68 (11.3) \\
\hline & Cohort B: Non-responders* & 60 & $54(13.8)$ \\
\hline \multirow[t]{2}{*}{ Chara, $2015^{12}$} & Responders* & 72 & $52.4(10.9)$ \\
\hline & Non-responders* & 62 & $52.1(10.9)$ \\
\hline Gupta, $2018^{13}$ & All* & 86 & $39(32-49)^{ \pm}$ \\
\hline Gwinnutt, $2017^{14}$ & All* & 65 & $57(47-66)^{ \pm}$ \\
\hline Haroon, $2008^{15}$ & All* & - & $44.5(36.5-53.5)^{ \pm}$ \\
\hline
\end{tabular}


DAS28 <3.2, change in DAS). Heterogeneity of predictors, type of analysis, definitions of clinical response and time points of clinical response measurements in the included articles prohibited pooling the data in an appropriate way. All quantitative information on predictors and predictive models, including information on confounders in case of adjusted analyses, is summarised in Supplementary Tables 1 and 2.

\section{Patient and disease characteristics as predictors}

Of all 7 patient and 22 disease characteristics, 14 predictors ( 3 and 11 , respectively) were also evaluated in at least one other cohort (Supplementary Table 1). The directions of the predictive effects of replicated predictors are shown in Table 3.

Four of these replicated predictors were described in multiple articles sharing details on statistical analyses and response outcomes, allowing combining the predictive value of these predictive factors. Results of the impact of age, female gender, smoking and DAS in these articles are shown in Figure 2.

Age was investigated in 4 studies, showing conflicting non-statistically significant associations with response (Figure 2a). 17,27,29,37 One of those 4 studies, that of Saevarsdottir et $a^{29}{ }^{29}$, used multiple response outcomes for their analyses: ACR70, ACR50, ACR20, DAS28 remission, SDAl remission and CDAI remission; and ordinal outcomes for EULAR, SDAI and CDAl response categories. Neither for these response outcomes, age was predictive, except for a positive association between age and higher CDAl response category (no values given).

As shown in figure $2 \mathrm{~b}$, female gender was a statistically significant predictor of nonresponse in one of four studies OR 0.553 (95\% confidence interval (Cl) 0.323-0.943)..$^{17}$ In a study by Saevarsdottir et $a^{29}$ using multiple response outcomes, negative statistically significant associations were found with EULAR response categories: (OR 0.54 (95\% CI

DAS28, mean (SD)\$ $\begin{gathered}\text { RF/ACPA } \\ \text { (positive \%) }\end{gathered} \quad \begin{gathered}\text { Disease/symptom } \\ \text { duration, mean (SD)\$ }\end{gathered}$ $89 / 68$

$\begin{array}{cc}5.2(1.0)^{\wedge} & - \\ 5.2(0.8)^{\wedge} & - \\ 5.9(1.0)^{\wedge} & - \\ 5.9(0.9)^{\wedge} & - \\ 3.4(0.5) & 92 / 89 \\ 3.6(0.8) & 92 / 87 \\ 5.9(5.4-6.6)^{ \pm \wedge} & 76 / 81 \\ 4.3(3.3-5.2)^{ \pm \#} & 68 / 63 \\ 6.5(5.5-7.2)^{ \pm} & -\end{array}$

Medication

MTX (Start dose (mg/week)/ administration route/strategy)

$\begin{array}{lcc}- & -/-/ \text { T2T } & \text { Naïve/None } \\ - & 15 \pm 4.9 /-/- & - \\ - & 20 \pm 4.4 /-/- & \\ - & 15 \pm 5.3 /-/- & \\ - & 15 \pm 5.4 /-/- & \text { Naïve/None } \\ \text { M (9) } & 10 / \text { oral / T2T } & \text { None/Concom } \\ M(9) & & \text { Naïve/Naïve } \\ M(7-48)^{ \pm} & 15 / \text { oral / T2T } & \end{array}$


Table 1. Continued

\begin{tabular}{|c|c|c|c|}
\hline \multirow{2}{*}{$\begin{array}{l}\text { Study } \\
1^{\text {st }} \text { Author, publication year }\end{array}$} & \multicolumn{3}{|l|}{ Baseline characteristics } \\
\hline & Which patients? & $\begin{array}{c}\text { Gender } \\
\text { (female), \% }\end{array}$ & $\begin{array}{c}\text { Age }(Y) \\
\text { mean }(S D)^{\$}\end{array}$ \\
\hline \multirow[t]{2}{*}{ Jenko, $2018^{16}$} & Cohort A* & 81 & $58(45-69)^{ \pm}$ \\
\hline & Cohort B* & 73 & $56(50-64)^{ \pm}$ \\
\hline \multirow[t]{2}{*}{ Kavanaugh, $2017^{17}$} & Responders* & - & - \\
\hline & Non-responders* & - & - \\
\hline Kooloos, $2010^{18}$ & All * & 69 & $54.6(13.3)$ \\
\hline \multirow[t]{2}{*}{ Levitsky, $2015^{19}$} & Survivin (biomarker) positive* & 68 & $56.0(43.0-62.3)^{ \pm}$ \\
\hline & Survivin (biomarker) negative* & 76 & $57.0(44.0-67.0)^{ \pm}$ \\
\hline Lopez-Rodriguez, $2018^{20}$ & All* & 81 & $52.7(3.7)$ \\
\hline \multirow[t]{3}{*}{ Michelsen, $2017^{21}$} & RA patients (starting MTX or starting TNFi) & & \\
\hline & Depressed/anxious & 75 & $53.5(13.2)$ \\
\hline & Not depressed/anxious & 62 & $55.3(13.8)$ \\
\hline Michelsen, 201722 & RA patients (starting MTX or starting TNFi) & 70 & $55.0(13.5)$ \\
\hline Murosaki, $2017^{23}$ & All* & 71 & $66.0(36.0-86.0) \ddagger$ \\
\hline Norvang, $2018^{24}$ & All* & 70 & $56.7(13.2)$ \\
\hline Patro, $2016^{25}$ & All* & 86 & $40(33-50)^{ \pm}$ \\
\hline Pelletier, $2017^{26}$ & All* & - & $58(12)$ \\
\hline Ponchel, $2013^{27}$ & Validation cohort* & - & - \\
\hline Rizvi, $2018^{28}$ & All* & 67 & $62.4(42.1-72.5)^{ \pm}$ \\
\hline Saevarsdottir, $2011^{29}$ & All* & 71 & $57(44-64)^{ \pm}$ \\
\hline Saevarsdottir, $2011^{30}$ & All* & 69 & $55(45-62)^{ \pm}$ \\
\hline Safy, $2018^{31}$ & FUNCTION cohort* & 80 & $50.0(13.1)$ \\
\hline \multirow[t]{4}{*}{ Seitz, $2002^{32}$} & $\mathrm{ACR}<20^{*}$ & 92 & $50.9(13.8)$ \\
\hline & ACR 20-50* & 100 & $52.2(5.7)$ \\
\hline & ACR 50-70* & 67 & $55.9(13.4)$ \\
\hline & $\mathrm{ACR}>70 *$ & 25 & $52.9(18.5)$ \\
\hline \multirow[t]{3}{*}{ Stuhlmüller, $2016^{33}$} & EULAR good response* & 46 & $53(20-77) \ddagger$ \\
\hline & EULAR moderate response* & 85 & $58(23-74)^{\ddagger}$ \\
\hline & EULAR non-response* & 77 & $57(35-77)^{\ddagger}$ \\
\hline Tan, $2016^{34}$ & All* & 92 & - \\
\hline Velarde-de la Cruz, $2016^{35}$ & RA patients (starting MTX or other DMARD) & 90 & $46.9(12.3)$ \\
\hline Wang, $2012^{36}$ & All* & 84 & $56.4(2.8)$ \\
\hline Wessels, $2006^{37}$ & All* & 69 & $54.6(13.3)$ \\
\hline Wessels, $2006^{38}$ & All* & 69 & $54.6(13.3)$ \\
\hline \multirow[t]{2}{*}{ Wessels, $2007^{39}$} & Responders* & 60 & $55.3(14)$ \\
\hline & Non-responders* & 79 & $53.6(13)$ \\
\hline
\end{tabular}

ACR: American College of Rheumatology; ACPA: anti-citrullinated protein antibodies; Concom: concomitant; DAS28; disease activity score assessing 28 joints; DMARDs: disease-modifying antirheumatic drugs; EULAR: European League Against Rheumatism; GCs: glucocorticoids; im: intramuscular; IQR: interquartile range; M: months; mg: milligram; MTX: methotrexate; RF: rheumatoid factor; sc: subcutaneous; T2T: treat-to-target; TNFi: tumor necrosis factor inhibitor; W: weeks; Y: years. 


\section{Medication}

DAS28, mean (SD)\$ $\begin{gathered}\text { RF/ACPA } \\ \text { (positive \%) }\end{gathered} \quad \begin{gathered}\text { Disease/symptom } \\ \text { duration, mean (SD)\$ }\end{gathered}$

MTX (Start dose (mg/week)/ administration route/strategy)

\begin{tabular}{|c|c|c|c|c|}
\hline $5.2(4.4-5.9)^{ \pm}$ & $69 / 74$ & - & $20(15-20)^{ \pm} /-/-$ & Naiive/ - \\
\hline $7.5(6.8-8.2)^{ \pm}$ & $85 / 71$ & - & $12.5(10-15)^{ \pm} /-/-$ & \\
\hline $5.6^{\#}$ & - & $6 \mathrm{M}$ & 7.5 / oral / step-up vs T2T & None/Concom \\
\hline $6.2^{\#}$ & - & $6 \mathrm{M}$ & & \\
\hline $4.5(0.8)^{\dagger}$ & $67 /-$ & $2 W(0-105) \ddagger$ & 7.5 / oral / T2T & None/Concom \\
\hline $5.8(5.1-6.4)^{ \pm}$ & $81 / 66$ & $5 M(4-8)^{ \pm}$ & 20 / oral / - & Naïve/Concom \\
\hline $5.7(5.0-6.4)^{ \pm}$ & $58 / 56$ & $5 \mathrm{M}(4-9)^{ \pm}$ & & \\
\hline $5.3(1.2)^{\wedge}$ & $61 / 55$ & - & $18.7 \pm 4.6 /-/-$ & - \\
\hline $4.8(1.3)^{\wedge}$ & - & $0.3 Y(0-5)^{ \pm}$ & - & Naiive/ - \\
\hline $4.5(1.4)^{\wedge}$ & - & $0.3 Y(0-5)^{ \pm}$ & & \\
\hline $5.0(1.4)^{\wedge}$ & - & $0.7 Y(0-6)^{ \pm}$ & - & Naïve/ - \\
\hline $5.0(3.2-8.1)^{\ddagger \#}$ & $52 / 53$ & $0.3 Y(0-25)^{\ddagger}$ & 4-6 / oral / step-up & None/Concom \\
\hline $5.2(1.1)^{\wedge}$ & $59 /-$ & $0.1 Y(0-1)^{ \pm}$ & - & Naïve/Concom \\
\hline $5.93(5.4-6.5)^{ \pm \wedge}$ & $82 / 79$ & - & 10 / oral / step-up & Naiive/ Naïve \\
\hline- & - & $6 \mathrm{M}(3)$ & - & - \\
\hline- & - & - & - & Naïve/ Naïve \\
\hline $5.3(4.2-6.2)^{ \pm}$ & - & $9 M(2-11)^{ \pm}$ & - & - \\
\hline $5.7(4.9-6.3)^{ \pm \wedge}$ & $49 / 64$ & $6 \mathrm{M}(4-8)^{ \pm}$ & 10 / oral / step-up & Naïve/Concom \\
\hline $5.6(4.8-6.3)^{ \pm}$ & $67 / 65$ & OY & - & Naïve/Concom \\
\hline $6.5(1.0)^{\wedge}$ & $89 / 86$ & OY $(0.5)$ & 7.5 / oral / T2T & None/Concom \\
\hline- & $54 / 15$ & $46 \mathrm{M}(46)$ & 15 / sc or im / stable & None/Concom \\
\hline- & $67 / 0$ & $96 \mathrm{M}(5 \mathrm{l})$ & & \\
\hline- & $55 / 30$ & $82 \mathrm{M}(78)$ & & \\
\hline- & $36 / 0$ & $48 \mathrm{M}(6 \mathrm{l})$ & & \\
\hline $5.6(3.3-7.9)^{\ddagger}$ & $69 / 58$ & $1 \mathrm{M}(0-12)^{\ddagger}$ & 15 / - / stable & Naïve/ - \\
\hline $6.8(5.1-8.3) \ddagger$ & $69 / 39$ & $1 M(0-241) \ddagger$ & & \\
\hline $5.0(3.9-6.7)^{\ddagger}$ & $77 / 39$ & $1 \mathrm{M}(0-6)^{\ddagger}$ & & \\
\hline $5.0(0.9)$ & $71 / 52$ & - & 10 / oral / step-up & Naïve/None \\
\hline $6.50(1.2)$ & $26 /-$ & $2 Y(3)$ & - & Naiive/ - \\
\hline $5.9(1.4)$ & - & $5 Y(2)$ & 10 / oral / - & None/None \\
\hline $4.5(0.8)^{\dagger}$ & $67 /-$ & $2 W(0-105)^{\ddagger}$ & 7.5 / oral / T2T & None/Concom \\
\hline $4.5(0.8)^{\dagger}$ & $67 /-$ & $2 W(0-105) \ddagger$ & 7.5 / oral / T2T & None/Concom \\
\hline $4.1(0.9)^{\dagger}$ & $62 / 44$ & $24 \mathrm{~W}(13-54)^{ \pm}$ & 7.5 / oral / Т2Т & None/Concom \\
\hline $4.7(0.7)^{\dagger}$ & $74 / 52$ & $25 W(15-42)^{ \pm}$ & & \\
\hline
\end{tabular}

*: RA patients starting MTX; \$: Unless specified otherwise; -: not provided/specified; : values given as median (IQR); ;: values given as median (range); \#: DAS28 using C-reactive protein; ${ }^{\wedge}$ : DAS28 using erythrocyte sedimentation rate; t: DAS at assessment of 44 joints; : reported as being naive for DMARDs/GCs and if not, if concomitant DMARDs/ GCs are used (started at least 4 weeks before initiation of MTX and kept in a stable dose), or if no concomitant DMARDs/GCs are given (none). 
Table 2. Risk of bias of included articles

\begin{tabular}{|c|c|c|c|c|c|c|c|}
\hline \multirow{2}{*}{$\begin{array}{l}\text { Study } \\
1^{\text {st }} \text { Author }\end{array}$} & \multirow[b]{2}{*}{$\begin{array}{l}\text { Publication } \\
\text { type }\end{array}$} & \multicolumn{2}{|l|}{ Risk of bias in } & \multirow[b]{2}{*}{$\begin{array}{l}\text { Prognostic } \\
\text { factor } \\
\text { measure- } \\
\text { ment }\end{array}$} & \multirow[b]{2}{*}{$\begin{array}{l}\text { Outcome } \\
\text { measure- } \\
\text { ment }\end{array}$} & \multirow[b]{2}{*}{$\begin{array}{l}\text { Study } \\
\text { confoun- } \\
\text { ding }\end{array}$} & \multirow[b]{2}{*}{$\begin{array}{l}\text { Statistical } \\
\text { analysis } \\
\text { and } \\
\text { reporting }\end{array}$} \\
\hline & & $\begin{array}{l}\text { Study } \\
\text { participation }\end{array}$ & $\begin{array}{l}\text { Study } \\
\text { attribution }\end{array}$ & & & & \\
\hline Bugattil & Abstr & + & + & $+/-$ & - & $+/-$ & + \\
\hline Carinill & Art & $+/-$ & $+/-$ & - & - & + & - \\
\hline Chara $^{12}$ & Art & - & $+/-$ & $+/-$ & - & $+/-$ & - \\
\hline Gupta $^{13}$ & Art & - & $+1-$ & - & - & $+1-$ & - \\
\hline Gwinnutt' ${ }^{14}$ & Abstr & $+/-$ & $+/-$ & $+/-$ & $+1-$ & $+1-$ & $+1-$ \\
\hline Haroon 15 & Art & $+/-$ & $+1-$ & $+1-$ & - & - & $+1-$ \\
\hline Jenkol6 & Art & $+/-$ & $+1-$ & - & $+/-$ & $+1-$ & - \\
\hline Kavanaugh ${ }^{17}$ & Abstr & $+/-$ & $+/-$ & - & + & $+/-$ & - \\
\hline Kooloos $^{18}$ & Art & - & $+1-$ & - & - & - & $+1-$ \\
\hline Levitsky $^{19}$ & Art & - & + & $+/-$ & - & $+/-$ & + \\
\hline Lopez-Rodriguez ${ }^{20}$ & Art & + & + & - & - & $+/-$ & $+1-$ \\
\hline Michelsen ${ }^{21}$ & Art & $+/-$ & + & $+/-$ & - & + & - \\
\hline Michelsen ${ }^{22}$ & Art & $+/-$ & + & - & - & + & - \\
\hline Murosaki $^{23}$ & Art & $+/-$ & $+1-$ & $+/-$ & $+/-$ & $+1-$ & $+1-$ \\
\hline Norvang $^{24}$ & Art & - & + & + & - & + & $+1-$ \\
\hline Patro 25 & Art & - & - & - & - & $+1-$ & $+1-$ \\
\hline Pelletier ${ }^{26}$ & Abstr & + & $+1-$ & $+/-$ & - & + & + \\
\hline Ponchel ${ }^{27}$ & Art & + & $+1-$ & - & - & + & $+1-$ \\
\hline $\mathrm{Rizvi}^{28}$ & Abstr & $+1-$ & + & - & $+1-$ & $+1-$ & $+1-$ \\
\hline Saevarsdottir ${ }^{29}$ & Art & - & $+1-$ & - & - & - & $+1-$ \\
\hline Saevarsdottir ${ }^{30}$ & Art & - & $+1-$ & $+1-$ & - & $+/-$ & $+1-$ \\
\hline Safy ${ }^{31}$ & Abstr & $+/-$ & - & - & - & - & $+/-$ \\
\hline Seitz ${ }^{32}$ & Art & $+/-$ & + & $+1-$ & $+/-$ & $+/-$ & + \\
\hline Stuhlmüller ${ }^{33}$ & Art & $+/-$ & + & + & $+1-$ & $+1-$ & $+1-$ \\
\hline $\operatorname{Tan}^{34}$ & Art & $+/-$ & - & - & - & - & - \\
\hline Velarde-de la Cruz ${ }^{35}$ & Art & $+/-$ & - & - & $+/-$ & + & $+/-$ \\
\hline Wang $^{36}$ & Art & - & + & - & - & $+1-$ & $+1-$ \\
\hline Wessels ${ }^{37}$ & Art & - & $+1-$ & - & - & - & $+1-$ \\
\hline Wessels $^{38}$ & Art & - & $+/-$ & - & - & - & + \\
\hline Wessels ${ }^{39}$ & Art & - & $+/-$ & - & - & - & $+/-$ \\
\hline
\end{tabular}

Quality of included articles was assessed using the QUIPS tool for prognostic studies. ${ }^{9}$ Risk of bias: -: low; +/-: moderate; +: high; Art: article, Abstr: abstract. 
0.35-0.86), adjusted OR 0.50 (95\% Cl 0.31-0.81)) and DAS remission (no values given) as outcomes.

Current smoking was investigated in two cohorts by Saevarsdottir et al (Figure 2c).29,30 Statistically significant and non-significant associations were found within these cohorts using different clinical outcomes of response: In one cohort there was a negative association between current smoking and ACR response categories (ACR50 and

\section{A. Age per 10 years}

(1)

\section{B. Female gender}

$$
\begin{array}{r}
\text { Kavanaugh et } a^{17} \\
\text { Stable LDA, 20-26W } \\
\text { Ponchel et } a^{27} \\
\text { DAS28 }<2.6,6 \mathrm{M} \\
\text { Saevarsdottir et } a^{29} \\
\text { ACR70 response, } 3-4 \mathrm{M} \\
\text { Wessels et } a^{\beta 7} \\
\text { DAS44 } \leq 2.4,6 \mathrm{M}
\end{array}
$$

\section{ACR70 response, 3-4M Saevarsdottir et aßo EULAR good response, 3M}

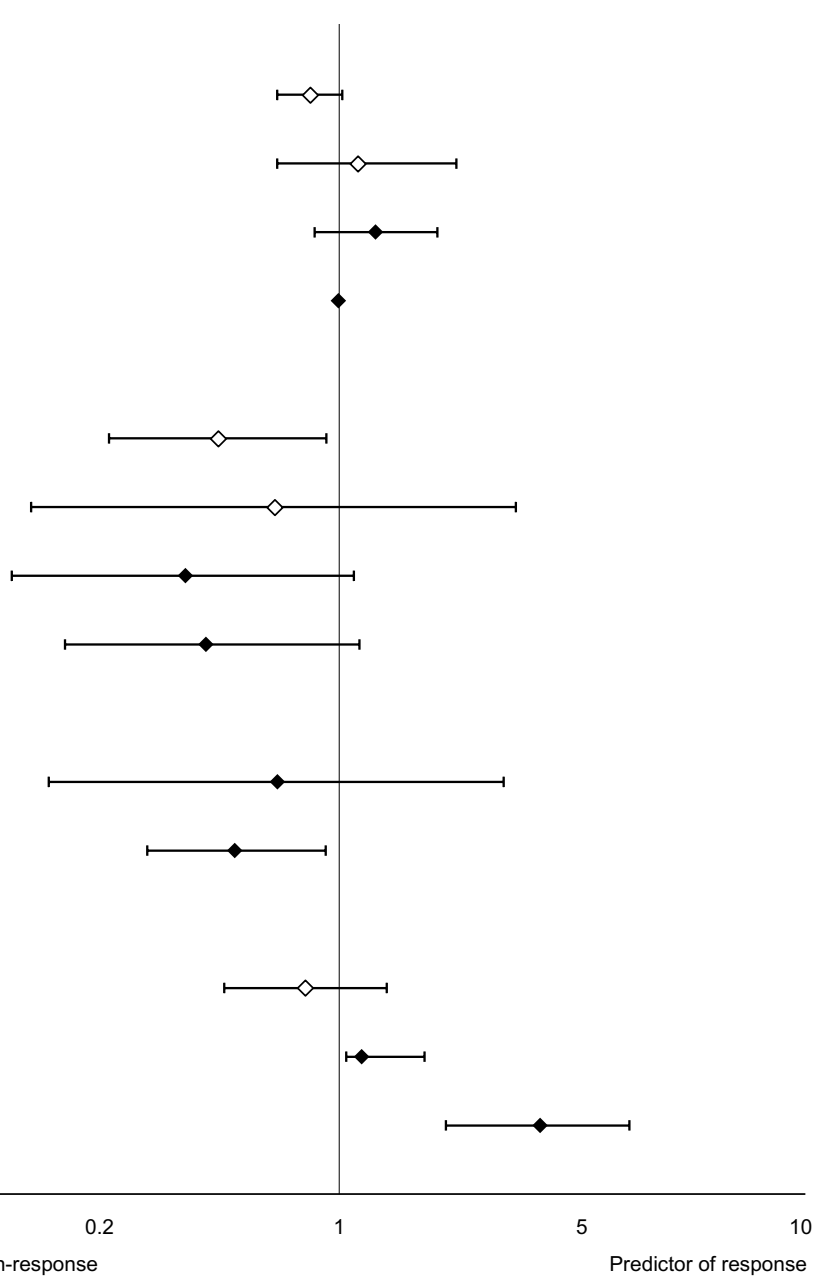

Figure 2. Associations between baseline predictors and response to methotrexate according to different binary outcome criteria and at different time points

Odds ratios are shown as diamonds: White diamonds represent univariate analyses; black diamonds multivariate analyses; and whiskers 95\% confidence intervals. Used response criteria and time points of clinical response are shown in grey type for each study.

ACR: American College of Rheumatology; DAS28: disease activity score assessing 28 joints; DAS44: disease activity score assessing 44 joints; EULAR: European League Against Rheumatism; LDA: low disease activity (definition not specified in article); M: months; W: weeks. 
ACR20: numbers not given; ACR70: adjusted OR 0.74 (95\%Cl 0.24-2.26)) and higher EULAR response category (adjusted OR 0.35 (95\% Cl 0.20-0.63)) ${ }^{29}$; In the other cohort, between current smoking with EULAR good response (adjusted OR 0.60 ( $95 \% \mathrm{Cl} 0.39-0.94)$ ) and DAS28 <2.6 (adjusted OR 0.66 (95\%Cl 0.40-1.08)). ${ }^{30}$ In addition, Wessels et al categorised patients according to smoking status and RF status. ${ }^{39} \mathrm{RF}$ positivity in combination with smoking was found to predict non-response (adjusted OR 0.1 (95\% Cl 0.0-0.4)).

Higher baseline DAS28 predicted response to MTX in a study by Tan et a ${ }^{\beta 4}$ (adjusted OR 1.12 (95\% Cl 1.04-1.53)) and higher baseline DAS44 predicted response in a study by Wessels et $a^{137}$ (adjusted OR 2.7 ( $95 \% \mathrm{Cl}$ 1.7-4.2), Figure 2d). In contrast, Saevarsdottir et $a^{29}$ found a higher baseline DAS28 to be a predictor of non-response, using EULAR response category and DAS remission as clinical outcomes (no values given). Ponchel et a ${ }^{27}$ assessed the association between DAS28 per unit as predictor and DAS28<2.6 as clinical response and found no statistically significant association (OR 0.85 (95\% Cl 0.57-1.27)).

RF positivity predicted non-response defined as DAS $<3.2$ by Bugatti et a ${ }^{10}$ (adjusted HR 0.61 (95\% Cl 0.39-0.95)), and non-response defined as DAS44 $\leq 2.4$ by Wessels et $a^{137}$ (adjusted OR 0.4 (95\% Cl 0.2-0.8)). Analyses of Bugatti et al were performed in ACPApositive RA patients; Wessels et al made no selection of RA patients. Three other studies found no statistically significant associations. ${ }^{27,29,33}$

Conflicting and/or non-statistically significant predictive values were found for other validated patient and disease characteristics: longer symptom duration, higher TJC, higher SJC, higher CRP, higher ESR, higher HAQ, presence of erosions, higher dose of MTX and ACPA positivity (Table 3, Supplementary Table 1).

\section{Genetic predictors and other predictive laboratory markers}

Out of 50 genetic predictors and 23 other laboratory markers, only 4 genetic predictors and 1 other laboratory marker were evaluated in more than one cohort (Table 3 , Supplementary Table 1).

The number of regulatory T-cells was found to be higher in responders versus nonresponders to MTX by Gupta et al ( $p<0.05$, AUC 0.66).. ${ }^{3}$ Ponchel et $a{ }^{27}$ could not replicate these findings and found no statistically significant association between regulatory T-cells and response to MTX (OR 1.05 (95\%Cl 0.85-1.31), adjusted OR 1.02 (95\% Cl 0.81-1.28)). Jenko et $a^{16}$ used a validation cohort for all associations in their study. Conflicting results were found regarding the prediction of clinical response to MTX at 6 months, defined as change in DAS28, with the genetic polymorphism adenosine monophosphate deaminase 1 (AMPDI) rsl7602729, involved in the adenosine pathway ( $\mathrm{B} 0.70$ ( $95 \% \mathrm{Cl} 0.01-$ 1.38, $\mathrm{p}$-value 0.047$)$ and $\mathrm{B}-0.75$ (95\%Cl-1.32- -0.18, $\mathrm{p}$-value 0.01$)$, respectively).

Statistically significant associations with clinical response could not be validated for gene polymorphisms coding for transporters nor for a gene polymorphism involved in the folate pathway. ${ }^{16}$ 
Table 3. Direction of predictive values of baseline predictors that were measured in multiple cohorts

\begin{tabular}{|c|c|c|c|c|c|c|}
\hline Predictor & $1^{\text {st }}$ Author & $\begin{array}{l}\text { Patients } \\
\text { (n) }\end{array}$ & $\begin{array}{l}\text { Time } \\
\text { point of } \\
\text { response }\end{array}$ & Response outcome & $\begin{array}{l}\text { Direction of } \\
\text { predictive } \\
\text { effect }\end{array}$ & $\begin{array}{l}\text { Type of } \\
\text { regression } \\
\text { analysis }\end{array}$ \\
\hline \multicolumn{7}{|c|}{ Patient characteristics } \\
\hline \multirow[t]{12}{*}{ Higher age } & Kavanaugh ${ }^{17}$ & 687 & W20-26 & Stable LDA & nss-neg & UV \\
\hline & Ponchel $^{27}$ & 50 & $6 \mathrm{M}$ & DAS28<2.6 & nss-pos & UV \\
\hline & Saevarsdottir ${ }^{29}$ & 322 & $3-4 M$ & EULAR response category & ss-pos & $U V+M V$ \\
\hline & & & & SDAI response category & nss-pos & MV \\
\hline & & & & CDAI response category & ss-pos & MV \\
\hline & & & & ACR70 response & nss-pos & MV \\
\hline & & & & ACR50 response & nss-pos & MV \\
\hline & & & & ACR20 response & nss-pos & MV \\
\hline & & & & DAS28 remission & nss-pos & MV \\
\hline & & & & SDAI remission & nss-pos & MV \\
\hline & & & & CDAl remission & nss-pos & MV \\
\hline & Wessels $^{37}$ & 186 & $6 \mathrm{M}$ & DAS44 $\leq 2.4$ & nss & MV \\
\hline \multirow[t]{12}{*}{ Female } & Kavanaugh"17 & 687 & W20-26 & Stable LDA & ss-neg & UV \\
\hline & Ponchel $^{27}$ & 50 & $6 \mathrm{M}$ & DAS28<2.6 & nss-neg & UV \\
\hline & Saevarsdottir ${ }^{29}$ & 322 & $3-4 M$ & EULAR response category & ss-neg & $U V+M V$ \\
\hline & & & & SDAI response category & nss-neg & MV \\
\hline & & & & CDAl response category & nss-neg & MV \\
\hline & & & & ACR70 response & nss-neg & MV \\
\hline & & & & ACR50 response & nss-neg & MV \\
\hline & & & & ACR20 response & nss-neg & MV \\
\hline & & & & DAS28 remission & ss-neg & MV \\
\hline & & & & SDAl remission & nss-neg & MV \\
\hline & & & & CDAl remission & nss-neg & MV \\
\hline & Wessels $^{37}$ & 186 & $6 \mathrm{M}$ & DAS44 $\leq 2.4$ & nss-neg & MV \\
\hline \multirow{11}{*}{$\begin{array}{l}\text { Current } \\
\text { smoking }\end{array}$} & Saevarsdottir ${ }^{29}$ & 322 & $3-4 M$ & EULAR response category & ss-neg & $U V+M V$ \\
\hline & & & & SDAI response category & ss-neg & MV \\
\hline & & & & CDAl response category & ss-neg & MV \\
\hline & & & & ACR70 response & nss-neg & MV \\
\hline & & & & ACR50 response & ss-neg & MV \\
\hline & & & & ACR20 response & ss-neg & MV \\
\hline & & & & DAS28 remission & nss-neg & MV \\
\hline & & & & SDAI remission & nss-neg & MV \\
\hline & & & & CDAl remission & nss-neg & MV \\
\hline & Saevarsdottir ${ }^{30}$ & 626 & $\begin{array}{l}3 \mathrm{M} \text { (range } \\
2.5-5 \mathrm{M})\end{array}$ & EULAR good response & ss-neg & MV \\
\hline & & & & DAS $<2.6$ & nss-neg & MV \\
\hline
\end{tabular}


Table 3. Continued

\begin{tabular}{|c|c|c|c|c|c|c|}
\hline Predictor & $1^{\text {st }}$ Author & $\begin{array}{l}\text { Patients } \\
(\mathrm{n})\end{array}$ & $\begin{array}{l}\text { Time } \\
\text { point of } \\
\text { response }\end{array}$ & Response outcome & $\begin{array}{l}\text { Direction of } \\
\text { predictive } \\
\text { effect }\end{array}$ & $\begin{array}{l}\text { Type of } \\
\text { regression } \\
\text { analysis }\end{array}$ \\
\hline \multicolumn{7}{|c|}{ Disease characteristics } \\
\hline \multirow{6}{*}{$\begin{array}{l}\text { Longer } \\
\text { symptom } \\
\text { duration }\end{array}$} & Ponchel $^{27}$ & 50 & $6 \mathrm{M}$ & DAS28<2.6 & nss-neg & UV \\
\hline & Saevarsdottir ${ }^{29}$ & 322 & $3-4 M$ & EULAR response category & ss-neg & $\begin{array}{l}\text { MV (nss in } \\
\text { UV) }\end{array}$ \\
\hline & & & & SDAl response category & nss-neg & MV \\
\hline & & & & CDAI response category & nss-neg & MV \\
\hline & & & & ACR20 response & nss-neg & MV \\
\hline & $\operatorname{Tan}^{34}$ & 69 & $12 \mathrm{~W}$ & EULAR good response & ss-pos & MV \\
\hline \multirow[t]{3}{*}{ Higher TJC } & Ponchel $^{27}$ & 50 & $6 \mathrm{M}$ & DAS28<2.6 & ss-neg & UV \\
\hline & $\operatorname{Rizvi}^{28}$ & - & $3 \mathrm{M}$ & $\triangle \mathrm{DAS} 28$ & nss & UV \\
\hline & $\operatorname{Rizvi}^{28}$ & - & $6 \mathrm{M}$ & $\triangle \mathrm{DAS} 28$ & ss-pos & UV \\
\hline \multirow[t]{4}{*}{ Higher SJC } & Ponchel ${ }^{27}$ & 50 & $6 \mathrm{M}$ & DAS28 $<2.6$ & nss-neg & UV \\
\hline & $\operatorname{Rizvi}^{28}$ & - & $3 \mathrm{M}$ & $\triangle \mathrm{DAS} 28$ & nss & UV \\
\hline & $\operatorname{Rizvi}^{28}$ & - & $6 \mathrm{M}$ & $\triangle \mathrm{DAS} 28$ & ss-pos & UV \\
\hline & $\operatorname{Tan}^{34}$ & 69 & $12 \mathrm{~W}$ & EULAR good response & ss-pos & MV \\
\hline \multirow[t]{4}{*}{ Higher DAS28 } & Ponchel $^{27}$ & 50 & $6 \mathrm{M}$ & DAS28<2.6 & nss-neg & UV \\
\hline & Saevarsdottir ${ }^{29}$ & 322 & $6 \mathrm{M}$ & EULAR response category & ss-neg & $U V+M V$ \\
\hline & & & & DAS28 remission & ss-neg & MV \\
\hline & $\operatorname{Tan}^{34}$ & 69 & $3-4 M$ & EULAR good response & ss-pos & MV \\
\hline Higher DAS44 & Wessels ${ }^{37}$ & 186 & $12 \mathrm{~W}$ & DAS44 $\leq 2.4$ & ss-pos & MV \\
\hline \multirow[t]{4}{*}{ Higher CRP } & Ponchel $^{27}$ & 50 & $6 \mathrm{M}$ & DAS28<2.6 & nss-neg & UV \\
\hline & $\mathrm{Rizvi}^{28}$ & - & $3 \mathrm{M}$ & $\triangle \mathrm{DAS} 28$ & nss & UV \\
\hline & $\mathrm{Rizvi}^{28}$ & - & $6 \mathrm{M}$ & $\triangle \mathrm{DAS} 28$ & nss & UV \\
\hline & $\operatorname{Tan}^{34}$ & 69 & $12 \mathrm{~W}$ & EULAR good response & nss-neg & MV \\
\hline \multirow[t]{3}{*}{ Higher ESR } & $\mathrm{Rizvi}^{28}$ & - & $3 \mathrm{M}$ & $\triangle \mathrm{DAS} 28$ & nss & UV \\
\hline & $\operatorname{Rizvi}^{28}$ & - & $6 \mathrm{M}$ & $\triangle \mathrm{DAS} 28$ & nss & UV \\
\hline & $\operatorname{Tan}^{34}$ & 69 & $12 \mathrm{~W}$ & EULAR good response & ss-pos & MV \\
\hline \multirow[t]{11}{*}{ Higher HAQ } & Kavanaugh $^{17}$ & 687 & W20-26 & Stable LDA & ss-pos & UV \\
\hline & Saevarsdottir ${ }^{29}$ & 322 & $3-4 M$ & EULAR response category & ss-neg & $U V+M V$ \\
\hline & & & & SDAl response category & nss-pos & MV \\
\hline & & & & CDAI response category & nss-neg & MV \\
\hline & & & & ACR70 response & nss & MV \\
\hline & & & & ACR50 response & nss & MV \\
\hline & & & & ACR20 response & nss-neg & MV \\
\hline & & & & DAS28 remission & ss-neg & MV \\
\hline & & & & SDAI remission & ss-neg & MV \\
\hline & & & & CDAl remission & nss-neg & MV \\
\hline & $\operatorname{Tan}^{34}$ & 69 & $12 \mathrm{~W}$ & EULAR good response & ss-neg & MV \\
\hline
\end{tabular}


Table 3. Continued

\begin{tabular}{|c|c|c|c|c|c|c|}
\hline Predictor & $1^{\text {st }}$ Author & $\begin{array}{l}\text { Patients } \\
\text { (n) }\end{array}$ & $\begin{array}{l}\text { Time } \\
\text { point of } \\
\text { response }\end{array}$ & Response outcome & $\begin{array}{l}\text { Direction of } \\
\text { predictive } \\
\text { effect }\end{array}$ & $\begin{array}{l}\text { Type of } \\
\text { regression } \\
\text { analysis }\end{array}$ \\
\hline \multirow{2}{*}{$\begin{array}{l}\text { Presence of } \\
\text { erosions }\end{array}$} & Jenko: cohort $A^{16}$ & 110 & $6 \mathrm{M}$ & $\triangle \mathrm{DAS} 28$ & ss-pos & UV \\
\hline & Jenko: cohort B ${ }^{16}$ & 133 & $6 \mathrm{M}$ & $\triangle \mathrm{DAS} 28$ & nss-pos & UV \\
\hline \multirow{2}{*}{$\begin{array}{l}\text { Higher dose } \\
\text { of MTX }\end{array}$} & Jenko: cohort $A^{16}$ & 110 & $6 \mathrm{M}$ & $\triangle \mathrm{DAS} 28$ & ss-pos & UV \\
\hline & Jenko: cohort B ${ }^{16}$ & 133 & $6 \mathrm{M}$ & $\triangle \mathrm{DAS} 28$ & ss-neg & UV \\
\hline \multirow[t]{6}{*}{ RF positive } & Bugattio & 360 & $6 \mathrm{M}$ & DAS28<3.2 & ss-neg & MV \\
\hline & Bugattil & 360 & $6 \mathrm{M}$ & DAS28<2.6 & ss-neg & MV \\
\hline & Ponchel $^{27}$ & 50 & $6 \mathrm{M}$ & DAS28<2.6 & nss-pos & UV \\
\hline & Saevarsdottir ${ }^{29}$ & 322 & $3-4 M$ & EULAR response category & nss-pos & $U V+M V$ \\
\hline & Stuhlmüller ${ }^{33}$ & 39 & $\begin{array}{l}3.7 \mathrm{M} \\
\text { (average) }\end{array}$ & EULAR good response & nss-pos & UV \\
\hline & Wessels ${ }^{37}$ & 186 & $6 \mathrm{M}$ & DAS44 $\leq 2.4$ & ss-neg & MV \\
\hline \multirow{3}{*}{$\begin{array}{l}\text { ACPA } \\
\text { positive }\end{array}$} & Ponchel $^{27}$ & 50 & $6 \mathrm{M}$ & DAS28 $<2.6$ & nss-neg & UV \\
\hline & Saevarsdottir ${ }^{29}$ & 322 & $3-4 M$ & EULAR response category & nss-pos & $U V+M V$ \\
\hline & Stuhlmüller ${ }^{33}$ & 38 & $\begin{array}{l}3.7 \mathrm{M} \\
\text { (average) }\end{array}$ & EULAR good response & nss-pos & UV \\
\hline \multicolumn{7}{|c|}{ Genetic predictors } \\
\hline \multirow{2}{*}{$\begin{array}{l}\text { SLC19Al } \\
\text { rsl051266 }\end{array}$} & Jenko: cohort $A^{16}$ & 110 & $6 \mathrm{M}$ & $\triangle \mathrm{DAS} 28$ & ss-neg & UV \\
\hline & Jenko: cohort B ${ }^{16}$ & 133 & $6 \mathrm{M}$ & $\triangle \mathrm{DAS} 28$ & nss-pos & UV \\
\hline \multirow{2}{*}{$\begin{array}{l}\text { SLOlBl } \\
\text { rsl051266 }\end{array}$} & Jenko: cohort $A^{16}$ & 110 & $6 \mathrm{M}$ & $\triangle \mathrm{DAS} 28$ & ss-pos & UV \\
\hline & Jenko: cohort B ${ }^{16}$ & 133 & $6 \mathrm{M}$ & $\triangle \mathrm{DAS} 28$ & nss-neg & UV \\
\hline \multirow{2}{*}{$\begin{array}{l}\text { TYMS } \\
\text { rs347430033 }\end{array}$} & Jenko: cohort $A^{16}$ & 110 & $6 \mathrm{M}$ & $\triangle \mathrm{DAS} 28$ & nss-pos & UV \\
\hline & Jenko: cohort B ${ }^{16}$ & 133 & $6 \mathrm{M}$ & $\triangle \mathrm{DAS} 28$ & nss-neg & UV \\
\hline \multirow{2}{*}{$\begin{array}{l}\text { AMPDI } \\
\text { rsl7602729 }\end{array}$} & Jenko: cohort $A^{16}$ & 110 & $6 \mathrm{M}$ & $\triangle \mathrm{DAS} 28$ & ss-pos & UV \\
\hline & Jenko: cohort B B & 133 & $6 M$ & $\triangle \mathrm{DAS} 28$ & ss-neg & UV \\
\hline
\end{tabular}

ACR: American College of Rheumatology; ACPA: anti-citrullinated protein antibodies; AMPDI: adenosine monophosphate deaminase l; CDAl response category: clinical disease activity index response category (no, minor, major); CRP: C-reactive protein; DAS28; disease activity score assessing 28 joints; DAS44: disease activity score assessing 44 joints; DMARDs: disease-modifying antirheumatic drugs; ESR: erythrocyte sedimentation rate; EULAR response category: European League Against Rheumatism response category (no, moderate, good); HAQ: health assessment questionnaire; LDA: low disease activity (definition not specified in article); M: months; MTX: methotrexate; MV: multivariate analysis (see Supplementary Table 2 for adjusted confounders); n: number; nss: predictor not statistically significant; nss-neg: non-statistically significant predictor of non-response; nss-pos: non-statistically significant predictor of response; RF: rheumatoid factor; SDAl response category: simplified disease activity index response category (no, minor, major); SJC: swollen joint count; SCLC19Al: solute carrier family 19 member 1; SLOIBI: solute carrier organic anion transporter family member 1Bl; ss-neg: statistically significant predictor of non-response; ss-pos: statistically significant predictor of response; TJC: tender joint count; TYMS: thymidylate synthase; UV: univariate analysis; W: weeks; $\Delta$ : change in. 


\section{Predictive models}

Different predictive models were extracted: 2 models contained patient and disease characteristics; 1 contained genetic predictors; 1 contained other laboratory markers; and 7 contained a combination of all types of predictors (Supplementary Table 2).

Two models were analysed in two cohorts. The model created by Jenko et al included both clinical characteristics and genetic predictors: DAS28 at diagnosis, MTX dose, presence of erosions and four gene polymorphisms coding for transporters and involved in the folate and adenosine pathway (SLC19A1 rs1051266, SLO1B1 rs1051266, TYMS rs347430033, AMPDI rsl7602729)..$^{16}$ This model had a PPV of 0.80 in the initial cohort and 0.82 in the validation cohort, but the NPV dropped from 0.80 in the initial cohort to 0.17 in the validation cohort. The authors explained the failed validation by differences in patients' characteristics and clinical management between the two cohorts.

The epigenetic model created by Carini et al included 5 chromosome conformation signatures, selected out of 13322 chromosomal interactions from 123 gene loci previously associated with RA." The final model contained IFNARI, IL-2IR, IL-23, IL-17A and CXCL13 loci. Sensitivity of the first models was $89.7 \%$, specificity $90.0 \%$ and AUC-ROC 0.90 , and the performance of the model in the validation cohort was $75.0 \%, 85.7 \%$ and 0.91 , respectively.

\section{Discussion}

In this extensive systematic literature review, 30 articles with a total of 102 possible predictors of clinical response to MTX treatment in RA patients were reviewed. Female gender, ${ }_{17,29}$ current smoking, ${ }^{29,30}$ and RF positivity ${ }^{10,37}$ appeared to be the most promising individual predictors of non-response. Statistically significant associations between these predictors and clinical response were found in multiple cohorts, but not in all studies. ${ }^{27,29,30,33,37}$ Seven studies combined clinical characteristics with genetic and other laboratory biomarkers in predictive models. Only one of these models was validated for clinical response at 3 to 6 months after start of MTX treatment. The NPV of this model dropped enormously in the validation cohort, challenging its general validity. ${ }^{16}$ Another validated model, including epigenetic markers but no clinical characteristics, was only validated in a small validation cohort, but might be promising to correctly identify responders and non-responders."

Current evidence on prediction of response to treatment with MTX does not show individual predictors with confirmed sufficient predictive value for use in individual patients. Promising epigenetic markers should first be combined with patient and disease characteristics to assess their additional value in daily practice. This makes that the unmet clinical need is still present. Reliable predictors could help to avoid periods with unnecessary active $\mathrm{RA}$, joint damage and might diminish the risk of patients becoming difficult-to-treat. ${ }^{40}$ 
Therefore, more studies are required that combine clinical characteristics with genetic and other laboratory predictors, and that focus on prediction of clinical response to MTX at 3 to 6 months after start of treatment. Until reliable predictors are found, other efforts should be made to optimally utilise the window of opportunity; in case of presence of prognostically unfavourable factors in RA patients, starting treatment with MTX in higher doses and/or with concomitant use of other DMARDs should be considered. ${ }^{1-3}$

To our knowledge, a systematic literature review on all types of predictors of clinical response to MTX and with a focus on baseline predictors of response at 3 to 6 months after start has not been performed before. Focussing on clinical response after 3 to 6 months is a strength of this study, because of the relevance of this period for daily practice, in line with treat-to-target strategies in current recommendations. ${ }^{12}$

Significant heterogeneity was found between studies regarding treatment regimens, type of predictors, predictive association measures used to describe the predictive effect, criteria and definitions used for clinical response and time points of clinical response. Additionally, different types of statistical analyses were performed and, in case of multivariate analyses, analyses were adjusted for different confounders. This heterogeneity warrants careful interpretation of the results and hampers drawing conclusions on the magnitude of the predictive effects. Furthermore, most of the included articles had a moderate risk of bias. These limitations should be taken into account when interpreting the results.

A former systematic review on all types of predictors for clinical response to MTX but without the specific time limit we used was performed in 2007.41 Drouin et al concluded that female, smoking, RF positive patients who have high disease activity at baseline are less likely to respond to treatment with MTX. These results are similar to ours, except for the predictive effect of high disease activity at baseline for less response to MTX. Drouin et $a$ l based their conclusion on findings by Wessels et $a^{139}$, Hoekstra et $a^{42}$ and Aletaha et $a l .{ }^{43}$ The first article was included in our review, the latter two were not because they examined clinical response at 48 weeks and 1 year, respectively. We found conflicting results regarding the predictive values of high DAS at baseline. ${ }^{27,29,34,37}$ All these latter articles were published after 2007, except for the article by Wessels et al. $^{37}$

MTX is believed to achieve its effect through different pathways of which folate antagonism and increasing adenosine signalling are thought to be the most important. ${ }^{44}$ Surprisingly, we did not find any reliable predictors of clinical response that were related to either the folate or adenosine pathway. In previous studies, the predictive value of baseline levels of erythrocyte folate has been assessed, both as an individual predictor and as part of a predictive model. ${ }^{45,46}$ These two studies were not included in our systematic literature review, as more than one-half of the study participants started other conventional synthetic DMARDs in addition to MTX. Starting additional DMARDs would have prohibited to evaluate the clinical response to MTX only and was therefore an exclusion criterion in 
our review.

In conclusion, female gender, current smoking and/or RF positivity appeared to be weak individual predictors of non-response to MTX. For the individual patient, no reliable predictors of clinical response to MTX at 3 to 6 months after treatment initiation were found. Future studies on prediction of response to MTX should focus on combining clinical characteristics with genetic and other laboratory biomarkers within this time span, to get closer to personalised medicine. 


\section{References}

1. Smolen JS, Landewé R, Bijlsma J, et al. EULAR recommendations for the management of rheumatoid arthritis with synthetic and biological disease-modifying antirheumatic drugs: 2016 update. Ann Rheum Dis 2017;76:96077.

2. Singh JA, Saag KG, Bridges SL, et al. 2015 American College of Rheumatology Guideline for the Treatment of Rheumatoid Arthritis. Arthritis Rheumatol 2016;68:1-26.

3. Vastesaeger $\mathrm{N}, \mathrm{Xu} \mathrm{S}$, Aletaha $\mathrm{D}$, et al. A pilot risk model for the prediction of rapid radiographic progression in rheumatoid arthritis. Rheumatology 2009;48:1114-21.

4. Smolen JS, Aletaha D. Rheumatoid arthritis therapy reappraisal: strategies, opportunities and challenges. Nat Rev Rheumatol 2015;11:27689.

5. Bakker MF, Jacobs JWG, Welsing PMJ, et al. Lowdose prednisone inclusion in a methotrexatebased, tight control strategy for early rheumatoid arthritis: A randomized trial. Ann Intern Med 2012;156:329-39.

6. Goekoop-Ruiterman YPM, De Vries-Bouwstra $\mathrm{JK}$, Allaart CF, et al. Clinical and radiographic outcomes of four different treatment strategies in patients with early rheumatoid arthritis (the best study): A randomized, controlled trial. Arthritis Rheum 2005;52:3381-90.

7. Boers $M$, Verhoeven AC, Markusse $H M$, et al. Randomised comparison of combined step-down prednisolone, methotrexate and sulphasalazine with sulphasalazine alone in early rheumatoid arthritis. Lancet 1997;350:30918.

8. Bijlsma JWJ, Welsing PMJ, Woodworth TG, et al. Early rheumatoid arthritis treated with tocilizumab, methotrexate, or their combination (U-Act-Early): a multicentre, randomised, double-blind, double-dummy, strategy trial. Lancet 2016;388:343-55.

9. Hayden JA, van der Windt DA, Cartwright JL, et al. Assessing bias in studies of prognostic factors. Ann. Intern. Med. 2013;158:280-6.

10. Bugatti S, Manzo A, Zanframundo $G$, et al. SAT0060 Response to conventional synthetic dmards differs depending on rheumatoid factor levels in anti-citrullinated positive patients with early rheumatoid arthritis. Ann Rheum Dis 2017;76:790-1.

11. Carini C, Hunter E, Ramadass AS, et al. Chromosome conformation signatures define predictive markers of inadequate response to methotrexate in early rheumatoid arthritis. $J$ Transl Med 2018;16:1-11.

12. Chara L, Sánchez-Atrio $A$, Pérez $A$, et al. The number of circulating monocytes as biomarkers of the clinical response to methotrexate in untreated patients with rheumatoid arthritis. J Transl Med 2015;13:1-10.

13. Gupta V, Katiyar S, Singh A, et al. CD39 positive regulatory $\mathrm{T}$ cell frequency as a biomarker of treatment response to methotrexate in rheumatoid arthritis. Int $\mathrm{J}$ Rheum Dis 2018;21:1548-56.

14. Gwinnutt JM, Hyrich $K L$, Lunt $M$, et al. Poor prognostic factors at the start of methotrexate therapy are not associated with worse treatment response: Results from the rheumatoid arthritis medication study. Arthritis Rheumatol 2017;69:75-7.

15. Haroon N, Srivastava R, Misra R, et al. A novel predictor of clinical response to methotrexate in patients with rheumatoid arthritis: A pilot study of in vitro T cell cytokine suppression. J Rheumatol 2008;35:975-8.

16. Jenko $B$, Tomšič $M$, Jekić $B$, et al. Clinical pharmacogenetic models of treatment response to methotrexate monotherapy in Slovenian and Serbian rheumatoid arthritis patients: Differences in patient's management may preclude generalization of the models. Front Pharmacol 2018;9:1-8.

17. Kavanaugh A, Vollenhoven RF van, Wolfe BA, et al. SAT0142 Predictors of inadequate response and rapid radiographic progression in patients with early rheumatoid arthritis receiving methotrexate: a post hoc analysis of 2 randomized, controlled trials of adalimumab. Ann Rheum Dis 2017;76:822-3.

18. Kooloos WM, Wessels JA., Straaten $T$ van der, et al. Functional polymorphisms and methotrexate treat- ment outcome in recent-onset rheumatoid arthritis. Pharmacogenomics 2010;11:399-404.

19. Levitsky $A$, Erlandsson MC, van Vollenhoven $\mathrm{RF}$, et al. Serum survivin predicts responses to treatment in active rheumatoid arthritis: A post hoc analysis from the SWEFOT trial. BMC Med 2015;13:1-10.

20. López-Rodríguez R, Ferreiro-Iglesias A, Lima $A$, et al. Replication study of polymorphisms associated with response to methotrexate in patients with rheumatoid arthritis. Sci Rep 2018;8:1-8.

21. Michelsen B, Kristianslund EK, Sexton J, et al. Do depression and anxiety reduce the likelihood of remission in rheumatoid arthritis and 
psoriatic arthritis? Data from the prospective multicentre NOR-DMARD study. Ann Rheum Dis 2017;76:1906-10.

22. Michelsen B, Kristianslund EK, Hammer $\mathrm{HB}$, et al. Discordance between tender and swollen joint count as well as patient's and evaluator's global assessment may reduce likelihood of remission in patients with rheumatoid arthritis and psoriatic arthritis: Data from the prospective multicentre NOR-DMARD study. Ann Rheum Dis 2017;76:708-11.

23. Murosaki T, Nagatani K, Sato T, et al. Prediction of the therapeutic response to methotrexate at 24 weeks by methotrexate-polyglutamates concentration in erythrocytes at 8 weeks in patients with rheumatoid arthritis. Mod Rheumatol 2017;27:411-6.

24. Norvang $V$, Sexton J, Kristianslund EK, et al. Predicting achievement of the treatment targets at 6 months from 3-month response levels in rheumatoid arthritis: data from reallife follow-up in the NOR-DMARD study. RMD Open 2018;4:e000773.

25. Patro PS, Singh A, Misra R, et al. Myeloid-related protein 8/14 levels in rheumatoid arthritis: Marker of disease activity and response to methotrexate. J Rheumatol 2016;43:731-7.

26. Pelletier $M$, Fortin $P R$, Longchamps $M-P$, et al. Plasma cytokines at diagnosis may predict non-response to methotrexate in patients with early rheumatoid arthritis. Arthritis Rheumatol 2017;69:3-5.

27. Ponchel F, Goëb V, Parmar R, et al. An immunological biomarker to predict MTX response in early RA. Ann Rheum Dis 2014;73:2047-53.

28. Rizvi S, Bukhari M. AB0267 Baseline predictors of response to methotrexate in early rheumatoid arthritis. Ann Rheum Dis 2018;77:1314.

29. Saevarsdottir $S$, Wallin $H$, Seddighzadeh $M$, et al. Predictors of response to methotrexate in early DMARD naive rheumatoid arthritis: results from the initial open-label phase of the SWEFOT trial. Ann Rheum Dis 2011;70:469-75.

30. Saevarsdottir S, Wedrén S, Seddighzadeh M, et al. Patients with early rheumatoid arthritis who smoke are less likely to respond to treatment with methotrexate and tumor necrosis factor inhibitors: Observations from the epidemiological investigation of rheumatoid arthritis and the Swedish Rheumatology Reg. Arthritis Rheum 2011;63:26-36.

31. Safy $M$, Jacobs JWG, Edwardes $M$, et al. SAT0169 No evidence that concomitant glucocorticoid therapy affects efficacy and safety of tocilizumab monotherapy in rheumatoid arthritis clinical trials. Ann Rheum Dis 2018;77:945.

32. Seitz $M$, Zwicker $M$, Villiger PM. Pretreatment cytokine profiles of peripheral blood mononuclear cells and serum from patients with rheumatoid arthritis in different American College of Rheumatology response groups to methotrexate. J Rheumatol 2003;30:28-35.

33. Stuhlmüller B, Mans K, Tandon N, et al. Genomic stratification by expression of HLA-DRB4 alleles identifies differential innate and adaptive immune transcriptional patterns - A strategy to detect predictors of methotrexate response in early rheumatoid arthritis. Clin Immunol 2016;171:50-61.

34. Tan W, Wang F, Guo D, et al. High serum level of haptoglobin is associated with the response of 12 weeks methotrexate therapy in recent-onset rheumatoid arthritis patients. Int J Rheum Dis 2016;19:482-9.

35. Velarde-de la Cruz EE, Sánchez-Hernández PE, Muñoz-Valle JF, et al. KIR2DL2 and KIR2DS2 as genetic markers to the methotrexate response in rheumatoid arthritis patients. Immunopharmacol Immunotoxicol 2016;38:303-9.

36. Wang Z, Chen Z, Yang S, et al. IH NMR-based metabolomic analysis for identifying serum biomarkers to evaluate methotrexate treatment in patients with early rheumatoid arthritis. Exp Ther Med 2012;4:165-71.

37. Wessels JAM, Kooloos WM, De Jonge $R$, et al. Relationship between genetic variants in the adenosine pathway and outcome of methotrexate treatment in patients with recent-onset rheumatoid arthritis. Arthritis Rheum 2006;54:2830-9.

38. Wessels JAM, De Vries-Bouwstra JK, Heijmans $B T$, et al. Efficacy and toxicity of methotrexate in early rheumatoid arthritis are associated with single-nucleotide polymorphisms in genes coding for folate pathway enzymes. Arthritis Rheum 2006;54:1087-95.

39. Wessels JAM, Van Der Kooij SM, Le Cessie S, et al. A clinical pharmacogenetic model to predict the efficacy of methotrexate monotherapy in recent-onset rheumatoid arthritis. Arthritis Rheum 2007;56:1765-75.

40. de Hair MJH, Jacobs JWG, Schoneveld JLM, et al. Difficult-to-treat rheumatoid arthritis: an area of unmet clinical need. Rheumatology (Oxford) 2017;57:1135-44.

41. Drouin J, Haraoui B. Predictors of clinical response and radiographic progression in 
patients with rheumatoid arthritis treated with methotrexate monotherapy. J Rheumatol 2010;37:1405-10.

42. Hoekstra $M$, van Ede $A E$, Haagsma $C J$, et al. Factors associated with toxicity, final dose, and efficacy of methotrexate in patients with rheumatoid arthritis. Ann Rheum Dis
2003;62:423-6.

43. Aletaha D, Funovits J, Keystone EC, et al. Disease activity early in the course of treatment predicts response to therapy after one year in rheumatoid arthritis patients. Arthritis Rheum 2007;56:3226-35. 


\section{Supplementary}

\section{Search strategy}

Pubmed

((RA[Title/Abstract] OR "rheumatoid arthritis"[Title/Abstract] OR "arthritis, rheumatoid"[Mesh]) AND (Methotrexate[tiab] OR MTX[tiab] OR "Methotrexate"[Mesh])) AND ((Predict*[tiab] OR Prognost*[tiab]) AND (Respons*[tiab] OR Remission[tiab] OR Efficacy[tiab] OR Effective[tiab] OR Effectiveness OR "Disease activity"[tiab] OR ACR20[tiab] OR "ACR 20"[tiab] OR ACR-50[tiab] OR "ACR 50"[tiab] OR ACR-70[tiab] OR "ACR 70"[tiab] OR DAS[tiab] OR DAS28[tiab] OR DAS44[tiab] OR CDAI[tiab] OR SDAI[tiab] OR HAQ[tiab] OR "health assessment questionnaire"[tiab]))

\section{Embase}

((RA OR 'rheumatoid arthritis' OR 'arthritis, rheumatoid'):ab,ti,kw AND (Methotrexate OR MTX):ab,ti,kw) AND (Predict* OR Prognost*):ab,ti,kw AND (Respons* OR Remission OR efficacy OR effective OR effectiveness OR 'disease activity' OR ACR-20 OR 'ACR 20' OR ACR-50 OR 'ACR 50' OR ACR-70 OR 'ACR 70' OR DAS OR DAS28 OR DAS44 OR CDAI OR SDAI OR HAQ OR 'health assessment'):ab,ti,kw

\section{Supplementary Tables}

Supplementary Table 1 'Quantitative information on predictors' and Supplementary Table 2 'Quantitative information on predictive models' can be accessed via: https://doi.org/10.1016/j.jbspin.2019.04.002. 



\section{CHAPTER 3}

The multi-biomarker disease activity score tracks response to rituximab treatment in rheumatoid arthritis patients: a post hoc analysis of three cohort studies

Arthritis Res Ther 2018;20:256.

Nadia M.T. Roodenrijs, Maria J.H. de Hair, Gill Wheater, Mohsen Elshahaly, Janneke Tekstra, Y.K. Onno Teng, Floris P.J.G. Lafeber, Ching Chang Hwang, Xinyu Liu, Eric H. Sasso, Jacob M. van Laar 


\section{Abstract}

\section{Objectives}

A multi-biomarker disease activity (MBDA) score has been validated as an objective measure of disease activity in rheumatoid arthritis (RA) and shown to track response to treatment with several disease-modifying antirheumatic drugs (DMARDs). The objective of this study was to evaluate the ability of the MBDA score to track response to treatment with rituximab.

\section{Methods}

Data were used from 57 RA patients from 3 cohorts treated with rituximab $1000 \mathrm{mg}$ and methylprednisolone $100 \mathrm{mg}$ at days 1 and 15. The MBDA score was assessed in serum samples obtained at baseline (BL) and 6 months. Spearman's rank correlation coefficients were calculated for BL values, 6-month values, and change from BL to 6 months $(\triangle)$, between MBDA score and the following measures: disease activity score assessing 28 joints (DAS28) using erythrocyte sedimentation rate (ESR) or high-sensitivity C-reactive protein (hsCRP), ESR, (hs)CRP, 28 swollen and tender joint counts (SJC28, TJC28), patient visual analogue scale for general health (VAS-GH), health assessment questionnaire (HAQ) and radiographic progression over 12 months using Sharp/van der Heijde score (SHS), as well as six bone turnover markers. Additionally, multivariable linear regression analyses were performed using these measures as dependent variable and the MBDA score as independent variable, with adjustment for relevant confounders. The association between $\triangle M B D A$ score and European League Against Rheumatism (EULAR) response at 6 months was assessed with adjustment for relevant confounders.

\section{Results}

At BL, the median MBDA score and DAS28-ESR were 54.0 (IQR 44.3-70.0) and 6.3 (IQR 5.47.1), respectively. MBDA scores correlated significantly with DAS28-ESR, DAS28-hSCRP, ESR and (hs)CRP at BL and 6 months. $\triangle M B D A$ score correlated significantly with changes in these measures. $\triangle M B D A$ score was associated with EULAR good or moderate response (adjusted OR 0.89, 95\% Cl 0.81-0.98, $\mathrm{p}=0.02$ ). Neither BL MBDA score nor $\triangle \mathrm{MBDA}$ score correlated statistically significantly with $\triangle \mathrm{SHS}(\mathrm{n}=11)$ or change in bone turnover markers ( $n=23)$, although $\triangle S H S \geq 5$ was observed in 5 (56\%) of nine patients with high MBDA scores.

\section{Conclusions}

We have shown, for the first time, that the MBDA score tracked disease activity in RA patients treated with rituximab and that change in MBDA score reflected the degree of treatment response. 


\section{Introduction}

Rheumatoid arthritis (RA) is the most common, chronic inflammatory joint disease, characterised by synovitis, joint damage and systemic immune and inflammatory manifestations. Achieving remission or low disease activity is the main treatment goal in order to prevent joint damage and disability! The European League Against Rheumatism (EULAR) and the American College of Rheumatology (ACR) recommend regular assessment of the level of disease activity., ${ }^{2,3}$ The disease activity score assessing 28 joints (DAS28) is one of the most frequently used composite scores for the assessment of disease activity in clinical studies of RA. ${ }^{4}$ However, the DAS28 has shortcomings that hamper its use in clinical practice. ${ }^{5}$ It does not include the ankles or feet, whereas these are common sites of inflammation in RA. Moreover, the DAS28 contains subjective components, making it highly variable between and within assessors and unreliable at the patient level. In addition, RA inflammation can be extra-articular, which is not readily detected by the DAS28. Thus, there is a need for an objective measure that reflects systemic disease activity and is sensitive to change. It would be of additional benefit if that measure could be used to predict radiographic progression.

The multi-biomarker disease activity (MBDA) score is based on biochemical markers only. It is thus more objective than the DAS28 and may potentially be a better indicator of systemic inflammation. The MBDA score is calculated with an algorithm that uses the concentrations of 12 serum protein biomarkers to produce a score, on a scale of 1 to 100 , that represents the level of disease activity in patients with RA. ${ }^{6}$ The MBDA score has been validated based on its correlation with DAS28 using C-reactive protein (CRP) and other clinical disease activity measures. ${ }^{78}$ The clinical validation of the MBDA score is supported by evidence that the MBDA score is a stronger predictor of radiographic progression than DAS28-CRP, and that it predicts radiographic progression when it is discordant with DAS28-CRP (e.g. when DAS28 is low and MBDA score is high). ${ }^{9}$

The MBDA score tracks response to a variety of disease-modifying antirheumatic drugs (DMARDs), including methotrexate ${ }^{10}$ (with and without prednisone"), tumour necrosis factor (TNF) inhibitors, ${ }^{12-14}$ abatacept ${ }^{13-15}$ and the Janus Kinase (JAK) inhibitor tofacitinib'16. The MBDA score has not yet been assessed in patients treated with rituximab.

Rituximab is an anti-CD20 monoclonal antibody. CD20 is expressed by pre-B and mature B-cells, which produce a number of pro-inflammatory cytokines, such as interleukin-6 (IL-6) and TNF. By depleting CD20+ B-cells, rituximab treatment leads to a decrease in these pro-inflammatory cytokines, ${ }^{17,18}$ thereby reducing clinical disease activity. IL-6 and TNF are 2 of the 12 biomarkers of the MBDA score. It is not known if the clinical response to rituximab is paralleled by changes in the biomarker profile of the MBDA score.

The purpose of the current study was to assess the ability of the MBDA score to measure disease activity upon and track response to treatment with rituximab and, if 
so, if this would be mainly explained by the objective component of the DAS28 (acute phase reactants). Furthermore, we investigated the ability of the MBDA score to predict radiographic progression and change in serum bone turnover markers upon rituximab treatment.

\section{Methods}

\section{Study population and treatment protocol}

We used data from three prospective cohort studies in which adult, refractory RA patients were treated with rituximab because of active disease despite conventional treatment (e.g. a combination of DMARDs, including maximum tolerable doses of a conventional synthetic (cs)DMARD and/or TNF inhibitor): one cohort from the Leiden University Medical Center (LUMC) ${ }^{19}$ and one from the University Medical Center (UMC) Utrecht, ${ }^{20}$ both in the Netherlands, and the HORUS cohort in the United Kingdom ${ }^{21}$. All patients with available serum samples were selected from the cohorts. Patients received rituximab $1000 \mathrm{mg}$ intravenously on days 1 and 15, after an infusion with intravenous methylprednisolone $100 \mathrm{mg}$. Patients were followed for at least one year from baseline. For the current study we used disease activity data from the first 6 months following rituximab infusion, to avoid potentially confounding effects from repeat rituximab infusions in some patients.

\section{Clinical assessments and serum samples}

Demographics, disease duration, smoking status (no or yes) and serum status for rheumatoid factor (RF) and for autoantibodies against citrullinated peptides (ACPA) were assessed at baseline. Swollen and tender joint counts assessing 28 joints (SJC28, TJC28), patient visual analogue scale (VAS) for general health (GH) and health assessment questionnaire (HAQ) were obtained for patients at baseline and 6 months, as were erythrocyte sedimentation rate (ESR), CRP and high-sensitivity (hs)CRP (the latter only in HORUS). The DAS28 was calculated using both ESR and hsCRP. EULAR response at 6 months was determined using DAS28-ESR. ${ }^{22}$ Radiographs of hands and feet were obtained at baseline and at 12 months (UMC Utrecht cohort) and radiographic progression was assessed using the Sharp/van der Heijde score (SHS) by one reader. Clinically important radiographic progression was defined as $\triangle S H S \geq 5 .{ }^{23}$ In the HORUS cohort serum bone formation markers (BAP (bone-specific alkaline phosphatase), PINP (procollagen type l amino-terminal propeptide), DKK1 (Dickkopf-1), sclerostin) and bone resorption markers (TRAP5b (tartrate-resistant acid phosphatase isoenzyme 5b), BCTX (Beta-isomerised carboxy terminal telopeptide of type I collagen)) were determined at baseline and at 6 months (Supplementary). 


\section{Determination of the MBDA score}

Serum samples were collected at baseline in all three cohorts, and at 6 months in the UMC Utrecht and HORUS cohorts. Samples were shipped frozen to Crescendo Bioscience, Inc. (South San Francisco, CA, USA) for measurement of the 12 MBDA biomarkers. The biomarkers represent inflammatory and destructive processes: vascular cell adhesion molecule-1 (VCAM-1), epidermal growth factor (EGF), vascular endothelial growth factor A (VEGF-A), IL-6, TNF receptor type 1 (TNF-RI), matrix metalloproteinase 1 (MMP1), MMP-3, human cartilage glycoprotein-39 (YKL-40), leptin, resistin, serum amyloid $A$ (SAA) and CRP. The MBDA biomarkers were measured by electrochemiluminescencebased multiplexed sandwich immunoassays (Meso Scale Discovery, Rockville, MD, USA) using the same types of reagents and instrument and the same algorithm as described previously. 6,7

\section{Statistical analyses}

Baseline characteristics were assessed using descriptive statistics. Differences between the 3 cohorts were analysed using one-way Analysis of Variance, Kruskal-Wallis Test or Chi-square test, as appropriate.

Spearman's rank correlations $(r)$ were analysed for values at baseline, at 6 months and for change from baseline to 6 months $(\triangle)$ between MBDA score and the following measures: DAS28-ESR, DAS28-hsCRP, ESR, CRP, hsCRP, SJC28, TJC28, VAS-GH, HAQ, SHS (UMC Utrecht cohort), bone turnover markers (HORUS cohort). Multivariable linear regression analyses were performed using these measures as dependent variable and the MBDA score as independent variable, with adjustment by age, gender, smoking status (no or yes), RF status, ACPA status, and cohort. Bone turnover markers were additionally adjusted for menopausal status (pre-menopausal or post-menopausal).24 Logistic regression analysis was performed to assess the association between baseline MBDA score or $\triangle M B D A$ score and EULAR response (good or moderate) at 6 months, with adjustment by the same covariates.

Two-sided p-values $<0.05$ were considered statistically significant. All statistical analyses were performed using IBM SPSS Statistics 21 software.

\section{Results}

\section{Patient characteristics at baseline}

Baseline characteristics were generally typical of those for patients with established RA starting rituximab treatment and were mostly similar between the three cohorts. SJC28, ESR, CRP and HAQ were statistically significantly different between the three cohorts (Table 1). Overall, $90 \%$ and $80 \%$ of patients were seropositive for RF or ACPA, respectively. 


\section{MBDA score and DAS28 at baseline and 6 months}

At baseline the median MBDA score was 54 (interquartile range (IQR) 44-70, n=52), with high (>44), moderate $(30-44)$ or low $(<30)^{7}$ scores observed in $40(77 \%), 7(13 \%)$ and 5 (10\%) patients, respectively. At 6 months the median MBDA score was 51 (IQR 39-58, $\mathrm{n}=42)$, with high, moderate or low scores observed in $26(62 \%), 11(26 \%)$ and 5 patients (12\%), respectively. The median $\triangle M B D A$ score was -7 (IQR -19-3, $n=42)$.

At baseline and at 6 months, the median values for DAS28-ESR were 6.3 (IQR 5.4-7.1, $n=51$ ) and 5.0 (IQR 4.2-6.2, $\mathrm{n}=45$ ), respectively, and the median $\triangle \mathrm{DAS} 28-\mathrm{ESR}$ was -1.0 (IQR - 2.0$-0.1, \mathrm{n}=42$ ). At baseline and at 6 months, the median values for DAS28-hsCRP were 5.8

Table 1. Patient characteristics at baseline

\begin{tabular}{|c|c|c|c|c|c|}
\hline & $\begin{array}{c}\text { All, } \\
\mathrm{n}=57\end{array}$ & $\begin{array}{c}\text { HORUS, } \\
n=26\end{array}$ & $\begin{array}{l}\text { UMC Utrecht, } \\
n=20\end{array}$ & $\begin{array}{c}\text { LUMC, } \\
\mathrm{n}=11\end{array}$ & $\begin{array}{c}\mathrm{p}- \\
\text { value }\end{array}$ \\
\hline Female, $\mathrm{n}(\%)$ & $41(72)$ & $22(85)$ & $12(60)$ & $7(64)$ & $0.15^{1}$ \\
\hline Age in years, mean (SD) & $56.6(11.2)$ & $59.3(10.8)$ & $56.7(11.6)$ & $50.1(9.5)$ & $0.07^{2}$ \\
\hline $\begin{array}{l}\text { Disease duration in years, median } \\
\text { (IQR) }\end{array}$ & $\begin{array}{l}11.5(6.3- \\
16.4)\end{array}$ & $9.9(4.1-14.4)$ & $13.4(8.4-17.6)$ & $13.0(5.2-15.5)$ & $0.46^{3}$ \\
\hline Current smoking, number (\%) & $20(35)$ & $10(38)$ & $8(40)$ & $2(18)$ & $0.42^{1}$ \\
\hline RF positive, number (\%) & $51(90)$ & $23(89)$ & $19(95)$ & $9(82)$ & $0.51^{1}$ \\
\hline ACPA positive, number (\%) & $44(80)$ & $19(79), n=24$ & $17(85)$ & $8(73)$ & 0.71 \\
\hline \multicolumn{6}{|l|}{ Menopausal status, females (\%) } \\
\hline Pre-menopausal & $14(25)$ & $6(23)$ & $5(25)$ & $3(27)$ & $0.30^{1}$ \\
\hline Post-menopausal & $27(47)$ & $16(62)$ & $7(35)$ & $4(36)$ & \\
\hline SJC28, median (IQR) & $9(4-16)$ & $9(4-15)$ & $12(8-19), n=19$ & $4(1-10), n=8$ & $0.02^{3}$ \\
\hline TJC28, median (IQR) & $15(10-23)$ & $16(11-25)$ & $14(8-17), n=19$ & $13(5-24), n=8$ & $0.35^{3}$ \\
\hline $\begin{array}{l}\text { VAS-GH, 0-100 mm (worst), } \\
\text { median (IQR) }\end{array}$ & $64(45-73)$ & $69(40-78)$ & $\begin{array}{c}57(46-69) \\
n=19\end{array}$ & $65(53-84), n=8$ & $0.36^{3}$ \\
\hline ESR, mm/h, median (IQR) & $37(21-51)$ & $32(12-41), n=24$ & $52(21-91), n=18$ & $32(29-44), n=7$ & $0.02^{3}$ \\
\hline CRP, mg/L, median (IQR) & $15(6-34)$ & $11(5-25), n=25$ & $29(11-50), n=18$ & $13(5-56), n=5$ & $0.02^{3}$ \\
\hline hsCRP, mg/L, median (IQR) & NA & $10(3-26)$ & NA & NA & NA \\
\hline DAS28-ESR, median (IQR) & $6.3(5.4-7.1)$ & $\begin{array}{l}6.2(5.0-7.2) \\
n=25\end{array}$ & $\begin{array}{c}6.6(5.8-7.1) \\
n=18\end{array}$ & $6.1(3.8-7.3), n=8$ & $0.64^{3}$ \\
\hline DAS28-hsCRP, median (IQR) & NA & $5.8(4.6-6.8)$ & NA & NA & NA \\
\hline MBDA score, median (IQR) & $54(44-70)$ & $51(44-67), n=25$ & $64(49-74)$ & $55(34-71), n=7$ & $0.15^{3}$ \\
\hline HAQ, median (IQR) & $1.8(1.4-2.1)$ & $1.9(1.7-2.1)$ & $1.5(1.1-1.9), n=11$ & $1.3(1.3-1.9), n=7$ & $0.02^{3}$ \\
\hline SHS, median (IQR) & $44(24-128)$ & NA & $61(29-142), n=19$ & $25(21-94), n=8$ & $0.34^{3}$ \\
\hline
\end{tabular}

ACPA: anti-citrullinated protein antibodies; CRP: C-reactive protein; DAS28: disease activity score assessing 28 joints; ESR: erythrocyte sedimentation rate; HAQ: health assessment questionnaire; hsCRP: high-sensitivity CRP; IQR: interquartile range; MBDA: multi-biomarker disease activity; mg/L: milligram/litre; mm/h: millimetre/hour; NA: not applicable; RF: rheumatoid factor; SD: standard deviation; SHS: Sharp/van der Heijde score; SJC28: swollen joint count assessing 28 joints; TJC28: tender joint count assessing 28 joints; VAS-GH: patient visual analogue scale for general health.

1. Differences between cohorts were analysed using Chi-Square Test; 2 . Differences between cohorts were analysed using one-way Analysis of Variance; 3. Differences between cohorts were analysed using Kruskal-Wallis Test. 
(IQR 4.6-6.8, $\mathrm{n}=26$ ) and 4.7 (IQR 3.8-6.2, $\mathrm{n}=26$ ), respectively, and the median $\triangle \mathrm{DAS} 28-$ hsCRP was -0.9 (IQR -1.6-0.1, n=26).

\section{Correlation between MBDA score and disease activity measures}

Correlations between MBDA score and DAS28 and their changes over time are shown in Figure 1. A significant Spearman's correlation was found between MBDA score and DAS28-ESR at baseline $(r=0.52, p<0.01)$ and at 6 months $(r=0.49, p<0.01)$. $\triangle M B D A$ score from baseline to 6 months was significantly correlated with $\triangle \mathrm{DAS} 28-E S R(r=0.60, p<0.01)$.

Similarly, the MBDA score was significantly correlated with DAS28-hsCRP at baseline $(r=0.51, p<0.01)$ and at 6 months $(r=0.45, p=0.03) . \triangle M B D A$ score from baseline to 6 months was significantly correlated with $\triangle \mathrm{DAS} 28-\mathrm{hsCRP}(r=0.48, \mathrm{p}=0.02)$.

MBDA score was significantly correlated with ESR, hSCRP and CRP, as was also true for their changes from baseline to 6 months (Table 2). Correlations were not significant between the MBDA score and SJC28, TJC28, VAS-GH or HAQ, except for $\triangle$ SJC28 and $\triangle V A S-G H$ from baseline to 6 months (Table 2).

The results of the multivariable regression analysis resembled those of the correlation analyses, except that the associations between $\triangle \mathrm{MBDA}$ score versus $\triangle \mathrm{ESR}$ and $\triangle \mathrm{SJC} 28$

A

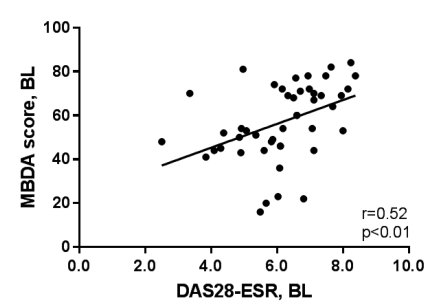

D

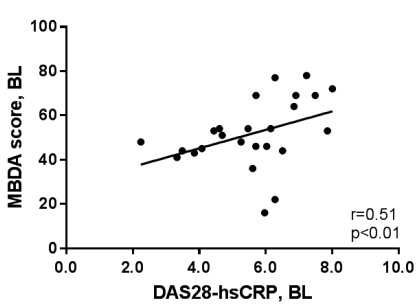

B

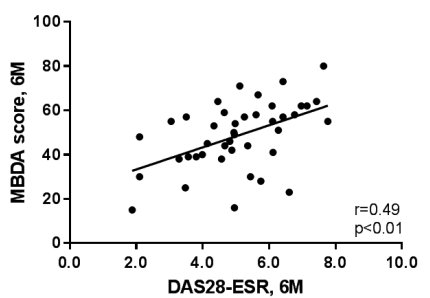

E

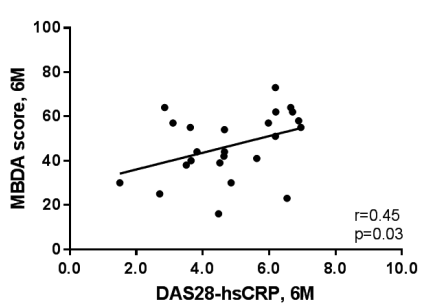

C

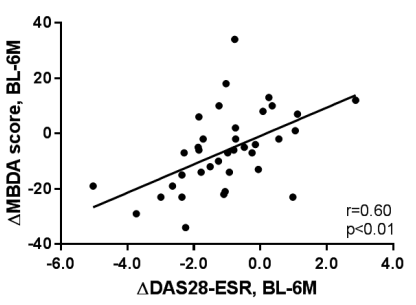

F

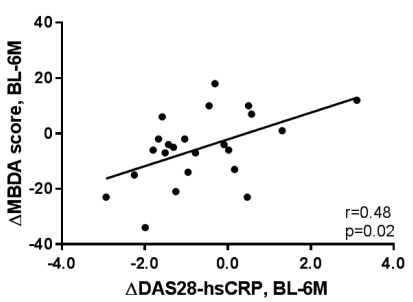

Figure 1. Correlation between MBDA score and DAS28

A. MBDA score versus DAS28-ESR at baseline $(n=46)$. B. MBDA score versus DAS28-ESR at 6 months $(n=42)$. C. $\triangle M B D A$ score vs $\triangle \mathrm{DAS28}-\mathrm{ESR}$, from baseline to 6 months $(n=38)$. D. MBDA score versus DAS28-hsCRP at baseline $(n=25)$. E. MBDA score versus DAS28-hsCRP at 6 months $(n=24)$. F. $\triangle M B D A$ score versus $\triangle D A S 28-h s C R P$, from baseline to 6 months $(n=23)$.

BL: baseline; DAS28: disease activity score assessing 28 joints; ESR: erythrocyte sedimentation rate; hsCRP: high-sensitivity C-reactive protein; M: months; MBDA: multi-biomarker disease activity; r: Spearman's rank correlation; $\triangle$ : change over time.

Negative change values represent improvement over 6 months. 
Table 2. Correlations and associations between the MBDA score and disease activity measures

\begin{tabular}{|c|c|c|c|c|c|c|}
\hline Measure & $\begin{array}{l}\text { Time point or period } \\
\text { for comparison with } \\
\text { MBDA score }\end{array}$ & $\begin{array}{l}\text { Number of } \\
\text { available } \\
\text { samples }\end{array}$ & $r$ & $\mathrm{p}$-value & B $(95 \% \mathrm{Cl})^{\prime}$ & $p$-value \\
\hline \multirow[t]{3}{*}{ DAS28-ESR } & $\mathrm{BL}$ & 46 & 0.52 & $<0.01$ & $0.05(0.02-0.07)$ & $<0.01$ \\
\hline & $6 \mathrm{M}$ & 42 & 0.49 & $<0.01$ & $0.06(0.02-0.09)$ & 0.01 \\
\hline & $\triangle$ & 38 & 0.60 & $<0.01$ & $0.05(0.01-0.08)$ & 0.02 \\
\hline \multirow[t]{3}{*}{ ESR } & $\mathrm{BL}$ & 44 & 0.75 & $<0.01$ & $1.20(0.71-1.70)$ & $<0.01$ \\
\hline & $6 \mathrm{M}$ & 42 & 0.66 & $<0.01$ & $0.81(0.36-1.26)$ & $<0.01$ \\
\hline & $\Delta$ & 37 & 0.48 & $<0.01$ & $0.57(-0.03-1.17)$ & 0.06 \\
\hline \multirow[t]{3}{*}{ DAS28-hsCRP2 } & $\mathrm{BL}$ & 25 & 0.51 & $<0.01$ & $0.06(0.02-0.10)$ & 0.01 \\
\hline & $6 \mathrm{M}$ & 24 & 0.45 & 0.03 & $0.06(0.02-0.10)$ & $<0.01$ \\
\hline & $\Delta$ & 23 & 0.48 & 0.02 & $0.05(0.00-0.09)$ & $<0.05$ \\
\hline \multirow[t]{3}{*}{$\mathrm{hsCRP}^{2}$} & $\mathrm{BL}$ & 25 & 0.80 & $<0.01$ & $1.24(0.72-1.76)$ & $<0.01$ \\
\hline & $6 \mathrm{M}$ & 24 & 0.80 & $<0.01$ & $0.75(0.41-1.10)$ & $<0.01$ \\
\hline & $\Delta$ & 23 & 0.71 & $<0.01$ & $0.90(0.60-1.21)$ & $<0.01$ \\
\hline \multirow[t]{3}{*}{ CRP } & $\mathrm{BL}$ & 46 & 0.75 & $<0.01$ & $1.07(0.62-1.52)$ & $<0.01$ \\
\hline & $6 \mathrm{M}$ & 40 & 0.76 & $<0.01$ & $0.82(0.58-1.06)$ & $<0.01$ \\
\hline & $\Delta$ & 37 & 0.59 & $<0.01$ & $0.68(0.18-1.19)$ & $<0.01$ \\
\hline \multirow[t]{3}{*}{$\mathrm{SJC} 28$} & $\mathrm{BL}$ & 48 & 0.15 & 0.32 & $0.10(-0.06-0.26)$ & 0.22 \\
\hline & $6 \mathrm{M}$ & 42 & 0.26 & 0.10 & $0.14(-0.01-0.28)$ & 0.06 \\
\hline & $\triangle$ & 40 & 0.42 & $<0.01$ & $0.12(-0.04-0.29)$ & 0.14 \\
\hline \multirow[t]{3}{*}{ TJC28 } & $\mathrm{BL}$ & 48 & 0.23 & 0.12 & $0.17(0.02-0.32)$ & 0.03 \\
\hline & $6 \mathrm{M}$ & 42 & 0.25 & 0.11 & $0.17(-0.01-0.34)$ & 0.06 \\
\hline & $\Delta$ & 40 & 0.28 & 0.08 & $0.04(-0.15-0.23)$ & 0.67 \\
\hline \multirow[t]{3}{*}{ VAS-GH } & $\mathrm{BL}$ & 48 & 0.20 & 0.18 & $0.34(-0.12-0.79)$ & 0.14 \\
\hline & $6 \mathrm{M}$ & 42 & 0.27 & 0.09 & $0.46(-0.08-0.99)$ & 0.09 \\
\hline & $\triangle$ & 40 & 0.36 & 0.02 & $0.74(0.08-1.40)$ & 0.03 \\
\hline \multirow[t]{3}{*}{ HAQ } & $\mathrm{BL}$ & 39 & 0.02 & 0.91 & $0.06(-0.06-0.02)$ & 0.30 \\
\hline & $6 \mathrm{M}$ & 41 & -0.03 & 0.85 & $-0.01(-0.01-0.01)$ & 0.84 \\
\hline & $\triangle$ & 34 & 0.19 & 0.28 & $0.00(-0.01-0.01)$ & 0.77 \\
\hline
\end{tabular}

BL: MBDA score and measure both at baseline; Cl: confidence interval; DAS28: disease activity score using 28 joints; ESR: erythrocyte sedimentation rate; HAQ: health assessment questionnaire; hsCRP: high-sensitivity C-reactive protein; MBDA: multi-biomarker disease activity; r: Spearman's rank correlation; SJC28: swollen joint count assessing 28 joints; TJC28: tender joint count assessing 28 joints; VAS-GH: patient visual analogue scale for general health; 6M: MBDA score and measure both at month 6; $\triangle$ : change in MBDA score and measure, both from baseline to month 6 .

1. ß: regression coefficient from multivariable linear regression analysis, after adjustment by age, gender, smoking status, RF status, ACPA status, and cohort; 2. HORUS cohort only. 
were not statistically significant and the association between MBDA score versus TJC28 at baseline was statistically significant (Table 2).

\section{Association between MBDA score and EULAR response}

At 6 months, 21 patients (48\%) were classified as non-, 19 patients (43\%) as moderate and 4 patients ( $9 \%$ ) as good EULAR responders. The distribution of values for $\triangle M B D A$ score within each EULAR response category is shown in Figure 2. $\triangle M B D A$ score from baseline to 6 months was significantly associated with EULAR response (good or moderate) versus non-response at 6 months (odds ratio (OR): 0.93 (95\% Cl 0.88-0.98, $\mathrm{p}=0.01$ ) per unit change in MBDA score, Figure 2). Adjusted by age, gender, smoking status, RF status, ACPA status and cohort, this association remained statistically significant (OR: 0.89 (95\% Cl 0.81-0.98, $\mathrm{p}=0.02$ ) per unit change in MBDA score).

The MBDA score at baseline was not associated with EULAR response (good or moderate) versus non-response at 6 months, with OR of $1.01(95 \% \mathrm{Cl} 0.98-1.05, \mathrm{p}=0.35)$ per unit MBDA score, even after adjustment by age, gender, smoking status, RF status, ACPA status, and cohort (OR: 1.03 (95\% Cl 0.98-1.08, $\mathrm{p}=0.27$ ) per unit MBDA score).

\section{Correlation between MBDA score and radiographic progression or bone turnover markers}

For the 11 patients with radiographs available at baseline and 12 months, all from the UMC Utrecht cohort, the median $\triangle \mathrm{SHS}$ was 3 (IQR -1-12). At baseline, low, moderate and high

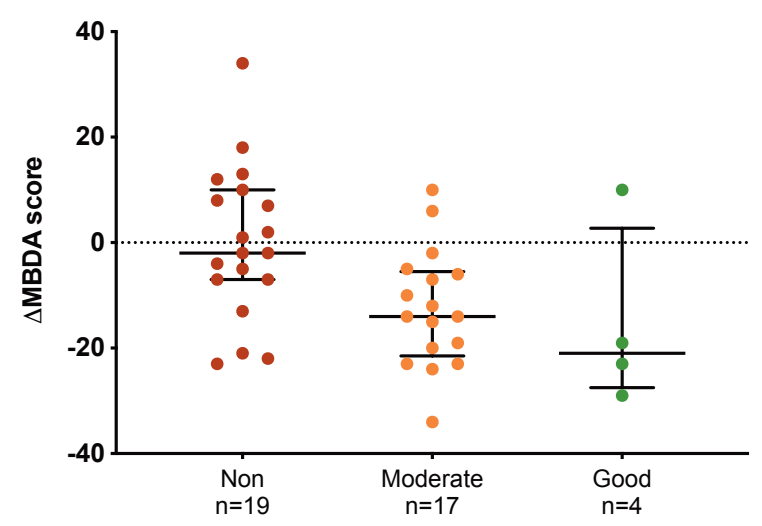

EULAR response category at 6 months

Figure 2. Change in MBDA score by EULAR response category at 6 months Individual patient values of $\triangle$ MBDA score from baseline to 6 months are shown as dots, grouped by EULAR response category at 6 months (EULAR non-, moderate, good response). Dark lines represent median values. Whiskers represent interquartile ranges $\left(25^{\text {th }}-75^{\text {th }}\right)$.

EULAR: European League Against Rheumatism; MBDA: multi-biomarker disease activity; n: number of patients; $\triangle$ : change. 
Table 3. Correlations and associations between the MBDA score and radiographic progression or bone turnover markers

\begin{tabular}{lllllll}
\hline Measure & $\begin{array}{l}\text { Time point or period } \\
\text { for comparison with } \\
\text { MBDA score }\end{array}$ & $\begin{array}{l}\text { Number of } \\
\text { available } \\
\text { samples }\end{array}$ & $r$ & p-value & $\beta(95 \% \mathrm{Cl})^{1}$ & $p$-value \\
\hline$\Delta \mathrm{SHS}(\mathrm{BL}-12 \mathrm{M})^{2}$ & $\mathrm{BL}$ & 11 & 0.19 & 0.57 & $0.34(-0.33-1.01)$ & 0.23 \\
& $6 \mathrm{M}$ & 11 & 0.18 & 0.60 & $0.18(-0.72-1.08)$ & 0.62 \\
& $\Delta$ & 11 & 0.19 & 0.57 & $-0.38(-1.35-0.60)$ & 0.34
\end{tabular}

\section{Bone turnover markers ${ }^{3}$}

\begin{tabular}{lllllll} 
BCTX & $\Delta$ & 23 & 0.22 & 0.31 & $1.10(-6.79-8.99)$ & 0.77 \\
PINP & $\Delta$ & 23 & -0.14 & 0.54 & $-0.31(-2.05-1.42)$ & 0.70 \\
BAP & $\Delta$ & 23 & -0.01 & 0.98 & $-0.01(-0.17-0.15)$ & 0.88 \\
TRAP5b & $\Delta$ & 23 & -0.20 & 0.37 & $0.00(-0.03-0.03)$ & 0.99 \\
DKK1 & $\Delta$ & 23 & 0.27 & 0.22 & $0.15(-0.40-0.69)$ & 0.57 \\
Sclerostin & $\Delta$ & 23 & 0.11 & 0.63 & $0.10(-0.37-0.56)$ & 0.66 \\
\hline
\end{tabular}

BAP: bone-specific alkaline phosphatase; ßCTX: Beta-isomerised carboxy terminal telopeptide of type I collagen; BL: MBDA score at baseline; Cl: confidence interval; DKKI: Dickkopf-1; MBDA: multi-biomarker disease activity; PINP: procollagen type 1 amino-terminal propeptide; r: Spearman's rank correlation; SHS: Sharp/van der Heijde score; TRAP5b: tartrate-resistant acid phosphatase isoenzyme 5b; 6M: MBDA score at month 6; $\triangle$ : change (for SHS: $\triangle M B D A$ score from baseline to month 6 and $\triangle$ SHS from baseline to month 12; for bone turnover markers: both $\triangle$ from baseline to month 6).

1. ß: regression coefficient from multivariable linear regression analysis, after adjustment by age, gender and/or menopausal status, smoking status, RF status, and ACPA status; 2. UMC Utrecht cohort only; 3. HORUS cohort only.

MBDA scores were observed in 1,1 and 9 patients, respectively. Radiographic progression $(\triangle \mathrm{SHS} \geq 5)$ in patients with low, moderate and high MBDA scores was observed in $0(0 \%), 0$ (0\%), and $5(56 \%)$ patients, respectively.

No significant Spearman's correlation was found between MBDA score or $\triangle M B D A$ score and $\triangle \mathrm{SHS}$ over 12 months, nor bone turnover markers (Table 3). Similar findings were obtained with multivariable regression analysis adjusted by age, gender (menopausal status for bone turnover markers), smoking status, RF status and ACPA status (Table 3).

\section{Discussion}

The MBDA score has been shown to track response to a variety of DMARDs. We found significant correlations between the MBDA score and DAS28-ESR as well as DAS28-hsCRP at baseline and at 6 months, and between $\triangle M B D A$ score and $\triangle D A S 28-E S R$ and $\triangle D A S 28-$ hsCRP from baseline to 6 months in patients treated with rituximab. Moreover, $\triangle M B D A$ score was significantly associated with EULAR response to rituximab treatment. This is the first time it has been shown that the MBDA score can be used to track disease activity in 
RA patients upon treatment with rituximab and that change in the MBDA score reflects response to rituximab treatment.

Our findings on the MBDA score are consistent with several previous studies in RA patients upon treatment with other cs-, biological, or targeted synthetic DMARDs. ${ }^{6-8,0-16}$ In our study, we additionally investigated if the MBDA score correlated with the individual components of the DAS28. We found correlations between the MBDA score or $\triangle M B D A$ score and ESR, hsCRP or their changes, but found limited correlations between the MBDA score and the other DAS28 components. The correlation between the MBDA score and the DAS28 thus seems predominantly dependent on the biochemical components of the DAS28, the ESR or (hs)CRP. It would be of interest to assess the additional value of the MBDA score above ESR or CRP alone, but the present study was not powered to analyse this. A larger study has reported that an increase in TJC, SJC and patient global assessment was paralleled by an increase in MBDA category; and that, in patients positive for either RF and/or ACPA, an MBDA score excluding CRP was a significant predictor of both DAS28-CRP, and of DAS28 without any CRP or ESR component. ${ }^{7}$

In addition, MBDA score appeared to be more sensitive for detecting inflammation than ESR or CRP. A study of 9135 RA patients with active disease found that ESR and CRP were normal in the majority. ${ }^{25}$ In other studies, MBDA score was often elevated in such patients ${ }^{26}$ and, when it was, risk of radiographic progression was increased. ${ }^{9,27}$ In patients with disproportionally high subjective disease activity components (e.g. high tender joint counts with low ESR or CRP) the MBDA score might be an important alternative disease activity measure. We could not address this hypothesis, as no patients with normal ESR or CRP (defined as $\leq 1 \mathrm{~mm} / \mathrm{h}$ or $\mathrm{mg} / \mathrm{L}$ ) were included in this study.

Previous studies have shown that the MBDA score was a significant predictor of radiographic progression, both in early and established RA. ${ }^{9,27-30}$ In the present study, all patients with clinically important radiographic progression ( $\triangle \mathrm{SHS} \geq 5$ ) had a high MBDA score at baseline. This result resembles the findings in previous studies. ${ }^{9,27-30}$ We did not find a significant Spearman's correlation between baseline MBDA score and $\triangle \mathrm{SHS}$ in patients treated with rituximab, possibly due to the small number of patients $(n=11)$ and the limited observation period.

B-cell depletion upon rituximab treatment has been shown to be most effective in RFpositive patients, ${ }^{31}$ and has been suggested to be associated with ACPA positivity. ${ }^{32}$ In future studies, it would be of interest to stratify the performance of the MBDA score in rituximab treated RA patients according to RF and ACPA status.

Other studies have shown that rituximab treatment increases bone formation and decreases bone resorption in RA patients. ${ }^{33,34}$ For example, a significant correlation was found between the changes of DAS28 and BCTX, 34 showing that the anti-inflammatory therapeutic response with rituximab and the anti-resorptive effect on bone might be related. In future, in larger studies with longer follow-up, it may be of interest to investigate 
the relationship between the MBDA score and bone turnover.

In conclusion, we have shown, for the first time, that the MBDA score correlated with DAS28 following treatment with the B-cell depleting agent rituximab and that $\triangle M B D A$ score reflected the treatment response. Our findings are consistent with previous research in RA patients treated with other DMARDs. 


\section{References}

1. Smolen JS, Aletaha D, McInnes IB. Rheumatoid arthritis. Lancet 2016;388:2023-38.

2. Smolen JS, Landewé R, Bijlsma J, et al. EULAR recommendations for the management of rheumatoid arthritis with synthetic and biological disease-modifying antirheumatic drugs: 2016 update. Ann Rheum Dis 2017;76:960-77.

3. Singh JA, Saag KG, Bridges SL, et al. 2015 American College of Rheumatology Guideline for the Treatment of Rheumatoid Arthritis. Arthritis Rheumatol 2016;68:1-26.

4. Gaujoux-Viala C, Mouterde G, Baillet A, et al. Evaluating disease activity in rheumatoid arthritis: Which composite index is best? A systematic literature analysis of studies comparing the psychometric properties of the DAS, DAS28, SDAI and CDAI. Jt Bone Spine 2012;79:149-55.

5. Jacobs JWG, Ten Cate DF, van Laar JM. Monitoring of rheumatoid arthritis disease activity in individual patients: still a hurdle when implementing the treat-to-target principle in daily clinical practice. Rheumatology 2015;54:959-61.

6. Centola M, Cavet G, Shen Y, et al. Development of a Multi-Biomarker Disease Activity Test for Rheumatoid Arthritis. PLoS One 2013;8.

7. Curtis JR, Van Der Helm-Van Mil AH, Knevel R, et al. Validation of a novel multibiomarker test to assess rheumatoid arthritis disease activity. Arthritis Care Res 2012;64:1794-803.

8. Hirata $S$, Dirven $L$, Shen $Y$, et al. A multibiomarker score measures rheumatoid arthritis disease activity in the best study. Rheumatology (Oxford) 2013;52:1202-7.

9. Li W, Sasso EH, van der Helm-van Mil AHM, et al. Relationship of multi-biomarker disease activity score and other risk factors with radiographic progression in an observational study of patients with rheumatoid arthritis. Rheumatology 2016;55:357-66.

10. Bakker MF, Cavet G, Jacobs JW, et al. Performance of a multi-biomarker score measuring rheumatoid arthritis disease activity in the CAMERA tight control study. Ann Rheum Dis 2012;71:1692-7.

11. Jurgens MS, Jacobs JW, Bijlsma JW, et al. The Multi-biomarker Disease Activity Test for Assessing Response to Treatment Strategies Using Methotrexate with or without Prednisone in the CAMERA Trial-II. 2014:81-98.

12. Hirata S, Li W, Defranoux N, et al. A multi- biomarker disease activity score tracks clinical response consistently in patients with rheumatoid arthritis treated with different anti-tumor necrosis factor therapies: A retrospective observational study. Mod Rheumatol 2015;25:344-9.

13. Fleischmann R, Connolly SE, Maldonado MA, et al. Brief Report: Estimating Disease Activity Using Multi-Biomarker Disease Activity Scores in Rheumatoid Arthritis Patients Treated With Abatacept or Adalimumab. Arthritis Rheumatol 2016;68:2083-9.

14. Curtis JR, Wright GC, Strand V, et al. Reanalysis of the Multi-Biomarker Disease Activity Score for Assessing Disease Activity in the Abatacept Versus Adalimumab Comparison in BiologicNaive Rheumatoid Arthritis Subjects with Background Methotrexate Study: Comment on the Article by Fleischmann. Arthritis Rheumatol 2017;69:863-5.

15. Haney D, Cavet G, Durez P, et al. Correlation of a Multi-Biomarker Disease Activity Response Assessment to Disease Activity Score 28 (C-Reactive Protein) Response Assessment and Omeract Ramris Scores in a PlaceboControlled Rheumatoid Arthritis Clinical Trial with Abatacept (ASSET). ACR Abstr 21592012.

16. Yamaoka K, Kubo S, Li W, et al. FRI0333 Effects of Tofacitinib Treatment on Leptin and other Components of the Multi-Biomarker Disease Activity Score in Patients with Rheumatoid Arthritis. Ann Rheum Dis 2014;73:507.2-508.

17. Shaw T, Quan J, Totoritis MC. B cell therapy for rheumatoid arthritis: The rituximab (antiCD20) experience. In: Annals of the Rheumatic Diseases. BMJ Publishing Group Ltd 2003. 559.

18. Mok CC. Rituximab for the treatment of rheumatoid arthritis: an update. Drug Des Devel Ther 2013;8:87.

19. Teng YO, Wheater $G$, Hogan $V E$, et al. Induction of long-term B-cell depletion in refractory rheumatoid arthritis patients preferentially affects autoreactive more than protective humoral immunity. Arthritis Res Ther 2012;14:57.

20. Teng $\mathrm{YKO}$, Tekstra J, Breedveld FC, et al. Rituximab fixed retreatment versus ondemand retreatment in refractory rheumatoid arthritis: Comparison of two B cell depleting treatment strategies. BMJ Publishing Group Ltd 2009.

21. Elshahaly $M$, Wheater $G$, Naraghi $K$, et al. Changes in bone density and bone turnover in patients with rheumatoid arthritis treated with rituximab, a B cell depleting monoclonal 
antibody (HORUS TRIAL). BMC Musculoskelet Disord 2013;14:A10.

22. van Riel PL, van Gestel $A M$, van de Putte $L B$. Development and validation of response criteria in rheumatoid arthritis: steps towards an international consensus on prognostic markers. Br J Rheumatol 1996;35 Suppl 2:4-7.

23. Bruynesteyn $K$, Heijde $V$ Der, Boers $M$, et al. Determination of the Minimal Clinically Important Difference in Rheumatoid Arthritis Joint Damage of the Sharp / van der Heijde and Larsen / Scott Scoring Methods by Clinical Experts and Comparison With the Smallest Detectable Difference. Arthritis Rheum 2002;46:913-20.

24. Curtis JR, Greenberg JD, Harrold LR, et al. Influence of obesity, age, and comorbidities on the multi-biomarker disease activity test in rheumatoid arthritis. Semin Arthritis Rheum 2018;47:472-7.

25. Kay J, Morgacheva $\mathrm{O}$, Messing SP, et al. Clinical disease activity and acute phase reactant levels are discordant among patients with active rheumatoid arthritis: Acute phase reactant levels contribute separately to predicting outcome at one year. Arthritis Res Ther 2014;16:R40.

26. Lee $Y C$, Hackett J, Frits $M$, et al. Multibiomarker disease activity score and C-reactive protein in a cross-sectional observational study of patients with rheumatoid arthritis with and without concomitant fibromyalgia. Rheumatology 2016;55:640-8.

27. van der Helm-van Mil AHM, Knevel R, Cavet G, et al. An evaluation of molecular and clinical remission in rheumatoid arthritis by assessing radiographic progression. Rheumatology 2013;52:839-46.

28. Markusse IM, Dirven L, Van Den Broek $M$, et al. A multibiomarker disease activity score for rheumatoid arthritis predicts radiographic joint damage in the best study. J Rheumatol 2014;41:2114-9.

29. Hirata S, Li W, Kubo S, et al. Association of the multi-biomarker disease activity score with joint destruction in patients with rheumatoid arthritis receiving tumor necrosis factoralpha inhibitor treatment in clinical practice. Mod Rheumatol 2016;26:850-6.

30. Hambardzumyan K, Bolce R, Saevarsdottir S, et al. Pretreatment multi-biomarker disease activity score and radiographic progression in early RA: Results from the SWEFOT trial. Ann Rheum Dis 2015;74:1102-9.

31. Benucci $M$, Manfredi $M$, Sarzi-Puttini $P$, et al. Predictive factors of response to rituximab therapy in rheumatoid arthritis: What do we know today? Autoimmun. Rev. 2010;9:801-3.

32. Cuppen BVJ, Welsing PMJ, Sprengers JJ, et al. Personalized biological treatment for rheumatoid arthritis: a systematic review with a focus on clinical applicability. Rheumatology 2016;55:826-39.

33. Zerbini CAF, Clark $\mathrm{P}$, Mendez-Sanchez L, et al. Biologic therapies and bone loss in rheumatoid arthritis. Osteoporos Int 2017;28:429-46.

34. Wheater $G$, Hogan VE, Teng $Y K O$, et al. Suppression of bone turnover by B-cell depletion in patients with rheumatoid arthritis. Osteoporos Int 2011;22:3067-72. 


\section{Supplementary}

\section{Determination of bone turnover markers}

All bone turnover markers were quantified following the manufacturer's guidelines and reference ranges (BAP: 1-75 $\mathrm{g} / \mathrm{L}$; PINP: premenopausal females 15.1-58.6 $\mathrm{Mg} / \mathrm{L}$, postmenopausal females 16.3-73.9 $\mathrm{\mu g} / \mathrm{L}$, males >50 years 15.1- $58.6 \mu \mathrm{g} / \mathrm{L}$; DKKl: 1.7-160pmol/L; sclerostin: 7.5-240pmol/L; TRAP5b: 0.5-10.0U/L; BCTX: premenopausal females 25-573 ng/L, postmenopausal females 104-1008 ng/L, males >50 years 0-854 $\mathrm{ng} / \mathrm{L}$ ). BAP was quantified in $50 \mu \mathrm{l}$ serum by chemiluminescence on the IDS iSYS analyser (ImmunoDiagnostic Systems - Boldon, Tyne and Wear, UK). PINP and BCTX were quantified by electrochemiluminescent immunoassay on the Elecsys 2010 analyser (Roche Diagnostics, Lewes, UK). DKK-1 and sclerostin levels were measured in 20 $\mathrm{\mu l}$ serum using a manual Biomedica ELISA, purchased from Oxford Biosystems Cadama (Wheatley, Oxford, UK), a UK distributor for Biomedica Medizinprodukte (Vienna, Austria). TRAP-5b was measured in 100 $\mathrm{ul}$ serum by a manual IDS ELISA (ImmunoDiagnostic Systems - Boldon, Tyne and Wear, UK). This method specifically measured TRAP isoform $5 b$ activity freshly liberated from osteoclasts. 



\section{CHAPTER 4}

\section{Characteristics of difficult-to-treat rheumatoid arthritis: results of an international survey}

Ann Rheum Dis 2018;77:1705-9.

Nadia M.T. Roodenrijs, Maria J.H. de Hair, Marlies C. van der Goes, Johannes W.G. Jacobs, Paco M.J. Welsing, Désirée van der Heijde, Daniel Aletaha, Maxime Dougados, Kimme L. Hyrich, Ian B. McInnes, Ulf Müller-Ladner, Ladislav Šenolt, Zoltán Szekanecz, Jacob M. van Laar, György Nagy, on behalf of the whole EULAR Task Force on development of EULAR recommendations for the management of difficult-to-treat rheumatoid arthritis 


\section{Abstract}

\section{Objectives}

Patients with difficult-to-treat rheumatoid arthritis (RA) remain symptomatic despite treatment according to current EULAR management recommendations. These focus on early phases of the disease and pharmacological management. We aimed to identify characteristics of difficult-to-treat RA and issues to be addressed in its workup and management that are not covered by current management recommendations.

\section{Methods}

An international survey was conducted among rheumatologists with multiple-choice questions on disease characteristics of difficult-to-treat RA. Using open questions, additional items to be addressed and items missing in current management recommendations were identified.

\section{Results}

Four hundred ten respondents completed the survey: $50 \%$ selected disease activity score assessing 28 joints $>3.2$ or presence of signs suggestive of active disease as characteristics of difficult-to-treat RA; $42 \%$ selected fatigue; $48 \%$ selected failure to $\geq 2$ conventional synthetic disease-modifying antirheumatic drugs (DMARDs) and $\geq 2$ biological/targeted synthetic DMARDs; $89 \%$ selected inability to taper glucocorticoids below 5 or $10 \mathrm{mg}$ prednisone equivalent daily. Interfering comorbidities, extra-articular manifestations and polypharmacy were identified as important issues missing in current management recommendations.

\section{Conclusions}

There is wide variation in concepts of difficult-to-treat RA. Several important issues regarding these patients are not addressed by current EULAR recommendations. 


\section{Introduction}

The European League Against Rheumatism (EULAR) recommendations and the American College of Rheumatology guidelines on management of rheumatoid arthritis (RA) focus on early phases of the disease and on pharmacological management.,2 These recommendations suggest intensifying the disease-modifying antirheumatic drug (DMARD) strategy, if improvement or the treatment target is not achieved within 3 or 6 months, respectively. Nevertheless, a significant proportion of patients remains symptomatic after several cycles of treatment, which makes them difficult-to-treat; this is a significant clinical problem in daily practice. ${ }^{3}$

A wide array of potential factors contributes to difficult-to-treat RA, such as DMARD resistance or intolerance, adverse reactions, treatment non-adherence and limited drug options due to comorbidities. Importantly, patients with RA may also remain symptomatic due to non-inflammatory factors, such as secondary osteoarthritis, pain syndromes, social and occupational decline and coping difficulties. All these may (in different combinations) play a role in individual patients and require specific management approaches, which should be addressed in management recommendations.

Currently, different concepts exist on difficult-to-treat RA, such as refractory, multidrug resistant or persistent RA, or concepts based on number of failed DMARDs and failed treatment goals. ${ }^{4-7}$ Depending on the criteria used, the estimated prevalence of difficultto-treat RA ranges from $5 \%$ to $20 \%{ }^{6}$

We aimed to identify characteristics of the concept of difficult-to-treat RA and to explore issues to be addressed in its workup and management that are not covered by current EULAR management recommendations.

\section{Methods}

\section{Online survey among rheumatologists}

An online survey (Supplementary File, set up by DvdH, GN, JWGJ, JMvL, MJHdH and PMJW) was conducted among rheumatologists (including rheumatologists-in-training). The survey was distributed by email in the network of the authors and by Emerging EULAR Network (EMEUNET), and it was asked to additionally forward it to other rheumatologists. The survey consisted of two questions regarding the background of the respondents (Where do you work? How many RA patients do you treat?).

Four multiple-choice questions addressed the perceived necessity of incorporating the following items, and their cut-offs, into the concept of difficult-to-treat RA: disease activity level; presence of fatigue; number of DMARDs that failed; and inability to taper glucocorticoid (GC) treatment. Only one response option could be selected at each 
multiple-choice item, which were selected as - according to expert opinion - being among the most frequent and relevant characteristics in clinical practice. Fatigue was selected as one of the most relevant patient-reported outcomes in RA. ${ }^{8}$

Three open questions were: 'Please define any additional characteristics and suggested criteria for difficult-to-treat RA'; 'Please mention additional clinical issues or comorbidities to be addressed in the workup and management of these patients'; and 'Please mention any clinically relevant situations which are not covered by the current RA EULAR recommendations'.

\section{Qualification and quantification of the responses to the open questions}

NMTR and MJHdH independently classified the responses to the open questions into categories (Supplementary File). This enabled summarising and quantifying. Categories were defined based on the responses that were given to the open questions. One response could fit multiple categories. 'Other' was used to classify characteristics that did not fit into one of the categories. Discrepancies in classification between NMTR and $\mathrm{MJHdH}$ were resolved by consensus.

\section{Statistical analyses}

All responses were evaluated using descriptive statistics, performed using IBM SPSS Statistics V.21 software.

\section{Results}

\section{Respondents}

Four hundred and ten respondents from 33 countries completed the survey between July 2017 and March 2018. Of the 385 respondents who filled out the name of their country, $96 \%$ was European (Figure 1). Twenty-five per cent of the respondents ( $n=7$ missing) treated $<100$ unique patients with RA, 42\% 100-300 patients, and 32\% >300 patients.

\section{Selected difficult-to-treat RA disease characteristics}

Fifty per cent of respondents selected 'disease activity score assessing 28 joints using erythrocyte sedimentation rate (DAS28-ESR) >3.2 OR presence of signs suggestive of active inflammatory disease activity with a DAS28-ESR $\leq 3.2^{\prime}$ as characteristics (Figure 2a). Fortytwo per cent included fatigue (Figure $2 b$ ). Forty-eight per cent selected ' $\geq 2$ conventional synthetic (cs) DMARDs AND $\geq 2$ biological (b) DMARDs or targeted synthetic (ts) DMARDs with different modes of action' for how many insufficiently effective antirheumatic drugs should at least have been applied (Figure 2c). Eighty-nine per cent selected inability to taper GCs $<5 \mathrm{mg}(43 \%)$ or $10 \mathrm{mg}(46 \%)$ prednisone or equivalent daily for more than 1 


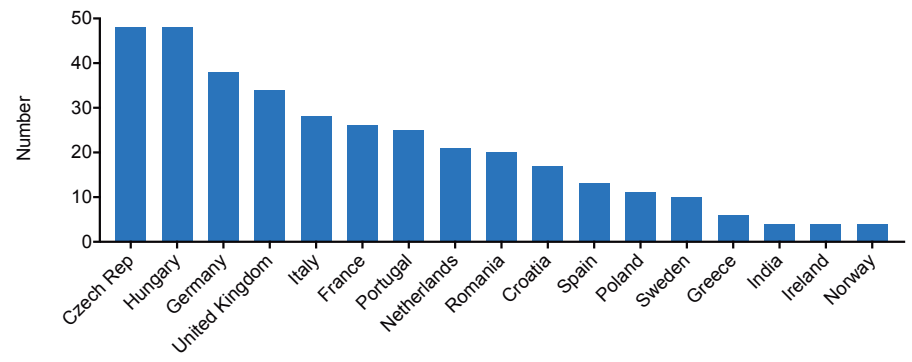

Figure 1. Number of respondents per country

Less than 4 (not shown): Austria, Belarus, Bulgaria, Denmark, Egypt, Estonia, Iceland, Israel, Kenya, Pakistan, Russia, Serbia, Slovakia, Slovenia, Tunisia and Turkey

A. What should be the definition for not well-controlled disease in the definition of difficult-to-treat RA?

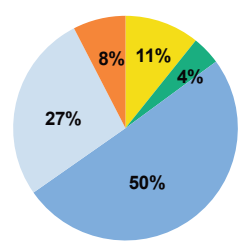

DAS28-ESR $>3.2$

DAS28-ESR $>5.1$

- DAS28-ESR $>3.2$ OR presence of signs suggestive of active inflammatory disease activity with a DAS28-ESR $\leq 3.2$

- DAS28-ESR $>5.1$ OR presence of signs suggestive of active inflammatory disease activity with a DAS28-ESR $\leq 5.1$

- Other

Total responses $=409$

C. Which and how many anti-rheumatic drugs should at least be tried with insufficient effect for the definition of difficult-to-treat RA?

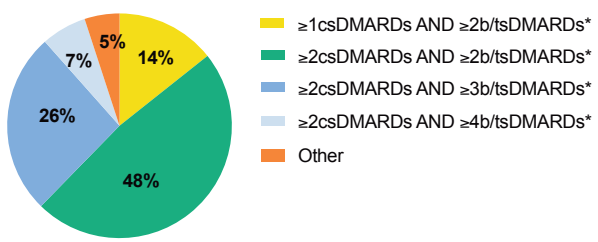

Total responses $=398$

\section{B. Would you include fatigue in the definition of not well-controlled disease?}

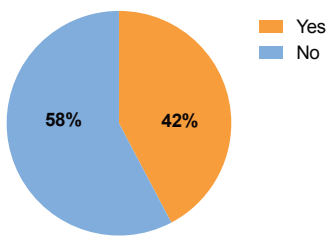

Total responses $=396$

D. Treatment with glucocorticoids should be mentioned in the criteria for difficult-to-treat RA as follows:

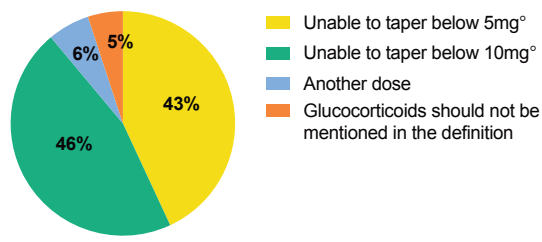

Total responses $=397$

\section{E. Additional characteristics for difficult-to-treat RA}

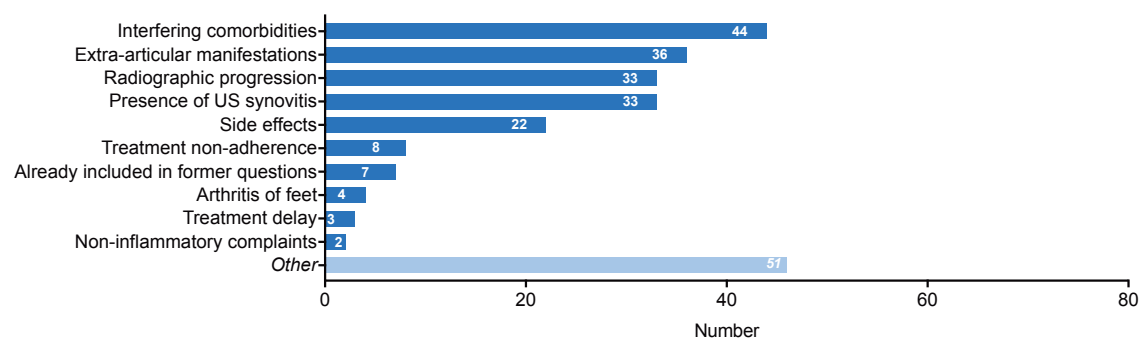

Figure 2. Difficult-to-treat RA characteristics

b/tsDMARDs: biological/targeted synthetic disease-modifying antirheumatic drugs; csDMARDs: conventional synthetic DMARDs; DAS28-ESR: disease activity score assessing 28 joints using erythrocyte sedimentation rate; RA: rheumatoid arthritis; US: ultrasonography; *: with different modes of action; ${ }^{\circ}$ : or equivalent daily for more than 1 year, irrespective of DMARD treatment. 
year, irrespective of DMARD treatment (Figure 2d), as difficult-to-treat RA characteristic.

\section{Additional difficult-to-treat RA characteristics}

Two hundred and forty-three additional characteristics of difficult-to-treat RA were given by 169 respondents (Figure 2e), most frequently categorised into 'interfering comorbidities' and 'extra-articular manifestations'. Examples are cardiovascular risk, malignancies, interstitial lung disease and vasculitis. A diversity of 'other' responses was given, for example, inflammation on MRI, morning stiffness and patient dissatisfaction.

\section{Interfering clinical issues and items missing in current EULAR recommendations, important to manage difficult-to-treat RA}

For interfering issues to be addressed in the workup and management of difficult-totreat RA, 396 suggestions were given by 170 respondents (Figure 3a), most frequently cardiovascular disease and extra-articular manifestations. Other interfering clinical issues were drug intolerance, smoking and chronic liver disease.

For issues not covered by the current EULAR recommendations, 64 were mentioned by 54 respondents (Figure 3b). These were most frequently classified as interfering comorbidities and extra-articular manifestations. Also issues regarding pharmacological

\section{A. Interfering clinical issues or comorbidities to be addressed in the workup and management of these patients}

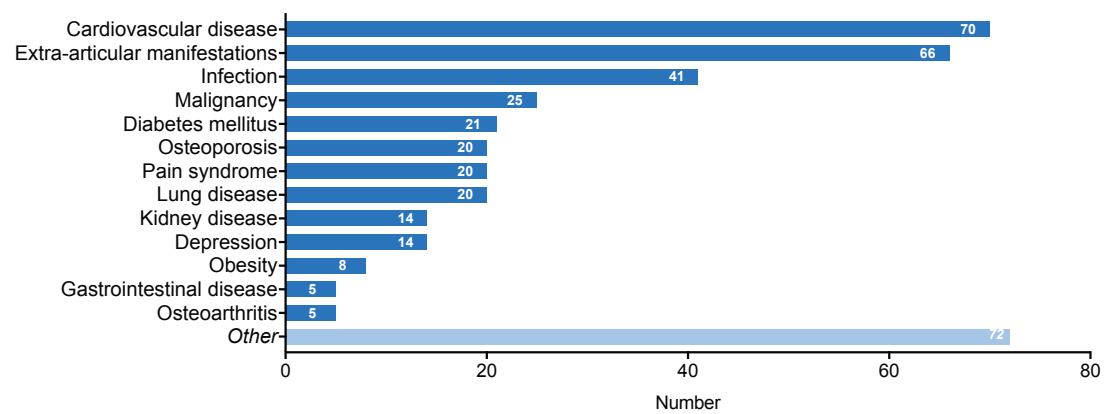

B. Clinically relevant situations which are not covered by the current RA EULAR recommendations

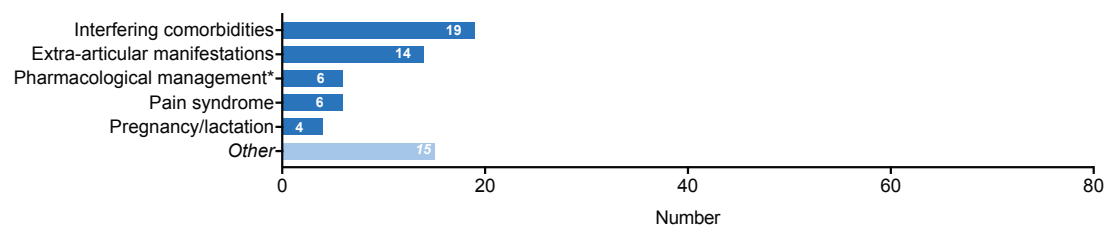

Figure 3. Interfering clinical issues and items missing in the current EULAR recommendations, important to manage difficult-to-treat RA

EULAR: European League Against Rheumatism; RA: rheumatoid arthritis.

*: e.g. tapering strategies, adverse events, polypharmacy. 
management (e.g. tapering regimen, adverse events and polypharmacy), pain syndromes and pregnancy and lactation were mentioned. Other items were ongoing joint destruction, coping problems and persistent single joint involvement.

\section{Discussion}

Our results show a wide variety in concepts of difficult-to-treat RA; active disease, failure to DMARD treatment and inability to taper GCs are considered main characteristics. Additional difficult-to-treat RA characteristics were mostly related to extra-articular manifestations and interfering comorbidities that may hamper assessment of disease activity or limit treatment possibilities. As items missing in current RA EULAR management recommendations, interfering comorbidities (especially cardiovascular disease, infection and malignancy), extra-articular manifestations, pharmacological management (e.g. tapering strategies, adverse events and polypharmacy) and pain syndromes were mentioned most frequently.

Of the factors mentioned as contributing to difficult-to-treat RA in this survey, for example treatment non-adherence, adverse events and coping strategies, exact prevalences are unknown. These should be determined in future research for an indication of their need to be included in management recommendations.

Our results mainly reflect how difficult-to-treat RA is experienced in European countries. Additional contributing factors to difficult-to-treat RA in countries outside Europe might be limited access to diagnostic tests, to rheumatologists and to DMARDs. These should be addressed in management recommendations as well.

Our study has limitations. The survey was distributed via email, and it was asked to forward it to other rheumatologists to increase the number of respondents. As a drawback, the total number of rheumatologists who received it is unknown. The four multiple-choice questions had pre-specified response options, limiting input to these questions but enabling the responses to be easily summarised and quantified. The open questions required a classification system for the responses; some responses were classified into two categories and there was a number of responses that was classified as 'other'. Additionally, the pre-specified multiple-choice questions may have biased the results of the open questions. However, by these open questions, we received a large inventory of issues that may need to be addressed in clinical practice.

The strengths of this study are the large number of respondents and of European countries represented by the respondents; the many suggestions of items that are not covered by the current EULAR RA management recommendations underline the unmet clinical need for this subpopulation of patients with RA.

Recently, a EULAR Task Force has been initiated on the development of recommendations 
for the comprehensive management of difficult-to-treat RA. The results of this survey will fuel discussions on items to include in the management recommendations of difficultto-treat RA.

In conclusion, the results of this survey underscore the difficulty in establishing an unambiguous concept of difficult-to-treat RA, which is seen as a heterogeneous condition not fully covered by current EULAR recommendations. The recently established EULAR Task Force will explore the management of difficult-to-treat RA further. 


\section{References}

1. Smolen JS, Landewé R, Bijlsma J, et al. EULAR recommendations for the management of rheumatoid arthritis with synthetic and biological disease-modifying antirheumatic drugs: 2016 update. Ann Rheum Dis 2017;76:960-77.

2. Singh JA, Saag KG, Bridges SL, et al. 2015 American College of Rheumatology Guideline for the Treatment of Rheumatoid Arthritis. Arthritis Rheumatol 2016;68:1-26.

3. de Hair MJH, Jacobs JWG, Schoneveld JLM, et al. Difficult-to-treat rheumatoid arthritis: an area of unmet clinical need. Rheumatology (Oxford) 2017;57:1135-44.

4. Unger M, Alasti F, Supp G, et al. The Good, the Bad and the Ugly - Refractory Rheumatoid
Arthritis in 2016. Arthritis Rheumatol 2016;68.

5. Miossec P. Introduction: 'Why is there persistent disease despite aggressive therapy of rheumatoid arthritis?'. Arthritis Res. Ther. 2014;16:113.

6. Kearsley-Fleet L, De Cock D, Watson K, et al. Refractory Disease in Rheumatoid Arthritis: Results from the British Society of Rheumatology Biologics Register for Rheumatoid Arthritis. Ann Rheum Dis 2017;76:960-77.

7. Buch $\mathrm{MH}$. Defining refractory rheumatoid arthritis. Ann Rheum Dis 2018;77:966-9.

8. Taylor PC, Moore A, Vasilescu R, et al. A structured literature review of the burden of illness and unmet needs in patients with rheumatoid arthritis: a current perspective. Rheumatol. Int. 2016;36:685-95. 


\section{Supplementary}

\section{Questionnaire}

QI: Where do you work? (city, country)

Q2: How many RA patients do you treat?
a. Less than 100
b. Between 100 and 300
c. More than 300
d. I'm not a physician

Q3: What should be the definition for not well-controlled disease in the definition of difficult-to-treat RA?

a. DAS28-ESR > 3.2

b. DAS28-ESR > 5.1

c. DAS28-ESR > 3.2 OR presence of signs suggestive of active inflammatory disease activity with a DAS28-ESR $\leq 3.2$

d. DAS28-ESR > 5.1 OR presence of signs suggestive of active inflammatory disease activity with a DAS28-ESR $\leq 5.1$

e. Other (please specify)

Q4: Would you include fatigue in the definition of not well-controlled disease?
a. Yes
b. No
c. Please elaborate (if applicable)
d. Other (please specify)

Q5: Which and how many antirheumatic drugs should at least be tried with insufficient effect (failed) for the definition of difficult-to-treat RA:

a. $\geq 1$ csDMARD AND $\geq 2 \mathrm{~b} / \mathrm{tsDMARDs}$ with different mode of action

b. $\geq 2$ csDMARDs AND $\geq 2$ b/tsDMARDs with different mode of action

c. $\geq 2$ csDMARDs AND $\geq 3 \mathrm{~b} /$ tsDMARDs with different mode of action

d. $\geq 2$ csDMARDs AND $\geq 4 \mathrm{~b} /$ tsDMARDs with different mode of action

e. Further suggestions (please specify)

Q6: Treatment with glucocorticoids should be mentioned in the criteria for difficult-totreat RA as follows:

a. Unable to taper glucocorticoids below $5 \mathrm{mg}$ prednisone or equivalent daily for more 
than 1 year, irrespective of DMARD treatment

b. Unable to taper glucocorticoids below $10 \mathrm{mg}$ prednisone or equivalent daily for more than 1 year, irrespective of DMARD treatment

c. Glucocorticoids should not be included in the criteria

d. Suggested glucocorticoid dose/duration of treatment (if other than above)

Q7: Please define any additional characteristics and suggested criteria for difficult-totreat RA

Q8: Please mention additional clinical issues or comorbidities to be addressed in the workup and management of these patients

Q9: Please mention any clinically relevant situations which are not covered by the current RA EULAR recommendations

\section{Categories per question}

We have created these categories to categorise the responses to the open questions. If not clearly stated if a disease/problem was meant as 'extra-articular manifestation' or as 'interfering comorbidity' it was classified according to both categories.

Q7: Please define any additional characteristics and suggested criteria for difficult-totreat RA

- Interfering comorbidities

- Extra-articular manifestations

- Radiographic progression

- Persistence of US synovitis

- Side effects

- Treatment non-adherence

- Already included in former questions

- Arthritis of the feet

- Treatment delay

- Non-inflammatory complaints

- Other 
Q8: Please mention additional clinical issues or comorbidities to be addressed in the workup and management of these patients

- Cardiovascular disease

- Extra-articular manifestations

- Infection

- Malignancy

- Diabetes Mellitus

- Osteoporosis

- Pain syndrome

- Lung disease

- Kidney disease

- Depression

- Obesity

- Gastrointestinal disease

- Osteoarthritis

- Other

Q9: Please mention any clinically relevant situations which are not covered by the current RA EULAR recommendations

- Interfering comorbidities

- Extra-articular manifestations

- Pharmacological management

- Pain syndrome

- Pregnancy/lactation

- Other 



\section{CHAPTER 5}

\section{EULAR definition of difficult-to-treat rheumatoid arthritis}

Ann Rheum Dis 2021;80:31-5.

György Nagy, Nadia M.T. Roodenrijs, Paco M.J. Welsing, Melinda Kedves, Attila Hamar, Marlies C. van der Goes, Alison Kent, Margot Bakkers, Etienne Blaas, Ladislav Šenolt, Zoltán Szekanecz, Ernest Choy, Maxime Dougados, Johannes W.G. Jacobs, Rinie Geenen, Johannes W.J Bijlsma, Angela Zink, Daniel Aletaha, Jan L.M. Schoneveld, Piet van Riel, Loriane Gutermann, Yeliz Prior, Elena Nikiphorou, Ferraccioli Gianfranco, Georg Schett, Kimme Hyrich, Ulf Müller-Ladner, Maya H. Buch, lain B. McInnes, Désirée van der Heijde,* Jacob M. van Laar* 


\section{Abstract}

\section{Objectives}

Despite treatment according to the current management recommendations, a significant proportion of patients with rheumatoid arthritis (RA) remain symptomatic. These patients can be considered to have 'difficult-to-treat RA'. However, uniform terminology and an appropriate definition are lacking. The Task Force in charge of the development of EULAR recommendations for the comprehensive management of difficult-to-treat rheumatoid arthritis aims to create recommendations for this underserved patient group. Herein, we present the definition of difficult-to treat RA, as the first step.

\section{Methods}

The Steering Committee drafted a definition with suggested terminology based on an international survey among rheumatologists. This was discussed and amended by the Task Force, including rheumatologists, nurses, health professionals and patients, at a face-to-face meeting until sufficient agreement was reached (assessed through voting).

\section{Results}

The following three criteria were agreed by all Task Force members as mandatory elements of the definition of difficult-to-treat RA: 1. treatment according to EULAR recommendation and failure of $\geq 2 \mathrm{~b} / \mathrm{tsDMARDs}$ (with different mechanisms of action) after failing csDMARD therapy (unless contraindicated); 2. presence of at least one of the following: at least moderate disease activity; signs and/or symptoms suggestive of active disease; inability to taper glucocorticoid treatment; rapid radiographic progression; RA symptoms that are causing a reduction in quality of life; 3 . the management of signs and/or symptoms is perceived as problematic by the rheumatologist and/or the patient.

\section{Conclusions}

The proposed EULAR definition for difficult-to-treat RA can be used in clinical practice, clinical trials and can form a basis for future research. 


\section{Introduction}

European League Against Rheumatism (EULAR) recommendations provide valuable guidance to direct the management of rheumatoid arthritis (RA). The treat-to-target (T2T) strategy advises an agreed disease activity target, remission or at least low disease activity, that can in turn inform responsive treatment escalation. ${ }^{1-3}$ However, a number of patients remain symptomatic despite recommended treatment changes reflecting the complex interplay of disease and wider patient and clinical factors that leads to the increasingly recognised term of 'difficult to treat RA'. 4-7

A recent international survey of rheumatologists highlighted the perceived management problems and features in this patient category; the results of which confirmed the unmet need of this subpopulation of RA patients. ${ }^{8}$ The survey indicated that in addition to new drugs, new management approaches are also needed for the optimal treatment of these patients. Consequently, a EULAR Task Force was established to derive comprehensive recommendations addressing unmet needs in the management of difficult-to-treat (D2T) RA. Uniform terminology and a clear definition for this patient group are lacking. In the current literature, different terms are used to describe this subpopulation of RA patients, e.g. severe, refractory, resistant to multiple drugs or treatments, established and difficult-to-treat. ${ }^{4-7}$ As an initial step in the development of the management recommendations for D2T RA, terminology and a definition of this complicated RA patient group was established by the Task Force, guided by the results of the survey. ${ }^{8}$

\section{Methods}

\section{Steering Committee and Task Force}

The Steering Committee of the Task Force included a convenor (GN), co-convenor (JMvL), two methodologists (PMJW/DvdH) a rheumatology postdoctoral fellow $(\mathrm{MJHdH})$ and three fellows (NMTR, MK and AH). The Task Force comprises 32 individuals (including the Steering Committee members) of which 25 members were present at the first Task Force meeting, which took place in August 2018. Among the Task Force members, there were 26 rheumatologists (including two EMerging EUlar Network (EMEUNET) representatives), two patient partners, one health professional, one psychologist, one pharmacist and one occupational therapist. All rheumatologists are experienced in the treatment of RA, the majority with significant experience in clinical trials and a proportion in outcomes research. Numerous Task Force members have a leading role in organising and evaluating patient registries. All Task Force members declared their potential conflicts of interest before the start of the project. 


\section{Survey}

An online survey was conducted among rheumatologists to identify characteristics of D2T RA; the survey was distributed by email via the authors' networks and by EMEUNET. The survey consisted of nine questions, including two general questions 'Where do you work? How many RA patients do you treat?', and four multiple-choice and three open questions regarding the definition of D2T RA. Four hundred and ten respondents from 33 countries completed the survey between July 2017 and March 2018, 96\% of the respondents were European. ${ }^{8}$

\section{Development of terminology and definition for D2T RA}

The Steering Committee created the first draft of the definition based on the results of the survey and on a scoping literature search that was performed to explore different definitions that currently have been used (by NMTR,MJHdH and PMJW, see Supplementary). The results of the survey, the proposed terminology and the draft definition were presented to the Task Force at the first Task Force meeting. The definition was divided into three parts: treatment failure history, characterisation of active/symptomatic disease and clinical perception.

\section{Agreement process}

After the presentation of the draft terminology and definition, the general concepts were discussed and amended. Thereafter, the detailed wording was discussed and amended until consensus was reached. A voting process was conducted for each item of the terminology and definition. In case no consensus was reached among the present Task Force members, the preferred version was selected by voting. Twenty-one Task Force members were present during this discussion and voting process. After the meeting, two versions of the definition were distributed among all Task Force members to select the final version.

\section{Results}

\section{Terminology}

At the first Task Force meeting, based on a scoping literature search and the suggestions of the Task Force members a variety of potential terms to describe this patient population were presented, including severe, refractory, multi-drug/treatment resistant and complex RA. None of these terms was deemed to cover the wide range of possible clinical scenarios which may be relevant for this patient population. Since 'difficult-totreat' is a widely accepted term in several fields including pulmonology, psychiatry and cardiology, $^{9-11}$ this terminology was finally proposed by the Steering Committee and 
unanimously endorsed by the Task Force (21/21 agreed by voting).

\section{Definition}

Thereafter, we sought to create a definition of D2T RA based on the results of the previously mentioned international survey ${ }^{8}$ and expert opinion. The Task Force agreed that both articular and extra-articular components should be considered and agreed to include the following criteria in the definition: 1. treatment failure history; 2. characterisation of active/symptomatic disease; 3 . clinical perception. All three criteria need to be present to confirm the state of D2T RA.

\section{Criterion \#1: Treatment failure history}

In the survey, $48 \%$ of the respondents selected ' $\geq 2$ conventional synthetic diseasemodifying antirheumatic drugs (csDMARDs) AND $\geq 2$ biological (b-) DMARDs or targeted synthetic (ts-) DMARDs with different mode of action' for the number and type of antirheumatic drugs that should have failed before a patient can be considered to have D2T RA. The Steering Committee initially proposed to include treatment duration in the definition 'Treatment according to the current standard of care/EULAR recommendations for $\geq 1$ year'. This was chosen so that D2T RA patients are in phase III of the current RA management recommendations, in which no recommendation is given other than to switch to another b/tsDMARD.' However, inclusion of a certain time period in the definition was not supported by all Task Force members (primarily in order to provide flexibility) and the Task Force voted against referral to a treatment duration period for the definition of D2T RA (19/21 agreed, 2 abstained).

All Task Force members agreed to include the number of DMARDs previously failed in the definition and to create the definition consistent with the current EULAR RA management recommendations. 'Failure of at least $2 \mathrm{~b} /$ tsDMARDs with different mode of action' was selected by the majority of the respondents of the survey. ${ }^{8}$ Although according to the current EULAR recommendations ${ }^{1}$ no prioritisation for switching mechanism of action versus cycling is stated, it was decided that before being classified as D2TRA, a patient should at least have failed two b/tsDMARDs with different mechanisms of action. Consequently, it was decided to select this cut-off by the Task Force. With this cut-off, patients had to have completed phase III of the recommendations at least once (i.e. they may also have been treated with multiple bDMARDs of a single class (e.g. several TNF-inhibitors) and also have failed another b/tsDMARD). Finally, all members agreed to select the following proposal: 'Treatment according to EULAR recommendation and failure of $\geq 2 \mathrm{~b} / \mathrm{tsDMARDs}$ with different mechanisms of action after failing CSDMARD therapy (unless contraindicated)' (21/21 agreed). This also indicates that if CSDMARD treatment is contraindicated, failure of $\geq 2 \mathrm{~b} / \mathrm{tsDMARDs}$ with different mechanisms of action is sufficient. 
Socioeconomic factors may limit the access to expensive DMARDS (e.g. in low income countries), therefore (with the agreement of all Task Force members) we have added to the first criterion: 'unless restricted by access to treatment due to socioeconomic factors'.

\section{Criterion \#2. Characterisation of active/symptomatic disease}

Fifty percent of the respondents of the international survey selected 'disease activity score assessing 28 joints using erythrocyte sedimentation rate (DAS28-ESR) >3.2 OR presence of signs suggestive of active inflammatory disease activity with a DAS28-ESR $\leq 3.2^{\prime}$ as a characteristic of D2T RA. Additionally, $95 \%$ of the respondents of the international survey suggested to include the inability to taper glucocorticoids (GCs) in the criteria of D2T RA. ${ }^{8}$ Therefore, the Steering Committee proposed the following characterisation of active/ symptomatic disease: 'Presence of active disease defined as $\geq 1$ of: 1. DAS28-ESR >3.2; 2 . Presence of signs suggestive of active RA; and/or 3. Inability to taper oral glucocorticoids (below $7.5 \mathrm{mg} /$ day prednisone or equivalent)'. At the Task Force meeting, it was decided to include not only DAS28, which was the only composite disease activity measure offered in the survey, but to rather use a more generic definition: 'at least moderate disease activity (according to validated composite measures including joint counts e.g. DAS28-ESR >3.2 or CDAI >10)' (21/21 agreed). In addition to clinical signs and symptoms, it was agreed that this clarification should also include imaging and biochemical markers suggestive of active disease.

Furthermore, all Task Force members agreed that not only patients with joint related problems should qualify to be defined as being D2T. Extra-articular manifestations, such as vasculitis, pericarditis, scleritis or glomerulonephritis may complicate the management of RA, and were therefore decided to be included in the definition. This resulted in the following wording: 'Signs (including acute phase reactants and imaging) and/or symptoms suggestive of active disease (joint related or other)' (21/21 agreed).

In the survey, $43 \%$ of the respondents selected to include 'unable to taper glucocorticoids below $5 \mathrm{mg}$ prednisone or equivalent daily' and 46\% selected 'unable to taper glucocorticoids below $10 \mathrm{mg}$ prednisone or equivalent daily' (in addition, another 6\% chose to include inability to taper GCs, although with a different, unspecified dose). ${ }^{8}$ At the Task Force meeting, it was decided to include the following definition as a compromise: 'Inability to taper oral glucocorticoids (below $7.5 \mathrm{mg} /$ day prednisone or equivalent)'. The Task Force voted to keep this item in the definition (19/2l agreed).

During the Task Force meeting, additional possible signs of active disease were explicitly suggested for inclusion in the definition. First, the Task Force agreed to include rapid radiographic disease in the definition as a possible feature of D2T RA, as this might be occasionally observed even in case of clinically inactive disease. The following was proposed: 'Rapid radiographic progression (with or without signs of active disease)' (21/21 agreed). Second, non-inflammatory disease was considered, since these complaints, 
e.g. concomitant fibromyalgia, might mimic inflammatory activity. Non-inflammatory disease may lead to several aforementioned characteristics of active/symptomatic disease. Furthermore, non-inflammatory disease might also lead to other clinically import complaints. Therefore, to ensure that patients with non-inflammatory complaints could be classified as having difficult-to-treat RA, it was suggested and unanimously agreed to add 'Well-controlled disease according to above standards, but still having RA symptoms that are causing a reduction in quality of life' (21/21 agreed).

The Task Force discussed whether to add fatigue to the definition, as this is one of the most common problems. ${ }^{12,13}$ Since fatigue can diminish quality of life, it was suggested to be already included in the definition. In accordance with the survey (58\% of respondents suggested not to include fatigue), ${ }^{8}$ all Task Force members agreed to leave out the explicit mentioning of fatigue from the definition of D2T RA (21/21 agreed).

\section{Criterion \#3: Clinical perception}

As a final criterion, the Steering Committee suggested to include 'The disease is perceived as problematic by the rheumatologist and/or the patient'. This suggests that only clinical scenarios which are judged as problematic (e.g. apparently ineffective treatment) are referred to as D2T RA. Since the definition is only applicable to patients in which a management problem is present, it was agreed to adapt the definition accordingly: 'The management of signs and/or symptoms is perceived as problematic by the

Table 1. Definition of difficult-to-treat RA

1. Treatment according to EULAR recommendations and failure of $\geq 2 \mathrm{~b} / \mathrm{tsDMARDs}$ (with different mechanisms of action)' after failing csDMARD therapy (unless contraindicated) ${ }^{2}$

2. Signs suggestive of active/progressive disease, defined as $\geq 1$ of:

a. At least moderate disease activity (according to validated composite measures including joint counts e.g. DAS28-ESR >3.2 or CDAl >10)

b. Signs (including acute phase reactants and imaging) and/or symptoms suggestive of active disease (joint related or other)

c. Inability to taper glucocorticoid treatment (below $7.5 \mathrm{mg} /$ day prednisone or equivalent)

d. Rapid radiographic progression (with or without signs of active disease) ${ }^{3}$

e. Well-controlled disease according to above standards, but still having RA symptoms that are causing a reduction in quality of life

3. The management of signs and/or symptoms is perceived as problematic by the rheumatologist and/or the patient

All three criteria need to be present in D2T RA.

b: biological; CDAl: clinical disease activity index; cs: conventional synthetic; DAS28-ESR: disease activity score assessing 28 joints using erythrocyte sedimentation rate; DMARD: disease-modifying antirheumatic drug; EULAR: European League Against Rheumatism; mg: milligram; RA: rheumatoid arthritis; ts: targeted synthetic.

1. Unless restricted by access to treatment due to socioeconomic factors.

2. If CSDMARD treatment is contraindicated, failure of $\geq 2 \mathrm{~b} / \mathrm{tsDMARDs}$ with different mechanisms of action is sufficient.

3. Rapid radiographic progression: change in van der Heijde-modified Sharp score $\geq 5$ points at 1 year.14 
rheumatologist and/or the patient'. There were some concerns that this criterion might be too subjective, especially for research. However, the focus of the recommendations should be on the clinical implications, which supports to include this criterion. All Task Force members agreed unanimously on this (21/21 agreed).

\section{Order}

Most Task Force members agreed to start the definition with the treatment failure history criterion instead of the characterisation of active/symptomatic disease. However, the group noted that starting with signs of active disease might be better focused on the patients' needs. Therefore, with the agreement of all Task Force members, it was decided to vote on the order of the two criteria, by which all Task Force members supported the first version of the definition (agreed 31/31 ( $\mathrm{AH}$, who joined the Task Force later, did not vote), Table 1).

\section{Discussion}

The treatment of the heterogeneous patient population that comprises D2T RA is often a clinical challenge for which practical management recommendations are needed. Several factors may complicate the management of these patients. Such factors include persistent inflammatory activity due to resistance of disease to DMARDs, limited drug options due to adverse drug reactions and/or comorbidities that preclude the use of DMARDs or treatment non-adherence. On the other hand, concomitant syndromes or diseases, such as fibromyalgia, osteoarthritis and psychosocial factors associated with poor coping, can result in non-inflammatory symptoms (e.g. pain) that can mimic inflammatory activity and therewith contribute to D2T RA. Currently, D2T RA EULAR management recommendations are under development, aiming to cover all inflammatory and non-inflammatory factors underlying D2T RA. These will include both pharmacological and non-pharmacological treatment options and will be complementary to the existing RA recommendations. ${ }^{1-3}$ As an essential initial step in the development of recommendations for D2T RA, the Task Force provided terminology and a definition of D2T RA.

The term difficult-to-treat (D2T) was selected because it was deemed to best capture the possible clinical scenarios. A definition of D2T RA, consisting of three criteria was agreed on by consensus by a multidisciplinary group of experts including patient representatives: 1. treatment failure history; 2 . characterisation of active/symptomatic disease; 3 . clinical perception. These elements were selected based on the results of the survey.

The second criterion has five subelements, reflecting all potential clinically meaningful indicators of active/symptomatic disease. In this definition, in accordance with recent 
recommendations, the term 'moderate disease activity according to validated composite measures including joint counts' was used.,3 However, these indices might not always include the affected joints (e.g. feet) or other signs of disease activity. ${ }^{15}$ The 'Signs (including acute phase reactants and imaging) and/or symptoms suggestive of active disease (joint related or other)' item covers all potentially affected joints, as well as extra-articular manifestations.

The acceptable GC dose for chronic use remains a matter of discussion, although there is a significant group of RA patients that is treated with GCs long term. Current EULAR RA recommendations suggest to consider using GCs, when initiating or changing CSDMARDs, but GCs should be tapered as rapidly as clinically feasible.' The EULAR Task Force in charge of evaluating the risk of long-term GC therapy suggested that the risk of harm is generally low at long-term doses of $\leq 5 \mathrm{mg}$ prednisone equivalent per day. ${ }^{16} \mathrm{In}$ the currently proposed definition of D2T RA, in accordance with the result of the survey, 'Inability to taper glucocorticoid treatment (below $7.5 \mathrm{mg} /$ day prednisone or equivalent)' is listed as a criterion. We realise that lower GC doses were suggested by other EULAR Task Forces, on the other hand, we believe that less stringent criteria will be more realistic to define the D2T patients, as inability to follow these criteria can indicate a management problem.

The Task Force felt that not only patients fulfilling criterion 1 and 3 with inflammatory activity should be able to be classified as having difficult-to-treat RA, but also those patients with non-inflammatory complaints. Coexisting non-inflammatory conditions may lead to a high clinical burden. These may mimic inflammatory activity by hampering proper grading of disease activity and 'falsely' elevating disease activity scores through rather subjective measures. ${ }^{5}$ Additionally, these symptoms (such as fatigue or pain) could reduce quality of life. Therefore, the second criterion 'Signs suggestive of active/ progressive disease' was deemed to cover the wide variety of patients with inflammatory activity and/or non-inflammatory complaints.

There are some limitations of this work. The definition of D2T RA needs to be validated. Rheumatologists' and patients' acceptance can, as a first step, be used as a sign of face validity. Furthermore, not all aspects of D2T RA may have been adequately captured by the currently proposed definition, although the criteria mentioned are agreed on by a large group of experts based on a survey involving $>400$ rheumatologists. A further complicating factor might be that, as also apparent from the definition, this patient group is rather heterogeneous and hence difficult to capture in one definition.

In conclusion, the principal goal of RA management is to achieve sustained remission or at least low disease activity following steps of the current EULAR recommendations.' A new management approach is necessary for D2T RA patients, in which this treatment goal is not achieved. Hopefully, the definition presented here will provide a robust and consistent identification of patients with D2T RA. In addition, this definition can provide a 
platform to define a group of similar patients for research. Further work is underway to provide detailed recommendations for the management of D2T RA. 


\section{References}

1. Smolen JS, Landewé RBM, Bijlsma JWJ, et al. EULAR recommendations for the management of rheumatoid arthritis with synthetic and biological disease-modifying antirheumatic drugs: 2019 update. Ann Rheum Dis 2020;79:685-99.

2. Singh JA, Saag KG, Bridges SL, et al. 2015 American College of Rheumatology Guideline for the Treatment of Rheumatoid Arthritis. Arthritis Rheumatol 2016;68:1-26.

3. Smolen JS, Breedveld FC, Burmester GR, et al. Treating rheumatoid arthritis to target: 2014 update of the recommendations of an international task force. Ann Rheum Dis 2016;75:3-15.

4. Kearsley-Fleet L, Davies R, De Cock D, et al. Biologic refractory disease in rheumatoid arthritis: Results from the British Society for Rheumatology Biologics Register for Rheumatoid Arthritis. Ann Rheum Dis 2018;77:1405-12.

5. de Hair MJH, Jacobs JWG, Schoneveld JLM, et al. Difficult-to-treat rheumatoid arthritis: an area of unmet clinical need. Rheumatology (Oxford) 2017;57:1135-44.

6. Bécède M, Alasti F, Gessl I, et al. Risk profiling for a refractory course of rheumatoid arthritis. Semin Arthritis Rheum 2019;49:211-7.

7. Buch $\mathrm{MH}$. Defining refractory rheumatoid arthritis. Ann Rheum Dis 2018;77:966-9.

8. Roodenrijs NMT, de Hair MJH, van der Goes $M C$, et al. Characteristics of difficult-to-treat rheumatoid arthritis: results of an international survey. Ann Rheum Dis 2018;77:1705-9.

9. Robinson DS, Campbell DA, Durham SR, et al.
Systematic assessment of difficult-to-treat asthma. Eur Respir J 2003;22:478-83.

10. Thase ME. Therapeutic alternatives for difficult-to-treat depression: A narrative review of the state of the evidence. CNS Spectr 2004;9:808-21.

11. Papademetriou V, Tsioufis C, Gradman A, et al. Difficult-to-treat or resistant hypertension: Etiology, pathophysiology, and innovative therapies. Int J Hypertens 2011;2011:438198.

12. Almeida C, Choy EHS, Hewlett S, et al. Biologic interventions for fatigue in rheumatoid arthritis. Cochrane database syst Rev 2016;2016:CD008334.

13. Katz $P$, Margaretten $M$, Trupin L, et al. Role of Sleep Disturbance, Depression, Obesity, and Physical Inactivity in Fatigue in Rheumatoid Arthritis. Arthritis Care Res 2016;68:81-90.

14. Fautrel B, Granger B, Combe B, et al. Matrix to predict rapid radiographic progression of early rheumatoid arthritis patients from the community treated with methotrexate or leflunomide: results from the ESPOIR cohort. Arthritis Res Ther 2012;14:R249.

15. Landewé R, Van Der Heijde D, Van Der Linden $\mathrm{S}$, et al. Twenty-eight-joint counts invalidate the DAS28 remission definition owing to the omission of the lower extremity joints: A comparison with the original DAS remission. Ann Rheum Dis 2006;65:637-41.

16. Strehl C, Bijlsma JWJ, De Wit M, et al. Defining conditions where long-term glucocorticoid treatment has an acceptably low level of harm to facilitate implementation of existing recommendations: Viewpoints from an EULAR task force. Ann Rheum Dis 2016;75:952-7. 


\section{Supplementary}

\section{Scoping literature review}

Aims

- To identify definitions of difficult-to-treat rheumatoid arthritis (D2T RA)

- To check if these are in accordance with the proposed definition of D2T RA

\section{Search}

\# 1 PubMed: (resistant[Title/Abstract] OR resistance[Title/Abstract] OR persistent[Title/ Abstract] OR persistence[Title/Abstract] OR refractory[Title/Abstract] OR refractoriness[Title/Abstract] OR difficult-to-treat[Title/Abstract] OR "difficult to treat"[Title/Abstract]) AND ("Rheumatoid arthritis"[Title/Abstract] OR "Arthritis, Rheumatoid"[Mesh])

Filter: Human, publication date $<5$ yr

\# 2 PubMed: (resistant[Title/Abstract] OR resistance[Title/Abstract] OR persistent[Title/ Abstract] OR persistence[Title/Abstract] OR refractory[Title/Abstract] OR refractoriness[Title/Abstract] OR difficult-to-treat[Title/Abstract] OR "difficult to treat"[Title/Abstract]) AND ("Rheumatoid arthritis"[Title/Abstract] OR "Arthritis, Rheumatoid"[Mesh])

Filter: Review, systematic review, publication date $<5$ years

Search date: July 3, 2018

Inclusion criteria

- Research in humans

- RA patients

- Defining D2T RA

Results

Out of 1023 unique articles, 60 articles were included in the review (Supplementary Figure 1). The definition of D2T RA (or synonyms) is often not explained.

Only three articles used the same number of failed disease-modifying antirheumatic drugs (DMARDs) as used in the proposed definition of D2T RA, i.e. $\geq 2$ biological (b-) DMARDs (see Supplementary Table, which can be accessed via: https://doi.org/10.1136/ annrheumdis-2020-217344). The study designs of these articles were: one randomised controlled trial, one cohort study and one editorial. The other 57 articles used a definition that was defined less explicitly (e.g. failure to conventional synthetic (cs-)DMARDs and bDMARDs) or was defined less strictly compared to the proposed definition of D2T RA. 


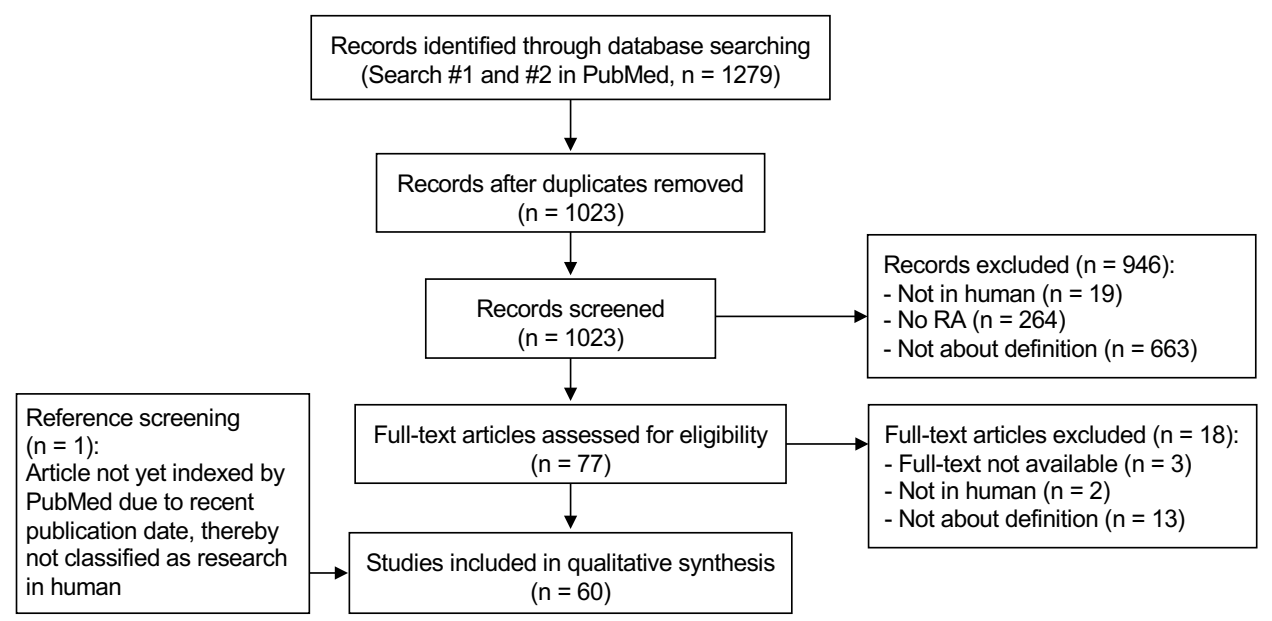

Supplementary Figure 1. Flow chart of search and selection of papers n: number; RA: rheumatoid arthritis. 



\section{CORRESPONDENCE RESPONSE}

Response to: 'Correspondence on 'EULAR definition of difficult-to-treat rheumatoid arthritis" by Novella-Navarro et al

Ann Rheum Dis 2020. doi: 10.1136/annrheumdis-2020-219535 [Epub ahead of print 4 Dec 2020]

Nadia M.T. Roodenrijs, Paco MJ. Welsing, Marlies C. van der Goes, Johannes W.G. Jacobs, Désirée van der Heijde, Jacob M. van Laar, György Nagy 
We read with great interest the correspondence of Novella-Navarro et al on our paper regarding the European League Against Rheumatism (EULAR) definition of difficultto-treat rheumatoid arthritis (D2T RA).1,2 We appreciate their acknowledgement of the need for uniform terminology and a uniform definition to describe the concept of D2T RA. Previously, this need had also been underlined by rheumatologists participating in an international survey. ${ }^{3}$ The use of heterogeneous terminology and definitions might hamper research and management of these patients. ${ }^{4-9}$

Novella-Navarro et al compared their definition of multi-refractory RA in their recently conducted retrospective study with the three criteria of the EULAR definition of D2T RA (Table 1).210 They classified RA patients as having 'multi-refractory' disease after failing $\geq 2$ biological and/or targeted synthetic disease-modifying antirheumatic drugs (b/ tsDMARDs) with different mechanisms of action or $\geq 3 \mathrm{~b} / \mathrm{tsDMARDs}$ with the same target, and as having 'non-refractory' disease if achieving low disease activity or remission on the first bDMARD. Noteworthy, $96 \%$ of their multi-refractory patients met the EULAR definition of D2T RA (Table 1), ${ }^{2}$ which supports the clinical usefulness of our definition.'

As some members of our Task Force also conducted a study in D2T RA patients," we feel it would be interesting to compare our results with those of Novella-Navarro et al, applying their slightly different criteria. ${ }^{10}$ In our cross-sectional study, consecutive RA patients, treated for at least one year, were prospectively enrolled and classified as having D2T RA if they fulfilled the EULAR definition (Table 1)! RA patients who did not fulfil

Table 1. Definition of difficult-to-treat RA

1. Treatment according to EULAR recommendations and failure of $\geq 2 \mathrm{~b} / \mathrm{tsDMARDs}$ (with different mechanisms of action)' after failing csDMARD therapy (unless contraindicated) ${ }^{2}$

2. Signs suggestive of active/progressive disease, defined as $\geq 1$ of:

a. At least moderate disease activity (according to validated composite measures including joint counts e.g. DAS28-ESR >3.2 or CDAl >10)

b. Signs (including acute phase reactants and imaging) and/or symptoms suggestive of active disease (joint related or other)

c. Inability to taper glucocorticoid treatment (below $7.5 \mathrm{mg} /$ day prednisone or equivalent)

d. Rapid radiographic progression (with or without signs of active disease) ${ }^{3}$

e. Well-controlled disease according to above standards, but still having RA symptoms that are causing a reduction in quality of life

3. The management of signs and/or symptoms is perceived as problematic by the rheumatologist and/or the patient

All three criteria need to be present in D2T RA.

b: biological; CDAl: clinical disease activity index; cs: conventional synthetic; DAS28-ESR: disease activity score assessing 28 joints using erythrocyte sedimentation rate; DMARD: disease-modifying antirheumatic drug; EULAR: European League Against Rheumatism; mg: milligram; RA: rheumatoid arthritis; ts: targeted synthetic.

1. Unless restricted by access to treatment due to socioeconomic factors.

2. If CSDMARD treatment is contraindicated, failure of $\geq 2 \mathrm{~b} / \mathrm{tsDMARDs}$ with different mechanisms of action is sufficient.

3. Rapid radiographic progression: change in van der Heijde-modified Sharp score $\geq 5$ points at 1 year..$^{2}$ 
all three criteria served as a control group.

Regarding the first criterion of the EULAR definition (Table 1), it should be noted that a specific number of failed csDMARDs is not included in the definition. Novella-Navarro et al showed that $95 \%$ of the multi-refractory patients (39 of 41 ) had been treated with $\geq 2$ csDMARDs. ${ }^{10}$ In our study, $89 \%$ of the D2T RA patients (46 of 52) failed $\geq 2$ csDMARDs." As cSDMARDs may be contraindicated and socioeconomic factors - including differences in the availability of (b/ts)DMARDs - may result in different treatment schedules between countries, the Task Force added two exceptions to the first criterion (Table 1)' enabling that the patients who it would concern could still fulfil the D2T RA definition.

As second criterion of the EULAR definition, patients should have 'signs suggestive of active/progressive disease (defined as $\geq 1$ of 5 prespecified items, Table 1).' One of the prespecified items is having 'at least moderate disease activity'. This was reflected in our study with D2T RA patients having a significantly higher DAS28-ESR compared with non-D2T RA patients (median (IQR): 4.1 (3.5-6.1) vs 2.5 (1.8-3.3), p<0.001)." In the paper of Novella-Navarro et al, disease activity scores when meeting the criteria of multirefractory RA are not described, prohibiting comparison of these results. ${ }^{10}$

The third criterion of the EULAR definition (Table 1)' was added to emphasise that the definition is only applicable to patients in whom a management problem is acknowledged. Novella-Navarro et al did not apply a similar criterion, although they found at the start of the first bDMARD statistically significantly worse levels of global patient assessment and functional disability in multi-refractory compared with in nonrefractory RA patients, potentially also indicating a management problem. ${ }^{10}$ In our study, the patients' burden of D2T RA was substantial too, indicated by statistically significantly worse levels of functional disability, quality of life, pain and fatigue compared with that of non-D2T RA." The Task Force discussed that this criterion might be too subjective and, therefore, might also be too complicated to integrate in research (specifically in retrospective studies). However, as the definition and management recommendations for D2T RA are primarily developed for clinical practice, eventually, the Task Force unanimously decided to add this criterion.

We agree with Novella-Navarro et al that identifying risk factors for developing D2T RA may be helpful to identify D2T RA patients. ${ }^{10}$ Identifying risk factors at RA onset may even help preventing development of D2T RA. D2T RA is a heterogeneous condition, in which various contributing factors can be present (e.g. concomitant fibromyalgia, treatment non-adherence, as described in our study).," By adequate management of these contributing factors, the risk of developing D2T RA may be diminished. In addition to the risk factors identified by Novella-Navarro et al, we identified lower socioeconomic status at RA onset as risk factor for developing D2T RA." Although potentially helpful, all identified risk and contributing factors should be validated in other cohorts of D2T RA patients before they can be implemented in management strategies in clinical 
practice.10,11

In summary, the EULAR definition of D2T RA, based on international consensus, has been established to unify terminology and the definition. Preferably, this definition will be used in future studies to classify D2T RA patients uniformly. However, we welcome studies such as that of Novella-Navarro et al to gain further insights into the intricate D2T RA state. Hopefully, all these initiatives, together with the EULAR recommendations for the management of D2T RA that are currently being developed, ${ }^{13-15}$ will eventually improve outcomes of D2T RA patients because that is what it is all about in the end. 


\section{References}

1. Nagy G, Roodenrijs NMT, Welsing PMJ, et al. EULAR definition of difficult-to-treat rheumatoid arthritis. Ann Rheum Dis 2020;80:31-5.

2. Novella Navarro M, Rodríguez C, Tornero C, et al. Correspondence on EULAR definition of difficult-to-treat rheumatoid arthritis by Nagy G, Roodenrijs NMT, Welsing PMJ, et al. Ann Rheum Dis 2020. Accepted for publication.

3. Roodenrijs NMT, de Hair MJH, van der Goes $\mathrm{MC}$, et al. Characteristics of difficult-to-treat rheumatoid arthritis: results of an international survey. Ann Rheum Dis 2018;77:1705-9.

4. de Hair MJH, Jacobs JWG, Schoneveld JLM, et al. Difficult-to-treat rheumatoid arthritis: an area of unmet clinical need. Rheumatology (Oxford) 2017;57:1135-44.

5. Buch $\mathrm{MH}$. Defining refractory rheumatoid arthritis. Ann Rheum Dis 2018;77:966-9.

6. Roodenrijs NM, de Hair MJ, van der Goes MC, et al. Correspondence to viewpoint 'Defining refractory rheumatoid arthritis' by Buch. Ann Rheum Dis 2019;78:e105.

7. Buch $\mathrm{MH}$. Response to 'Correspondence to viewpoint 'Defining refractory rheumatoid arthritis' by Buch' by Roodenrijs et al. Ann Rheum Dis 2019;78:e106.

8. Kearsley-Fleet L, Davies R, De Cock D, et al. Biologic refractory disease in rheumatoid arthritis: Results from the British Society for Rheumatology Biologics Register for Rheumatoid Arthritis. Ann Rheum Dis 2018;77:1405-12.

9. Bécède $M$, Alasti $F$, Gessl I, et al. Risk profiling for a refractory course of rheumatoid arthritis. Semin Arthritis Rheum 2019;49:211-7.
10. Novella-Navarro M, Plasencia C, Tornero C. Clinical predictors of multiple failure to biological therapy in patients with rheumatoid arthritis. Arthritis Res Ther 2020;22:284.

11. Roodenrijs NMT, van der Goes MC, Welsing PMJ, et al. Difficult-to-treat rheumatoid arthritis: contributing factors and burden of disease. Rheumatology 2020:keaa860.

12. Fautrel B, Granger B, Combe B, et al. Matrix to predict rapid radiographic progression of early rheumatoid arthritis patients from the community treated with methotrexate or leflunomide: results from the ESPOIR cohort. Arthritis Res Ther 2012;14:R249.

13. Roodenrijs NMT, Kedves M, Hamar A, et al. THUO110 Diagnostic issues in difficult-to-treat rheumatoid arthritis: preliminary results of a systematic literature review informing the 2020 EULAR recommendations for the management of difficult-to-treat rheumatoid arthritis. Ann Rheum Dis 2020;79:265-6.

14. Roodenrijs NMT, Hamar A, Kedves $M$, et al. SAT0052 Therapeutic strategies in difficultto-treat rheumatoid arthritis: preliminary results of a systematic literature review informing the 2020 EULAR recommendations for the management of difficult-to-treat rheumatoid arthritis. Ann Rheum Dis 2020;79:953-953.

15. Roodenrijs NMT, Hamar A, Kedves $M$, et al. FRI0047 Strategies regarding goal setting and self-management in difficult-to-treat rheumatoid arthritis: preliminary results of a systematic literature review informing the 2020 EULAR recommendations for the management of difficult-to-treat rheumatoid arthritis. Ann Rheum Dis 2020;79:595-595. 



\section{CHAPTER 6}

Immunological mechanisms underlying

DMARD inefficacy in difficult-to-treat

rheumatoid arthritis: a narrative review with

systematic literature search

Manuscript in preparation.

Nadia M.T. Roodenrijs, Paco M.J. Welsing, Joel van Roon, Jan L.M. Schoneveld, Marlies C. van der Goes, György Nagy, Michael J Townsend, Jacob M. van Laar 


\section{Abstract}

Management of rheumatoid arthritis (RA) patients has significantly improved over the past decades. However, a substantial proportion of patients is difficult-to-treat (D2T), these patients remain symptomatic after failing several biological and/or targeted synthetic disease-modifying antirheumatic drugs (b/tsDMARDs).

Multiple factors can contribute to D2T RA, including treatment non-adherence, comorbidities and co-existing mimicking diseases (e.g. fibromyalgia). Additionally, all currently available (b/ts)DMARDs may be truly ineffective ('true' refractory RA) and/or lead to unacceptable side effects. For this last group, more insight is needed into the underlying immunological mechanisms to optimise pharmacological management. In this narrative review based on a systematic literature search, an overview of underlying mechanisms is presented, focussing on immunology. Potential scenarios are discussed including the influence of different levels of gene expression regulation and clinical characteristics. Although the exact underlying mechanisms remain largely unknown, the identified heterogeneity between individual patients supports the assumption that (D2T) RA is a syndrome involving different pathogenic mechanisms. 


\section{Introduction}

With the introduction of biological and targeted synthetic disease-modifying antirheumatic drugs (b/tsDMARDs), outcomes of rheumatoid arthritis (RA) have significantly improved. ${ }^{1-3}$ However, there remains a substantial proportion of patients who can be classified as having 'difficult-to-treat (D2T)' RA. D2T RA has recently been defined as patients who remain symptomatic after failing at least two b/tsDMARD with different mechanisms of action (Table 1). ${ }^{4}$ The unmet need for these patients has been underlined by rheumatologists across Europe. ${ }^{5}$

The term 'refractory' RA is frequently used to describe D2T RA patients and may be incorrect in some cases. ${ }^{4,6-8}$ Multiple factors may contribute to the persistence of symptoms and/or signs in D2T RA patients, underscoring its heterogeneity and the difficulty in treating these patients (Figure 1).,6-11 Only if all currently available (b/ts)DMARDs are truly ineffective, 'true' refractory RA is present.

Mechanisms underlying DMARD inefficacy as well as (unacceptable) side effects in D2T RA are largely unknown. ${ }^{6,9,11}$ To be able to optimise treatment strategies, including the discovery of new therapeutic drug targets, more insight is needed into these underlying mechanisms in D2T RA patients.

The aim of this narrative review based on a systematic literature search was to explore if 'true' refractory RA exists and, if so, how frequent it is, and to explore and summarise

Table 1. EULAR definition of difficult-to-treat RA ${ }^{4}$

1. Treatment according to EULAR recommendations and failure of $\geq 2 \mathrm{~b} / \mathrm{tsDMARDs}$ (with different mechanisms of action)' after failing csDMARD therapy (unless contraindicated) ${ }^{2}$

2. Signs suggestive of active/progressive disease, defined as $\geq 1$ of:

a. At least moderate disease activity (according to validated composite measures including joint counts e.g. DAS28-ESR >3.2 or CDAl >10)

b. Signs (including acute phase reactants and imaging) and/or symptoms suggestive of active disease (joint related or other)

c. Inability to taper glucocorticoid treatment (below $7.5 \mathrm{mg} /$ day prednisone or equivalent)

d. Rapid radiographic progression (with or without signs of active disease) ${ }^{3}$

e. Well-controlled disease according to above standards, but still having RA symptoms that are causing a reduction in quality of life

3. The management of signs and/or symptoms is perceived as problematic by the rheumatologist and/or the patient

All three criteria need to be present in D2T RA.

b: biological; CDAl: clinical disease activity index; cs: conventional synthetic; DAS28-ESR: disease activity score assessing 28 joints using erythrocyte sedimentation rate; DMARD: disease-modifying antirheumatic drug; EULAR: European League Against Rheumatism; mg: milligram; RA: rheumatoid arthritis; ts: targeted synthetic.

1. Unless restricted by access to treatment due to socioeconomic factors.

2. If CSDMARD treatment is contraindicated, failure of $\geq 2 \mathrm{~b} / \mathrm{tsDMARD}$ with different mechanisms of action is sufficient.

3. Rapid radiographic progression: change in van der Heijde-modified Sharp score $\geq 5$ points at 1 year..$^{2}$ 


\section{DIFFICULT-TO-TREAT RHEUMATOID ARTHRITIS}

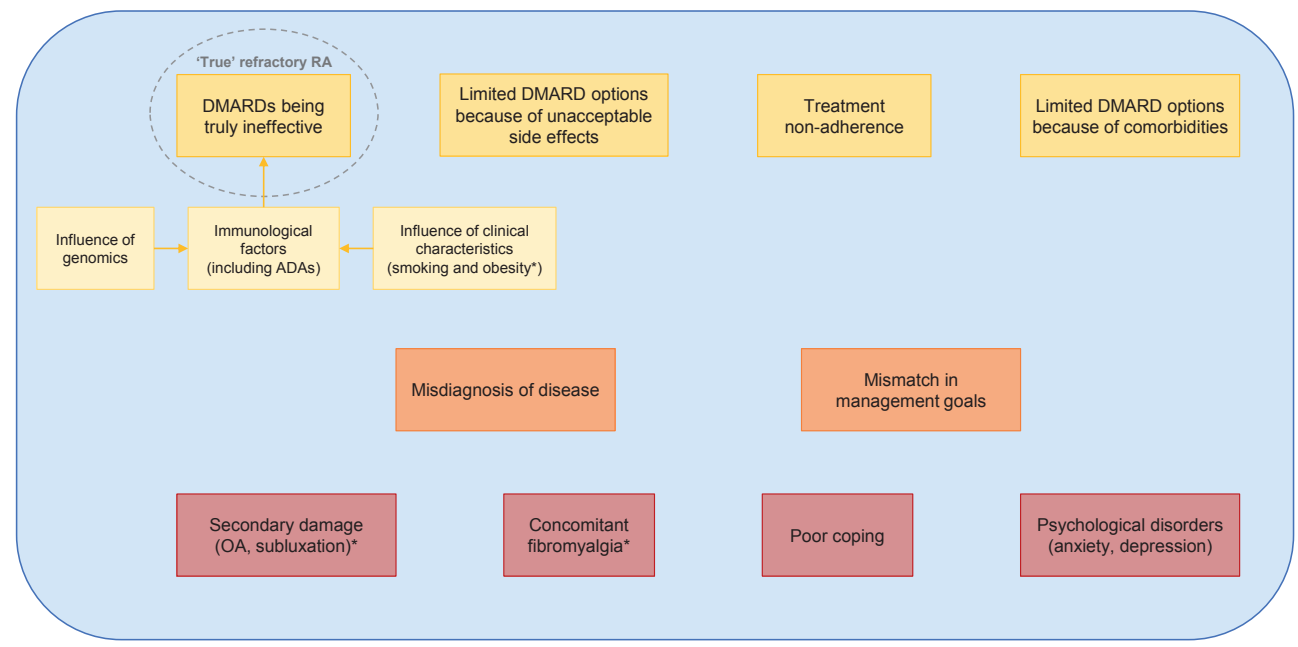

Figure 1. Factors contributing to difficult-to-treat rheumatoid arthritis

Multiple factors that potentially contribute to difficult-to-treat RA are presented. ${ }^{9,10}$ All contributing factors, except for misdiagnosis of disease, could coexist. 'true' refractory RA is only present if all currently available DMARDs are truly ineffective.

Factors in yellow may result in persistent inflammation; Factors in orange may result in non-inflammatory symptoms and/or persistent inflammation; Factors in red may result in non-inflammatory symptoms. *: These factors may additionally hamper proper grading of inflammatory disease activity.

ADAs: anti-drug antibodies; OA: osteoarthritis; RA: rheumatoid arthritis.

potential mechanisms underlying DMARD inefficacy as well as (unacceptable) side effects in D2T RA patients with a focus on immunological mechanisms that might mediate resistance.

\section{Methods}

\section{Research questions}

The systematic literature search was conducted following the EULAR Standardised Operating Procedures. ${ }^{13}$ The research questions were formulated and approved by the Task Force on management recommendations for D2T RA. ${ }^{14}$ The questions focussed on (a) the frequency of 'true' refractory RA, and (b) reasons for DMARDs being ineffective or toxic (including side effects) in D2T RA (Supplementary File). ${ }^{15}$

\section{Search strategy}

The databases of PubMed and Embase were searched for papers in English until November 2020. Additionally, the conference abstracts of EULAR and ACR from 2017 to 2020 
were screened. In addition to terms for RA, terms for potential underlying mechanisms, outcome measures and DMARDs were included (Supplementary File). A search limit was set to the last seven years. This cut-off was chosen because D2T RA is a new concept given the recent introduction of numerous $\mathrm{b} / \mathrm{tsDMARDs}$.

\section{Selection of studies, data extraction and analyses}

First, titles and abstracts were screened by NMTR and PMJW according to a set list of selection criteria (Supplementary File). Articles regarding RA patients who had been treated with at least one b/tsDMARD were eligible for inclusion. Articles were selected if they reported the frequency of refractory RA and/or an association between an underlying mechanism and DMARD inefficacy or (unacceptable) side effects. Second, full-text versions were screened. Articles were screened in duplicate, until the percentage of conflicts was below 5\%. Disagreements were discussed until consensus was reached. Information was extracted from the included articles using a predetermined format.

As many different underlying mechanisms exist and can be studied, results are summarised descriptively and additional context is narratively added.

\section{Results}

\section{Study characteristics}

The systematic literature search resulted in 3801 unique papers. After title, abstract and full text screening, 115 papers were selected (Figure 2).6,16-129

\section{Frequency of 'true' refractory RA}

The frequency of refractory RA was reported in two studies with different definitions and follow-up periods (Table 2).6,16 Both studies defined refractory RA as failure of at least two bDMARDs. Only the cohort study explicitly reported that these should be of different mechanisms of action, ${ }^{16}$ as in the D2T RA definition. ${ }^{4}$ In this cohort study, $6 \%$ started a third bDMARD with a different mechanism of action after a median of 8 years. In the narrative review, it was estimated that $20 \%$ of patients progress to a third bDMARD during their disease course. ${ }^{6}$ Although methodology used in these studies and reported incidence measures are different (i.e. different definitions of refractory RA (using a low hurdle response criterion in the narrative review; and using bDMARDs with another mechanism of action in the cohort study)), results may indicate that over lifetime a considerable part of RA patients become refractory to multiple ( $\geq 2)$ bDMARDs.

Nevertheless, not all currently available b/tsDMARDs have been tried in these patients nor other factors potentially contributing to D2T RA (Figure 1) have been excluded in these studies and, therefore, it remains formally unknown whether 'true' refractory RA 


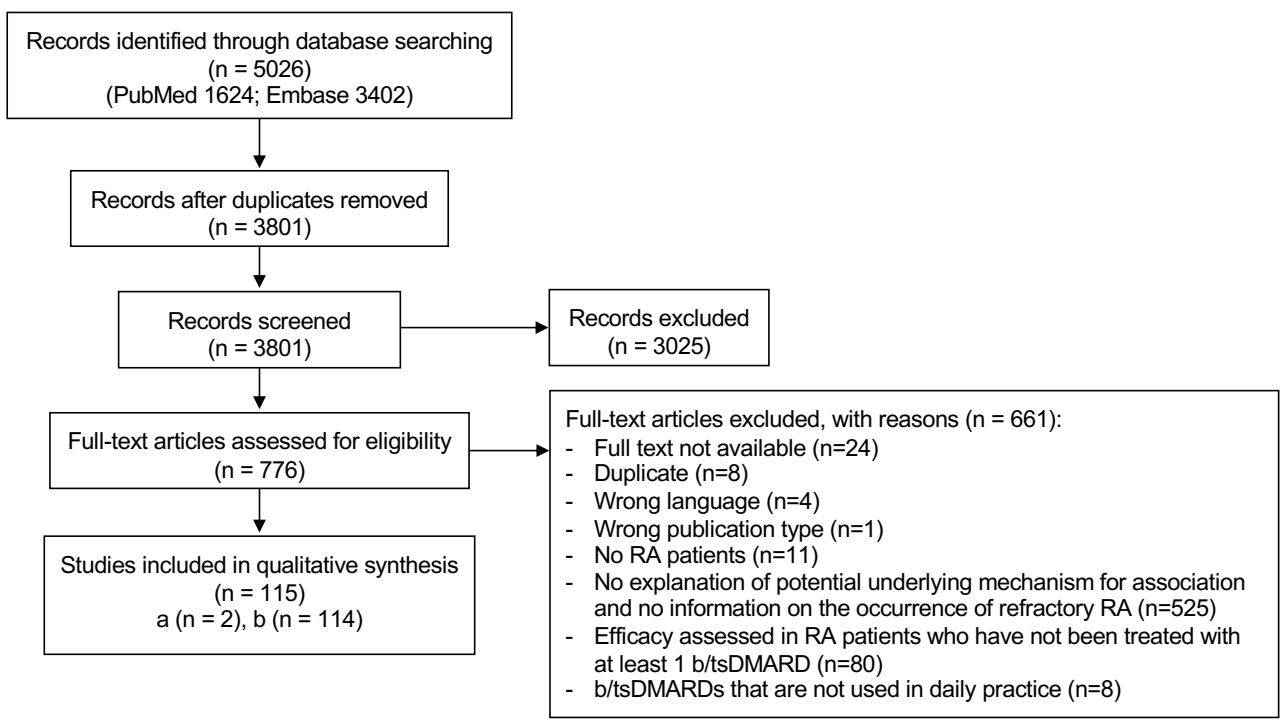

Figure 2. Flow chart of search and selection of papers

The questions focussed on ( $a$ ) the frequency of 'true' refractory RA, and (b) reasons for DMARDs being ineffective or toxic in D2T RA.

b/tsDMARD: biological/targeted synthetic disease-modifying antirheumatic drugs; n: number of studies; RA: rheumatoid arthritis.

really exists. Likely, the frequency of 'true' refractory RA will be much lower than estimated above, since 'true' refractory RA only represents a subgroup of these patients.

\section{Immunology underlying inefficacy}

Numerous studies have been performed on associations between treatment response to specific drugs and mechanisms underlying inefficacy. These do not only highlight the mechanism of action of the drug, but also reveal potential pathogenic pathways in non-responders when comparing them with responders. Although no studies have been performed in D2T RA patients (with 'true' refractory RA) specifically, the identified studies may aid in unravelling mechanisms underlying DMARD inefficacy in 'true' refractory RA. Below, we will discuss some examples as identified in the literature search (see also Figure 3).

The innate and the adaptive immune system

Individual differences in the role of the innate versus the adaptive immune system in the pathogenesis of RA have been suggested to explain differences in DMARD (in-)efficacy. ${ }^{6}$ Several studies showed the relation between good response to tumour necrosis factors inhibitors (TNFis) and the innate immune system, probably because innate cells are the main source of TNF. In a study in patients treated with TNFi, at baseline, genes associated 
Table 2. Papers on the frequency of 'true' refractory RA

\begin{tabular}{|c|c|c|c|c|}
\hline Paper & Design & $\begin{array}{l}\text { Description of } \\
\text { population }\end{array}$ & $\begin{array}{l}\text { Description of } \\
\text { refractory RA }\end{array}$ & $\begin{array}{l}\text { Frequency of 'true' } \\
\text { refractory RA }\end{array}$ \\
\hline Buch, $2018^{6}$ & $\begin{array}{l}\text { Narrative review } \\
\text { based on RCT data } \\
\text { (with low hurdle } \\
\text { response endpoints) }\end{array}$ & NA & $\begin{array}{l}\text { Failure of } \geq 2 \\
\text { bDMARDs }\end{array}$ & $\begin{array}{l}\text { Almost } 20 \% \text { progress } \\
\text { to a 3rd bDMARD } \\
\text { (estimation) }\end{array}$ \\
\hline Kearsley-Fleet, $2018^{16}$ & Cohort (BSRBR-RA) & $\begin{array}{l}\text { Patients with RA } \\
\text { starting first-line TNFi } \\
\text { from } 2001 \text { to } 2014 \\
\text { ( } n=13,502 ; 111,034 \\
\text { person years). }\end{array}$ & $\begin{array}{l}\text { Starting } 3^{\text {rd }} \text { class } \\
\text { of bDMARD } \\
\text { (with different } \\
\text { mechanisms of } \\
\text { action) }\end{array}$ & $\begin{array}{l}6 \%(n=867) \\
\text { developed } \\
\text { refractory RA } \\
\text { (median duration } 8 \\
\text { years) }\end{array}$ \\
\hline
\end{tabular}

bDMARD: biological disease-modifying antirheumatic drug; BSRBR-RA: British Society for rheumatology biologics register for RA; n: number; NA: not applicable; RA: rheumatoid arthritis; RCT: randomised controlled trial; TNFi: tumour necrosis factor inhibitor.

with innate immune cells were expressed at higher levels in good responders, while genes associated with adaptive immune cells were expressed at higher levels in nonresponders. ${ }^{48}$ Similarly, a pre-treatment myeloid phenotype (predominantly innate cells, i.e. macrophage and NF-kB process dominated) was associated with good response to TNFi in a study using synovial biopsies. ${ }^{26}$ Additionally, higher baseline levels of synovial macrophages (i.e. specific innate cells) have been associated with better response to TNFi. In another study, higher baseline levels of TNFRl expressing monocytes (i.e. specific innate cells) were associated with better response to etanercept. ${ }^{84}$

Contrary, good response to tocilizumab (an interleukin-6 receptor (IL-6R) antagonist) has been associated with the adaptive immune system. In the aforementioned synovial biopsy study, a pre-treatment lymphoid phenotype (predominantly adaptive cells, i.e. B cell- and plasmablast-dominated) was associated with good response to tocilizumab.43 However, the association between the innate or adaptive immune system and treatment response is not always as clear-cut as described above. For instance, higher baseline levels of innate cells (i.e. CD3-CD56+ natural killer (NK) cells) have also been associated with good response to tocilizumab. ${ }^{39}$ The innate and adaptive immune systems are interacting on various levels and can therefore not be seen as distinct. Their interaction is important, as innate immune cells are needed to trigger a specific immune response by adaptive cells. Cytokines, such as TNFa and IL-6, are crucial in this interaction, which also explains why both drugs can influence innate and adaptive immune cells. ${ }^{83}$ The importance of TNFa and IL-6 in the pathogenesis of RA is underlined by the success of bDMARDs targeting these cytokines (i.e. TNFi, tocilizumab and sarilumab).19,46,90 However, in D2T RA patients who fail both drugs due to true inefficacy, other immunological factors should underlie the persistence of inflammatory disease activity.

\section{T cells}

Decrease of self-tolerance by reduced frequencies of regulatory T cells (Tregs) is thought 

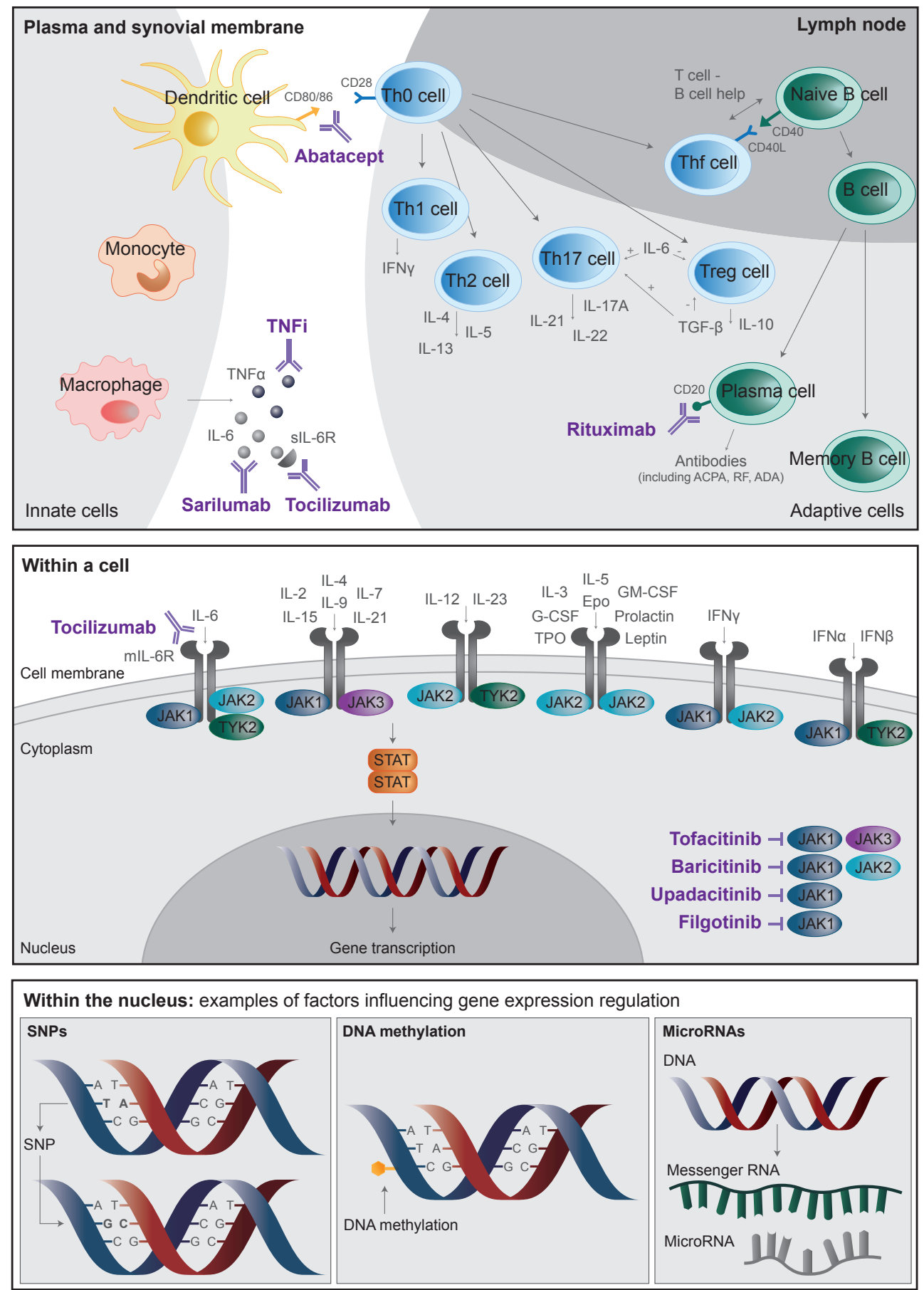

Figure 3. A simplified overview of innate and adaptive immune processes and intracellular signalling pathways in RA as well as potential factors influencing gene expression regulation 
Figure 3. Continued

In the plasma and synovial membrane (white and light grey) and lymph node (dark grey) a simplified overview of different innate and adaptive immune cells is shown as well as their interactions that lead to a multitude of proinflammatory mediators (including cytokines) that play a key role. Within the cell, different signalling pathways (e.g. JAK/STAT) that contribute to cell activation are shown. Biological and targeted synthetic disease-modifying antirheumatic drugs approved for the treatment of RA are shown in purple as well as the mechanisms/mediators they target.

Within the nucleus, examples of factors influencing gene expression regulation are presented. SNPs in the genetic code can enhance or repress gene transcription and affect the function of genes, if the SNP results in an amino acid change. Epigenetic modification can render the DNA more or less accessible to transcription factors. For instance, DNA methylation at CPG sites can repress genes. Additionally, microRNAs are another mode of gene expression regulation. MicroRNAs bind to messenger RNA products of target genes and block gene translation into proteins.

ACPA: anti-citrullinated protein antibodies; ADA: anti-drug antibodies; DNA: deoxyribonucleic acid; EPO: erythropoietin; IFN: interferon; IL: interleukin; JAK: janus kinase; G-CSF: granulocyte colony-stimulating factor; GM-CSF: Granulocyte-macrophage colony-stimulating factor; mIL-6R: membrane-bound IL-6 receptor; RA: rheumatoid arthritis; RF: rheumatoid factor; RNA: ribonucleic acid; slL-6R: soluble IL-6 receptor; SNP; singlenucleotide polymorphisms; STAT: signal transducer and activator of transcription; Th cell: T helper cell; Thf cell: follicular T helper cell; TNF: tumour necrosis factor; TNFi: tumour necrosis factor inhibitor; Treg cell: regulatory T cell; TPO: thrombopoietin.

to play a major role in the pathogenesis of RA and has also been related to DMARD (in-) efficacy. ${ }^{140}$ In patients treated with tocilizumab, an increase in Tregs during treatment has been associated with achieving remission. ${ }^{73} \mathrm{IL}-6$ decreases the differentiation of Tregs by inhibiting the expression of a specific transcriptional factor (FoxP3). Tocilizumab prevents this by blocking IL-6R, resulting in an increase in Tregs. This was also reflected in another study, in which increased Helios expression (a transcription factor, which is selectively induced in FoxP3+ Tregs and is inhibited by IL-6) in CD4+ Tregs after treatment with tocilizumab was associated with good response. ${ }^{20} \mathrm{~A}$ relation between Tregs and treatment response has also been found in patients treated with abatacept (which inhibits T cell costimulation). In responders to abatacept, increased activity of Tregs after treatment was found. ${ }^{89}$ Abatacept may increase the number of Tregs by enhancing early growth response gene 2 (EGR2) expression, which is responsible for negative regulation of T cell activation. ${ }^{141}$ Although these studies emphasise the heterogeneity in the underlying mechanisms between responders and non-responders, no findings were related to pathways that are still active in non-responders to these drugs.

Furthermore, the decreased self-tolerance by reduced frequencies of Tregs contributes to an expansion of Thl and Thl7 cells because the differentiation of Thl7 cells is stimulated by the same signals that inhibit Treg differentiation (e.g. IL-6 and TGF- $\beta$ ). ${ }^{140,142}$ In a study in patients treated with TNFi, high baseline levels of IL-17A producing Thl7 cells were associated with non-response. ${ }^{34}$ Targeting these Thl7 cells may therefore be a potential drug target in 'true' refractory RA. However, clinical trials in RA patients with molecules targeting IL-17A showed insufficient benefit of these drugs, ${ }^{143}$ although this may be a result of selecting an inappropriate patient population and does not directly mean that these 
drugs are not beneficial in 'true' refractory RA patients. Nevertheless, IL-17A serves as an amplifier of inflammation, ${ }^{144}$ so it is likely that IL-17A targeting drugs need to be combined with a TNFi or another cytokine-targeted therapy to be effective. ${ }^{145}$ This combination may therefore specifically be useful in 'true' refractory RA.

\section{B cells}

In addition to T cells, B cells are other key cells of the adaptive immune system. Higher baseline levels of (synovial) B cells may result in a more favourable response to rituximab (which directly targets CD20+ B cells). ${ }^{19}$ However, higher baseline levels of activated peripheral memory B cells (CD95+ Ki67) have been associated with non-response to rituximab.42 Depletion of these cells is less effective, as these cells reside in sites such as lymph nodes and do not circulate in the peripheral blood. Whether other b/tsDMARDs may influence these cells is unknown. Presence of these activated memory B cells may therefore underlie DMARD inefficacy in 'true' refractory RA.

Furthermore, the presence of synovial lymphoid aggregates (containing B an T cells), which resemble the characteristics of secondary lymphoid organs, has been associated with worse response to TNFi. ${ }^{46}$ These lymphoid aggregates produce TNFa, resulting in higher TNFa levels, 147,148 which may at least partly explain this association. Additionally, the presence of these lymphoid aggregates has been associated with more refractory disease, potentially suggesting that these can also underlie DMARD inefficacy in 'true' refractory RA.

\section{Anti-drug antibodies}

The presence of ADAs is a well-accepted mechanism underlying DMARD inefficacy. They neutralise bDMARDs resulting in subtherapeutic serum drug levels (i.e. immunogenicity) $6,85,9,99,106,108,126$ ADAs are mainly IgM and IgG antibodies, produced by $B$ cells, although IgE antibodies may also occur. ${ }^{28,91}$ ADAs are more frequently found during treatment with non-fully human-derived antibodies: the chimeric TNFi (infliximab) and humanized antibodies (TNFis: adalimumab, certolizumab pegol and golimumab).28,85,26 However, ADAs have also been found for other bDMARDs: etanercept (another TNFi), abatacept, rituximab and tocilizumab. $28,30,131$ Frequencies differ, although numbers up to $53 \%$ for infliximab and 30\% for adalimumab have been reported. ${ }^{28,32}$ For adalimumab, certolizumab pegol, golimumab and infliximab, ADAs mostly target the Fab part, which directly neutralises the drug..$^{91}$ ADAs can also target the Fc part, which does not neutralise the drug, but can result in deceased circulating levels due to enhanced pharmacokinetic clearance of these immune complexes.

Several factors may increase the risk of bDMARD immunogenicity. Subcutaneous and intramuscular administration may result in a higher occurrence of ADAs due to differences in antigen presentation by dendritic cells (DCs).30,85,99,133 Additionally, a longer 
dose interval has been linked to a higher occurrence of ADAs, as this could trigger a secondary immune response. ${ }^{24,85,99,133}$ Patients with higher disease activity levels may also be more prone to ADA formation. Here, DCs may increase their costimulatory proteins, which decreases the threshold for T cell stimulation and triggers the formation of ADAs. This may even trigger ADAs for bDMARDs that are less immunogenetic. ${ }^{134,135}$ Genetics, e.g. IL-10 polymorphisms, have also been associated with ADAs. ${ }^{106,136}$

Addition of methotrexate to bDMARDs has been shown to reduce the presence of ADAs and is associated with better response. 31,51,06 This may be explained by the suppression of early $T$ and $B$ cell expansion resulting in reduced ADA formation, but could also be due to the additional anti-inflammatory effect of methotrexate itself.

\section{Autoantibodies}

Inefficacy of bDMARDs has also been linked to seropositivity for anti-citrullinated protein antibodies (ACPA) and/or rheumatoid factor (RF). Presence of these autoantibodies has been related to differences in synovial tissue phenotypes, with B cell-rich synovitis associated with increased levels of autoantibodies. ${ }^{137,138}$ Seropositive patients were found to have a more favourable response to rituximab and abatacept compared to seronegative patients. ${ }^{50,53,100}$ This may be explained by the influence of these treatments on autoantibody producing B cells, contrary to cytokine-targeted therapies, such as TNFi and tocilizumab. ${ }^{53,39}$

\section{Intracellular signalling pathways}

Apart from mechanisms that explain DMARD inefficacy on the cellular level, DMARD inefficacy has been associated with intracellular signalling pathways. The successful introduction of janus kinase inhibitors (JAKi) reflects the role of the JAK/signal transducer and activator of transcription (STAT) signalling pathway in RA. ${ }^{149,150}$ The JAK/STAT signalling pathway consists of 4 JAK and 7 STAT proteins and facilitates the transmission of multiple chemical signals (e.g. cytokines) from outside the cell to the nucleus, resulting in the activation of genes through transcription. ${ }^{151}$

The JAK/STAT signalling pathway has also been related to bDMARD (in-)efficacy. For example, higher baseline levels of STAT-1 and -3 phosphorylation after ex vivo cytokine stimulation of leukocytes (with interferon-y (IFNy), IL-10, IL-4 and IL-2) have been associated with better clinical response to tocilizumab. ${ }^{94}$ STATI and STAT3 regulate Thl7 differentiation and may become exhausted after persistent activation by high IL-6 signals through IL6R. ${ }^{94,52}$ By blocking IL-6R with tocilizumab, these STATs become available again for other cytokines (e.g. IFNy) resulting in restored T cell balance. In another study in differently treated patients (TNFi, tocilizumab, rituximab), this was confirmed for STATI and also found for STAT6. ${ }^{77}$ STAT6 promotes expression of several Th2-specific transcription factors resulting in production of Th2 cytokines (e.g. IL-4, IL-5, IL-13) and Treg cell response.153 
Again, the pathways that are still active in non-responders remain unknown.

\section{Heterogeneity in underlying immunology}

The differences in the immunology underlying b/tsDMARD inefficacy emphasise the heterogeneity in the pathogenesis of individual RA patients. This heterogeneity is also present in synovial tissue of patients with early RA ${ }^{154,155}$ and patients who failed csDMARDs. ${ }^{156-158}$ In recent studies in early RA, three pre-treatment phenotypes have been identified: Iympho-myeloid (i.e. B cells and myeloid cells (innate and adaptive cells)), diffuse-myeloid (i.e. myeloid lineage predominance (innate cells)) and pauci-immune (i.e. stromal cells, few immune cells). ${ }^{154,155}$ The lympho-myeloid phenotype was associated with poorer prognosis: worse radiographic outcomes and a higher proportion of patients requiring bDMARDs at 12-month follow-up. ${ }^{155}$ This phenotype may thus be an early indicator of 'true' refractory RA, although studies in relation to bDMARD response still need to be performed.

Recently, the results of the R4RA trial have been published..$^{59}$ In this study, patients were treated with tocilizumab and rituximab, stratified using synovial biopsies at baseline in B-cell poor and B-cell rich patients. In patients histologically classified as B-cell poor and in those classified as B-cell rich, no statistically significantly benefit of either tocilizumab or rituximab was found. However, when patients were classified as B-cell poor based on RNA sequencing, significant higher response rates at 16 weeks were found for tocilizumab compared with rituximab. Not only may this indicate that RNA sequencing could be more sensitive than histology to assess the underlying immunology and may have a role in selecting the appropriate (b/ts)DMARD strategy, this also further highlights the heterogeneity in the underlying immunology in RA.

\section{Influence of (epi-)genetics}

In addition to cellular and molecular differences that contribute to (in-)efficacy, differences in genetics and epigenetics can underlie DMARD inefficacy. Epigenetic and genetic heterogeneity strongly affect gene expression, which can strongly influence immunological constitution and contribute to DMARD inefficacy as another independent layer (Figure 3). Although the majority of the findings is not (well) validated, the identified evidence reveals influences of gene expression regulation at multiple levels of genetic organisation (see also Supplementary Table 1).

\section{Single-nucleotide polymorphisms}

Single-nucleotide polymorphisms (SNPS) in the genetic code may be related to differences in DMARD (in-)efficacy. These SNPs can either be located in regulatory or coding regions of genes.

Alterations in the regulatory region can enhance or repress genetranscription. For example, 
a more favourable response to TNFi treatment was found in carriers of the rs2836249194ins/del ATTG polymorphism in the NF-kBl promoter, while homozygous carriers of the T-allele of the rs187084-1486T>C SNP in the toll-like receptor (TLR)9 promoter showed a less favourable response. ${ }^{56}$ TLRs are part of the innate immune response and promote an increase in inflammatory chemokines, cytokines and cell adhesion molecules via NFkB (a transcription factor). ${ }^{160,161}$

Variations in the coding regions of genes may lead to amino-acid substitution in the corresponding proteins and affect their function. The Fcy receptor regulates immune responses through the interaction with antibodies (including bDMARDs) and is expressed on all immune cells. In FCGR3A, within the gene for the Fcy receptor 3B, the rs396991 SNP $596 T>G$ nucleotide substitution causes an amino acid change of phenylalanine into valine. The valine isoform is considered to have a higher affinity to lgG than the phenylalanine isoform and correlates with a stronger immunological response. ${ }^{67,96}$ Carriers of one or more of the valine alleles had a better treatment response to rituximab, 67,96 while carriers of the FCGR3A 596T-genotype (lower affinity genotype) had a better response to tocilizumab. 67

\section{Epigenetics}

Epigenetic modification can render the DNA more or less accessible to transcription factors. These alterations could influence DMARD (in-)efficacy in individual patients. For instance, DNA methylation at CPG sites represses genes, whilst hypomethylation results in a permissive chromatin structure. For etanercept, five differentially methylated DNA positions at baseline have been identified to differ between responders and nonresponders, although this could not be confirmed in another study. ${ }^{162,163}$

MicroRNAs are another mode of gene expression regulation. ${ }^{164,65}$ These non-coding short single-stranded RNA molecules act by base pairing to messenger RNA products of target genes. The resulting double-stranded RNA molecules are recognised by the cell's machinery and targeted for degradation. Depending on the mode of action of the target gene, microRNAs could enhance or inhibit certain pathways. The microRNA expression profile of patients could therefore constitute another level at which differences in the DNA sequence can account for altered DMARD efficacy.

The expression of several microRNAs in RA patients who failed multiple bDMARDs (not further specified) has been compared with RA patients who failed csDMARDs, patients having low disease activity and healthy controls. ${ }^{52}$ A cluster of microRNA-23a, $-24-2$, and -27a was significantly reduced in patients who failed multiple bDMARDs and in those who failed csDMARDs. Interestingly, microRNA-23a and $-27 a$ were found to mediate the regulatory loop of IL-6 and their expression was found to be repressed by cytokines of the JAK/STAT signalling pathway (e.g. IFN-y and GM-CSF). Additionally, cells lacking microRNA-23a and $-27 a$ expressed higher levels of pro-inflammatory cytokines (e.g. 
TNFa and IL-6). Whether these patients failed bDMARDs interfering with these cytokines was not described.

\section{Influence of clinical characteristics}

\section{Smoking}

In addition to being a risk factor for the development of RA, smoking has also been associated with more severe disease and DMARD inefficacy. ${ }^{36,166}$ In multiple studies, smoking has been associated with reduced responsiveness to bDMARDs, especially to TNFis. ${ }^{78,167-170}$

Several mechanisms may explain the association between smoking and TNFi inefficacy. ${ }^{36}$ Smoking has been associated with higher levels of (IgA) RF and ACPA, a poor prognostic factor for response, by promoting the citrullination of proteins. ${ }^{2,17-174}$ Furthermore, smoking has been associated with higher levels of inflammatory cytokines: higher ratios of TNFa/ soluble TNF receptor ${ }^{175}$ and higher levels of serum soluble IL-2R.176,177 Additionally, a higher basal metabolism resulting in increased drug metabolism has been found among smokers. ${ }^{178}$ Therefore, higher doses of drugs may be needed in these patients. ${ }^{179}$

Furthermore, several (epi-)genetics have been associated with the relation between smoking and TNFi inefficacy. Higher expression levels of SEMA6B (a protein resulting in signal transduction of fibroblast growth factor-receptor-1 and -2 (FGFRl-2) and vascular endothelial growth factor-receptor 2 (VEGFR2)) and GPR15 (a chemo attractant for T cells on the cell surface of monocytes and neutrophils) are increased in smokers, potentially increasing T cell involvement and synovial vascular proliferation. ${ }^{37}$ In another study, the T allele of NLRP3(rs4612666) was associated with non-response to TNFi in current smokers. Smoking may result in the production of reactive oxygen species leading to increased expression and activation of the NLRP3 inflammasome, an intracellular innate immune sensor, which releases strong pro-inflammatory cytokines (IL-1ß and IL-18).112

\section{Obesity}

Obese patients may respond less favourably to TNFi. ${ }^{18,28}$ A first explanation may be the higher distribution volume and different pharmacokinetics in obese patients. 18,28 However, the same association has been found for infliximab, which dosage is adjusted for body weight. In early RA, the association between inefficacy and obesity was even found with infliximab doses up to 10 milligrams per kilogram. ${ }^{180}$ The increased level of adipocytes as a result of more fatty tissue may be another explanation.18 Adipocytes release adipokines, including leptin that is known to induce the expression of pro-inflammatory cytokines, such as TNFa and IL-6. The impact of obesity on synovial tissue has recently been shown. ${ }^{181}$ Even in obese RA patients who achieved remission after TNFi treatment, a higher degree of residual synovitis (CD68+ macrophages, CD20+ B cells, CD3+ T cells) was found compared to normal weight patients. 
Furthermore, the higher level of Fc-receptors in omental adipocytes may play a role. ${ }^{182,183}$ Infliximab is bound by these receptors, which may also explain why the association between obesity and inefficacy of infliximab is even stronger than that with certolizumab pegol that lacks the Fc fragment. ${ }^{128}$

\section{Immunology underlying (unacceptable) side effects}

Mechanisms underlying (unacceptable) side effects were generally found to be directly related to the mechanism of action of DMARDs (Table 3). For example, lack of immunity to infections can occur during treatment with tsDMARDs leading to reactivation of latent viral infections, e.g. herpes zoster infection. ${ }^{72,86,10,102,184}$ In this respect, different underlying mechanisms related to the JAK/STAT signalling pathway have been suggested: inhibition of STAT5 signalling resulting in impairment of Tregs or inhibition of the IFN-related pathway that is associated with innate antiviral surveillance. $22,86,102,15,184$

Additionally, the FCGR3B NAI/NAl genotype, within the gene for the Fcy receptor 3B, has been associated with infusion reactions to adalimumab and infliximab, as it may alter immune cellular function resulting in systemic hypersensitivity. ${ }^{20,85}$ An association between the presence of ADAs and hypersensitivity to infliximab has also been reported, ${ }^{28,85}$ although it is currently unknown why hypersensitivity only occurs in some patients.

Furthermore, two SNPs have been suggested to underlie the association between obesity and toxicity (not further specified) to combined treatment with etanercept and methotrexate. ${ }^{64}$ This suggests that clinical characteristics may also be an additional trigger.

\section{Future perspective}

Despite insights from studies on immunological mechanisms underlying (b/ts)DMARD (in-)efficacy, studies assessing the pathways that are still active in non-responders are scarce and studies in 'true' refractory RA patients who failed all currently available b/ tsDMARDs are lacking. Although some hints were found for a role of Thl7 cells, activated memory B cells (CD95+ Ki67) and synovial lymphoid aggregates, it remains unknown which exact mechanisms are responsible for the persistence of inflammatory disease activity in 'true' refractory RA. Likely, a combination of factors plays a role. In addition to individual differences in DMARD (in-)efficacy that underline the heterogeneity in the pathogenesis of (D2T) RA, individual differences in (epi-)genetics and clinical characteristics add an additional layer of complexity to this heterogeneity.

Future studies assessing mechanisms underlying DMARD inefficacy in 'true' refractory RA patients are needed, comparing mechanisms in D2T with those in non-D2T RA patients. 
Table 3. Examples of immunological mechanisms underlying (unacceptable) side effects

\begin{tabular}{|c|c|c|c|}
\hline Drug & $\begin{array}{l}\text { Description of } \\
\text { (unacceptable) side } \\
\text { effects }\end{array}$ & Mechanism & Ref \\
\hline \multicolumn{4}{|c|}{ Immunological mechanisms } \\
\hline TNFi & (Opportunistic) infections & Reduced recruitment and activation of macrophages & 28,21 \\
\hline TNFi & (Intracellular) infections & Lower level of TNFa & 60 \\
\hline TNFi & Fungal infections & Lower levels of IFNy & 22 \\
\hline $\begin{array}{l}\text { TNFi (INX, } \\
\text { ADM) }\end{array}$ & $\begin{array}{l}\text { Tuberculosis (higher risk } \\
\text { than with ETN) }\end{array}$ & $\begin{array}{l}\text { Induction of apoptosis in TNF-expressing macrophages and } \\
\text { T cells, and inhibition of both TNFRp55 and TNFRp75 }\end{array}$ & 22 \\
\hline TNFi & Lupus-like disease & $\begin{array}{l}\text { Exposure of nuclear antigens through the induction of } \\
\text { apoptosis of inflammatory cells }\end{array}$ & 28 \\
\hline TNFi & Lupus-like disease & Presence of ADAs & 60 \\
\hline TNFi (INX) & Hypersensitivity reactions & Presence of ADAs & 28,85 \\
\hline TNFi & TNFi-induced psoriasis & Higher levels of IFNa & 60 \\
\hline Tocilizumab & Neutropenia & Decreased neutrophil recruitment from the bone marrow & 28 \\
\hline Rituximab & Infusion reactions & $\begin{array}{l}\text { IgE mediated hypersensitivity resulting from the release } \\
\text { of histamine, leukotrienes, and prostaglandins from mast } \\
\text { cells, and cytokine-release by immune cells (B and NK cells) } \\
\text { resulting from the binding of the Fc portion of rituximab to } \\
\text { the FcyR3A/CDI6 }\end{array}$ & 98 \\
\hline TsDMARD & $\begin{array}{l}\text { Reactivation of latent viral } \\
\text { infections }\end{array}$ & $\begin{array}{l}\text { Inhibition of STAT5 and/or inhibition of the IFN-related } \\
\text { pathway }\end{array}$ & $\begin{array}{l}72,86, \\
102,184\end{array}$ \\
\hline GC & $\begin{array}{l}\text { Higher risk of GC-induced } \\
\text { osteoporosis }\end{array}$ & Inhibition of osteoblast differentiation & 59,76 \\
\hline GC & $\begin{array}{l}\text { Reduced muscle mass } \\
\text { and strength }\end{array}$ & Reduction in anabolic protein synthesis & 59 \\
\hline \multicolumn{4}{|c|}{ Influence of (epi-)genetics } \\
\hline MTX & Hepatic enzyme elevation & $\mathrm{ABCB} 13435 \mathrm{C}>\mathrm{T}$ and $\mathrm{ABCB} 13435 \mathrm{C}>\mathrm{T}$ & 58 \\
\hline \multirow[t]{5}{*}{ MTX } & $\begin{array}{l}\text { MTX-related } \\
\text { gastrointestinal disorders }\end{array}$ & ABCC2 rs2273697 & 105 \\
\hline & $\begin{array}{l}\text { MTX-related skin and } \\
\text { subcutaneous tissue, } \\
\text { gastrointestinal and } \\
\text { overall toxicity }\end{array}$ & ABCC2 rs4148396 & \\
\hline & $\begin{array}{l}\text { MTX-related hepatobiliary } \\
\text { disorders }\end{array}$ & ABCC2 rs7080681 & \\
\hline & $\begin{array}{l}\text { Toxicity: not further } \\
\text { specified }\end{array}$ & MS rsl805087: G homozygotes & \\
\hline & $\begin{array}{l}\text { Toxicity: not further } \\
\text { specified }\end{array}$ & $\begin{array}{l}\text { ADORA2a rs2236624C }>T \text {, rs2267076T }>C, \text { rs2298383C }>T \text {, } \\
\text { rs3761422C }>T \text { and } r s 5760410 G>A\end{array}$ & \\
\hline \multirow[t]{2}{*}{ MTX } & Gastrointestinal toxicity & RFCl G80A 80GG wild type genotype & 107 \\
\hline & $\begin{array}{l}\text { Overall MTX-related } \\
\text { toxicity }\end{array}$ & C3435T: carriers of at least one 3435T variant allele & \\
\hline \multirow[t]{2}{*}{ MTX } & $\begin{array}{l}\text { Toxicity: not further } \\
\text { specified }\end{array}$ & MTHFR 677T allele & 118 \\
\hline & $\begin{array}{l}\text { Toxicity: not further } \\
\text { specified }\end{array}$ & GGH 401CC genotype & \\
\hline
\end{tabular}


Table 3. Continued

\begin{tabular}{|c|c|c|c|}
\hline Drug & $\begin{array}{l}\text { Description of toxicity } \\
\text { (including side effects) }\end{array}$ & Mechanism & Ref \\
\hline MTX & $\begin{array}{l}\text { Hepatotoxicity and } \\
\text { alopecia }\end{array}$ & RFC-1 80AA genotype & 118 \\
\hline MTX & $\begin{array}{l}\text { Gastrointestinal, hepato- } \\
\text { and overall toxicity }\end{array}$ & MTHFR (C677T) & 32 \\
\hline Sulfasalazine & $\begin{array}{l}\text { Toxicity: not further } \\
\text { specified }\end{array}$ & $\begin{array}{l}\text { N-acetyl transferase } 2 \text { (NAT2*5A, NAT2*5B, NAT2*5C, NAT2*6 } \\
\text { and NAT2*7) }\end{array}$ & 32 \\
\hline Azathioprine & $\begin{array}{l}\text { Toxicity: not further } \\
\text { specified }\end{array}$ & $\begin{array}{l}\text { Thiopurine methyl-transferase gene (TPMT*2(G238C), } \\
\text { TPMT*3A(G460AandA719G), and TPMT*3C(A719G)) }\end{array}$ & 32 \\
\hline Leflunomide & $\begin{array}{l}\text { Toxicity: not further } \\
\text { specified }\end{array}$ & $\begin{array}{l}\text { CYPIA2-163C>A rs762551, CYP2C19*17allele rs12248560, } \\
\text { CYP2C9*3 homozygotes }\end{array}$ & 32 \\
\hline $\begin{array}{l}\text { TNFi (ADM, } \\
\text { IFX) }\end{array}$ & Hypersensitivity reactions & FCGR3BNAI/NAl genotype & 20,185 \\
\hline \multicolumn{4}{|c|}{ Influence of clinical characteristics } \\
\hline MTX + ETN & $\begin{array}{l}\text { Toxicity: not further } \\
\text { specified }\end{array}$ & Obese carriers of CMYA5 rsl2651804 or CCL2 rs1504582 & 64 \\
\hline
\end{tabular}

ADA: anti-drug antibodies; ADM: adalimumab; ETN: etanercept; IFN: interferon; INX: infliximab; GC: glucocorticoids; MTX: methotrexate; ref: reference; TNF(i): tumour necrosis factor (inhibitor); tsDMARD: targeted synthetic diseasemodifying antirheumatic drug.

To this end, combined analyses of blood and synovial tissue, molecular profiling using different technological platforms (e.g. proteomics, transcriptomics, metabolomics) and (epi-)genetic analyses are detrimental. ${ }^{186-189}$

Before these analyses will be conducted, the origin of the signs and symptoms of D2T RA patients should be ascertained (Figure 1). Recently, three subgroups of established RA patients (although not fulfilling the D2T RA definition) ${ }^{4}$ were identified using synovial histologic features and RNA sequencing data: high inflammatory, mixed and low inflammatory. ${ }^{190}$ The low inflammatory subgroup had high pain scores suggesting a noninflammatory origin of the symptoms. Therefore, careful assessment of inflammation is important to select the appropriate ('true' refractory RA) patients, as non-inflammatory factors may confound findings.

In addition to a comparison of mechanisms in D2T and in non-D2T RA, a deeper understanding of the pathogenesis of RA might also help in unravelling mechanisms that are still active in 'true' refractory RA. For example, deep single cell profiling of the synovium has shown that key cell lineages, such as synovial macrophages, are represented in functionally distinct populations, with levels of MerTK+ macrophage populations that express negative regulators of inflammation inversely correlating with the risk of clinical flare, suggesting a role for these cells. ${ }^{191}$ Additionally, the Accelerating Medicine Partnership - a public-private partnership established to identify and validate promising targets for therapeutics - has identified 18 synovial key cells involved in the pathogenesis 
of RA: innate cells (e.g. IL-1ß pro-inflammatory monocytes), adaptive cells (e.g. PDCDI+ peripheral and follicular Th cells) and THYl(CD90)+HLA-DRAhi sublining fibroblasts. ${ }^{192}$ Studies on associations with treatment response and clinical phenotypes will follow and may provide additional useful insights.

Furthermore, studies on mechanisms underlying (in-)efficacy of newer treatment options may aid in increasing our understanding of mechanisms underlying failure of multiple b/tsDMARDs. For instance, cellular therapies (such as tolerogenic DC transfer) and therapies selectively stimulating Tregs (e.g. using low-dose IL-2 therapy), which aim to restore immune tolerance, are currently under evaluation and may be promising in 'true' refractory RA. ${ }^{193-195}$ Additionally, vagus nerve stimulation has recently been introduced as therapeutic option for patients who failed at least two b/tsDMARDs with different mechanisms of action. ${ }^{196,197}$ Vagus nerve stimulation activates the inflammatory reflex, which plays a role in the regulation of innate and adaptive immunity through the activation of the cholinergic anti-inflammatory pathway. When conducting such pharmacological studies, the heterogeneity in the pathogenesis of RA patients should be considered. As these new drugs may only be beneficial for a subgroup of RA patients, this heterogeneity may confound efficacy outcomes and could result in the incorrect conclusion that these new drugs are not beneficial. Therefore, selecting the most appropriate patient population (i.e. 'true' refractory RA patients) may help to discover novel pharmacological strategies.

\section{Conclusions}

The presented heterogeneity in immunological mechanisms substantiates the assumption that RA is a syndrome, in which the pathogenesis differs between individuals. Differences in (epi-)genetic predisposition and clinical characteristics further contribute to this complex interplay. D2T RA should specifically be seen as heterogeneous: not only different immunological mechanisms may underlie DMARD inefficacy, but additional contributing factors may also result in the persistence of signs and symptoms suggestive of active disease. Due to the lack of evidence in patients who failed multiple b/tsDMARDs, the exact immunological mechanisms that are still active and can potentially be targeted in 'true' refractory RA, a subgroup of D2T RA patients in whom DMARDs are truly ineffective, as well as whether 'true' refractory RA really exists remain unknown. Future studies will be needed to increase our understanding and ultimately improve outcomes of D2T RA patients. 


\section{References}

1. Smolen JS, Aletaha D, Mclnnes IB. Rheumatoid arthritis. Lancet 2016;388:2023-38.

2. Smolen JS, Landewé RBM, Bijlsma JWJ, et al. EULAR recommendations for the management of rheumatoid arthritis with synthetic and biological disease-modifying antirheumatic drugs: 2019 update. Ann Rheum Dis 2020;79:685-99.

3. Singh JA, Saag KG, Bridges SL, et al. 2015 American College of Rheumatology Guideline for the Treatment of Rheumatoid Arthritis. Arthritis Rheumatol 2016;68:1-26.

4. Nagy G, Roodenrijs NMT, Welsing PMJ, et al. EULAR definition of difficult-to-treat rheumatoid arthritis. Ann Rheum Dis 2021;80:31-5.

5. Roodenrijs NMT, de Hair MJH, van der Goes $\mathrm{MC}$, et al. Characteristics of difficult-to-treat rheumatoid arthritis: results of an international survey. Ann Rheum Dis 2018;77:1705-9.

6. Buch $\mathrm{MH}$. Defining refractory rheumatoid arthritis. Ann Rheum Dis 2018;77:966-9.

7. Roodenrijs NM, de Hair MJ, van der Goes MC, et al. Correspondence to viewpoint 'Defining refractory rheumatoid arthritis' by Buch. Ann Rheum Dis 2019;78:el05.

8. Buch MH. Response to 'Correspondence to viewpoint 'Defining refractory rheumatoid arthritis' by Buch' by Roodenrijs et al. Ann Rheum Dis 2019;78:el06.

9. de Hair MJH, Jacobs JWG, Schoneveld JLM, et al. Difficult-to-treat rheumatoid arthritis: an area of unmet clinical need. Rheumatology (Oxford) 2017;57:1135-44.

10. Roodenrijs NMT, van der Goes MC, Welsing PMJ, et al. Difficult-to-treat rheumatoid arthritis: contributing factors and burden of disease. Rheumatology 2020:keaa860.

11. Buch $\mathrm{MH}$, Eyre S, McGonagle D. Persistent inflammatory and non-inflammatory mechanisms in refractory rheumatoid arthritis. Nat Rev Rheumatol 2021;17:17-33.

12. Fautrel B, Granger B, Combe B, et al. Matrix to predict rapid radiographic progression of early rheumatoid arthritis patients from the community treated with methotrexate or leflunomide: results from the ESPOIR cohort. Arthritis Res Ther 2012;14:R249.

13. van der Heijde D, Aletaha D, Carmona L, et al. 2014 Update of the EULAR standardised operating procedures for EULAR-endorsed recommendations. Ann Rheum Dis 2015;74:813.
14. Nagy G, Roodenrijs NMT, Welsing PMJ, et al. EULAR recommendations for the management of difficult-to-treat rheumatoid arthritis. Manuscript in preparation.

15. Thompson M, Tiwari A, Fu R, et al. A Framework To Facilitate the Use of Systematic Reviews and Meta-Analyses in the Design of Primary Research Studies. Rockville, MD Agency Healthc. Res. Qual. 2012.http://www.ncbi.nlm. nih.gov/books/ NBK83621/ (accessed 13 Nov 2019).

16. Kearsley-Fleet L, Davies R, De Cock D, et al. Biologic refractory disease in rheumatoid arthritis: Results from the British Society for Rheumatology Biologics Register for Rheumatoid Arthritis. Ann Rheum Dis 2018;77:1405-12.

17. Bluett J, Barton A. Precision Medicine in Rheumatoid Arthritis. Rheum Dis Clin North Am 2017;43:377-87.

18. Toussirot É, Michel F, Binda D, et al. The role of leptin in the pathophysiology of rheumatoid arthritis. Life Sci 2015;140:29-36.

19. Townsend MJ. Molecular and cellular heterogeneity in the Rheumatoid Arthritis synovium: clinical correlates of synovitis. Best Pract Res Clin Rheumatol 2014;28:539-49.

20. Tsukamoto $M$, Kameda $\mathrm{H}$, Ohshige $\mathrm{T}$, et al. Fcy receptor $3 \mathrm{~B}$ polymorphism is associated with hypersensitivity reactions to adalimumab in Japanese patients with rheumatoid arthritis. Mod Rheumatol 2017;27:778-81.

21. Uno K, Yoshizaki K, Iwahashi $M$, et al. Pretreatment Prediction of Individual Rheumatoid Arthritis Patients' Response to Anti-Cytokine Therapy Using Serum Cytokine/ Chemokine/Soluble Receptor Biomarkers. PLoS One 2015;10:e0132055.

22. Vashisht $P, O^{\prime}$ dell J. Not all TNF inhibitors in rheumatoid arthritis are created equal: important clinical differences. Expert Opin Biol Ther 2017;17:989-99.

23. Vomero $M$, Manganelli $V$, Barbati $C$, et al. Reduction of autophagy and increase in apoptosis correlates with a favorable clinical outcome in patients with rheumatoid arthritis treated with anti-TNF drugs. Arthritis Res Ther 2019;21:39.

24. Vultaggio A, Nencini F, Pratesi $S$, et al. Manifestations of Antidrug Antibodies Response: Hypersensitivity and Infusion Reactions. J Interferon Cytokine Res 2014;34:946-52.

25. Wielińska J, Kolossa K, Świerkot J, et al. Polymorphisms within the RANK and RANKL 
Encoding Genes in Patients with Rheumatoid Arthritis: Association with Disease Progression and Effectiveness of the Biological Treatment. Arch Immunol Ther Exp (Warsz) 2020;68:24.

26. Wijbrandts CA, Tak PP. Prediction of Response to Targeted Treatment in Rheumatoid Arthritis. Mayo Clin Proc 2017;92:1129-43.

27. Wright HL, Thomas HB, Moots RJ, et al. Interferon gene expression signature in rheumatoid arthritis neutrophils correlates with a good response to TNFi therapy. Rheumatology (Oxford) 2015;54:188-93.

28. Boyman O, Comte D, Spertini F. Adverse reactions to biologic agents and their medical management. Nat Rev Rheumatol 2014;10:612-27.

29. Wright HL, Cox T, Moots RJ, et al. Neutrophil biomarkers predict response to therapy with tumor necrosis factor inhibitors in rheumatoid arthritis. J Leukoc Biol 2017;101:785-95.

30. Wu C, Wang S, Xian $P$, et al. Effect of AntiTNF Antibodies on Clinical Response in Rheumatoid Arthritis Patients: A MetaAnalysis. Biomed Res Int 2016;2016:7185708.

31. $\mathrm{Xu} \mathrm{Z}$, Davis HM, Zhou H. Clinical impact of concomitant immunomodulators on biologic therapy: Pharmacokinetics, immunogenicity, efficacy and safety. J Clin Pharmacol 2015;55:560-74.

32. Zhang LL, Yang $S$, Wei W, et al. Genetic polymorphisms affect efficacy and adverse drug reactions of DMARDs in rheumatoid arthritis. Pharmacogenet Genomics 2014;24:531-8.

33. Zhang $\mathrm{KX}$, IP $\mathrm{CK}$, Chung SK, et al. Drugresistance in rheumatoid arthritis: the role of p53 gene mutations, $A B C$ family transporters and personal factors. Curr Opin Pharmacol 2020;54:59-71.

34. Bystrom J, Clanchy $\mathrm{Fl}$, Taher $\mathrm{TE}$, et al. Response to Treatment with TNFa Inhibitors in Rheumatoid Arthritis Is Associated with High Levels of GM-CSF and GM-CSF+ T Lymphocytes. Clin Rev Allergy Immunol 2017;53:265-76.

35. Canet LM, Cáliz R, Lupiañez CB, et al. Genetic variants within immune-modulating genes influence the risk of developing rheumatoid arthritis and anti-TNF drug response: a twostage case-control study. Pharmacogenet Genomics 2015;25:432-43.

36. Chang $\mathrm{K}$, Yang $\mathrm{SM}$, Kim $\mathrm{SH}$, et al. Smoking and rheumatoid arthritis. Int $\mathrm{J}$ Mol Sci 2014;15:22279-95.

37. Cuppen BVJ, Rossato M, Fritsch-Stork RDE, et al. RNA sequencing to predict response to TNF-a inhibitors reveals possible mechanism for nonresponse in smokers. Expert Rev Clin Immunol 2018;14:623-33.

38. Daien Cl, Gailhac S, Mura T, et al. High levels of memory $B$ cells are associated with response to a first tumor necrosis factor inhibitor in patients with rheumatoid arthritis in a longitudinal prospective study. Arthritis Res Ther 2014;16:R95.

39. Daïen $\mathrm{Cl}$, Gailhac S, Audo R, et al. High levels of natural killer cells are associated with response to tocilizumab in patients with severe rheumatoid arthritis. Rheumatology (Oxford) 2015;54:601-8.

40. Dávila-Fajardo $\mathrm{CL}$, Márquez A, PascualSalcedo D, et al. Confirmation of $-174 \mathrm{G} / \mathrm{C}$ interleukin-6 gene promoter polymorphism as a genetic marker predicting antitumor necrosis factor treatment outcome. Pharmacogenet Genomics 2014;24:1-5.

41. Dávila-Fajardo CL, van der Straaten T, BaakPablo R, et al. FcGR genetic polymorphisms and the response to adalimumab in patients with rheumatoid arthritis. Pharmacogenomics 2015;16:373-81.

42. Adlowitz DG, Barnard J, Biear JN, et al. Expansion of Activated Peripheral Blood Memory B Cells in Rheumatoid Arthritis, Impact of $B$ Cell Depletion Therapy, and Biomarkers of Response. PloS One 2015;10:e0128269.

43. Dennis G, Holweg CT, Kummerfeld SK, et al. Synovial phenotypes in rheumatoid arthritis correlate with response to biologic therapeutics. Arthritis Res Ther 2014;16:R90.

44. Derambure C, Dzangue-Tchoupou G, Berard $C$, et al. Pre-silencing of genes involved in the electron transport chain (ETC) pathway is associated with responsiveness to abatacept in rheumatoid arthritis. Arthritis Res Ther 2017;19:109.

45. Díaz-Torné C, Ortiz de Juana MA, Geli C, et al. Rituximab-induced interleukin-15 reduction associated with clinical improvement in rheumatoid arthritis. Immunology 2014;142:354-62.

46. Diaz-Torne $\mathrm{C}$, Ortiz MDA, Moya $\mathrm{P}$, et al. The combination of IL-6 and its soluble receptor is associated with the response of rheumatoid arthritis patients to tocilizumab. Semin Arthritis Rheum 2018;47:757-64.

47. Eektimmerman F, Swen JJ, Böhringer S, et al. Pathway analysis to identify genetic variants associated with efficacy of adalimumab in rheumatoid arthritis. Pharmacogenomics 
2017;18:945-53.

48. Farutin $\mathrm{V}$, Prod'homme $\mathrm{T}$, McConnell $\mathrm{K}$, et al. Molecular profiling of RA patients suggests a differential involvement of adaptive and innate cell populations in response to anti-TNF treatment. Arthritis Rheumatol 2017;69:(suppl 10).

49. Ferreiro-Iglesias A, Montes A, Perez-Pampin E, et al. Replication of PTPRC as genetic biomarker of response to TNF inhibitors in patients with rheumatoid arthritis. Pharmacogenomics J 2016;16:137-40.

50. Finckh A, Courvoisier D, Gottenberg J, et al. Does seropositivity influence differentially drug discontinuation of biologic antirheumatic agents with non-anti-TNF mode of action? Ann Rheum Dis 2017;76:567.

51. Friedman B, Cronstein B. Methotrexate mechanism in treatment of rheumatoid arthritis. Jt bone spine 2019;86:301-7.

52. Frleta-Gilchrist $M$, Mclntyre D, Elmesmari $A$, et al. JAK/STAT mediated inhibition of MIR23a/24-2/27a cluster potentiates activation of CDI4+ monocytes in treatment-resistant RA. Arthritis Rheumatol 2017;69:(suppl 10).

53. Alivernini S, Galeazzi M, Peleg $\mathrm{H}$, et al. Is ACPA positivity the main driver for rheumatoid arthritis treatment? Pros and cons. Autoimmun Rev 2017;16:1096-102.

54. Funahashi K, Koyano S, Echizen $\mathrm{H}$, et al. Whole genome analysis on the genetic backgrounds associated with the secondary failure to etanercept in patients with rheumatoid arthritis. Mod Rheumatol 2017;27:271-7.

55. Gazeau P, Alegria GC, Devauchelle-Pensec V, et al. Memory B Cells and Response to Abatacept in Rheumatoid Arthritis. Clin Rev Allergy Immunol 2017;53:166-76.

56. Gębura K, Świerkot J, Wysoczańska B, et al. Polymorphisms within Genes Involved in Regulation of the NF-kB Pathway in Patients with Rheumatoid Arthritis. Int J Mol Sci 2017;18.

57. Ghannam K, Martinez Gamboa L, Kedor C, et al. Response to abatacept is associated with the inhibition of proteasome ßli expression in $T$ cells of patients with rheumatoid arthritis. RMD open 2020;6.

58. Hakamata J, Hashiguchi $M$, Kaneko $Y$, et al. Risk factors for abnormal hepatic enzyme elevation by methotrexate treatment in patients with rheumatoid arthritis: A hospital based-cohort study. Mod Rheumatol 2018;28:611-20.

59. Hardy RS, Raza K, Cooper MS. Therapeutic glucocorticoids: mechanisms of actions in rheumatic diseases. Nat Rev Rheumatol 2020;16:133-44.

60. Her $\mathrm{M}$, Kavanaugh A. Alterations in immune function with biologic therapies for autoimmune disease. J Allergy Clin Immunol 2016;137:19-27.

61. Honne K, Hallgrímsdóttir I, Wu C, et al. A longitudinal genome-wide association study of anti-tumor necrosis factor response among Japanese patients with rheumatoid arthritis. Arthritis Res Ther 2016;18:12.

62. Ingawale DK, Mandlik SK, Patel SS. An emphasis on molecular mechanisms of antiinflammatory effects and glucocorticoid resistance. J Complement Integr Med 2015;12:1-13.

63. Iwaszko M, Świerkot J, Kolossa K, et al. Influence of CD94 and NKG2A variants on susceptibility to rheumatoid arthritis and efficacy of antiTNF treatment. Jt bone spine 2016;83:75-9.

64. Aslibekyan S, Sha J, Redden DT, et al. Genebody mass index interactions are associated with methotrexate toxicity in rheumatoid arthritis. Ann Rheum Dis 2014;73:785-6.

65. Iwaszko M, Świerkot J, Kolossa K, et al. Influence of NKG2D Genetic Variants on Response to Anti-TNF Agents in Patients with Rheumatoid Arthritis. Genes (Basel) 2018;9.

66. Iwaszko $M$, Świerkot J, Dratwa $M$, et al. Association of MICA-129Met/Val polymorphism with clinical outcome of anti-TNF therapy and MICA serum levels in patients with rheumatoid arthritis. Pharmacogenomics J 2020.

67. Jiménez Morales $A$, Maldonado-Montoro $M$, Martínez de la Plata JE, et al. FCGR2A/FCGR3A Gene Polymorphisms and Clinical Variables as Predictors of Response to Tocilizumab and Rituximab in Patients With Rheumatoid Arthritis. J Clin Pharmacol 2019;59:517-31.

68. Juge P-A, Gazal S, Constantin A, et al. Variants of genes implicated in type 1 interferon pathway and $\mathrm{B}$-cell activation modulate the EULAR response to rituximab at 24 weeks in rheumatoid arthritis. RMD open 2017;3:e000448.

69. Julià A, Fernandez-Nebro A, Blanco F, et al. A genome-wide association study identifies a new locus associated with the response to anti-TNF therapy in rheumatoid arthritis. Pharmacogenomics J 2016;16:147-50.

70. Julià A, Ávila $\mathrm{G}$, Celis R, et al. Lower peripheral helper $\mathrm{T}$ cell levels in the synovium are associated with a better response to anti-TNF therapy in rheumatoid arthritis. Arthritis Res 
Ther 2020;22.

71. Kasama T, Isozaki T, Takahashi R, et al. Clinical effects of tocilizumab on cytokines and immunological factors in patients with rheumatoid arthritis. Int Immunopharmacol 2016;35:301-6.

72. Kato M. New insights into IFN-y in rheumatoid arthritis: role in the era of JAK inhibitors. Immunol Med 2020;43:72-8.

73. Kikuchi J, Hashizume $M$, Kaneko $Y$, et al Peripheral blood CD4(+)CD25(+)CDI27(low) regulatory $T$ cells are significantly increased by tocilizumab treatment in patients with rheumatoid arthritis: increase in regulatory $T$ cells correlates with clinical response. Arthritis Res Ther 2015;17:10.

74. Krintel SB, Dehlendorff $\mathrm{C}$, Hetland $\mathrm{ML}$, et al. Prediction of treatment response to adalimumab: a double-blind placebocontrolled study of circulating microRNA in patients with early rheumatoid arthritis. Pharmacogenomics J 2016;16:141-6.

75. Aterido A, Cañete JD, Tornero J, et al. A Combined Transcriptomic and Genomic Analysis Identifies a Gene Signature Associated With the Response to AntiTNF Therapy in Rheumatoid Arthritis. Front Immunol 2019;10:1459.

76. Kuchuk NO, Hoes JN, Bijlsma JW, et al. Glucocorticoid-induced osteoporosis: an overview. Int J Clin Rheumtol 2014;9:311-26.

77. Kuuliala K, Kuuliala A, Koivuniemi R, et al. STAT6 and STATI Pathway Activation in Circulating Lymphocytes and Monocytes as Predictor of Treatment Response in Rheumatoid Arthritis. PLoS One 2016;11:e0167975.

78. Kuzuya K, Saeki Y, Hashimoto J, et al. Smoking reduces efficacy of biologics differently by target cytokines in rheumatoid arthritis. Arthritis Rheumatol 2017;69:(suppl 10).

79. Liu Y, Han Y, Qu H, et al. Correlation of microRNA expression profile with clinical response to tumor necrosis factor inhibitor in treating rheumatoid arthritis patients: A prospective cohort study. J Clin Lab Anal 2019;33.

80. Luxembourger C, Ruyssen-Witrand A, Ladhari $\mathrm{C}$, et al. A single nucleotide polymorphism of IL6-receptor is associated with response to tocilizumab in rheumatoid arthritis patients. Pharmacogenomics J 2019;19:368-74.

81. Maldonado-Montoro $M$, Cañadas-Garre $M$, González-Utrilla A, et al. Genetic and clinical biomarkers of tocilizumab response in patients with rheumatoid arthritis. Pharmacol Res 2016;111:264-71.
82. Manuel Sánchez-Maldonado J, Martínez-

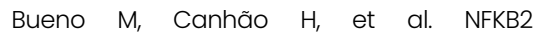
polymorphisms associate with the risk of developing rheumatoid arthritis and response to TNF inhibitors: Results from the REPAIR consortium. Sci Rep 2020;10:4316.

83. McInnes IB, Buckley CD, Isaacs JD. Cytokines in rheumatoid arthritis - shaping the immunological landscape. Nat Rev Rheumatol 2016;12:63-8.

84. Meusch $U$, Krasselt $M$, Rossol $M$, et al. In vitro response pattern of monocytes after tmTNF reverse signaling predicts response to antiTNF therapy in rheumatoid arthritis. J Transl Med 2015;13:256.

85. Mok CC, Tsai WC, Chen DY, et al. Immunogenicity of anti-TNF biologic agents in the treatment of rheumatoid arthritis. Expert Opin Biol Ther 2016;16:201-11.

86. Atzeni $F$, Talotta $R$, Nucera $V$, et al. Adverse events, clinical considerations and management recommendations in rheumatoid arthritis patients treated with JAK inhibitors. Expert Rev Clin Immunol 2018;14:945-56.

87. Montes A, Perez-Pampin E, Narváez J, et al. Association of FCGR2A with the response to infliximab treatment of patients with rheumatoid arthritis. Pharmacogenet Genomics 2014;24:238-45.

88. Naka T, Fujimoto M. LRG is a novel inflammatory marker clinically useful for the evaluation of disease activity in rheumatoid arthritis and inflammatory bowel disease. Immunol Med 2018;41:62-7.

89. Nakachi S, Sumitomo S, Tsuchida $Y$, et al. Interleukin-10-producing LAG3+ regulatory $T$ cells are associated with disease activity and abatacept treatment in rheumatoid arthritis. Arthritis Res Ther 2017;19:97.

90. Neregård $\mathrm{P}$, Krishnamurthy $\mathrm{A}$, Revu $\mathrm{S}$, et al. Etanercept decreases synovial expression of tumour necrosis factor- $a$ and lymphotoxin- $a$ in rheumatoid arthritis. Scand J Rheumatol 2014;43:85-90.

91. Niccoli L, Nannini C, Blandizzi C, et al. Personalization of biologic therapy in patients with rheumatoid arthritis: Less frequently accounted choice-driving variables. Ther Clin Risk Manag 2018;14:2097-11.

92. Nishimoto $T$, Seta $N$, Anan R, et al. A single nucleotide polymorphism of TRAFl predicts the clinical response to anti-TNF treatment in Japanese patients with rheumatoid arthritis. Clin Exp Rheumatol;32:211-7. 
93. Oliver J, Plant D, Webster AP, et al. Genetic and genomic markers of anti-TNF treatment response in rheumatoid arthritis. Biomark Med 2015;9:499-512.

94. Ortiz MA, Diaz-Torné $\mathrm{C}$, Hernández M V , et al. IL-6 blockade reverses the abnormal STAT activation of peripheral blood leukocytes from rheumatoid arthritis patients. Clin Immunol 2015;158:174-82.

95. Oryoji K, Yoshida K, Kashiwado Y, et al. Shared epitope positivity is related to efficacy of abatacept in rheumatoid arthritis. Ann Rheum Dis 2018;77:1234-6.

96. Pál I, Szamosi S, Hodosi K, et al. Effect of Fcyreceptor 3a (FCGR3A) gene polymorphisms on rituximab therapy in Hungarian patients with rheumatoid arthritis. RMD open 2017;3:e000485.

97. Avila-Pedretti G, Tornero J, Fernández-Nebro A, et al. Variation at FCGR2A and functionally related genes is associated with the response to anti-TNF therapy in rheumatoid arthritis. PLoS One 2015;10:e0122088.

98. Paul F, Cartron G. Infusion-related reactions to rituximab: frequency, mechanisms and predictors. Expert Rev Clin Immunol 2019;15:383-9.

99. Prado MS, Bendtzen K, Andrade LEC. Biological anti-TNF drugs: immunogenicity underlying treatment failure and adverse events. Expert Opin Drug Metab Toxicol 2017;13:985-95.

100. Pratt AG, Isaacs JD. Seronegative rheumatoid arthritis: pathogenetic and therapeutic aspects. Best Pract Res Clin Rheumatol 2014;28:651-9.

101. Rajasimhan S, Pamuk O, Katz JD. Safety of Janus Kinase Inhibitors in Older Patients: A Focus on the Thromboembolic Risk. Drugs and Aging 2020;37:551-8.

102. Reddy V, Cohen S. JAK Inhibitors: What Is New? Curr. Rheumatol. Rep. 2020;22.

103. Rodríguez-Carrio J, Alperi-López M, López P, et al. Heterogeneity of the Type I Interferon Signature in Rheumatoid Arthritis: A Potential Limitation for Its Use As a Clinical Biomarker. Front Immunol 2017;8:2007.

104. Romano E, Terenzi R, Manetti M, et al. Disease activity improvement in rheumatoid arthritis treated with tumor necrosis factor-a inhibitors correlates with increased soluble Fas levels. J Rheumatol 2014;41:1961-5.

105. Romão VC, Lima $A$, Bernardes $M$, et al. Three decades of low-dose methotrexate in rheumatoid arthritis: can we predict toxicity? Immunol Res 2014;60:289-310.
106. Rubbert-Roth A, Szabó $M Z$, Kedves $M$, et al. Failure of anti-TNF treatment in patients with rheumatoid arthritis: The pros and cons of the early use of alternative biological agents. Autoimmun Rev 2019;18.

107. Samara SA, Irshaid YM, Mustafa KN. Association of MDRl C3435T and RFCl G80A polymorphisms with methotrexate toxicity and response in Jordanian rheumatoid arthritis patients. Int $\mathrm{J}$ Clin Pharmacol Ther 2014;52:746-55.

108. Bastida C, Ruíz V, Pascal M, et al. Is there potential for therapeutic drug monitoring of biologic agents in rheumatoid arthritis? $\mathrm{Br} J$ Clin Pharmacol 2017;83:962-75.

109. Schotte $H$, Schmidt $H$, Gaubitz $M$, et al. Interleukin-6 promoter haplotypes are associated with etanercept response in patients with rheumatoid arthritis. Clin Rheumatol 2015;34:2021-8.

110. Schotte $\mathrm{H}$, Schlüter $\mathrm{B}$, Schmidt $\mathrm{H}$, et al. Putative IL-10 Low Producer Genotypes Are Associated with a Favourable Etanercept Response in Patients with Rheumatoid Arthritis. PLoS One 2015;10:e0130907.

111. Sellam J, Marion-Thore S, Dumont F, et al. Use of whole-blood transcriptomic profiling to highlight several pathophysiologic pathways associated with response to rituximab in patients with rheumatoid arthritis: data from a randomized, controlled, open-label trial. Arthritis Rheumatol 2014;66:2015-25.

112. Sode J, Vogel U, Bank S, et al. Anti-TNF treatment response in rheumatoid arthritis patients is associated with genetic variation in the NLRP3-inflammasome. PLoS One 2014;9:e100361.

113. Sode J, Vogel U, Bank S, et al. Genetic Variations in Pattern Recognition Receptor Loci Are Associated with Anti-TNF Response in Patients with Rheumatoid Arthritis. PLoS One 2015;10:e0139781.

114. Sode J, Vogel U, Bank S, et al. Confirmation of an IRAK3 polymorphism as a genetic marker predicting response to anti-TNF treatment in rheumatoid arthritis. Pharmacogenomics $\mathrm{J}$ 2018;18:81-6.

115. Spiliopoulou A, Colombo M, Plant D, et al. Association of response to TNF inhibitors in rheumatoid arthritis with quantitative trait loci for CD40 and CD39. Ann Rheum Dis 2019;78:1055-61.

116. Sweeney SR, Kavanaugh A, Lodi A, et al. Metabolomic profiling predicts outcome of rituximab therapy in rheumatoid arthritis. 
RMD open 2016;2:e000289.

117. Swierkot J, Bogunia-Kubik K, Nowak B, et al. Analysis of associations between polymorphisms within genes coding for tumour necrosis factor (TNF)-alpha and TNF receptors and responsiveness to TNF-alpha blockers in patients with rheumatoid arthritis. Jt bone spine 2015;82:94-9.

118. Świerkot J, Ślęzak $R$, Karpiński $P$, et al. Associations between single-nucleotide polymorphisms of RFC-1, GGH, MTHFR , TYMS, and TCll genes and the efficacy and toxicity of methotrexate treatment in patients with rheumatoid arthritis. Pol Arch Med Wewn 2015;125:152-61.

119. Bek S, Bojesen AB, Nielsen $J$, et al. Systematic review and meta-analysis: pharmacogenetics of anti-TNF treatment response in rheumatoid arthritis. Pharmacogenomics J 2017;17:403-11.

120. Takatori $\mathrm{H}$, Kawashima H, Matsuki A, et al. Helios Enhances Treg Cell Function in Cooperation With FoxP3. Arthritis Rheum 2015;67:1491-502.

121. Takayanagi N. Biological agents and respiratory infections: Causative mechanisms and practice management. Respir Investig 2015;53:185-200.

122. Talotta R, Berzi A, Atzeni F, et al. Paradoxical Expansion of Thl and Thl7 Lymphocytes in Rheumatoid Arthritis Following Infliximab Treatment: a Possible Explanation for a Lack of Clinical Response. J Clin Immunol 2015;35:550-7.

123. Teitsma XM, Jacobs JWG, Mokry $M$, et al. Identification of differential co-expressed gene networks in early rheumatoid arthritis achieving sustained drug-free remission after treatment with a tocilizumab-based or methotrexate-based strategy. Arthritis Res Ther 2017;19:170.

124. Teitsma XM, Yang $W$, Jacobs JWG, et al. Baseline metabolic profiles of early rheumatoid arthritis patients achieving sustained drug-free remission after initiating treat-to-target tocilizumab, methotrexate, or the combination: insights from systems biology. Arthritis Res Ther 2018;20:230.

125. Teitsma XM, Jacobs JWG, Concepcion $\mathrm{AN}$, et al. Explorative analyses of protein biomarkers in patients with early rheumatoid arthritis achieving sustained drug-free remission after treatment with tocilizumabor methotrexate-based strategies: from transcriptomics to proteomics. Clin Exp Rheumatol 2018;36:976-83.
126. Ternant $\mathrm{D}$, Bejan-Angoulvant $\mathrm{T}$, Passot $C$, et al. Clinical Pharmacokinetics and Pharmacodynamics of Monoclonal Antibodies Approved to Treat Rheumatoid Arthritis. Clin Pharmacokinet 2015;54:1107-23.

127. Thomson TM, Lescarbeau RM, Drubin DA, et al. Blood-based identification of nonresponders to anti-TNF therapy in rheumatoid arthritis. BMC Med Genomics 2015;8:26.

128. Tolusso B, Alivernini S, Gigante $M R$, et al. Biomolecular features of inflammation in obese rheumatoid arthritis patients: management considerations. Expert Rev Clin Immunol 2016;12:751-62.

129. Torices $S$, Julia $A$, Muñoz $P$, et al. A functional variant of TLRIO modifies the activity of NFkB and may help predict a worse prognosis in patients with rheumatoid arthritis. Arthritis Res Ther 2016;18:221.

130. Mclnnes IB, Schett $G$. The pathogenesis of rheumatoid arthritis. $N$ Engl J Med 2011;365:2205-19.

131. Safford M, Collins S, Lutz MA, et al. Egr-2 and Egr-3 are negative regulators of $T$ cell activation. Nat Immunol 2005;6:472-80.

132. Kimura A, Kishimoto T. IL-6: Regulator of Treg/ Th17 balance. Eur J Immunol 2010;40:1830-5.

133. Kerschbaumer A, Sepriano A, Smolen JS, et al. Efficacy of pharmacological treatment in rheumatoid arthritis: a systematic literature research informing the 2019 update of the EULAR recommendations for management of rheumatoid arthritis. Ann Rheum Dis 2020.

134. van den Berg WB, Miossec P. IL-17 as a future therapeutic target for rheumatoid arthritis. Nat Rev Rheumatol 2009;5:549-53.

135. Glatt S, Taylor PC, Mcinnes IB, et al. Efficacy and safety of bimekizumab as add-on therapy for rheumatoid arthritis in patients with inadequate response to certolizumab pegol: a proof-of-concept study. Ann Rheum Dis 2019;78:1033-40.

136. Cañete JD, Celis R, Moll C, et al. Clinical significance of synovial lymphoid neogenesis and its reversal after anti-tumour necrosis factor a therapy in rheumatoid arthritis. Ann Rheum Dis 2009;68:751-6.

137. Klimiuk PA, Goronzy JJ, Björnsson J, et al. Tissue cytokine patterns distinguish variants of rheumatoid synovitis. Am J Pathol 1997;151:13119.

138. Klimiuk PA, Sierakowski S, Latosiewicz R, et al. Circulating tumour necrosis factor $a$ and soluble tumour necrosis factor receptors in patients with different patterns of rheumatoid 
synovitis. Ann Rheum Dis 2003;62:472-5.

139. Burmester GR, Choy E, Kivitz A, et al. Low immunogenicity of tocilizumab in patients with rheumatoid arthritis. Ann Rheum Dis 2017;76:1078-85.

140. Strand V, Balsa A, Al-Saleh J, et al. Immunogenicity of Biologics in Chronic Inflammatory Diseases: A Systematic Review. BioDrugs 2017;31:299-316.

141. Mehta P, Manson JJ. What is the Clinical Relevance of TNF Inhibitor Immunogenicity in the Management of Patients With Rheumatoid Arthritis? Front Immunol 2020;11.

142. Van Schouwenburg PA, Rispens T, Wolbink GJ. Immunogenicity of anti-TNF biologic therapies for rheumatoid arthritis. Nat Rev Rheumatol 2013;9:164-72.

143. do Prado $A D$, Bisi MC, Piovesan $D M$, et al. Ultrasound power Doppler synovitis is associated with plasma IL-6 in established rheumatoid arthritis. Cytokine 2016;83:27-32.

144. AtzeniF, TalottaR, SalaffiF, etal.Immunogenicity and autoimmunity during anti-TNF therapy. Autoimmun Rev 2013;12:703-8.

145. Bartelds GM, Wijbrandts CA, Nurmohamed $M T$, et al. Anti-adalimumab antibodies in rheumatoid arthritis patients are associated with interleukin-10 gene polymorphisms. Arthritis Rheum 2009;60:2541-2.

146. Rivellese F, Humby F, Bugatti S, et al. B Cell Synovitis and Clinical Phenotypes in Rheumatoid Arthritis: Relationship to Disease Stages and Drug Exposure. Arthritis Rheumatol 2020;72:714-25.

147. Lewis MJ, Barnes MR, Blighe K, et al. Molecular Portraits of Early Rheumatoid Arthritis Identify Clinical and Treatment Response Phenotypes. Cell Rep 2019;28:2455-2470.e5.

148. Finckh A, Ciurea A, Brulhart L, et al. Which subgroup of patients with rheumatoid arthritis benefits from switching to rituximab versus alternative anti-tumour necrosis factor (TNF) agents after previous failure of an anti-TNF agent? Ann Rheum Dis 2010;69:387-93.

149. O'Shea JJ, Gadina M. Selective Janus kinase inhibitors come of age. Nat Rev Rheumatol 2019;15:74-5.

150. Choy EH. Clinical significance of Janus Kinase inhibitor selectivity. Rheumatology (Oxford). 2019;58:953-62.

151. Aaronson DS, Horvath CM. A road map for those who don't know JAK-STAT. Science (80) 2002;296:1653-5.

152. Jones GW, Greenhill CJ, Williams JO, et al. Exacerbated inflammatory arthritis in response to hyperactive gpl30 signalling is independent of IL-17A. Ann Rheum Dis 2013;72:1738-42.

153. Goenka S, Kaplan MH. Transcriptional regulation by STAT6. Immunol Res 2011;50:8796.

154. Humby F, Lewis $M$, Ramamoorthi $N$, et al. Synovial cellular and molecular signatures stratify clinical response to csDMARD therapy and predict radiographic progression in early rheumatoid arthritis patients. Ann Rheum Dis 2019;78:761-72.

155. Lliso-Ribera G, Humby $F$, Lewis $M$, et al. Synovial tissue signatures enhance clinical classification and prognostic/treatment response algorithms in early inflammatory arthritis and predict requirement for subsequent biological therapy: Results from the pathobiology of early arthritis cohort (PEA. Ann Rheum Dis 2019;78:1642-52.

156. van der Pouw Kraan TCTM, van Gaalen FA, Huizinga TWJ, et al. Discovery of distinctive gene expression profiles in rheumatoid synovium using cDNA microarray technology: Evidence for the existence of multiple pathways of tissue destruction and repair. Genes Immun 2003;4:187-96.

157. Van der Pouw Kraan TCTM, Van Gaalen FA, Kasperkovitz $P$ V., et al. Rheumatoid arthritis is a heterogeneous disease: Evidence for differences in the activation of the STAT1 pathway between rheumatoid tissues. Arthritis Rheum 2003;48:2132-45.

158. Van Baarsen LGM, Wijbrandts CA, Timmer TCG, et al. Synovial tissue heterogeneity in rheumatoid arthritis in relation to disease activity and biomarkers in peripheral blood. Arthritis Rheum 2010;62:1602-7.

159. Humby F, Durez $\mathrm{P}$, Buch $\mathrm{MH}$, et al. Rituximab versus Tocilizumab in anti-TNF inadequate responder patients with Rheumatoid Arthritis (R4RA): a stratified, biopsy-driven, multicentre, randomised, open label, controlled clinical trial - 16 week outcomes. Lancet (In Press 2021;397:305-17.

160. Drexler SK, Foxwell BM. The role of Toll-like receptors in chronic inflammation. Int J Biochem Cell Biol 2010;42:506-18.

161. Liu T, Zhang L, Joo D, et al. NF-kB signaling in inflammation. Signal Transduct Target Ther 2017;2:1-9.

162. Plant D, Webster A, Nair N, et al. Differential Methylation as a Biomarker of Response to Etanercept in Patients With Rheumatoid Arthritis. Arthritis Rheumatol 2016;68:1353-60. 
163. Tao W, Concepcion AN, Vianen M, et al. Multiomics and machine learning accurately predicts clinical response to Adalimumab and Etanercept therapy in patients with rheumatoid arthritis. Arthritis Rheumatol 2020:art.41516.

164. He L, Hannon GJ. MicroRNAs: Small RNAs with a big role in gene regulation. Nat Rev Genet 2004;5:522-31.

165. Wang J, Yan S, Yang J, et al. Non-coding RNAs in Rheumatoid Arthritis: From Bench to Bedside. Front Immunol 2020;10:3129.

166. Westhoff $G$, Rau R, Zink A. Rheumatoid arthritis patients who smoke have a higher need for DMARDs and feel worse, but they do not have more joint damage than non-smokers of the same serological group. Rheumatology (Oxford) 2008;47:849-54.

167. Abhishek A, Butt S, Gadsby K, et al. Anti-TNF-a agents are less effective for the treatment of rheumatoid arthritis in current smokers. J Clin Rheumatol 2010;16:15-8.

168. Hyrich KL, Watson KD, Silman $A J$, et al. Predictors of response to anti-TNF-alpha therapy among patients with rheumatoid arthritis: results from the British Society for Rheumatology Biologics Register. Rheumatology (Oxford) 2006;45:1558-65.

169. Mattey DL, Brownfield A, Dawes PT. Relationship between pack-year history of smoking and response to tumor necrosis factor antagonists in patients with rheumatoid arthritis. J Rheumatol 2009;36:1180-7.

170. Söderlin MK, Petersson IF, Geborek P. The effect of smoking on response and drug survival in rheumatoid arthritis patients treated with their first anti-TNF drug. Scand J Rheumatol 2012;41:1-9.

171. Bobbio-Pallavicini F, Caporali R, Alpini C, et al. High IgA rheumatoid factor levels are associated with poor clinical response to tumour necrosis factor a inhibitors in rheumatoid arthritis. Ann Rheum Dis 2007;66:302-7.

172. Braun-Moscovici Y, Markovits D, Zinder O, et al. Anti-cyclic citrullinated protein antibodies as a predictor of response to anti-tumor necrosis factor-alpha therapy in patients with rheumatoid arthritis. J Rheumatol 2006;33:497-500.

173. Lee DM, Phillips R, Hagan EM, et al. Quantifying anti-cyclic citrullinated peptide titres: Clinical utility and association with tobacco exposure in patients with rheumatoid arthritis. Ann Rheum Dis 2009;68:201-8.
174. Potter C, Hyrich KL, Tracey A, et al. Association of rheumatoid factor and anti-cyclic citrullinated peptide positivity, but not carriage of shared epitope or PTPN22 susceptibility variants, with anti-tumour necrosis factor response in rheumatoid arthritis. Ann Rheum Dis 2009;68:69-74.

175. Glossop JR, Dawes PT, Mattey DL. Association between cigarette smoking and release of tumour necrosis factor and its soluble receptors by peripheral blood mononuclear cells in patients with rheumatoid arthritis. Rheumatology 2006;45:1223-9.

176. Kuuliala A, Nissinen R, Kautiainen $\mathrm{H}$, et al. Low circulating soluble interleukin 2 receptor level predicts rapid response in patients with refractory rheumatoid arthritis treated with infliximab. Ann Rheum Dis 2006;65:26-9.

177. Park SJ, Shin Jl. The effect of smoking on response and drug survival in rheumatoid arthritis patients treated with their first anti-TNF drug: Comments on the article by Soderlin et al. Scand J Rheumatol 2012;41:411-2.

178. Metsios GS, Stavropoulos-Kalinoglou A, Nevill $A M$, et al. Cigarette smoking significantly increases basal metabolic rate in patients with rheumatoid arthritis. Ann Rheum Dis 2008;67:70-3.

179. Westhoff G, Rau R, Zink A. Rheumatoid arthritis patients who smoke have a higher need for DMARDs and feel worse, but they do not have more joint damage than non-smokers of the same serological group. Rheumatology 2008;47:849-54.

180. Heimans L, Van Den Broek M, Le Cessie S, et al. Association of high body mass index with decreased treatment response to combination therapy in recent-onset rheumatoid arthritis patients. Arthritis Care Res 2013;65:1235-42.

181. Alivernini S, Tolusso B, Gigante MR, et al. Overweight/obesity affects histological features and inflammatory gene signature of synovial membrane of Rheumatoid Arthritis. Sci Rep 2019;9:1-12.

182. Palming J, Gabrielsson BG, Jennische E, et al. Plasma cells and FC receptors in human adipose tissue-lipogenic and antiinflammatory effects of immunoglobulins on adipocytes. Biochem Biophys Res Commun 2006;343:43-8.

183. Wojtal KA, Rogler G, Scharl M, et al. Fc Gamma Receptor CD64 Modulates the Inhibitory Activity of Infliximab. PLoS One 2012;7:e43361.

184. Lortholary O, Fernandez-Ruiz M, Baddley JW, 
et al. Infectious complications of rheumatoid arthritis and psoriatic arthritis during targeted and biological therapies: a viewpoint in 2020. Ann Rheum Dis 2020;79:1532-43.

185. Okuyama A, Nagasawa H, Suzuki K, et al. Fcy receptor IIIb polymorphism and use of glucocorticoids at baseline are associated with infusion reactions to infliximab in patients with rheumatoid arthritis. Ann Rheum Dis 2011;70:299-304.

186. Orr C, Sousa E, Boyle DL, et al. Synovial tissue research: A state-of-the-art review. Nat Rev Rheumatol 2017;13:463-75.

187. Donlin L, Rao D, Wei K, et al. High dimensional analyses of cells dissociated from cryopreserved synovial tissue. Methods high-dimensonal Anal cells dissociated from cyropreserved synovial tissue 2018:284844.

188. Donlin LT, Park SH, Giannopoulou E, et al. Insights into rheumatic diseases from nextgeneration sequencing. Nat Rev Rheumatol 2019;15:327-39.

189. Winthrop $\mathrm{KL}$, Weinblatt $M E$, Bathon $\mathrm{J}$, et al. Unmet need in rheumatology: reports from the Targeted Therapies meeting 2019. Ann Rheum Dis 2020;79:88-93.

190. Orange $D E$, Agius $P$, DiCarlo $E F$, et al. Identification of Three Rheumatoid Arthritis Disease Subtypes by Machine Learning Integration of Synovial Histologic Features and RNA Sequencing Data. Arthritis Rheumatol 2018;70:690-701.

191. Alivernini S, MacDonald L, Elmesmari A, et al. Distinct synovial tissue macrophage subsets regulate inflammation and remission in rheumatoid arthritis. Nat Med 2020;26:1295306.

192. Zhang F, Wei K, Slowikowski K, et al. Defining inflammatory cell states in rheumatoid arthritis joint synovial tissues by integrating single-cell transcriptomics and mass cytometry. Nat Immunol 2019;20:928-42.

193. Jansen MAA, Spiering R, Broere F, et al. Targeting of tolerogenic dendritic cells towards heatshock proteins: a novel therapeutic strategy for autoimmune diseases? Immunology 2018;153:51-9.

194. Eden W Van, Jansen MAA, Ludwig IS, et al. Heat shock proteins can be surrogate autoantigens for induction of antigen specific therpeutic tolerance in rheumatoid arthritis. Front Immunol 2019;10:279.

195. Wu R, Li N, Zhao X, et al. Low-dose Interleukin-2: Biology and therapeutic prospects in rheumatoid arthritis. Autoimmun Rev 2020;19:102645.

196. Koopman F, Musters A, Backer M, et al. Vagus nerve stimulation in patients with rheumatoid arthritis: 24 month safety an efficacy. Arthritis Rheum 2019;70:(suppl 10).

197. Genovese MC, Gaylis NB, Sikes D, et al. Safety and efficacy of neurostimulation with a miniaturised vagus nerve stimulation device in patients with multidrug-refractory rheumatoid arthritis: a two-stage multicentre, randomised pilot study. Lancet Rheumatol 2020;2:e527-38. 


\section{Supplementary}

\section{Supplementary File}

\section{a. Frequency of 'true' refractory RA \\ Clinical question}

Does 'true' refractory RA really exist and how frequent is it?

\section{b. Underlying mechanisms for unsuccessful treatment outcomes}

\section{Clinical question}

What are reasons that drugs are not successful (such as adverse events, pharmacology, interaction with other drugs)?

Epidemiological question

What are reasons for DMARD treatment not being successful in RA patients?

- P: RA patients who start DMARD treatment

- I: Presence of underlying mechanisms for unsuccessful treatment outcome (e.g. pathways, adverse events, pharmacology, interaction with other drugs, 'true' refractory RA?)

- C: Absence of above mechanisms

- O: Therapeutic success of drug treatment

\section{Search in short}

Rheumatoid arthritis AND General terms for mechanisms/specific types of mechanisms of interest AND General terms for outcomes, and often used outcome measures AND General terms for treatment, and specific DMARDs

\section{Search in full}

PubMed: (RA[tiab] OR "rheumatoid arthritis"[tiab] OR "arthritis, rheumatoid"[Mesh]) AND (pathway[tiab] OR pathways[tiab] OR mechanism[tiab] OR mechanisms[tiab] OR interaction[tiab] OR interactions[tiab] OR "drug interactions"[Mesh] OR immunologic[tiab] OR immunological[tiab] OR "immunologic factors"[Mesh] OR genetic[tiab] OR "signal transduction"[tiab] OR "signal transduction"[Mesh] OR metabolic[tiab]) AND (therapeutic success[tiab] OR DAS[tiab] OR DAS28[tiab] OR DAS44[tiab] OR CDAI[tiab] OR SDAI[tiab] OR Response[tiab] OR Responsiveness[tiab] OR ACR20[tiab] OR ACR50[tiab] OR ACR70[tiab] OR "ACR 20"[tiab] OR "ACR 50"[tiab] OR "ACR 70"[tiab] OR "disease activity"[tiab] OR efficacy[tiab] OR effectiveness[tiab] OR toxic*[tiab] OR safety[tiab] OR tolerability[tiab] OR tolerable[tiab] OR "adverse events"[tiab] OR "Adverse event"[tiab] OR "Adverse effect"[tiab] 
OR "Adverse effects"[tiab] OR "Adverse reaction"[tiab] OR "Adverse reactions"[tiab] OR "Side effect"[tiab] OR "Side effects"[tiab] OR "Adverse drug reaction"[tiab] OR "Adverse drug reactions"[tiab] OR "Drug event"[tiab] OR "Drug events"[tiab] OR "Drug-Related Side Effects and Adverse Reactions"[Mesh]) AND (DMARD[tiab] OR DMARDs[tiab] OR "disease modifying antirheumatic drug" [tiab] OR "disease modifying antirheumatic drugs"[tiab] OR "disease modifying anti-rheumatic drug" [tiab] OR "disease modifying anti-rheumatic drugs"[tiab] OR "Antirheumatic Agents"[Mesh] OR Methotrexate[tiab] OR MTX[tiab] OR "Methotrexate"[Mesh] OR Leflunomide[tiab] OR "leflunomide"[Supplementary Concept] OR Sulfasalazine[tiab] OR "Sulfasalazine"[Mesh] OR Salazosulfapyridine[tiab] OR Hydroxychloroquine [tiab] OR Chloroquine[tiab] OR "Chloroquine"[Mesh] OR Isoxazole[tiab] OR "Isoxazoles"[Mesh] OR Gold[tiab] OR "Gold"[Mesh] OR "Gold compounds"[Mesh] OR Azathioprine[tiab] OR "Azathioprine"[Mesh] OR Cyclosporine[tiab] OR "Cyclosporine"[Mesh] OR Penicillamine[tiab] OR "Penicillamine"[Mesh] OR Cyclophosphamide[tiab] OR "Cyclophosphamide"[Mesh] OR "Mycophenolic acid"[tiab] OR "Mycophenolic acid"[Mesh] OR Pyrroles[Tiab] OR "Pyrroles"[Mesh] OR Chlorambucil[tiab] OR "Chlorambucil"[Mesh] OR Mycophenolate[tiab] OR "Mycophenolic acid"[Mesh] OR Minocycline[tiab] OR "Minocycline"[Mesh] OR Tofacitinib[tiab] OR "tofacitinib"[Supplementary Concept] OR Baricitinib[tiab] OR "baricitinib"[Supplementary Concept] OR Filgotinib[tiab] OR "GLPG0634"[Supplementary Concept] OR Peficitinib[tiab] OR "peficitinib"[Supplementary Concept] OR Decernotinib[tiab] OR "2-((2-(1H-pyrrolo(2,3-b)pyridin-3-yl)pyrimidin-4yl)amino)-2-methyl-N-(2,2,2-trifluoroethyl)butanamide"[Supplementary Concept] OR Ruxolitinib[tiab] OR " INCB018424"[Supplementary Concept] OR Upadacitinib[tiab] OR "Biological Products"[Mesh] OR Infliximab[tiab] OR "Infliximab"[Mesh] OR Golimumab[tiab] OR Adalimumab[tiab] OR "Adalimumab"[Mesh] OR Certolizumab[tiab] OR "Certolizumab Pegol"[Mesh] OR Etanercept[tiab] OR "Etanercept"[Mesh] OR Abatacept[tiab] OR "Abatacept"[Mesh] OR Tocilizumab[tiab] OR "tocilizumab"[Supplementary Concept] OR Anakinra[tiab] OR "Interleukin 1 Receptor Antagonist Protein"[Mesh] OR Rituximab[tiab] OR "Rituximab"[Mesh] OR Secukinumab[tiab] OR "secukinumab"[Supplementary Concept] OR Ustekinumab[tiab] OR Ustekinumab[Mesh] OR Brodalumab[tiab] OR Brodalumab[Supplementaryconcept] ORIxekizumab[tiab] ORIxekizumab[Supplementary concept] OR Plasmapheresis[tiab] OR Plasmapheresis[Mesh] OR "Intravenous immunoglobulin"[tiab] OR "Intravenous immunoglobulins"[tiab] OR "Intravenous antibodies"[tiab] OR IVIG[tiab] OR "Immunoglobulins, intravenous"[Mesh])

Search in Embase is available on request.

\section{Exclusion criteria}

- Duplicate

- Language other than English

- Animal study 
- Wrong publication type (e.g., Editorial, abstract not of ACR or EULAR of past 2 years)

- No RA patients

- No patients who have been treated with at least $1 \mathrm{~b} /$ tsDMARD

- No association between underlying mechanisms for (un)successful DMARD treatment and clinical therapeutic success of DMARD treatment (defined by physician, patient, or combined disease activity scores) AND no information on the occurrence of 'refractory' RA

- b/tsDMARDs that are not used in daily practice

- Other

Predetermined items for data extraction

- Study:

- Ist Author

- Title

- Publication year

- Publication type (original study/SLR/narrative review/abstract)

- Study design (if applicable)

- RA diagnosis according to [classification criteria]

- General description of population

- Baseline characteristics:

- Number of patients

- Gender (female)

- Age (years)

- DAS28 (or another composite index)

- RF positivity

- ACPA positivity

- Disease duration (days/months/years)

- DMARD history

- Current DMARD therapy

- Determinant \& outcome:

- Question a:

- Description of population

- Total population (n)

- Description of refractory RA

- Frequency (n (\%))

- Question b: 
- Description of DMARD treatment

- Category of DMARD

- Description of (un)successfulness

- Category of unsuccessfulness

- Time point of response/treatment failure

- Category of underlying mechanism (immunology, (epi-)genetics, clinical characteristics, other)

- Associated underlying mechanism

- Explanation of underlying mechanism 
Supplementary Table 1. Examples of (epi-)genetics influencing immunological mechanisms underlying DMARD (in-)efficacy

\begin{tabular}{|c|c|c|c|}
\hline (Epi-)genetics & DMARD & $\begin{array}{l}\text { Direction of association of } \\
\text { presence/level of (epi-) } \\
\text { genetics with response }\end{array}$ & Ref \\
\hline \multicolumn{4}{|l|}{ SNPS } \\
\hline CD40LG rs1126535 & TNFi (ADM) & + & 47 \\
\hline CD69 rsl1052877-A allele & Tocilizumab & + & 81 \\
\hline CHUK rsl1591741 & TNFi (ADM, CZP, ETN, GLM, INX) & $+/-$ & 114 \\
\hline $\begin{array}{l}\text { ENTPD gene (encoding CD39) SNPs (higher } \\
\text { genotypic score for the expression of the } \\
\text { ectonucleotidase CD39 on CD4 T cells) }\end{array}$ & TNFi (ADM, CZP, ETN, GLM, INX) & - & 115 \\
\hline FcGR2A HI3IR polymorphism & TNFi (INX) & - & 87 \\
\hline FcGR2A & TNFi (INX) & - (in ACPA positive patients) & 97 \\
\hline FcGR2A & TNFi (ADM) & + & 97 \\
\hline FcGR2a-R* allele (low affinity) & TNFi (ADM) & + & 41 \\
\hline FcGR2A rsl801274 & TNFi & + & 119 \\
\hline FcGR2A rsl801274-TT & Rituximab & + & 67 \\
\hline $\begin{array}{l}\text { FcGR2A rsl801274 SNP 13IR }>\text { H allele and } \\
\text { FcGR3A rs396991SNP 596T>G allele: } \\
\text { combination }\end{array}$ & TNFi (ADM) & + & 41 \\
\hline FcGR3A rs396991-TT & Tocilizumab & + & 67 \\
\hline FcGR3A rs396991-G & Rituximab & + & 67 \\
\hline FcGR3A V allele & Rituximab & + & 96 \\
\hline GALNT18 rs4910008-C allele & Tocilizumab & + & 81 \\
\hline GFRAl rsl679568-AG/AA & TNFi (ADM, ETN, INX) & + & 61 \\
\hline IFNG rs2069705C & TNFi & $+/-$ & 35 \\
\hline IFNG rs2430561 & TNFi (ADM, CZP, ETN, GLM, INX) & + & 112 \\
\hline IL-6 -174G/C (rsl800795) & TNFi (ADM, ETN, INX) & + & 40 \\
\hline IL-6 promoter (-174G) haplotype & TNFi (ETN) & + & 109 \\
\hline IL-6R rsl208353 AA-genotype & Tocilizumab & + & 80 \\
\hline IL-10 promoter $-2849 \mathrm{G}>\mathrm{A}$ & TNFi (ETN) & + & 110 \\
\hline IL-10 rsl80089 & TNFi (ADM, ETN, INX) & + & 49 \\
\hline IL-12B rs6887695 & TNFi (ADM, CZP, ETN, GLM, INX) & $+1-$ & 113 \\
\hline IL-18 rsl87238: baseline values (GC, GC/CC) & TNFi (ADM, CZP, ETN, GLM, INX) & $+/-$ & 113 \\
\hline IL-18 rs10754558: baseline values (CG, CG/GG) & TNFi (ADM, CZP, ETN, GLM, INX) & $+/-$ & 113 \\
\hline $\begin{array}{l}\text { IRF5 rs2004640, SPPI rs9138 and TNFSFI3B } \\
\text { rs9514828: combination }\end{array}$ & Rituximab & + & 68 \\
\hline MAP3K7, BACH2 rs284515-AG/GG & TNFi (ADM, ETN, INX) & + & 61 \\
\hline MED15 rsl13878252 & TNFi (ETN) & + & 69 \\
\hline MICA rsl051792-GG genotype & TNFi (ADM, CZP, ETN, INX) & - & 66 \\
\hline MYD88 rs7744 & TNFi (ADM, CZP, ETN, GLM, INX) & $+1-$ & 114 \\
\hline NLRP3 rs4612666 & TNFi (ADM, CZP, ETN, GLM, INX) & + & 112 \\
\hline
\end{tabular}


Supplementary Table 1. Continued

\begin{tabular}{|c|c|c|c|}
\hline (Epi-)genetics & DMARD & $\begin{array}{l}\text { Direction of association of } \\
\text { presence/level of (epi-) } \\
\text { genetics with response }\end{array}$ & Ref \\
\hline NLPR3 rs461266 & TNFi (ADM, CZP, ETN, GLM, INX) & - & 114 \\
\hline NLRP3 rs4612666-T & TNFi (ADM, CZP, ETN, GLM, INX) & - (in current smokers) & 112 \\
\hline NKG2A rs7301582- C/CC & TNFi (ADM, CZP, ETN, INX) & - & 63 \\
\hline NKG2D rs225336-GG, rs1049174-CC & TNFi (ADM, CZP, ETN, INX) & - & 65 \\
\hline NKG2D rs225336, rs1049174 heterozygous & TNFi (ADM, CZP, ETN, INX) & + & 65 \\
\hline NF-KB (including IL33 and STAT5A) and EMR4P & TNFi (ETN) & + & 109 \\
\hline NF-kB1 rs28362491-94ins/del ATTG & TNFi (ADM, CZP, ETN, INX) & + & 56 \\
\hline NF-kB2rsl056890T allele & TNFi (ADM, ETN, GLM, INX) & + & 82 \\
\hline NF-kBIB rs3136645, rs9403 & TNFi (ADM, CZP, ETN, GLM, INX) & $+1-$ & 114 \\
\hline NF-kBIB rs9403 & TNFi & - & 119 \\
\hline $\begin{array}{l}\text { NLRP3 rs10754558: variant allele carriers (CG, } \\
\text { CG/GG) }\end{array}$ & TNFi (ADM, CZP, ETN, GLM, INX) & $+/-$ & 113 \\
\hline $\begin{array}{l}\text { PDEA3A-SLC01Cl rs379421, C > T } \\
\text { polymorphism }\end{array}$ & TNFi (ADM, ETN, INX) & - & 17,93 \\
\hline PTPRC rsl0919563 & TNFi (ADM, ETN, INX) & + & $\begin{array}{l}17,33, \\
49\end{array}$ \\
\hline RANKL rs7988338 GG homozygote & TNFi & + & 25 \\
\hline RANKL rs7325635 GG homozygote & TNFi & - & 25 \\
\hline STK10 rs2279515, rs2306963, rs3111491 & TNFi (ETN) & $\begin{array}{l}\text { - (secondary non- } \\
\text { response) }\end{array}$ & 54 \\
\hline TANK rsl267067 & TNFi (ADM) & + & 47 \\
\hline TLR1 rs4833095: homozygous carriers CC & TNFi (ADM, CZP, ETN, GLM, INX) & $+/-$ & 113 \\
\hline TLR5 rs5744174: allele carriers TC, CC, TC/CC & TNFi (ADM, CZP, ETN, GLM, INX) & + (in RF negative patients) & 113 \\
\hline TLRI0 1473T variant & TNFi (INX) & - & 129 \\
\hline$T N F-a-857 C>T,-308 G>A,-238 G>A$ & TNFi (ADM, CZP, ETN, INX) & + & 117 \\
\hline TNFAIP3 rs2230926 & TNFi (ADM) & + & 47 \\
\hline TNF promoter region (-308G/ASNP) - GG & TNFi & + & 32 \\
\hline TNF promoter region (-308G/ASNP) - AA & TNFi & - & 32 \\
\hline TNFRSFIB 196T >G & TNFi & + & 32 \\
\hline TRAF1 rsl6860-GG genotype & TNFi (ADM, ETN, INX) & - & 92 \\
\hline TRAFl rs3761847 & TNFi & + & 33,119 \\
\hline VEGFA rs25648 & TNFi (ADM) & + & 47 \\
\hline WDR27 rs75908454-CT/CC & TNFi (ADM, ETN, INX) & + & 61 \\
\hline $\begin{array}{l}\text { Gene coexpression modules (18 genes: } \\
\text { rs72681642, rs3104464, rsl2135530, rs10782647, } \\
\text { rs4562674, rs } 11165922, \text { chr5:67561207, } \\
\text { chr8:12854804, rs2454329, rs62187579, } \\
\text { rsl40900228, rs } 11165917, \text { rsl519149, rs10061960, } \\
\text { rs } 1550805, \text { rs } 9677007, \text { rs } 4949803, \text { rs } 74105112)\end{array}$ & $\begin{array}{l}\text { TNFi (ADM) } \\
\text { TNFi (ETN) }\end{array}$ & $\begin{array}{l}+ \\
+/-\end{array}$ & 75 \\
\hline
\end{tabular}


Supplementary Table 1. Continued

\begin{tabular}{|c|c|c|c|}
\hline (Epi-)genetics & DMARD & $\begin{array}{l}\text { Direction of association of } \\
\text { presence/level of (epi-) } \\
\text { genetics with response }\end{array}$ & Ref \\
\hline
\end{tabular}

\section{Epigenetics}

Differential methylation of LRPAPI

TNFi (ETN) cg04857395, cg26401028, cg16426293, cg 03277049, cgl2226028

Lower CD40 methylation and higher CD40 transcription

TNFi (ADM, CZP, ETN, GLM, INX) +

miR-886.3p (high expression) with $m i R-22$

TNFi (ADM)

Multiple bDMARDs (not further specified)

miR-23a 24-a 27a cluster (repressed)

TNFi (ETN)

miR-146a-5p (high expression)

let-7a-5p (low expression)

\section{Other examples of differences in gene expression}

\begin{abstract}
CD14, CD36, CD46, CD47, CD163, and CD16: higher levels of associated cell surface markers
\end{abstract}

COX7B, COXI1, UQCRC2, NDUFU3, NDUFS1: upregulated

Cytokine signalling involved in inflammation, including TNF and NF-kB pathways: lower gene expression, while having higher levels of specific metabolic activities associated lipolysis and fatty acid oxidation

CX3CLl: higher levels of expression

PIK3CD: higher levels of expression

GPR15: higher levels of expression

GR-B: increased expression

Granulocyte migration genes: most overrepresented

HDAC2: reduced expression

HLA-DRBl shared epitope

IFN-response genes (IFI6: IFNa-inducible protein 6, MX2: myxovirus resistance 2, OASL: 2'-5'-oligoadenylate synthetase-like gene): increased expression levels

IFN-response genes: increase expression

IFN pathway: downregulation

IFN-regulated genes in neutrophils (CMPK2, IFI44L, IFITIB, IFI6, LY6E, OASl, OAS2, OAS3, RSAD2, USP18): higher expression

MDRI: increased expression

Metallothionein-1G gene: increased expression levels

Neutrophil granule protein genes: AZUI, BPI, CEACAM8, CRISP3, CTSG, DEFA4, ELANE, LCN2, LTF, MMP8, MPO, RNASE2, RNASE3

$\begin{array}{lcc}\text { TNFi (ADM, INX) } & + & 48 \\ \text { Abatacept + MTX } & + & 44 \\ \text { TNFi (INX) } & - & 127\end{array}$

TNFi (ADM, ETN, INX)

TNFi (ADM, ETN)

GC

Tocilizumab

+ (sustained free

GC

Abatacept

Tocilizumab remission)

Rituximab

TNFi (ADM, ETN, GLM)

\section{$+$}

$+$

GC

Tocilizumab

TNFi (ADM, ETN, GLM) 
Supplementary Table 1. Continued

\begin{tabular}{|c|c|c|c|}
\hline (Epi-)genetics & DMARD & $\begin{array}{l}\text { Direction of association of } \\
\text { presence/level of (epi-) } \\
\text { genetics with response }\end{array}$ & Ref \\
\hline $\begin{array}{l}\text { Nuclear-transcribed mRNA catabolic } \\
\text { process, nonsense-mediated decay genes: } \\
\text { most overrepresented }\end{array}$ & Tocilizumab + MTX & $\begin{array}{l}+ \text { (sustained free } \\
\text { remission) }\end{array}$ & 123,125 \\
\hline $\begin{array}{l}\text { Proteasome immunosubunit ßli in CD4+ and } \\
\text { CD8+ T cells: significant reduction in gene } \\
\text { expression }\end{array}$ & Abatacept & + & 57 \\
\hline SEMA6B: higher levels of expression & TNFi (ADM, ETN) & - & 37 \\
\hline Synovial myeloid gene signature expression & TNFi & + & 43 \\
\hline
\end{tabular}

ACPA: anti-citrullinated protein antibodies; ADM: adalimumab; CZP: certolizumab pegol; DMARDs: diseasemodifying antirheumatic drugs; ETN: etanercept; GC: glucocorticoids; GLM: golimumab; INX: infliximab; MTX: methotrexate; ref: reference; RF: rheumatoid factor; SNP: Single-nucleotide polymorphisms; TNFi: tumour necrosis factor inhibitor; +: association with response; -: association with non-response; +/-: no association. 



\section{CHAPTER 7}

Diagnostic issues in difficult-to-treat rheumatoid arthritis: a systematic literature review informing the EULAR recommendations for the management of difficult-to-treat rheumatoid arthritis

RMD Open 2021;7:e001511.

Nadia M.T. Roodenrijs, Melinda Kedves, Attila Hamar, György Nagy, Jacob M. van Laar, Désirée van der Heijde, Paco M.J. Welsing 


\section{Abstract}

\section{Objectives}

To summarise the evidence on diagnostic issues in difficult-to-treat rheumatoid arthritis (D2T RA) informing the European League Against Rheumatism recommendations for the management of D2T RA.

\section{Methods}

A systematic literature review (SLR) was performed regarding the optimal confirmation of a diagnosis of rheumatoid arthritis (RA) and of mimicking diseases and the assessment of inflammatory disease activity. PubMed and Embase databases were searched up to December 2019. Relevant papers were selected and appraised.

\section{Results}

Eighty-two (82) papers were selected for detailed assessment. The identified evidence had several limitations: 1) no studies were found including D2T RA patients specifically, and only the minority of studies included RA patients in whom there was explicit doubt about the diagnosis of RA or presence of inflammatory activity; 2) mostly only correlations were reported, not directly useful to evaluate the accuracy of detecting inflammatory activity in clinical practice; 3) heterogenous, and often suboptimal, reference standards were used; 4) (thus) only very few studies had a low risk of bias.

To ascertain a diagnosis of RA or relevant mimicking disease, no diagnostic test with sufficient validity and accuracy was identified. To ascertain inflammatory activity in RA patients in general and in those with obesity and fibromyalgia, ultrasonography (US) was studied most extensively and was found to be the most promising diagnostic test.

\section{Conclusions}

This SLR highlights the scarcity of high-quality studies regarding diagnostic issues in D2T RA. No diagnostic tests with sufficient validity and accuracy were found to confirm nor exclude the diagnosis of RA nor its mimicking diseases in D2T RA patients. Despite the lack of high-quality direct evidence, US may have an additional value to assess the presence of inflammatory activity in D2T RA patients, including those with concomitant obesity or fibromyalgia. 


\section{Introduction}

Treatment options for rheumatoid arthritis (RA) have largely expanded and treatment strategies have improved over the past decades. Nowadays, many patients reach remission or low disease activity when following the current European League Against Rheumatism (EULAR) recommendations and/or American College of Rheumatology (ACR) guideline for the management of RA. ${ }^{12}$ However, there is still a substantial proportion of RA patients that remains symptomatic even though they have been treated according to these recommendations. This patient group is referred to as having 'difficult-to-treat (D2T) RA'. This disease state is expected to affect $5 \%$ to $20 \%$ of all RA patients, depending on the specific definition used. ${ }^{3-5}$ D2T RA has recently been defined as patients who failed at least two biological/targeted synthetic disease-modifying antirheumatic drugs (b/ tsDMARDs) with different mechanisms of action after failing conventioanl synthetic (cs) DMARD therapy. ${ }^{6}$ Additionally, patients should have signs and/or symptoms suggestive of active disease, which is perceived as problematic by the patient and/or rheumatologist. The unmet need for these patients was previously underlined by an international survey that was conducted among rheumatologists.' Consequently, the importance has been acknowledged by EULAR with the approval of a Task Force on the development of management recommendations for D2T RA.

In D2T RA patients, DMARD therapy is frequently changed in routine daily practice in case of signs and/or symptoms suggestive of active disease. ${ }^{4}$ However, D2T RA is a heterogeneous disease state and various factors could contribute to the persistence of these signs and/or symptoms: factors related to inflammation (e.g. having underlying immunologic disease mechanisms driving 'true' refractory disease or treatment nonadherence), factors of non-inflammatory origin (e.g. concomitant fibromyalgia) or both. ${ }^{4,7}$ All these contributing factors may require different pharmacological and nonpharmacological therapeutic strategies, ${ }^{4}$ which are reviewed in a separate systematic literature review (SLR). ${ }^{9}$

Importantly, intensificationorotherchanges inDMARD therapytoreduce inflammationmay only be appropriate in patients with insufficient response to therapy due to inflammatory RA activity. ${ }^{4}$ Symptoms of other diseases, e.g. psoriatic arthritis and polyarticular gouty arthritis, may mimic RA possibly leading to misdiagnosis of the disease. ${ }^{4,8,10}$ Additionally, coexistence of certain circumstances, e.g. obesity, pain syndromes and osteoarthritis, may hamper proper grading of disease activity by influencing diagnostic measures. ${ }^{4,8}$ Therefore, in D2T RA, it will be important to ascertain the diagnosis of RA and the presence of inflammatory RA activity before adjusting therapeutic strategies.

The aim of this SLR was firstly to explore and summarise how to optimally confirm the diagnosis of RA in a D2T RA patient and how to optimally diagnose and rule out alternative or coexisting mimicking diseases. In addition, this SLR focussed on the assessment of 
the presence of inflammatory activity in D2T RA patients and in those with comorbidities that may influence this assessment. This SLR, together with the other SLR focussing on therapeutic strategies in D2T RA, ${ }^{9}$ was conducted to inform the EULAR recommendations for the management of D2T RA.

\section{Methods}

\section{Research questions}

This SLR was conducted following the EULAR Standardised Operating Procedures." Three clinical questions on diagnostic issues in D2T RA patients were proposed by the fellow (NMTR), co-methodologist (PMJW) and postdoctoral fellow (MJHdH), and then approved by the Steering Committee (GN (convenor), JMvL (co-convenor), DvdH (methodologist), MK (fellow)). At the first Task Force meeting, which was held in August 2018, the questions were discussed, amended and then approved by the whole Task Force.

The clinical questions were focussed on diagnostic techniques for (1) the confirmation of the diagnosis of RA or a relevant differential diagnosis (either as alternative or coexisting mimicking disease), (2a) the assessment of inflammatory activity in RA patients, and (2b) the assessment of inflammatory activity in RA patients with comorbidities that might influence the assessment of inflammatory activity. Mimicking diseases deemed of interest were gouty arthritis, calcium pyrophosphate deposition disease, psoriatic arthritis, spondyloarthritis, polymyalgia rheumatica, systemic lupus erythematosus, reactive arthritis, paraneoplastic syndromes, osteoarthritis and fibromyalgia. Comorbidities of interest that might influence the assessment of inflammatory activity were infections, malignancies, obesity, pain syndromes (including fibromyalgia), osteoarthritis, subluxations and joint dislocations. The clinical questions were transformed into epidemiological questions using the 'Patients, Indicator test, Comparison test (i.e. reference standard), Outcome (PICO) format' (Supplementary File).'2

\section{Search strategy}

The databases of PubMed and Embase were searched for papers in English until December 2018 for search 1 and December 2019 for search 2. Additionally, the conference abstracts of EULAR and ACR were screened, from 2017 until 2018 for search 1 and from 2017 until 2019 for search 2. Advice regarding the set-up of the search strategy was provided by two experienced librarians of Utrecht University (FPW and PHW).

The first search focussed on the diagnosis of RA and relevant differential diagnoses. In addition to terms for RA and terms related to diagnostic studies, terms for misdiagnosis and common alternative and coexisting mimicking diseases were included (search details in Supplementary File). During the Task Force meeting, it was agreed to perform a 
limited search on recent literature on this topic as not much research on (mis-)diagnosis relevant to our project was expected to be present and to have a more focussed approach given the many clinical questions on D2T RA that were defined by the Task Force. Therefore, a search limit was set to the last ten years and reference screening was not performed.

The second search focussed on the assessment of inflammatory activity. In addition to terms for RA and terms related to diagnostic studies, terms for D2T RA and comorbidities that might influence assessment of inflammatory activity, general terms for inflammation and specific tests to assess inflammatory activity were included (search details in Supplementary File). A search limit was set to the last ten years. In addition, the reference lists of selected papers were manually screened. References published in the year 2000 and later were eligible for inclusion. This cut-off was chosen because of the introduction of bDMARDs around this time and, herewith, the beginning of a new diagnostic and therapeutic landscape regarding acceptable disease activity in the field of RA.

\section{Selection of studies}

First, titles and abstracts were screened in duplicate by the fellows (NMTR and MK) according to a set list of selection criteria (Supplementary File) until the percentage of conflicts was below $5 \%$. In case of conflicts or when in doubt, eligibility was discussed with the co-methodologist (PMJW). Second, all full-text versions of the selected papers were screened in duplicate by the fellows (NMTR and MK). Disagreements were discussed with the co-methodologist (PMJW) until consensus was reached.

As specific evidence on D2T RA patients was expected to be scarce, we decided not to focus on D2T RA patients only, but on a broader population of RA patients. Regarding the diagnosis of RA and relevant differential diagnoses, papers were eligible when focussing on patients clinically diagnosed with RA and suspected of a mimicking disease, patients suspected of RA according to the classification criteria in whom a new diagnostic test was evaluated, or patients suspected of RA, but not satisfying classification criteria. Regarding the assessment of inflammatory activity, we decided to only exclude papers when the study population included treatment naïve RA patients. Additionally, in this population, diagnostic tests beyond currently used reference standards had to be evaluated.

\section{Data extraction and quality assessment}

Information on study design, patient characteristics, index test, reference standard and diagnostic outcomes were extracted from the included papers using a predetermined format (Supplementary File).

Risk of bias (ROB) and applicability of the included original papers were assessed using the Quality Assessment of Diagnostic Accuracy Studies tool version 2 (QUADAS-2)13 and highest RoB as found among categories was reported here (low, moderate, high). 
For SLRs, RoB was assessed using 'A MeaSurement Tool to Assess systematic Reviews' version 2 (AMSTAR-2) and overall RoB was reported according to its scoring system (low, moderate, high, critically high) ${ }^{14}$

One important item included in RoB assessment is the reference standard used. For the diagnosis of RA and relevant differential diagnoses, we deemed a clinical diagnosis according to a rheumatologist as the appropriate reference standard. For the assessment of the presence of inflammatory activity, the preferred reference standard differed between study populations. For the general established RA population, we considered validated composite disease activity indices (e.g. DAS28 or CDAI) as appropriate to assess the presence of inflammatory activity at patient level, and the clinical assessment of swelling in the joint at joint level (i.e. in a specific joint). In patients in whom there is explicit doubt about the presence of inflammatory activity (including patients with mimicking diseases), the traditional measures are not trustworthy. Therefore, in studies assessing this population we considered (scores based on) established imaging measures as a more appropriate reference standard.

Data extraction and quality assessment were performed in duplicate by the fellows (NMTR and $\mathrm{AH}$ ) until the number of conflicts was below $5 \%$. Disagreements and remaining doubts were discussed with the co-methodologist (PMJW) until consensus was reached.

\section{Statistical analyses}

Extracted data were summarised descriptively regarding study and patient characteristics and reported diagnostic association measures. Preferably, sensitivity, specificity, positive predictive value (PPV), negative predictive value (NPV), likelihood ratios (LRs) and odds ratios (ORs) were reported. If these were not available, other association measures (typically (Pearson or Spearman) correlation coefficients) were reported, although these measures do not well reflect diagnostic accuracy of measures and thus provide lower quality of evidence. ${ }^{5}$ Pooling of results was considered based on clinical and statistical homogeneity.

\section{Results}

\section{Study characteristics}

The first search regarding the diagnosis of RA and relevant differential diagnoses yielded 2111 unique papers. Title and abstract screening resulted in 337 papers, which were fully reviewed. Four of them fulfilled the selection criteria and were included for data extraction (Figure la). One additional paper was included via the search on the assessment of inflammatory activity as this paper focussed on the diagnosis of RA. Of these five papers, one paper regarded the optimal confirmation of a diagnosis of RA ${ }^{16}$ and four papers the 


\section{confirmation of a coexisting mimicking disease in RA patients. ${ }^{17-20}$}

The second search on the assessment of inflammatory disease activity resulted in 3858 unique papers. After title and abstract screening, 237 papers were selected for fulltext screening and 45 papers were selected for inclusion. Additionally, 32 papers were

A. (Mis-)diagnosis of RA and relevant differential diagnoses

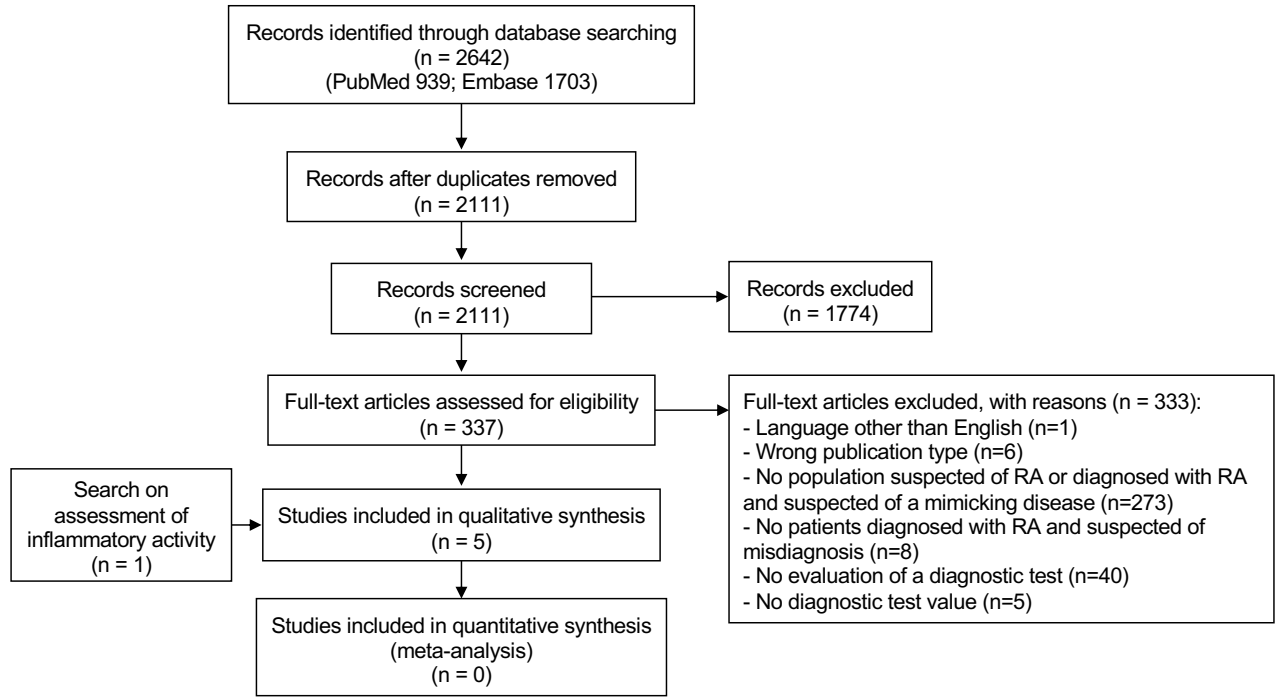

B. The assessment of inflammatory activity in (2a) RA patients, and (2b) RA patients with comorbidities that might influence the assessment

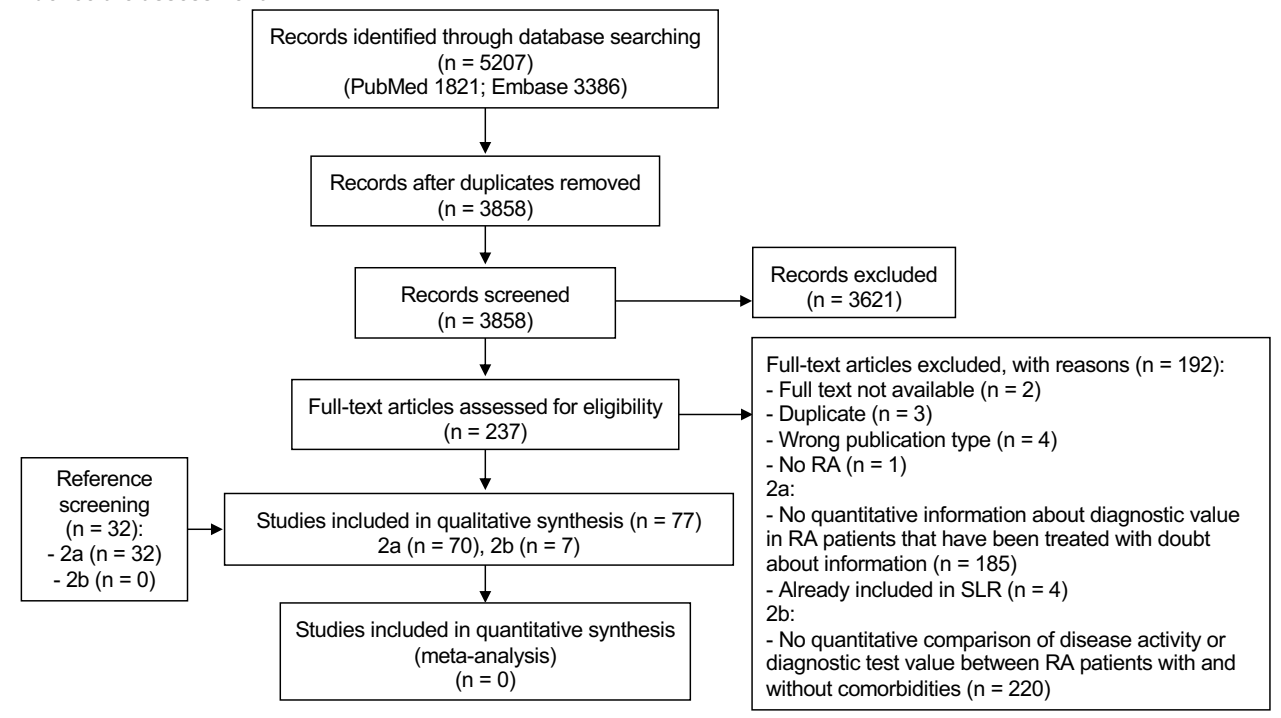

Figure 1. Flow charts of search and selection of papers

n: number of papers; RA: rheumatoid arthritis; SLR: systematic literature review. 
selected via reference screening (Figure lb). Seventy of 77 papers were selected for the assessment of inflammatory activity in general, ${ }^{21-90}$ the seven remaining papers studied the assessment of inflammatory activity in RA patients with specific comorbidities. ${ }^{91-97}$ Heterogeneity in diagnostic tests, diagnostic association measures and reference standards used prohibited pooling the data in an appropriate way. The majority of studies regarding the assessment of inflammatory activity reported correlations only, instead of the preferred diagnostic association measures (i.e. sensitivity, specificity, PPV, NPV, LRs or ORs). All quantitative information on diagnostic tests is summarised in Supplementary Tables 1-5.

Most studies were found to have a moderate or high RoB. For confirmation of the diagnosis of RA, predominantly because the cut-off for the optimal sensitivity and specificity was selected by using a receiver operating characteristic (ROC) curve analysis of the data of the same patient cohorts (i.e. no predefined cut-off). For assessment of inflammatory activity, predominantly because the reference standard used was not optimal. The patient flow and timing of index test versus reference standard were generally described clearly and were appropriate in most studies.

Overall concerns about applicability for the majority of studies were moderate or high, mainly since the patient populations of included studies did not contain (D2T) RA patients in whom there was explicit doubt about the diagnosis of RA or the presence of inflammatory activity (RoB assessment and concerns regarding applicability per paper in Supplementary Tables 1-5).

\section{Optimal confirmation of the diagnosis of RA}

One study (low RoB) was found assessing the confirmation of the diagnosis of RA, in patients with a self-reported diagnosis (Table 1, Supplementary Table 1).16 Index tests that were assessed in this study were the ACR 1987 classification criteria ${ }^{98}$ and adapted versions of these criteria by including synovitis on ultrasonography (US), erosions on US or X-rays, and rheumatoid factor (RF) and anti-citrullinated protein antibodies (ACPA)

Table 1. Papers on the confirmation of the diagnosis of RA

\begin{tabular}{|c|c|c|c|}
\hline Paper & Design & Study population & Diagnostic test for RA \\
\hline $\begin{array}{l}\text { Pedersen, } \\
2014^{16}\end{array}$ & CS & $\begin{array}{l}\text { Patients with self- } \\
\text { reported RA }(n=51)\end{array}$ & $\begin{array}{l}\text { ACR } 1987 \text { criteria } \\
\text { Adapted ACR } 1987 \text { criteria adding: } \\
\text { - GS synovitis, US erosions, RF } \\
\text { - GS synovitis, US erosions, ACPA } \\
\text { - GS synovitis, erosions on X-ray, ACPA } \\
\text { - PD synovitis, US erosions, ACPA } \\
\text { RAMRIS scale for synovitis (MCP joints 2-5) } \\
\text { RAMRIS scale for synovitis (combined wrist and MCP joints 2-5) }\end{array}$ \\
\hline
\end{tabular}

ACPA: anti-citrullinated protein antibody; ACR: American College of Rheumatology; Cl: confidence interval; CS: cross-sectional; GS: Grey scale; L: low (green); MCP: metacarpophalangeal; n: number of patients; NR: not reported; PD: power doppler; RA: rheumatoid arthritis; RAMRIS: Rheumatoid arthritis MRI scoring system; RF: rheumatoid factor; 
positivity in various combinations. Additionally, the RA magnetic resonance imaging (MRI) scoring system (RAMRIS) scale ${ }^{99}$ for synovitis was assessed as an alternative for the ACR 1987 classification criteria. The reference standard in this study was the clinical diagnosis made by one rheumatologist after a retrospectively conducted review of all available relevant evidence (except for outcomes of MRI, US and ACPA). The ACR 1987 criteria were found to have a sensitivity of $44 \%$ and specificity of $94 \%$. Using the adapted ACR 1987 criteria including Grey scale (GS) synovitis on US, erosions on US, and RF, the sensitivity increased to $72 \%$ at the expense of a minor decrease in specificity to $91 \%$. Using the RAMRIS scale for the assessment of synovitis in metacarpophalangeal (MCP) joints 2 to 5 , resulted in a sensitivity of $69 \%$ and a specificity of $100 \%$ (Table 1 ).

\section{Diagnosis of alternative or coexisting mimicking diseases in RA patients}

Four papers were found on coexisting mimicking diseases in RA, papers on alternative mimicking diagnoses in RA patients were not found. Three of four papers reported on fibromyalgia as a coexisting mimicking disease (Table 2, Supplementary Table 2). In all three papers, the cut-off for the optimal sensitivity and specificity was selected by using a ROC analysis of the data of the same patient cohorts, resulting in a high RoB. The first study assessed the Fibromyalgia Rapid Screening Tool (FiRST) as a diagnostic test for fibromyalgia in consecutive patients suspected of RA or a mimicking disease." Using the clinical diagnosis of fibromyalgia according to a rheumatologist as reference standard, a sensitivity of $83 \%$ and specificity of $88 \%$ were found. In the second study, a score, derived using the individual components of the DAS28 and rearranging the formula (Table 2), was used to assess a diagnosis of fibromyalgia. ${ }^{20}$ Using the diagnosis of fibromyalgia according to the 2010 criteria, ${ }^{100}$ a sensitivity of $81 \%$ and specificity of $80 \%$ were found. The third study used a case-control design, in which microRNA let-7a, $-21-5 p$, -143 and -103a-3p were assessed to diagnose concomitant fibromyalgia in established RA patients..$^{18}$ MicroRNA-143 was found to be downregulated in patients with concomitant fibromyalgia. A sensitivity and specificity of $90 \%$ and $70 \%$ were found.

Reference standard ${ }^{\ddagger}$

Clinical diagnosis according to a rheumatologist

\begin{tabular}{lccc} 
Time interval & Sens, \%(95\%Cl) & Spec, \% (95\%Cl) & RoB \\
\hline NR & $44(22-69)$ & $94(81-99)$ & $\mathrm{L}$ \\
& $72(47-90)$ & $91(77-83)$ & \\
$72(47-90)$ & $87(73-97)$ \\
$56(31-79)$ & $91(77-98)$ \\
$39(17-64)$ & $100(90-100)$ \\
$69(39-91)$ & $100(73-100)$ \\
$62(32-86)$ & $94(73-100)$ & \\
\hline
\end{tabular}

RoB: risk of bias: patient selection/index test/reference standard/flow and timing, highest as found, colour shows highest risk of bias among categories; sens: sensitivity; spec: specificity; US: ultrasonography; †: a clinical diagnosis according to a rheumatologist was deemed as the appropriate reference standard. 
The only study with a low RoB, was a cross-sectional study reporting on bacterial infections as a mimicking disease in RA patients who presented with a flare (Table 2, Supplementary Table 2). ${ }^{19}$ The reference standard for the presence of bacterial infections was the agreed diagnosis by physicians based on symptoms, bacterial culture tests, imaging and response to antibiotic therapy. Erythrocyte sedimentation rate (ESR) >15 $\mathrm{mm} / \mathrm{h}$ was found to have the highest sensitivity with $98 \%$. Procalcitonin $\geq 0.5 \mathrm{ng} / \mathrm{ml}$ was found to have the highest specificity with $98 \%$.

\section{Assessment of inflammatory activity}

Seventy papers evaluated the assessment of inflammatory activity in RA patients at patient and/or joint level. ${ }^{21-90}$ Fifty-eight different diagnostic tests were analysed: 51 biomarkers, six imaging measures and one used histology (Supplementary Table 3 and 4). Different reference standards were used for inflammatory activity: composite indices (DAS28, CDAl, SDAI), clinical assessment (swollen joint count (SJC(28/32/66)), tender joint count (TJC(28/32/66))) and imaging measures (US, MRI, folate scan). No studies with a low or moderate RoB were found that evaluated a diagnostic test in a population in whom there was explicit doubt about the presence of inflammatory activity and that

Table 2. Papers on the diagnoses of mimicking diseases in RA patients

\begin{tabular}{|c|c|c|c|}
\hline Paper & Design & Study population & Diagnostic test for mimicking disease \\
\hline Fan, $2016^{17}$ & CS & $\begin{array}{l}\text { Established RA ( } n=279) \text {, with final } \\
\text { diagnosis: } \\
\text { - RA with concomitant diagnosis of } \\
\text { FM according to ACR criteria }(n=52) \\
\text { or to rheumatologist }(n=93) \\
\text { - RA without FM (according to ACR } \\
\text { criteria: } n=227 \text {; or to rheumatologist: } \\
n=186)\end{array}$ & Fibromyalgia Rapid Screening Tool (FiRST) $\geq 5$ (/6) \\
\hline $\begin{array}{l}\text { Ghib, } \\
2018^{\wedge 18}\end{array}$ & $\mathrm{CC}$ & $\begin{array}{l}\text { Established RA }(n=20) \text { : } \\
\text { - RA with FM }(n=10) \\
\text { - RA without FM but with VAS-pain } \\
\geq 50(n=10)\end{array}$ & MicroRNA-143 >1.0 \\
\hline $\begin{array}{l}\text { Salaffi, } \\
2018^{20}\end{array}$ & CS & $\begin{array}{l}\text { Established RA }(n=292) \text {, with final } \\
\text { diagnosis: } \\
\text { - RA with FM }(n=43) \\
\text { - RA without FM }(n=249)\end{array}$ & $\begin{array}{l}\text { Derived DAS28 patient-reported components } \\
(\text { DAS28-P)* }>0.6312 \\
*(0.56 \times \sqrt{ } \text { TJC }+0.014 \times G H) / D A S 28\end{array}$ \\
\hline Sato, $2012^{19}$ & CS & $\begin{array}{l}\text { Established RA }(n=118) \text {, with final } \\
\text { diagnosis: } \\
\text { - Flare of RA }(n=18) \\
\text { - Non-infectious complications } \\
(n=23) \\
\text { - Non-bacterial infection }(n=15) \\
\text { - Bacterial infection }(n=62)\end{array}$ & $\begin{array}{l}\text { Procalcitonin } \geq 0.5 \mathrm{ng} / \mathrm{ml} \\
\text { Procalcitonin } \geq 0.2 \mathrm{ng} / \mathrm{ml} \\
\text { WBC count }>8500 / \mathrm{m} 3 \\
\text { CRP } \geq 0.3 \mathrm{mg} / \mathrm{dl} \\
\text { ESR }>15 \mathrm{~mm} / \mathrm{h} \\
\text { Procalcitonin } \geq 0.5 \mathrm{ng} / \mathrm{ml}+\text { WBC count }>8500 / \mathrm{m} 3\end{array}$ \\
\hline
\end{tabular}

ACR: American College of Rheumatology; CC: case control; Cl: confidence interval; CRP: C-reactive protein; CS: cross-sectional; dl: decilitre; DAS28: disease activity score assessing 28 joints; ESR: erythrocyte sedimentation rate; FM: fibromyalgia; GH: global health; H: high (red); h: hour; L: low (green); m³; cubic metre; mm: millimetre; ml: millilitre; n: number of patients; ng: nanogram; NR: not reported; RA: rheumatoid arthritis; RoB: risk of bias: patient 
also reported appropriate diagnostic association measures.

\section{Patient level}

In papers at patient level, 57 different diagnostic tests were assessed (Supplementary Table 3). Seventeen biomarkers and two imaging measures (US sum scores and optical spectral transmission (OST) measures) were assessed in more than one study using the same reference standard per diagnostic test (Table 3). The majority of papers at patient level reported correlation measures only.

Only one study (moderate RoB) explicitly evaluated patients in whom there was doubt about the presence of inflammation, although this study did not report appropriate diagnostic association measures. ${ }^{25}$ In patients who had symptoms suggestive of inflammatory joint pain, weak or non-statistically significant correlations were found between DAS28 and US sum scores (US sum scores of hands and feet: $r=0.14$; US sum scores of MTP joints: $r=0.03$ ). In established RA patients in whom there was not explicit doubt about the presence of inflammation, moderate to strong correlations between US sum scores and composite indices were found in eight other papers (range of $r: 0.40$ to 0.70 , statistically significant (s) in 6 of 8 papers (2 low RoB, 5 moderate RoB, 1 high

Within period

of hospital

admission

$\begin{array}{lc}26 & 98 \\ 34 & 89 \\ 66 & 59 \\ 70 & 5 \\ 98 & 9 \\ 21 & 1\end{array}$

selection/index test/reference standard/flow and timing, highest as found, colour shows highest risk of bias among categories; sens: sensitivity; spec: specificity; TJC: tender joint count; VAS: visual analogue scale; WBC: white blood cell; ^: abstract; *: 95\% Cl, if reported; ‡: a clinical diagnosis according to a rheumatologist was deemed as the appropriate reference standard. 
Table 3. Concise summary of papers on the assessment of inflammatory activity at patient level

\section{Papers/design}

54 papers (4 SLRs (\#14/22/7/14), 46 CS,

$2 \mathrm{CS}^{\wedge}, 2$ CC) $)^{22-29,34,35,37-40,43-50,52-58,61-65,68-80,82-88}$

\section{RA patients:}

- General RA population, $n=9686+N R$

- Suspected of or diagnosed with RA, $n=40$

- Established RA, $n=46$

- ACPA positive, $\mathrm{n}=72$

- Active RA, $n=40$

- With musculoskeletal symptoms, n=27

- With at least 1 painful or swollen joint,

$\mathrm{n}=42$

- With knee effusion, $n=30$

- With at least 1 joint amenable to biopsy,

$\mathrm{n}=15$

- Who were referred for examination of

hands and feet, $n=46$

Replicated diagnostic tests (\#: $n$ ) Reference standards (\#: $n$ )

Biomarkers:

MBDA score (2: 3936); miR-

146a (1 SLR: 638); ACPA (8: 568);

Neutrophile lymphocyte ratio (3:

523); Platelet lymphocyte ratio (2:

421); Leptin (6: 404); IL-6 (4: 373); VEGF (5: 344); MMP-3 (2: 173); IL-17

(2: 121+NR); TNF(a) (2: 185); RF (2:

165); Fibrinogen (2: 152); Resistin

(2: 141); IL-2 (2: 111); IL-4 (2: 111); IL-10

(2: 111)

Imaging (sum scores):

US all types (9: 2060+NR): US GS (2:

57), US PD (5: 646); OST measures

(3: 171)
DAS28 (51: 8656+NR); CDAI

(7: 4186); SDAI (6: 4140);

Composite score, not further

specified (1: 1307)

\section{Clinical assessment as reference standard}

20 papers (17 CS, $\left.2 \mathrm{CS}^{\wedge}, 1 \mathrm{CC}\right)^{24,26-}$

$30,35,43,47,52,57,63,65,66,68,72,74,75,82,88$

RA patients:

- General population, $n=1002$

- ACPA positive, $n=72$

- Established RA, $n=46$

- With knee effusion, $n=30$

- With at least 1 joint amenable to biopsy,

$\mathrm{n}=15$

- With at least 1 painful or swollen joint,

$\mathrm{n}=42$

\section{Imaging as reference standard}

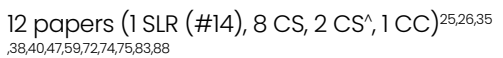

RA patients:

- General population, n=1795

- Who were referred for examination of

hands and feet $(n=46)$

- With at least 1 painful or swollen joint, $\mathrm{n}=42$
Biomarkers:

IL-6 (2: 205); VEGF (2: 205); ACPA (3: 28/32/66 (20: 1207)

181); Leptin (2: 87)

Imaging (sum scores):

US (5: 299); OST measure (2: 109)

Biomarkers:

IL-6 (2: 207); VEGF (3: 277)

Imaging (sum scores):

US (4: $110+N R$ ); OST measures (2:

109)
US (11: 1865); MRI (2: 1325) 
Following replicated diagnostic tests with DAS28 as reference standard:

- MBDA score: pooled $r=0.41$ ( $\mathrm{s}, 1 \mathrm{SLR}) ; \mathrm{r}=0.52$ ( $\mathrm{s}, 1 \mathrm{CS})$

SLR concludes: 'The MBDA demonstrates moderate convergent validity with DAS28-CRP and DAS28ESR, but weaker correlations with SDAI, CDAl, and RAPID3.'

- miR-146a: pooled $r=0.434(\mathrm{~s})$

SLR concludes: 'Circulating and synovial tissue/fluid miR-146a levels are high in patients with RA, and circulating miR-146a levels positively correlate with ESR.'

- IL-17: pooled $r=0.335$ ( $s, 1$ SLR); r =0.373 ( $s, 1$ CS)

SLR concludes: 'Circulating IL-17 level is related to the disease activity of RA.'

- ACPA: $r=-0.126-0.437$ ( $s$ in \#2; ns in \#6)

- Neutrophile lymphocyte ratio: $r=0.192-0.345$ ( $\mathrm{s}$ in \#1; ns in \#1)

- Platelet lymphocyte ratio: $r=0.22-0.352(s, \# 2)$

- Leptin: $r=-0.111-0.513$ ( $s$ in \#4; ns in \#2)

- IL-6: $r=0.31-0.409(\mathrm{~s}, \# 4)$

- VEGF: $r=0.10-0.6527$ (s, in \#2; ns in \#3)

- MMP-3: $r=0.30-0.674(s, \# 3)$

- TNF(a): $r=-0.01-0.213$ (s in \#l; ns in \#1))

- RF: $r=0.265-0.3(s, \# 2)$

- Fibrinogen: $r=0.373-0.55(\mathrm{~s}, \# 2)$

- Resistin: $r=0.403-0.44(s, \# 2)$

- IL-2: $r=-0.08--0.005(n s, \# 2)$

- IL-4: $r=-0.004-0.191(n s, \# 2)$

- IL-10: r=-0.11-0.226 (ns, \#2)

Following replicated imaging measures with composite index as reference standard:

- US (all types): $r=0.03,0.40-0.70$ ( $s$ in \#7; NR in \#2)

- US (GS): $r=0.251-0.70(s, \# 2)$

- US (PD): $r=0.5043-0.72$ ( $\mathrm{s}$ in \#5; ns in \#1)

- OST measure: $r=0.06-0.42$ ( $\mathrm{s}$ in \#2; $\mathrm{ns}$ in \#1)

SLR concludes: 'Ultrasonography can be regarded as a valuable tool for globally examining the extent of synovitis in RA. However, it is presently difficult to determine a minimal number of joints to be included in a global ultrasonography score. Further validation of proposed scores is needed.'

Following diagnostic tests with SJC as reference standard:

- IL-6: $r=0.39-0.41(s, \# 2)$

- VEGF: $r=0.13-0.14(\mathrm{~ns}, \# 2)$

- ACPA: $r=-0.051(n s, \# 3)$

- Leptin: $r=-0.046(n s, \# 1)$

- US: $r=0.3270-0.78(s, \# 4)$

- OST measure: $\mathrm{r}=0.30-0.50(\mathrm{~s}, \# 2)$

Following diagnostic tests with TJC as reference standard:

- IL-6: r=0.09- 0.14 (ns, \#2)

- VEGF: $r=-0.03-0.15$ (ns, \#2)

- ACPA: $r=0-0.144(n s, \# 3)$

- Leptin: $r=0.072-0.59$ ( $\mathrm{s}$ in \#l; ns in \#2)

- US: $r=0.25-0.65(s, \# 4)$

- OST measure: $r=-0.02-0.25$ ( $\mathrm{s}$ in \#1; ns in \#1)

Following diagnostic tests with US as reference standard:

- IL-6: $r=0.23-0.49$ ( $\mathrm{s}$ in \#2; ns in \#1)

- VEGF: $r=-0.10-0.4824$ ( $\mathrm{s}$ in \#2; ns in \#1)

- US (PD in 6 joints vs PD in 12 joints): $r=0.03-0.935$ ( $s$ in \#3; ns in \#1)

- OST measure: $r=0.54-0.64(s, \# 2)$

SLR concludes: 'Ultrasonography can be regarded as a valuable tool for globally examining the

extent of synovitis in RA. However, it is presently difficult to determine a minimal number of joints to be included in a global ultrasonography score. Further validation of proposed scores is needed.' 
Table 3. Continued

ACPA: anti-citrullinated protein antibody; CC: case control; CDAl: clinical disease activity index; CS: cross-sectional; DAS28: disease activity score assessing 28 joints; ESR: erythrocyte sedimentation rate; GS: Grey scale; H: high (red); IL: interleukin; L: Iow (green); M: moderate (yellow); MBDA: multi-biomarker disease activity; miRNA: micro ribonucleic acid; MMP-3: matrix metalloproteinase-3; MRl: magnetic resonance imaging; n: number of patients; NR: not reported; ns: not significant; OST: optical spectral transmission; PD: power Doppler; r: correlation coefficient; RA: rheumatoid arthritis; RF: rheumatoid factor; RoB: risk of bias: patient selection/index test/reference standard/ flow and timing, highest as found, colour shows highest risk of bias among categories; s: significant; SDAl: simplified disease activity index; SJC: swollen joint count; SLR: systematic literature review; TJC: tender joint count; TNF: tumour necrosis factor; US: ultrasonography; VEGF: vascular endothelial growth factor; \#: number of studies; ^: abstract; ‡: For the general established RA population, validated composite disease activity indices (e.g. DAS28 or CDAI) were deemed as appropriate to assess the presence of inflammatory activity at patient level. In patients in whom there is explicit doubt about the presence of inflammatory activity, the traditional measures are not trustworthy. Therefore, in studies assessing this population we considered scores based on established imaging measures as a more appropriate reference standard.

RoB)). ${ }^{24,27,35,40,74,78,80,83}$ One of these papers was a SLR, in which the authors concluded that US can be a valuable tool to globally assess the extent of synovitis, although it is presently difficult to determine a minimal number of joints to be included in an US sum score. ${ }^{40}$

Only four papers reported an appropriate diagnostic association measure and had a low or moderate RoB, although these papers assessed established RA patients in whom there was not explicit doubt about the presence of inflammation. ${ }^{49,55,84,85}$ All four papers had a moderate RoB and assessed a different biomarker using DAS28 as a reference standard: high-sensitivity cardiac troponin (DAS28>5.1: PPV 21.2\%, NPV 94.6\%), human neutrophil peptides 1-3 (DAS28>2.6: sensitivity 72\%, specificity $70.6 \%$ ), anti-citrullinated protein antibodies (ACPA, DAS28 not further specified: OR 2.0,95\% $\mathrm{Cl}$ 1.004-3.983), and matrix metalloproteinase-3 (MMP-3, DAS28>3.2: sensitivity $93.2 \%$; specificity $82.8 \%$ ). Of these biomarkers, ACPA and MMP-3 were assessed in more than one study, although only correlation coefficients were reported in the other papers (ACPA, $\mathrm{r}:-0.13-0.44$, s in 1 of 7 papers ( 6 moderate RoB, 1 high RoB); MMP-3, r: 0.30 and 0.61 , s in 2 of 2 papers ( $1 \mathrm{low}$ RoB, 1 moderate RoB)).22,35,52,53,62-65,73

Additionally, the SLR about the multi-biomarker disease activity (MBDA) score (including 22 studies, moderate RoB) reported that in three of four papers the MBDA score discriminated between low versus moderate/high disease activity (MBDA 230 ). ${ }^{34,102-105}$ The appropriate diagnostic association measures were not reported in the SLR and could only be calculated in one of these three papers (DAS28-CRP $\geq 2.7$ (at the 6 month visit (i.e. non-treatment naïve patients): sensitivity $69 \%$, specificity $64 \%$ ). ${ }^{102}$ Furthermore, moderate statistically significant correlations were reported between the MBDA score and DAS28CRP (r: 0.41 (pooled r, SLR) and 0.52, both moderate RoB). 34,56

\section{Joint level}

At joint level, 15 different diagnostic tests were assessed (Table 4, Supplementary Table 
4). Four diagnostic tests (clinically swollen joints, optical spectral transmission (OST) measures, US and MRI) were assessed in more than one study with the same reference standard used per diagnostic test. In none of the studies, there was explicit doubt about the presence of inflammatory activity.

Almost all studies had a high RoB, predominantly because the cut-off for the optimal sensitivity and specificity was selected by using a ROC curve analysis of the data of the same patient cohort or because the reference standard was not appropriate. The only paper with a moderate RoB was a SLR (including 14 studies), which was performed without critical flaws ${ }^{67}$ In this SLR, synovitis of different joints was assessed with US as diagnostic test and MRI as a reference standard. However, the reference standard used in this SLR (i.e. MRI) was regarded as inappropriate to assess the presence of inflammatory activity in the general established RA population, which hampers its applicability.

Using the reference standard deemed appropriate to us (i.e. clinical diagnosis of swelling of a joint), three papers were found assessing OST (high RoB).42,72,88 Each study used different diagnostic association measures to report the diagnostic value of OST measures in different joints (sensitivity $37-59 \%$ and specificity $86-93 \%$; PPV $46 \%$ and NPV $86 \%$; area under the ROC 0.88).

\section{Assessment of inflammatory activity in RA patients with comorbidities}

Studies assessing diagnostic tests for the assessment of inflammatory activity in RA patients with a specific comorbidity that may influence the assessment were found for obesity and fibromyalgia (Table 5, Supplementary Table 5).

Inflammatory activity in RA patients with and without obesity was assessed in four papers (patient level: 2 moderate RoB, 1 high RoB; joint level: 1 moderate RoB). ${ }^{91,92,96,97}$ In the first study at patient level with moderate RoB, an US sum score of 28 joints and a DAS28 in which SJC was based on US assessment were compared with traditional SJC28 and DAS28. ${ }^{96}$ In patients with a BMI below 25, no significant differences were found between the US-based and traditional measures. In patients with a BMI above 25, the US28 sum score was significantly higher than SJC28 (mean difference in patients with BMI 25-30: 1.818, $\mathrm{p}=0.001 ; \mathrm{BMl}>30$ : 1.600, $\mathrm{p}=0.049$ ). While comparing US-DAS28 with DAS28, US-DAS28 was only statistically significantly higher than DAS28 in patients with a BMI between 25 and 30 (Table 5). In the other study at patient level with moderate RoB, lower extremity SJC (only joints below the waist) was found to be increased in patients with a BMl above 30, corrected for patient and physician global disease activity, ESR and TJC (OR 1.633, $\mathrm{p}=0.005) .{ }^{97}$ This association was less clear for SJC44 (OR 1.765, $\mathrm{p}=0.090$ ), suggesting that upper extremity assessment is not significantly influenced by obesity. In the study at joint level (moderate RoB), clinical assessment of a joint being swollen was found to be overestimated in patients with obesity. ${ }^{91}$ The probability of synovitis according to US decreased per higher BMl category (BMl<25, BMl 25-30, BMl >30), corrected for age, 
Table 4. Concise summary of papers on the assessment of inflammatory activity at joint level

Papers/design

4 papers (3 CS, 1 CC) $)^{21,42,72,88}$

341 RA patients

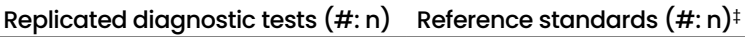

Clinical evaluation according to physician (2: 132); Clinically swollen joint (2: 109); Clinically tender joint (1: 50)

US (8: 526); FolateScan (1: 40); MRI (4: 295)

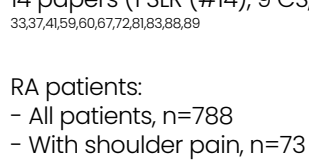

- With shoulder pain, $n=73$

\author{
Clinical assessment. \\ Clinically tender joints (2: 102); \\ Clinically swollen joints (3: 163) \\ Biomarkers: \\ IL-2, IL-4, IL-6, IL-10, IL-17, TNF, IFN, \\ VEGF (1: 64) \\ Other imaging measure than \\ reference standard: \\ OST measures (5: 222); US (3: 456); \\ MRI (1: 19); Contrast-enhanced \\ MRI (1: 43); Fluorescence optical \\ imaging (1: 18)
}

\section{Histology as reference standard}

1 paper (CS) ${ }^{27}$

RA patients with at least 1 joint amenable to biopsy, $n=15$
Imaging:

US (GS and PD; 1: 15)
Krenn index of cellular inflammation (1: 15); Krenn lining layer score (1: 15); Inflammatory cell infiltrates (1: 15)

CC: case control; CS: cross-sectional; GS: Grey scale; H: high; IFN: interferon; IL: interleukin; L: low; M: moderate; MCP: metacarpophalangeal; MRI: magnetic resonance imaging; n: number of patients; NPV: negative predictive value; NR: not reported; OST: optical spectral transmission; PD: power Doppler; PIP: proximal interphalangeal; PPV: positive predictive value; r: correlation coefficient; RA: rheumatoid arthritis; RoB: risk of bias: patient selection/index test/reference standard/flow and timing, highest as found, colour shows highest risk of bias among categories; s: significant; sens: sensitivity; SLR: systematic literature review; spec: specificity; TNF: tumour necrosis factor; US:

gender and clinical assessment of a joint being swollen (OR BMl $0.52(95 \% \mathrm{Cl} 0.30-0.93$, $\mathrm{p}=0.03)$ ).

Three papers evaluated the assessment of inflammatory activity in RA patients with and without fibromyalgia at patient level (2 moderate RoB, 1 high RoB).92-94 The first study with moderate RoB assessed the correlation of composite indices with 7-joint US scores. ${ }^{93}$ Statistically significant correlations were found with 7-joint US scores based on GS in patients with and without fibromyalgia (range of $r: 0.36$ to 0.43 and 0.39 to 0.57, respectively). Using 7-joint US scores based on power Doppler (PD), a significant correlation was found in patients without fibromyalgia (range of $\mathrm{r}: 0.35$ to 0.38 ), although correlations were found not to be statistically significant in patients with fibromyalgia 
OST measures as diagnostic test with following types of clinical assessment as reference standard:

- Clinically swollen: Sens 37-42\% (\#1); spec 86-93\% (\#1)

- Clinically swollen and/or tender: PPV: 46-50\% (\#1); NPV: 78-85\% (\#1)

- Clinical evaluation according to physician: $r=0.63, p<0.0001$ (\#1)

Treatment influenced on the basis of US findings: $51.7 \%(\# 1)$

Clinically swollen joints (\#2):

- Hand and foot joints: NPV $40 \%(\# 1)$

- Hand joints: sens $41 \%$, spec $93 \%$ (\#1)

OST measure as diagnostic test with US as reference standard (\#5):

- Hand joints: sens 29-91\%, spec 24-93\% (\#4)

- Wrist: sens 39\%, spec $87 \%$ (\#1)

- MCP: sens $70 \%$, spec $74 \%(\# 1)$

- PIP: sens 29-83\%, spec 64-89\% (\#3)

US as diagnostic test with MRI as reference standard (\#2 of which 1 SLR): sens 64-91\%, spec 60-94\%. - SLR concludes: 'US is a valid and reproducible technique for detecting synovitis in the wrist and finger joints. It may be considered for routine use as part of the standard diagnostic tools in RA.'

None of the other diagnostic tests were replicated using the same diagnostic accuracy measures.

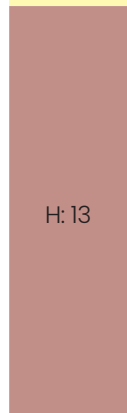

US (GS) as diagnostic test with following histology measures as reference standard:

- Krenn index of cellular inflammation $(r=0.65, p<0.01)$

- Krenn lining layer score $(r=0.52, p<0.05)$

US (PD) as diagnostic test with following histology measures as reference standard:

- Krenn index of cellular inflammation ( $r=0.34$, s NR)

- Krenn lining layer score $(r=0.48$, s NR)

ultrasonography; VEGF: vascular endothelial growth factor; vs: versus; \#: number of studies; ^: abstract; ‡: For the general established RA population, the clinical assessment of swelling in the joint was deemed as appropriate to assess the presence of inflammatory activity at joint level (i.e. in a specific joint). In patients in whom there is explicit doubt about the presence of inflammatory activity, the traditional measures are not trustworthy. Therefore, in studies assessing this population we considered established imaging measures as a more appropriate reference standard.

(range of $\mathrm{r}: 0.01$ to 0.12 ). In the other study with moderate RoB, statistically significant correlations were found between swollen joint counts and 7-point US scores for synovitis and for tenosynovitis based on GS and PD in patients without fibromyalgia (range of r: 0.44 to 0.57). ${ }^{95}$ Again, correlations were not statistically significant in patients with fibromyalgia (r: not given).

\section{Discussion}

In this SLR, evidence was sought regarding the optimal confirmation of RA and relevant 
Table 5. Concise summary of papers on the assessment of inflammatory activity in RA patients with comorbidities that may influence the assessment

\begin{tabular}{l}
\hline Papers/design \\
\hline Obesity \\
At patient level \\
3 papers $(3 \mathrm{CS})^{92,96,97}$ \\
RA patients, $\mathrm{n}=756$ : \\
- BMl $<25, \mathrm{n}=287+\mathrm{NR}$ \\
- BMl $25-30, \mathrm{n}=33+\mathrm{NR}$ \\
$-\mathrm{BMl}>30, \mathrm{n}=79+\mathrm{NR}$
\end{tabular}

At joint level

1 paper (1 CS) $)^{91}$

RA patients, $\mathrm{n}=43$ :

- $\mathrm{BMI}<25, \mathrm{n}=17$

- BMI 25-30, n=12

- BMl >30, n=14

\section{Fibromyalgia}

At patient level

3 papers $\left(2 \mathrm{CS}^{\wedge}, 1 \mathrm{CC}\right)^{93-95}$

RA patients, $n=239$

- Without fibromyalgia, $n=161$

- With fibromyalgia, $n=78$
Replicated diagnostic tests (\#: $n$ ) Reference standards (\#: $n$ )

\author{
- MBDA score (1: 357) \\ - Adjusted MBDA score (1:190 \\ MBDAoriginal + 67.175 - (0.79*age) \\ $-(1.74 * \mathrm{BMl})+(0.018 * \mathrm{age} * \mathrm{BMI})$ \\ - US: sum score of 28 joints (1: 76) \\ - US-DAS28 (SJC based on - US \\ findings, $1: 76$ ) \\ - SJC44 (1: 323) \\ - Lower extremity SJC (1: 323)
}

Clinically swollen joint (1: 43)
- CDAl (1: 357)

- MBDA score (1: 190)

- SJC28 (1:76)

- DAS28 (1: 76)

- ACR core set measures (patient/physician global, ESR, TJC; l: 323)

\author{
- SJC (1: 39) \\ - DAS28 (1: 72) \\ - CDAI (1: 72) \\ - SDAI (1: 72) \\ - Modified DAS28 (1: 130) \\ - Modified CDAI (1: 130) \\ - Modified SDAI (1: 130) \\ Modified: excluding TJC and \\ patient's global assessment of \\ disease activity
}

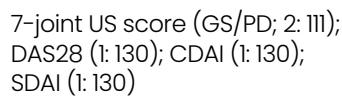
DAS28 (1: 130); CDAI (1: 130); $\operatorname{SDAI}(1: 130)$

ACR: American College of Rheumatology; BMl: body mass index; CC: case-control; CDAl: clinical disease activity index; Cl: confidence interval; CRP: C-reactive protein; CS: cross-sectional; DAS28: disease activity score assessing 28 joints; ESR: erythrocyte sedimentation rate; GS: Grey scale; H: high (red); L: low (green); M: moderate (yellow); MBDA: multi-biomarker disease activity; n: number of patients; NR: not reported; ns: not significant; OR: odds ratio; PD: power Doppler; r: correlation coefficient; RA: rheumatoid arthritis; RoB: risk of bias: patient selection/index test/ 
- MBDA as diagnostic test with CDAl as reference standard:

$\mathrm{BMl}<25$ : $r=0.33, p=0.0004$; BMl 25-30: $r=0.28, p=0.002 ; B M l>30: r=-0.02, p=0.80$

- Adjusted MBDA as diagnostic test with MBDA as reference standard:

All BMl categories: $r=0.91, p<0.00001$ (BMl categories and adjusted MBDA with CDAl as reference standard: NR)

- US28 sum score as diagnostic test with SJC28 as reference standard, mean difference (i.e. US28 sum score higher than SJC28):

BMI <25: 0.429 ( $p=0.467)$; BMI 25-30: 1.818 ( $p=0.001)$; BMI >30: $1.600(p=0.049)$

- US-DAS28 as diagnostic test with DAS28 as reference standard, mean difference (i.e. US-DAS28 higher than DAS28):

$\mathrm{BMI}<25$ : $0.014(p=0.812) ; B M I 25-30: 0.175(p=0.002) ; B M l>30: 0.011(p=0.894)$

In case of obesity (BMI >30), SJC increases correcting for ACR core set measures (patient/physician global, ESR, TJC):

- Lower extremity SJC: OR $1.633(p=0.005)$

- SJC44: OR $1.037(p=0.090)$

Per higher BMl category the chance of synovitis according to US decreased correcting for age, gender and clinically swollen joints (i.e. the SJC overestimates disease activity in obese patients): - OR BMI 0.52 ( $95 \%$ Cl 0.30-0.93, p=0.03)

Correlation coefficient in patients without vs with fibromyalgia with 7-joint US score (GS/PD) as reference standard:

- DAS28 (GS): $r=0.39(p<0.05)$ vs $r=0.36(p<0.05)$

- DAS28 (PD): $r=0.35(p<0.05)$ vs $r=0.12(n s)$

- CDAI (GS): $r=0.57(p<0.05)$ vs $r=0.43(p<0.05)$

- CDAI (PD): $r=0.37$ ( $p<0.05)$ vs $r=0.01$ (ns)

- SDAI (GS): $r=0.57(p<0.05)$ vs $r=0.38(p<0.05)$

- SDAI (PD): $r=0.38$ ( $p<0.05$ ) vs $r=0.01$ (ns)

- SJC (GS) - synovitis: $r=0.44(p=0.015)$ vs ns

- SJC (PD) - synovitis $r=0.47(p=0.008)$ vs ns

- SJC (GS) - tenosynovitis: $r=0.57(p=0.001)$ vs ns

- SJC (PD) - tenosynovitis: $r=0.46(p=0.011)$ vs ns

In patients with fibromyalgia a discrepancy between traditional and modified composite scores originates, with higher traditional scores in these patients.

Mean increment ( $95 \% \mathrm{Cl}, \mathrm{p}$-value), adjusted for age, sex and nodular disease:

- DAS28-ESR: 1.50 (0.60-2.40, 0.001)

- DAS28-CRP: $1.55(0.63-2.48,0.001)$

- CDAl: 10.78 (3.23-18.34, 0.006)

- SDAl: 11.34 (3.80-18.89, 0.0158)

reference standard/flow and timing, highest as found, colour shows highest risk of bias among categories; sens: SDAl: simplified disease activity index; SJC: swollen joint count; TJC: tender joint count; US: ultrasonography; \#: number of studies; ${ }^{\wedge}$ : abstract; $;:$ In patients with comorbidities that may influence the assessment of inflammatory activity, the traditional measures may not be trustworthy. Therefore, in studies assessing this population we considered (scores based on) established imaging measures as a more appropriate reference standard. 
differential diagnoses as well as the assessment of inflammatory activity in D2T RA patients in whom there was doubt about the diagnosis or the presence of inflammatory activity. Several limitations were found in the selected evidence. First, no studies were identified including D2T RA patients specifically and only the minority of studies included RA patients in whom there was explicit doubt about the diagnosis of RA or about the presence of inflammatory activity. Second, a heterogeneous collection of diagnostic tests was evaluated using different association measures, hampering pooling of results. Third, only very few studies with a low RoB were found. Additional limitations were found in the evidence regarding the assessment of inflammatory activity in D2T RA patients. Mostly, only correlation measures were reported, which are not directly appropriate to assess a test for indicating the presence or absence of inflammatory disease activity in clinical practice (although a strong correlation is likely a prerequisite). Furthermore, major heterogeneity was found in reference standards used in these studies, reflecting the lack of a true gold standard to assess inflammatory activity. Taking all the above-mentioned limitations into account, the identified evidence should be regarded as indirect for the population of D2T RA patients and the results should be interpreted carefully.

Limited evidence was found to consider specific diagnostic tests to confirm or rule out the diagnosis of RA or relevant differential diagnoses. None of the diagnostic tests in the studies regarding the diagnosis of RA or relevant differential diagnoses were replicated, limiting the validity of the results. The only study with a low RoB showed that adapted ACR 1987 criteria (including GS synovitis, US erosions, RF) and RAMRIS scale of MCP joints had an additional value above the traditional ACR 1987 criteria to rule out the diagnosis of RA (sensitivity $69 \%$ and $72 \%$, respectively, compared with $42 \%$ ), although probably still too low to rule out RA with sufficient certainty. ${ }^{16}$ Moreover, classification criteria, such as the ACR 1987 criteria, should only be applied after a diagnosis is made and are inappropriate to make a diagnosis, making these results not applicable to ascertain the diagnosis of RA in clinical practice. ${ }^{106}$ Furthermore, in the other study with a low RoB in RA patients who presented with a flare, ESR $<15 \mathrm{~mm} / \mathrm{h}$ was shown to be able to rule out and procalcitonin $\geq 0.5 \mathrm{ng} / \mathrm{ml}$ to confirm bacterial infection as a mimicking disease..$^{19}$ Some studies were found assessing the diagnosis of (concomitant) fibromyalgia, although all these studies had a high RoB. ${ }^{17,18,20}$

As 'best available direct evidence' to assess the presence of inflammatory activity in RA patients in whom there was explicit doubt about the presence of inflammatory activity, only one study was identified, having a moderate RoB. In this study, only weak and statistically non-significant correlations were reported between an US sum score and DAS28. ${ }^{25}$ In the general population of RA patients who are not treatment naive, US was studied most extensively among all diagnostic tests in papers with low to moderate RoB. ${ }^{24,27,35,40,67,74,78,83}$ All papers reported moderate to strong correlations between DAS28 and US sum scores, although also here appropriate diagnostic association measures 
were not reported. These moderate to strong correlations in the general RA population together with the absence of at least a moderate correlation in patients in whom there is explicit doubt about the presence of inflammatory activity (and thus in in whom traditional measures may not be trusted), suggest that uS may have an additional value in these patients. However, the optimal number of joints to include in an US sum score to assess inflammatory activity at patient level differed per study and is currently unclear. ${ }^{40}$ This limitation hampers the current use of an US sum score in clinical practice.

As the 'best available indirect evidence' to assess the presence of inflammatory activity in RA patients in whom there was not explicit doubt about the presence of inflammatory activity, MMP-3 and the MBDA score were studied most extensively in studies reporting the appropriate diagnostic association measures with low to moderate RoB. ${ }^{34,35,56,64}$ However, for MMP-3, no validated cut-off was found ${ }^{35,64}$ and, for the MBDA score, the cut-off could not be validated in all studies, $102-105,107$ limiting the applicability for use in daily practice. At joint level, studies with low to moderate RoB assessing US as well as other diagnostic tests with the preferred reference standard at joint level (i.e. clinically swollen joints) were not found.

Presence of obesity and fibromyalgia in RA patients was found to hamper proper grading of disease activity using traditional composite indices.91,94,96,97 Presence of fibromyalgia led to overestimation of disease activity compared with US and modified composite indices, while the influence of obesity on the assessment of disease activity was conflicting between studies. Two studies reported an overestimation of disease activity using traditional composite indices compared with US, at least in the joints of the lower extremities.91,97 On the contrary, the presence of obesity was found to lead to underestimation of inflammatory activity using SJC compared with a US-based SJC in another study. ${ }^{96}$ In obese patients, composite indices may not only be influenced by the SJC, but also by acute phase reactants. Acute phase reactants may be elevated through the production of inflammatory mediators from adipocytes, resulting in increased composite indices in obese patients. ${ }^{4}$

US was studied most extensively to assess the presence of inflammatory activity in patients with concomitant obesity or fibromyalgia, in studies having a moderate RoB. In these patients, correlations between US and composite indices were weaker or not statistically significant anymore compared with patients without these comorbidities. This suggests that US may have an additional value to traditional measures to assess inflammatory RA activity in patients with these comorbidities. ${ }^{91,93,95}$ No studies were found regarding other comorbidities that might influence assessment of inflammatory disease activity.

A previous EULAR project has focussed on the development of the EULAR recommendations for the use of imaging of the joints in RA and there is some overlap with our SLR (recommendation 3: 'Ultrasound and MRI are superior to clinical examination 
in the detection of joint inflammation; these techniques should be considered for a more accurate assessment of inflammation'). ${ }^{108}$ The statement regarding US is consistent with the findings of our SLR. However, the results of our SLR do not clearly indicate the usefulness of MRI. Most studies on MRI were not included in our SLR, predominantly because they were focussed on treatment naive RA patients, were published before the year 2000 or assessed the change in inflammatory activity instead of the presence of inflammatory activity as relevant for our question. ${ }^{109-118}$

In addition to the limitations in the evidence that was found, this SLR has some limitations itself. Although an extensive literature search has been performed, relevant papers might have been missed. Regarding the diagnosis of RA and relevant differential diagnoses, it was chosen to perform a limited search focussing on the last ten years and not to perform reference screening because not much relevant evidence was presently expected before this time and to enable focussing more on the other clinical questions regarding D2T RA where more relevant literature was expected. After the second Task Force meeting was postponed due to the COVID-19 outbreak, it was decided not to update the search for this specific question because of the same abovementioned reasons. Regarding the assessment of inflammatory activity, the search focussed on the last ten years, although references of selected papers were also screened and relevant papers published from the year 2000 were selected because of the introduction of bDMARDs around this time point. Additionally, for this search, we focussed on nontreatment naive RA patients resulting in the exclusion of papers focussing on RA patients in the early phase of the disease. However, we felt this was well-justifiable as D2T RA patients are by definition established RA patients and evidence on early RA was deemed too indirect for our present work. Although, as above decisions could be considered limitations, it should be stressed that choices were made by the Task Force, including experienced clinicians, researchers and methodologists and with input from experienced librarians. Therefore, we think the methodological stringency of this SLR and its focus on established RA patients in the present diagnostic and therapeutic era, have resulted in a comprehensive overview of the current literature.

Further guidance on the diagnostic issues in D2T RA, including the clinical implications of the results, will be provided by the EULAR Task Force on D2T RA in their recommendations for the management of D2T RA, which will be published soon."19 Additionally, a research agenda will be provided including topics that should be addressed in future studies.

In conclusion, this SLR highlights the scarcity of evidence on the optimal confirmation or ruling out of a diagnosis of RA and relevant differential diagnoses in D2T RA patients. Therefore, textbook knowledge on potential alternative and/or coexisting mimicking diseases remains highly relevant. When currently used clinical measures may not be trusted as in D2T RA patients, US may have some additional value to assess the presence of inflammatory activity in these patients as well as in those with concomitant obesity or 
fibromyalgia. However, more high-quality studies addressing D2T RA patients in whom there is reasonable doubt about the diagnosis and about the presence of inflammatory activity are required. 


\section{References}

1. Smolen JS, Landewé RBM, Bijlsma JWJ, et al. EULAR recommendations for the management of rheumatoid arthritis with synthetic and biological disease-modifying antirheumatic drugs: 2019 update. Ann Rheum Dis 2020;79:685-99.

2. Singh JA, Saag KG, Bridges SL, et al. 2015 American College of Rheumatology Guideline for the Treatment of Rheumatoid Arthritis. Arthritis Rheumatol 2016;68:1-26.

3. Buch $\mathrm{MH}$. Defining refractory rheumatoid arthritis. Ann Rheum Dis 2018;77:966-9.

4. de Hair MJH, Jacobs JWG, Schoneveld JLM, et al. Difficult-to-treat rheumatoid arthritis: an area of unmet clinical need. Rheumatology (Oxford) 2017;57:1135-44.

5. Kearsley-Fleet L, Davies R, De Cock D, et al. Biologic refractory disease in rheumatoid arthritis: Results from the British Society for Rheumatology Biologics Register for Rheumatoid Arthritis. Ann Rheum Dis 2018;77:1405-12.

6. Nagy G, Roodenrijs NMT, Welsing PMJ, et al. EULAR definition of difficult-to-treat rheumatoid arthritis. Ann Rheum Dis 2021;80:31-5.

7. Roodenrijs NMT, de Hair MJH, van der Goes $\mathrm{MC}$, et al. Characteristics of difficult-to-treat rheumatoid arthritis: results of an international survey. Ann Rheum Dis 2018;77:1705-9.

8. Roodenrijs NMT, van der Goes MC, Welsing $\mathrm{PMJ}$, et al. Difficult-to-treat rheumatoid arthritis: contributing factors and burden of disease. Rheumatology 2020:keaa860.

9. Roodenrijs NMT, Hamar A, Kedves $M$, et al. Pharmacological and non-pharmacological therapeutic strategies in difficult-to-treat rheumatoid arthritis: a systematic literature review informing the EULAR recommendations for the management of difficult-to-treat rheumatoid arthritis. RMD open 2021;7:e001512.

10. Paalanen K, Puolakka K, Nikiphorou $E$, et al. Is seronegative rheumatoid arthritis true rheumatoid arthritis? A nationwide cohort study. Rheumatology (Oxford) 2020:keaa623.

11. van der Heijde D, Aletaha D, Carmona L, et al. 2014 Update of the EULAR standardised operating procedures for EULAR-endorsed recommendations. Ann Rheum Dis 2015;74:813.

12. Thompson M, Tiwari A, Fu R, et al. A Framework To Facilitate the Use of Systematic Reviews and Meta-Analyses in the Design of Primary
Research Studies. Rockville, MD Agency Healthc. Res. Qual. 2012.http://www.ncbi.nlm. nih.gov/books/ NBK83621/ (accessed 13 Nov 2019).

13. Whiting PF, Rutjes AWS, Westwood ME, et al. Quadas-2: A revised tool for the quality assessment of diagnostic accuracy studies. Ann Intern Med 2011;155:529-36.

14. Shea BJ, Reeves BC, Wells G, et al. AMSTAR 2: A critical appraisal tool for systematic reviews that include randomised or non-randomised studies of healthcare interventions, or both. BMJ 2017;358:j4008.

15. Morgan CJ, Aban I. Methods for evaluating the agreement between diagnostic tests. J Nucl Cardiol 2016;23:511-3.

16. Pedersen JK, Lorenzen T, Ejbjerg B, et al. Low-field magnetic resonance imaging or combined ultrasonography and anti-cyclic citrullinated peptide antibody improve correct classification of individuals as established rheumatoid arthritis: Results of a population-based, cross-sectional study. BMC Musculoskelet Disord 2014;15:268.

17. Fan A, Tournadre A, Pereira B, et al. Performance of Fibromyalgia Rapid Screening Tool (FiRST) to detect fibromyalgia syndrome in rheumatic diseases. Rheumatology (Oxford) 2016;55:1746-50.

18. Ghib LJ, Cojocneanu-Petric R, Budisan L, et al. THU0518 The diagnostic value of selected micrornas in patients with fibromyalgia associated with rheumatoid arthritis: a pilot study. Ann Rheum Dis 2018;77:463.3-464.

19. Sato H, Tanabe N, Murasawa A, et al. Procalcitonin is a specific marker for detecting bacterial infection in patients with rheumatoid arthritis. I Rheumatol 2012;39:1517-23.

20. Salaffi F, Di Carlo $M$, Carotti $M$, et al. The subjective components of the Disease Activity Score 28-joints (DAS28) in rheumatoid arthritis patients and coexisting fibromyalgia. Rheumatol Int 2018;38:1911-8.

21. Agrawal S, Bhagat SS, Dasgupta B. Improvement in diagnosis and management of musculoskeletal conditions with onestop clinic-based ultrasonography. Mod Rheumatol 2009;19:53-6.

22. Algergawy SA, Abd El-Sabour M, Osman AS, et al. Early diagnostic and prognostic values of anti-cyclic citrullinated peptide antibody and cartilage oligomeric matrix protein in rheumatoid arthritis. Egypt $\mathrm{J}$ Immunol 2013;20:11-20. 
23. Bustos Rivera-Bahena C, Xibillé-Friedmann D-X, González-Christen J, et al. Peripheral blood leptin and resistin levels as clinical activity biomarkers in Mexican Rheumatoid Arthritis patients. Reumatol Clínica 2016;12:323-6.

24. Ceponis A, Onishi M, Bluestein HG, et al. Utility of the ultrasound examination of the hand and wrist joints in the management of established rheumatoid arthritis. Arthritis Care Res 2014;66:236-44.

25. Ciurtin C, Brown G, Cotton A, et al. THU0138 Das 28 correlated poorly with the objective evidence of inflammation as detected by ultrasound (US) examination of hands and feet in patients with established rheumatoid arthritis (RA). Ann Rheum Dis 2017;76:253.1-253.

26. do Prado $A D$, Bisi $M C$, Piovesan $D M$, et al. Ultrasound power Doppler synovitis is associated with plasma IL-6 in established rheumatoid arthritis. Cytokine 2016;83:27-32.

27. Filer A, Mandelin Al, DiCarlo E, et al. Histological and Clinical Correlates of Ultrasound Measures of Joint Inflammation: Analysis of RA Tissue Obtained By Ultrasound Guided Biopsy in Phase 1 of the Accelerating Medicines Partnership RA Network - ACR Meeting Abstracts. Arthritis Rheumatol 2017;69.

28. Gunaydin R, Kaya T, Atay A, et al. Serum leptin levels in rheumatoid arthritis and relationship with disease activity. South Med J 2006;99:1078-83.

29. Ha YJ, Kang EJ, Lee SW, et al. Usefulness of serum leucine-rich alpha-2 glycoprotein as a disease activity biomarker in patients with rheumatoid arthritis. J Korean Med Sci 2014;29:1199-204.

30. Hammer HB, Sexton J, Michelsen B, et al. FRI0042 Tender joints have low agreement with patient's evaluation of spontaneous joint pain, joint swelling and ultrasound verified synovitis in patients with established rheumatoid arthritis. Ann Rheum Dis 2018;77:567.3-568.

31. Hermann KGA, Backhaus $M$, Schneider $U$, et al. Rheumatoid Arthritis of the Shoulder Joint: Comparison of Conventional Radiography, Ultrasound, and Dynamic ContrastEnhanced Magnetic Resonance Imaging. Arthritis Rheum 2003;48:3338-49.

32. Inamo J, Kaneko Y, Sakata K, et al. THU0162 Residual synovitis in ankles and feet detected by ultrasonography in patients with rheumatoid arthritis. Ann Rheum Dis 2018;77:300.2-300.
33. Amitai I, Werner S, Schicke B, et al. Comparison of photo optical imaging with musculoskeletal ultrasound and clinical examination in the assessment of inflammatory activity in proximal interphalangeal joints in rheumatoid arthritis and osteoarthritis. J Rheumatol 2015;42:1595-602.

34. Johnson TM, Register KA, Schmidt CM, et al. Correlation of the Multi-Biomarker Disease Activity Score with Rheumatoid Arthritis Disease Activity Measures: A Systematic Review and Meta-Analysis. Arthritis Care Res (Hoboken) 2018;71:1459-72.

35. Kawashiri SY, Kawakami A, Iwamoto N, et al. The power Doppler ultrasonography score from 24 synovial sites or 6 simplified synovial sites, including the metacarpophalangeal joints, reflects the clinical disease activity and level of serum biomarkers in patients with rheumatoid arthritis. Rheumatology (Oxford) 2011;50:962-5.

36. Kawashiri SY, Suzuki T, Nishino A, et al. Automated Breast Volume Scanner, a new automated ultrasonic device, is useful to examine joint injuries in patients with rheumatoid arthritis. Mod Rheumatol 2015;25:837-41.

37. Krabbe $\mathrm{S}$, Ammitzbøll-Danielsen $\mathrm{M}$, Østergaard $M$, et al. Sensitivity and specificity of optical spectral transmission imaging in detecting joint inflammation in rheumatoid arthritis. Ann Rheum Dis 2016;75:632-3.

38. Kurosaka $D$, Hirai $K$, Nishioka $M$, et al. Clinical significance of serum levels of vascular endothelial growth factor, angiopoietin-l, and angiopoietin-2 in patients with rheumatoid arthritis. J Rheumatol 2010;37:1121-8.

39. Lee SW, Park MC, Park YB, et al. Measurement of the serum leptin level could assist disease activity monitoring in rheumatoid arthritis. Rheumatol Int 2007;27:537-40.

40. Mandl P, Naredo E, Wakefield RJ, et al. A systematic literature review analysis of ultrasound joint count and scoring systems to assess synovitis in rheumatoid arthritis according to the OMERACT filter. J Rheumatol 2011;38:2055-62.

41. Matteson EL, Lowe VJ, Prendergast FG, et al. Assessment of disease activity in rheumatoid arthritis using a novel folate targeted radiopharmaceutical FolatescanTM. Clin Exp Rheumatol 2009;27:253-9.

42. Meier AJL. Potential of optical spectral transmission measurements for joint inflammation measurements in rheumatoid 
arthritis patients. J Biomed Opt 2012;17:081420.

43. Metawi SA, Abbas D, Kamal MM, et al. Serum and synovial fluid levels of interleukin-17 in correlation with disease activity in patients with RA. Clin Rheumatol 2011;30:1201-7.

44. Andrés Cerezo L, Šumová B, Prajzlerová K, et al. Calgizzarin (S100All): a novel inflammatory mediator associated with disease activity of rheumatoid arthritis. Arthritis Res Ther 2017;19:79.

45. Milman N, Karsh J, Booth RA. Correlation of a multi-cytokine panel with clinical disease activity in patients with rheumatoid arthritis. Clin Biochem 2010;43:1309-14.

46. Ni $M$, Wei $W$, Wang $Y$, et al. Serum levels of calreticulin in correlation with disease activity in patients with rheumatoid arthritis. J Clin Immunol 2013;33:947-53.

47. Nordal $\mathrm{HH}$, Brokstad $\mathrm{KA}$, Solheim $\mathrm{M}$, et al. Calprotectin (S100A8/A9) has the strongest association with ultrasound-detected synovitis and predicts response to biologic treatment: Results from a longitudinal study of patients with established rheumatoid arthritis. Arthritis Res Ther 2017;19:3.

48. Olama SM, Senna MK, Elarman M. Synovial/ Serum leptin ratio in rheumatoid arthritis: The association with activity and erosion. Rheumatol Int 2012;32:683-90.

49. Önder B, Kurtaran A, Kimyon S, et al. Association of anti-CCP positivity with serum ferritin and DAS-28. Rheumatol Int 2009;30:223-7.

50. Oner SY, Volkan O, Oner C, et al. Serum leptin levels do not correlate with disease activity in rheumatoid arthritis. Acta Reumatol Port 2015;2015:50-4.

51. Ostendorf B, Peters R, Dann P, et al. Magnetic resonance imaging and miniarthroscopy of metacarpophalangeal joints: Sensitive detection of morphologic changes in rheumatoid arthritis. Arthritis Rheum 2001;44:2492-502.

52. Papadopoulos NG, Tsiaousis GZ, PavlitouTsiontsi A, et al. Does the presence of anti-CCP autoantibodies and their serum levels influence the severity and activity in rheumatoid arthritis patients? Clin Rev Allergy Immunol 2008;34:11-5.

53. Predeteanu D, Varzaru L, Balanescu A, et al. Anti-cyclic citrullinated peptide antibodies-activity markers in rheumatoid arthritis. J Med Life 2009;2:36-41.

54. Rodríguez-Carrio J, Alperi-López M, López P, et al. Red cell distribution width is associated with cardiovascular risk and disease parameters in rheumatoid arthritis. Rheumatol (United Kingdom) 2014;54:641-6.

55. Avouac J, Meune C, Chenevier-Gobeaux C, et al. Inflammation and disease activity are associated with high circulating cardiac markers in rheumatoid arthritis independently of traditional cardiovascular risk factors. J Rheumatol 2014;41:248-55.

56. Roodenrijs NMT, de Hair MJH, Wheater G, et al. The multi-biomarker disease activity score tracks response to rituximab treatment in rheumatoid arthritis patients: a post-hoc analysis of three cohort studies. Arthritis Res Ther 2018;20:256.

57. Rooney $\mathrm{T}$, Scherzer R, Shigenaga JK, et al. Levels of plasma fibrinogen are elevated in well-controlled rheumatoid arthritis. Rheumatology (Oxford) 2011;50:1458-65.

58. Sahebari $M$, Mirfeizi $Z$, Rezaieyazdi $Z$, et al. 25(OH) vitamin $D$ serum values and rheumatoid arthritis disease activity (DA S28 ESR). Casp J Intern Med 2014;5:148-14855.

59. Schäfer VS, Hartung W, Hoffstetter $P$, et al. Quantitative assessment of synovitis in patients with rheumatoid arthritis using fluorescence optical imaging. Arthritis Res Ther 2013;15:R124.

60. Scheel AK, Backhaus $M$, Klose AD, et al. First clinical evaluation of sagittal laser optical tomography for detection of synovitis in arthritic finger joints. Ann Rheum Dis 2005;64:239-45.

61. Šenolt L, Housa D, Vernerová Z, et al. Resistin in rheumatoid arthritis synovial tissue, synovial fluid and serum. Ann Rheum Dis 2007;66:45863.

62. Serdaroğlu $M$, Çakırbay $H$, Değer $O$, et al. The association of anti-CCP antibodies with disease activity in rheumatoid arthritis. Rheumatol Int 2008;28:965-70.

63. Shah BG, Quershi HJ, Zafar U. Serum anti-CCP antibody and its correlation with disease activity in local Pakistani rheumatoid arthritis patients. Pakistan J Med Heal Sci 2014;8:1041-4.

64. Skacelova $M$, Hermanova $Z$, Horak $P$, et al. Higher levels of matrix metalloproteinase -3 in patients with RA reflect disease activity and structural damage. Biomed Pap 2017;161:296302.

65. Sockalingam S, Khuan CS, Sthaneshwar P. Prevalence of anti cyclic citrullinated peptide antibodies in Malaysian rheumatoid arthritis patients and its correlation with disease activity. Int J Rheum Dis 2009;12:211-5.

66. Axelsen MB, Eshed I, Duer-jensen $A$, et al. 
Whole-body MRI assessment of disease activity and structural damage in rheumatoid arthritis: First step towards an MRI joint count. Rheumatol (United Kingdom) 2014;53:845-53.

67. Takase-Minegishi K, Horita N, Kobayashi K, et al. Diagnostic test accuracy of ultrasound for synovitis in rheumatoid arthritis: systematic review and meta-analysis. Rheumatology (Oxford) 2018;57:49-58.

68. Targońska-Stepniak B, Majdan M, Dryglewska M. Leptin serum levels in rheumatoid arthritis patients: Relation to disease duration and activity. Rheumatol Int 2008;28:585-91.

69. Tekeoğlu I, Gürol G, Harman H, et al. Overlooked hematological markers of disease activity in rheumatoid arthritis. Int $\mathrm{J}$ Rheum Dis 2016;19:1078-82.

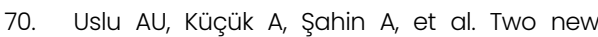
inflammatory markers associated with Disease Activity Score-28 in patients with rheumatoid arthritis: Neutrophil-lymphocyte ratio and platelet-lymphocyte ratio. Int J Rheum Dis 2015;18:731-5.

71. Valle $Y$, Ledezma-Lozano IY, Torres-Carrillo $\mathrm{N}$, et al. Circulating TNFRI and TNFRII levels correlated with the disease activity score (DAS28) in rheumatoid arthritis. Scand J Rheumatol 2009;38:332-5.

72. van Onna $M$, Ten Cate $D F$, Tsoi $K L$, et al. Assessment of disease activity in patients with rheumatoid arthritis using optical spectral transmission measurements, a noninvasive imaging technique. Ann Rheum Dis 2016;75:511-8.

73. Vanichapuntu $M$, Phuekfon $P$, Suwannalai $P$, et al. Are anti-citrulline autoantibodies better serum markers for rheumatoid arthritis than rheumatoid factor in Thai population? Rheumatol Int 2010;30:755-9.

74. Vlad V, Berghea F, Libianu S, et al. Ultrasound in rheumatoid arthritis - Volar versus dorsal synovitis evaluation and scoring. BMC Musculoskelet Disord 2011;12:124.

75. Witt $M$, Frielinghausen $J$, Mueller $R$, et al. Evaluation of a Novel Semi-Automated Ultrasound System for the Detection of Synovitis: A Prospective Study involving 45 Patients with Rheumatoid Arthritis. Ultrasound Int Open 2016;02:El17-23.

76. Yildirim K, Karatay S, Melikoglu MA, et al. Associations between acute phase reactant levels and disease activity score (DAS28) in patients with rheumatoid arthritis. Ann Clin Lab Sci 2004;34:423-6.

77. Bae SC, Lee YH. MiR-146a levels in rheumatoid arthritis and their correlation with disease activity: a meta-analysis. Int. J. Rheum. Dis. 2018;21:1335-42.

78. Yokota K, Tsuzuki Wada T, Akiyama $Y$, et al. Detection of synovial inflammation in rheumatic diseases using superb microvascular imaging: Comparison with conventional power Doppler imaging. Mod Rheumatol 2018;28:327-33.

79. Zengin $\mathrm{O}$, Onder $\mathrm{ME}$, Kalem $\mathrm{A}$, et al. New inflammatory markers in early rheumatoid arthritis. Z Rheumatol 2018;77:144-50.

80. Zufferey P, Brulhart L, Tamborrini G, et al. Ultrasound evaluation of synovitis in RA: Correlation with clinical disease activity and sensitivity to change in an observational cohort study. Jt Bone Spine 2014;81:222-7.

81. Abdelzaher MG, Tharwat S, AbdElkhalek A, et al. Ultrasound versus magnetic resonance imaging in the evaluation of shoulder joint pathologies in a cohort of rheumatoid arthritis patients. Int J Rheum Dis 2019;22:215864.

82. Myngbay A, Bexeitov $\mathrm{Y}$, Adilbayeva A, et al. CTHRCl: A New Candidate Biomarker for Improved Rheumatoid Arthritis Diagnosis. Front Immunol 2019;10:1353.

83. Ngai $\mathrm{Ng} \mathrm{S}$, Bjørndal Axelsen $\mathrm{M}$, Ostergaard $M$, et al. OP0135 How well does whole body magnetic resonance imaging agree with whole body ultrasound in the assessment of joint inflammation in rheumatoid arthritis patients. Ann Rheum Dis 2019;78:143.

84. Okcu M, Oktayoglu P, Mete N, et al. A useful marker in the assessment of remission and activation of disease in patients with rheumatoid arthritis: Serum human neutrophil peptides 1-3. J Back Musculoskelet Rehabil 2018;31:1145-50.

85. Tuncer T, Kaya A, Gulkesen A, et al. Matrix metalloproteinase-3 levels in relation to disease activity and radiological progression in rheumatoid arthritis. Adv Clin Exp Med 2019;28:665-70.

86. Yuan $\mathrm{Z}-\mathrm{C}$, Wang $\mathrm{J}-\mathrm{M}$, Huang $\mathrm{A}-\mathrm{F}$, et al. Elevated expression of interleukin-37 in patients with rheumatoid arthritis. Int $\mathrm{J}$ Rheum Dis 2019;22:1123-9.

87. Zhang $X$, Yuan $Y$, Pan $Z$, et al. Elevated circulating $\mathrm{IL}-17$ level is associated with inflammatory arthritis and disease activity: A meta-analysis. Clin Chim Acta 2019;496:7683.

88. Besselink NJ, van der Meijde P, Rensen WHJJ, et al. Optical spectral transmission to assess 
inflammation in hand and wrist joints of rheumatoid arthritis patients. Rheumatology (Oxford) 2018;57:865-72.

89. Boesen $M$, Ellegaard $K$, Boesen $L$, et al. Ultrasound doppler score correlates with OMERACT RAMRIS bone marrow oedema and synovitis score in the wrist joint of patients with rheumatoid arthritis. Ultraschall der Medizin 2012;33:E166-72.

90. Bonfiglioli KR, Chakr R, Lima R, et al. SAT0632 Ultrasound in the management of rheumatoid arthritis using a novel pragmatic algorithm: a multicentric observational study. Ann Rheum Dis 2018;77:1167.2-1168.

91. Bauer EM, Ben-Artzi A, Duffy EL, et al. Jointspecific assessment of swelling and power Doppler in obese rheumatoid arthritis patients. BMC Musculoskelet Disord 2017;18:99.

92. Curtis JR, Greenberg JD, Harrold LR, et al. Influence of obesity, age, and comorbidities on the multi-biomarker disease activity test in rheumatoid arthritis. Semin Arthritis Rheum 2018;47:472-7.

93. Da Silva Chakr RM, Brenol JCT, Behar $M$, et al. Is ultrasound a better target than clinical disease activity scores in Rheumatoid Arthritis with fibromyalgia? A case-control study. PLoS One 2015;10:e0118620.

94. Sosa J, Karina Perez S, Julia Santa Cruz M, et al. M-DAS28, M-SDAl and M-CDAl Performance in a Cohort of RA Patients with and without Concomitant Fibromyalgia. Arthritis Rheumatol 2017;69:suppl 10.

95. Tamas M, Ghib L-J, Bondor C, et al. THU0156 Clinical assessment versus ultrasonography in patients with rheumatoid arthritis treated with biological agents - the impact of concomitant fibromyalgia. Ann Rheum Dis 2017;76:260.1-260.

96. Goossens J, Coustet B, Palazzo E, et al. Overweight and obesity affect clinical assessment of synovitis in rheumatoid arthritis: comparison of ultrasonography and clinical exam. Clin Exp Rheumatol 2019;37:4954.

97. Ranganath VK, Duffy EL, Garg VK, et al. Obesity Impacts Swelling of Ankle and Foot Joints in Early Rheumatoid Arthritis Patients. J Clin Rheumatol 2019;25:e8-11.

98. Arnett FC, Edworthy SM, Bloch DA, et al. The american rheumatism association 1987 revised criteria for the classification of rheumatoid arthritis. Arthritis Rheum 1988;31:315-24.

99. Østergaard M, Peterfy C, Conaghan P, et al.
OMERACT Rheumatoid Arthritis Magnetic Resonance Imaging Studies. Core set of MRI acquisitions, joint pathology definitions, and the OMERACT RA-MRI scoring system. J Rheumatol 2003;30:1385-6.

100. Wolfe F, Clauw DJ, Fitzcharles $M-A$, et al. The American College of Rheumatology preliminary diagnostic criteria for fibromyalgia and measurement of symptom severity. Arthritis Care Res (Hoboken) 2010;62:600-10.

101. Wolfe F, Clauw DJ, Fitzcharles M-AA, et al. Fibromyalgia criteria and severity scales for clinical and epidemiological studies: A modification of the ACR preliminary diagnostic criteria for fibromyalgia. J Rheumatol 2011;38:1113-22.

102. Bakker MF, Cavet G, Jacobs JW, et al. Performance of a multi-biomarker score measuring rheumatoid arthritis disease activity in the CAMERA tight control study. Ann Rheum Dis 2012;71:1692-7.

103. Curtis JR, Van Der Helm-Van Mil AH, Knevel R, et al. Validation of a novel multibiomarker test to assess rheumatoid arthritis disease activity. Arthritis Care Res 2012;64:1794-803.

104. Hirata S, Dirven L, Shen Y, et al. A multibiomarker score measures rheumatoid arthritis disease activity in the best study. Rheumatology (Oxford) 2013;52:1202-7.

105. Fleischmann R, Connolly SE, Maldonado MA, et al. Brief Report: Estimating Disease Activity Using Multi-Biomarker Disease Activity Scores in Rheumatoid Arthritis Patients Treated With Abatacept or Adalimumab. Arthritis Rheumatol 2016;68:2083-9.

106. Aggarwal R, Ringold S, Khanna $\mathrm{D}$, et al. Distinctions between diagnostic and classification criteria? Arthritis Care Res (Hoboken) 2015;67:891-7.

107. Davis JM. Editorial: The Multi-Biomarker Disease Activity Test for Rheumatoid Arthritis: Is It a Valid Measure of Disease Activity? Arthritis Rheumatol 2016;68:2061-6.

108. Colebatch AN, Edwards CJ, Østergaard $M$, et al. EULAR recommendations for the use of imaging of the joints in the clinical management of rheumatoid arthritis. Ann Rheum Dis 2013;72:804-14.

109. Calisir C, Murat Aynaci Al, Korkmaz C. The accuracy of magnetic resonance imaging of the hands and feet in the diagnosis of early rheumatoid arthritis. Jt Bone Spine 2007;74:362-7.

110. Emery P, Van Der Heijde D, Østergaard M, et 
al. Exploratory analyses of the association of MRI with clinical, laboratory and radiographic findings in patients with rheumatoid arthritis. Ann Rheum Dis 2011;70:2126-30.

111. Forslind $\mathrm{K}$, Johanson $\AA$, Larsson $\mathrm{EM}$, et al. Magnetic resonance imaging of the fifth metatarsophalangeal joint compared with conventional radiography in patients with early rheumatoid arthritis. Scand J Rheumatol 2003;32:131-7.

112. Forslind $\mathrm{K}$, Larsson EM, Eberhardt $\mathrm{K}$, et al. Magnetic resonance imaging of the knee: $A$ tool for prediction of joint damage in early rheumatoid arthritis? Scand J Rheumatol 2004;33:154-61.

113. Goupille P, Roulot B, Akoka S, et al. Magnetic resonance imaging: A valuable method for the detection of synovial inflammation in rheumatoid arthritis. J Rheumatol 2001;28:3540.

114. Haavardsholm EA, Østergaard M, Hammer $\mathrm{HB}$, et al. Monitoring anti-TNFa treatment in rheumatoid arthritis: Responsiveness of magnetic resonance imaging and ultrasonography of the dominant wrist joint compared with conventional measures of disease activity and structural damage. Ann
Rheum Dis 2009;68:1572-9.

115. Roimicher L, Lopes FPPL, de Souza SAL, et al. (99m)Tc-anti-TNF-a scintigraphy in RA: a comparison pilot study with MRI and clinical examination. Rheumatology (Oxford) 2011;50:2044-50.

116. Tamai $M$, Kawakami A, Iwamoto $N$, et al. Comparative study of the detection of joint injury in early-stage rheumatoid arthritis by magnetic resonance imaging of the wrist and finger joints and physical examination. Arthritis Care Res 2011;63:436-9.

117. Tonolli-Serabian I, Poet $\mathrm{JL}$, Dufour $\mathrm{M}$, et al. Magnetic resonance imaging of the wrist in rheumatoid arthritis: Comparison with other inflammatory joint diseases and control subjects. Clin Rheumatol 1996;15:137-42.

118. Wakefield RJ, Freeston JE, O'Connor P, et al. The optimal assessment of the rheumatoid arthritis hindfoot: A comparative study of clinical examination, ultrasound and high field MRI. Ann Rheum Dis 2008;67:1678-82.

119. Nagy G, Roodenrijs NMT, Welsing PMJ, et al. EULAR recommendations for the management of difficult-to-treat rheumatoid arthritis. Manuscript in preparation. 


\section{Supplementary}

\section{Supplementary File}

1. The confirmation of the diagnosis of RA or relevant differential diagnoses of an alternative or mimicking disease

Clinical question

How do we optimally confirm an RA diagnosis?

Epidemiological question

How can we confirm a diagnosis of RA in difficult-to-treat RA patients?

- P: Difficult-to-treat RA patients with doubt about diagnosis or doubt about RA as origin of complaints

- I: Diagnostic 'tests' for RA as well as differential diagnoses

- C: RA or non-RA/relevant differential diagnosis

- O: Diagnostic test value of 'tests' to establish RA/relevant differential diagnosis

\section{Search in short}

Rheumatoid arthritis AND Terms for misdiagnosis and common mimicking diseases (gout, CPPD, PSA, SpA, fibromyalgia, OA, PMR, SLE, paraneoplastic syndromes, reactive arthritis) AND Terms for diagnostic outcomes/association measures

\section{The assessment of inflammatory activity in RA patients}

\section{$2 a$. The assessment of inflammatory activity in RA patients}

Clinical question

How do we evaluate the level of inflammation of RA activity in difficult-to-treat RA patients?

\section{Epidemiological question}

How can we evaluate the presence of inflammatory RA activity in difficult-to-treat RA?

- P: Difficult-to-treat RA patients

- I: Diagnostic 'tests' to establish inflammatory activity

- C: Inflammatory activity or not (remission) according to a reference standard

- O: Diagnostic test value of the 'test(s)' to establish inflammatory activity

$2 \mathrm{~b}$. The assessment of inflammatory activity in RA patients with comorbidities that might influence the assessment

Clinical question 
How do comorbidities influence the assessment of RA?

Epidemiological question

How do comorbidities influence the assessment of RA disease activity?

- P: Difficult-to-treat RA patients

- I: Comorbidities (that may influence disease activity assessment)

- C: No comorbidities

- O: Diagnostic test value of combined disease activity score, APR or imaging to establish disease activity/comparison with reference standard in those with/without comorbidity

Search in short

Rheumatoid arthritis AND Terms for difficult-to-treat RA and comorbidities that may influence disease activity assessment AND General terms for inflammation AND Specific tests to assess inflammation AND Diagnostic query of PubMed extended with relevant diagnostic terms

\section{Other}

Searches in full, exclusion criteria, predetermined items for data extraction and Supplementary Tables 1-7 can be accessed via: https://10.1136/rmdopen-2020-001511. 



\section{CHAPTER 8}

Pharmacological and non-pharmacological therapeutic strategies in difficult-totreat rheumatoid arthritis: a systematic literature review informing the EULAR recommendations for the management of difficult-to-treat rheumatoid arthritis

RMD Open 2021;7:e001512.

Nadia M.T. Roodenrijs, Attila Hamar, Melinda Kedves, György Nagy, Jacob M. van Laar, Désirée van der Heijde, Paco M.J. Welsing 


\section{Abstract}

\section{Objectives}

To summarise, by a systematic literature review (SLR), the evidence regarding pharmacological and non-pharmacological therapeutic strategies in difficult-totreat rheumatoid arthritis (D2T RA), informing the EULAR recommendations for the management of D2T RA.

\section{Methods}

PubMed, Embase and Cochrane databases were searched up to December 2019. Relevant papers were selected and appraised.

\section{Results}

Two hundred seven papers studied therapeutic strategies. Limited evidence was found on effective and safe disease-modifying antirheumatic drugs (DMARDs) in patients with comorbidities and other contraindications that limit DMARD options (patients with obesity, hepatitis B and C, risk of venous thromboembolisms, pregnancy and lactation). In patients who previously failed biological (b-)DMARDs, all currently used b/targeted synthetic (ts-)DMARDs were found to be more effective than placebo. In patients who previously failed a tumour necrosis factor inhibitor (TNFi), there was a tendency of nonTNFi bDMARDs to be more effective than TNFis. Generally, effectiveness decreased in patients who previously failed a higher number of bDMARDs. Additionally, exercise, psychological, educational and self-management interventions were found to improve non-inflammatory complaints (mainly functional disability, pain, fatigue), education to improve goal setting, and self-management programs, educational and psychological interventions to improve self-management.

The identified evidence had several limitations: 1) no studies were found in D2T RA patients specifically, 2) heterogeneous outcome criteria were used, and 3) most studies had a moderate or high risk of bias.

\section{Conclusions}

This SLR underscores the scarcity of high-quality evidence on the pharmacological and non-pharmacological treatment of D2T RA patients. Effectiveness of b/tsDMARDs decreased in RA patients who had failed a higher number of bDMARDs and a subsequent $\mathrm{b} / \mathrm{tsDMARD}$ of a previously not targeted mechanism of action was somewhat more effective. Additionally, a beneficial effect of non-pharmacological interventions was found for improvement of non-inflammatory complaints, goal setting and self-management. 


\section{Introduction}

Therapeutic strategies for patients with rheumatoid arthritis (RA) have significantly improved over the past decades. However, there is still a substantial proportion of patients that remains symptomatic, even though they have been treated according to the current European League Against Rheumatism (EULAR) recommendations and/or American College of Rheumatology (ACR) guideline for the management of RA.,2 This patient group is referred to as having 'difficult-to-treat (D2T) RA'. This disease state is estimated to affect $5 \%$ to $20 \%$ of all RA patients, depending on the specific definition

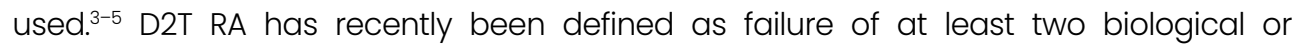
targeted synthetic disease-modifying antirheumatic drugs (b/tsDMARDs) with different mechanisms of action (MOA), in patients who are still having complaints which may be indicative of active disease and which is perceived as problematic by patient and/or rheumatologist. ${ }^{6}$ An international survey that was conducted among rheumatologists showed the unmet need for this patient population. ${ }^{7}$ Consequently, the importance has been acknowledged by EULAR with the approval of a Task Force in charge of the development of management recommendations for D2T RA.

D2T RA is a highly heterogeneous disease state. ${ }^{4}$ Patients could be symptomatic due to inflammatory activity: e.g. refractory disease (having underlying immunologic disease mechanisms driving multi-drug resistant or 'true' refractory disease), having active disease because they cannot be adequately treated (i.e. having limited drug options because of contraindications, such as comorbidities and/or (risk of) adverse events) or having persistent inflammatory activity due to non-adherence. In addition, patients could be symptomatic due to non-inflammatory factors, e.g. concomitant osteoarthritis and fibromyalgia., $3,4,9$ One of the abovementioned factors could be present, although inflammatory activity and non-inflammatory complaints frequently seem to coexist in daily practice. ${ }^{9}$

Furthermore, in D2T RA patients, a mismatch in goal setting between patients and health care professionals as well as suboptimal self-management could negatively impact treatment outcomes and illness perception. ${ }^{10}$ Patients' management goals could be unrealistic, e.g. aiming to return to all normal activities of daily living, while this is not always achievable because of, for example, the presence of joint damage. To be able to align treatment goals and to optimise self-management, it will be important to identify mismatches in treatment goals and suboptimal self-management to achieve the most optimal effect from available therapeutic strategies.

Currently, RA management recommendations endorse to switch to another b/tsDMARD in symptomatic patients who failed at least one previous b/tsDMARD and could possibly classify as having D2T RA.' This therapeutic strategy leads to a trial-and-error approach in D2T RA patients, as the origin of complaints remains unclear. ${ }^{4}$ Furthermore, 
prioritisation of b/tsDMARDs and non-pharmacological interventions are lacking in current recommendations. Additionally, no recommendations are currently available for RA patients with limited drug options because of contraindications, those with predominantly non-inflammatory complaints (e.g. pain, fatigue, reduced function and quality of life), suboptimal self-management, and for those in whom treatment goals are unclear or do not match with the health care professional.

Before switching to yet another DMARD, thorough evaluation of the origin of the complaints is needed to be able to choose the most appropriate treatment option. It will be needed to ascertain the diagnosis of RA and to evaluate alternative or coexisting mimicking diseases. Furthermore, it will be important to assess the presence or absence of inflammatory activity. Optimal diagnostic test for these diagnostic issues are reviewed in a separate systematic literature review (SLR)."

The aim of this SLR was first to explore and summarise pharmacological and nonpharmacological therapeutic strategies in D2T RA patients that could be used to treat inflammatory activity and non-inflammatory complaints. Furthermore, this SLR focussed on the optimisation, and therefore also the identification, of a mismatch in goal setting between patients and health care professionals and of suboptimal self-management. This SLR was conducted to inform the EULAR recommendations for the management of D2T RA.

\section{Methods}

\section{Research questions}

This SLR was conducted following the EULAR Standardised Operating Procedures (SOP).12 Seven clinical questions on therapeutic strategies and the identification of suboptimal goal setting and self-management in D2T RA patients were proposed by the fellow (NMTR), co-methodologist (PMJW) and postdoctoral fellow (MJHdH), and then approved by the Steering Committee (GN (convenor), JMvL (co-convenor), DvdH (methodologist), MK (fellow)). At the first Task Force meeting, the questions were discussed, amended and then approved by the whole Task Force.

The clinical questions were focussed on pharmacological and non-pharmacological therapeutic strategies for (la) patients with limited DMARD choices because of adverse events, comorbidities or other contraindications, ( $1 \mathrm{~b}$ ) patients who failed $\geq 2 \mathrm{~b} / \mathrm{tsDMARD}$ with different MOA, (lc) patients with predominantly non-inflammatory complaints; and additionally on (2a) the identification and (2b) optimisation of a mismatch in goal setting, and $(2 \mathrm{c})$ the identification and (2d) optimisation of suboptimal self-management. The clinical questions were transformed into epidemiological questions using the 'Patients, Intervention (index test for diagnostic question), Comparator (reference test), Outcome 
(PICO) format' (Supplementary File).13

\section{Search strategy}

The databases of PubMed, Embase and Cochrane were searched for papers in English until December 2019 for search 1 and December 2018 for search 2. Additionally, the conference abstracts of EULAR and ACR were screened, from 2017 until 2019 for search 1 and from 2017 until 2018 for search 2. Advice regarding the set-up of the search strategy was provided by two experienced librarians of Utrecht University (FPW and PHW).

The first search focussed on the pharmacological and non-pharmacological therapeutic strategies for RA patients with limited DMARD choices, who previously failed $\mathrm{b} /$ tsDMARDs or those with predominantly non-inflammatory complaints. In addition to terms for RA and terms related to therapeutic studies, terms were included for difficultto-treat disease, adverse events, fatigue, pregnancy and comorbidities that may limit DMARD choices (infections: hepatitis B virus (HBV), hepatitis C virus (HCV), human immunodeficiency virus (HIV), tuberculosis (TBC); malignancies; lung disease: fibrosis, asthma, chronic obstructive pulmonary disease (COPD); cardiovascular (CV) disease: hypertension, cardiomyopathy; hyperlipidaemia; chronic kidney dysfunction; chronic liver dysfunction; liver enzyme elevation; osteoporosis; diabetes mellitus; thrombosis; depression; anxiety; Supplementary File). Furthermore, terms were included for specific DMARDs, glucocorticoids (GCs), nonsteroidal anti-inflammatory drugs (NSAIDs) and non-pharmacological treatment options. A search limit was set to the last ten year. In addition, the reference lists of selected papers were manually screened. References published in the year 2000 and later were eligible for inclusion. This cut-off was chosen because of the introduction of bDMARDs around this time and, herewith, the beginning of a new therapeutic landscape regarding available treatment strategies in the field of RA. Moreover, as failure of $\geq 2 \mathrm{~b} / \mathrm{tsDMARD}$ with different MOA is part of the D2T RA definition, RA patients could not fulfil the definition before this time point.

The second search focussed on the identification and optimisation of suboptimal goal setting and self-management. In this search, terms for RA as well as terms for management goals and self-management were included (Supplementary File). No terms for difficult-to-treat patients were included, as studies in other RA patients were also considered to be relevant as indirect evidence for these specific questions, since limited evidence was expected. Additionally, no terms on specific outcomes were included as many outcomes could be of interest and they may be described in many different ways.

\section{Selection of studies}

First, titles and abstracts were screened in duplicate by the fellows (first search: NMTR and MK; second search: NMTR and $\mathrm{AH}$ ) according to a predefined list of selection criteria (Supplementary File) until the percentage of conflicts was below $5 \%$. In case of conflicts 
or when in doubt, eligibility was discussed with the co-methodologist (PMJW). Second, all full-text versions of the selected papers were screened in duplicate by the fellows (first search: NMTR, and MK or AH; second search: NMTR and AH). Disagreements were discussed with the co-methodologist (PMJW) until consensus was reached.

Following the EULAR SOP, SLRs of sufficient quality could be selected in addition to original studies.12 The original studies of the selected SLRs were excluded to avoid duplicate evidence. Additionally, the most recent SLR was selected in case of fully overlapping evidence in two or more SLRS.

As evidence for D2T RA patients specifically was expected to be scarce, for the question on RA patients who previously failed $\geq 2$ b/tsDMARDs with different MOA (1b), it was decided to select papers with patients who failed $\geq 1 \mathrm{~b} /$ tsDMARD. For the question on RA patients with non-inflammatory complaints (lc), papers specifically including patients with active disease, e.g. according to composite indices, were excluded and only papers regarding patients with non-inflammatory complaints (reduced function and quality of life, and presence of pain and fatigue) or in unselected populations (i.e. not specifically active disease) reporting on these outcomes were selected. Regarding the question on a mismatch in goal setting between patients and health care professionals (2a), studies comparing the frequencies of the importance of specific treatment goals between patients and health care professionals were also selected, as these may highlight important goals that may not match between patients and health care professionals.

\section{Data extraction and quality assessment}

Information on study design, patient characteristics, interventions, comparators and outcomes (including relevant descriptive statistics and/or occurrence and association measures) were extracted from the included papers using a predetermined format (Supplementary File).

Risk of bias (RoB) of the original papers was assessed using the Cochrane Collaboration's risk of bias tool, ${ }^{14}$ and highest RoB as found was reported here (low, moderate, high). For the questions on the identification of suboptimal goal setting (2a) and self-management (2c), risk of bias was assessed using the Quality Assessment of Diagnostic Accuracy Studies tool version 2 (QUADAS-2), 15 and highest RoB as found was reported (Iow, moderate, high). For SLRs, 'A MeaSurement Tool to Assess systematic Reviews version 2' (AMSTAR-2) was used and overall RoB was reported according to the AMSTAR-2 scoring system (low, moderate, high, critically high).16

Data extraction and quality assessment were performed in duplicate by the fellows (NMTR and $\mathrm{AH}$ ) until the number of conflicts was below $5 \%$. Disagreements and remaining doubts were discussed with the co-methodologist (PMJW) until consensus was reached.

\section{Statistical analyses}


Extracted data were summarised descriptively regarding the main reported or calculable association measures for the therapeutic questions (e.g. odds ratio (OR), mean outcome in intervention and comparator group) and the diagnostic evaluations (e.g. sensitivity, specificity, likelihood ratio).

Pooling of results was performed in case of sufficient clinical and statistical homogeneity as determined by the Steering Committee. For the questions on therapeutic strategies for RA patients with predominantly non-inflammatory complaints (lc) and optimisation of self-management ( $2 \mathrm{~d}$ ), effect sizes (using Cohen's d) were calculated or extracted to be able to compare results over different outcomes and scoring methods.

\section{Results}

\section{Study characteristics}

The first search regarding pharmacological as well as non-pharmacological strategies resulted in 5885 unique papers. After title and abstract screening, 1165 papers were selected for full-text screening and 121 papers were finally deemed eligible for inclusion. Additionally, 36 papers were selected via reference screening and hand search (Figure la). Thirty-two papers were selected for therapeutic strategies in patients with limited DMARD options, ${ }^{17-48} 73$ for patients who failed $\geq 1$ b/tsDMARD ${ }^{49-121}$ and 50 for patients with predominantly non-inflammatory complaints. ${ }^{122-171}$

The second search regarding goal setting and self-management yielded 1385 unique papers. Title and abstract screening resulted in 236 papers, and 38 papers were selected for data extraction. Four additional papers were selected via reference screening (Figure lb). Three ${ }^{172-174}$ and four papers ${ }^{175-178}$ were selected for the identification and optimisation, respectively, of a mismatch ingoal setting between patients and health care professionals. Five $^{173,179-182}$ and 31 papers ${ }^{122,25,126,130-132,135,137,144,145,148,152,153,159,160,162,167,183-196}$ were selected for the identification and optimisation, respectively, of suboptimal self-management.

None of the studies in search 1 or 2 included D2T RA patients specifically, resulting in a lower applicability of the results overall. Heterogeneity in study populations, therapeutic strategies, outcome criteria and association measures prohibited pooling the data in an appropriate way. All quantitative information regarding study characteristics, therapeutic strategies and outcomes is summarised in Supplementary Tables 1-7.

The overall RoB was moderate or high in the majority of studies. Studies were considered as having a high RoB, because of their study design (i.e. observational studies), subanalyses of randomised controlled trials (RCTs) were performed (not a priori planned or not based on stratified groups), or blinding of participants was not performed (as in the majority of studies regarding non-pharmacological strategies). Studies were assessed as having a moderate RoB, due to insufficient reporting of the randomisation process and/or 
A. Therapeutic strategies in (1a) RA patients with limited drug options due to contraindications, (1b) RA patients who failed $\geq 1$ b/tsDMARD, and (1c) RA patients with predominantly non-inflammatory complaints

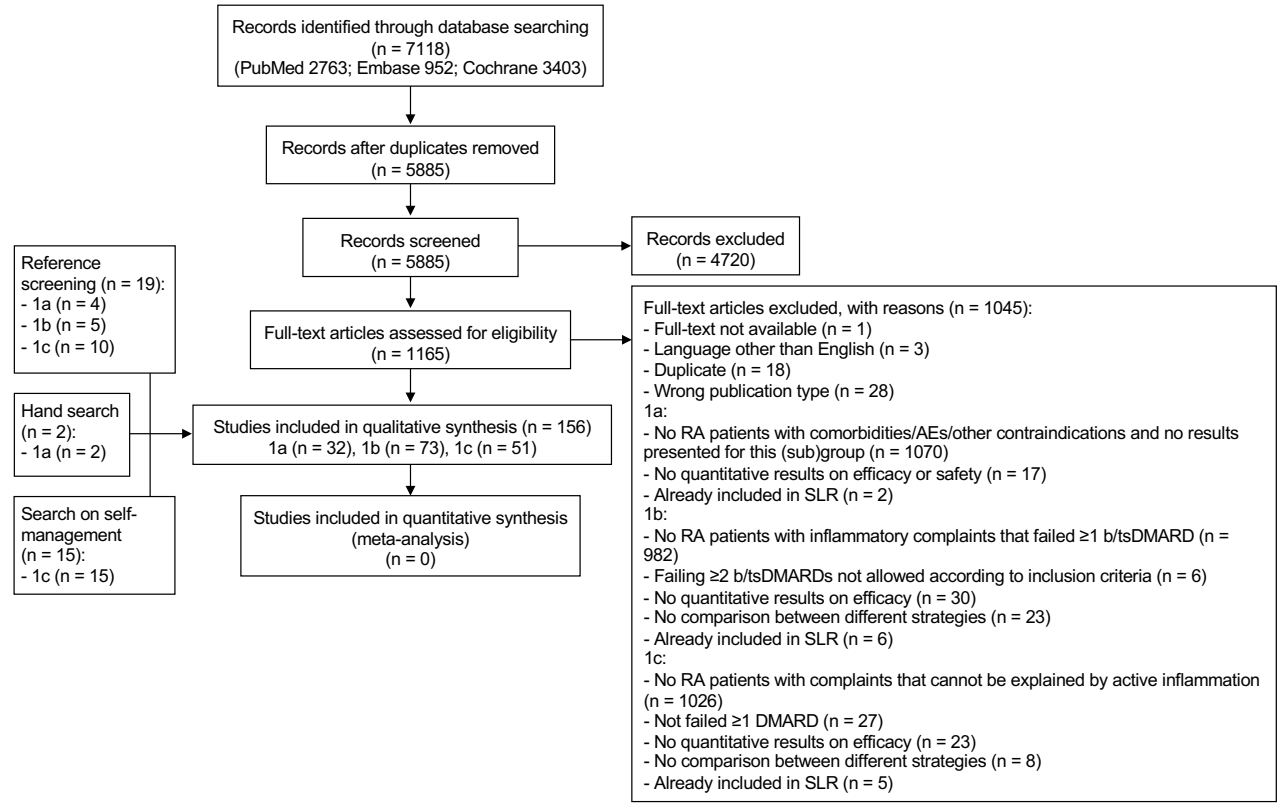

B. (2a) The identification of a mismatch in goal setting and (2b) optimisation of goal setting, and (2c) the identification and (2d) optimisation of suboptimal self-management.

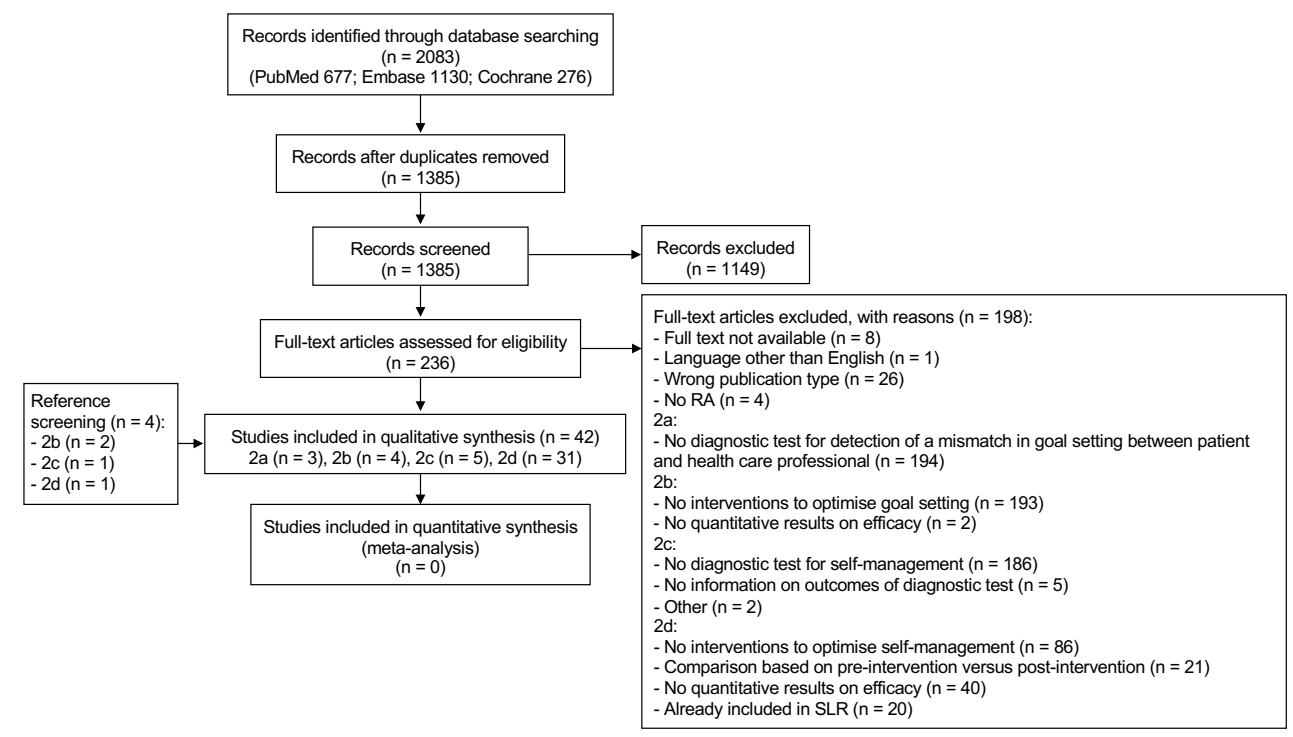

Figure 1. Flow charts of search and selection of papers AEs: adverse events; n: number of papers; RA: rheumatoid arthritis; SLR: systematic literature review. 
allocation concealment. Detailed RoB assessment is shown in Supplementary Tables 1-7.

\section{RA patients with limited drug options}

Thirty-two papers (6 SLRs, 9 RCTs, 17 observational studies; 1 low RoB, 7 moderate RoB, 24 high RoB) were selected comparing efficacy and/or safety of DMARDs in RA patients with limited DMARD options due to a comorbidity and/or another contraindication: HBV, HCV, hepatic disease, pulmonary disease, CV disease, obesity, osteoporosis/ osteopenia, renal disease, extra-articular manifestations, pregnancy, psychological disease (Supplementary Table 1).17-48 No evidence was identified comparing the efficacy and/or safety of DMARDs in RA patients with gastrointestinal disease, HIV, (latent) TBC, malignancies or previously experienced adverse events related to the treatment.

Only the efficacy of infliximab in RA patients with obesity was assessed in more than one cohort of RA patients. In both papers (high RoB), infliximab (3mg/ $/ \mathrm{kg}$ ) was found to be less effective in patients with a body mass index (BMI) >30 than in those with BMI $<30$ (disease activity score assessing 28 joints based on erythrocyte sedimentation rate (DAS28-ESR) remission at 12 months (M): $0 \%$ vs $22.4 \%, \mathrm{p}=0.01,28$ Change in DAS28 $\geq 1.2$ from baseline (BL) until 16 weeks (W): $58.1 \%$ vs $46.7 \%, \mathrm{p}=0.04) .{ }^{33}$ No differences in RA patients with $\mathrm{BMl}>30$ compared with those with $\mathrm{BMl}<30$ were found for treatment with adalimumab, etanercept or rituximab (in single observational studies: DAS28 based on C-reactive protein (-CRP) <2.6 at 12M, adalimumab: $14.8 \%$ vs 30.1\%, p=0.08; DAS28-CRP <2.6 at 12M, etanercept: $27.6 \%$ vs $36.2 \%, p=0.44$; ACR50 response at $24 \mathrm{~W}$, rituximab: $55.7 \%$ vs $49.1-$ $53.4 \%$, not significant). ${ }^{48}$ In patients with obesity, no studies comparing different treatment options were identified.

Safety of DMARD use in patients with a comorbidity was assessed in a SLR or in more than one cohort of RA patients with $\mathrm{HBV}, \mathrm{HCV}$, pregnancy/lactation and in those at risk for venous thromboembolisms (VTES). In patients with active HBV, a relatively low rate of HBV reactivation was found using bDMARDs in one SLR (of 21 studies, moderate RoB) and two observational studies (high RoB) compared with patients with inactive HBV (tumour necrosis factor inhibitor (TNFi), SLR: $10.7 \%$ vs $2.6 \%$; tocilizumab: $0 \%$ vs $4.8 \%$; abatacept: $0 \%$ vs $0 \%)^{20,22,39}$ The authors of the SLR concluded that antiviral prophylaxis would be recommended in patients with active HBV infection. ${ }^{20}$

In an observational study (high RoB) in patients with HCV undergoing treatment with TNFi, liver disease developed more frequently compared with patients without HCV (development of liver injury within $1 Y$ : $10 \%$ vs $1.23 \%, p=0.099$ ), ${ }^{34}$ a difference that did not reach statistical significance in this relatively small sample size $(n=101)$. The authors of the SLR (of 37 studies, high RoB) concluded that the safety profile of TNFi in the setting of HCV infection seemed to be acceptable.18

Evidence regarding safe DMARD use before and during pregnancy and during lactation was found in the 2020 ACR guideline and 2016 EULAR points to consider (of 53 and 319 
studies, respectively, moderate RoB). ${ }^{27,42}$ These recommendations have been based on extensive SLRs and summarise the evidence per DMARD. In patients before and during pregnancy and lactation, no safety issues have been identified for antimalarials, sulfasalazine, azathioprine, ciclosporin, tacrolimus and glucocorticoids. Of bDMARDs, TNFis (especially certolizumab pegol) and rituximab appear without identified safety issues. Three additional papers (1 SLR (of 84 studies, moderate RoB), 2 observational studies (high RoB)) resembled the findings of these recommendations. ${ }^{23,30,36}$ In the SLR (of 3 studies on this topic, moderate RoB) regarding the safety of DMARDs informing the 2019 EULAR RA management recommendations, the safety of tsDMARDs in RA patients and especially those at risk for VTEs was summarised. ${ }^{43}$ In these patients, an increased risk of VTEs was found for tofacitinib and baricitinib, specifically when using the higher doses of $10 \mathrm{mg}$ and $4 \mathrm{mg}$, respectively. Evidence on other tsDMARDs is not yet available.

\section{RA patients who failed $\geq 1 \mathrm{~b} /$ tsDMARD}

\section{Failure of $\geq 2$ bDMARDs}

Nine papers were found regarding the efficacy of $b /$ tsDMARDs in patients who failed $\geq 2$ bDMARDs (7 RCTs (subanalyses), 2 observational studies; 1 moderate RoB, 8 high RoB; Supplementary Table 2). In six of these papers, the efficacy of tocilizumab, tofacitinib, baricitinib, upadacitinib and filgotinib, respectively, versus placebo was assessed using ACR20 response at 12 to 24 weeks as an outcome (graphically summarised in Figure 2). ${ }^{55,64,72,80,95,120}$ ACR20 response favoured therapy with b/tsDMARD in all papers. The other study (high RoB) assessed the efficacy of mavrilimumab versus golimumab in a small population of 6 patients who previously failed 2 TNFis excluding golimumab (ACR20 response at 24W 66.7\% vs 0\%). ${ }^{119}$ In two observational studies (high RoB), the efficacy of an alternative TNFi versus rituximab was assessed and rituximab was found to be more effective (DAS28-ESR at 6M 4.54 vs 3.91, $p=0.021$; change in DAS28 from BL until 6M-0.75 vs -1.31 , not significant). ${ }^{53,66}$

\section{Failure of $\geq 1 \mathrm{~b} /$ tsDMARD}

Thirty-one papers (7 SLRs, 23 RCTs, 1 observational study; 8 low RoB, 16 moderate RoB, 7 high RoB) assessed the efficacy of b/tsDMARDs in patients who failed $\geq 1$ bDMARD versus placebo (Supplementary Table 2). ${ }^{50,52,56,57,61,67,68,70-72,73-77,79,80,85,92,94,95,97,98,103,104,108,109,111,113,115,116}$ A significant higher efficacy was found for the following b/tsDMARDs compared with placebo: alternative TNFi, abatacept, rituximab, tocilizumab, ixekizumab, ocrelizumab, olokizumab, sarilumab, secukinumab, sirukumab, tofacitinib, baricitinib, upadacitinib and filgotinib. No benefit in efficacy was found for other bDMARDs compared with placebo, which are not approved for RA (atacicept, fostamatinib and tabalumab).

Thirty-eight papers (7 SLRs, 6 RCTs, 25 observational studies; 2 low RoB, 7 moderate RoB, 


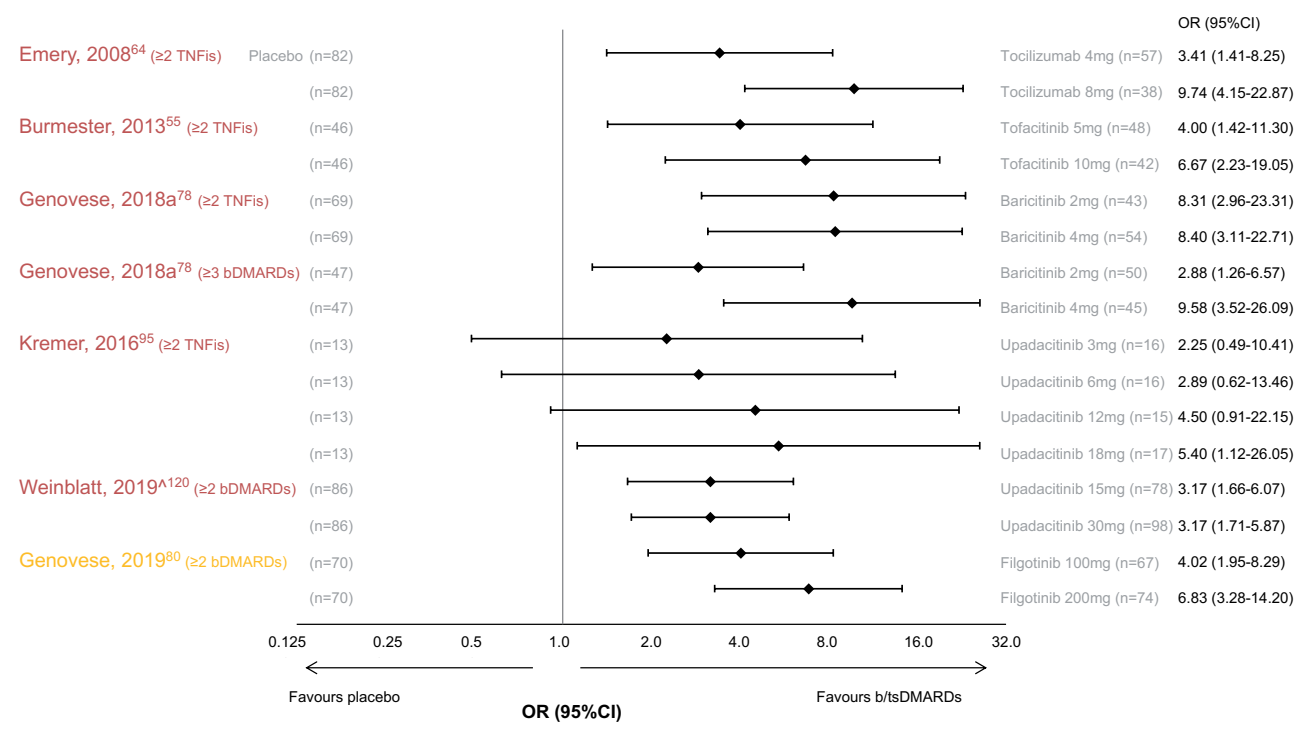

Figure 2. ACR20 response at 12 to 24 weeks in RA patients who failed $\geq 2$ bDMARDs

(m)ACR: (modified) American College of Rheumatology; bDMARD: biological synthetic disease-modifying antirheumatic drug; Cl: confidence interval; n: number of patients; OR: odds ratio; RA: rheumatoid arthritis; TNFi: tumour necrosis factor inhibitor; ${ }^{\wedge}$ : abstract.

Colours of author names according to risk of bias: font in yellow: moderate risk of bias; font in red: high risk of bias. Odds ratios are shown as diamonds and whiskers represent $95 \% \mathrm{Cl}$.

29 high RoB) compared the efficacy of different b/tsDMARDs in patients who failed $\geq 1 \mathrm{~b} /$

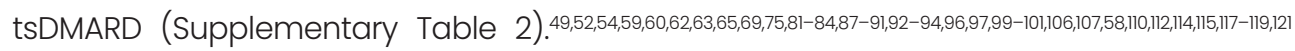
Only one study (high RoB) explicitly included a mixed population of patients who failed a bDMARD or a tsDMARD, ${ }^{69}$ all other papers only included patients who failed $\geq 1$ bDMARD. In 30 of 38 papers, patients explicitly failed a TNFi. Patients failed rituximab in three papers, ${ }^{62.114,177}$ tocilizumab in two papers, ${ }^{49,100}$ a non-TNFi in one paper, ${ }^{101}$ and a mix of TNFis and non-TNFi bDMARDs in two papers. ${ }^{96,110}$

Six papers (4 SLRs (of 9, 24,6 and 4 papers (partly overlapping)), 1 RCT and 1 observational study; 3 moderate RoB, 3 high RoB) assessed the efficacy of alternative TNFis versus nonTNFi bDMARDs as a group, in patients who failed a TNFi. $52.82,83,94,9,118$ Non-TNFi bDMARDs were found to be more effective in two original studies using different outcome criteria (EULAR good/moderate response at $52 \mathrm{~W} 43 \%$ vs $60 \%, \mathrm{p}=0.0006$ (high RoB); ${ }^{33}$ change in clinical disease activity index (CDAl) from BL until 1 year ( $\mathrm{Y}$ ) -4.81 vs $-7.54, \mathrm{p}=0.037$ (high RoB) ${ }^{18}$ ). Two of four SLRs (of 24 and 6 studies (partly overlapping); moderate RoB) concluded that there was a tendency of non-TNFi bDMARDs to be more effective than TNFis, after failure of a TNFi. $52,82,94,99$

Three papers assessed the efficacy of an alternative TNFi versus abatacept, conflicting results were found regarding the superiority of abatacept in patients who failed a TNFi 
(1 SLR (of 6 studies), 1 observational study; 1 moderate RoB, 1 high RoB), 88,94 and some advantage was found for TNFi to be more effective than abatacept in patients who failed tocilizumab (1 observational study; high RoB). ${ }^{49}$ There was a numerical advantage of rituximab to be more effective than TNFi in patients who failed a TNFi in six of ten papers (statistically significant in at least one of the response criteria in 5 papers (2 SLRs (of 24 and 6 studies (partly overlapping)), 1 RCT, 7 observational studies; 2 moderate RoB, 8 high RoB)).54,59,65,81,82,86,93,94,107,112 Additionally, there was a numerical advantage of tocilizumab in patients who failed a TNFi in three of four papers (statistically significant in at least one of the response criteria in 3 papers (2 SLRs (of 24 and 6 studies (partly overlapping)), 2 observational studies; 2 moderate RoB, 3 high RoB)), 82,90,91,94 a numerical advantage of tocilizumab in patients who failed rituximab in two of two papers (statistically significant in at least one of the response criteria in 1 paper (2 observational studies; 2 high RoB)) $)^{114,117}$ and no advantage of TNFi nor tocilizumab in patients who failed a bDMARD (not further specified (1 observational study; high RoB)). ${ }^{96}$ Five of the abovementioned papers reported an (modified) ACR50 response as outcome and are graphically summarised in Figure 3. ${ }^{54,86,88,90,94}$

Six papers compared the efficacy of different alternative TNFis in patients who failed a TNFi and concluded that there was insufficient evidence to prioritise (1 SLR (of 9 studies), 1 RCT, 4 observational studies; 1 low RoB, 5 high RoB).52,58-60,106,121 When directly comparing different non-TNFi bDMARDs, there was a numerical advantage of tocilizumab to be more effective than abatacept in patients who failed a TNFi in six of seven papers (statistically significant in at least one of the response criteria in 3 papers (1 SLR (of 4 studies), 1 RCT, 5 observational studies; 1 moderate RoB, 6 high RoB)).63,83,84,89,91,97,101 Additionally, there was

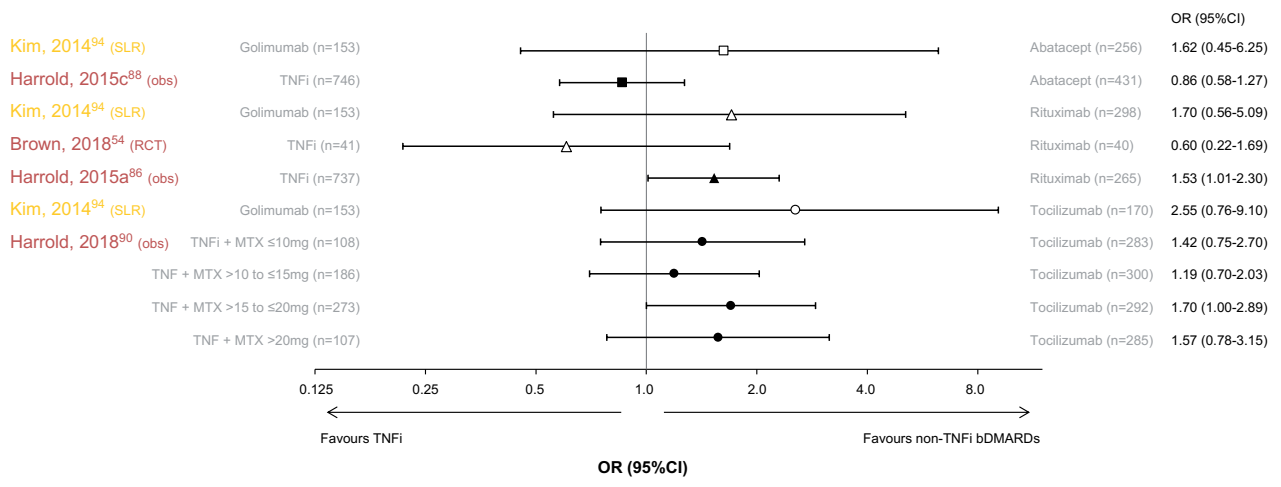

Figure 3. TNFi vs non-TNFi bDMARDs: (m)ACR50 response at 6 to 12 months in RA patients who failed $\geq 1$ TNFi (m)ACR: (modified) American College of Rheumatology; bDMARD: biological disease-modifying antirheumatic drug; Cl: confidence interval; mg: milligram; MTX: methotrexate; n: number of patients; obs: observational study; OR: odds ratio; TNFi: tumour necrosis factor inhibitor; SLR: systematic literature review.

Colours of author names according to risk of bias: font in yellow: moderate risk of bias; font in red: high risk of bias. Odds ratios are shown as a Abatacept; $\boldsymbol{\Delta}$ Rituximab; • Tocilizumab. White symbols represent univariate analyses, black symbols multivariate analyses and whiskers $95 \% \mathrm{Cl}$. 
a numerical advantage of tocilizumab to be more effective than abatacept in patients who failed rituximab in three of three papers (statistically significant in at least one of the response criteria in 2 papers (3 observational studies; 3 high RoB)).62,14,17 Furthermore, there was some advantage of tocilizumab to be more effective than rituximab in patients who failed a TNFi (4 papers) or a non-TNFi (1 paper) in five of five papers (statistically significant in at least one of the response criteria in all papers (2 SLRs (of 24 and 4 studies (partly overlapping)), 1 RCT, 2 observational studies; 2 moderate RoB, 3 high RoB)). ${ }^{82-84,97,101}$ Additionally, a numerical advantage of rituximab to be more effective than abatacept was found in patients who failed a TNFi in three of three papers (statistically significant in at least one of the response criteria in 1 paper (1 SLR (of 4 studies), 1 RCT, 1 observational study; 1 moderate RoB; 2 high RoB)). ${ }^{54,84,97}$

Three papers compared the efficacy between tsDMARDs and different bDMARDs (2 SLRs (of 4 and 5 studies (partly overlapping)), 1 observational study; 2 moderate RoB, 1 high RoB). $69,97,15$ There was a numerical advantage of rituximab to be more effective than tofacitinib in patients who failed a TNFi two of two papers, although statistical significance was not reached. ${ }^{97,115}$ Conflicting results were found regarding superiority in efficacy of the other bDMARDs assessed (TNFi, abatacept, tocilizumab) versus tofacitinib. No studies comparing bDMARDs versus other tsDMARDs were identified nor studies comparing different tsDMARDs.

Comparison of efficacy of b/tsDMARDs between RA patients who previously failed a different number of bDMARDs

Nine papers compared the efficacy of a b/tsDMARD between patients who previously failed an increasing number of bDMARDs (8 RCTs (subanalyses), 1 observational study; 1 moderate RoB, 8 high RoB; Supplementary Table 2). ${ }^{55,57,64,78,80,86,102,105,120}$ Seven studies used ACR20 response as an outcome, a graphical summary is shown in Figure 4. ${ }^{55,57,64,78,80,105,120}$ For golimumab, tocilizumab $4 \mathrm{mg}$ (intravenous), tofacitinib $5 \mathrm{mg}$ and baricitinib $2 \mathrm{mg}$, the efficacy numerically decreased with an increasing number of previously failed bDMARDs. 55,57,64,78,105 For upadacitinib and filgotinib, this tendency in decreased efficacy was less clear, ${ }^{80,120}$ as well as for the higher doses of tocilizumab (intravenous), tofacitinib and baricitinib (Figure 4). $55,57,64,78$

Two studies reported other response criteria than the ACR20 response. In these studies, the efficacy of abatacept and rituximab also numerically decreased with an increasing number of previously failed bDMARDs (abatacept: change in DAS28-CRP from BL until $6 \mathrm{M}$, failure of 1 TNFi vs 3 TNFis: $-2.1 \%$ vs $-1.7 \%$, statistically significant (high RoB); 102 rituximab: CDAl at 12M (not corrected for baseline values), failure of 1 TNFi vs $\geq 2$ TNFis: 13.2 vs 18.3, significance not reported (high RoB) ${ }^{87}$ ).

\section{RA patients with predominantly non-inflammatory complaints}




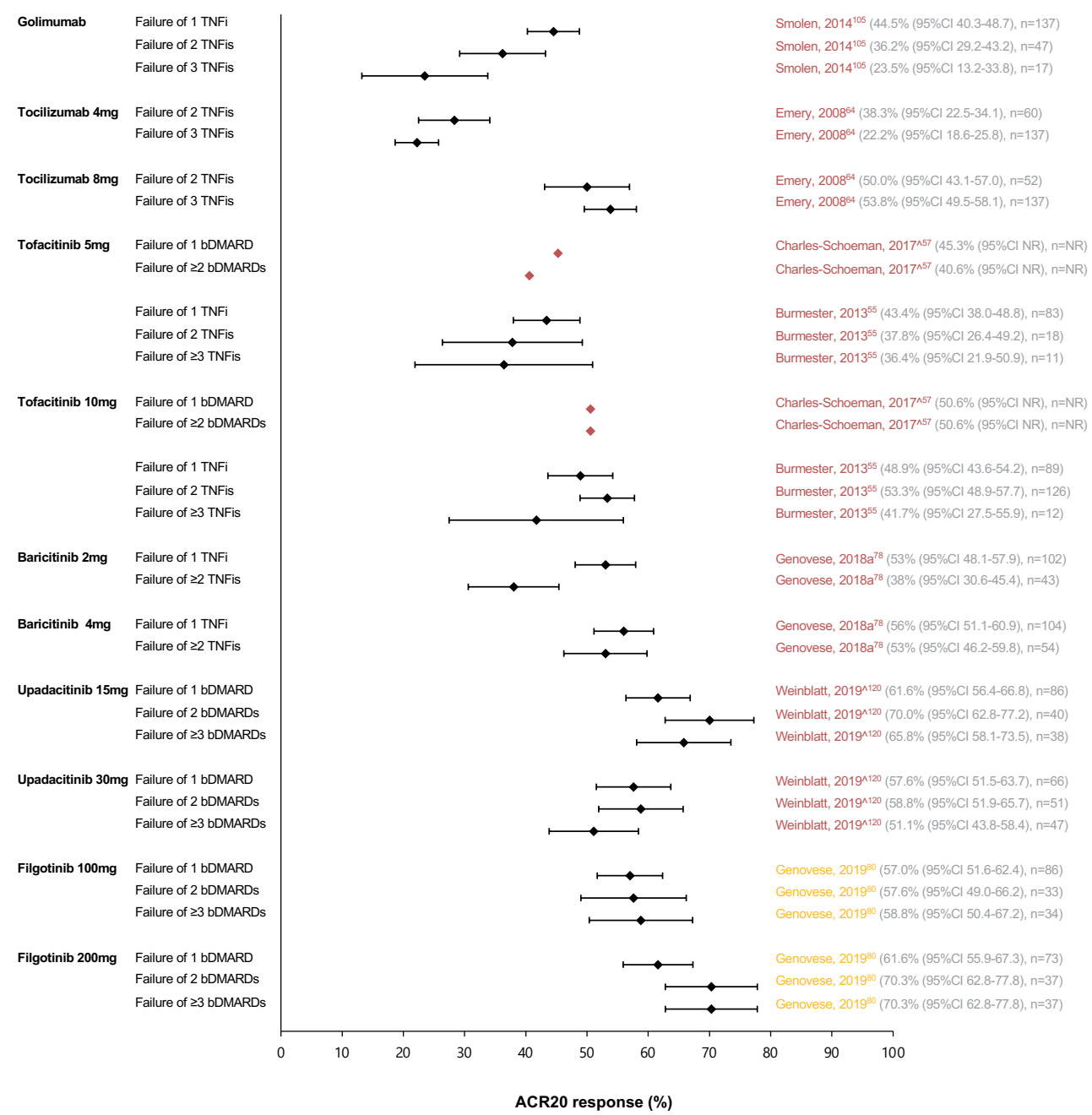

Figure 4. Comparison of ACR20 response at 3 months to 24 weeks in studies comparing RA patients with different number of previously failed bDMARDs

ACR: American College of Rheumatology; bDMARD: biological disease-modifying antirheumatic drug; Cl: confidence interval; n: number of patients; NR: not reported; OR: odds ratio; RA: rheumatoid arthritis; TNFi: tumour necrosis factor inhibitor; ${ }^{\wedge}$ : abstract.

Colours of author names according to risk of bias: font in yellow: moderate risk of bias; font in red: high risk of bias. Black diamond with whiskers: OR with 95\% Cl; Red diamond: OR, 95\%Cls are not reported.

Fifty papers (21 SLRs (on different topics, although partly overlapping), 27 RCTs, 2 observational studies; 12 low RoB, 11 moderate RoB, 27 high RoB) were found regarding RA patients with non-inflammatory complaints (Supplementary Table 3). ${ }^{22-17}$ Heterogeneous interventions were assessed, while the control intervention was mostly usual care or waiting list. Different outcome criteria were used to assess efficacy. The number of 
papers with statistically significant benefit of the intervention compared with control per category of intervention and per outcome, including their effect size (if reported or calculable), is shown in Table 1.

Only eight papers specifically selected patients with non-inflammatory complaints (pain: 4 (1 SLR (of 11 studies), 3 RCTs; 1 low RoB, 2 moderate RoB, 1 high RoB); $143,151,156,170$ fatigue: 3 (3 RCTs; 3 high RoB); $137,143,146$ psychological problems: 1 (RCT; high RoB); 138 difficulties in performing daily activities: 1 (RCT; high RoB) ${ }^{168}$ ). Although patients in these studies were selected on the presence of non-inflammatory complaints, DAS28 still ranged from 2.82 to 5.85 (not reported in 3 studies, Supplementary Table 3). Therefore, the presence of inflammatory activity could not be excluded. The results of these papers resembled the findings of the other studies, which enrolled an unselected population of RA patients and reported on outcomes regarding non-inflammatory complaints.

Exercise, education, self-management programs and intensification of patient care were found to improve function. Dietary, psychological interventions and self-management programs were found to reduce pain, as well as pharmacological interventions (ketoprofen patch and celecoxib). Psychological and self-management programs were found to reduce fatigue. No (sufficient) benefit in any of the abovementioned complaints was found for alternative medicine, cryotherapy and balneotherapy.

\section{Identification and optimisation of a mismatch in goal setting between RA patients and health care professionals}

Identification of a mismatch in goal setting

Three studies (all with a cross-sectional design; 2 high RoB, l qualitative study) were found regarding the identification of a mismatch in goal setting between patients and health care professionals (Supplementary Table 4). ${ }^{172-174}$ A diagnostic test to identify a mismatch was not identified. All studies compared two different populations regarding the importance of treatment goals: RA patients and clinicians in two studies, and RA patients with high and low disease activity in the other study. In the qualitative study, patients expressed a desire for clinicians to look beyond clinical markers and to consider patient reported outcomes. ${ }^{172}$ In the other study comparing RA patients and clinicians (high RoB), patients and clinicians had the same treatment goals regarding complaints, medication use and daily activity. ${ }^{173}$ Most treatment goals, such as reduction in inflammation, pain and fatigue, were more frequently scored as important by clinicians (patients versus clinicians: $50 \%$ vs $74 \%, 67 \%$ vs $88 \%$ and $46 \%$ vs $62 \%$, respectively). Only 'see more/other physicians to help RA management' was more frequently scored as important by RA patients, although the difference was small (13 vs 9\%). In the study comparing RA patients with high and those with low disease activity (high RoB), improvement in arthritis was more frequently scored as important by patients with high disease activity. ${ }^{174}$ On the other hand, improvement in morning stiffness was more frequently scored as important 
Table 1. Papers on therapeutic strategies for non-inflammatory complaints, including effect sizes

\begin{tabular}{|c|c|c|c|c|c|c|}
\hline \multirow{2}{*}{$\begin{array}{l}\text { Type of } \\
\text { intervention }\end{array}$} & \multirow[t]{2}{*}{ Outcome } & \multirow[b]{2}{*}{$\begin{array}{l}\text { Benefit of intervention } \\
\text { compared with } \\
\text { control' in } n \text { of } n \\
\text { selected papers } \\
\text { (if applicable: of which } n \\
\text { of } n \text { SLRs) }\end{array}$} & \multirow{2}{*}{$\begin{array}{l}\text { Effect size for } \\
\text { papers with } \\
\text { benefit of } \\
\text { intervention } \\
\text { (if available) }\end{array}$} & \multicolumn{3}{|c|}{$\operatorname{RoB}(n)$} \\
\hline & & & & $\mathrm{L}$ & M & $\mathrm{H}$ \\
\hline \multirow[t]{10}{*}{$\begin{array}{l}\text { Exercise }{ }^{129,136,165,166,171,} \\
137,140,147,153,154,157-159\end{array}$} & Function & $7 / 8^{(4 / 4)}$ & $\begin{array}{c}0.39 ; 0.46 ; 0.73 ; \\
0.88\end{array}$ & 2 & 2 & 4 \\
\hline & Pain & $4 / 10^{(2 / 7)}$ & $0.99^{3} ; 1.02$ & 3 & 3 & 4 \\
\hline & QoL & $2 / 3^{(0 / 1)}$ & 0.90 & 0 & 1 & 2 \\
\hline & Fatigue & $2 / 4^{(1 / 2)}$ & 0.37 & 0 & 2 & 2 \\
\hline & Other: & & & & & \\
\hline & $\begin{array}{l}\text { - Aerobic capacity and muscle } \\
\text { strength }\end{array}$ & $1 / 2^{(1 / 1)}$ & $0.47-0.99^{3}$ & 1 & 0 & 1 \\
\hline & - Range of motion & $1 / 2^{(1 / 1)}$ & - & 0 & 1 & 1 \\
\hline & - Grip function & $0 / 1^{(0 / 1)}$ & & 1 & 0 & 0 \\
\hline & - Global impact of disease & $1 / 1^{(1 / 1)}$ & - & 0 & 1 & 0 \\
\hline & - Mood & $0 / 1^{(0 / 1)}$ & & 0 & 1 & 0 \\
\hline \multirow[t]{4}{*}{ Diet $^{127,129}$} & Function & No papers & & & & \\
\hline & Pain & $2 / 2^{(2 / 2)}$ & - & 2 & 0 & 0 \\
\hline & QoL & No papers & & & & \\
\hline & Fatigue & No papers & & & & \\
\hline \multirow{7}{*}{$\begin{array}{l}\text { Psychological } \\
\text { interventions }{ }^{133,134,} \\
\text { 138,146,162,165 }\end{array}$} & Function & $1 / 2^{(1 / 1)}$ & - & 0 & 1 & 1 \\
\hline & Pain & $3 / 5^{(3 / 3)}$ & $0.177^{3}$ & 1 & 2 & 2 \\
\hline & QoL & No papers & & & & \\
\hline & Fatigue & $4 / 4^{(2 / 2)}$ & 0.16 & 1 & 1 & 2 \\
\hline & Other: & & & & & \\
\hline & - Overall efficacy & $1 / 1^{(1 / 1)}$ & - & 0 & 1 & 0 \\
\hline & - Negative mood and anxiety & $1 / 1$ & $0.31-0.46$ & 0 & 0 & 1 \\
\hline \multirow[t]{5}{*}{ Education'128,29,159 } & Function & $1 / 1^{(1 / 1)}$ & - & 1 & 0 & 0 \\
\hline & Pain & $0 / 1$ & & 0 & 0 & 1 \\
\hline & QoL & No papers & & & & \\
\hline & Fatigue & $0 / 1$ & & 0 & 0 & 1 \\
\hline & Other: Mood & $1 / 1^{(1 / 1)}$ & - & 0 & 1 & 0 \\
\hline \multirow{4}{*}{$\begin{array}{l}\text { Self- } \\
\text { management } \\
\text { programs } \\
122,125,167,126,132,135,137,144 \\
148,152,160\end{array}$} & Function & $2 / 7^{(2 / 3)}$ & - & 1 & 2 & 4 \\
\hline & Pain & $3 / 9^{(3 / 4)}$ & - & 2 & 2 & 5 \\
\hline & QoL & $0 / 2^{(0 / 1)}$ & & 1 & 0 & 1 \\
\hline & Fatigue & $4 / 4^{(3 / 3)}$ & 0.37 & 1 & 2 & 1 \\
\hline \multirow{6}{*}{$\begin{array}{l}\text { Alternative } \\
\text { medicine } \\
143,150,155,158\end{array}$} & Function & No papers & & & & \\
\hline & Pain & $2 / 3^{(0 / 1)}$ & $1.44-2.00$ & 1 & 1 & 1 \\
\hline & QoL & No papers & & & & \\
\hline & Other: & & & & & \\
\hline & - Range of motion & $1 / 1$ & $0.49-14.37$ & 0 & 0 & 1 \\
\hline & - Morning stiffness & $1 / 1^{(1 / 1)}$ & - & 1 & 0 & 0 \\
\hline
\end{tabular}


Table 1. Continued

\begin{tabular}{|c|c|c|c|c|c|c|}
\hline \multirow{2}{*}{$\begin{array}{l}\text { Type of } \\
\text { intervention }\end{array}$} & \multirow[t]{2}{*}{ Outcome } & \multirow{2}{*}{$\begin{array}{c}\text { Benefit of intervention } \\
\text { compared to control' } \\
\text { in } n \text { of } n \text { selected } \\
\text { papers } \\
\text { (if applicable: of which } n \\
\text { of } n \text { SLRs) }\end{array}$} & \multirow{2}{*}{$\begin{array}{l}\text { Effect size for } \\
\text { papers with } \\
\text { benefit of } \\
\text { intervention }{ }^{2} \\
\text { (if available) }\end{array}$} & \multicolumn{3}{|c|}{$\operatorname{RoB}(n)$} \\
\hline & & & & L & M & $\mathrm{H}$ \\
\hline \multirow{2}{*}{$\begin{array}{l}\text { Alternative } \\
\text { medicine }{ }^{143,150,155,158}\end{array}$} & Other: & & & & & \\
\hline & - Patient global assessment & $1 / 1^{(1 / 1)}$ & - & 0 & 1 & 0 \\
\hline \multirow[t]{4}{*}{ Cryotherapy ${ }^{142,149}$} & Function & $0 / 2$ & & 0 & 0 & 2 \\
\hline & Pain & $0 / 2$ & & 0 & 0 & 2 \\
\hline & QoL & No papers & & & & \\
\hline & Fatigue & $0 / 1$ & & 0 & 0 & 1 \\
\hline \multirow{5}{*}{$\begin{array}{l}\text { Balneotherapy²3, } \\
124,164,165\end{array}$} & Function & $1 / 3^{(0 / 1)}$ & - & 0 & 1 & 2 \\
\hline & Pain & $1 / 3^{(0 / 1)}$ & 0.88 & 0 & 1 & 2 \\
\hline & QoL & $0 / 1$ & & 0 & 0 & 1 \\
\hline & Fatigue & $0 / 1$ & & 0 & 0 & 1 \\
\hline & Other: Global impact of disease & $0 / 1^{(0 / 1)}$ & & 0 & 1 & 0 \\
\hline \multirow{4}{*}{$\begin{array}{l}\text { Intensification } \\
\text { of patient } \\
\text { care }^{130,131,145,161,168,169}\end{array}$} & Function & $2 / 4$ & 0.29 & 0 & 0 & 4 \\
\hline & Pain & $1 / 2$ & - & 0 & 0 & 2 \\
\hline & QoL & $0 / 3$ & & 0 & 0 & 3 \\
\hline & Fatigue & $1 / 2^{(0 / 1)}$ & - & 1 & 0 & 1 \\
\hline \multirow{5}{*}{$\begin{array}{l}\text { Pharmacological } \\
\text { (non-DMARD) })^{139,141,} \\
15,156,163,170\end{array}$} & Function & $0 / 1^{(0 / 1)}$ & & 1 & 0 & 0 \\
\hline & Pain & $2 / 6^{(1 / 4)}$ & $0.15^{\text {ketoprofen patch }}$ & 3 & 3 & 0 \\
\hline & QoL & $0 / 2^{(0 / 2)}$ & & 1 & 1 & 0 \\
\hline & Fatigue & No papers & & & & \\
\hline & Other: Patient global assessment & $1 / 1^{(1 / 1)}$ & - & 1 & 0 & 0 \\
\hline
\end{tabular}

H: high (red); L: low (green); M: moderate (yellow); n: number of papers; RA: rheumatoid arthritis; RoB: risk of bias; SLR: systematic literature review.

1. Mostly usual care or wait list; 2. Cohen's d, if different outcome measures were used the range in effect sizes over these measures is reported; 3. Pooled effect size, reported in SLR; 4. Combination of non-pharmacological interventions.

by patients with low disease activity.

\section{Optimisation of goal setting}

Four studies (1 RCT, 3 observational studies; all high RoB) were found regarding the optimisation of goal setting. ${ }^{175-178}$ All studies used a web-based education tool as the intervention (Supplementary Table 5). Different selections of RA patients were included: patients with active disease, patients who were unsure about starting treatment with methotrexate, patients who were starting b/tsDMARDs or an unselected population. RA knowledge improved statistically significantly using the intervention in all studies. In one study, in an unselected population of RA patients, willingness to try a b/tsDMARD 
was assessed and improved statistically significantly. ${ }^{175}$ In another study, in patients starting b/tsDMARDs, certainty in choosing a DMARD option was assessed and increased statistically significantly. ${ }^{178}$

\section{Identification and optimisation of self-management}

Identification of suboptimal self-management

Five papers were found regarding the identification of suboptimal self-management: two assessed the importance of factors associated with successful self-management comparing RA patients and clinicians (2 with a cross-sectional design; 1 low RoB, 1 high $\mathrm{ROB})^{173,81}$ and three assessed a diagnostic measure (1 SLR (of 15 studies), 2 cross-sectional studies; all moderate RoB; Supplementary Table 6). 179,180,182

In both descriptive studies regarding the importance of factors associated with optimal self-management, most factors were more frequently scored as important by clinicians than by RA patients (e.g. a discussion about self-management, more/longer visits, education about psychosocial needs, activities of daily living, sexual concerns). ${ }^{173,181}$ Patients expressed a desire for a more important role of pharmacists and nutritionists in arthritis education, and for more education on the disease, diagnostic process and nutrition. ${ }^{181}$

In the SLR regarding diagnostic measures, the Arthritis Self-Efficacy Scale (ASES) and RA self-efficacy scale (RASE) were found to be assessed in more than one cohort of RA patients. Evidence for the RASE suggested that this measure is multidimensional, which is not adequately represented in the scoring. Therefore, ASES was concluded to be the most reliable test, although its methodological weakness was acknowledged by the authors (i.e. the content validity and whether it adequately reflects self-efficacy was not explicitly addressed during development). ${ }^{179}$ In another study, the Modified Rheumatology Attitude Index correlated with the combined questionnaire for functional impairment and quality of life, pain score, patient global assessment and functional impairment questionnaire. ${ }^{180}$ In a study assessing two cohorts of RA patients, the Brief Resilient Coping scale also significantly correlated with different other measures. ${ }^{182}$

\section{Optimisation of self-management}

Thirty-one papers (7 SLRs (on different topics, although partly overlapping), 23 RCT, 1 observational study; 5 low Rob, 3 moderate RoB, 23 high RoB) were found regarding the optimisation of self-management (Supplementary Table 7). No papers specifically selected patients with suboptimal self-management. Different outcomes were found to describe self-management for which different outcome measures were used and heterogeneous interventions were assessed (Table 2). The number of papers with statistically significant benefit of the intervention compared with control (mostly usual care or waiting list) per outcome and per type of intervention, including their effect size (if 
Table 2. Papers on the optimisation of self-management, including effect sizes

\begin{tabular}{|c|c|c|c|c|c|c|}
\hline \multirow[t]{2}{*}{ Outcome } & \multirow[t]{2}{*}{ Type of intervention } & \multirow{2}{*}{$\begin{array}{l}\text { Benefit of intervention } \\
\text { compared with } \\
\text { control' in } n \text { of } n \\
\text { selected papers } \\
\text { (if applicable: of which } n \\
\text { of } n \text { SLRs) }\end{array}$} & \multirow{2}{*}{$\begin{array}{l}\text { Effect size for } \\
\text { papers with } \\
\text { benefit of } \\
\text { intervention }{ }^{2} \\
\text { (if available) }\end{array}$} & \multicolumn{3}{|c|}{$\operatorname{RoB}(n)$} \\
\hline & & & & L & M & $\mathrm{H}$ \\
\hline \multirow{12}{*}{$\begin{array}{l}\text { Self-efficacy } 122,125 \\
152,159,160,162,167,183,185-188, \\
126,189-196,130-132,137,144,145 \\
148\end{array}$} & \multirow[t]{3}{*}{ Self-management programs ${ }^{4}$} & \multirow[t]{3}{*}{$12 / 13^{(4 / 4)}$} & $0.18-0.39 ; 0.23-$ & 2 & \multirow[t]{3}{*}{2} & 9 \\
\hline & & & $0.53 ; 0.49^{3} ; 7.52-$ & & & \\
\hline & & & 8.25 & & & \\
\hline & Education & $6 / 6^{(1 / /)}$ & $\begin{array}{l}0.05-0.17 ; 0.22- \\
0.59 ; 1.23\end{array}$ & 0 & 1 & 5 \\
\hline & Psychological & $2 / 2^{(1 / 1)}$ & $0.20-0.35 ; 0.45$ & 1 & 0 & 1 \\
\hline & \multicolumn{3}{|l|}{ Other: } & & & \\
\hline & - Exercise & $1 / 1$ & $0.44-1.06$ & 0 & 0 & 1 \\
\hline & - Nurse-led follow-up & $1 / 1^{(1 / 1)}$ & - & 1 & 0 & 0 \\
\hline & - Eszopiclone 3mg & $1 / 1$ & $0.05-0.37$ & 0 & 1 & 0 \\
\hline & $\begin{array}{l}\text { - Assistive technology (eyedrop } \\
\text { dispenser) }\end{array}$ & $1 / 1^{(1 / 1)}$ & - & 1 & 0 & 0 \\
\hline & $\begin{array}{l}\text { - Patient reported outcome based } \\
\text { tele-health follow-up }\end{array}$ & $0 / 1$ & & 0 & 0 & 1 \\
\hline & $\begin{array}{l}\text { - Direct access to hospital review } \\
\text { through helpline }\end{array}$ & $0 / 1$ & & 0 & 0 & 1 \\
\hline \multirow{6}{*}{$\begin{array}{l}\text { Anxiety } 137,145,162,183,184 \\
187,191-193\end{array}$} & Self-management programs ${ }^{4}$ & $1 / 1$ & $0.69-0.71$ & 0 & 0 & 1 \\
\hline & Education & $1 / 3$ & 0.039 & 0 & 0 & 3 \\
\hline & Psychological & $1 / 2^{(1 / 1)}$ & $0.17^{3}$ & 1 & 0 & 1 \\
\hline & \multicolumn{3}{|l|}{ Other: } & & & \\
\hline & - Relaxation therapy & $0 / 1$ & & 0 & 0 & 1 \\
\hline & $\begin{array}{l}\text { - Direct access to hospital review } \\
\text { through helpline }\end{array}$ & $0 / 1$ & & 0 & 0 & 1 \\
\hline \multirow{6}{*}{$\begin{array}{l}\text { Depressive } \\
\text { symptoms s'132,137, } \\
\text { 193,145,152,162,183,184,187,19,192 }\end{array}$} & Self-management programs ${ }^{4}$ & $0 / 2$ & & 0 & 0 & 2 \\
\hline & Education & $0 / 3$ & & 0 & 0 & 3 \\
\hline & Psychological & $3 / 3^{(2 / 2)}$ & $0.15-0.33^{3} ; 0.65$ & 2 & 0 & 1 \\
\hline & \multicolumn{3}{|l|}{ Other: } & & & \\
\hline & - Relaxation therapy & $0 / 1$ & & 0 & 0 & 1 \\
\hline & $\begin{array}{l}\text { - Direct access to hospital review } \\
\text { through helpline }\end{array}$ & $0 / 1$ & & 0 & 0 & 1 \\
\hline \multirow{4}{*}{$\begin{array}{l}\text { RA knowledge } \\
167,183,185,189,193\end{array}$} & Self-management programs ${ }^{4}$ & $1 / 1$ & $0.34-0.47$ & 0 & 0 & 1 \\
\hline & Education & $2 / 3^{(1 / 1)}$ & 0.84 & 0 & 1 & 2 \\
\hline & Psychological & No papers & & & & \\
\hline & Other: Web-based rehabilitation & $1 / 1^{(1 / 1)}$ & - & 1 & 0 & 0 \\
\hline
\end{tabular}

H: high (red); L: low (green); M: moderate (yellow); n: number of papers; RA: rheumatoid arthritis; RoB: risk of bias; SLR: systematic literature review.

1. Mostly usual care or wait list; 2. If different outcome measures were used the range in effect sizes over these measures is reported; 3. Pooled effect size, reported in SLR; 4. Combination of non-pharmacological interventions. 
reported or calculable), is shown in Table 2.

Improvement in self-efficacy was found using self-management programs, educational and psychological interventions, exercise programs, nurse-led follow-up, eszopiclone $3 \mathrm{mg}$ (in RA patients with insomnia) and assistive technology (eye drop dispenser in RA patients with concomitant Sjögren's syndrome). Psychological interventions were found to reduce anxiety and depression. Education and web-based rehabilitation were found to improve RA knowledge. Benefit of the interventions was also found for other outcomes: self-management programs and cognitive behavioural therapy to reduce helplessness; self-management programs to improve autonomous motivation and self-management behaviour, and to reduce psychological distress; and psychological interventions to improve coping.

\section{Discussion}

Inthis SLR, evidence is summarised regarding pharmacological and non-pharmacological therapeutic strategies informing the EULAR Task Force in charge of the development of recommendations for the management of D2T RA. Several limitations were found in the selected available evidence. First, no studies were found evaluating therapeutic strategies in patients who fulfilled the definition of D2T RA. ${ }^{6}$ Therefore, typically, evidence regarding pharmacological therapeutic strategies needed to be extrapolated from studies in patients with active disease who failed at least one bDMARD, which was a TNFi in almost all studies, instead of two b/tsDMARDs with a different MOA as in the definition. Additionally, most studies on non-pharmacological strategies were performed in an unselected population of RA patients instead of in D2T RA patients with predominantly non-inflammatory complaints, in whom the absence of inflammatory activity could not be ascertained. Second, heterogeneity in patient populations, interventions, comparators and outcome criteria hampered pooling of efficacy outcomes. Third, only very few studies with low RoB were found. Considering these limitations, the results should be interpreted cautiously for D2T RA patients.

Regarding pharmacological interventions for D2T RA patients, limited evidence (of low to moderate quality) was found on the efficacy and safety of DMARDs for patients with limited DMARD options due to contraindications regarding obesity, $\mathrm{HBV}, \mathrm{HCV}$, pregnancy, lactation and those at risk for VTEs. In patients with obesity, infliximab may be less effective compared with in patients with a normal BMl, and adalimumab, etanercept and rituximab may be less affected by BMI. ${ }^{28,33,48}$ For patients with HBV, TNFi, abatacept and tocilizumab were found to be relatively safe. 20,22,39 For patients with HCV, TNFi was found to be a relatively safe treatment option. ${ }^{18,34}$ For patients before and during pregnancy and during lactation, no safety issues have been identified for several DMARDs, which 
are described in the 2020 ACR guideline and 2016 EULAR points to consider. ${ }^{27,42}$ In patients at risk for VTEs, an increased risk of VTEs was found during treatment with tsDMARDs, especially in the higher doses. ${ }^{43}$ In these patients, other DMARDs may be preferred.

In patients who failed at least two bDMARDs, several b/tsDMARDs (tocilizumab, tofacitinib, baricitinib, upadacitinib and filgotinib) were found to be more effective than placebo. 55,64,72,80,95,120 In patients who failed at least one bDMARD, a benefit in efficacy compared with placebo was found for all currently used b/tsDMARDs. ${ }^{52,56,57,67,68,76,77,79,80,85}$ $94,95,97,98,104,108,115,116$ Despite this benefit in efficacy compared with placebo, generally, the extent of the beneficial effect of b/tsDMARDs was found to become less when patients failed a higher number of previous bDMARDs. ${ }^{55,57,64,7,86,102,105}$ This tendency was less convincing for upadacitinib and filgotinib and for the higher doses of tocilizumab (intravenous), tofacitinib and baricitinib.55,57,64,78,80,120 Although this may suggest a preference for these b/ tsDMARDs (in these higher doses) in D2T RA patients, it may also be related to the more recent introduction of these drugs with a novel MOA and the timing of their application in therapeutic strategies. If, for instance, a TNFi would have been applied in patients who previously failed increasing numbers of tsDMARDs, the beneficial effect of TNFi may also have been less dependent on the number of previously failed tsDMARDs. However, for tocilizumab, tofacitinib and baricitinib, the tendency of decreasing efficacy with an increasing number of previously failed bDMARDs was not apparent for their higher doses and only for their lower doses. ${ }^{55,57,64,78}$ This may indicate that not only their more recent introduction and the novel MOA play a role. Additionally, the studies on tofacitinib, upadacitinib and filgotinib enrolled patients who failed different bDMARDs and not only TNFis. ${ }^{57,80,120}$ Nevertheless, future studies should assess to what extent this tendency is related to these specific b/tsDMARDs or to the order of their application in therapeutic strategies. Furthermore, a tendency was found for non-TNFi bDMARDs to be more effective than TNFis in patients who failed at least one TNFi, although insufficient evidence was identified to prioritise different non-TNFi b/tsDMARDs. ${ }^{49,52,54,59,65,81-83,86,88,90,99,93,94,96,99,107,112,14,117,118}$ These findings could indicate that non-TNFi bDMARDs and tsDMARDs may be somewhat more effective in comparison to another TNFi in D2T RA patients. Specifically, if these nonTNFi b/tsDMARDs are of a previously not targeted MOA.

Regarding non-pharmacological interventions for D2T RA patients, exercise, education, psychological and self-management interventions were found to be of additional benefit to improve non-inflammatory complaints (mainly functional disability, pain and fatigue). $22,125,129,132-138,140,44,146-148,152-154,157,126,158-160,162,165-167,171$ It may be expected that nonpharmacological interventions do not become less effective in patient failing a higher number of previous bDMARDs as much as pharmacological interventions as described above. Therefore, the additional benefit of non-pharmacological interventions might be even higher in D2T RA patients. However, no formal evidence was found to support this. Furthermore, education was found to improve goal setting and self-management. ${ }^{175-178}$ 
Additionally, self-management programs, education and psychological interventions were found to improve different aspects of self-management, namely self-efficacy, anxiety, depression and RA knowledge.122,125,167,183,185-192,132,193,195,137,144,148,152,159,160,162 Before goal setting between patients and health care professionals as well as self-management can be improved, a mismatch in goal setting and suboptimal self-management should be identified. No accurate measures were found to identify a mismatch in goal setting, although patients expressed a desire to take their quality of life goals more explicitly into account. ${ }^{172}$ ASES was found to be the most extensively studied tool to identify the level of self-management, although validated cut-offs are not available, hampering its use in clinical practice as a diagnostic instrument. ${ }^{179,197}$

This SLR was aimed to supplement the evidence as summarised for the current EULAR recommendations for the management of RA.' In the SLR informing the current RA management recommendations, insufficient evidence was found to prioritise different b/tsDMARDs. ${ }^{198}$ In contrast, in our SLR a tendency was found of non-TNFi bDMARDs to be more effective than TNFis in RA patients who failed at least one TNFi. This difference is explained by the inclusion of observational studies and network meta-analyses in our SLR, while these study designs were specifically excluded from the SLR informing the RA management recommendations. However, we felt it was necessary not to exclude this evidence from our SLR, as direct comparisons in RCTs are lacking in D2T RA patients. By all means, careful interpretation of the outcomes of these types of studies is warranted, also as results need to be extrapolated to the D2T RA patient population.

Although many therapeutic clinical questions were assessed in this SLR, not all therapeutic strategies for all potential factors contributing to D2T RA have been addressed. At the first meeting of the D2T RA Task Force, treatment non-adherence and lifestyle interventions were also discussed as topics of interest. As there are currently two ongoing EULAR projects related to these issues in patients with rheumatic and musculoskeletal diseases, it was decided to refer to the evidence found in their SLRs for the D2T RA recommendations in consultation with the Steering Committees of these Task Forces. Therefore, these topics are not included in this SLR.

In clinical practice, the heterogeneity of D2T RA should be considered when choosing the optimal therapeutic strategy for the individual D2T RA patient. As a myriad of factors may contribute to the D2T RA state, ${ }^{4,9}$ the therapeutic strategy should be individually tailored and may consist of multiple (pharmacological and non-pharmacological) interventions. Further guidance on this will be provided by the EULAR Task Force on D2T RA in their recommendations for the management of D2T RA, including their interpretation of the clinical implications of the results, which will be published soon. ${ }^{199}$ Additionally, the heterogeneity of D2T RA should be considered in future studies as not all therapeutic strategies will be useful for all D2T RA patients. The EULAR Task Force on D2T RA will also provide a research agenda, in which further guidance on topics of interest will be given. ${ }^{199}$ 
In addition to the limitations in the evidence that was found, this SLR has some limitations itself. Although an extensive literature search has been performed, relevant papers might have been missed due to the choices that have been made in the search strategy. For the search on pharmacological and non-pharmacological therapeutic strategies, the publication year 2000 was chosen as a cut-off because of the introduction of bDMARDs around this time point. For the subquestion on patients with predominantly noninflammatory complaints, it was chosen to focus on the often-reported outcomes of function, pain, quality of life and fatigue. For the subquestions on goal setting and selfmanagement, it was chosen to focus on RA patients only, while studies in patients with other chronic diseases may also provide useful information. In our opinion, these choices were mandatory to most efficiently create an overview of the current literature on the most important outcomes in the present therapeutic landscape. Nevertheless, these choices need to be reassessed in case the D2T RA recommendations, and consequently the SLRs, will be updated. Additionally, we chose to report effect sizes to compare results over different outcomes and scoring methods. However, effect sizes were not extractable for all studies, which hampers interpretation and pooling of results. The reported effect sizes varied widely for some outcomes (e.g. alternative medicine to improve range of motion: 0.49 -14.37), which may indicate publication bias. Although the possibility of publication bias was assessed within the RoB assessment of the SLRs ${ }^{16}$ and the level of evidence of the recommendations, interpreting this bias, especially with a limited number of studies, is difficult and, therefore, the effect sizes should be interpreted cautiously. In conclusion, this SLR underscores the scarcity of (high-quality) evidence on the optimal treatment of D2T RA patients. As D2T RA is a newly defined disease state, all evidence is to an extent indirect. Limited evidence was found on effective and/or safe DMARDs for patients with limited DMARD options due to contraindications. In patients who previously failed bDMARDs, all currently used b/tsDMARDs were found to be more effective than placebo. However, generally, effectiveness of b/tsDMARDs decreased in patients who had failed a higher number of bDMARDs and subsequent b/tsDMARDs of a previously not targeted MOA appear to be more effective. Furthermore, a beneficial effect of nonpharmacological interventions, specifically education, was found for improvement of non-inflammatory complaints (function, pain, fatigue), goal setting and selfmanagement (self-efficacy, anxiety, depression, RA knowledge). 


\section{References}

1. Smolen JS, Landewé RBM, Bijlsma JWJ, et al. EULAR recommendations for the management of rheumatoid arthritis with synthetic and biological disease-modifying antirheumatic drugs: 2019 update. Ann Rheum Dis 2020;79:685-99.

2. Singh JA, Saag KG, Bridges SL, et al. 2015 American College of Rheumatology Guideline for the Treatment of Rheumatoid Arthritis. Arthritis Rheumatol 2016;68:1-26.

3. Buch $\mathrm{MH}$. Defining refractory rheumatoid arthritis. Ann Rheum Dis 2018;77:966-9.

4. de Hair MJH, Jacobs JWG, Schoneveld JLM, et al. Difficult-to-treat rheumatoid arthritis: an area of unmet clinical need. Rheumatology (Oxford) 2017;57:1135-44.

5. Kearsley-Fleet L, Davies R, De Cock D, et al. Biologic refractory disease in rheumatoid arthritis: Results from the British Society for Rheumatology Biologics Register for Rheumatoid Arthritis. Ann Rheum Dis 2018;77:1405-12.

6. Nagy G, Roodenrijs NMT, Welsing PMJ, et al. EULAR definition of difficult-to-treat rheumatoid arthritis. Ann Rheum Dis 2021;80:31-5.

7. Roodenrijs NMT, de Hair MJH, van der Goes $\mathrm{MC}$, et al. Characteristics of difficult-to-treat rheumatoid arthritis: results of an international survey. Ann Rheum Dis 2018;77:1705-9.

8. Roodenrijs NM, de Hair MJ, van der Goes MC, et al. Correspondence to viewpoint 'Defining refractory rheumatoid arthritis' by Buch. Ann Rheum Dis 2019;78:e105.

9. Roodenrijs NMT, van der Goes MC, Welsing PMJ, et al. Difficult-to-treat rheumatoid arthritis: contributing factors and burden of disease. Rheumatology 2020:keaa860.

10. Strand V, Wright GC, Bergman MJ, et al. Patient Expectations and Perceptions of Goalsetting Strategies for Disease Management in Rheumatoid Arthritis. J Rheumatol 2015;42:2046-54.

11. Roodenrijs NMT, Kedves M, Hamar A, et al. Diagnostic issues in difficult-totreat rheumatoid arthritis: a systematic literature review informing the 2020 EULAR recommendations for the management of difficult-to-treat rheumatoid arthritis. RMD open 2021. Accepted for publication.

12. van der Heijde D, Aletaha D, Carmona L, et al. 2014 Update of the EULAR standardised operating procedures for EULAR-endorsed recommendations. Ann Rheum Dis 2015;74:813.

13. Thompson M, Tiwari A, Fu R, et al. A Framework To Facilitate the Use of Systematic Reviews and Meta-Analyses in the Design of Primary Research Studies. Rockville, MD Agency Healthc. Res. Qual. 2012.http://www.ncbi.nlm. nih.gov/books/ NBK83621/ (accessed 13 Nov 2019).

14. Higgins JPT, Altman DG, Gøtzsche PC, et al. The Cochrane Collaboration's tool for assessing risk of bias in randomised trials. BMJ 2011;343:d5928.

15. Whiting PF, Rutjes AWS, Westwood ME, et al. Quadas-2: A revised tool for the quality assessment of diagnostic accuracy studies. Ann Intern Med 2011;155:529-36.

16. Shea BJ, Reeves BC, Wells G, et al. AMSTAR 2: A critical appraisal tool for systematic reviews that include randomised or non-randomised studies of healthcare interventions, or both. BMJ 2017;358:j4008.

17. Abramkin A, Lisitsyna $T$, Veltishchev $D$, et al. THU0127 The dynamics of mental disorders frequency in complex dmards, biologics and antidepressants treatment of rheumatoid arthritis patients. Ann Rheum Dis 2017;76:249.1249.

18. Brunasso AMG, Puntoni M, Gulia A, et al. Safety of anti-tumour necrosis factor agents in patients with chronic hepatitis $\mathrm{C}$ infection: $\mathrm{A}$ systematic review. Rheumatology (Oxford) 2011;50:1700-11.

19. Götestam Skorpen $C$, Hoeltzenbein $M$, Tincani $A$, et al. The EULAR points to consider for use of antirheumatic drugs before pregnancy, and during pregnancy and lactation. Ann Rheum Dis 2016;75:795-810.

20. Gremese E, Carletto A, Padovan $M$, et al. Obesity and reduction of the response rate to anti-tumor necrosis factor a in rheumatoid arthritis: An approach to a personalized medicine. Arthritis Care Res 2013;65:94-100.

21. Hang $Y$, Qin $X$, Ren $T$, et al. Baicalin reduces blood lipids and inflammation in patients with coronary artery disease and rheumatoid arthritis: A randomized, double-blind, placebo-controlled trial. Lipids Health Dis 2018;17:146.

22. Hoeltzenbein $M$, Beck $E$, Rajwanshi $R$, et al. Tocilizumab use in pregnancy: Analysis of a global safety database including data from clinical trials and post-marketing data. Semin Arthritis Rheum 2016;46:238-45.

23. lannone F, La Montagna $G$, Bagnato $G$, et 
al. Safety of etanercept and methotrexate in patients with rheumatoid arthritis and hepatitis C virus infection: A multicenter randomized clinical trial. J Rheumatol 2014;41:286-92.

24. Ikonomidis I, Tzortzis S, Andreadou I, et al. Increased beneft of interleukin-1 inhibition on vascular function, myocardial deformation, and twisting in patients with coronary artery disease and coexisting rheumatoid arthritis. Circ Cardiovasc Imaging 2014;7:619-28.

25. Klaasen R, Wijbrandts CA, Gerlag DM, et al. Body mass index and clinical response to infliximab in rheumatoid arthritis. Arthritis Rheum 2011;63:359-64.

26. Lin KM, Cheng TT, Lin JC, et al. Tumor necrosis factor-a antagonist therapy for concomitant rheumatoid arthritis and hepatitis $C$ virus infection: a case series study. Clin Rheumatol 2015;34:1039-46.

27. Mori S, Yoshitama $T$, Hidaka $T$, et al. Effectiveness and safety of tocilizumab therapy for patients with rheumatoid arthritis and renal insufficiency: A real-life registry study in Japan (the ACTRA-RI study). Ann. Rheum. Dis. 2015;74:627-30.

28. Mouyis M, Flint JD, Giles IP. Safety of antirheumatic drugs in men trying to conceive: A systematic review and analysis of published evidence. Semin Arthritis Rheum 2019;48:91120.

29. Burmester $G$, Lin $Y$, Mangan E, et al. SAT0202 Efficacy and safety of sarilumab monotherapy versus adalimumab monotherapy in patients with active rheumatoid arthritis in the phase 3 monarch study, including subpopulations. Ann Rheum Dis 2017;76:849.1-849.

30. Nakamura T, Higashi Sl, Tomoda K, et al. Effectiveness of etanercept VS cyclophosphamide as treatment for patients with amyloid a amyloidosis secondary to rheumatoid arthritis. Rheumatology (Oxford) 2012;51:2064-9.

31. Nakamura Y, Suzuki T, Yoshida T, et al. Vitamin d and calcium are required during denosumab treatment in osteoporosis with rheumatoid arthritis. Nutrients 2017;9:E428.

32. Padovan $M$, Filippini $M$, Tincani $A$, et al. Safety of Abatacept in Rheumatoid Arthritis with Serologic Evidence of Past or Present Hepatitis B Virus Infection. Arthritis Care Res 2016;68:738-43.

33. Papalopoulos I, Fanouriakis A, Kougkas N, et al. Liver safety of non-tumour necrosis factor inhibitors in rheumatic patients with past hepatitis B virus infection: An observational, controlled, long-term study. Clin Exp Rheumatol 2018;36:102-9.

34. Ruscitti P, Masedu F, Alvaro S, et al. Antiinterleukin-1 treatment in patients with rheumatoid arthritis and type 2 diabetes (TRACK): A multicentre, open-label, randomised controlled trial. PLoS Med 2019;16:el002901.

35. Sammaritano LR, Bermas BL, Chakravarty EE, et al. 2020 American College of Rheumatology Guideline for the Management of Reproductive Health in Rheumatic and Musculoskeletal Diseases. Arthritis Rheumatol (Hoboken, NJ) 2020.

36. Sepriano A, Kerschbaumer A, Smolen JS, et al. Safety of synthetic and biological DMARDs: a systematic literature review informing the 2019 update of the EULAR recommendations for the management of rheumatoid arthritis. Ann Rheum Dis 2020.

37. Shin K, Park SH, Park W, et al. Monthly Oral Ibandronate Reduces Bone Loss in Korean Women With Rheumatoid Arthritis and Osteopenia Receiving Long-term Glucocorticoids: A 48-week Doubleblinded Randomized Placebo-controlled Investigator-initiated Trial. Clin Ther 2017;39:268-278.e2.

38. Strand V, Hagino O, Guillonneau S, et al. SAT0207 Depressive symptoms in patients with rheumatoid arthritis in sarilumab target and mobility trials and impact of treatment. Ann Rheum Dis 2018;77:964.1-964.

39. Suissa $S$, Hudson $M$, Dell'Aniello $S$, et al. Comparative safety of abatacept in rheumatoid arthritis with COPD: A real-world population-based observational study. Semin Arthritis Rheum 2019;49:366-72.

40. Cantini F, Boccia S, Goletti D, et al. HBV reactivation in patients treated with antitumor necrosis factor-alpha (TNF-a) agents for rheumatic and dermatologic conditions: A systematic review and meta-analysis. Int $\mathrm{J}$ Rheumatol 2014;2014:926836.

41. Weinblatt $M$, Combe $B$, Covucci $A$, et al. Safety of the selective costimulation modulator abatacept in rheumatoid arthritis patients receiving background biologic and nonbiologic disease-modifying antirheumatic drugs: A one-year randomized, placebo-controlled study. Arthritis Rheum 2006;54:2807-16.

42. Yoo D-H, Park W, Suh $\mathrm{C}-\mathrm{H}$, et al. Rituximab is effective in the treatment of rheumatoid 
arthritis irrespective of body mass index; up to 48 weeks results from phase 3 study. Arthritis Rheumatol 2017;69:suppl 10.

43. Chen $\mathrm{YM}$, Chen $\mathrm{HH}$, Chen $\mathrm{YH}$, et al. A comparison of safety profiles of tumour necrosis factor a inhibitors and rituximab therapy in patients with rheumatoid arthritis and chronic hepatitis C. Ann. Rheum. Dis. 2015;74:626-7.

44. Chen LF, Mo YQ, Jing J, et al. Short-course tocilizumab increases risk of hepatitis $B$ virus reactivation in patients with rheumatoid arthritis: a prospective clinical observation. Int J Rheum Dis 2017;20:859-69.

45. Clowse MEB, Feldman SR, Isaacs JD, et al. Pregnancy Outcomes in the Tofacitinib Safety Databases for Rheumatoid Arthritis and Psoriasis. Drug Saf 2016;39:755-62.

46. Combe B, Balsa A, Sarzi-Puttini P, et al. Efficacy and safety data based on historical or preexisting conditions at baseline for patients with active rheumatoid arthritis who were treated with baricitinib. Ann Rheum Dis 2019;78:1135-8.

47. Deodhar A, Bitman B, Yang Y, et al. The effect of etanercept on traditional metabolic risk factors for cardiovascular disease in patients with rheumatoid arthritis. Clin Rheumatol 2016;35:3045-52.

48. Genovese MC, Burmester GR, Hagino O, et al. SAT0121 Effect of sarilumab on glycosylated hemoglobin in patients with rheumatoid arthritis and diabetes. Ann Rheum Dis 2019;78:1128-9.

49. Akiyama $M$, Kaneko $\mathrm{Y}$, Kondo $\mathrm{H}$, et al. Comparison of the clinical effectiveness of tumour necrosis factor inhibitors and abatacept after insufficient response to tocilizumab in patients with rheumatoid arthritis. Clin Rheumatol 2016;35:2829-34.

50. Aletaha D, Bingham CO, Tanaka Y, et al. Efficacy and safety of sirukumab in patients with active rheumatoid arthritis refractory to antiTNF therapy (SIRROUND-T): a randomised, double-blind, placebo-controlled, parallelgroup, multinational, phase 3 study. Lancet 2017;389:1206-17.

51. Chatzidionysiou K, Vollenhoven RFV. Rituximab versus anti-TNF in patients who previously failed one TNF inhibitor in an observational cohort. Scand J Rheumatol 2013;42:190-5.

52. Cohen G, Courvoisier N, Cohen JD, et al. The efficiency of switching from infliximab to etanercept and vice-versa in patients with rheumatoid arthritis. Clin Exp Rheumatol
2015;23:795-800.

53. Cohen S, Tuckwell K, Katsumoto TR, et al. OP0025 Fenebrutinib compared to placebo and adalimumab in patients with inadequate response to either methotrexate therapy or prior tnf therapy: phase 2 study. Ann Rheum Dis 2019;78:80-1.

54. Das S, Vital EM, Horton S, et al. Abatacept or tocilizumab after rituximab in rheumatoid arthritis? An exploratory study suggests non-response to rituximab is associated with persistently high IL-6 and better clinical response to IL-6 blocking therapy. Ann Rheum Dis 2014;73:909-12.

55. Elmedany SH, Mohamed AE, Galil SMA. Efficacy and safety profile of intravenous tocilizumab versus intravenous abatacept in treating female Saudi Arabian patients with active moderate-to-severe rheumatoid arthritis. Clin Rheumatol 2019;38:2109-17.

56. Emery $\mathrm{P}$, Keystone $\mathrm{E}$, Tony $\mathrm{HP}$, et al. IL-6 receptor inhibition with tocilizumab improves treatment outcomes in patients with rheumatoid arthritis refractory to anti-tumour necrosis factor biologicals: Results from a 24-week multicentre randomised placebocontrolled trial. Ann Rheum Dis 2008;67:151623.

57. Emery P, Gottenberg JE, Rubbert-Roth A, et al. Rituximab versus an alternative TNF inhibitor in patients with rheumatoid arthritis who failed to respond to a single previous TNF inhibitor: SWITCH-RA, a global, observational, comparative effectiveness study. Ann Rheum Dis 2015;74:979-84.

58. Finckh A, Ciurea A, Brulhart L, et al. Which subgroup of patients with rheumatoid arthritis benefits from switching to rituximab versus alternative anti-tumour necrosis factor (TNF) agents after previous failure of an anti-TNF agent? Ann Rheum Dis 2010;69:387-93.

59. Fleischmann R, Kremer J, Cush J, et al. Placebo-controlled trial of tofacitinib monotherapy in rheumatoid arthritis. N Engl J Med 2012;367:495-507.

60. Fleischmann R, van Adelsberg $\mathrm{J}$, Lin $\mathrm{Y}$, et al. Sarilumab and Nonbiologic DiseaseModifying Antirheumatic Drugs in Patients With Active Rheumatoid Arthritis and Inadequate Response or Intolerance to Tumor Necrosis Factor Inhibitors. Arthritis Rheumatol 2017;69:277-90.

61. Al-Gareeb AlA, Gorial Fl, Mahmood AS. Niclosamide as an adjuvant to etanercept in treatment patients with active rheumatoid 
arthritis: an 8-week randomized controlled pilot study. Clin Rheumatol 2018;37:2633-41.

62. Fleischmann RM, Genovese MC, Enejosa J V, et al. Safety and effectiveness of upadacitinib or adalimumab plus methotrexate in patients with rheumatoid arthritis over 48 weeks with switch to alternate therapy in patients with insufficient response. Ann Rheum Dis 2019;78:1454-62.

63. Genovese MC, Kavanaugh A, Weinblatt ME, et al. An oral Syk kinase inhibitor in the treatment of rheumatoid arthritis: A three-month randomized, placebo-controlled, phase ॥ study in patients with active rheumatoid arthritis that did not respond to biologic agents. Arthritis Rheum 2011;63:337-45.

64. Genovese MC, Kinnman N, De La Bourdonnaye $G$, et al. Atacicept in patients with rheumatoid arthritis and an inadequate response to tumor necrosis factor antagonist therapy: Results of a phase II, randomized, placebocontrolled, dose-finding trial. Arthritis Rheum 2011;63:1793-803.

65. Genovese MC, Fleischmann RM, Greenwald M, et al. Tabalumab, an anti-BAFF monoclonal antibody, in patients with active rheumatoid arthritis with an inadequate response to TNF inhibitors. Ann Rheum Dis 2013;72:1461-8.

66. Genovese MC, Greenwald M, Cho CS, et al. A phase II randomized study of subcutaneous ixekizumab, an anti-interleukin-17 monoclonal antibody, in rheumatoid arthritis patients who were naive to biologic agents or had an inadequate response to tumor necrosis factor inhibitors. Arthritis Rheumatol 2014;66:1693-704.

67. Genovese MC, Van Der Heijde DM, Keystone EC, et al. A phase III, multicenter, randomized, double-blind, placebo-controlled, parallelgroup study of 2 dosing regimens of fostamatinib in patients with rheumatoid arthritis with an inadequate response to a tumor necrosis factor-a antagonist. J Rheumatol 2014;41:2120-8.

68. Genovese MC, Fleischmann R, Furst D, et al. Efficacy and safety of olokizumab in patients with rheumatoid arthritis with an inadequate response to TNF inhibitor therapy: Outcomes of a randomised Phase llb study. Ann Rheum Dis 2014;73:1607-15.

69. Genovese MC, Kremer J, Zamani O, et al. Baricitinib in patients with refractory rheumatoid arthritis. $N$ Engl J Med 2016;374:1243-52.

70. Genovese MC, Mangan EK, Fay J, et al.
Improvements in Remission and Low Disease Activity Are Achieved with Ongoing Sarilumab Treatment, in Patients with Rheumatoid Arthritis in 2 Phase 3 Studies - ACR Meeting Abstracts. Arthritis Rheumatol 2017;69:Suppl 10.

71. Genovese MC, Kremer JM, Kartman CE, et al. Response to baricitinib based on prior biologic use in patients with refractory rheumatoid arthritis. Rheumatology (Oxford) 2018;57:900-8.

72. Alivernini S, Laria A, Gremese E, et al. ACR70disease activity score remission achievement from switches between all the available biological agents in rheumatoid arthritis: A systematic review of the literature. Arthritis Res Ther 2009;11:R163.

73. Genovese MC, Fleischmann R, Combe B, et al. Safety and efficacy of upadacitinib in patients with active rheumatoid arthritis refractory to biologic disease-modifying anti-rheumatic drugs (SELECT-BEYOND): a double-blind, randomised controlled phase 3 trial. Lancet 2018;391:2513-24.

74. Genovese MC, Kalunian K, Gottenberg JE, et al. Effect of Filgotinib vs Placebo on Clinical Response in Patients With Moderate to Severe Rheumatoid Arthritis Refractory to DiseaseModifying Antirheumatic Drug Therapy: The FINCH 2 Randomized Clinical Trial. JAMA 2019;322:315-25.

75. Gomez-Reino JJ, Maneiro JR, Ruiz J, et al. Comparative effectiveness of switching to alternative tumour necrosis factor (TNF) antagonists versus switching to rituximab in patients with rheumatoid arthritis who failed previous TNF antagonists: The MIRAR Study. Ann Rheum Dis 2012;71:1861-4.

76. González-Vacarezza N, Alemán A, González $G$, et al. Rituximab and tocilizumab for the treatment of rheumatoid arthritis. Int $\mathrm{J}$ Technol Assess Health Care 2014;30:282-8.

77. Gottenberg JE, Brocq O, Perdriger A, et al. NonTNF-targeted biologic vs a second antiTNF drug to treat rheumatoid arthritis in patients with insufficient response to a first anti-TNF drug: A randomized clinical trial. JAMA - J Am Med Assoc 2016;316:1172-80.

78. Gottenberg J-E, Morel J, Perrodeau E, et al. Comparative effectiveness of rituximab, abatacept, and tocilizumab in adults with rheumatoid arthritis and inadequate response to TNF inhibitors: prospective cohort study. BMJ 2019;364:167.

79. Greenwald MW, Shergy WJ, Kaine JL, et al. 
Evaluation of the safety of rituximab in combination with a tumor necrosis factor inhibitor and methotrexate in patients with active rheumatoid arthritis: Results from a randomized controlled trial. Arthritis Rheum 2011;63:622-32.

80. Harrold LR, Reed GW, Magner R, et al. Comparative effectiveness and safety of rituximab versus subsequent anti-tumor necrosis factor therapy in patients with rheumatoid arthritis with prior exposure to anti-tumor necrosis factor therapies in the United States Corrona registry. Arthritis Res Ther 2015;17:256.

81. Harrold LR, Reed GW, Shewade A, et al. Effectiveness of rituximab for the treatment of rheumatoid arthritis in patients with prior exposure to anti-TNF: Results from the CORRONA registry. J Rheumatol 2015;42:10908.

82. Harrold LR, Reed GW, Kremer JM, et al. The comparative effectiveness of abatacept versus anti-tumour necrosis factor switching for rheumatoid arthritis patients previously treated with an anti-tumour necrosis factor. Ann Rheum Dis 2015;74:430-6.

83. Blom M, Kievit W, Donders ART, et al. Effectiveness of a third tumor necrosis factor-凶-blocking agent compared with rituximab after failure of 2 TNF-blocking agents in rheumatoid arthritis. J Rheumatol 2011;38:2355-61.

84. Harrold LR, Reed GW, Solomon $\mathrm{DH}$, et al. Comparative effectiveness of abatacept versus tocilizumab in rheumatoid arthritis patients with prior TNFi exposure in the US Corrona registry. Arthritis Res Ther 2016;18:280.

85. Harrold LR, Reed GW, Best J, et al. Real-world Comparative Effectiveness of Tocilizumab Monotherapy vs. Tumor Necrosis Factor Inhibitors with Methotrexate in Patients with Rheumatoid Arthritis. Rheumatol Ther 2018;5:507-23.

86. Hirabara S, Takahashi N, Fukaya N, et al Clinical efficacy of abatacept, tocilizumab, and etanercept in Japanese rheumatoid arthritis patients with inadequate response to anti-TNF monoclonal antibodies. Clin Rheumatol 2014;33:1247-54.

87. Huang $Y$, Fan $Y$, Liu Y, et al. Efficacy and safety of secukinumab in active rheumatoid arthritis with an inadequate response to tumor necrosis factor inhibitors: a meta-analysis of phase III randomized controlled trials. Clin Rheumatol 2019;38:2765-76.
88. Kekow J, Mueller-Ladner U, Schulze-Koops H. Rituximab is more effective than second antiTNF therapy in rheumatoid arthritis patients and previous TNFa blocker failure. Biol Targets Ther 2012;6:191-9.

89. Kim HL, Lee MY, Park SY, et al. Comparative effectiveness of cycling of tumor necrosis factor-a (TNF-a) inhibitors versus switching to non-TNF biologics in rheumatoid arthritis patients with inadequate response to TNF-a inhibitor using a Bayesian approach. Arch Pharm Res 2014;37:662-70.

90. Kremer JM, Emery P, Camp HS, et al. A Phase llb Study of ABT-494, a Selective JAK-1 Inhibitor, in Patients With Rheumatoid Arthritis and an Inadequate Response to Anti-Tumor Necrosis Factor Therapy. Arthritis Rheumatol 2016;68:2867-77.

91. Lauper K, Nordström DC, Pavelka K, et al. Comparative effectiveness of tocilizumab versus TNF inhibitors as monotherapy or in combination with conventional synthetic disease-modifying antirheumatic drugs in patients with rheumatoid arthritis after the use of at least one biologic disease-modifyin. Ann Rheum Dis 2018;77:1276-82.

92. Lee $\mathrm{YH}$, Bae SC. Comparative efficacy and safety of tocilizumab, rituximab, abatacept and tofacitinib in patients with active rheumatoid arthritis that inadequately responds to tumor necrosis factor inhibitors: a Bayesian network meta-analysis of randomized controlled tri. Int $\mathrm{J}$ Rheum Dis 2016;19:1103-11.

93. Malottki K, Barton $\mathrm{P}$, Tsourapas $\mathrm{A}$, et al. Adalimumab, etanercept, infiximab, rituximab and abatacept for the treatment of rheumatoid arthritis after the failure of a tumour necrosis factor inhibitor: A systematic review and economic evaluation. Health Technol Assess (Rockv) 2011;15:1-300.

94. Brown S, Everett CC, Naraghi K, et al. Alternative tumour necrosis factor inhibitors (TNFi) or abatacept or rituximab following failure of initial TNFi in rheumatoid arthritis: The SWITCH RCT. Health Technol Assess (Rockv) 2018;22:1280.

95. Nam JL, Takase-Minegishi K, Ramiro S, et al. Efficacy of biological disease-modifying antirheumatic drugs: A systematic literature review informing the 2016 update of the EULAR recommendations for the management of rheumatoid arthritis. Ann Rheum Dis 2017;76:1108-13.

96. Ogata A, Tanaka Y, Ishii T, et al. A randomized, 
double-blind, parallel-group, phase III study of shortening the dosing interval of subcutaneous tocilizumab monotherapy in patients with rheumatoid arthritis and an inadequate response to subcutaneous tocilizumab every other week: Results of. Mod Rheumatol 2018;28:76-84.

97. Pascart T, Philippe $P$, Drumez $E$, et al. Comparative efficacy of tocilizumab, abatacept and rituximab after non-TNF inhibitor failure: results from a multicentre study. Int J Rheum Dis 2016;19:1093-102.

98. Schiff M, Pritchard C, Huffstutter JE, et al. The 6-month safety and efficacy of abatacept in patients with rheumatoid arthritis who underwent a washout after anti-tumour necrosis factor therapy or were directly switched to abatacept: The ARRIVE trial. Ann Rheum Dis 2009;68:1708-14.

99. Schiff $M$, Combe B, Dörner $T$, et al. Efficacy and safety of tabalumab, an anti-BAFF monoclonal antibody, in patients with moderate-to-severe rheumatoid arthritis and inadequate response to TNF inhibitors: Results of a randomised, double-blind, placebo-controlled, phase 3 study. RMD Open 2015;1:e000037.

100. Singh JA, Hossain A, Tanjong Ghogomu E, et al. Biologics or tofacitinib for people with rheumatoid arthritis unsuccessfully treated with biologics: A systematic review and network meta-analysis. Cochrane Database Syst. Rev. 2017;2017.

101. Smolen JS, Kay J, Matteson EL, et al. Insights into the efficacy of golimumab plus methotrexate in patients with active rheumatoid arthritis who discontinued prior anti-tumour necrosis factor therapy: Post-hoc analyses from the GO-AFTER study. Ann Rheum Dis 2014;73:1811-8.

102. Smolen JS, Burmester GR, Combe B, et al. Head-to-head comparison of certolizumab pegol versus adalimumab in rheumatoid arthritis: 2-year efficacy and safety results from the randomised EXXELERATE study. Lancet 2016;388:2763-74.

103. Soliman MM, Hyrich $\mathrm{KL}$, Lunt $\mathrm{M}$, et al. Rituximab or a second anti-tumor necrosis factor therapy for rheumatoid arthritis patients who have failed their first anti-tumor necrosis factor therapy? Comparative analysis from the British Society for Rheumatology Biologics Register. Arthritis Care Res 2012;64:1108-15.

104. Strand V, Burmester GR, Zerbini CAF, et al. Tofacitinib with methotrexate in third-line treatment of patients with active rheumatoid arthritis: Patient-reported outcomes from a phase III trial. Arthritis Care Res 2015;67:47583.

105. Burmester GR, Blanco R, Charles-Schoeman $C$, et al. Tofacitinib (CP-690,550) in combination with methotrexate in patients with active rheumatoid arthritis with an inadequate response to tumour necrosis factor inhibitors: A randomised phase 3 trial. Lancet 2013;381:451-60.

106. Tak PP, Mease PJ, Genovese MC, et al. Safety and efficacy of ocrelizumab in patients with rheumatoid arthritis and an inadequate response to at least one tumor necrosis factor inhibitor: Results of a forty-eightweek randomized, double-blind, placebocontrolled, parallel-group phase III trial. Arthritis Rheum 2012;64:360-70.

107. Takahashi N, Fujibayashi T, Kida D, et al. Concomitant methotrexate and tacrolimus augment the clinical response to abatacept in patients with rheumatoid arthritis with a prior history of biological DMARD use. Rheumatol Int 2015;35:1707-16.

108. Takeuchi T, Tanaka Y, Yamanaka $\mathrm{H}$, et al. Efficacy and safety of olokizumab in Asian patients with moderate-to-severe rheumatoid arthritis, previously exposed to anti-TNF therapy: Results from a randomized phase II trial. Mod Rheumatol 2016;26:15-23.

109. Torrente-Segarra V, Acosta Pereira A, Morla R, et al. VARIAR Study: Assessment of short-term efficacy and safety of rituximab compared to an tumor necrosis factor alpha antagonists as second-line drug therapy in patients with rheumatoid arthritis refractory to a first tumor necrosis factor alpha antagonist. Reumatol Clínica 2016;12:319-22.

110. Van Vollenhoven RF, Wax S, Li Y, et al. Safety and efficacy of atacicept in combination with rituximab for reducing the signs and symptoms of rheumatoid arthritis: A phase II, randomized, double-blind, placebocontrolled pilot trial. Arthritis Rheumatol 2015;67:2828-36.

ו1. Vial G, Pouilly A De, Scouppe L, et al. FRI0234 Choice of biologic therapy following rituximab: influencing factors in a french multicenter cohort of rheumatoid arthritis. Ann Rheum Dis 2017;76:573.2-574.

112. Vieira $\mathrm{MC}$, Zwillich $\mathrm{SH}$, Jansen JP, et al. Tofacitinib Versus Biologic Treatments in Patients With Active Rheumatoid Arthritis Who Have Had an Inadequate Response to Tumor Necrosis Factor Inhibitors: Results 
From a Network Meta-analysis. Clin Ther 2016;38:2628-2641.e5.

113. Vital EM, Dass S, Buch MH, et al. An extra dose of rituximab improves clinical response in rheumatoid arthritis patients with initial incomplete B cell depletion: a randomised controlled trial. Ann Rheum Dis 2015;74:1195201.

114. Walker UA, Jaeger VK, Chatzidionysiou K, et al. Rituximab done: What's next in rheumatoid arthritis? A European observational longitudinal study assessing the effectiveness of biologics after rituximab treatment in rheumatoid arthritis. Rheumatology (Oxford) 2015;55:230-6.

115. Wei W, Knapp K, Wang L, et al. Treatment Persistence and Clinical Outcomes of Tumor Necrosis Factor Inhibitor Cycling or Switching to a New Mechanism of Action Therapy: Realworld Observational Study of Rheumatoid Arthritis Patients in the United States with Prior Tumor Necrosis Factor. Adv Ther 2017;34:193652.

116. Charles-Schoeman C, Burmester G, Nash P, et al. Efficacy and safety of tofacitinib following inadequate response to conventional synthetic or biological disease-modifying antirheumatic drugs. Ann Rheum Dis 2016;75:1293-301.

117. Weinblatt ME, McInnes IB, Kremer JM, et al. A Randomized Phase llb Study of Mavrilimumab and Golimumab in Rheumatoid Arthritis. Arthritis Rheumatol 2018;70:49-59.

118. Weinblatt M, Thomson G, Chen K, et al. FRIOI7l Clinical responses in patients with inadequate response to bdmards upon treatment with upadacitinib. Ann Rheum Dis 2019;78:759.

119. Zhang F,MaC.Comparison of the effectiveness on intra-articular and subcutaneous TNF inhibitor in rheumatoid arthritis patients. Clin Rheumatol 2018;37:199-204.

120. Charles-Schoeman C, Kremer J, Krishnaswami S, et al. THU0185 Comparison of tofacitinib safety and efficacy in rheumatoid arthritis patients with inadequate response to conventional synthetic dmards, or to one or more biological dmards. Ann Rheum Dis 2017;76:271.3-272.

121. Chatzidionysiou K, Askling J, Eriksson J, et al. Effectiveness of TNF inhibitor switch in RA: Results from the national Swedish register. Ann Rheum Dis 2015;74:890-6.

122. Albano MG, Giraudet-Le Quintrec J-SS, Crozet $\mathrm{C}$, et al. Characteristics and development of therapeutic patient education in rheumatoid arthritis: analysis of the 2003-2008 literature. Joint Bone Spine 2010;77:405-10.

123. Allam NM, Koura GMR, Alrawaili SM, et al. The effect of siwan therapy in management of patients with rheumatoid arthritis: A single blind randomized controlled trial. Biomed Res 2018;29:1400-6.

124. DiRenzo D, Crespo-Bosque M, Gould N, et al. Systematic Review and Meta-analysis: Mindfulness-Based Interventions for Rheumatoid Arthritis. Curr Rheumatol Rep 2018;20:75.

125. Dissanayake RK, Bertouch J V. Psychosocial interventions as adjunct therapy for patients with rheumatoid arthritis: A systematic review. Int J Rheum Dis 2010;13:324-34.

126. Dixon KE, Keefe FJ, Scipio CD, et al. Psychological interventions for arthritis pain management in adults: A meta-analysis. Heal Psychol 2007;26:241-50.

127. El Miedany Y, El Gaafary M, El Arousy N, et al. Arthritis education: the integration of patientreported outcome measures and patient self-management. Clin Exp Rheumatol 2012;30:899-904.

128. Eversden L, Maggs F, Nightingale $P$, et al. A pragmatic randomised controlled trial of hydrotherapy and land exercises on overall well being and quality of life in rheumatoid arthritis. BMC Musculoskelet Disord 2007;8:23.

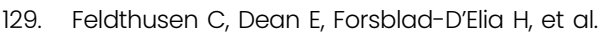
Effects of Person-Centered Physical Therapy on Fatigue-Related Variables in Persons With Rheumatoid Arthritis: A Randomized Controlled Trial. Arch Phys Med Rehabil 2016;97:26-36.

130. Ferwerda $M$, Van Beugen S, Van Middendorp $\mathrm{H}$, et al. A tailored-guided internet-based cognitive-behavioral intervention for patients with rheumatoid arthritis as an adjunct to standard rheumatological care: Results of a randomized controlled trial. Pain 2017;158:86878.

131. Fidahic $M$, Jelicic Kadic $A$, Radic $M$, et al. Celecoxib for rheumatoid arthritis. Cochrane Database Syst Rev 2017;2017.

132. Figen A, Geçene M, Gündüz R, et al. LongTerm Effects of Comprehensive Inpatient Rehabilitation on Function and Disease Activity in Patients with Chronic Rheumatoid Arthritis and Ankylosing Spondylitis. Turkish J Rheumatol 2011;26:135-44.

133. Fitzcharles MA, Ste-Marie PA, Häuser W, et al. Efficacy, Tolerability, and Safety of Cannabinoid Treatments in the Rheumatic 
Diseases: A Systematic Review of Randomized Controlled Trials. Arthritis Care Res 2016;68:681-8.

134. Annegret F, Thomas F. Long-term benefits of radon spa therapy in rheumatic diseases: Results of the randomised, multi-centre IMuRa trial. Rheumatol Int 2013;33:2839-50.

135. Gizińska M, Rutkowski R, Romanowski W, et al. Effects of Whole-Body Cryotherapy in Comparison with Other Physical Modalities Used with Kinesitherapy in Rheumatoid Arthritis. Biomed Res Int 2015;2015:1-7.

136. Gok Metin Z, Ozdemir L. The Effects of Aromatherapy Massage and Reflexology on Pain and Fatigue in Patients with Rheumatoid Arthritis: A Randomized Controlled Trial. Pain Manag Nurs 2016;17:140-9.

137. Hammond A, Young A, Kidao R. A randomised controlled trial of occupational therapy for people with early rheumatoid arthritis. Ann Rheum Dis 2004;63:23-30.

138. Hewlett S, Kirwan J, Pollock J, et al. Patient initiated outpatient follow up in rheumatoid arthritis: six year randomised controlled trial. BMJ 2005;330:171.

139. Hewlett S, Almeida C, Ambler N, et al. Group cognitive-behavioural programme to reduce the impact of rheumatoid arthritis fatigue: the RAFT RCT with economic and qualitative evaluations. Health Technol Assess 2019;23:1130.

140. Hurkmans E, Van Der Giesen FJ, Vlieland TPMV, et al. Dynamic exercise programs (aerobic capacity and/or muscle strength training) in patients with rheumatoid arthritis. Cochrane Database Syst Rev 2009;2009:CD006853.

141. Iversen MD, Hammond A, Betteridge N. Selfmanagement of rheumatic diseases: state of the art and future perspectives. Ann Rheum Dis 2010;69:955-63.

142. Jastrząbek R, Straburzyńska-Lupa A, Rutkowski $R$, et al. Effects of different local cryotherapies on systemic levels of TNF- $\mathrm{a}$, IL-6, and clinical parameters in active rheumatoid arthritis. Rheumatol Int 2013;33:2053-60.

143. Jiang $G$, Wan $B$, Huang $W$, et al. Influence of acupotomy loosing on IL-6, IL-10 and TNF- $a$ in synovial fluid of rheumatoid arthritis patients with elbow joint stiffness. World J Acupunct Moxibustion 2018;28:91-6.

144. Kawai S, Uchida E, Kondo M, et al. Efficacy and safety of ketoprofen patch in patients with rheumatoid arthritis: A randomized, doubleblind, placebo-controlled study. J Clin Pharmacol 2010;50:1171-9.
145. Anvar N, Matlabi H, Safaiyan A, et al. Effectiveness of self-management program on arthritis symptoms among older women: A randomized controlled trial study. Health Care Women Int 2018;39:1326-39.

146. Knittle K, De Gucht V, Hurkmans E, et al. Targeting motivation and self-regulation to increase physical activity among patients with rheumatoid arthritis: a randomised controlled trial. Clin Rheumatol 2015;34:231-8.

147. Lau YN, Ng J, Lee SY, et al. A brief report on the clinical trial on neural mobilization exercise for joint pain in patients with rheumatoid arthritis. Z Rheumatol 2019;78:474-8.

148. Lee MS, Pittler MH, Ernst E. Tai chi for rheumatoid arthritis: Systematic review. Rheumatology (Oxford) 2007;46:1648-51.

149. Lee JA, Son MJ, Choi J, et al. Bee venom acupuncture for rheumatoid arthritis: A systematic review of randomised clinical trials. BMJ Open. 2014;4:e006140.

150. Lee YC, Massarotti E, Edwards RR, et al. Effect of milnacipran on pain in patients with rheumatoid arthritis with widespread pain: A randomized blinded crossover trial. J Rheumatol 2016;43:38-45.

151. Macedo AM, Oakley SP, Panayi GS, et al. Functional and work outcomes improve in patients with rheumatoid arthritis who receive targeted, comprehensive occupational therapy. Arthritis Care Res 2009;61:1522-30.

152. Macfarlane GJ, Paudyal P, Doherty $M$, et al. A systematic review of evidence for the effectiveness of practitioner-based complementary and alternative therapies in the management of rheumatic diseases: Rheumatoid arthritis. Rheumatology (Oxford) 2012;51:1707-13.

153. Manning $V L$, Hurley $M V_{\text {., Scott }} \mathrm{DL}$, et al. Education, self-management, and upper extremity exercise training in people with rheumatoid arthritis: a randomized controlled trial. Arthritis Care Res (Hoboken) 2014;66:21727.

154. Mollard E, Michaud K. A Mobile App With Optical Imaging for the Self-Management of Hand Rheumatoid Arthritis: Pilot Study. JMIR mHealth uHealth 2018;6:el2221.

155. Muñoz-Fernández S, Aguilar MD, Rodríguez A, et al. Evaluation of the impact of nursing clinics in the rheumatology services. Rheumatol Int 2016;36:1309-17.

156. Baxter S V., Hale LA, Stebbings S, et al. Walking is a Feasible Physical Activity for People with Rheumatoid Arthritis: A Feasibility 
Randomized Controlled Trial. Musculoskeletal Care 2016;14:47-56.

157. Prothero L, Barley E, Galloway J, et al. The evidence base for psychological interventions for rheumatoid arthritis: A systematic review of reviews. Int J Nurs Stud 2018;82:20-9.

158. Richards BL, Whittle SL, Buchbinder R. Antidepressants for pain management in rheumatoid arthritis. Cochrane Database Syst Rev 2011.

159. Santos I, Cantista P, Vasconcelos C, et al. Balneotherapy and rheumatoid arthritis: A randomized control trial. Isr Med Assoc J 2016;18:474-8.

160. Santos EJF, Duarte C, Marques A, et al. Effectiveness of non-pharmacological and non-surgical interventions for rheumatoid arthritis: an umbrella review. JBI database Syst Rev Implement reports 2019;17:1494-531.

161. Siqueira US, Orsini Valente LG, De Mello MT, et al. Effectiveness of Aquatic Exercises in Women with Rheumatoid Arthritis: A Randomized, Controlled, 16-Week Intervention-The HydRA Trial. Am J Phys Med Rehabil 2017;96:167-75.

162. Srikesavan C, Bryer C, Ali U, et al. Web-based rehabilitation interventions for people with rheumatoid arthritis: A systematic review. J Telemed Telecare 2019;25:263-75.

163. Tijhuis GJ, Zwinderman AH, Hazes JMW, et al. A randomized comparison of care provided by a clinical nurse specialist, an inpatient team, and a day patient team in rheumatoid arthritis. Arthritis Rheum 2002;47:525-31.

164. Wang J, Zou X, Zhou L, et al. Patient satisfaction after nurse-led care in Chinese patients with rheumatoid arthritis: A China study. Biomed Res 2017;28:4972-8.

165. Whittle SL, Richards BL, Husni E, et al. Opioid therapy for treating rheumatoid arthritis pain. Cochrane Database Syst Rev 2011.

166. Williams MA, Srikesavan $\mathrm{C}$, Heine PJ, et al. Exercise for rheumatoid arthritis of the hand. Cochrane Database Syst Rev 2018;2018.

167. Cameron M, Gagnier JJ, Chrubasik S. Herbal therapy for treating rheumatoid arthritis. Cochrane Database Syst Rev 2011:CD002948.

168. Carandang K, Pyatak EA, Vigen CLP. Systematic Review of Educational Interventions for Rheumatoid Arthritis. Am J Occup Ther 2016;70:7006290020pl.

169. Christie A, Jamtvedt G, Dahm KT, et al Effectiveness of Nonpharmacological and Nonsurgical Interventions for Patients With Rheumatoid Arthritis: An Overview of Systematic Reviews. Phys Ther 2007;87:1697-
715.

170. de Thurah A, Esbensen BA, Roelsgaard IK, et al. Efficacy of embedded nurse-led versus conventional physician-led follow-up in rheumatoid arthritis: a systematic review and meta-analysis. RMD open 2017;3:e000481.

171. de Thurah A, Stengaard-Pedersen K, Axelsen $M$, et al. Tele-Health Followup Strategy for Tight Control of Disease Activity in Rheumatoid Arthritis: Results of a Randomized Controlled Trial. Arthritis Care Res (Hoboken) 2018;70:35360.

172. Barton JL, Hulen E, Schue A, et al. Experience and Context Shape Patient and Clinician Goals For Treatment of Rheumatoid Arthritis: A Qualitative Study. Arthritis Care Res (Hoboken) 2018;70:1614-20.

173. Gibofsky A, Galloway J, Kekow J, et al. Comparison of patient and physician perspectives in the management of rheumatoid arthritis: results from global physician- and patient-based surveys. Health Qual Life Outcomes 2018;16:211.

174. Torikai E, Suzuki D, Matsuyama Y. AB0222 Differences of disease impression and treatment expectation in rheumatoid arthritis patients with different disease activity. Ann Rheum Dis 2018;77:1295.

175. Fraenkel L, Peters $E$, Charpentier $P$, et al. Decision tool to improve the quality of care in rheumatoid arthritis. Arthritis Care Res (Hoboken) 2012;64:977-85.

176. Fraenkel L, Matzko CK, Webb DE, et al. Use of Decision Support for Improved Knowledge, Values Clarification, and Informed Choice in Patients With Rheumatoid Arthritis. Arthritis Care Res (Hoboken) 2015;67:1496-502.

177. Li LC, Adam PM, Backman CL, et al. Proof-ofconcept study of a Web-based methotrexate decision aid for patients with rheumatoid arthritis. Arthritis Care Res (Hoboken) 2014;66:1472-81.

178. Li LC, Shaw CD, Lacaille $D$, et al. Effects of a Web-Based Patient Decision Aid on Biologic and Small-Molecule Agents for Rheumatoid Arthritis: Results From a Proof-ofConcept Study. Arthritis Care Res (Hoboken) 2018;70:343-52.

179. Garratt AM, Løchting I, Smedslund $G$, et al. Measurement properties of instruments assessing self-efficacy in patients with rheumatic diseases. Rheumatology (Oxford) 2014;53:1161-71.

180. Palmer D, El Miedany Y. Self-helplessness in arthritis: an important but overlooked index. 
Br J Nurs 2010;19:965, 968-71.

181. Silvers IJ, Hovell MF, Weisman $\mathrm{MH}$, et al. Assessing physician/patient perceptions in rheumatoid arthritis. A vital component in patient education. Arthritis Rheum 1985;28:300-7.

182. Sinclair VG, Wallston KA. The development and psychometric evaluation of the Brief Resilient Coping Scale. Assessment 2004;11:94-101.

183. Barlow $\mathrm{JH}$, Wright CC. Knowledge in patients with rheumatoid arthritis: a longer term follow-up of a randomized controlled study of patient education leaflets. $\mathrm{Br} \mathrm{J}$ Rheumatol 1998;37:373-6.

184. Barsky AJ, Ahern DK, Orav EJ, et al. A randomized trial of three psychosocial treatments for the symptoms of rheumatoid arthritis. Semin Arthritis Rheum 2010;40:222-32.

185. Bell MJ, Lineker SC, Wilkins AL, et al. A randomized controlled trial to evaluate the efficacy of community based physical therapy in the treatment of people with rheumatoid arthritis. J Rheumatol 1998;25:231-7.

186. Breedland I, van Scheppingen C, Leijsma $M$, et al. Effects of a group-based exercise and educational program on physical performance and disease self-management in rheumatoid arthritis: a randomized controlled study. Phys Ther 2011;91:879-93.

187. Hewlett S, Ambler N, Almeida C, et al. Selfmanagement of fatigue in rheumatoid arthritis: a randomised controlled trial of group cognitive-behavioural therapy. Ann Rheum Dis 2011;70:1060-7.

188. Hosseini Moghadam M, Jahanbin I, Nazarinia MA. The Effect of Educational Program on Self-efficacy of Women with Rheumatoid Arthritis: A Randomized Controlled Clinical Trial. Int J community based Nurs midwifery 2018;6:12-20.

189. Lineker SC, Bell MJ, Wilkins AL, et al. Improvements following short term home based physical therapy are maintained at one year in people with moderate to severe rheumatoid arthritis. J Rheumatol 2001;28:1658.
190. Lorig KR, Ritter PL, Laurent DD, et al. The internetbased arthritis self-management program: a one-year randomized trial for patients with arthritis or fibromyalgia. Arthritis Rheum 2008;59:1009-17.

191. Niedermann K, de Bie RA, Kubli R, et al. Effectiveness of individual resource-oriented joint protection education in people with rheumatoid arthritis. A randomized controlled trial. Patient Educ Couns 2011;82:42-8.

192. Niedermann K, Buchi S, Ciurea A, et al. Six and 12 months' effects of individual joint protection education in people with rheumatoid arthritis: a randomized controlled trial. Scand J Occup Ther 2012;19:360-9.

193. Riemsma RP, Taal E, Brus HL, et al. Coordinated individual education with an arthritis passport for patients with rheumatoid arthritis. Arthritis Care Res 1997;10:238-49.

194. Roth T, Price JM, Amato DA, et al. The effect of eszopiclone in patients with insomnia and coexisting rheumatoid arthritis: a pilot study. Prim Care Companion J Clin Psychiatry 2009;11:292-301.

195. Siu AMH, Chui DYY. Evaluation of a community rehabilitation service for people with rheumatoid arthritis. Patient Educ Couns 2004;55:62-9.

196. Tuntland H, Kjeken I, Nordheim L, et al. The Cochrane review of assistive technology for rheumatoid arthritis. Eur J Phys Rehabil Med 2010;46:261-8.

197. Lorig K, Chastain RL, Ung E, et al. Development and evaluation of a scale to measure perceived self-efficacy in people with arthritis. Arthritis Rheum 1989;32:37-44.

198. Kerschbaumer A, Sepriano A, Smolen JS, et al. Efficacy of pharmacological treatment in rheumatoid arthritis: a systematic literature research informing the 2019 update of the EULAR recommendations for management of rheumatoid arthritis. Ann Rheum Dis 2020.

199. Nagy G, Roodenrijs NMT, Welsing PMJ, et al. EULAR recommendations for the management of difficult-to-treat rheumatoid arthritis. Manuscript in preparation. 


\section{Supplementary}

\section{Supplementary File}

1. Pharmacological and non-pharmacological therapeutic strategies for difficult-totreat RA patients

la. Therapeutic strategies in patients with limited DMARD options

Clinical question

- What are the management options when drug choices are limited to treat persistent inflammation in D2T RA?

- What are the management options for patients with comorbidities?

Epidemiological question

What are effective management options when DMARD choices are limited, because of adverse events, comorbidity or contraindications, to treat persistent inflammation in D2T RA?

- P: D2T RA patients with inflammatory activity and limited DMARD options

- I: Management options (including non-pharmacological therapy)

- C: No or different management options

- O: Efficacy and/or safety/tolerability

lb. Therapeutic strategies in patients who failed $\geq 1 \mathrm{~b} / \mathrm{tsDMARD}$

Clinical question

What are the management options for refractory RA?

Epidemiological question

What are effective management options for refractory RA?

- P: D2T RA with inflammation not explained by other causes (refractory RA)

- I: Management options (including non-pharmacological therapy)

- C: No or different management options

- O: Reduction of RA inflammation

lc. Therapeutic strategies in patients with predominantly non-inflammatory complaints Clinical question

What are the management options for D2TRA patients that primarily have noninflammatory complaints (e.g. fatigue, pain, reduced quality of life and physical functioning)?

Epidemiological question 
What are effective management options for RA patients with persistent complaints that are not explained by active inflammation?

- P: D2T RA patients with persistent complaints not explained by active inflammation

- I: Management options (including non-pharmacological therapy)

- C: No or different management options

- O: Reduction in (specific) complaints

\section{Search in short}

Rheumatoid arthritis AND Terms for difficult-to-treat RA, terms for fatigue, and terms for adverse events, and comorbidities that may limit DMARD choices AND General terms for treatment, and specific DMARDs, GCs, NSAIDs, non-pharmacological treatment options AND General terms for efficacy and tolerability, and often used outcome measures AND Adjusted Pubmed therapeutic query (to focus more generally on comparative studies not only RCTs)

2. Identification and optimisation of suboptimal goal setting and suboptimal selfmanagement

2a. Identification of a mismatch in goal setting between patient and health care professional

Clinical question

How to address a mismatch between management goals of patients and rheumatologists?

\section{Epidemiological question}

How to identify a mismatch between management goals of patients and health care professionals in difficult to treat RA patients?

- P:D2T RA patients where a mismatch between their management goals and the health care professional's management goals is suspected

- I: diagnostic/screening 'tests'/characteristics for mismatch of management goals

- C: Presence/level/absence of a mismatch of management goals according to a reference standard

- O: Diagnostic test value of the techniques to establish a mismatch of management goals

$2 b$. Optimisation of a mismatch in goal setting between patient and health care professional

Clinical question

How to optimise the goal setting by the patient and rheumatologist? 
Epidemiological question

How to optimise shared goal setting by the difficult to treat RA patient and health care professional?

- P: D2T RA patients with a mismatch between management goal of patient and health care professional

- I: Interventions to optimize goals setting by patient and health care professional

- C: No or another intervention

- O: Reduction of complaints

\section{2c. Identification of suboptimal self-management}

Clinical question

How to detect suboptimal self-management?

Epidemiological question

How to detect suboptimal self-management in difficult to treat RA patients?

- P: D2T RA patients who are suspected of suboptimal self-management

- I: diagnostic/screening 'tests'/characteristics for self-management

- C: Presence/level/absence of (adequate) self-management according to a reference standard

- O: Diagnostic test value of the techniques to establish (adequate) self-management

\section{$2 \mathrm{~d}$. Optimisation of suboptimal self-management}

Clinical question

How to improve self-management to improve complaints?

Epidemiological question

How to improve self-management in difficult to treat RA patients to improve complaints?

- P: D2T RA patient with suboptimal self-management

- I: Interventions to improve self-management

- C: No or another intervention

- O: Reduction of complaints

Search in short

Rheumatoid arthritis AND Terms for management goals and self-management

\section{Other}

Searches in full, exclusion criteria, predetermined items for data extraction and Supplementary Tables 1-7 can be accessed via: https://doi.org/rmdopen-2020-001512. 



\section{CHAPTER 9}

\section{EULAR recommendations for the management of difficult-to-treat rheumatoid arthritis}

Manuscript submitted to EULAR for review and approval.

György Nagy*, Nadia M.T. Roodenrijs*, Paco M.J. Welsing, Melinda Kedves, Attila Hamar, Marlies C. van der Goes, Alison Kent, Margot Bakkers, Polina Pchelnikova, Etienne Blaas, Ladislav Šenolt, Zoltán Szekanecz, Ernest Choy, Maxime Dougados, Johannes W.G. Jacobs, Rinie Geenen, Johannes W.J Bijlsma, Angela Zink, Daniel Aletaha, Jan L.M. Schoneveld, Piet van Riel, Sophie Dumas, Yeliz Prior, Elena Nikiphorou, Ferraccioli Gianfranco, Georg Schett, Kimme Hyrich, Ulf Müller-Ladner, Maya H. Buch, lain B. McInnes, Désirée van der Heijde, ${ }^{+}$ Jacob M. van Laar $^{+}$ 


\section{Abstract}

\section{Objectives}

To develop evidence-based European Alliance of Associations for Rheumatology (EULAR) recommendations for the management of difficult-to-treat rheumatoid arthritis (D2T RA).

\section{Methods}

A EULAR Task Force was initiated comprising 34 individuals: 26 rheumatologists, three patient partners, one rheumatology nurse, one rheumatology occupational therapist, one psychologist and two pharmacists. Two systematic literature reviews were performed addressing ten clinical questions around diagnostic challenges, and pharmacological and non-pharmacological therapeutic strategies in D2T RA. Subsequently, recommendations were formulated based on the identified evidence and expert opinion. Strength of recommendations (SoR, scale A-D: A typically consistent level 1 studies, D level 5 evidence or inconsistent studies) and level of agreement (LoA, scale 0-10: 0 completely disagree, 10 completely agree) with recommendations by the Task Force members were determined.

\section{Results}

Two overarching principles and ten recommendations were defined regarding the confirmation of the diagnosis of RA, assessing the presence or absence of inflammatory disease activity, pharmacological interventions to reduce inflammatory disease activity (including those with comorbidities) and non-pharmacological interventions to improve treatment adherence, functional disability, pain, fatigue, goal setting and selfefficacy. The SoR varied from level C to D. The LOA with the overarching principles and recommendations was generally high (ranging from 8.4 to 9.6).

\section{Conclusions}

These D2T RA management recommendations will support health care professionals and patients to deliver a holistic management approach and more personalised pharmacological and non-pharmacological therapeutic strategies. High-quality evidence was scarce. A research agenda was created to guide future research. 


\section{Introduction}

The treatment options for rheumatoid arthritis (RA) have significantly expanded with the introduction of biological and targeted synthetic disease-modifying antirheumatic drugs (b/tsDMARDs)! The first evidence-based European League Against Rheumatism (EULAR, from 2021 European Alliance of Associations for Rheumatology) recommendations for the management of RA were published in 2010, ${ }^{2}$ and updated in $2013,{ }^{3} 2016^{4}$ and $2019 .{ }^{5}$ The American College of Rheumatology $(\mathrm{ACR}){ }_{1}^{6}$ the Panamerican League of Associations for Rheumatology (PANLAR) 7 and the Asia Pacific League of Associations for Rheumatology $(\mathrm{APLAR})^{8}$ developed similar recommendations, with a principal focus on pharmacological therapy. These recommendations along with the implementation of treat-to-target (T2T) and tight control strategies offer valuable treatment guidance, 5,9 specifically in the early phase of the disease, leading to improved outcomes for the majority of RA patients. However, there is still a significant proportion of RA patients who do not reach low disease activity or remission and/or remain symptomatic after several cycles of conventional synthetic (cs), b and/or tsDMARDs, even though their treatment is being conducted

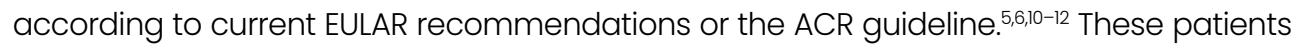
may be classified as having 'difficult-to-treat (D2T)' disease. Optimal management of these patients is a significant challenge in clinical practice, and hitherto, no guidance has been provided for the management of this heterogeneous patient population.

The unmet clinical need of D2T RA has been highlighted in an international survey. ${ }^{13}$ The heterogeneity of D2T RA was characterised by various factors that could contribute to the disease state, e.g. non-adherence, interfering comorbidities and non-inflammatory symptoms. In addition, the need for uniform terminology and a uniform definition was expressed, as well as explicit management recommendations (including nonpharmacological therapeutic strategies). Specific recommendations addressing D2T RA patients would be able to address all contributing factors systematically. Therefore, a EULAR Task Force was convened to develop recommendations for the management of D2T RA. These recommendations are complementary to the current EULAR RA recommendations. ${ }^{5}$ Furthermore, to minimise overlap, during the SLRS and the formulation of the recommendations we predominantly focussed on topics not addressed previously and refer to several published ${ }^{5,4-22}$ and ongoing EULAR projects where appropriate.

\section{Methods}

\section{Steering Committee and Task Force}

After approval by the EULAR executive committee, the convenor (GN) and co-convenor $(\mathrm{JMVL})$ formed the Steering Committee and Task Force. The process was conducted 
following the EULAR Standardised Operating Procedures (SOP). ${ }^{23}$ In addition to the (co-) convenors, the Steering Committee of the Task Force included a methodologist (DvdH), a co-methodologist (PMJW), a rheumatology postdoctoral fellow (MJHdH) and three fellows (NMTR, MK and AH). The Task Force comprised 34 individuals, including the Steering Committee members. Among the Task Force members were 26 rheumatologists (including two EMerging EUlar Network (EMEUNET) representatives), three patient partners, one rheumatology nurse, one rheumatology occupational therapist, one psychologist and two pharmacists. All rheumatologists were experienced in the treatment of RA, the majority with significant experience in clinical trials and some also in outcomes research and patient registries. All Task Force members declared their potential conflicts of interest before the start of the project. Two of the Task Force members (MJHdH (postdoctoral fellow) and LG (pharmacist)) left the Task Force during the process, due to new positions, and did not attend the second Task Force meeting.

\section{Target audience}

In accordance with the EULAR SOP, the primary target audience of these recommendations are health care professionals (HCPS) and patients (and their carers). ${ }^{23}$ Additionally, these recommendations can serve to highlight unmet needs in D2T RA and, therefore, secondarily also target policy officers, pharmaceutical and health insurance companies.

\section{Definition}

As an initial step in the development of management recommendations, a definition and a uniform term for the patient population to which the recommendations apply had to be established. The Steering Committee proposed terminology and created a first draft of a definition, guided by the results of the international survey and a scoping literature review. ${ }^{13}$ These were discussed with the whole Task Force and amended during the first Task Force meeting (held in August 2018). The final terminology and definition were chosen by a voting process. All Task Force members agreed with 'difficult-totreat RA' as the term and the final definition (Table 1). Further details are described in a separate paper. ${ }^{24}$

\section{Clinical questions and systematic literature reviews}

The first step of the process was the formulation of clinical questions for the systematic literature reviews (SLRS) by the Steering Committee. Clinical questions that could result in D2T RA were discussed and included diagnostic challenges and therapeutic strategies. The Steering Committee agreed that confirming the diagnosis of RA and the presence or absence of inflammatory RA activity are important first steps in the assessment and subsequent management of D2T RA. Therefore, clinical questions were formulated to focus on techniques for the confirmation of the diagnosis of RA and/or a relevant 
differential diagnosis (either as alternative (i.e. misdiagnosis) or coexisting mimicking disease), and the assessment of inflammatory activity in RA patients in general and in those with specific comorbidities that may influence this assessment. Thereafter, the Steering Committee agreed that it is necessary to consider treatment adherence and, if present, to optimise this and, therefore, also formulated a clinical question on adherence. Then, therapeutic clinical questions were formulated focussing on pharmacological and non-pharmacological therapeutic strategies for different aspects of D2T RA: patients with limited DMARD choices because of adverse events, comorbidities or other contraindications; patients in whom $\geq 2 \mathrm{~b} /$ tsDMARD with different mechanisms of action (MOA) failed; patients with predominantly non-inflammatory complaints (not directly related to inflammation); the therapeutic role of lifestyle interventions; the optimisation of goal setting between patients and HCPs as well as self-management. To optimise goal setting and self-management, it was agreed that it would be necessary to first identify a mismatch in goal setting and suboptimal self-management, respectively. These topics were therefore also addressed in clinical questions. All questions were discussed and finalised during the first Task Force meeting.

SLRs on these questions were performed by the fellows (NMTR, MK and AH) under supervision of the co-methodologist (PMJW) in accordance with the EULAR SOP. ${ }^{23}$ As other ongoing EULAR projects were already focussing on adherence and lifestyle factors, it was decided not to perform separate SLRs on these topics, but to refer to the respective

Table 1. Definition of difficult-to-treat RA ${ }^{24}$

1. Treatment according to EULAR recommendations and failure of $\geq 2 \mathrm{~b} /$ tsDMARDs (with different mechanisms of action)' after failing csDMARD therapy (unless contraindicated) ${ }^{2}$

2. Signs suggestive of active/progressive disease, defined as $\geq 1$ of:

a. At least moderate disease activity (according to validated composite measures including joint counts e.g. DAS28-ESR >3.2 or CDAI >10)

b. Signs (including acute phase reactants and imaging) and/or symptoms suggestive of active disease (joint related or other)

c. Inability to taper glucocorticoid treatment (below $7.5 \mathrm{mg} /$ day prednisone or equivalent)

d. Rapid radiographic progression (with or without signs of active disease) ${ }^{3}$

e. Well-controlled disease according to above standards, but still having RA symptoms that are causing a reduction in quality of life

3. The management of signs and/or symptoms is perceived as problematic by the rheumatologist and/or the patient

All three criteria need to be present in D2T RA.

b: biological; CDAl: clinical disease activity index; cs: conventional synthetic; DAS28-ESR: disease activity score assessing 28 joints using erythrocyte sedimentation rate; DMARD: disease-modifying antirheumatic drug; EULAR: European League Against Rheumatism; mg: milligram; RA: rheumatoid arthritis; ts: targeted synthetic.

1. Unless restricted by access to treatment due to socioeconomic factors.

2. If csDMARD treatment is contraindicated, failure of $\geq 2 \mathrm{~b} / \mathrm{tsDMARDs}$ with different mechanisms of action is sufficient.

3. Rapid radiographic progression: change in van der Heijde-modified Sharp score $\geq 5$ points at 1 year ${ }^{25}$ or a similar progression in another validated scoring method. 
SLRs and recommendations. 22,26 For the other questions, PubMed, Embase and Cochrane bibliographic databases were searched for relevant papers until December 2019, as well as EULAR and ACR conference abstracts from 2017 up to and including 2019. Relevant papers were selected and critically appraised. Results were summarised, including assessment of risk of bias (ROB). ${ }^{23}$ Further details on the methodology and results of the SLRs are published separately. ${ }^{27,28}$

\section{Consensus finding}

Based on the results of the SLRS, draft overarching principles and recommendations were proposed. The Steering Committee discussed the results of the SLRs comprehensively and further amended the overarching principles and recommendations.

The results of the SLRs as well as the proposed overarching principles and recommendations were presented to the Task Force at the second Task Force meeting. This face-to-face meeting was initially planned for March 2020, but was in the end held virtually due to COVID-19 over three sessions in September and October 2020. Twentyfive, 30 and 27 Task Force members, respectively, participated in these online meetings. After the presentations, all overarching principles and recommendations were discussed and amended.

After these discussions, a voting process was applied per recommendation. In round l, a majority of at least $75 \%$ was required to accept the recommendation. If this was not achieved, the recommendation was discussed and amended and subjected to the second ballot. In round 2, a majority of at least $66 \%$ was required to accept the rephrased recommendation. If this was not achieved, the recommendation was discussed and amended again and subjected to the third ballot. In round 3, a majority of at least $50 \%$ was required to accept the rephrased recommendation. If this was not achieved, the recommendation was rejected.

After the meeting, the level of evidence (LOE) and strengths of recommendations (SOR) according to the Oxford Centre for Evidence-Based Medicine system were determined.29 Then, the agreed overarching principles and recommendations were distributed among all Task Force members via email to assess their level of agreement (LoA) for each recommendation. LoA was anonymously scored on a scale from 0 to 10 (0: completely disagree; 10: completely agree). LOA is shown as mean (standard deviation (SD)) and as the proportion of Task Force members with a LOA of at least 8. Additionally, a research agenda was created.

All Task Force members reviewed the draft of the manuscript. After incorporating all comments, the manuscript was submitted to the EULAR Standing Committee on Clinical Affairs and the EULAR Executive Committee for review and approval. After incorporating their comments, the final version of the manuscript was submitted to the journal. 


\section{Results}

\section{General aspects}

The discussion of the Task Force resulted in two overarching principles and ten recommendations (Table 2). The LoE ranged from 3 to 5 and the SoR ranged from $\mathrm{C}$ to $D$, predominantly because high-quality evidence derived in the population of interest was scarce. The LOA was generally high and ranged from 8.4 to 9.6. The order of recommendations was presented in what was considered as logical sequence, in particular the first two recommendations, which serve as a basis for all subsequent items. Below, a point-by-point discussion is presented, explaining the reasoning behind the different topics and the supporting evidence.

\section{Overarching principles}

The Task Force formulated overarching principles, based on expert opinion, to provide a general conceptual framework.

A.These recommendations pertain to patients who fulfil the definition of D2T RA and are underpinned by the EULAR recommendations for the management of RA including the overarching principles (LOA 9.6 (1.0)).5,24

This principle emphasises the relationship between these recommendations and the EULAR definition of D2T RA, as these recommendations apply to RA patients fulling this definition. ${ }^{24}$ In addition, all overarching principles and recommendations of the EULAR recommendations for the management of RA also apply to D2T RA patients. ${ }^{5}$ Patients who fail at least two b/tsDMARDs with different MOA, and can therefore potentially be classified as having D2T RA, fall in phase III of the management algorithm of the 2019 EULAR RA management recommendations. From this point forwards, the D2T RA recommendations can be seen as a supplement in which further guidance is provided on factors contributing to the D2T RA state and for possible pharmacological and nonpharmacological therapeutic strategies. The Task Force unanimously agreed with this overarching principle ( $100 \%$ agreed, ${ }^{\text {st }}$ round, $n=27$ ).

B. Presence or absence of inflammation should be established to guide pharmacological and non-pharmacological interventions (LOA 9.5 (1.3)).

The Task Force emphasised that confirming the presence of inflammatory RA disease activity is essential and should be done prior to adjustment of DMARD therapy. If the persistence of signs and/or symptoms is not caused by inflammatory disease activity, DMARD therapy will in all probability be ineffective and may eventually (seemingly) lead to the apparent failure of multiple (b/ts)DMARDs. A myriad of factors may contribute to the D2T RA state and result in the persistence of signs and/or symptoms suggestive of 
Table 2. EULAR recommendations for the management of difficult-to-treat RA

LoE SoR

LOA

Mean $\geq 8 / 10$

(SD) (\%)

\section{Overarching principles}

A. These recommendations pertain to patients who fulfil the definition of D2T RA NA NA $\quad 9.6(1.0) \quad 97$ and are underpinned by the EULAR recommendations for the management of RA including the overarching principles.,24

B. Presence or absence of inflammation should be established to guide NA NA 9.5 (1.3) 91 pharmacological and non-pharmacological interventions.

\section{Recommendations}

1. If a patient has a presumed D2T RA, the possibility of misdiagnosis and/or the $5 \quad$ D $\quad 9.3(1.2) \quad 91$ presence of a coexistent mimicking disease should be considered as a first step.

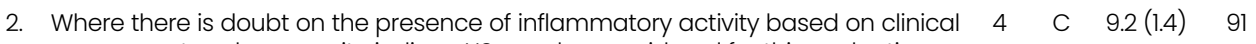
assessment and composite indices, US may be considered for this evaluation.

3. Composite indices and clinical evaluation should be interpreted with caution in $* 5 \quad * D \quad 9.2(1.3) \quad 88$ the presence of comorbidities, in particular obesity and fibromyalgia, as these \#4 \# C may directly heighten inflammatory activity and/or overestimate disease activity.

4. Treatment adherence should be discussed and optimised within the process of $\quad 5 \quad$ D $\quad 9.5(1.0) \quad 97$ shared decision making.

5. After failure of a second or subsequent b/tsDMARD, ${ }^{*}$ and particularly after two $* 4 * C \quad 9.2(1.3) \quad 94$ TNFi failures, ${ }^{*}$ treatment with a b/tsDMARD with a different target should be ${ }^{*} 3{ }^{*} \mathrm{C}$ considered.

6. If a third or subsequent b/tsDMARD is being considered, the maximum dose, as $3 \quad$ C $\quad 8.4(1.8) \quad 75$ found effective and safe in appropriate testing, should be used.

7. In patients with concomitant HBV/HCV, b/tsDMARDs can be used, and *4 ${ }^{*} \mathrm{C} \quad 8.9(1.4) \quad 88$ concomitant antiviral prophylaxis should be considered in close collaboration \# 5 \# D with the hepatologist."

8. In addition to pharmacological treatment, non-pharmacological interventions $* 3 \quad *$ C $\quad 9.4(1.2) \quad 97$ (i.e. exercise, ${ }^{*}$ psychological, ${ }^{*}$ educational* and self-management interventions*) * 4 \# D should be considered to optimise management of functional disability, pain, and fatigue.

9. Appropriate education and support should be offered to patients to directly $4 \begin{array}{lllll}4 & \text { C } & 9.4(1.2) & 97\end{array}$ inform their choices of treatment goals and management.

10. Consider offering self-management programs, relevant education, and $3 \quad$ C $\quad 9.1$ (1.7) 91 psychological interventions to optimise patient's ability to manage their disease confidently (i.e. self-efficacy).

b: biological; D2T: difficult-to-treat; DMARD: disease-modifying antirheumatic drug; EULAR: European Alliance of Associations for Rheumatology; HBV: hepatitis B virus; HCV: hepatitis C virus; LoA: levels of agreement; LoE: levels of evidence (according to the standards of the Oxford Centre for Evidence Based Medicine);29 RA: rheumatoid arthritis; SoR: strengths of recommendation (according to the standards of the Oxford Centre for Evidence Based Medicine); ${ }^{29}$ TNFi: tumour necrosis factor inhibitor; ts: targeted synthetic; US: ultrasonography.

In case the LOE and SoR differed within a recommendation, differences in LoE and SoR are shown using * and \#. Relevant mimicking diseases, for instance, crystal arthropathies, polymyalgia rheumatica, psoriatic arthritis, spondyloarthritis, Still's disease, systemic lupus erythematosus (SLE), Rhupus syndrome, vasculitis, idiopathic inflammatory myopathies, remitting symmetric seronegative synovitis and pitting oedema (RS3PE), reactive arthritis (e.g. parvo B19, Rubella, Whipple's disease, HBV and HCV infections), paraneoplastic syndromes, osteoarthritis, and fibromyalgia.

Relevant comorbidities, for instance, infections, malignancies, polymyalgia rheumatica, osteoarthritis, subluxations, joint dislocations. 
active disease.11,2,30 Some of these contributing factors may mimic inflammation related to RA, while the associated complaints are actually of non-inflammatory origin, such as concomitant fibromyalgia, osteoarthritis and/or psychological conditions. ${ }^{11,30}$ Factors that may contribute to the persistence of inflammatory activity include treatment nonadherence and comorbidities (e.g. infections or malignancies).1130 Therefore, even when the presence of inflammatory activity has been ascertained, the presence and role of these factors should be considered. All of these contributing factors may require different pharmacological and non-pharmacological management strategies. Thus, their identification is essential to guide appropriate therapeutic interventions and, therefore, D2T RA clearly warrants a holistic management approach. This overarching principle was accepted in the second round of the voting process ( $78 \%$ agreed, $2^{\text {nd }}$ round, $n=24$ ).

\section{Recommendations}

1. If a patient has a presumed D2T RA, the possibility of misdiagnosis and/or the presence of a coexistent mimicking disease should be considered as a first step (LOE 5, SOR D, LOA $9.3(1.3))$.

The Task Force unanimously agreed that an accurate RA diagnosis is the cornerstone of appropriate management. Therefore, reconsidering the diagnosis should be performed as a first step. In the SLR, very few studies could be identified on this clinically relevant item. ${ }^{27,31-35}$ Consequently, this recommendation is based on expert opinion, reinforced by indirect evidence.

During the discussions at the Task Force meeting, it was mentioned that misdiagnosis (i.e. an alternative disease mimic) may be more common in seronegative disease. ${ }^{36,37}$ However, misdiagnosis should be considered in all D2T RA patients. A variety of diseases may mimic ongoing RA disease activity, such as: crystal arthropathies, polymyalgia rheumatica, psoriatic arthritis, spondyloarthritis, Still's disease, systemic lupus erythematosus (SLE), Rhupus (RA-lupus) syndrome, idiopathic inflammatory myopathies, vasculitis, remitting symmetric seronegative synovitis and pitting oedema (RS3PE), reactive arthritis (e.g. parvo B19, Rubella, Whipple's disease, hepatitis B and C virus (HBV/ $\mathrm{HCV}$ ) infections), paraneoplastic syndromes, osteoarthritis and fibromyalgia. ${ }^{1,38}$ Furthermore, the Task Force agreed that in addition to misdiagnosis, the co-existence of another condition needs to be considered. These coexisting diseases may also underlie current signs and/or symptoms suggestive of active RA. The Task Force unanimously

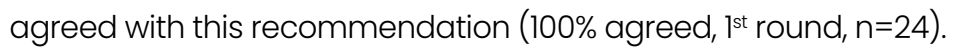

The question on misdiagnosis can also be viewed in the context of current management approaches. According to the widely accepted concept of the 'window of opportunity', efficacious management in the early phase of RA can lead to sustained benefit. ${ }^{39}$ Accordingly, early diagnosis and immediate initiation of DMARD therapy as soon as the diagnosis of RA is established form the base of the current EULAR recommendations 
and ACR guideline. ${ }^{5,6}$ However, early diagnosis might be associated with more equivocal symptoms and a higher risk of misdiagnosis. ${ }^{40}$ Misdiagnosis and, consequently, overtreatment limit treatment efficacy and may increase the risk of side effects, resulting in unnecessary health care costs and eventually the development of seemingly D2T RA.

2. Where there is doubt on the presence of inflammatory activity based on clinical assessment and composite indices, ultrasonography may be considered for this evaluation (LOE 4, SOR C, LOA 9.2 (1.4)).

This recommendation is linked closely to overarching principle $B$ that states that the presence or absence of inflammatory RA activity should drive treatment decisions. To be able to evaluate the presence or absence of inflammatory disease activity, a reliable test should be used. In daily practice, composite indices (at patient level) and the clinical evaluation of a joint being swollen (at joint level) are most frequently used to assess the presence of inflammatory disease activity and their use is also endorsed in current RA management recommendations. ${ }^{5}$ However, in D2T RA patients in whom there is doubt about the presence of inflammation, due to coexisting conditions mimicking inflammatory RA activity (see also recommendation \#1), these traditional measures may be difficult to interpret.

Despite an extensive literature search, limited (high-quality) evidence was found on diagnostics that can be used to assess the presence or absence of inflammatory disease activityin this patient group. ${ }^{27}$ When traditional measures arechallenging, ultrasonography (US) appears to be the most feasible measure to detect inflammatory activity both in D2T RA patients in general and in those with conditions that can compound assessment, such as obesity or concomitant fibromyalgia. In the general population of RA patients (where composite indices can be considered to be reliable), moderate to strong correlations were reported between US sum scores and composite indices on a group level. ${ }^{41-48}$ In a study in established RA patients in whom there was explicit doubt about the presence of inflammation, only weak and non-statistically significant correlations between US sum scores and composite indices were found. ${ }^{49}$ This suggests that US may be better related to ' true' inflammatory activity in these patients and may have an additional value in D2T RA patients in whom doubt about the presence of inflammatory activity exists. However, the minimal number of joints that should be included in an US assessment is unclear, ${ }^{44}$ which hampers the use of a sum score to determine the overall level of disease activity in daily practice. Of note, no studies were found on tests in patients with comorbidities that may influence the assessment of disease activity.

The evidence for biomarkers (e.g. miR-146, fibrinogen, resistin, matrix metallopeptidase 3 (MMP-3), interleukin 6 (IL-6), and multi-biomarker disease activity (MBDA) score) and other imaging measures (e.g. optical spectral transmission measures) is currently not convincing. ${ }^{27,43,50-64}$ The quality of this evidence was only low to moderate and no evidence 
could be identified on their role in patients in whom there was explicit doubt about the presence of inflammatory activity resulting in indirectness. These limitations hamper the current use of these biomarkers and imaging modalities in daily practice.

Considering the identified evidence, the Task Force agreed that US currently seems the best measure to assess inflammatory activity in the joints in D2T RA patients in case of doubt based on the assessment using traditional composite indices and/or clinical evaluation. The Task Force unanimously agreed with this recommendation (100\% agreed, $1^{\text {st }}$ round, $\left.n=24\right)$.

3. Composite indices and clinical evaluation should be interpreted with caution in the presence of comorbidities, * in particular obesity and fibromyalgia, " as these may directly heighten inflammatory activity and/or overestimate disease activity ( ${ }^{*} \operatorname{LOE} 5$, SOR D, * LOE 4, SOR C, LOA 9.2 (1.3)).

As mentioned previously, the appropriate evaluation of the presence or absence of inflammatory disease activity is essential for the optimal management of RA as this should drive appropriate treatment strategies (overarching principle B). In daily practice, composite indices are most frequently used to evaluate the presence of inflammatory disease activity. The use of composite indices is also endorsed in EULAR RA management recommendations. ${ }^{5}$ However, symptoms of coexisting conditions may mimic inflammatory RA activity and could therefore hamper accurate assessment of inflammatory disease activity (see also recommendations \#1 and 2).

Although the Task Force was unanimous in its opinion that numerous comorbidities might influence the assessment of inflammatory disease activity, substantial evidence was only found for obesity and fibromyalgia. ${ }^{27,65-68}$ These two conditions may also frequently coexist, further complicating the accurate assessment of inflammatory disease activity. In addition to obesity and fibromyalgia, it was discussed that other comorbidities (especially those increasing acute phase reactants: e.g. infections, malignancies or polymyalgia rheumatica) may also lead to the misclassification of inflammatory RA activity, although no substantial evidence was identified to support this. In addition, no evidence was identified regarding the impact of osteoarthritis, subluxation or joint dislocations on clinical evaluation of joints. ${ }^{27}$ Despite this limited evidence, the Task Force agreed that this recommendation should refer to all potential comorbidities that may influence the evaluation of inflammatory disease activity. The Task Force unanimously agreed with this recommendation (100\% agreed, lst round, $n=24)$.

4.Treatment adherence should be discussed and optimised within the process of shared decision making (LOE 5, SOR D, LOA 9.5 (1.0)).

In RA, drug non-adherence rates reportedly vary between $30 \%$ and $80 \%{ }^{26,69-71}$ and, recently, these rates have been shown to be substantially higher in D2T compared with non-D2T 
RA patients. ${ }^{30}$ Adherence to treatment is essential in disease management: an optimal effect of treatment can only be achieved if a patient adheres to treatment instructions. Suboptimal adherence can be detrimental, as it is associated with higher disease activity levels, which may result in inappropriate treatment switches and reduced quality of life. ${ }^{72-76}$ Specifically a D2T RA patient who does not adhere to treatment instructions and in whom inflammatory disease activity persists could exhaust all currently available (b/ts)DMARDs. Therefore, the Task Force unanimously agreed that adherence should be addressed as a standalone recommendation. As another EULAR project has recently provided detailed recommendations for the detection, assessment and management of non-adherence in people with rheumatic and musculoskeletal diseases (RMDs), it was chosen to refer to their SLR and recommendations instead of performing a separate $\mathrm{SLR}^{22,26}$

According to the World Health organization (WHO) definition, adherence to long-term therapy is 'the extent to which a person's behaviour - taking medication, following a diet, and/or executing lifestyle changes - corresponds with agreed recommendations from a health care provider'.77 In accordance with this definition, the Task Force agreed to use 'treatment adherence' instead of 'drug adherence', as the recommendation also applies to non-pharmacological strategies.

The Task Force agreed that a discussion about suboptimal or non-adherence is essential to optimise adherence: the current level of adherence should be evaluated as well as the patient's perspective on adherence. There is no gold standard for identifying nonadherence: a discussion with the patient is usually informative and questionnaires (easy to apply, although this self-reported measure tends to overreport adherence) or serum and/or urine drug level measurements (which provide direct information but might be expensive and difficult to interpret due to differences in individual drug metabolism) can also be used. ${ }^{26,78,79}$ If suboptimal adherence is present, this might be explained by various factors: both unintentional (e.g. forgetting to take the prescribed drugs) and intentional non-adherence (driven by a decision not to take the prescribed drugs, e.g. due to fear of side effects) are common in RA. ${ }^{69,79,80}$ The patient's evaluation of the risk-benefit ratio of the selected drug(s) is also of paramount importance. Treatment adherence should therefore be discussed to be able to optimise adherence. In addition to physicians, other HCPs, such as rheumatology nurses, psychologists and pharmacists, may also be involved in these discussions.

Furthermore, shared decision making is clearly vital to optimise adherence. ${ }^{26,79}$ In this context, the quality of the relationship between the patient and the HCP is important. ${ }^{81,82}$ As non-adherence is a precarious topic, the patient should be made to feel safe and supported to discuss all aspects. In addition to these discussion between patients and HCPs, the Task Force deemed that providing appropriate education, especially in case of intentional non-adherence, might also be useful and could strengthen the 
process of shared decision making (see also recommendations \#9 and 10). ${ }^{26,79}$ This recommendation was accepted in the first round of the voting process ( $96 \%$ agreed, $1^{\text {st }}$ round, $\mathrm{n}=28$ ).

5. After failure of a second or subsequent b/tSDMARD,* and particularly after two TNFi failures," treatment with a b/tsDMARD with a different target should be considered (* LOE 4, SOR C, " LOE 3, SOR C, LOA 9.2 (1.3)).

Increasing numbers of $b /$ tsDMARDs (with different MOA), from five molecular drug target families, are now available for the treatment of RA: tumour necrosis factor (TNF), IL-6, CD80/86, CD20 and janus kinase (JAK). ${ }^{83}$ As treatment options, switching within the same class as well as switching to a drug with a different target can be effective and are both endorsed in RA management recommendations. ${ }^{5,27,83}$ However, a considerable proportion of RA patients fail at least two b/tsDMARDs with different MOA, which may result in having D2T RA. ${ }^{10,11,84}$ In routine daily practice, DMARD therapy is frequently changed - generally based on a trial-and-error approach - when signs and/or symptoms suggestive of active disease are present," while anti-inflammatory therapy should only be intensified if inflammatory RA activity has been ascertained and is not explained by other potentially contributing factors, e.g. treatment non-adherence or comorbidities (overarching principle B). The Task Force therefore agreed that more guidance in pharmacological therapeutic strategies is needed.

In the SLR, only limited evidence was identified on pharmacological therapeutic strategies in RA patients in whom at least two b/tsDMARDs (specifically with different MOA) failed. ${ }^{28}$ In the identified clinical trials in RA patients in whom multiple b/tsDMARDs failed, it was generally not possible to disentangle clearly the reasons for previous DMARD failure, e.g. toxicity, lack of efficacy or other factors potentially contributing to D2T RA. This resulted in the inclusion of heterogeneous patient populations, complicating the interpretation of outcomes of these studies.

After failure of at least two b/tsDMARDs, some evidence was identified regarding the beneficial effect of treatment with a b/tsDMARD with a different target. ${ }^{28}$ This evidence indicated that a third or fourth b/tsDMARD (i.e. tocilizumab, tofacitinib, baricitinib, upadacitinib, filgotinib) is more effective than placebo. ${ }^{85-90}$ However, no preference can be given to any of these DMARDs. In patients in whom at least one bDMARD failed, TNFi, abatacept and rituximab were more effective than placebo, ${ }^{83,91-95}$ although direct evidence was lacking about the efficacy as third and fourth bDMARD compared to placebo. ${ }^{28}$ In patients in whom previously a higher number of bDMARDs failed, the extent of the beneficial effect of several b/tsDMARDs (i.e. TNFi and the lower doses of tocilizumab,

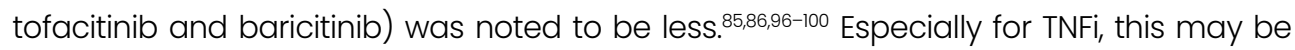
related to the timing of their application in treatment strategies and may not be related to their MOA, although evidence to support this is lacking. ${ }^{28}$ Furthermore, a tendency was 
identified for non-TNFis to be more efficacious than TNFis in patients in whom at least

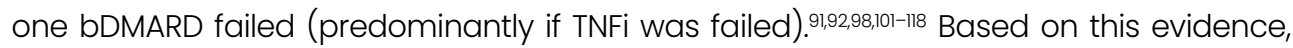
our current recommendation proposes to switch to a b/tsDMARD of a different target, after failure of a second or subsequent b/tsDMARD and, particularly, after failure of two TNFis. This recommendation was accepted in the first round of the voting process ( $96 \%$ agreed, $1^{\text {st }}$ round, $\mathrm{n}=24$ ).

The Task Force emphasised that the current recommendation is in line with the 2019 EULAR RA recommendation on b/tsDMARD switches (\#10: 'If a bDMARD or tsDMARD has failed, treatment with another bDMARD or a tsDMARD should be considered; if one TNFi therapy has failed, patients may receive an agent with another MOA or a second TNFi.'). Our recommendation adds the following: first, another b/tsDMARD should be used after failure of the second or subsequent $b /$ tsDMARD; second, $a$ b/tsDMARD with a different target is preferred after failure of a second or subsequent b/tsDMARD. ${ }^{5}$

The Task Force also discussed the efficacy of DMARD combination therapy. In this regard, we refer to the 2019 RA EULAR recommendations, as no additional evidence was identified for D2T RA (\#9: 'bDMARDs and tsDMARDs should be combined with a csDMARD; in patients who cannot use csDMARDs as comedication, IL-6 pathway inhibitors and tsDMARDs may have some advantages compared with other bDMARDs'). ${ }^{5}$

6. If a third or subsequent b/tsDMARD is being considered, the maximum dose, as found effective and safe in appropriate testing, should be used (LOE 3, SOR C, LOA 8.4 (1.8)).

In the SLR, the extent of the beneficial effect of b/tsDMARDs was generally found to be less in patients in whom a higher number of previous bDMARDs failed. ${ }^{28}$ This tendency was less convincing for upadacitinib and filgotinib, and for the higher doses of tocilizumab (intravenously administered), tofacitinib and baricitinib. ${ }^{85,86,88,90,99,100}$ This suggests that the higher doses of intravenous tocilizumab, and tofacitinib and baricitinib may be preferred in patients in whom previously a higher number of bDMARDs failed. ${ }^{85,86,99,100}$ The Task Force agreed that the evidence supports the use of these higher doses from the beginning rather than dose escalation. Of note, this higher dose should only be used where appropriate, i.e. not in patients in whom contraindications for this higher dose are present.

In addition, it was argued that this recommendation might be more informative by including the names of the specific b/tsDMARD (baricitinib and tocilizumab, and not tofacitinib, as tofacitinib is not licensed at higher doses than $5 \mathrm{mg}$ twice daily). The following wording was accepted ( $95 \%$ agreed, $1^{\text {st }}$ round, $n=22$ ): 'If a second or subsequent $\mathrm{b} /$ tsDMARD has failed, and baricitinib or iv tocilizumab are being considered, the higher licensed dose should be used if appropriate'. However, it was also discussed that explicitly mentioning drug names (i.e. baricitinib and tocilizumab) should be avoided in management recommendations as novel evidence may emerge for other drugs. 
Therefore, the Steering Committee initiated a new voting after the Task Force meeting regarding this recommendation without explicit drug names. The Task Force members agreed to change the wording of the recommendation and to exclude the drug names resulting in the current recommendation ( $94 \%$ agreed, $2^{\text {nd }}$ round, $n=32$ ).

7. In patients with concomitant HBV/HCV, b/tsDMARDs can be used, * and concomitant antiviral prophylaxis should be considered in close collaboration with the hepatologist\# (* LOE 4, SOR C, " LOA 5, SOR D, LOA 8.9 (1.4)).

In clinical practice, adverse events, comorbidities, other coexisting conditions and other contraindications may significantly limit treatment options, potentially contributing to the D2T state. ${ }^{1,13}$ An extensive literature search was performed to find appropriate evidence on safe and efficacious therapies in patients with such contraindications. ${ }^{28}$ Limited evidence was identified: no studies were identified for RA patients with human immunodeficiency virus (HIV), gastrointestinal disease, latent tuberculosis, and malignancies; and only limited evidence was identified for RA patients with extra-articular manifestations, hepatic disease, osteoporosis, psychological disease, pulmonary disease, and renal disease. More than one study per intervention was identified only for RA patients with hepatitis B virus (HBV), hepatitis C virus (HCV), cardiovascular disease (CVD), obesity, and before and during pregnancy and lactation.

Substantial evidence was only identified related to HBV and HCV infection. ${ }^{28}$ According to the identified evidence, TNFi, abatacept and tocilizumab may be considered in patients with $\mathrm{HBV}^{119-121}$ and TNFi in patients with $\mathrm{HCV}$. ${ }^{122,123}$ No evidence was identified on other $\mathrm{b} /$ tsDMARDs, although this does not indicate that these b/tsDMARDs are not safe to use. Therefore, the Task Force voted not to include specific bDMARDs in the recommendation (83\% agreed, $n=24$ ). Furthermore, the Task Force agreed that concomitant antiviral prophylaxis should be considered, ${ }^{119}$ and that the treatment should be conducted in close collaboration with the hepatologist. The Task Force unanimously agreed with this recommendation (100\% agreed, $\mathrm{l}^{\text {st }}$ round, $\mathrm{n}=24$ ).

8. In addition to pharmacological treatment, non-pharmacological interventions (i.e. exercise, ${ }^{*}$ psychological, " educational* and self-management interventions*) should be considered to optimise management of functional disability, pain, and fatigue (* LOE 3, SOR C, " LOE 4, SOR C, LOA 9.4 (1.2)).

Pharmacological DMARD therapy remains the mainstay of RA treatment when inflammatory RA disease activity is present, ${ }^{5}$ as DMARDs suppress inflammation and may prevent (or at least will inhibit progression of) irreversible joint damage. ${ }^{83}$ However, a wide spectrum of factors, in addition to those resulting in inflammatory RA activity, may contribute to the persistence of signs and/or symptoms, although these are not directly related to inflammation (i.e. non-inflammatory symptoms, including functional disability, 
pain and fatigue) 11,30 Therefore, the Task Force agreed that individually tailored nonpharmacological interventions are also important components of the management of D2T RA. $11,28,30$

The SLR focussed on non-DMARD interventions to improve non-inflammatory complaints in RA patients who do not clearly have active disease (e.g. defined as DAS28-ESR >3.2), to reduce the impact of inflammation on the results. ${ }^{28}$ At the Task Force meeting, it was noted that it is not always possible to disentangle inflammatory and non-inflammatory symptoms in clinical practice. The Task Force agreed that non-pharmacological interventions should be considered in all D2T RA patients, as the burden in all D2T RA patients is substantial (i.e. worse levels of functional disability, quality of life, pain and fatigue), ${ }^{30}$ and not only in those patients without inflammatory RA activity.

In the SLR, evidence was found regarding the beneficial effect of exercise, education, psychological and self-management interventions to improve pain, fatigue and functional disability, while substantial evidence regarding the role of nonpharmacological interventions to improve quality of life was lacking. ${ }^{28}$ Benefit of exercise in RA is well-established ${ }^{124}$ and was specifically found to improve physical functioning. A wide range of physical activities might be advised in accordance with the patients' status, e.g. aerobic exercises, water-based dynamic exercises, muscle strengthening or hand exercises. ${ }^{125-137}$ Additionally, psychological interventions could be applied, specifically to reduce pain and fatigue, e.g. cognitive behavioural therapy and interventions focussing on stress management. . $35,138-142$ Furthermore, patient education can assist patients in learning about their disease and management options (see also recommendations \#4, 9 and 10) $)^{14}$ and was specifically found to improve physical functioning. ${ }^{132}$ Education can be provided one-on-one, but also in group sessions promoting patients to learn from each other. Lastly, self-management programs can be applied. These programs are typically a combination of different non-pharmacological interventions (e.g. exercise and education) and were found to optimise the management of pain, fatigue and functional disability (see also recommendations \#9 and 10). ${ }^{29,143-152}$

since no single treatment modality offers overwhelming improvement of symptoms, ideally, a package of care (i.e. multimodal treatment) should be considered in accordance with the patient's needs and preferences. This individually tailored multimodal treatment can be provided by different members of the rheumatology team (e.g. rheumatologists, rehabilitation physicians, rheumatology nurses, physiotherapists, occupational therapists, psychologists, pharmacists, podiatrists). The Task Force unanimously agreed with this recommendation (100\% agreed, $1^{\text {st }}$ round, $n=29$ ).

9. Appropriate education and support should be offered to patients to directly inform their choices of treatment goals and management (LOE 4, SOR C, LOA 9.4 (1.2)).

Setting treatment goals has a central role in the management of RA. In the current EULAR 
RA management recommendations, therapeutic strategies can be adjusted if there is no improvement at 3 months or if the treatment target is not achieved at 6 months (recommendation \#3). ${ }^{5}$ Preferably, this treatment target is reaching clinical remission or at least low disease activity. However, these treatment targets may be unrealistic to achieve for D2T RA patients, considering their disease history, accrued joint damage and other factors that may contribute to the D2T RA state." Applying these classic treatment targets may therefore result in unnecessary DMARD switches and associated complaints. Therefore, the Task Force agreed that, in D2T RA in particular, treatment goals should be tailored to the individual patient.

A discussion between the patient and the HCP on the feasibility of these patienttailored treatment goals seems highly relevant to avoid unrealistic goals as well as a mismatch in goal setting between patients and HCPs, which could negatively impact disease outcomes." In the SLR, no diagnostic test to identify a mismatch in treatment goals (between HCP and RA patient) was found. ${ }^{28}$ The Task Force agreed that treatment goals should be discussed to be able to identify a mismatch in treatment goals and to optimise goal setting in shared decision making.

Additionally, the Task Force agreed that HCPs should educate patients by sharing relevant information to inform their choices. The risks and benefits of different management options should be discussed as well as the feasibility of certain treatment goals. In the SLR, web-based education tools were found to improve patients' knowledge and certainty in treatment decisions. ${ }^{28,153-156}$ These educational tools could be used in addition to providing information in discussions. As perceptions on treatment goals and management may change over time, education should be offered continuously as an integral part of the long-term partnership between patients and HCPs.

Furthermore, the HCP should emphasise that patients can participate in the process of decision making. A dialogue (using bidirectional instead of unidirectional communication) between the patient and the HCP is sometimes challenging, but certainly indispensable to promote shared decision making. ${ }^{81,82}$ If patients will be sufficiently educated, they will eventually be able to make informed choices about treatment goals and management, and become an equal partner in the process of shared decision making. This recommendation was accepted in the first round of the voting process ( $89 \%$ agreed, $1^{\text {st }}$ round, $\mathrm{n}=28$ ).

10. Consider offering self-management programs, relevant education, and psychological interventions to optimise patient's ability to manage their disease confidently (i.e. selfefficacy; LOE 3, SOR C, LOA 9.1 (1.7)).

Self-efficacy refers to patients' ability to control or manage various aspects of their disease and has a major role in the well-being of patients. ${ }^{157}$ Self-efficacy beliefs determine how individuals think, feel and act, and are an important aspect of self-management. People 
with low self-efficacy quickly give up their goals when faced with difficulties and are at higher risk of worse levels of pain, fatigue, depression, anxiety and stress. ${ }^{157-159}$ All this may contribute to the D2T RA state.1,30 In contrast, a strong sense of self-efficacy improves human performance and well-being in several ways, promotes the accomplishment of challenging goals and supports commitment to them. ${ }^{157}$ Improved self-efficacy may not only improve disease outcomes, but may also improve many aspects of health behaviour, including mental well-being, treatment adherence and willingness to change lifestyle factors. Therefore, the Task Force agreed that strengthening self-efficacy is specifically important in D2T RA.

The first step in improving self-efficacy might be to identify the level of self-efficacy. In the SLR, the Arthritis Self-Efficacy Scale (ASES), a tool to measure perceived self-efficacy to cope with the disease ${ }^{160}$ was found as a reliable measure. ${ }^{28,161}$ However, Task Force members noted that the ASES is too general to evaluate self-efficacy. ${ }^{161}$ Therefore, the Task Force decided not to formulate a standalone recommendation regarding its application ( $89 \%$ agreed, $n=27$ ), although there was consensus that the ASES could be used in daily practice as a screening instrument and to assess the change in self-efficacy over time. Eventually, the Task Force felt that it is difficult to clearly define which level of self-efficacy is suboptimal and agreed that offering interventions to improve self-efficacy could be beneficial for all D2T RA patients.

In the SLR, self-management programs, educational and psychological interventions were found to have a beneficial effect to improve self-efficacy. ${ }^{28}$ Additionally, some evidence was identified suggesting that patients would like more education on disease processes. ${ }^{28,162,163}$ Educational interventions, e.g. individual education, a group education program or education through a mobile app, were specifically beneficial to improve

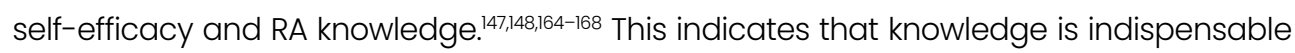
to improve self-efficacy. Psychological interventions, e.g. cognitive behavioural therapy or relaxation therapy, were not only beneficial to improve self-efficacy, but may also reduce symptoms related to anxiety and depression. 14,144,69 Self-management programs (i.e. typically a combination of different non-pharmacological interventions) were also found to be effective in improving self-efficacy. ${ }^{129,36,144-146,148-15,170-174}$ Additionally, mobile health applications can be considered to improve self-management. ${ }^{175}$

The Task Force thoroughly debated if these interventions should be offered to every patient (mandatory) or should be considered only (optional). After substantial discussion, the Task Force agreed that offering self-management programs should be optional (agreed $82 \%, \mathrm{n}=28$ ). If a patient agrees to try and improve their self-efficacy, the type of intervention should be determined based on the patient's status and preferences, in line with the concept of shared decision making. This recommendation was accepted in the first round of the voting process ( $96 \%$ agreed, $1^{\text {st }}$ round, $n=28$ ). 


\section{Additional discussions}

Some other comorbidities and coexisting conditions, not explicit part of the recommendations due to the lack of (sufficient) evidence, were also discussed at the Task Force meeting.

In the SLR on safe and efficacious therapies in RA patients with contraindications, ${ }^{28}$ evidence was also identified on venous thromboembolisms (VTEs). In RA patients using tsDMARDs, specifically in high doses, in whom risk factors for VTE are present, higher frequencies of VTEs were reported..$^{176}$ The Task Force unanimously agreed that in patients at risk for VTEs, tsDMARDs - specifically at high doses - should be used with caution. As this item is already covered in the 2019 EULAR RA management recommendations ${ }^{5}$ and as this increased risk of VTEs is not specific for D2T RA patients, the Task Force unanimously decided not to include this item as a standalone recommendation (no formal voting). Nevertheless, the increased risk of VTEs should be considered as a factor limiting treatment options, specifically for D2T RA patients with VTE risk factors.

Furthermore, therapeutic strategies during pregnancy and lactation were discussed. Recommendations about safe DMARDs before and during pregnancy and lactation are already published as 2016 EULAR points to consider and as 2020 ACR guideline.177,178 Few additional studies were identified, of which the results resembled those of these recommendations. ${ }^{28,79-181}$ Therefore, the Task Force agreed not to formulate a separate recommendation on this item and to refer to the already existing recommendations (no formal voting)! ${ }^{177,178}$ Most relevant for D2T RA patients, TNFis (especially certolizumab pegol) and rituximab are best studied among $\mathrm{b} /$ tsDMARDs and appear without identified safety issues. ${ }^{177,178}$

The Task Force discussed and agreed that there are additional clinically meaningful contraindications of some therapies resulting in limited treatment options, e.g. tocilizumab in case of diverticulitis or JAK-inhibitors in case of repeated herpes zoster infections. ${ }^{176}$ However, no substantial clinical evidence was identified about safe and/ or efficacious therapies for patients with these conditions ${ }^{28}$ and, therefore, no specific recommendations were formulated on these items.

Obesity, as a coexisting condition, was the subject of intense debate during the meeting. Although obesity does not result in limited drug options, treatment efficacy might be different in obese patients.1182 The identified evidence suggested that intravenously administered infliximab may be less effective in patients with a body mass index (BMI) above 30 compared with in those with a BMl below 30.183,184 Therefore, it was suggested to include a recommendation on this item. The Task Force voted whether this should be a standalone recommendation or not. The first vote did not clearly indicate the preference of the Task Force (formulate a separate recommendation on this item $58 \%, n=24$ ). Further discussion followed, in which it was added that notable evidence was identified, where the evidence for several other comorbidities was lacking or very limited. On the 
other hand, the identified evidence did still only cover two studies with a high RoB ${ }^{183,184}$ The repeated vote clearly indicated not to formulate a separate recommendation on this item (formulate a separate recommendation on this item $12 \%, n=24$ ).

\section{Research agenda}

The Task Force created a research agenda containing research questions that are considered most relevant to address (Table 3).

\section{Discussion}

Outcomes of RA patients have improved significantly over the past decades. The introduction of new DMARDs, the updated EULAR/ACR classification criteria, ${ }^{38}$ the treat-totarget approach, ${ }^{9}$ the definitions of remission ${ }^{185}$ and RA management recommendations ${ }^{5}$ have promoted early diagnosis and treatmentaccording to strategic algorithms with clear therapeutic targets of remission or at least low disease activity. The recommendations for early RA ${ }^{186}$ focussed on the initial treatment and provided further management support together with additional recommendations and points to consider, e.g. on cardiovascular disease risk management, 15 detection of comorbidities, ${ }^{16}$ use of imaging ${ }^{17}$ management of pain ${ }^{19}$ and patient education. ${ }^{14}$ The 'window of opportunity' hypothesis supports early

Table 3. Research agenda

1. How can we optimally confirm a diagnosis of RA in D2T RA patients?

2. Which reference standard should be used to assess the presence or absence of inflammation in D2T RA patients, in whom there is doubt after assessment by traditional measures?

3. What is the role of synovial biopsies in the assessment of the presence or absence of inflamma-tion in D2T RA?

4. Is any of the b/tsDMARDs superior to treat inflammatory disease activity in D2T RA?

5. Could treatment history be used to stratify b/tsDMARD treatment in D2T RA?

6. Could synovial tissue analyses be used to stratify b/tsDMARD treatment in D2T RA?

7. Which DMARD is preferred in D2T RA patients with specific adverse events, comorbidities (in-cluding extraarticular manifestations), other coexisting conditions and other contraindications that limit DMARD options?

8. Could the development of the D2T RA state be prevented by adequate management of the potentially contributing factors in an earlier phase of RA?

9. Could the D2T RA state ameliorate if potentially contributing factors are adequately addressed?

10. Does 'true' refractory RA (patients in whom (b/ts)DMARDs are truly ineffective) really exist?

11. Which immunological mechanisms and/or pathways underlie inefficacy to multiple b/tsDMARDs in D2T RA?

12. How does smoking impact D2T RA?

13. How does obesity impact D2T RA? And which treatment is preferred in D2T RA patients with obesity?

b/tsDMARDs: biological or targeted synthetic disease-modifying antirheumatic drugs; D2T: difficult-to-treat; RA: rheumatoid arthritis. 
and aggressive treatment, this evolution was essential to provide improved care and outcomes for RA patients. $^{39}$

Despite these developments, a significant proportion of patients remain symptomatic, which may make their disease 'difficult-to-treat'.113 This especially applies to patients with long-standing disease, frequently with substantial structural damage and various comorbidities. ${ }^{30}$ This clinically relevant unmet need motivated our Task Force to create a definition of D2T RA ${ }^{24}$ and to provide detailed management recommendations on diagnostic challenges and pharmacological and non-pharmacological therapeutic strategies for this underserved subpopulation of RA patients.

Clearly, D2TRA should be seen as a heterogeneous disease state. ${ }^{11-13,30}$ D2T RA is an umbrella concept as a wide variety of factors may contribute to the D2T RA state and could lead to difficulties in its management. Patients in whom (b/ts)DMARDs are ineffective (multidrug resistant or 'true refractory' RA) only represent a subgroup of this heterogeneous patient population ${ }^{10}$ and, therefore, the heterogeneity of D2T RA should be acknowledged in daily clinical practice. Identification of all factors potentially contributing to D2T RA is essential in order to tailor management strategies to the individual patient and warrants a holistic management approach.

The D2T RA recommendations provide guidance to HCPs and patients, from the point onwards where the RA management recommendations do not provide any specific help. ${ }^{5}$ A logical order to present the recommendations has been discussed and agreed, starting when patients fulfil the definition of D2T RA. ${ }^{24}$ The first step in the management of D2T RA (as well as the first recommendation) is reconsidering the diagnosis of RA, followed by the assessment of the presence or absence of inflammatory disease activity and the evaluation of treatment non-adherence. These recommendations enable a holistic management approach, in which the heterogeneity of D2T RA is acknowledged and all factors potentially contributing to the persistence of signs and/or symptoms suggestive of active disease are considered. Then, the recommendations promote individually tailored therapeutic interventions by addressing therapeutic aspects of b/tsDMARD selection (also in patients with comorbidities and coexisting conditions, including HBV and $\mathrm{HCV}$ ) and non-pharmacological interventions to improve adherence, functional disability, pain, fatigue, goal setting and self-efficacy.

Although the Task Force aimed to cover all potential aspects of D2T RA, not all relevant topics have been addressed in the SLRs because of the considerable overlap with previous or ongoing EULAR projects (e.g. treatment non-adherence, lifestyle factors, pain syndromes, osteoarthritis, see below). For a few topics, the Task Force members felt that the theme is particularly relevant in the context of D2T RA. For instance, education is already addressed in separate EULAR recommendations, ${ }^{14}$ but also has an important role in the D2T RA recommendations (\#4, 8-10).

Treatment non-adherence is common in patients with RMDs and may also contribute 
to the D2T RA state.1130,77,79 Non-adherence has therefore also been addressed in the D2T RA recommendations (\#4). Additional guidance on treatment non-adherence can be found in the recently published EULAR points to consider for the detection, assessment and management of non-adherence in people with RMDs. ${ }^{22}$

Lifestyle factors, including diet, lack of exercise, smoking and alcohol consumption, might also be associated with D2T disease.11,87 Therefore, the management of lifestyle factors in D2T RA patients was raised as a clinically relevant issue at our first Task Force meeting and resulted in the formulation of a research question on this topic. However, an ongoing EULAR project is focussing on lifestyle behaviour recommendations to prevent progression of RMDs and will be published soon. The Task Force therefore decided to refer to these points to consider for the management of these factors, as evidence in D2T RA patients specifically was expected to be lacking.

Additionally, concomitant fibromyalgia and other pain syndromes as well as osteoarthritis may coexist in D2T RA patients and may (partly) explain the persistence of sign and/or symptoms suggestive of active disease. ${ }^{1,30}$ The importance of these conditions was also discussed at the first Task Force meeting. Because previous EULAR projects have focussed on these conditions, it was decided to refer to their recommendations. Guidance on the management of these coexisting conditions can be found in the 'EULAR revised recommendations for the management of fibromyalgia', 18 'EULAR recommendations for the health professional's approach to pain management in inflammatory arthritis and osteoarthritis', '19 '2018 update of the EULAR recommendations for the management of hand osteoarthritis' 20 and 'EULAR recommendations for the non-pharmacological core management of hip and knee osteoarthritis'.2

One of the main conclusions of the SLRs is the lack of direct evidence and the scarcity of high-quality indirect evidence regarding D2T RA. ${ }^{27,28}$ The lack of direct evidence is not surprising, considering the recent establishment of the EULAR definition of D2T RA. ${ }^{24}$ However, indirect evidence (i.e. in RA patients in whom at least two b/tsDMARDs failed, especially with different MOA) was also scarce and the quality was generally low to moderate. ${ }^{27,28}$ This lack of (high-quality) direct evidence should be seen as a limitation of these recommendations and, therefore, future studies addressing D2T RA patients specifically are needed. Importantly, the heterogeneity of D2T RA should be considered when conducting such studies, as not all management strategies will be helpful in all D2T RA patients. Selecting the appropriate patient population will therefore be crucial in order to obtain relevant results (see also Table 3). As, hopefully, new evidence regarding D2T RA will emerge, the recommendations on the management of D2T RA need to be updated within three to five years.

These recommendations can only improve the management of D2T RA, and therewith the outcomes of D2T RA patients, if they will be implemented successfully. To this end, the D2T RA recommendations will be disseminated across national and international 
societies, both by presentations at congresses and online. Additionally, a slide deck will be provided on the EULAR website.

In summary, the evidence as identified in the SLRs together with expert opinion have resulted in a comprehensive set of overarching principles and recommendations for the management of D2T RA, promoting a holistic management approach and individually tailored pharmacological and non-pharmacological therapeutic strategies. Although high-quality evidence was scarce, these recommendations will provide assistance to HCPs and patients in the management of D2T RA patients. A research agenda was created to support future research in this emerging field. 


\section{References}

1. Smolen JS, Aletaha D, McInnes IB. Rheumatoid arthritis. Lancet 2016;388:2023-38.

2. Smolen JS, Landewé $\mathrm{R}$, Breedveld $\mathrm{FC}$, et al. EULAR recommendations for the management of rheumatoid arthritis with synthetic and biological disease-modifying antirheumatic drugs. Ann Rheum Dis 2010;69:964-75.

3. Smolen JS, Landewé $\mathrm{R}$, Breedveld FC, et al. EULAR recommendations for the management of rheumatoid arthritis with synthetic and biological disease-modifying antirheumatic drugs: 2013 update. Ann Rheum Dis 2014;73:492-509.

4. Smolen JS, Landewé R, Bijlsma J, et al. EULAR recommendations for the management of rheumatoid arthritis with synthetic and biological disease-modifying antirheumatic drugs: 2016 update. Ann Rheum Dis 2017;76:960-77.

5. Smolen JS, Landewé RBM, Bijlsma JWJ, et al. EULAR recommendations for the management of rheumatoid arthritis with synthetic and biological disease-modifying antirheumatic drugs: 2019 update. Ann Rheum Dis 2020;79:685-99.

6. Singh JA, Saag KG, Bridges SL, et al. 2015 American College of Rheumatology Guideline for the Treatment of Rheumatoid Arthritis. Arthritis Rheumatol 2016;68:1-26.

7. Brenol C V., Nava JIG, Soriano ER. Proper management of rheumatoid arthritis in Latin America. What the guidelines say? Clin Rheumatol 2015;34:51-5.

8. Lau CS, Chia F, Dans L, et al. 2018 update of the APLAR recommendations for treatment of rheumatoid arthritis. Int $\mathrm{J}$ Rheum Dis 2019;22:357-75.

9. Smolen JS, Breedveld FC, Burmester GR, et al. Treating rheumatoid arthritis to target: 2014 update of the recommendations of an international task force. Ann Rheum Dis 2016;75:3-15.

10. Buch $\mathrm{MH}$. Defining refractory rheumatoid arthritis. Ann Rheum Dis 2018;77:966-9.

11. de Hair MJH, Jacobs JWG, Schoneveld JLM, et al. Difficult-to-treat rheumatoid arthritis: an area of unmet clinical need. Rheumatology (Oxford) 2017;57:1135-44.

12. Buch $\mathrm{MH}$, Eyre S, McGonagle D. Persistent inflammatory and non-inflammatory mechanisms in refractory rheumatoid arthritis. Nat Rev Rheumatol 2021;17:17-33.
13. Roodenrijs NMT, de Hair MJH, van der Goes $\mathrm{MC}$, et al. Characteristics of difficult-to-treat rheumatoid arthritis: results of an international survey. Ann Rheum Dis 2018;77:1705-9.

14. Zangi $H A$, Ndosi $M$, Adams $J$, et al. EULAR recommendations for patient education for people with inflammatory arthritis. Ann Rheum Dis 2015;74:954-62.

15. Agca R, Heslinga SC, Rollefstad S, et al. EULAR recommendations for cardiovascular disease risk management in patients with rheumatoid arthritis and other forms of inflammatory joint disorders: 2015/2016 update. Ann Rheum Dis 2016;76:17-28.

16. Baillet A, Gossec L, Carmona L, et al. Points to consider for reporting, screening for and preventing selected comorbidities in chronic inflammatory rheumatic diseases in daily practice: A EULAR initiative. Ann Rheum Dis 2016;75:965-73.

17. Colebatch AN, Edwards CJ, Østergaard $M$, et al. EULAR recommendations for the use of imaging of the joints in the clinical management of rheumatoid arthritis. Ann Rheum Dis 2013;72:804-14.

18. Macfarlane $G J$, Kronisch $C$, Dean $L E$, et al. EULAR revised recommendations for the management of fibromyalgia. Ann Rheum Dis 2017;76:318-28.

19. Geenen $\mathrm{R}$, Overman $\mathrm{CL}$, Christensen $\mathrm{R}$, et al. EULAR recommendations for the health professional's approach to pain management in inflammatory arthritis and osteoarthritis. Ann Rheum Dis 2018;77:797-807.

20. Kloppenburg M, Kroon FPB, Blanco FJ, et al. 2018 update of the EULAR recommendations for the management of hand osteoarthritis. Ann Rheum Dis 2019;78:16-24.

21. Fernandes L, Hagen KB, Bijlsma JWJ, et al. EULAR recommendations for the nonpharmacological core management of hip and knee osteoarthritis. Ann Rheum Dis 2013;72:1125-35.

22. Ritschl V, Stamm TA, Aletaha D, et al. 2020 EULAR points to consider for the prevention, screening, assessment and management of non-adherence to treatment in people with rheumatic and musculoskeletal diseases for use in clinical practice. Ann Rheum Dis 2020; annrheumdis-2020-218986.

23. van der Heijde $D$, Aletaha D, Carmona $L$, et al. 2014 Update of the EULAR standardised operating procedures for EULAR-endorsed recommendations. Ann Rheum Dis 2015;74:813. 
24. Nagy G, Roodenrijs NMT, Welsing PMJ, et al. EULAR definition of difficult-to-treat rheumatoid arthritis. Ann Rheum Dis 2021;80:31-5.

25. Fautrel B, Granger B, Combe B, et al. Matrix to predict rapid radiographic progression of early rheumatoid arthritis patients from the community treated with methotrexate or leflunomide: results from the ESPOIR cohort. Arthritis Res Ther 2012;14:R249.

26. RitschIV, Stamm TA, Aletaha D, et al. Prevention, screening, assessing and managing of nonadherent behaviour in people with rheumatic and musculoskeletal diseases: systematic reviews informing the 2020 EULAR points to consider. RMD Open 2020;6:e001432.

27. Roodenrijs NMT, Kedves $M$, Hamar A, et al. Diagnostic issues in difficult-totreat rheumatoid arthritis: a systematic literature review informing the 2020 EULAR recommendations for the management of difficult-to-treat rheumatoid arthritis. RMD open 2021;7:e001511.

28. Roodenrijs NMT, Hamar A, Kedves $M$, et al. Pharmacological and non-pharmacological therapeutic strategies in difficult-to-treat rheumatoid arthritis: a systematic literature review informing the EULAR recommendations for the management of difficult-to-treat rheumatoid arthritis. RMD open 2021;7:e001512.

29. OCEBM Levels of Evidence Working Group, Oxford Centre for Evidence-Based Medicine. The Oxford 2011 Levels of Evidence. 2011.https:// www.cebm.net/2016/05/ocebm-levels-ofevidence/

30. Roodenrijs NMT, van der Goes MC, Welsing PMJ, et al. Difficult-to-treat rheumatoid arthritis: contributing factors and burden of disease. Rheumatology 2020:keaa860.

31. Pedersen JK, Lorenzen T, Ejbjerg B, et al. Low-field magnetic resonance imaging or combined ultrasonography and anti-cyclic citrullinated peptide antibody improve correct classification of individuals as established rheumatoid arthritis: Results of a population-based, cross-sectional study. BMC Musculoskelet Disord 2014;15:268.

32. Fan A, Tournadre A, Pereira B, et al. Performance of Fibromyalgia Rapid Screening Tool (FiRST) to detect fibromyalgia syndrome in rheumatic diseases. Rheumatology (Oxford) 2016;55:1746-50.

33. Ghib LJ, Cojocneanu-Petric R, Budisan L, et al. THU0518 The diagnostic value of selected micrornas in patients with fibromyalgia associated with rheumatoid arthritis: a pilot study. Ann Rheum Dis 2018;77:463.3-464.

34. Salaffi $F$, Di Carlo $M$, Carotti $M$, et al. The subjective components of the Disease Activity Score 28-joints (DAS28) in rheumatoid arthritis patients and coexisting fibromyalgia. Rheumatol Int 2018;38:1911-8.

35. Sato $\mathrm{H}$, Tanabe $\mathrm{N}$, Murasawa $\mathrm{A}$, et al. Procalcitonin is a specific marker for detecting bacterial infection in patients with rheumatoid arthritis. J Rheumatol 2012;39:1517-23.

36. Paalanen K, Puolakka K, Nikiphorou E, et al. Is seronegative rheumatoid arthritis true rheumatoid arthritis? A nationwide cohort study. Rheumatology (Oxford) 2020:keaa623.

37. Nikiphorou E, Sjöwall C, Hannonen P, et al. Longterm outcomes of destructive seronegative (rheumatoid) arthritis - Description of four clinical cases. BMC Musculoskelet Disord 2016;17.

38. Aletaha D, Neogi T, Silman AJ, et al. 2010 Rheumatoid arthritis classification criteria: An American College of Rheumatology/ European League Against Rheumatism collaborative initiative. Ann Rheum Dis 2010;69:1580-8.

39. Burgers LE, Raza K, Van Der Helm-Van Mil $\mathrm{AH}$. Window of opportunity in rheumatoid arthritis-Definitions and supporting evidence: From old to new perspectives. RMD Open 2019;5:V.

40. Landewé RBM. Overdiagnosis and overtreatment in rheumatology: A little caution is in order. Ann Rheum Dis 2018;77.

41. Ceponis A, Onishi M, Bluestein HG, et al. Utility of the ultrasound examination of the hand and wrist joints in the management of established rheumatoid arthritis. Arthritis Care Res 2014;66:236-44.

42. Filer A, Mandelin Al, DiCarlo E, et al. Histological and Clinical Correlates of Ultrasound Measures of Joint Inflammation: Analysis of RA Tissue Obtained By Ultrasound Guided Biopsy in Phase 1 of the Accelerating Medicines Partnership RA Network - ACR Meeting Abstracts. Arthritis Rheumatol 2017;69.

43. Kawashiri SY, Kawakami A, Iwamoto N, et al. The power Doppler ultrasonography score from 24 synovial sites or 6 simplified synovial sites, including the metacarpophalangeal joints, reflects the clinical disease activity and level of serum biomarkers in patients with rheumatoid arthritis. Rheumatology (Oxford) 2011;50:962-5. 
44. Mandl P, Naredo E, Wakefield RJ, et al. A systematic literature review analysis of ultrasound joint count and scoring systems to assess synovitis in rheumatoid arthritis according to the OMERACT filter. J Rheumatol 2011;38:2055-62.

45. Yokota K, Tsuzuki Wada T, Akiyama Y, et al. Detection of synovial inflammation in rheumatic diseases using superb microvascular imaging: Comparison with conventional power Doppler imaging. Mod Rheumatol 2018;28:327-33.

46. Vlad V, Berghea F, Libianu S, et al. Ultrasound in rheumatoid arthritis - Volar versus dorsal synovitis evaluation and scoring. BMC Musculoskelet Disord 2011;12:124.

47. Ngai $\mathrm{Ng} \mathrm{S}$, Bjørndal Axelsen $\mathrm{M}$, Ostergaard $M$, et al. OP0135 How well does whole body magnetic resonance imaging agree with whole body ultrasound in the assessment of joint inflammation in rheumatoid arthritis patients. Ann Rheum Dis 2019;78:143.

48. Takase-Minegishi K, Horita N, Kobayashi K, et al. Diagnostic test accuracy of ultrasound for synovitis in rheumatoid arthritis: systematic review and meta-analysis. Rheumatology (Oxford) 2018;57:49-58.

49. Ciurtin C, Brown G, Cotton A, et al. THU0138 Das 28 correlated poorly with the objective evidence of inflammation as detected by ultrasound (US) examination of hands and feet in patients with established rheumatoid arthritis (RA). Ann Rheum Dis 2017;76:253.1-253.

50. Šenolt L, Housa D, Vernerová Z, et al. Resistin in rheumatoid arthritis synovial tissue, synovial fluid and serum. Ann Rheum Dis 2007;66:45863.

51. Skacelova $M$, Hermanova $Z$, Horak $P$, et al. Higher levels of matrix metalloproteinase- 3 in patients with RA reflect disease activity and structural damage. Biomed Pap 2017;161:296302.

52. Tuncer T, Kaya A, Gulkesen A, et al. Matrix metalloproteinase-3 levels in relation to disease activity and radiological progression in rheumatoid arthritis. Adv Clin Exp Med 2019;28:665-70.

53. Yildirim K, Karatay S, Melikoglu MA, et al. Associations between acute phase reactant levels and disease activity score (DAS28) in patients with rheumatoid arthritis. Ann Clin Lab Sci 2004;34:423-6.

54. van Onna $\mathrm{M}$, Ten Cate $\mathrm{DF}$, Tsoi $\mathrm{KL}$, et al. Assessment of disease activity in patients with rheumatoid arthritis using optical spectral transmission measurements, a noninvasive imaging technique. Ann Rheum Dis 2016;75:511-8.

55. Meier AJL. Potential of optical spectral transmission measurements for joint inflammation measurements in rheumatoid arthritis patients. J Biomed Opt 2012;17:081420.

56. Besselink NJ, van der Meijde P, Rensen WHJJ, et al. Optical spectral transmission to assess inflammation in hand and wrist joints of rheumatoid arthritis patients. Rheumatology (Oxford) 2018;57:865-72.

57. Bae SC, Lee YH. MiR-146a levels in rheumatoid arthritis and their correlation with disease activity: a meta-analysis. Int. J. Rheum. Dis. 2018;21:1335-42.

58. Bustos Rivera-Bahena C, Xibillé-Friedmann D-X, González-Christen J, et al. Peripheral blood leptin and resistin levels as clinical activity biomarkers in Mexican Rheumatoid Arthritis patients. Reumatol Clínica 2016;12:323-6.

59. do Prado AD, Bisi MC, Piovesan DM, et al. Ultrasound power Doppler synovitis is associated with plasma IL-6 in established rheumatoid arthritis. Cytokine 2016;83:27-32.

60. Johnson TM, Register KA, Schmidt CM, et al. Correlation of the Multi-Biomarker Disease Activity Score with Rheumatoid Arthritis Disease Activity Measures: A Systematic Review and Meta-Analysis. Arthritis Care Res (Hoboken) 2018;71:1459-72.

61. Milman N, Karsh J, Booth RA. Correlation of a multi-cytokine panel with clinical disease activity in patients with rheumatoid arthritis. Clin Biochem 2010;43:1309-14.

62. Nordal $H H$, Brokstad $K A$, Solheim $M$, et al. Calprotectin (S100A8/A9) has the strongest association with ultrasound-detected synovitis and predicts response to biologic treatment: Results from a longitudinal study of patients with established rheumatoid arthritis. Arthritis Res Ther 2017;19:3.

63. Roodenrijs NMT, de Hair MJH, Wheater G, et al. The multi-biomarker disease activity score tracks response to rituximab treatment in rheumatoid arthritis patients: a post-hoc analysis of three cohort studies. Arthritis Res Ther 2018;20:256.

64. Rooney $T$, Scherzer R, Shigenaga JK, et al. Levels of plasma fibrinogen are elevated in well-controlled rheumatoid arthritis. Rheumatology (Oxford) 2011;50:1458-65.

65. Bauer EM, Ben-Artzi A, Duffy EL, et al. Jointspecific assessment of swelling and power 
Doppler in obese rheumatoid arthritis patients. BMC Musculoskelet Disord 2017;18:99.

66. Sosa J, Karina Perez S, Julia Santa Cruz M, et al. M-DAS28, M-SDAl and M-CDAl Performance in a Cohort of RA Patients with and without Concomitant Fibromyalgia. Arthritis Rheumatol 2017;69:suppl 10.

67. Ranganath VK, Duffy EL, Garg VK, et al. Obesity Impacts Swelling of Ankle and Foot Joints in Early Rheumatoid Arthritis Patients. J Clin Rheumatol 2019;25:e8-11.

68. Goossens J, Coustet B, Palazzo $\mathrm{E}$, et al. Overweight and obesity affect clinical assessment of synovitis in rheumatoid arthritis: comparison of ultrasonography and clinical exam. Clin Exp Rheumatol 2019;37:4954.

69. Van Den Bemt BJF, Zwikker HE, Van Den Ende CHM. Medication adherence in patients with rheumatoid arthritis: A critical appraisal of the existing literature. Expert Rev Clin Immunol 2012;8:337-51.

70. Murage $\mathrm{M}$, Tongbram $\mathrm{V}$, Feldman $\mathrm{S}$, et al. Medication adherence and persistence in patients with rheumatoid arthritis, psoriasis, and psoriatic arthritis: a systematic literature review. Patient Prefer Adherence 2018;12:1483503.

71. López-Medina C, Moltó A, Gérald F, et al. Assessment of the adherence to diseasemodifying drugs in patients with chronic inflammatory rheumatic diseases: Results of a survey of 1594 patients. Jt Bone Spine 2019;86:610-4.

72. Contreras-Yáñez I, Ponce De León S, Cabiedes $J$, et al. Inadequate therapy behavior is associated to disease flares in patients with rheumatoid arthritis who have achieved remission with diseasemodifying antirheumatic drugs. Am J Med Sci 2010;340:282-90.

73. De Vera MA, Mailman J, Galo JS. Economics of non-adherence to biologic therapies in rheumatoid arthritis topical collection on health economics and quality of life. Curr Rheumatol Rep 2014;16.

74. Salaffi $F$, Carotti $M$, Di Carlo $M$, et al. Adherence to anti-tumor necrosis factor therapy administered subcutaneously and associated factors in patients with rheumatoid arthritis. J Clin Rheumatol 2015;21:419-25.

75. Pasma A, Schenk C V., Timman R, et al. Non-adherence to disease-modifying antirheumatic drugs is associated with higher disease activity in early arthritis patients in the first year of the disease. Arthritis Res Ther 2015;17.

76. Pasma A, Schenk C, Timman R, et al. Does nonAdherence to DMARDs influence hospitalrelated healthcare costs for early arthritis in the first year of treatment? PLoS One 2017;12.

77. Sabaté E. WHO | Adherence to long-term therapies: evidence for action. World Health Organization 2015.

78. Lam WY, Fresco P. Medication Adherence Measures: An Overview. Biomed Res Int 2015;2015:1-12.

79. Gossec L, Molto A, Romand X, et al. Recommendations for the assessment and optimization of adherence to diseasemodifying drugs in chronic inflammatory rheumatic diseases: A process based on literature reviews and expert consensus. Jt Bone Spine 2019;86:13-9.

80. Joplin S, Van Der Zwan R, Joshua F, et al. Medication adherence in patients with rheumatoid arthritis: The effect of patient education, health literacy, and musculoskeletal ultrasound. Biomed Res Int 2015;2015.

81. Makoul G, Clayman ML. An integrative model of shared decision making in medical encounters. Patient Educ Couns 2006;60:30112.

82. Dy SM, Purnell TS. Key concepts relevant to quality of complex and shared decisionmaking in health care: A literature review. Soc Sci Med 2012;74:582-7.

83. Kerschbaumer A, Sepriano A, Smolen JS, et al. Efficacy of pharmacological treatment in rheumatoid arthritis: a systematic literature research informing the 2019 update of the EULAR recommendations for management of rheumatoid arthritis. Ann Rheum Dis 2020.

84. Kearsley-Fleet L, Davies R, De Cock D, et al. Biologic refractory disease in rheumatoid arthritis: Results from the British Society for Rheumatology Biologics Register for Rheumatoid Arthritis. Ann Rheum Dis 2018;77:1405-12.

85. Emery $\mathrm{P}$, Keystone $\mathrm{E}$, Tony $\mathrm{HP}$, et al. IL-6 receptor inhibition with tocilizumab improves treatment outcomes in patients with rheumatoid arthritis refractory to anti-tumour necrosis factor biologicals: Results from a 24-week multicentre randomised placebocontrolled trial. Ann Rheum Dis 2008;67:151623.

86. Burmester GR, Blanco R, Charles-Schoeman 
C, et al. Tofacitinib (CP-690,550) in combination with methotrexate in patients with active rheumatoid arthritis with an inadequate response to tumour necrosis factor inhibitors: A randomised phase 3 trial. Lancet 2013;381:451-60.

87. Genovese MC, Fleischmann RM, Greenwald M, et al. Tabalumab, an anti-BAFF monoclonal antibody, in patients with active rheumatoid arthritis with an inadequate response to TNF inhibitors. Ann Rheum Dis 2013;72:1461-8.

88. Genovese MC, Kalunian K, Gottenberg JE, et al. Effect of Filgotinib vs Placebo on Clinical Response in Patients With Moderate to Severe Rheumatoid Arthritis Refractory to DiseaseModifying Antirheumatic Drug Therapy: The FINCH 2 Randomized Clinical Trial. JAMA 2019;322:315-25.

89. Kremer JM, Emery P, Camp HS, et al. A Phase llb Study of ABT-494, a Selective JAK-1 Inhibitor, in Patients With Rheumatoid Arthritis and an Inadequate Response to Anti-Tumor Necrosis Factor Therapy. Arthritis Rheumatol 2016;68:2867-77.

90. Weinblatt M, Thomson G, Chen K, et al. FRIOl71 Clinical responses in patients with inadequate response to bdmards upon treatment with upadacitinib. Ann Rheum Dis 2019;78:759.

91. Alivernini S, Laria A, Gremese E, et al. ACR70disease activity score remission achievement from switches between all the available biological agents in rheumatoid arthritis: A systematic review of the literature. Arthritis Res Ther 2009;11:R163.

92. Kim HL, Lee MY, Park SY, et al. Comparative effectiveness of cycling of tumor necrosis factor-a (TNF-a) inhibitors versus switching to non-TNF biologics in rheumatoid arthritis patients with inadequate response to TNF-a inhibitor using a Bayesian approach. Arch Pharm Res 2014;37:662-70.

93. Lee $\mathrm{YH}, \mathrm{Bae}$ SC. Comparative efficacy and safety of tocilizumab, rituximab, abatacept and tofacitinib in patients with active rheumatoid arthritis that inadequately responds to tumor necrosis factor inhibitors: a Bayesian network meta-analysis of randomized controlled tri. Int J Rheum Dis 2016;19:1103-11.

94. Malottki K, Barton P, Tsourapas A, et al. Adalimumab, etanercept, infiximab, rituximab and abatacept for the treatment of rheumatoid arthritis after the failure of a tumour necrosis factor inhibitor: A systematic review and economic evaluation. Health
Technol Assess (Rockv) 2011;15:1-300.

95. Singh JA, Hossain A, Tanjong Ghogomu E, et al. Biologics or tofacitinib for people with rheumatoid arthritis unsuccessfully treated with biologics: A systematic review and network meta-analysis. Cochrane Database Syst. Rev. 2017;2017.

96. Smolen JS, Kay J, Matteson EL, et al. Insights into the efficacy of golimumab plus methotrexate in patients with active rheumatoid arthritis who discontinued prior anti-tumour necrosis factor therapy: Post-hoc analyses from the GO-AFTER study. Ann Rheum Dis 2014;73:1811-8.

97. Schiff M, Pritchard C, Huffstutter JE, et al. The 6-month safety and efficacy of abatacept in patients with rheumatoid arthritis who underwent a washout after anti-tumour necrosis factor therapy or were directly switched to abatacept: The ARRIVE trial. Ann Rheum Dis 2009;68:1708-14.

98. Harrold LR, Reed GW, Magner R, et al. Comparative effectiveness and safety of rituximab versus subsequent anti-tumor necrosis factor therapy in patients with rheumatoid arthritis with prior exposure to anti-tumor necrosis factor therapies in the United States Corrona registry. Arthritis Res Ther 2015;17:256.

99. Charles-Schoeman C, Kremer J, Krishnaswami S, et al. THU0185 Comparison of tofacitinib safety and efficacy in rheumatoid arthritis patients with inadequate response to conventional synthetic dmards, or to one or more biological dmards. Ann Rheum Dis 2017;76:271.3-272.

100. Genovese MC, Kremer JM, Kartman CE, et al. Response to baricitinib based on prior biologic use in patients with refractory rheumatoid arthritis. Rheumatology (Oxford) 2018;57:900-8.

101. Chatzidionysiou K, Vollenhoven RFV. Rituximab versus anti-TNF in patients who previously failed one TNF inhibitor in an observational cohort. Scand J Rheumatol 2013;42:190-5.

102. Emery P, Gottenberg JE, Rubbert-Roth A, et al. Rituximab versus an alternative TNF inhibitor in patients with rheumatoid arthritis who failed to respond to a single previous TNF inhibitor: SWITCH-RA, a global, observational, comparative effectiveness study. Ann Rheum Dis 2015;74:979-84.

103. Gomez-Reino JJ, Maneiro JR, Ruiz J, et al. Comparative effectiveness of switching to alternative tumour necrosis factor (TNF) antagonists versus switching to rituximab in 
patients with rheumatoid arthritis who failed previous TNF antagonists: The MIRAR Study. Ann Rheum Dis 2012;71:1861-4.

104. Kekow J, Mueller-Ladner U, Schulze-Koops H. Rituximab is more effective than second antiTNF therapy in rheumatoid arthritis patients and previous TNFa blocker failure. Biol Targets Ther 2012;6:191-9.

105. Soliman MM, Hyrich KL, Lunt M, et al. Rituximab or a second anti-tumor necrosis factor therapy for rheumatoid arthritis patients who have failed their first anti-tumor necrosis factor therapy? Comparative analysis from the British Society for Rheumatology Biologics Register. Arthritis Care Res 2012;64:1108-15.

106. Torrente-Segarra V, Acosta Pereira A, Morla R, et al. VARIAR Study: Assessment of short-term efficacy and safety of rituximab compared to an tumor necrosis factor alpha antagonists as second-line drug therapy in patients with rheumatoid arthritis refractory to a first tumor necrosis factor alpha antagonist. Reumatol Clínica 2016;12:319-22.

107. Harrold LR, Reed GW, Best J, et al. Real-world Comparative Effectiveness of Tocilizumab Monotherapy vs. Tumor Necrosis Factor Inhibitors with Methotrexate in Patients with Rheumatoid Arthritis. Rheumatol Ther 2018;5:507-23.

108. Hirabara S, Takahashi N, Fukaya N, et al. Clinical efficacy of abatacept, tocilizumab, and etanercept in Japanese rheumatoid arthritis patients with inadequate response to anti-TNF monoclonal antibodies. Clin Rheumatol 2014;33:1247-54.

109. Lauper K, Nordström DC, Pavelka K, et al. Comparative effectiveness of tocilizumab versus TNF inhibitors as monotherapy or in combination with conventional synthetic disease-modifying antirheumatic drugs in patients with rheumatoid arthritis after the use of at least one biologic disease-modifyin. Ann Rheum Dis 2018;77:1276-82.

110. Vial G, Pouilly A De, Scouppe L, et al. FRI0234 Choice of biologic therapy following rituximab: influencing factors in a french multicenter cohort of rheumatoid arthritis. Ann Rheum Dis 2017;76:573.2-574.

111. Walker UA, Jaeger VK, Chatzidionysiou K, et al. Rituximab done: What's next in rheumatoid arthritis? A European observational longitudinal study assessing the effectiveness of biologics after rituximab treatment in rheumatoid arthritis. Rheumatology (Oxford) 2015;55:230-6.
112. Gottenberg JE, Brocq O, Perdriger A, et al. NonTNF-targeted biologic vs a second antiTNF drug to treat rheumatoid arthritis in patients with insufficient response to a first anti-TNF drug: A randomized clinical trial. JAMA - J Am Med Assoc 2016;316:1172-80.

113. Wei W, Knapp K, Wang $L$, et al. Treatment Persistence and Clinical Outcomes of Tumor Necrosis Factor Inhibitor Cycling or Switching to a New Mechanism of Action Therapy: Realworld Observational Study of Rheumatoid Arthritis Patients in the United States with Prior Tumor Necrosis Factor. Adv Ther 2017;34:193652.

114. González-Vacarezza N, Alemán A, González $G$, et al. Rituximab and tocilizumab for the treatment of rheumatoid arthritis. Int $\mathrm{J}$ Technol Assess Health Care 2014;30:282-8.

115. Nam JL, Takase-Minegishi K, Ramiro S, et al. Efficacy of biological disease-modifying antirheumatic drugs: A systematic literature review informing the 2016 update of the EULAR recommendations for the management of rheumatoid arthritis. Ann Rheum Dis 2017;76:1108-13.

116. Akiyama $\mathrm{M}$, Kaneko $\mathrm{Y}$, Kondo $\mathrm{H}$, et al. Comparison of the clinical effectiveness of tumour necrosis factor inhibitors and abatacept after insufficient response to tocilizumab in patients with rheumatoid arthritis. Clin Rheumatol 2016;35:2829-34.

117. Harrold LR, Reed GW, Kremer JM, et al. The comparative effectiveness of abatacept versus anti-tumour necrosis factor switching for rheumatoid arthritis patients previously treated with an anti-tumour necrosis factor. Ann Rheum Dis 2015;74:430-6.

118. Brown S, Everett CC, Naraghi K, et al. Alternative tumour necrosis factor inhibitors (TNFi) or abatacept or rituximab following failure of initial TNFi in rheumatoid arthritis: The SWITCH RCT. Health Technol Assess (Rockv) 2018;22:1280.

119. Cantini F, Boccia S, Goletti D, et al. HBV reactivation in patients treated with antitumor necrosis factor-alpha (TNF-a) agents for rheumatic and dermatologic conditions: A systematic review and meta-analysis. Int J Rheumatol 2014;2014:926836.

120. Padovan M, Filippini M, Tincani A, et al. Safety of Abatacept in Rheumatoid Arthritis with Serologic Evidence of Past or Present Hepatitis B Virus Infection. Arthritis Care Res 2016;68:738-43.

121. Chen LF, Mo YQ, Jing J, et al. Short-course 
tocilizumab increases risk of hepatitis B virus reactivation in patients with rheumatoid arthritis: a prospective clinical observation. Int J Rheum Dis 2017;20:859-69.

122. Lin KM, Cheng TT, Lin JC, et al. Tumor necrosis factor-a antagonist therapy for concomitant rheumatoid arthritis and hepatitis $\mathrm{C}$ virus infection: a case series study. Clin Rheumatol 2015:34:1039-46.

123. Brunasso AMG, Puntoni M, Gulia A, et al. Safety of anti-tumour necrosis factor agents in patients with chronic hepatitis $C$ infection: $A$ systematic review. Rheumatology (Oxford) 2011;50:1700-11.

124. Rausch Osthoff A-K, Niedermann K, Braun J, et al. 2018 EULAR recommendations for physical activity in people with inflammatory arthritis and osteoarthritis. Ann Rheum Dis 2018;77:1251-60.

125. Eversden L, Maggs F, Nightingale P, et al. A pragmatic randomised controlled trial of hydrotherapy and land exercises on overall well being and quality of life in rheumatoid arthritis. BMC Musculoskelet Disord 2007;8:23.

126. Figen A, Geçene $M$, Gündüz $R$, et al. LongTerm Effects of Comprehensive Inpatient Rehabilitation on Function and Disease Activity in Patients with Chronic Rheumatoid Arthritis and Ankylosing Spondylitis. Turkish J Rheumatol 2011;26:135-44.

127. Williams MA, Srikesavan C, Heine PJ, et al. Exercise for rheumatoid arthritis of the hand. Cochrane Database Syst Rev 2018;2018.

128. Lau YN, Ng J, Lee SY, et al. A brief report on the clinical trial on neural mobilization exercise for joint pain in patients with rheumatoid arthritis. Z Rheumatol 2019;78:474-8.

129. Feldthusen C, Dean E, Forsblad-D'Elia $H$, et al. Effects of Person-Centered Physical Therapy on Fatigue-Related Variables in Persons With Rheumatoid Arthritis: A Randomized Controlled Trial. Arch Phys Med Rehabil 2016;97:26-36.

130. Macedo AM, Oakley SP, Panayi GS, et al. Functional and work outcomes improve in patients with rheumatoid arthritis who receive targeted, comprehensive occupational therapy. Arthritis Care Res 2009;61:1522-30.

131. Siqueira US, Orsini Valente LG, De Mello MT, et al. Effectiveness of Aquatic Exercises in Women with Rheumatoid Arthritis: A Randomized, Controlled, 16-Week Intervention-The HydRA Trial. Am J Phys Med Rehabil 2017;96:167-75.

132. Christie A, Jamtvedt $G$, Dahm KT, et al. Effectiveness of Nonpharmacological and Nonsurgical Interventions for Patients With Rheumatoid Arthritis: An Overview of Systematic Reviews. Phys Ther 2007;87:1697715.

133. Hurkmans E, Van Der Giesen FJ, Vlieland TPMV, et al. Dynamic exercise programs (aerobic capacity and/or muscle strength training) in patients with rheumatoid arthritis. Cochrane Database Syst Rev 2009;2009:CD006853.

134. Lee MS, Pittler MH, Ernst E. Tai chi for rheumatoid arthritis: Systematic review. Rheumatology (Oxford) 2007;46:1648-51.

135. Santos EJF, Duarte C, Marques A, et al. Effectiveness of non-pharmacological and non-surgical interventions for rheumatoid arthritis: an umbrella review. JBI database Syst Rev Implement reports 2019;17:1494-531.

136. Manning VL, Hurley $M V_{\text {., }}$ Scott $D L$, et al. Education, self-management, and upper extremity exercise training in people with rheumatoid arthritis: a randomized controlled trial. Arthritis Care Res (Hoboken) 2014;66:21727.

137. Macfarlane GJ, Paudyal P, Doherty $M$, et al. A systematic review of evidence for the effectiveness of practitioner-based complementary and alternative therapies in the management of rheumatic diseases: Rheumatoid arthritis. Rheumatology (Oxford) 2012;51:1707-13.

138. Hewlett S, Almeida C, Ambler N, et al. Group cognitive-behavioural programme to reduce the impact of rheumatoid arthritis fatigue: the RAFT RCT with economic and qualitative evaluations. Health Technol Assess 2019;23:1130.

139. Ferwerda $M$, Van Beugen S, Van Middendorp $\mathrm{H}$, et al. A tailored-guided internet-based cognitive-behavioral intervention for patients with rheumatoid arthritis as an adjunct to standard rheumatological care: Results of a randomized controlled trial. Pain 2017;158:86878.

140. Dixon KE, Keefe FJ, Scipio CD, et al. Psychological interventions for arthritis pain management in adults: A meta-analysis. Heal Psychol 2007;26:241-50.

141. Prothero L, Barley E, Galloway J, et al. The evidence base for psychological interventions for rheumatoid arthritis: A systematic review of reviews. Int J Nurs Stud 2018;82:20-9.

142. Dissanayake RK, Bertouch J V. Psychosocial interventions as adjunct therapy for patients with rheumatoid arthritis: A systematic review. Int J Rheum Dis 2010;13:324-34. 
143. Baxter S V., Hale LA, Stebbings S, et al. Walking is a Feasible Physical Activity for People with Rheumatoid Arthritis: A Feasibility Randomized Controlled Trial. Musculoskeletal Care 2016;14:47-56.

144. DiRenzo D, Crespo-Bosque M, Gould N, et al. Systematic Review and Meta-analysis: Mindfulness-Based Interventions for Rheumatoid Arthritis. Curr Rheumatol Rep 2018;20:75.

145. Hammond A, Young A, Kidao R. A randomised controlled trial of occupational therapy for people with early rheumatoid arthritis. Ann Rheum Dis 2004;63:23-30.

146. Knittle K, De Gucht V, Hurkmans E, et al. Targeting motivation and self-regulation to increase physical activity among patients with rheumatoid arthritis: a randomised controlled trial. Clin Rheumatol 2015;34:231-8.

147. Mollard E, Michaud K. A Mobile App With Optical Imaging for the Self-Management of Hand Rheumatoid Arthritis: Pilot Study. JMIR mHealth uHealth 2018;6:el2221.

148. Albano MG, Giraudet-Le Quintrec J-SS, Crozet C, et al. Characteristics and development of therapeutic patient education in rheumatoid arthritis: analysis of the 2003-2008 literature. Joint Bone Spine 2010;77:405-10.

149. Iversen MD, Hammond A, Betteridge N. Selfmanagement of rheumatic diseases: state of the art and future perspectives. Ann Rheum Dis 2010;69:955-63.

150. Srikesavan C, Bryer C, Ali U, et al. Web-based rehabilitation interventions for people with rheumatoid arthritis: A systematic review. J Telemed Telecare 2019;25:263-75.

151. Anvar N, Matlabi $H$, Safaiyan A, et al. Effectiveness of self-management program on arthritis symptoms among older women: A randomized controlled trial study. Health Care Women Int 2018;39:1326-39.

152. El Miedany $Y$, El Gaafary M, El Arousy N, et al. Arthritis education: the integration of patientreported outcome measures and patient self-management. Clin Exp Rheumatol 2012;30:899-904.

153. Fraenkel L, Peters $E$, Charpentier $P$, et al. Decision tool to improve the quality of care in rheumatoid arthritis. Arthritis Care Res (Hoboken) 2012;64:977-85.

154. Fraenkel L, Matzko CK, Webb DE, et al. Use of Decision Support for Improved Knowledge, Values Clarification, and Informed Choice in Patients With Rheumatoid Arthritis. Arthritis Care Res (Hoboken) 2015;67:1496-502.
155. Li LC, Adam PM, Backman CL, et al. Proof-ofconcept study of a Web-based methotrexate decision aid for patients with rheumatoid arthritis. Arthritis Care Res (Hoboken) 2014;66:1472-81.

156. Li LC, Shaw CD, Lacaille $D$, et al. Effects of a Web-Based Patient Decision Aid on Biologic and Small-Molecule Agents for Rheumatoid Arthritis: Results From a Proof-ofConcept Study. Arthritis Care Res (Hoboken) 2018;70:343-52

157. Taal E, Rasker JJ, Wiegman O. Patient education and self-management in the rheumatic diseases: A self-efficacy approach. Arthritis Care Res (Hoboken) 1996;9:229-38.

158. Barlow JH, Cullen LA, Rowe IF. Educational preferences, psychological well-being and self-efficacy among people with rheumatoid arthritis. Patient Educ Couns 2002;46:11-9.

159. Bandura A. Self-Efficacy. In: The Corsini Encyclopedia of Psychology. Hoboken, NJ, USA: : John Wiley \& Sons, Inc. 2010. 1-3.

160. Lorig K, Chastain RL, Ung E, et al. Development and evaluation of a scale to measure perceived self-efficacy in people with arthritis. Arthritis Rheum 1989;32:37-44.

161. Garratt AM, Løchting I, Smedslund $G$, et al. Measurement properties of instruments assessing self-efficacy in patients with rheumatic diseases. Rheumatology (Oxford) 2014;53:1161-71.

162. Gibofsky A, Galloway J, Kekow J, et al. Comparison of patient and physician perspectives in the management of rheumatoid arthritis: results from global physician- and patient-based surveys. Health Qual Life Outcomes 2018;16:211.

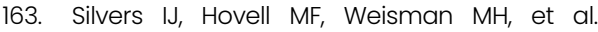
Assessing physician/patient perceptions in rheumatoid arthritis. A vital component in patient education. Arthritis Rheum 1985;28:300-7.

164. Barlow $\mathrm{JH}$, Wright CC. Knowledge in patients with rheumatoid arthritis: a longer term follow-up of a randomized controlled study of patient education leaflets. Br J Rheumatol 1998;37:373-6.

165. Hosseini Moghadam M, Jahanbin I, Nazarinia MA. The Effect of Educational Program on Self-efficacy of Women with Rheumatoid Arthritis: A Randomized Controlled Clinical Trial. Int J community based Nurs midwifery 2018;6:12-20.

166. Niedermann K, de Bie RA, Kubli $R$, et al. Effectiveness of individual resource-oriented 
joint protection education in people with rheumatoid arthritis. A randomized controlled trial. Patient Educ Couns 2011;82:42-8.

167. Niedermann K, Buchi S, Ciurea A, et al. Six and 12 months' effects of individual joint protection education in people with rheumatoid arthritis: a randomized controlled trial. Scand J Occup Ther 2012;19:360-9.

168. Riemsma RP, Taal E, Brus HL, et al. Coordinated individual education with an arthritis passport for patients with rheumatoid arthritis. Arthritis Care Res 1997;10:238-49.

169. Hewlett S, Ambler N, Almeida C, et al. Selfmanagement of fatigue in rheumatoid arthritis: a randomised controlled trial of group cognitive-behavioural therapy. Ann Rheum Dis 2011;70:1060-7.

170. Lineker SC, Bell $\mathrm{MJ}$, Wilkins $\mathrm{AL}$, et al. Improvements following short term home based physical therapy are maintained at one year in people with moderate to severe rheumatoid arthritis. J Rheumatol 2001;28:1658.

171. Breedland I, van Scheppingen C, Leijsma $M$, et al. Effects of a group-based exercise and educational program on physical performance and disease self-management in rheumatoid arthritis: a randomized controlled study. Phys Ther 2011;91:879-93.

172. Lorig KR, Ritter PL, Laurent DD, et al. The internetbased arthritis self-management program: a one-year randomized trial for patients with arthritis or fibromyalgia. Arthritis Rheum 2008;59:1009-17.

173. Siu AMH, Chui DYY. Evaluation of a community rehabilitation service for people with rheumatoid arthritis. Patient Educ Couns 2004;55:62-9.

174. Bell MJ, Lineker SC, Wilkins AL, et al. A randomized controlled trial to evaluate the efficacy of community based physical therapy in the treatment of people with rheumatoid arthritis. J Rheumatol 1998;25:231-7.

175. Najm A, Nikiphorou E, Kostine M, et al. EULAR points to consider for the development, evaluation and implementation of mobile health applications aiding self-management in people living with rheumatic and musculoskeletal diseases. RMD Open 2019;5.

176. Sepriano A, Kerschbaumer A, Smolen JS, et al. Safety of synthetic and biological DMARDs: a systematic literature review informing the 2019 update of the EULAR recommendations for the management of rheumatoid arthritis. Ann Rheum Dis 2020.
177. Götestam Skorpen $\mathrm{C}$, Hoeltzenbein M, Tincani $A$, et al. The EULAR points to consider for use of antirheumatic drugs before pregnancy, and during pregnancy and lactation. Ann Rheum Dis 2016;75:795-810.

178. Sammaritano LR, Bermas BL, Chakravarty EE, et al. 2020 American College of Rheumatology Guideline for the Management of Reproductive Health in Rheumatic and Musculoskeletal Diseases. Arthritis Rheumatol (Hoboken, NJ) 2020.

179. Clowse MEB, Feldman SR, Isaacs JD, et al. Pregnancy Outcomes in the Tofacitinib Safety Databases for Rheumatoid Arthritis and Psoriasis. Drug Saf 2016;39:755-62.

180. Hoeltzenbein $M$, Beck E, Rajwanshi $R$, et al. Tocilizumab use in pregnancy: Analysis of a global safety database including data from clinical trials and post-marketing data. Semin Arthritis Rheum 2016;46:238-45.

181. Mouyis M, Flint JD, Giles IP. Safety of antirheumatic drugs in men trying to conceive: $A$ systematic review and analysis of published evidence. Semin Arthritis Rheum 2019;48:91120.

182. Nikiphorou E, Norton S, Young A, et al. The association of obesity with disease activity, functional ability and quality of life in early rheumatoid arthritis: Data from the early rheumatoid arthritis study/early rheumatoid arthritis network UK prospective cohorts. Rheumatol (United Kingdom) 2018;57:1194202.

183. Gremese E, Carletto A, Padovan $M$, et al. Obesity and reduction of the response rate to anti-tumor necrosis factor a in rheumatoid arthritis: An approach to a personalized medicine. Arthritis Care Res 2013;65:94-100.

184. Klaasen R, Wijbrandts CA, Gerlag DM, et al. Body mass index and clinical response to infliximab in rheumatoid arthritis. Arthritis Rheum 2011;63:359-64.

185. Felson DT, Smolen JS, Wells G, et al. American College of Rheumatology/European League Against Rheumatism provisional definition of remission in rheumatoid arthritis for clinical trials. Ann Rheum Dis 2011;70:404-13.

186. Combe B, Landewe R, Daien Cl, et al. 2016 update of the EULAR recommendations for the management of early arthritis. Ann Rheum Dis 2017;76:948-59.

187. Lahiri M, Luben RN, Morgan C, et al. Using lifestyle factors to identify individuals at higher risk of inflammatory polyarthritis (results from the European Prospective Investigation 
of Cancer-Norfolk and the Norfolk Arthritis Register-the EPIC-2-NOAR Study). Ann Rheum
Dis 2014;73:219-26. 



\section{CHAPTER 10}

\section{Difficult-to-treat rheumatoid arthritis: contributing factors and burden of disease}

Rheumatology 2020. doi: 10.1093/rheumatology/keaa860 [Epub ahead of print 17 Dec 2020]

Nadia M.T. Roodenrijs, Marlies C. van der Goes, Paco M.J. Welsing, Janneke Tekstra, Floris P.J.G. Lafeber, Johannes W.G. Jacobs, Jacob M. van Laar 


\section{Abstract}

\section{Objectives}

Treatment of difficult-to-treat (D2T) RA patients is generally based on trial-and-error and can be challenging due to a myriad of contributing factors. We aimed to identify risk factors at RA onset, contributing factors and the burden of disease.

\section{Methods}

Consecutive RA patients were enrolled and categorised as D2T, according to the EULAR definition, or not (controls). Factors potentially contributing to D2T RA and burden of disease were assessed. Risk factors at RA onset and factors independently associated with D2T RA were identified by logistic regression. D2T RA subgroups were explored by cluster analysis.

\section{Results}

Fifty-two RA patients were classified as D2T and 100 as non-D2T.

Lower socioeconomic status at RA onset was found as an independent risk factor for developing D2T RA (OR 1.97 (95\% Cl 1.08-3.61)). Several contributing factors were independently associated with D2T RA, occurring more frequently in D2T than in non-D2T patients: limited drug options because of adverse events ( $94 \%$ vs $57 \%$ ) or comorbidities (69\% vs $37 \%$, mismatch in patient's and rheumatologist's wish to intensify treatment (37\% vs 6\%), concomitant fibromyalgia (38\% vs 9\%) and poorer coping (worse levels). Burden of disease was significantly higher in D2T RA patients.

Three subgroups of D2T RA patients were identified: 1. 'non-adherent dissatisfied patients'; 2. patients with 'pain syndromes and obesity'; 3. patients closest to the concept of 'true refractory RA'.

\section{Conclusions}

This comprehensive study on D2T RA shows multiple contributing factors, a high burden of disease and the heterogeneity of D2T RA. These findings suggest that these factors should be identified in daily practice in order to tailor therapeutic strategies further to the individual patient. 


\section{Introduction}

Despite significant improvement in the treatment of rheumatoid arthritis (RA) over the past decades, up to twenty percent of RA patients suffers from difficult-to-treat disease. ${ }^{1-3}$ A European League Against Rheumatism (EULAR) Task Force has recently defined difficult-to-treat (D2T) RA as patients having persistency of symptoms and/or signs despite failure of at least two biological or targeted synthetic disease-modifying antirheumatic drugs (b/tsDMARDs) with different mechanisms of action (full definition in Table 1). ${ }^{4,5}$

Changes in the treatment of D2T RA patients are generally based on trial-and-error, as specific management recommendations for these patients are lacking except for switching to another b/tsDMARD after failure of a first one. ${ }^{4}$ DMARD switches are indicated in case of inflammatory RA activity, but patients' signs and symptoms do not always simply represent active RA. In each D2T RA patient, one or multiple factor(s) could contribute to the disease state: factors potentially influencing inflammatory activity and/or resulting in non-inflammatory complaints.' All these contributing factors require different management strategies.

Inflammatory activity may persist due to multidrug resistance, wherein smoking, alcohol use, obesity and immunologic mechanisms (including anti-drug antibodies) may play a role.,.$^{2,-10}$ Furthermore, inflammatory activity can persist in case of suboptimal

Table 1. EULAR definition of difficult-to-treat RA ${ }^{5}$

1. Treatment according to EULAR recommendations and failure of $\geq 2 \mathrm{~b} /$ tsDMARDs (with different mechanisms of action)' after failing csDMARD therapy (unless contraindicated) ${ }^{2}$

2. Signs suggestive of active/progressive disease, defined as $\geq 1$ of:

a. At least moderate disease activity (according to validated composite measures including joint counts e.g. DAS28-ESR >3.2 or CDAI >10)

b. Signs (including acute phase reactants and imaging) and/or symptoms suggestive of active disease (joint related or other)

c. Inability to taper glucocorticoid treatment (below $7.5 \mathrm{mg} /$ day prednisone or equivalent)

d. Rapid radiographic progression (with or without signs of active disease) ${ }^{3}$

e. Well-controlled disease according to above standards, but still having RA symptoms that are causing a reduction in quality of life

3. The management of signs and/or symptoms is perceived as problematic by the rheumatologist and/or the patient

All three criteria need to be present in D2T RA.

b: biological; CDAl: clinical disease activity index; cS: conventional synthetic; DAS28-ESR: disease activity score assessing 28 joints using erythrocyte sedimentation rate; DMARD: disease-modifying antirheumatic drug; EULAR: European League Against Rheumatism; mg: milligram; RA: rheumatoid arthritis; ts: targeted synthetic.

1. Unless restricted by access to treatment due to socioeconomic factors.

2. If csDMARD treatment is contraindicated, failure of $\geq 2 \mathrm{~b} / \mathrm{tsDMARDs}$ with different mechanisms of action is sufficient.

3. Rapid radiographic progression: change in van der Heijde-modified Sharp score $\geq 5$ points at 1 year." 
treatment, due to limited treatment options because of (recurring) adverse events or contraindications related to comorbidities as well as treatment non-adherence..$^{2,13}$ On the other hand, factors such as concomitant gouty arthritis, obesity, osteoarthritis or fibromyalgia, may mimic inflammatory RA complaints (or even the disease itself), resulting in misdiagnosis and/or hampering of proper grading of RA activity. ${ }^{14-18}$ Finally, depression, anxiety, poor coping strategies and unrealistic management goals may result in (non-inflammatory) complaints not directly related to RA nor its inflammatory activity.1,19-22 Only if immunological mechanisms contribute to the D2T RA state, patients may suffer from 'true refractory RA'.2,23,24

An international survey showed the need for a more holistic approach in this D2T patient population. ${ }^{25}$ Therefore, unravelling the heterogeneity of D2T RA as well as the occurrence and importance of specific factors contributing to D2T RA is crucial in order to facilitate more appropriate therapeutic strategies. This will aid in developing a structured and individualised management approach for D2T RA patients, which includes pharmacological as well as non-pharmacological therapies. Thereby, the burden of disease for individual patients could be decreased as well as the socioeconomic impact for the society.

The aim of this study was therefore to characterise D2T RA - by identifying risk factors at RA onset and potentially contributing inflammatory and non-inflammatory factors - and to determine the burden of disease.

\section{Methods}

\section{Study design and participants}

Consecutive RA patients, fulfilling the 2010 American College of Rheumatology (ACR)/ EULAR classification criteria for $\mathrm{RA}^{26}$ and treated according to the current standard of care (treat-to-target) for at least one year, were prospectively recruited for a cross-sectional study at the Department of Rheumatology \& Clinical Immunology of University Medical Center (UMC) Utrecht. Enrolment was conducted from February 2019 to August 2020. RA patients were classified as having D2T RA if they fulfilled all criteria of the EULAR definition (Table 1). ${ }^{5}$ RA patients who did not fulfil all three criteria served as a control group.

This study was approved by the medical ethics committee of the UMC Utrecht and performed according to the Helsinki declaration. Written informed consent from all participants was obtained.

\section{Study procedures}

Patientand disease characteristics, potentiallycontributing factors (knownfrom literature)' and burden of disease were assessed during an extensive single study visit examination 
with questionnaires. Characteristics were (socio-)demographics, disease duration, smoking and alcohol status, medical history (including extra-articular manifestations, ${ }^{27}$ comorbidities according to EULAR comorbidity domains ${ }^{28}$ and Charlson Comorbidity Index $(\mathrm{CCl})^{29}$, and rheumatoid factor (RF) and anti-citrullinated protein antibodies (ACPA) status. Information on previous and current use of DMARDs, glucocorticoids and pain killers, including reasons of failing previous DMARDs (adverse events, comorbidities) was recorded. Presence of fibromyalgia was assessed following ACR 2010 Fibromyalgia criteria. ${ }^{30}$ A physical examination was performed including assessment of swollen and tender joint count (SJC/TJC), and height and weight. C-reactive protein (CRP) and erythrocyte sedimentation rate (ESR) were measured. X-rays of hands and feet of the past two years were collected, or performed if not yet available, to assess the presence of joint erosions. If there was doubt about the presence of synovitis, osteoarthritis and/or subluxation or crystal arthritis at time of the study visit, ultrasonography, $\mathrm{X}$-ray or synovial fluid aspiration, respectively, were performed as additional diagnostic investigations.

The questionnaires addressed treatment adherence (medication adherence reporting scale (MARS-5)), ${ }^{31}$ management goals and wish to adapt treatment (self-developed questionnaire), anxiety and depression (hospital anxiety and depression scale (HADS)), ${ }_{1}^{32}$ coping strategies (brief Illness perception questionnaire (IPQ) ${ }^{33}$ and helplessness items from Illness Cognition Questionnaire (ICQ) for chronic diseases), ${ }^{34}$ and burden of disease (physical functioning (health assessment questionnaire (HAQ)), ${ }_{15}^{35}$ quality of life (Euro Quality of Life using 5 Dimensions (EQ-5D)), ${ }^{36}$ pain (visual analogue scale (VAS)), fatigue (Functional Assessment of Chronic Illness Therapy (FACIT)), ${ }_{1}^{37}$ and physical activity (short International Physic Activity Questionnaire (IPAQ))).38

Additionally, the rheumatologist filled out a questionnaire regarding the wish to adapt treatment. Furthermore, a list of supplied drugs in the past six months was requested from the pharmacy, to identify discrepancies between prescribed and supplied drugs.

\section{Statistical analyses}

Patient characteristics, potentially contributing factors and burden of disease were summarised using descriptive statistics and compared between (subgroups of) D2T and non-D2T RA patients. To this end, independent T-test or one-way analysis of variance was used for continuous parameters, and Mann-Whitney $U$ or Kruskal-Wallis test as their distribution free alternatives. Fisher's exact or chi-square test was used to compare binary or categorical parameters, respectively.

Multivariable logistic regression was performed using D2T RA as dependent variable and risk factors at RA onset (age at disease onset, gender, RF and ACPA positivity, and education level as surrogate for socioeconomic status) as independent variables. Multivariable penalised logistic regression by Lasso method, as appropriate given the relatively small sample size of the study in relation to the number of variables, were used 
to explore which of the potentially contributing factors (independent variables, Table 5) were independently associated with having D2T RA (dependent variable).

To explore if distinct groups among D2T RA patients might exist, $\mathrm{k}$-medoid cluster analysis using partitioning around medoids algorithm was performed using all factors potentially contributing to D2T RA (17 variables, Table 5). This method of cluster analysis was chosen as all variables were of interest and we wanted to use continuous as well as categorical variables in this analysis, which is not feasible in e.g. hierarchical cluster analysis. The silhouette width, calculated based on Gower distances, was compared for several solutions regarding the number of clusters (2-17). The silhouette width reflects cluster validity and encompasses two clustering criteria: separation (i.e. average distance to the closest other cluster) and compactness (i.e. average within cluster distance). If the highest/optimal silhouette widths were comparable for several solutions, the clinical relevance of solutions (based on descriptive statistics of the different groups) by expert opinion (NMTR, MCVdG, PMJW, FPJGL, JMvL) was used to derive the final solution.

Statistical analyses were performed using IBM SPSS Statistics version 24 (IBM Corp, Armonk, NY, USA) and R version 4.0.0 (R Foundation for Statistical Computing, Vienna, Austria), package 'cluster' and 'Rtsne'.

\section{Results}

\section{Patient enrolment}

During the study period, 1070 RA patients were identified at our outpatient department, of whom 144 patients failed $\geq 2 \mathrm{~b} /$ tsDMARDs with different mechanisms of action. Of those 144 patients, 76 were willing to participate and 52 of these patients fulfilled D2T RA criteria. Additionally, 100 non-D2T RA patients were recruited.

\section{Patient characteristics}

Patient characteristics were broadly similar for patients classified as having D2T and non-D2T (Table 2). Age of disease onset was statistically significantly lower in D2T patients (mean 41.9 vs 47.8, $\mathrm{p}=0.01$ ). D2T RA patients had statistically significantly higher disease activity scores (median DAS28-ESR 4.1 vs 2.5, $\mathrm{p}<0.001$ ).

Of extra-articular manifestations, interstitial lung disease was more common in D2T RA patients (10 vs $2 \%, p=0.047$, supplementary Figure la). A higher median number of comorbidities was found in D2T than in non-D2T RA patients ( $2 \mathrm{vs} 1, \mathrm{p}<0.001$ ). Recurrent infections (54\% vs 15\%), osteoporosis (37\% vs 17\%) and gastrointestinal disease (19\% vs 7\%) were statistically significantly more frequent in D2T RA patients (Supplementary Figure lb). 
Table 2. Patient characteristics: (socio-)demographics and disease characteristics

\begin{tabular}{|c|c|c|c|}
\hline & D2T RA $(n=52)$ & Non-D2T RA $(n=100)$ & $\mathrm{p}$-value \\
\hline \multicolumn{4}{|l|}{ (Socio-)demographics } \\
\hline $\mathrm{Age}^{ \pm}$ & $60.2(11.4)$ & $64.5(10.9)$ & $0.03^{1}$ \\
\hline Female, \% & 73 & 72 & $1.00^{2}$ \\
\hline \multicolumn{4}{|l|}{ Cohabitation, \% } \\
\hline Alone & 21 & 20 & \multirow{5}{*}{$0.55^{3}$} \\
\hline With partner, without children & 46 & 55 & \\
\hline With partner and children & 23 & 20 & \\
\hline With children, without partner & 8 & 5 & \\
\hline Other & 2 & 0 & \\
\hline Having children, $\%$ & 77 & 82 & $0.52^{4}$ \\
\hline \multicolumn{4}{|l|}{ Education, \% } \\
\hline None & 2 & $0^{\ddagger}$ & \multirow{4}{*}{$0.13^{3}$} \\
\hline Primary school & 6 & 2 & \\
\hline Secondary school/secondary vocational education & 58 & 49 & \\
\hline High vocational education/university & 34 & 49 & \\
\hline Work participation, \% & 14 & & \multirow{6}{*}{$0.09^{3}$} \\
\hline Paid work, not work disabled & 6 & $23^{\ddagger}$ & \\
\hline Paid work and partly work disabled & 31 & 2 & \\
\hline Fully work disabled & 31 & 17 & \\
\hline Retired & 19 & 44 & \\
\hline Other & 19 & 14 & \\
\hline \multicolumn{4}{|l|}{ Disease characteristics } \\
\hline Disease duration, years & $17.0(9.0-25.0)$ & $14.0(8.0-24.0)$ & $0.33^{4}$ \\
\hline Age at onset, years ${ }^{ \pm}$ & $41.9(12.4)$ & $47.8(15.6)$ & $0.01^{1}$ \\
\hline RF positivity, \% & 75 & 65 & $0.27^{2}$ \\
\hline ACPA positivity, $\%$ & 73 & 65 & $0.36^{2}$ \\
\hline Joint erosions, \% & 69 & 59 & $0.29^{2}$ \\
\hline Extra-articular manifestations, $\%$ & 48 & 46 & $0.87^{2}$ \\
\hline Joint surgeries, \% & 63 & 49 & $0.12^{2}$ \\
\hline Prostheses, \% & 29 & 21 & $0.32^{2}$ \\
\hline DAS28-ESR & $4.1(3.5-6.1)$ & $2.5(1.8-3.3)$ & $<0.001^{4}$ \\
\hline SJC44 & $5(2-8)$ & $0(0-0)$ & $<0.001^{4}$ \\
\hline TJC44 & $15(6-25)$ & $3(0-10)$ & $<0.001^{4}$ \\
\hline VAS-GH & $59(40-76)$ & $21(5-52)$ & $<0.001^{4}$ \\
\hline $\mathrm{ESR}, \mathrm{mm} / \mathrm{h}$ & $10(5-27)$ & $7(3-14)$ & $0.06^{4}$ \\
\hline $\mathrm{CRP}, \mathrm{mg} / \mathrm{L}$ & $4(1-17)$ & $2(1-5)$ & $0.08^{4}$ \\
\hline \multicolumn{4}{|l|}{ Comorbidities } \\
\hline Number according to EULAR domains ${ }^{28}$ & $2(1-3)$ & $1(0-1)$ & $<0.001^{4}$ \\
\hline Index according to $\mathrm{CCl}^{29}$ & $63(57-72)$ & $68(59-75)$ & $0.06^{4}$ \\
\hline
\end{tabular}


Table 2. Continued

difficult-to-treat; DAS28-ESR: disease activity score assessing 28 joints using erythrocyte sedimentation rate; ESR: erythrocyte sedimentation rate; n: number; RF: rheumatoid factor; SJC44: swollen joint count assessing 44 joints; TJC44: tender joint count assessing 44 joints; VAS-GH: visual analogue score assessing global health (0-100, higher score reflects worse global health); $: \mathrm{n}=99$.

Numbers are median (interquartile range), unless stated otherwise; ${ }^{ \pm}$: mean (standard deviation).

Differences between groups were analysed using: 1. Independent T-test; 2. Fisher's exact test; 3. Chi-square test; 4. Mann-Whitney U test.

Table 3. Medication

\begin{tabular}{|c|c|c|c|}
\hline & D2T RA $(n=52)$ & Non-D2T RA $(n=100)$ & $\mathrm{p}$-value \\
\hline \multicolumn{4}{|l|}{ Current drugs } \\
\hline DMARDs, number & $2(1-2)$ & $1(1-2)$ & 0.071 \\
\hline $\operatorname{csDMARD}(\mathrm{s}), \%$ & 71 & 86 & $0.03^{2}$ \\
\hline bDMARD, \% & 52 & 39 & $0.17^{2}$ \\
\hline tsDMARD, \% & 23 & 0 & $<0.01^{2}$ \\
\hline Glucocorticoid therapy, \% & 52 & 16 & $<0.001^{2}$ \\
\hline Dose, mg/day prednisone or equivalent daily, in users & $7.5(5.0-15.0)$ & $5.0(5.0-7.5)$ & $0.02^{1}$ \\
\hline Pain killers, \% & 94 & 64 & $<0.001^{2}$ \\
\hline Regular use of paracetamol, \% & 25 & 11 & $0.03^{2}$ \\
\hline Regular use of NSAIDs, $\%$ & 40 & 19 & $<0.01^{2}$ \\
\hline Regular use of opioids, $\%$ & 12 & 5 & $0.19^{2}$ \\
\hline \multicolumn{4}{|l|}{ Failed DMARDs } \\
\hline csDMARDs, number & $3(3-5)$ & $2(1-3)$ & $<0.001^{1}$ \\
\hline b/tsDMARDs, number & $4(3-6)$ & $0(0-1)$ & $<0.001^{\prime}$ \\
\hline with different mechanisms of action, number & $3(2-4)$ & $0(0-0)$ & $<0.001^{1}$ \\
\hline \multicolumn{4}{|l|}{ Time periods } \\
\hline From diagnosis to start of $\mathrm{l}^{\mathrm{st}} \mathrm{b} / \mathrm{tsDMARD}$ (years) & $5.5(1.6-14.0)$ & $5.7(1.8-13.9), n=57$ & $0.79^{\prime}$ \\
\hline \multicolumn{4}{|l|}{ From start of $1^{\text {st }}$ to failure of } \\
\hline $1^{\text {st }}$ & $2.1(1.0-5.9)$ & $4.0(0.8-7.2), n=35$ & $0.91^{1}$ \\
\hline $2^{\text {nd }}$ & $4.5(2.2-10.2)$ & $8.6, n=3$ & $0.11^{1}$ \\
\hline $3^{\text {rd }}$ & $8.1(4.8-11.2), \mathrm{n}=34$ & & \\
\hline $4^{\text {th }}$ & $10.8(3.9-15.3), n=22$ & & \\
\hline $5^{\text {th }}$ & $12.8(5.7-16.9), n=11$ & & \\
\hline $\begin{array}{l}6^{\text {th }} \mathrm{b} / \mathrm{ts} D M A R D \text { with different mechanisms of action } \\
\text { (years) }\end{array}$ & $11.5, \mathrm{n}=2$ & & \\
\hline
\end{tabular}

b: biological; cs: conventional synthetic; D2T: difficult-to-treat; DMARD: disease-modifying antirheumatic drug; mg: milligram; n: number; NSAIDs: non-steroidal anti-inflammatory drugs; RA: rheumatoid arthritis; ts: targeted synthetic.

Numbers are median (interquartile range). Differences between groups were analysed using: 1. Mann-Whitney $U$ test; 2. Fisher's exact test. 


\section{Medication}

Current drugs

CsDMARDs were less and tsDMARDs were more frequently prescribed to D2T RA patients, although the median number of total current DMARDs did not differ statistically significantly (Table 3). Glucocorticoids were more commonly prescribed and with a higher dose among D2T RA than among non-D2T patients (52 vs 16\%, p<0.001; median dose in users: 7.5 vs $5.0 \mathrm{mg} /$ day prednisone or equivalent daily, respectively, $p=0.02$ ). Pain killers (paracetamol, non-steroidal anti-inflammatory drugs (NSAIDs) and/or opioids) were statistically significantly more frequently prescribed to D2T RA patients (94 vs 64\%, $\mathrm{p}<0.001)$.

\section{Failed DMARDs}

The number of failed cs- and b/tsDMARDs was statistically significantly higher in D2T than in non-D2T RA patients (Table 3). Following the definition of D2T RA, all D2T patients failed $\geq 2 \mathrm{~b} /$ tsDMARDs with different mechanisms of action, while this was only $3 \%$ for nonD2T RA patients. D2T RA patients failed a median number of $3 \mathrm{~b} / \mathrm{tsDMARDs}$ with different mechanisms of action (IQR 2-4), and 4 different b/tsDMARDs in general (IQR 3-6). The median duration from starting a first $b /$ tsDMARD to failing a second class of $b / t s D M A R D s$ was 4.5 years in D2T RA patients (IQR 2.2-10.2, Table 3).

\section{Risk factors at RA onset for developing D2T RA}

Lower socioeconomic status (i.e. lower level of education) at RA onset was independently associated with D2T RA (OR $1.97(95 \% \mathrm{Cl} 1.08-3.61, \mathrm{p}=0.03)$ ). No statistically significant associations were found for the other potential risk factors in multivariable analysis (Table 4).

\section{Factors contributing to D2T RA}

Several contributing factors were statistically significantly more frequently found among

Table 4. Risk factors at RA onset (independently) associated with development of D2T RA

\begin{tabular}{lcc}
\hline & $\begin{array}{c}\text { Univariable OR } \\
(95 \% \mathrm{Cl}, \mathrm{p} \text {-value })\end{array}$ & $\begin{array}{c}\text { Multivariable OR } \\
(95 \% \mathrm{Cl}, \mathrm{p} \text {-value })\end{array}$ \\
\hline Higher age of onset & $0.97(0.95-0.996,0.02)$ & $0.98(0.95-1.00,0.05)$ \\
Female gender & $0.95(0.45-2.01,0.89)$ & $1.04(0.46-2.34,0.92)$ \\
RF positivity & $1.62(0.76-3.42,0.21)$ & $1.47(0.56-3.88,0.43)$ \\
ACPA positivity & $1.46(0.70-3.06,0.31)$ & $1.01(0.39-2.64,0.98)$ \\
Lower socioeconomic status (lower level of education) & $1.93(1.07-3.47,0.03)$ & $1.97(1.08-3.61,0.03)$ \\
\hline ACPA: anti-citrullinated protein antibodies; Cl: confidence interval; D2T: difficult-to-treat; OR: odds ratio; RA: & &
\end{tabular}


Table 5. Factors contributing to D2T RA

\begin{tabular}{|c|c|c|c|}
\hline & D2T RA $(n=52)$ & Non-D2T RA $(n=100)$ & $\mathrm{p}$-value \\
\hline Smoking, \% & 19 & 11 & 0.22 \\
\hline Alcohol use, \% & 52 & 76 & $<0.01^{1}$ \\
\hline Number per week, in users & $3.0(1.0-7.0)$ & $4.0(0.5-7.0)$ & $0.87^{2}$ \\
\hline Overweight or obesity, \% & 62 & 60 & $1.00^{1}$ \\
\hline BMl & $27(22-31)$ & $26(23-29)$ & $0.65^{2}$ \\
\hline Presence of concomitant gouty arthritis/CPPD, \% & 12 & 3 & 0.091 \\
\hline \multicolumn{4}{|l|}{ Presence of limited drug options because of: } \\
\hline - Adverse events, \% & 94 & 57 & $<0.001^{1}$ \\
\hline - Comorbidities\#, \% & 69 & 37 & $<0.001^{1}$ \\
\hline \multicolumn{4}{|l|}{ Treatment non-adherence, according to: } \\
\hline - Level of MARS-5 & $24(21-25)$ & $24(23-25) \ddagger$ & $0.16^{2}$ \\
\hline $\begin{array}{l}\text { - Discrepancy in prescribed and supplied DMARDs } \\
\text { and GCs, \% }\end{array}$ & 40 & $22^{\ddagger}$ & $0.02^{1}$ \\
\hline \multicolumn{4}{|l|}{$\begin{array}{l}\text { Mismatch between patient and rheumatologist in wish } \\
\text { to intensify treatment: }\end{array}$} \\
\hline - Patient's wish to intensify, and not rheumatologist, \% & 37 & $6^{\ddagger}$ & $<0.001^{1}$ \\
\hline - Rheumatologist's wish to intensify, and not patient, \% & 13 & $9 \ddagger$ & $0.41^{1}$ \\
\hline Secondary osteoarthritis, \% & 77 & 68 & 0.27 \\
\hline Subluxation, \% & 23 & 19 & 0.671 \\
\hline Fibromyalgia, \% & 38 & 9 & $<0.001^{1}$ \\
\hline Depression, probable, \% & 15 & $4^{\ddagger}$ & 0.02 \\
\hline Level & $6(3-10)$ & $2(1-5)^{\ddagger}$ & $<0.001^{2}$ \\
\hline Anxiety, probable, \% & 17 & $7 \ddagger$ & $0.09^{1}$ \\
\hline Level & $7(2-9)$ & $4(2-7)^{\ddagger}$ & $0.02^{2}$ \\
\hline \multicolumn{4}{|l|}{ Poor coping strategies: } \\
\hline - Level of helplessness (ICQ) & $13(10-17)$ & $9(7-13) \ddagger$ & $<0.001^{2}$ \\
\hline - Level of threatening view of illness (IPQ) & $47(36-57)$ & $35(27-44) \ddagger$ & $<0.001^{2}$ \\
\hline
\end{tabular}

BMl: body mass index; CPPD: calcium pyrophosphate deposition disease; D2T: difficult-to-treat; DMARD: diseasemodifying antirheumatic drug; ICQ: illness cognition questionnaire; IPQ: illness perception questionnaire; IQR: interquartile range; MARS-5: medication adherence reporting scale; n: number; RA: rheumatoid arthritis; \#: Recurrent infections, malignancies, moderate to severe heart failure, moderate to severe renal insufficiency, moderate to severe liver failure; ${ }^{\ddagger}: \mathrm{n}=99$.

Overweight/obesity according to BMl above 25. Level of treatment non-adherence according to medication adherence reporting scale (5-25, higher score reflects higher level of adherence). ${ }^{31}$ Fibromyalgia according to ACR 2010 criteria. $^{30}$ Depression and anxiety according to hospital anxiety and depression scale (0-21, score >10 reflects probable depression or anxiety). ${ }^{2}$ Level of illness perception according to Illness Perception Questionnaire (0-80, higher score reflects more threatening view of illness). ${ }^{33}$ Level of helplessness according to Illness Cognition Questionnaire for chronic diseases (6-24, higher score reflects higher level of helplessness).34

Numbers are median (interquartile range). Differences between groups were analysed using: 1. Fisher's exact test; 2. Mann-Whitney U test. 
D2T than among non-D2T RA patients (Table 5). These were higher frequencies of limited drug options because of AEs (94\% vs 57\%) or because of comorbidities (69\% vs $37 \%$ ), treatment non-adherence (discrepancy in prescribed and supplied drugs: $40 \%$ vs $22 \%$ ) and a mismatch in the wish to intensify treatment strategies between patient and rheumatologists (patient's wish to intensify treatment: $37 \%$ vs $6 \%$ ). Additionally, fibromyalgia and depression were more commonly found in D2T RA patients (38\% vs 9\%, and $15 \%$ vs $4 \%$, respectively), as well as higher levels of anxiety and poorer coping (Table 5).

In multivariable penalised logistic regression, limited drug options because of AEs and comorbidities, a mismatch between patient and rheumatologist in wish to intensify treatment (patient's wish to intensify treatment in contrast to rheumatologist), fibromyalgia, and poorer coping (higher levels of threatening illness perception) were independently and statistically significantly associated with D2T RA (Supplementary Table 1).

\section{Burden of disease}

A statistically significantly higher burden of disease was found in D2T compared to in non-D2T RA patients, indicated by lower physical functioning and quality of life and worse levels of fatigue and pain (Table 6). A statistically significantly lower level of physical

Table 6. Clinical burden of D2T RA

\begin{tabular}{|c|c|c|c|}
\hline & D2T RA $(n=52)$ & Non-D2T RA $(n=99)$ & $\mathrm{p}$-value \\
\hline Physical functioning (HAQ), level & $1.8(1.4-2.1)$ & $1.0(0.5-1.4)$ & $<0.001^{1}$ \\
\hline \multicolumn{4}{|l|}{ Quality of Life (EQ-5D-5L) } \\
\hline Index (Dutch tariff) & $0.62(0.31-0.77)$ & $0.81(0.68-0.85)$ & $<0.001^{1}$ \\
\hline VAS & $51(26-65)$ & $75(60-85)$ & $<0.001^{1}$ \\
\hline Fatigue (FACIT-F), level & $27(18-34)$ & $39(29-45)$ & $<0.001^{1}$ \\
\hline Pain (VAS-pain), level & $58(30-80)$ & $23(8-49)$ & $<0.001^{1}$ \\
\hline \multicolumn{4}{|l|}{ Physical activity (IPAQ), \% } \\
\hline Low & 35 & 13 & \\
\hline Moderate & 23 & 37 & $<0.01^{2}$ \\
\hline High & 42 & 50 & \\
\hline Total hours of sitting per week & $46(28-63)$ & $42(28-56)$ & $0.75^{1}$ \\
\hline
\end{tabular}

D2T: difficult-to-treat; EQ-5D: Euro Quality of Life using 5 Dimensions (0-1, higher score reflects higher quality of life); ${ }_{1}^{36}$ FACIT-F: Functional Assessment of Chronic Illness Therapy Fatigue (0-52, higher score reflects lower level of fatigue);; HAQ: health assessment questionnaire (0-3, higher score reflects lower level of physical functioning):35 IPAQ: international physical activity questionnaire; ${ }^{38} \mathrm{n}$ : number; RA: rheumatoid arthritis; VAS: visual analogue scale (0-100, higher score reflects better quality of life); VAS-pain: visual analogue scale to assess pain (0-100, higher score reflects higher level of pain).

Numbers are median (interquartile range). Differences between groups were analysed using: 1. Mann-Whitney $U$ test; 2. Chi-square test. 
activity per week was found in D2T compared to non-D2T RA patients.

\section{Subgroups of D2T RA}

The highest silhouette width was found for thirteen clusters (0.17, Supplementary Figure 2). As seventeen variables were used as input for cluster analysis, each cluster mainly represented one individual variable and clinical relevance was considered to be poor. After assessing the clinical relevance of all proposed numbers of clusters with a silhouette width above 0.15 , three clusters were chosen as the optimal number in which the clusters were sufficiently distinct.

The three subgroups of D2T RA patients are visualised in Figure 1 and Supplementary Figure 3 (details on potentially contributing factors in Supplementary Table 2). Subgroup one $(n=17)$ and two $(n=8)$ both comprised patients in whom non-inflammatory contributing factors (i.e. depression and poorer coping strategies) were more common. In addition, in subgroup one, higher frequencies of treatment non-adherence and patient dissatisfaction with treatment were found (patient's wish to intensify treatment in contrast to the rheumatologist). In subgroup two, in addition to depression and poorer coping, fibromyalgia and anxiety were more frequently found, as well as smoking and obesity.

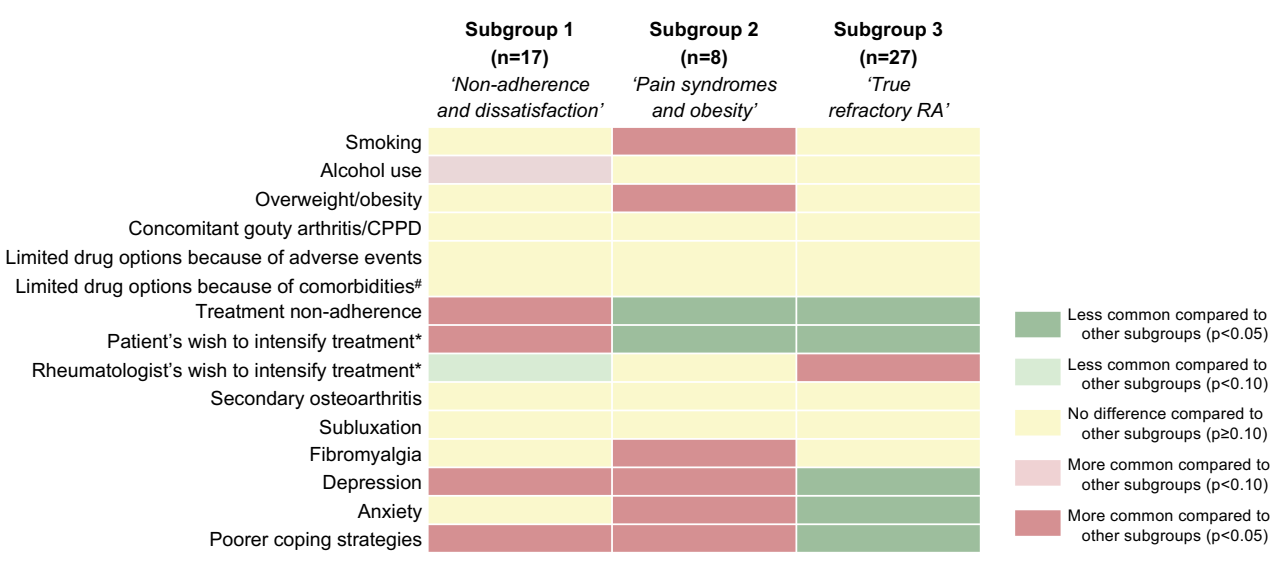

Figure 1. Subgroups of D2T RA

CPPD: calcium pyrophosphate deposition disease; D2T: difficult-to-treat; n: number; RA: rheumatoid arthritis; \#: Recurrent infections, malignancies, moderate to severe heart failure, moderate to severe renal insufficiency, moderate to severe liver failure; *: to intensify treatment, in contrast to rheumatologist or patient, respectively.

Overweight/obesity according to body mass index above 25. Treatment non-adherence according to level of medication adherence reporting scale and discrepancy between prescribed and supplied disease-modified antirheumatic drugs and glucocorticoids. ${ }^{31}$ Fibromyalgia according to ACR 2010 criteria. ${ }^{30}$ Depression and anxiety according to hospital anxiety and depression scale (0-21, score >10 reflects probable depression or anxiety).32 Poor coping strategies according to level of illness perception according to Illness Perception Questionnaire (0-80, higher score reflects more threatening view of illness) and level of helplessness according to Illness Cognition Questionnaire for chronic diseases (6-24, higher score reflects higher level of helplessness).33,34

Differences between groups were analysed using: Fisher's exact or Mann-Whitney U test, as appropriate. 
The third subgroup $(n=27)$ comprised patients in whom treatment non-adherence, depression, anxiety, and poorer coping strategies were less common. Therefore, this subgroup may be considered to be as closest to the concept of 'true refractory RA'. In this subgroup, the rheumatologist's wish to intensify treatment (in contrast to the patient's wish) was more frequently found.

Work participation and TJC44 were statistically significantly different between the three subgroups (Supplementary Table 3). Patients in subgroup one were more frequently work disabled compared to patients in the other subgroups (subgroup 1: $42 \%$; subgroup 2: $25 \%$; subgroup 3: $26 \% ; p=0.03$ ). TJC44 was statistically significantly higher in subgroup two, in which fibromyalgia was more common (median (IQR): subgroup 1: 12 (6-21); subgroup 2: 34 (22-39); subgroup 3: 15 (8-25); $p=0.02)$. In subgroup three, erosions were numerically more frequent, although statistical significance was not reached (subgroup l: 59\%; subgroup 2: 57\%; subgroup 3: 78\%; $p=0.33$ ).

\section{Discussion}

In this first study on the recently established D2T RA definition, ${ }^{5}$ multiple factors contributing to D2T RA were identified accompanied by a higher burden of disease in D2T RA patients. Of these factors, limited drug options because of AEs or comorbidities, a mismatch between patient's and rheumatologist's wish to intensify treatment, fibromyalgia, and poorer coping were independently associated with D2T RA. Lower socioeconomic status at RA onset was found as an independent risk factor for developing D2T RA. Three subgroups of D2T RA patients were identified: 1. 'non-adherent dissatisfied patients'; 2. patients with 'pain syndromes and obesity'; 3. patients who may be considered as closest to the concept of 'true refractory RA'.

In prior studies regarding RA patients who can be considered as having D2T RA, different criteria regarding treatment failure were used (e.g. failure of $\geq 2$ csDMARDs or $\geq 2$ bDMARDs not specifically with different mechanisms of action). ${ }^{39-41}$ In another study, patients who were exposed to $\geq 3$ bDMARDs with different mechanisms of action were studied, resembling the D2T RA definition. ${ }^{3}$ However, the other criteria of the definition were not taken into account (i.e. signs suggestive of presently active/progressive disease and management being perceived as problematic by the rheumatologist and/or patient). Therefore, direct comparisons of the results to other studies are not possible.

In this study, lower socioeconomic status at RA onset was identified as an independent risk factor for developing D2T RA. The crucial role of social determinants in treatment failure was also discussed in a recent review. ${ }^{42}$ Lower socioeconomic status is known to be associated with higher burden of disease and a higher risk of mortality. ${ }^{43-46}$ In the aforementioned study regarding patients exposed to $\geq 3$ bDMARDs with different 
mechanisms of action, lower socioeconomic status has also been found to be associated with being bDMARD refractory at onset of bDMARD treatment. ${ }^{3}$ Therefore, our suggestion would be to monitor RA patients with lower socioeconomic status more intensively and to be alert for the presence or development of factors contributing to D2T RA. By adequate management when these contributing factors develop, the risk of acquiring D2T RA may potentially be diminished. Future studies will be needed to evaluate the effectiveness of this strategy.

Treatment non-adherence was found as one of the factors contributing to D2T RA (according to a discrepancy in prescribed and supplied drugs). However, the level of adherenceaccordingtoaself-reported questionnairedidnot differstatisticallysignificantly between the two RA groups. Conflicting results of various assessment methods of nonadherence have previously been described and especially self-reported questionnaires are known to overreport adherence levels. ${ }^{47}$ Therefore, despite these conflicting results, treatment non-adherence probably is an important problem in D2T RA patients. Serum drug levels may more accurately assess adherence, although individual's specific drug metabolism and 'white coat' adherence (i.e. improved treatment adherence around clinic visit appointments) could complicate their interpretation. ${ }^{47}$ Hence, a perfect measure of adherence does not yet exist and, therefore, a multi-measure approach combining these measures is currently advised. ${ }^{47}$ Furthermore, studies on the role of therapeutic drug monitoring (TDM, i.e. systematic regular assessments of serum drug levels to guide dosing and optimise treatment) 48 in D2T RA patients could give additional insights into the importance of non-adherence as a contributing factor and could also help to determine whether TDM would be a relevant management strategy.

For several contributing factors (e.g. limited drug options because of comorbidities and fibromyalgia), it is difficult to assess whether these factors are either underlying the disease state, have developed as a result of having D2T RA, or both. Long-term observational studies, starting at disease onset, will be needed to resolve these issues. Nevertheless, the current presence of these contributing factors as well as the higher burden of disease in D2T RA patients indicates that these factors should be addressed in management strategies. ${ }^{21,49}$

The clinically most interesting identified contributing factors are the ones that are modifiable (i.e. treatment non-adherence, a mismatch between patient's and rheumatologist's wish to intensify treatment, depression, anxiety and poorer coping), as adequate management of these may ameliorate the D2T RA state. Although it remains debatable to what extent these factors are modifiable, addressing them adequately could at least result in reduction of associated complaints. Several non-pharmacological therapeutic strategies have been shown to be beneficial in the general population of RA patients: education, psychological and self-management interventions have been found to diminish non-inflammatory complaints and problems (pain, fatigue and functional 
disability) as well as treatment non-adherence, and to improve goal setting and different aspects of self-management (self-efficacy, anxiety, depression and RA knowledge).21,49,50 Although high-quality evidence of a beneficial effect of these non-pharmacological therapeutic strategies is still lacking in D2T RA patients, ${ }^{2,49}$ these strategies could be useful for those in whom these modifiable contributing factors are present.

The heterogeneity of D2T RA is underlined by the three subgroups we identified. The subgroup with relatively lower frequencies of contributing factors may be considered as having D2T RA closest to the concept of 'true refractory RA'.2 Here, contributing factors associated with D2T RA have a minor role and D2T RA may predominantly be driven by immunological mechanisms resulting in the D2T RA state.,22,23 The rheumatologist's wish to intensify treatment (where no further treatment options exist) and erosions being most common in this subgroup, support this suggestion. Anti-drug antibodies were not assessed in our study and may therefore still play a role in this subgroup of D2T RA patients. 1.2 Other immunological mechanisms underlying 'true refractory RA' are largely unknown, although several hypotheses exist.1.2 It would be interesting to compare immunological mechanisms that are still active between D2T and non-D2T RA patients. To this end, analyses of synovial biopsies, biomarker studies, whole genome sequencing and epigenetic analyses may be useful. ${ }^{51-53}$ Ultimately, a better understanding of immunological mechanisms may facilitate the development of new pharmacological strategies and may also contribute to the ongoing classification of subgroups of D2T RA. Notably, DAS28-ESR did not differ between D2T RA subgroups, although the highest numerical value was found in the subgroup comprising patients with 'pain syndromes and obesity'. This is probably due to the highest TJC and highest level of ESR found in this subgroup, which may be explained by the higher occurrences of fibromyalgia and obesity, respectively. This finding stresses earlier suggestions that composite indices may not be optimal to assess inflammatory disease activity in individual D2T RA patients, as contributing factors may also result in elevated values.1,7,54,55 To ascertain inflammatory disease activity in the general established population of RA patients, ultrasonography has an additional value and, therefore, it could also be an additional tool in D2T RA patients. ${ }^{17,56}$ However, no formal evidence currently exists to support this suggestion, as studies specifically in D2T RA patients are lacking. ${ }^{17}$ Future studies should demonstrate the role of ultrasonography in these patients.

One of the strengths of this study is the prospectively conducted enrolment of patients resulting in almost no missing data, although not all invited patients decided to participate. Another strength is the use of the recently established EULAR definition of D2T RA, ${ }^{5}$ which is meant to align research in this important area. However, this has also resulted in the exclusion of patients who cannot yet classify as having D2T RA according to the EULAR definition (i.e. have not yet failed $\geq 2 \mathrm{~b} /$ tsDMARDs, while these are not strictly contraindicated), ${ }^{5}$ while their management is already perceived as a struggle in daily 
practice. Furthermore, the relatively small sample size may be considered as a limitation, especially for the exploratory subgroup analysis and the multivariable logistic regression assessing potentially contributing factors, although our use of penalised logistic regression may have minimised the risk of overfitting. Nevertheless, before implementing the results - and specifically those of the subgroup analysis - in daily practice, the results should be replicated in another cohort of D2T RA patients.

In conclusion, multiple factors contributing to D2T RA were identified, e.g. limited DMARD options because of adverse events and comorbidities, fibromyalgia and poorer coping, accompanied by a higher burden of disease. Lower socioeconomic status at RA onset was found to be an independent risk factor for developing D2T RA. Three subgroups of D2T RA patients could be identified, underscoring the heterogeneity of the D2T RA state. The occurrence of factors contributing to the disease state, the burden of disease and the heterogeneity in D2T RA patients suggest that these factors should be identified in daily practice in order to tailor therapeutic strategies further to the individual patient. 


\section{References}

1. de Hair MJH, Jacobs JWG, Schoneveld JLM, et al. Difficult-to-treat rheumatoid arthritis: an area of unmet clinical need. Rheumatology (Oxford) 2017;57:1135-44.

2. Buch $\mathrm{MH}$. Defining refractory rheumatoid arthritis. Ann Rheum Dis 2018;77:966-9.

3. Kearsley-Fleet L, Davies R, De Cock D, et al. Biologic refractory disease in rheumatoid arthritis: Results from the British Society for Rheumatology Biologics Register for Rheumatoid Arthritis. Ann Rheum Dis 2018;77:1405-12.

4. Smolen JS, Landewé RBM, Bijlsma JWJ, et al. EULAR recommendations for the management of rheumatoid arthritis with synthetic and biological disease-modifying antirheumatic drugs: 2019 update. Ann Rheum Dis 2020;79:685-99.

5. Nagy G, Roodenrijs NMT, Welsing PMJ, et al. EULAR definition of difficult-to-treat rheumatoid arthritis. Ann Rheum Dis 2021;80:31-5.

6. Chang $\mathrm{K}$, Yang $\mathrm{SM}$, Kim $\mathrm{SH}$, et al. Smoking and rheumatoid arthritis. Int $\mathrm{J}$ Mol Sci 2014;15:22279-95.

7. Westhoff G, Rau R, Zink A. Rheumatoid arthritis patients who smoke have a higher need for DMARDs and feel worse, but they do not have more joint damage than non-smokers of the same serological group. Rheumatology (Oxford) 2008;47:849-54.

8. Maxwell JR, Gowers IR, Moore DJ, et al. Alcohol consumption is inversely associated with risk and severity of rheumatoid arthritis. Rheumatology 2010;49:2140-6.

9. Imhof A, Froehlich $\mathrm{M}$, Brenner $\mathrm{H}$, et al. Effect of alcohol consumption on systemic markers of inflammation. Lancet 2001;357:763-7.

10. Van Schouwenburg PA, Rispens T, Wolbink GJ. Immunogenicity of anti-TNF biologic therapies for rheumatoid arthritis. Nat Rev Rheumatol 2013;9:164-72.

11. Fautrel B, Granger B, Combe B, et al. Matrix to predict rapid radiographic progression of early rheumatoid arthritis patients from the community treated with methotrexate or leflunomide: results from the ESPOIR cohort. Arthritis Res Ther 2012;14:R249.

12. Xue $A L$, Wu SY, Jiang $L$, et al. Bone fracture risk in patients with rheumatoid arthritis. Med (United States) 2017;96.

13. Harrold LR, Andrade SE. Medication Adherence of Patients with Selected Rheumatic
Conditions: A Systematic Review of the Literature. Semin Arthritis Rheum 2009;38:396402.

14. Ruiz-Medrano E, Espinosa-Ortega HF, ArceSalinas CA. The effect of concomitant hand osteoarthritis on pain and disease activity in patients with rheumatoid arthritis. Clin Rheumatol 2019;38:2709-16.

15. George MD, Baker JF. The Obesity Epidemic and Consequences for Rheumatoid Arthritis Care. Curr Rheumatol Rep 2016;18:1-9.

16. Naranjo A, Ojeda S, Francisco F, et al. Fibromyalgia in patients with rheumatoid arthritis is associated with higher scores of disability. Ann Rheum Dis 2002;61:660-1.

17. Ranzolin A, Brenol JCT, Bredemeier $M$, et al. Association of concomitant fibromyalgia with worse disease activity score in 28 joints, health assessment questionnaire, and short form 36 scores in patients with rheumatoid arthritis. Arthritis Care Res 2009;61:794-800.

18. Roodenrijs NMT, Kedves M, Hamar $A$, et al. THUO110 Diagnostic issues in difficult-to-treat rheumatoid arthritis: preliminary results of a systematic literature review informing the 2020 EULAR recommendations for the management of difficult-to-treat rheumatoid arthritis. Ann Rheum Dis 2020;79:265-6.

19. Zhang L, Cai P, Zhu W. Depression has an impact on disease activity and healthrelated quality of life in rheumatoid arthritis: A systematic review and meta-analysis. Int J Rheum Dis 2020;23:285-93.

20. Machin AR, Babatunde O, Haththotuwa R, et al. The association between anxiety and disease activity and quality of life in rheumatoid arthritis: a systematic review and metaanalysis. Clin Rheumatol 2020;39:1471-82.

21. Englbrecht $M$, Kruckow $M$, Araujo $E$, et al. The Interaction of Physical Function and Emotional Well-being in Rheumatoid ArthritisWhat is the Impact on Disease Activity and Coping? Semin Arthritis Rheum 2013;42:48291.

22. Roodenrijs NMT, Hamar A, Kedves $M$, et al. FRI0047 Strategies regarding goal setting and self-management in difficult-to-treat rheumatoid arthritis: preliminary results of a systematic literature review informing the 2020 EULAR recommendations for the management of difficult-to-treat rheumatoid ar. Ann Rheum Dis 2020;79:595-595.

23. Roodenrijs NM, de Hair MJ, van der Goes MC, et al. Correspondence to viewpoint 'Defining refractory rheumatoid arthritis' by Buch. Ann 
Rheum Dis 2019;78:el05.

24. Buch MH. Response to 'Correspondence to viewpoint 'Defining refractory rheumatoid arthritis' by Buch' by Roodenrijs et al. Ann Rheum Dis 2019;78:el06.

25. Roodenrijs NMT, de Hair MJH, van der Goes $\mathrm{MC}$, et al. Characteristics of difficult-to-treat rheumatoid arthritis: results of an international survey. Ann Rheum Dis 2018;77:1705-9.

26. Aletaha D, Neogi T, Silman AJ, et al. 2010 Rheumatoid arthritis classification criteria: An American College of Rheumatology/ European League Against Rheumatism collaborative initiative. Ann Rheum Dis 2010;69:1580-8.

27. Carmona L, González-Alvaro I, Balsa A, et al. Rheumatoid arthritis in Spain: occurrence of extra-articular manifestations and estimates of disease severity. Ann Rheum Dis 2003;62:897-900.

28. Radner H, Chatzidionysiou K, Nikiphorou E, et al. 2017 EULAR recommendations for a core data set to support observational research and clinical care in rheumatoid arthritis. Ann Rheum Dis 2018;77:476-9.

29. Charlson ME, Pompei $\mathrm{P}$, Ales $\mathrm{KL}$, et al. A new method of classifying prognostic comorbidity in longitudinal studies: development and validation. J Chronic Dis 1987;40:373-83.

30. Wolfe F, Clauw DJ, Fitzcharles M-AA, et al. Fibromyalgia criteria and severity scales for clinical and epidemiological studies: A modification of the ACR preliminary diagnostic criteria for fibromyalgia. J Rheumatol 2011;38:1113-22.

31. Thompson K, Kulkarni J, Sergejew AA. Reliability and validity of a new Medication Adherence Rating Scale (MARS) for the psychoses. Schizophr Res 2000;42:241-7.

32. Zigmond AS, Snaith RP. The hospital anxiety and depression scale. Acta Psychiatr Scand 1983;67:361-70.

33. Broadbent E, Petrie KJ, Main J, et al. The Brief Illness Perception Questionnaire. J Psychosom Res 2006;60:631-7.

34. Evers AWM, Kraaimaat FW, Van Lankveld W, et al. Beyond unfavorable thinking: The illness cognition questionnaire for chronic diseases. J Consult Clin Psychol 2001;69:1026-36.

35. Maska L, Anderson J, Michaud K. Measures of functional status and quality of life in rheumatoid arthritis: Health Assessment Questionnaire Disability Index (HAQ), Modified Health Assessment Questionnaire (MHAQ), Multidimensional Health Assessment
Questionnaire (MDHAQ), Health Assessment. Arthritis Care Res (Hoboken) 2011;63:S4-13.

36. EuroQol Group. EuroQol--a new facility for the measurement of health-related quality of life. Health Policy 1990;16:199-208.

37. Cella D, Yount S, Sorensen M, et al. Validation of the Functional Assessment of Chronic Illness Therapy Fatigue Scale relative to other instrumentation in patients with rheumatoid arthritis. J Rheumatol 2005;32:811-9.

38. Craig $\mathrm{CL}$, Marshall $\mathrm{AL}$, Str $\mathrm{M} M \mathrm{MS}$, et al. International Physical Activity Questionnaire: 12-Country Reliability and Validity. Med Sci Sport Exerc 2003;35:1381-95.

39. Takanashi S, Kaneko Y, Takeuchi T. FRI0079 Characteristics of difficult-to-treat rheumatoid arthritis. Ann Rheum Dis 2020;79:612-3.

40. Batko B, UrbańskiK, Świerkot J, et al. Comorbidity burden and clinical characteristics of patients with difficult-to-control rheumatoid arthritis. Clin Rheumatol 2019;38:2473-81.

41. Richard L, Gonde H, Devauchelle A, et al. SAT0108 Characteristics of multiresistant patients to biological treatments in rheumatoid arthritis, and associated factors: an observational and retrospective study in 385 patients. Ann Rheum Dis 2019;78:1120-1.

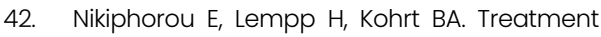
failure in inflammatory arthritis: time to think about syndemics? Rheumatology (Oxford) 2019;58:1526-33.

43. Group ES. Socioeconomic deprivation and rheumatoid disease: What lessons for the health service? Ann Rheum Dis 2000;59:7949.

44. Jacobi CE, Mol GD, Boshuizen HC, et al. Impact of socioeconomic status on the course of rheumatoid arthritis and on related use of health care services. Arthritis Rheum 2003;49:567-73.

45. Marra CA, Lynd LD, Esdaile JM, et al. The impact of low family income on self-reported health outcomes in patients with rheumatoid arthritis within a publicly funded health-care environment. Rheumatology 2004;43:1390-7.

46. Verstappen SMM. The impact of socioeconomic status in rheumatoid arthritis. Rheumatology 2016;56:1051-2.

47. Lam WY, Fresco P. Medication Adherence Measures: An Overview. Biomed Res Int 2015;2015:1-12.

48. Perry $M$, Abdullah $A$, Frleta $M$, et al. The potential value of blood monitoring of biologic drugs used in the treatment of 
rheumatoid arthritis. Ther Adv Musculoskelet Dis 2020;12:1759720X2090485.

49. Roodenrijs NMT, Hamar A, Kedves $M$, et al. SAT0052 Therapeutic strategies in difficultto-treat rheumatoid arthritis: preliminary results of a systematic literature review informing the 2020 EULAR recommendations for the management of difficult-to-treat rheumatoid arthritis. Ann Rheum Dis 2020;79:953-953.

50. Gossec L, Molto A, Romand X, et al. Recommendations for the assessment and optimization of adherence to diseasemodifying drugs in chronic inflammatory rheumatic diseases: A process based on literature reviews and expert consensus. Jt Bone Spine 2019;86:13-9.

51. Orr C, Sousa E, Boyle DL, et al. Synovial tissue research: A state-of-the-art review. Nat Rev Rheumatol 2017;13:463-75.

52. Donlin LT, Park SH, Giannopoulou E, et al. Insights into rheumatic diseases from nextgeneration sequencing. Nat Rev Rheumatol
2019;15:327-39.

53. Winthrop $\mathrm{KL}$, Weinblatt $\mathrm{ME}$, Bathon $\mathrm{J}$, et al. Unmet need in rheumatology: reports from the Targeted Therapies meeting 2019. Ann Rheum Dis 2020;79:88-93.

54. Gaujoux-Viala C, Mouterde G, Baillet A, et al. Evaluating disease activity in rheumatoid arthritis: Which composite index is best? A systematic literature analysis of studies comparing the psychometric properties of the DAS, DAS28, SDAI and CDAI. Jt Bone Spine 2012;79:149-55.

55. Jacobs JWG, Ten Cate DF, van Laar JM. Monitoring of rheumatoid arthritis disease activity in individual patients: still a hurdle when implementing the treat-totarget principle in daily clinical practice. Rheumatology 2015;54:959-61.

56. Colebatch AN, Edwards CJ, Østergaard $M$, et al. EULAR recommendations for the use of imaging of the joints in the clinical management of rheumatoid arthritis. Ann Rheum Dis 2013;72:804-14. 


\section{Supplementary}

\section{A. Extra-articular manifestations}

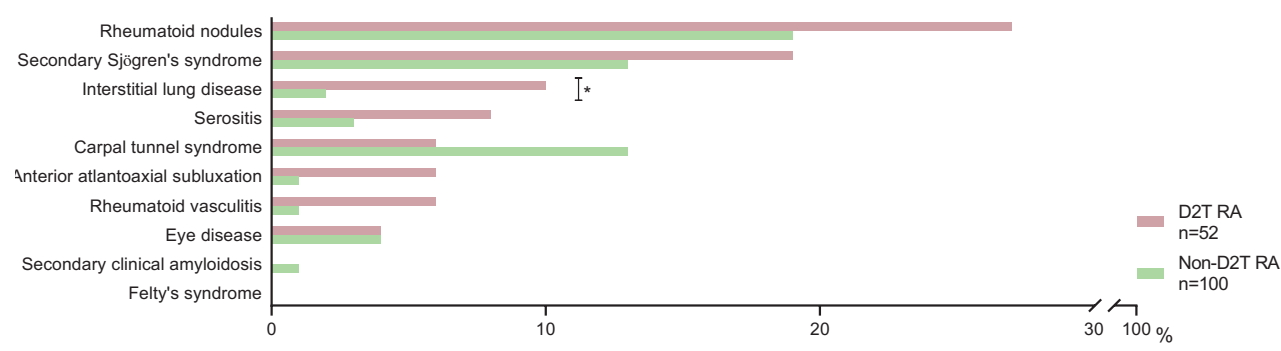

B. Comorbidities (previously diagnosed), according to EULAR domains ${ }^{28}$

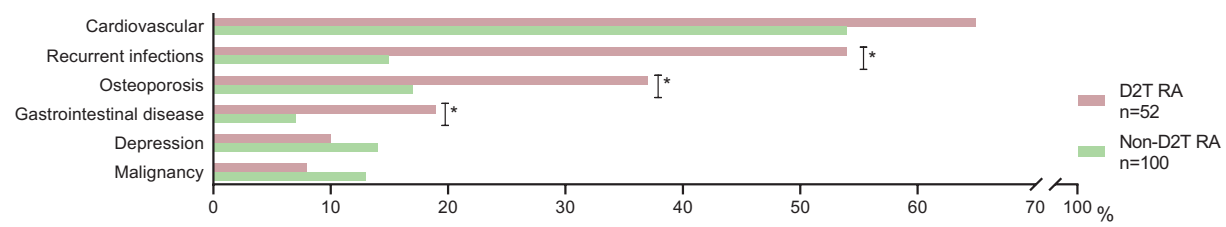

Supplementary Figure 1. Extra-articular manifestations and comorbidities D2T: difficult-to-treat; EULAR: European League Against Rheumatism; RA: rheumatoid arthritis; *: p<0.05. Differences between groups were analysed using Fisher's exact test.

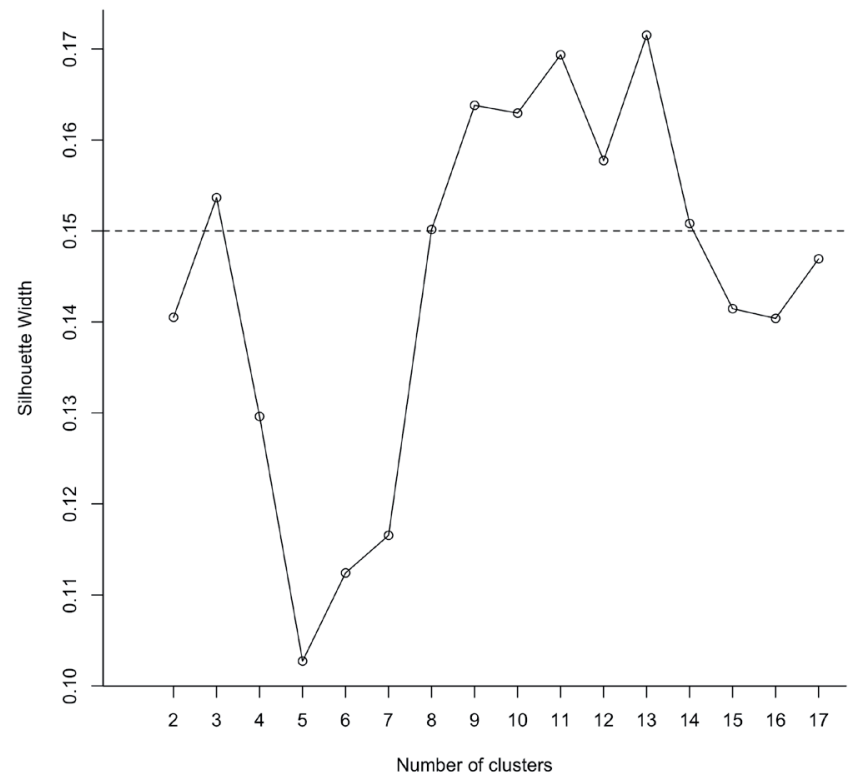

Supplementary Figure 2. Silhouette figure of cluster analyses of subgroups of D2T RA

The silhouette width is an estimate of the average distance between clusters. The silhouette width ranges from -1 to +1 . A higher value represents individuals in the same cluster being as similar as possible and in the different clusters as distinct as possible. 


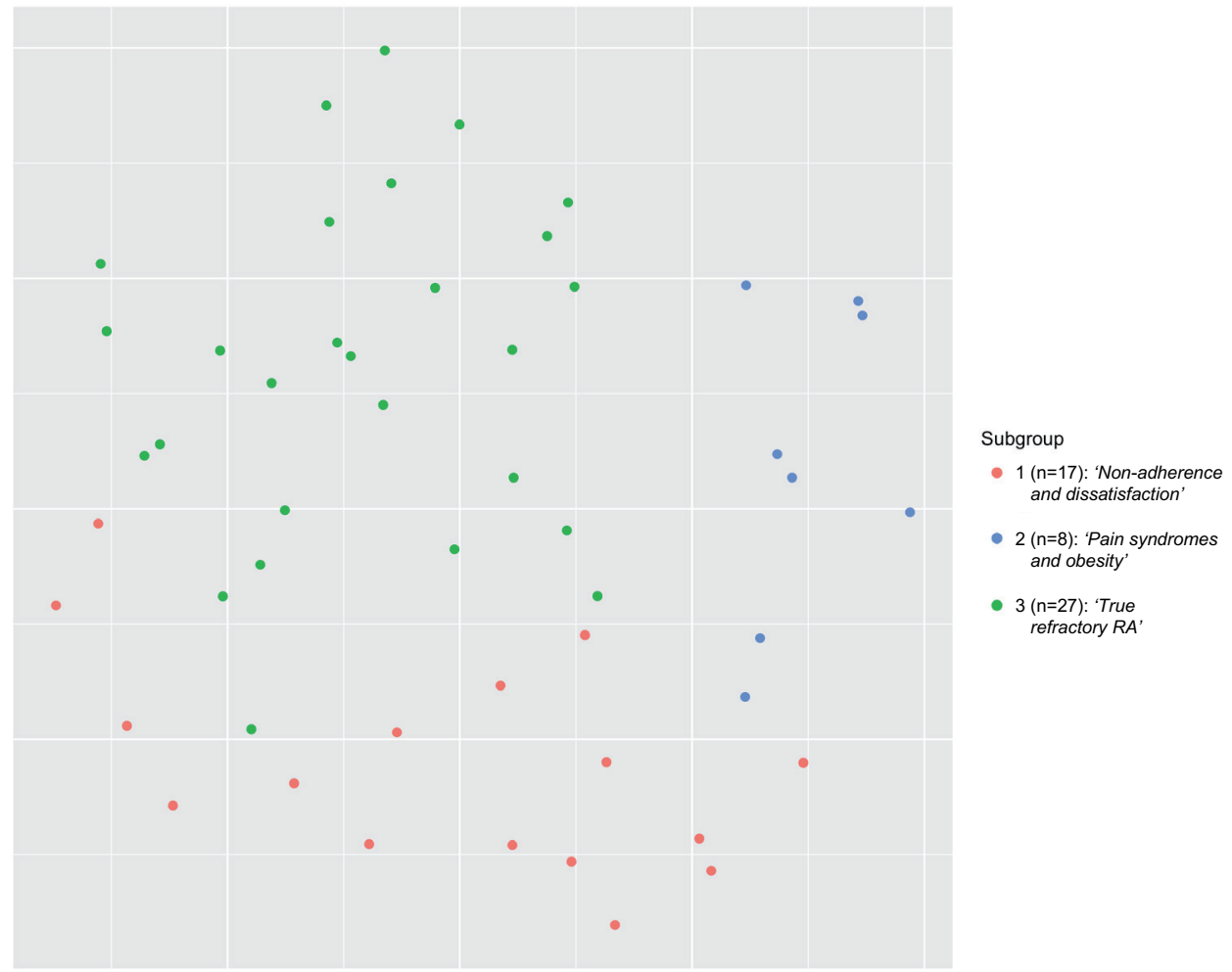

Supplementary Figure 3. Cluster plot of three subgroups of D2T RA

Visualisation in a lower dimensional space of three subgroups of D2T RA, using Gower distances in partitioning around medoids clustering.

D2T: difficult-to-treat; n: number; RA: rheumatoid arthritis. 
Supplementary Table 1. Contributing factors (independently) associated with D2T RA

\begin{tabular}{|c|c|c|c|}
\hline & $\begin{array}{c}\text { Univariable OR } \\
\text { (95\%Cl, p-value) }\end{array}$ & $\begin{array}{l}\text { Multivariable OR } \\
\text { (95\%Cl, p-value)' }\end{array}$ & $\begin{array}{c}\text { Penalised } \\
\mathrm{OR}^{2}\end{array}$ \\
\hline Current smoking & $1.93(0.76-4.89,0.17)$ & $2.86(0.71-11.47,0.14)$ & - \\
\hline Higher level of alcohol use, units/week & $0.96(0.89-1.02,0.19)$ & $0.92(0.86-0.99,0.03)$ & - \\
\hline Higher BMl & $1.02(0.95-1.09,0.60)$ & $0.98(0.89-1.09,0.75)$ & - \\
\hline Concomitant gouty arthritis/CPPD & $3.13(0.84-11.64,0.09)$ & $1.38(0.21-8.88,0.74)$ & - \\
\hline \multicolumn{4}{|l|}{ Limited drug options because of: } \\
\hline$-\mathrm{AES}$ & $12.32(3.60-42.20,<0.001)$ & $7.42(1.54-35.74,0.01)$ & 2.31 \\
\hline - Comorbidities $\#$ & $3.83(1.87-7.83,<0.001)$ & $3.51(1.28-9.64,0.02)$ & 1.48 \\
\hline \multicolumn{4}{|l|}{ Non-adherence: } \\
\hline $\begin{array}{l}\text { - Higher level of non-adherence according to } \\
\text { MARS-5 }\end{array}$ & $1.12(0.97-1.32,0.12)$ & $1.27(1.01-1.61,0.04)$ & - \\
\hline $\begin{array}{l}\text { - Discrepancy in prescribed and supplied } \\
\text { DMARDs }\end{array}$ & $2.52(1.21-5.24,0.01)$ & $1.51(0.50-4.59,0.47)$ & - \\
\hline \multicolumn{4}{|l|}{$\begin{array}{l}\text { Mismatch between patient and rheumatologist } \\
\text { in wish to intensify treatment: }\end{array}$} \\
\hline - Wish patient to intensify treatment & $9.02(3.32-24.52,<0.001)$ & $7.59(2.10-27.38,<0.01)$ & 2.30 \\
\hline - Wish rheumatologist to intensify treatment & $1.57(0.55-4.50,0.40)$ & $3.53(0.74-16.74,0.11)$ & - \\
\hline Osteoarthritis & $1.57(0.73-3.39,0.25)$ & $1.63(0.52-5.07,0.40)$ & - \\
\hline Subluxation & $1.28(0.57-2.89,0.55)$ & $1.56(0.49-4.92,0.45)$ & - \\
\hline Fibromyalgia & $6.32(2.61-15.29,<0.001)$ & $3.18(0.95-10.60,0.06)$ & 1.83 \\
\hline Depression & $4.32(1.23-15.11,0.02)$ & $1.23(0.14-10.56,0.85)$ & - \\
\hline Anxiety & $2.75(0.96-7.88,0.06)$ & $1.41(0.24-8.26,0.70)$ & - \\
\hline \multicolumn{4}{|l|}{ Poor coping: } \\
\hline - Higher level of helplessness (ICQ) & $1.19(1.09-1.29,<0.001)$ & $0.94(0.78-1.14,0.55)$ & - \\
\hline - Higher level of threatening view of illness (IPQ) & $1.08(1.04-1.11,<0.001)$ & $1.08(1.00-1.16,0.05)$ & 1.02 \\
\hline
\end{tabular}

BMl: body mass index; CPPD: calcium pyrophosphate deposition disease; D2T: difficult-to-treat; DMARD: diseasemodifying antirheumatic drug; ICQ: illness cognition questionnaire; IPQ: illness perception questionnaire; IQR: interquartile range; MARS-5: medication adherence reporting scale; n: number; OR: odds ratio; \#: Recurrent infections, malignancies, moderate to severe heart failure, moderate to severe renal insufficiency, moderate to severe liver failure.

Level of treatment non-adherence according to medication adherence reporting scale (5-25, higher score reflects higher level of adherence).31 Fibromyalgia according to ACR 2010 criteria. ${ }^{30}$ Depression and anxiety according to hospital anxiety and depression scale (0-2l, score $>10$ reflects probable depression or anxiety). ${ }^{32}$ Level of illness perception according to IIIness Perception Questionnaire (0-80, higher score reflects more threatening view of illness)..3 Level of helplessness according to Illness Cognition Questionnaire for chronic diseases (6-24, higher score reflects higher level of helplessness). ${ }^{34}$

Analyses using: 1. Multivariable logistic regression, model is overfitted due to the number of variables in relation to the sample size; 2. Penalised logistic regression by Lasso method. 
Supplementary Table 2. Factors contributing to the disease state in subgroups of D2T RA

\begin{tabular}{|c|c|c|c|}
\hline & $\begin{array}{c}\text { Subgroup } 1 \\
(\mathrm{n}=17) \\
\text { 'Non-adherence } \\
\text { and dissatisfaction' }\end{array}$ & 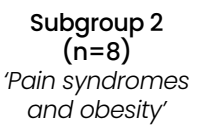 & $\begin{array}{l}\text { Subgroup } 3 \\
(\mathrm{n}=27) \\
\text { 'True } \\
\text { refractory } R A \text { ' }\end{array}$ \\
\hline Smoking, \% & 6 & 75 & 11 \\
\hline Alcohol use, number per week & $0(1-1)$ & $0(0-1)$ & $0(0-1)$ \\
\hline Overweight or obesity, \% & 53 & 100 & 56 \\
\hline BMl & $25(22-30)$ & $30(27-35)$ & $26(21-31)$ \\
\hline Presence of concomitant gouty arthritis/CPPD, \% & 18 & 13 & 7 \\
\hline \multicolumn{4}{|l|}{ Presence of limited drug options because of: } \\
\hline - Adverse events, \% & 100 & 88 & 93 \\
\hline - Comorbidities\#, \% & 77 & 50 & 70 \\
\hline \multicolumn{4}{|l|}{ Treatment non-adherence, according to: } \\
\hline - Level of MARS- 5 & $25(22-25)$ & $23(20-23)$ & $24(22-25)$ \\
\hline $\begin{array}{l}\text { - Discrepancy in prescribed and supplied DMARDs and } \\
\text { GCs, \% }\end{array}$ & 71 & 25 & 26 \\
\hline \multicolumn{4}{|l|}{$\begin{array}{l}\text { Mismatch between patient and rheumatologist in wish } \\
\text { to intensify treatment: }\end{array}$} \\
\hline - Patient's wish to intensify, and not rheumatologist, \% & 82 & 0 & 19 \\
\hline - Rheumatologist's wish to intensify, and not patient, \% & 0 & 0 & 26 \\
\hline Secondary osteoarthritis, \% & 71 & 75 & 81 \\
\hline Subluxation, \% & 23 & 13 & 26 \\
\hline Fibromyalgia, \% & 24 & 75 & 37 \\
\hline Depression, probable, \% & 35 & 25 & 0 \\
\hline Level & $10(4-12)$ & $10(9-14)$ & $4(2-6)$ \\
\hline Anxiety, probable, \% & 24 & 50 & 4 \\
\hline Level & $8(4-11)$ & $11(9-13)$ & $3(2-7)$ \\
\hline \multicolumn{4}{|l|}{ Poor coping strategies: } \\
\hline - Level of helplessness (ICQ) & $17(13-19)$ & $18(15-20)$ & $11(9-13)$ \\
\hline - Level of threatening view of illness (IPQ) & $55(45-60)$ & $55(49-65)$ & $39(31-47)$ \\
\hline
\end{tabular}

BMl: body mass index; CPPD: calcium pyrophosphate deposition disease; D2T: difficult-to-treat; DMARD: diseasemodifying antirheumatic drug; ICQ: illness cognition questionnaire; IPQ: illness perception questionnaire; IQR: interquartile range; MARS-5: medication adherence reporting scale; n: number; OR: odds ratio; \#: Recurrent infections, malignancies, moderate to severe heart failure, moderate to severe renal insufficiency, moderate to severe liver failure.

Overweight/obesity according to BMl above 25. Level of treatment non-adherence according to medication adherence reporting scale (5-25, higher score reflects higher level of adherence). ${ }^{31}$ Fibromyalgia according to ACR 2010 criteria. $^{30}$ Depression and anxiety according to hospital anxiety and depression scale (0-21, score >10 reflects probable depression or anxiety). ${ }^{2}$ Level of illness perception according to Illness Perception Questionnaire (0-80, higher score reflects more threatening view of illness)..$^{33}$ Level of helplessness according to Illness Cognition Questionnaire for chronic diseases (6-24, higher score reflects higher level of helplessness). ${ }^{34}$ 
Supplementary Table 3. Patient characteristics of subgroups of D2T RA

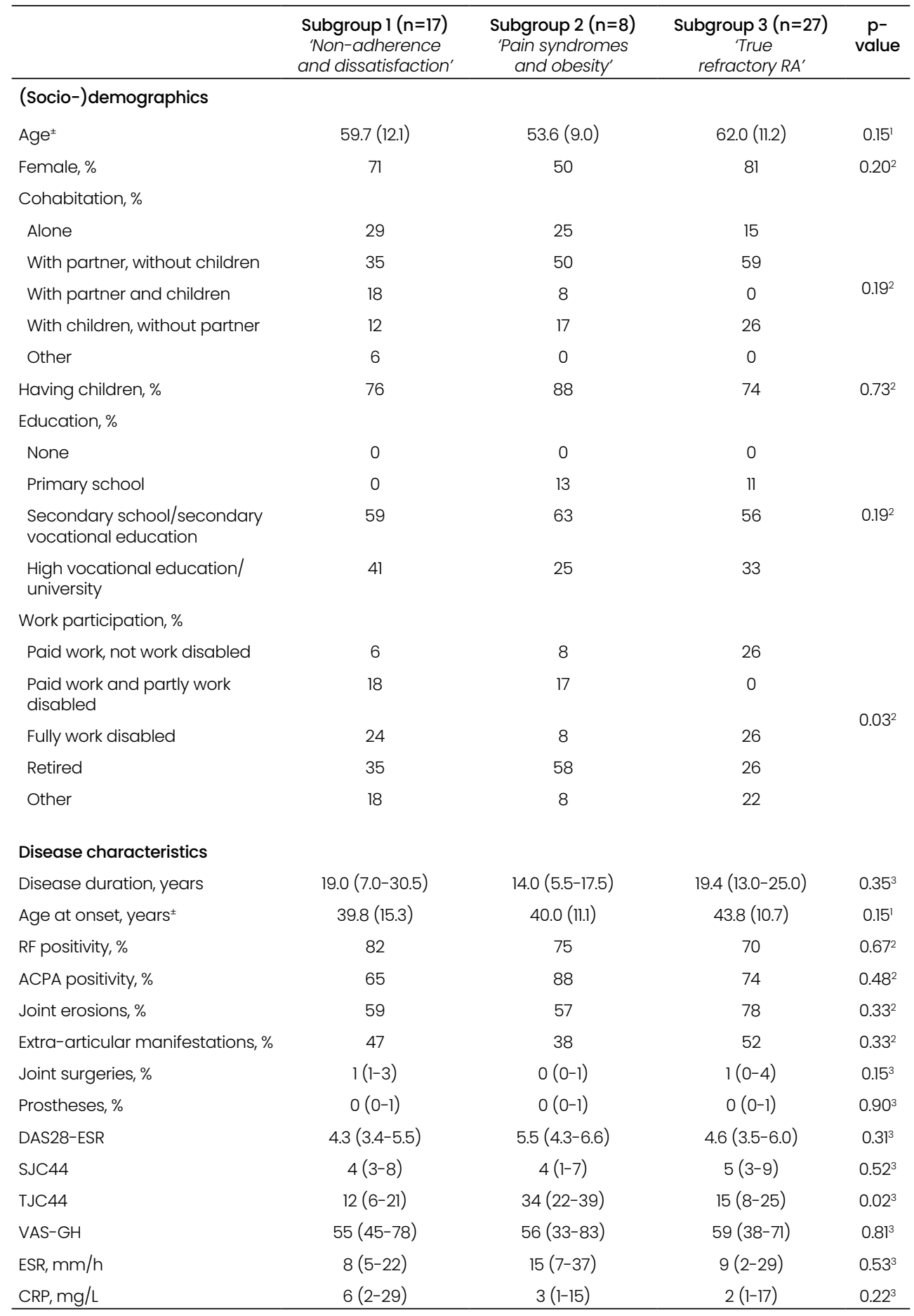


Supplementary Table 3. Continued

\begin{tabular}{|c|c|c|c|c|}
\hline & $\begin{array}{l}\text { Subgroup } 1(n=17) \\
\text { 'Non-adherence } \\
\text { and dissatisfaction' }\end{array}$ & $\begin{array}{l}\text { Subgroup } 2(\mathrm{n}=8) \\
\text { 'Pain syndromes } \\
\text { and obesity' }\end{array}$ & $\begin{array}{c}\text { Subgroup } 3(n=27) \\
\text { 'True } \\
\text { refractory } R A^{\prime}\end{array}$ & $\begin{array}{c}\mathrm{p}- \\
\text { value }\end{array}$ \\
\hline \multicolumn{5}{|l|}{ Comorbidities } \\
\hline $\begin{array}{l}\text { Number according to EULAR } \\
\text { domains }^{28}\end{array}$ & $2(2-3)$ & $2(1-3)$ & $2(1-2)$ & $0.12^{3}$ \\
\hline Index according to $\mathrm{CCl}^{29}$ & $64(60-73)$ & $56(52-64)$ & $63(60-77)$ & $0.13^{3}$ \\
\hline \multicolumn{5}{|c|}{$\begin{array}{l}\text { ACPA: anti-citrullinated protein antibodies; CRP: reactive protein C; D2T: difficult-to-treat; DAS28-ESR: disease } \\
\text { activity score assessing } 28 \text { joints using erythrocyte sedimentation rate; ESR: erythrocyte sedimentation rate; mg: } \\
\text { milligram; n: number; RF: rheumatoid factor; SJC44: swollen joint count assessing } 44 \text { joints; TJC44: tender joint count } \\
\text { assessing } 44 \text { joints; VAS-GH: visual analogue score assessing global health (0-100, higher score reflects worse } \\
\text { global health). }\end{array}$} \\
\hline
\end{tabular}





\section{CHAPTER 11}

\section{Health care utilisation and economic burden of difficult-to-treat rheumatoid arthritis: a cost of illness study}

Rheumatology 2021. doi: 10.1093/rheumatology/keab078 [Epub ahead of print 27 Jan 2021]

Nadia M.T. Roodenrijs, Paco M.J. Welsing, Marlies C. van der Goes, Janneke Tekstra, Floris P.J.G. Lafeber, Johannes W.G. Jacobs, Jacob M. van Laar 


\section{Abstract}

\section{Objectives}

To determine the impact of difficult-to-treat rheumatoid arthritis (D2T RA) on (costs related to) health care utilisation, other resource use and work productivity.

\section{Methods}

Data regarding health care utilisation, other resource use and work productivity of 52 D2T (according to the EULAR definition) and 100 non-D2T RA patients were collected via a questionnaire and an electronic patient record review during a study visit. Annual costs were calculated and compared between groups. Multivariable linear regression analysis was performed to assess whether having D2T RA was associated with higher costs.

\section{Results}

Mean (95\% confidence interval) annual total costs were $€ 37,605(€ 27,689-50,378)$ for D2T and $€ 19,217$ ( $€ 15,647-22,945)$ for non-D2T RA patients $(p<0.001)$. D2T RA patients visited their rheumatologist more frequently, were more often admitted to day care facilities, underwent more laboratory tests and used more drugs (specifically tsDMARDs), compared with non-D2T RA patients ( $p<0.01$ ). In D2T RA patients, the main contributors to total costs were informal help of family and friends $(28 \%)$, drugs (26\%) and loss of work productivity (16\%).

After adjustment for physical functioning (health assessment questionnaire (HAQ)), having D2T RA was no longer statistically significantly associated with higher total costs. HAQ was the only independent determinant of higher costs in multivariable analysis.

\section{Conclusions}

The economic burden of D2T RA is significantly higher than that of non-D2T RA, indicated by higher health care utilisation and higher annual total costs. Functional disability is a key determinant of higher costs in RA. 


\section{Introduction}

Health care resource use, including that for rheumatoid arthritis (RA), is high and rising. Nowadays, treat-to-target and tight control treatment strategies have led to a higher proportion of RA patients using biological and targeted synthetic disease-modifying antirheumatic drugs (b/tsDMARDs), especially in earlier phases of their disease. ${ }^{2-4}$ Additionally, diagnostic modalities, such as ultrasonography and MRI, are increasingly implemented in clinical practice. ${ }^{5}$ Although the introduction of these management strategies comes with additional costs, clinical outcomes for the majority of RA patients have significantly improved over the past decades. ${ }^{6,7}$ Concurrently, costs related to work productivity loss (often referred to as indirect costs) due to the morbidity of the individual or their relatives may have decreased.

In a recent systematic literature review (SLR) on the cost-of-illness of RA in the United States, annual costs directly related to health care utilisation were estimated at $\$ 12,509$ for all RA patients, annual costs for bDMARDs users were even higher with $\$ 36,053 .{ }^{8}$ Another recent SLR regarding costs in the bDMARD era across the world found annual costs directly related to health care utilisation and other resource use (e.g. travel costs to medical facilities; the combination of these costs is often referred to as direct costs) ranging from $\$ 401$ to even $\$ 67,306$ per RA patient. ${ }^{9}$ Additionally, annual total costs (i.e. health care utilisation, other resource use and productivity loss) were found to range from $\$ 2,407$ to a maximum of $\$ 83,845$ per RA patient. The hypothesis that costs related to productivity loss might have decreased due to higher work productivity could not be confirmed and only a shift in costs directly related to health care utilisation was found, namely higher costs related to drugs and lower costs related to hospitalisation. ${ }^{9}$

Despite the introduction of modern management strategies, there remains a significant proportion of RA patients that does not benefit from treatment with several b/tsDMARDs. An estimated $5 \%$ to $20 \%$ of RA patients can be classified as having difficult-to-treat (D2T) RA. ${ }^{10-13}$ D2T RA is often a multifactorial disease state: one or multiple contributing factors may lead to persistence of symptoms and/or signs suggestive of active disease." Three subgroups of D2T RA patients have previously been identified, reflecting the heterogeneity of this disease state: 1. 'non-adherent dissatisfied patients'; 2 . patients with 'pain syndromes and obesity'; 3. patients with RA closest to the concept of 'true refractory RA'.14 $^{\prime}$

In routine clinical practice, factors contributing to D2T RA are unclear in the majority of patients..$^{15}$ Therefore, changes in the treatment of individual D2T RA patients are generally based on trial-and-error, increasing the risk of unnecessary and inappropriate use of expensive b/tsDMARDs. Additionally, fibromyalgia, depression and anxiety as well as lower quality of life and functional disability have been found to be significantly more frequent in D2T than in non-D2T RA patients. ${ }^{14}$ These factors are known to be associated 
with increased health care costs. ${ }^{16-18}$ Hence, costs associated with D2T RA might be substantially higher than costs associated with non-D2T RA.

Knowledge on health care utilisation patterns and costs of D2T RA is relevant for policy makers, health insurance and pharmaceutical companies. ${ }^{9}$ More insight into cost-ofillness of D2T RA could underline the need for specific management strategies aiming to improve outcomes for D2T RA patients and to limit the economic burden. Cost-of-illness studies are also interesting to set priorities for care innovations. Additionally, they provide necessary input for economic evaluations of (new) interventions for this subpopulation of RA patients.

The aim of this study was to determine the impact of D2T RA on (costs related to) health care utilisation, other resource use and work productivity.

\section{Methods}

\section{Participants}

Consecutive RA outpatients, fulfilling the 2010 American College of Rheumatology/ European League Against Rheumatism (ACR/EULAR) classification criteria for RA ${ }^{19}$ and treated according to the current standard of care (treat-to-target) for at least one year, were recruited for a cross-sectional study into factors potentially contributing to D2T RA. Patients were enrolled from February 2019 to August 2020 at the Department of Rheumatology \& Clinical Immunology of the University Medical Center Utrecht, the Netherlands. Participating patients were classified as having D2T RA if they fulfilled all three criteria of the new EULAR definition, ${ }^{13}$ in short: 1. previous failure of $\geq 2 \mathrm{~b} / \mathrm{tsDMARDs}$ with different mechanisms of action; 2. symptoms and signs suggestive of active/ progressive disease; 3. management perceived as problematic by the rheumatologist and/or patient. The participating RA patients who did not meet all three D2T RA criteria served as a control group. Further details regarding participants, study procedures and contributing factors have been described previously. ${ }^{14}$

\section{Health care utilisation, other resource use and work productivity}

Information on patient and disease characteristics was collected during a single study visit. ${ }^{14}$ The utilisation of DMARDs, pain killers, and laboratory tests in the past year was extracted from the electronic patient record and checked with the participants during the study visit.

Additionally, participants filled out the 'Dutch health care utilisation and work participation questionnaire', as used in previous studies. ${ }^{16,20-23}$ The questionnaire includes the following categories regarding health care utilisation related to RA in the past three months: admission to health care facilities (hospital including surgeries and admissions to 
intensive care unit, rehabilitation center and nursing home), consultations with health care professionals (physicians and paramedics), diagnostic tests (imaging measures), help from a district nurse and formal home help. Furthermore, questions regarding other resource use in the past three months are included: devices needed to perform daily activities, informal help from family and friends and additional costs related to RA (e.g. complementary medicines, travel cost). The questionnaire also assesses work productivity, including work participation, absence due to illness in the past three months and being assessed as work disabled.

\section{Estimations of costs}

Costs (in euros) were estimated following the Methods and Guidelines for Economic Evaluations in Health Care using 2019 Dutch standard prices as much as possible (using the price index, Supplementary Table 1-2). ${ }^{20}$ Costs were calculated from the societal perspective, i.e. not limited to reimbursed costs (as would be the health insurance perspective). ${ }^{20}$ For devices needed to perform daily activities, adaptations in and around the house and additional costs, patient-reported prices were used. For informal help from family and friends, the price of home help was used as a surrogate price for replacement. The costs in the past three months were multiplied by 4 to estimate the annual costs. For medication, the official drug prices were used and in case of multiple prices per drug, the lowest price was used (Supplementary Table 3). ${ }^{20}$

Additionally, costs of productivity loss were calculated..$^{20}$ Absence due to illness was classified as incidental or structural absence. For incidental absence, the exact number of days was used (multiplied by 4 to reflect annual absence) and costs were calculated using Dutch average wages. For structural absence, costs were estimated using the friction cost method. This method is based on the assumption that the costs of structural productivity loss are only incurred during the friction period (85 days in 2019), which is the time period that is needed to fully replace the individual. For patients assessed as becoming work disabled during the past year, productivity loss was also calculated using the friction method following the same method as for structural absence. For these patients, the costs of a disability pension (i.e. minimum wage) were added for the remaining days (i.e. 365 - 85 (friction period) $=280$ days). For patients who were work disabled for more than one year, no productivity loss was applied following the assumption that these patients' productivity had already been replaced. For these patients, the annual costs of a disability pension were used (Supplementary Table 1). ${ }^{9}$

\section{Statistical analyses}

Patient characteristics and different aspects of health care utilisation, other resource use and work productivity as well as related total costs were evaluated using descriptive statistics. As utilisation and costs were highly skewed, bootstrapping (1000 drawings) was 
used to estimate a mean value with 95\% confidence interval (Cl). Additionally, median (interquartile range (IQR)) was reported. Differences in these variables between D2T and non-D2T RA patients were tested for statistical significance using independent T-, MannWhitney $U$, Fisher's exact or chi-square test, as appropriate. Differences in total costs between the three previously identified subgroups of D2T RA were tested for statistical significance using Kruskal-Wallis test. ${ }^{14}$

Furthermore, multivariable linear regression was used to determine whether D2T RA patients incurred higher total costs (total costs: dependent variable) than non-D2T patients, correcting for population characteristics (i.e. age, gender, disease duration, number of comorbidities: independent variables). As total costs were highly skewed, natural log transformation was performed. Additionally, an analysis adjusted for previously identified determinants of costs (i.e. disease activity score, physical functioning, quality of life, presence of fibromyalgia, depression or anxiety) ${ }^{16-18}$ was performed to evaluate whether an association with higher costs remained. If determinants were statistically significantly associated with higher costs $(p<0.05)$, it was also assessed whether their influence was different for D2T and non-D2T RA patients by adding interaction terms to the model.

Two-sided tested $p$-values $<0.05$ were considered statistically significant. Due to limited missing data (Tables 1-4), no imputation was performed. Due to the exploratory design of the study, analyses were not corrected for multiple testing. ${ }^{24-26}$ Statistical analyses were performed using IBM SPSS Statistics version 24 (IBM Corp, Armonk, NY, USA).

\section{Results}

\section{Patient characteristics}

Of the enrolled patients, 52 had D2T RA and 100 non-D2T RA. Most patient characteristics did not differ between D2T and non-D2T RA patients (Table 1, as described previously ${ }^{14}$ ). DAS28-ESR was significantly higher in D2T RA patients, mirroring the D2T RA definition (median (IQR): D2T 4.1 (3.5-5.9), non-D2T 2.5 (1.8-3.3), p<0.001). A higher number of comorbidities was found in D2T RA patients, as well as a higher clinical burden.

\section{Health care utilisation}

During the past three months, D2T RA patients visited their rheumatologist more frequently than non-D2T RA patients (mean number of visits (95\%Cl): D2T 1.6 (1.4-1.9), non-D2T 1.2 (1.11.3), $\mathrm{p}<0.01$, Table 2). Additionally, D2T RA patients were more often admitted to day care facilities (mean number of days (95\%Cl): D2T 0.6 (0.3-0.9), non-D2T 0.2 (0.1-0.3), p<0.01), and underwent more laboratory tests (mean (95\%Cl): D2T 2.1 (1.6-2.7), non-D2T 1.5 (1.3-1.7), $\mathrm{p}<0.01)$, compared with non-D2T RA patients. 
Table 1. Patient characteristics: (socio-)demographics and disease characteristics

\begin{tabular}{|c|c|c|c|}
\hline & D2T RA $(n=52)$ & Non-D2T RA $(n=100)$ & $\mathrm{p}$-value \\
\hline \multicolumn{4}{|l|}{ (Socio-)demographics } \\
\hline $\mathrm{Age}^{ \pm}$ & $60.2(11.4)$ & $64.5(10.9)$ & $0.03^{1}$ \\
\hline Female, \% & 73 & 72 & $1.00^{2}$ \\
\hline \multicolumn{4}{|l|}{ Cohabitation, \% } \\
\hline Alone & 21 & 20 & \multirow{5}{*}{$0.55^{3}$} \\
\hline With partner, without children & 46 & 55 & \\
\hline With partner and children & 23 & 20 & \\
\hline With children, without partner & 8 & 5 & \\
\hline Other & 2 & 0 & \\
\hline \multicolumn{4}{|l|}{ Education, \% } \\
\hline None & 2 & $0 \ddagger$ & \multirow{4}{*}{$0.13^{3}$} \\
\hline Primary school & 6 & 2 & \\
\hline Secondary school/secondary vocational education & 58 & 49 & \\
\hline High vocational education/university & 34 & 49 & \\
\hline \multicolumn{4}{|l|}{ Disease characteristics } \\
\hline Disease duration, years & $17.0(9.0-25.0)$ & $14.0(8.0-24.0)$ & $0.33^{4}$ \\
\hline RF positivity, \% & 75 & 65 & $0.27^{2}$ \\
\hline ACPA positivity, $\%$ & 73 & 65 & $0.36^{2}$ \\
\hline Presence of joint erosions, $\%$ & 69 & 59 & $0.29^{2}$ \\
\hline DAS28-ESR & $4.1(3.5-6.1)$ & $2.5(1.8-3.3)$ & $<0.001^{4}$ \\
\hline Physical functioning (HAQ), level ${ }^{\#}$ & $1.8(1.4-2.1)$ & $1.0(0.5-1.4)$ & $<0.001^{4}$ \\
\hline \multicolumn{4}{|l|}{ Quality of Life (EQ-5D-5L)\# } \\
\hline Index (Dutch tariff) & $0.62(0.31-0.77)$ & $0.81(0.68-0.85)$ & $<0.001^{4}$ \\
\hline VAS & $51(26-65)$ & $75(60-85)$ & $<0.001^{4}$ \\
\hline Comorbidities, number according to EULAR domains ${ }^{27}$ & $2(1-3)$ & $1(0-1)$ & $<0.001^{4}$ \\
\hline Fibromyalgia, \% & 38 & 9 & $<0.001^{2}$ \\
\hline Depression, probable, \%\# & 15 & 4 & $0.02^{2}$ \\
\hline Level & $6(3-10)$ & $2(1-5)$ & $<0.001^{4}$ \\
\hline Anxiety, probable, \%\# & 17 & 7 & $0.09^{2}$ \\
\hline Level & $7(2-9)$ & $4(2-7)$ & $0.02^{4}$ \\
\hline
\end{tabular}

ACPA: anti-citrullinated protein antibodies; D2T: difficult-to-treat; DAS28-ESR: disease activity score assessing 28 joints using erythrocyte sedimentation rate; EQ-5D: Euro Quality of Life using 5 Dimensions (0-1, higher score reflects higher quality of life);28 EULAR: European League Against Rheumatism; HAQ: health assessment questionnaire (0-3, higher score reflects higher level of functional disability); ${ }^{29} \mathrm{n}$ : number; RF: rheumatoid factor; VAS: visual analogue scale (0-100, higher score reflects better quality of life); \#: missing for 1 non-D2T RA patient.

Fibromyalgia according to ACR 2010 criteria. $^{30}$ Depression and anxiety according to hospital anxiety and depression scale (0-21, score >10 reflects probable depression or anxiety).31

Numbers are median (interquartile range), unless stated otherwise; $\ddagger$ : mean (standard deviation). Differences between groups were analysed using: 1. Independent T-test; 2. Fisher's exact test; 3. Chi-square test; 4. MannWhitney U test. 
Table 2. Health care utilisation during the past three months

\begin{tabular}{|c|c|c|c|c|c|}
\hline & \multicolumn{2}{|c|}{ D2T RA $(n=52)$} & \multicolumn{2}{|c|}{ Non-D2T RA $(n=100)$} & \multirow{2}{*}{$\begin{array}{c}\mathrm{p}- \\
\text { value }\end{array}$} \\
\hline & Mean $(95 \% \mathrm{Cl})$ & Median (IQR)^ & Mean $(95 \% \mathrm{Cl})$ & Median (IQR)^ & \\
\hline \multicolumn{6}{|l|}{ Consultations with $\mathrm{HCPs}^{\#}$} \\
\hline Rheumatologist, number & $1.6(1.4-1.9)$ & $1.0(1.0-2.0)$ & $1.2(1.1-1.3)$ & $1.0(1.0-1.0)$ & $<0.01^{1}$ \\
\hline Rheumatology nurse, number & $0.5(0.4-0.8)$ & $0.0(0.0-1.0)$ & $0.5(0.4-0.7)$ & $0.0(0.0-1.0)$ & $0.56^{1}$ \\
\hline General practitioner, number & $1.0(0.7-1.4)$ & $1.0(0.0-2.0)$ & $1.1(0.8-1.4)$ & $1.0(0.0-2.0)$ & $0.83^{1}$ \\
\hline Other medical specialist, number & $2.3(1.4-3.3)$ & $1.0(0.0-3.0)$ & $2.2(1.7-2.7)$ & $2.0(0.0-3.0)$ & $0.84^{1}$ \\
\hline Other paramedic, number & $5.1(3.1-7.4)$ & $1.5(0.0-8.5)$ & $4.7(3.1-6.6)$ & $0.0(0.0-6.0)$ & $0.61^{1}$ \\
\hline Psychosocial support, number & $0.9(0.3-1.7)$ & $0.0(0.0-0.0)$ & $0.6(0.2-1.1)$ & $0.0(0.0-0.0)$ & $0.23^{1}$ \\
\hline District nurse, hours/week & $3.6(0.8-7.0)$ & $0.0(0.0-0.0)$ & $1.6(0.0-4.1)$ & $0.0(0.0-0.0)$ & 0.091 \\
\hline Formal house help, hours/week & $12.3(7.0-18.5)$ & $0.0(0.0-26.0)$ & $10.5(6.1-15.9)$ & $0.0(0.0-0.0)$ & $0.28^{1}$ \\
\hline \multicolumn{6}{|l|}{ Admission to health care facilities" } \\
\hline \multicolumn{6}{|l|}{ Hospital, days } \\
\hline General ward & $1.5(0.1-4.1)$ & $0.0(0.0-0.0)$ & $0.2(0.0-0.4)$ & $0.0(0.0-0.0)$ & $0.15^{1}$ \\
\hline Intensive care unit & $0.0(0.0-0.0)$ & $0.0(0.0-0.0)$ & $0.0(0.0-0.1)$ & $0.0(0.0-0.0)$ & $0.31^{1}$ \\
\hline Day care & $0.6(0.3-0.9)$ & $0.0(0.0-1.0)$ & $0.2(0.1-0.3)$ & $0.0(0.0-0.0)$ & $<0.01$ \\
\hline $\begin{array}{l}\text { Rehabilitation center or nursing home, } \\
\text { days }\end{array}$ & $0.0(0.0-0.0)$ & $0.0(0.0-0.0)$ & $0.0(0.0-0.0)$ & $0.0(0.0-0.0)$ & $1.00^{1}$ \\
\hline Surgery, number & $0.0(0.0-0.0)$ & $0.0(0.0-0.0)$ & $0.0(0.0-0.1)$ & $0.0(0.0-0.0)$ & $0.15^{1}$ \\
\hline \multicolumn{6}{|l|}{ Diagnostic tests } \\
\hline $\begin{array}{l}\text { Laboratory tests, number of } \\
\text { administrations }\end{array}$ & $2.1(1.6-2.7)$ & $2.0(1.0-2.0)$ & $1.5(1.3-1.7)$ & $1.0(1.0-2.0)$ & $<0.01^{\prime}$ \\
\hline \multicolumn{6}{|l|}{ Imaging measures, number"\# } \\
\hline - x-ray & $1.5(1.0-2.0)$ & $1.0(0.0-2.0)$ & $1.4(1.1-1.6)$ & $1.0(0.0-2.0)$ & $0.90^{1}$ \\
\hline - Ultrasonography & $0.4(0.2-0.7)$ & $0.0(0.0-1.0)$ & $0.4(0.2-0.6)$ & $0.0(0.0-0.0)$ & $0.17^{1}$ \\
\hline - СТ & $0.3(0.1-0.5)$ & $0.0(0.0-0.0)$ & $0.1(0.0-0.2)$ & $0.0(0.0-0.0)$ & 0.09 \\
\hline$-M R I$ & $0.3(0.1-0.5)$ & $0.0(0.0-0.0)$ & $0.1(0.0-0.2)$ & $0.0(0.0-0.0)$ & $0.17^{1}$ \\
\hline \multicolumn{6}{|l|}{ Drugs } \\
\hline DMARDs, number & $1.7(1.4-1.9)$ & $2.0(1.0-2.0)$ & $1.5(1.3-1.6)$ & $1.0(1.0-2.0)$ & 0.071 \\
\hline $\operatorname{csDMARD}(\mathrm{s}), \%$ & \multicolumn{2}{|c|}{71} & \multicolumn{2}{|c|}{86} & $0.03^{2}$ \\
\hline bDMARD, \% & \multicolumn{2}{|c|}{52} & \multicolumn{2}{|c|}{39} & $0.17^{2}$ \\
\hline tsDMARD, \% & \multicolumn{2}{|c|}{23} & \multicolumn{2}{|c|}{0} & $<0.01^{2}$ \\
\hline Glucocorticoid therapy, \% & \multicolumn{2}{|c|}{52} & \multicolumn{2}{|c|}{16} & $<0.001^{2}$ \\
\hline Pain killers, \% & \multicolumn{2}{|c|}{94} & \multicolumn{2}{|c|}{64} & $<0.001^{2}$ \\
\hline Regular use of paracetamol, $\%$ & \multicolumn{2}{|c|}{25} & \multicolumn{2}{|c|}{11} & $0.03^{2}$ \\
\hline Regular use of NSAIDs, $\%$ & \multicolumn{2}{|c|}{40} & \multicolumn{2}{|c|}{19} & $<0.01^{2}$ \\
\hline Regular use of opioids, $\%$ & \multicolumn{2}{|c|}{12} & \multicolumn{2}{|c|}{5} & $0.19^{2}$ \\
\hline
\end{tabular}

b: biological; Cl: confidence interval; cs: conventional synthetic; CT: computed tomography; D2T: difficult-totreat; DMARD: disease-modifying antirheumatic drug; HCP: health care professional; IQR: interquartile range; MRI: magnetic resonance imaging; n: number; NSAIDs: non-steroidal anti-inflammatory drugs; RA: rheumatoid arthritis; ts: targeted synthetic; ${ }^{*}$ : missing for 1 non-D2T RA patient; ${ }^{\wedge}$ : unless percentages are shown. 
Table 2. Continued

Other paramedic: physiotherapist, hydrotherapist, occupational therapist, exercise therapist, podiatrist; Psychosocial support: Social worker, psychologist, psychotherapist, psychiatrist.

Differences between groups were analysed using: 1. Mann-Whitney $U$ test; 2 . Fisher's exact test. $95 \% \mathrm{Cl}$ is based on bootstrapping. P-values should be interpreted with caution, given the high number of tests without correction for multiple testing.

CSDMARDs, which in general are the cheapest DMARDs, were more frequently prescribed to non-D2T than to D2T RA patients ( $86 \%$ vs $71 \%$, respectively, $p=0.03$ ), while the more expensive tsDMARDs were only prescribed to D2T RA patients. Other health care utilisation did not differ statistically significantly between the groups (Table 2).

\section{Work productivity}

Thirty-seven D2T and 55 non-D2T RA patients were of working age (i.e. younger than 67 years). Of these patients, $43 \%$ of D2T and 31\% of non-D2T RA patients were fully work disabled $(p=0.23)$, for a median of 13 years (IQR 5-22) and 11 years (IQR 4-25), respectively $(p=0.81)$.

Of patients of working age, $45 \%$ of D2T and $44 \%$ of non-D2T RA patients had a paid job, although $30 \%$ and $8 \%$ of these patients, respectively, were still partially work disabled. D2T RA patients with a paid job and who were not partially work disabled worked a median of 24 hours a week (IQR 20-40) and non-D2T patients 30 hours (IQR 20-38, p=0.66). Of patients with a paid job, $20 \%$ of D2T and $34 \%$ of non-D2T RA patients had been absent from work during the past three months $(p=0.69)$, with a median number of 2 and 4 days absent, respectively $(p=0.04)$.

In none of D2T and in 2\% of non-D2T RA patients, the partner of the patient worked less hours a week to take care of the patient $(p=0.55)$. Further details on work productivity are shown in Supplementary Table 4.

\section{Estimations of costs}

Mean annual total costs $(95 \% \mathrm{Cl})$ were $€ 37,605$ (€27,689-50,378) for D2T and $€ 19,217$ ( $€ 15,647-22,945)$ for non-D2T RA patients ( $p<0.001$, Table 3, Figure 1). Costs directly related to health care utilisation and other resource use were significantly higher in D2T than in non-D2T RA patients.

In D2T RA patients, main contributors to total costs were informal help of family and friends (28\%), drugs (26\%) and costs related to productivity loss (16\%). In non-D2T RA patients, the same main contributors were found, although with a different ranking: drugs (27\%), costs related to productivity loss (24\%) and informal help of family and friends (17\%).

\section{Health care utilisation and costs in subgroups of D2T RA}

Health care utilisation and costs in the three subgroups of D2T RA that were identified 
previously (1. 'non-adherent dissatisfied patients'; 2. patients with 'pain syndromes and obesity'; 3. patients with RA that can be considered as closest to the concept of 'true refractory RA') ${ }^{14}$ are shown in Figure 1, Supplementary Table 5 and 6.

Total costs did not differ statistically significantly between subgroups of D2T RA (mean (95\%Cl)): subgroup 1 'non-adherent dissatisfied patients' €48,536 (€22,184-81,772),

Table 3. Estimations of annual mean costs in euros per patient, of having D2T RA or not

\begin{tabular}{|c|c|c|c|c|c|}
\hline & \multicolumn{2}{|c|}{ D2T RA $(n=52)$} & \multicolumn{2}{|c|}{ Non-D2T RA $(n=100)^{\#}$} & \multirow{2}{*}{$\begin{array}{c}\mathrm{p}- \\
\text { value }\end{array}$} \\
\hline & Mean $(95 \% \mathrm{Cl})$ & Median (IQR) & Mean $(95 \% \mathrm{Cl})$ & Median (IQR) & \\
\hline \multicolumn{6}{|l|}{$\begin{array}{l}\text { Costs directly related to health care } \\
\text { utilisation }(€)\end{array}$} \\
\hline $\begin{array}{l}\text { Consultations with health care } \\
\text { professionals }\end{array}$ & $\begin{array}{c}4077 \\
(2449-6075)\end{array}$ & $\begin{array}{c}1229 \\
(0-5121)\end{array}$ & $\begin{array}{l}2970(2016- \\
4175)\end{array}$ & $\begin{array}{c}846 \\
(0-3331)\end{array}$ & \\
\hline - $\quad$ Admission to health care facilities & $\begin{array}{c}3792 \\
(1041-9044)\end{array}$ & $\begin{array}{c}0 \\
(0-1296)\end{array}$ & $\begin{array}{l}1445(305- \\
2971)\end{array}$ & $\begin{array}{c}0 \\
(0-0)\end{array}$ & \\
\hline - Diagnostic tests & $\begin{array}{c}1524 \\
(953-2329)\end{array}$ & $\begin{array}{c}994 \\
(344-1422)\end{array}$ & $932(714-1209)$ & $\begin{array}{c}456 \\
(344-1068)\end{array}$ & \\
\hline - Drugs & $\begin{array}{c}10850 \\
(5748-13888)\end{array}$ & $\begin{array}{c}9727 \\
(8200-11262)\end{array}$ & $\begin{array}{l}5175(3873- \\
6474)\end{array}$ & $\begin{array}{c}745 \\
(435-11812)\end{array}$ & \\
\hline $\begin{array}{l}\text { Total costs directly related to health } \\
\text { care utilisation }\end{array}$ & $\begin{array}{c}19119 \\
(14972-24968)\end{array}$ & $\begin{array}{c}16256 \\
(10849-21908)\end{array}$ & $\begin{array}{c}10478 \\
(8289-12940)\end{array}$ & $\begin{array}{c}6943 \\
(1601-16612)\end{array}$ & $<0.001$ \\
\hline \multicolumn{6}{|l|}{ Costs related to other resource use $(€)$} \\
\hline $\begin{array}{l}\text { - Devices needed to perform daily } \\
\text { activities and adaptations in and } \\
\text { around the house }\end{array}$ & $\begin{array}{c}714 \\
(87-171)\end{array}$ & $\begin{array}{c}0 \\
(0-0)\end{array}$ & $\begin{array}{c}269 \\
(67-519)\end{array}$ & $\begin{array}{c}0 \\
(0-0)\end{array}$ & \\
\hline $\begin{array}{l}\text { - Informal help from family and } \\
\text { friends }\end{array}$ & $\begin{array}{c}10495 \\
(4924-18559)\end{array}$ & $\begin{array}{c}3886 \\
(0-11103)\end{array}$ & $\begin{array}{c}3236 \\
(2058-4661)\end{array}$ & $\begin{array}{c}0 \\
(0-3331)\end{array}$ & \\
\hline $\begin{array}{l}\text { - Additional costs (e.g. alternative } \\
\text { therapies and travel costs) }\end{array}$ & $\begin{array}{c}1254 \\
(663-1963)\end{array}$ & $\begin{array}{c}360 \\
(0-1133)\end{array}$ & $\begin{array}{c}629 \\
(411-902)\end{array}$ & $\begin{array}{c}150 \\
(0-524)\end{array}$ & \\
\hline $\begin{array}{l}\text { Total costs related to other resource } \\
\text { use }\end{array}$ & $\begin{array}{c}12463 \\
(6843-20491)\end{array}$ & $\begin{array}{c}5084 \\
(912-13314)\end{array}$ & $\begin{array}{c}4134 \\
(2869-5560)\end{array}$ & $\begin{array}{c}820 \\
(20-5251)\end{array}$ & $<0.001$ \\
\hline \multicolumn{6}{|l|}{ Costs related to productivity loss $(€)$} \\
\hline - Incidental absence due to illness & $57(10-161)$ & $0(0-0)$ & 650 (111-1497) & $0(0-0)$ & \\
\hline - $\quad$ Structural absence due to illness ${ }^{\ddagger}$ & $0(0-0)$ & $0(0-0)$ & $645(122-1416)$ & $0(0-0)$ & \\
\hline - Disability pension & $\begin{array}{c}5966 \\
(3556-8531)\end{array}$ & $\begin{array}{c}0 \\
(0-19390)\end{array}$ & $\begin{array}{c}3355 \\
(2003-4815)\end{array}$ & $\begin{array}{c}0 \\
(0-0)\end{array}$ & \\
\hline Total costs related to productivity loss & $\begin{array}{c}6023 \\
(1244-3633)\end{array}$ & $\begin{array}{c}0 \\
(0-19390)\end{array}$ & $\begin{array}{c}4646(2943- \\
6507)\end{array}$ & $\begin{array}{c}0 \\
(0-1692)\end{array}$ & 0.32 \\
\hline Total costs $(€)$ & $\begin{array}{c}37605 \\
(27689-50378)\end{array}$ & $\begin{array}{c}23991 \\
(14678-48109)\end{array}$ & $\begin{array}{c}19217 \\
(15647-22945)\end{array}$ & $\begin{array}{c}14107 \\
(3650-32361)\end{array}$ & $<0.001$ \\
\hline
\end{tabular}

Cl: confidence interval; D2T: difficult-to-treat; n: number; IQR: interquartile range; RA: rheumatoid arthritis; \#: missing for 1 non-D2T RA patient, except costs related to laboratory measures and drugs; ;: i.e. costs related to productivity loss using the friction cost method, only applicable to patients with structural absence (>85 consecutive days per year) due to illness or to those who became work disabled in the past year.

Differences between groups were analysed using Mann-Whitney U Test. 95\% Cl is based on bootstrapping. 


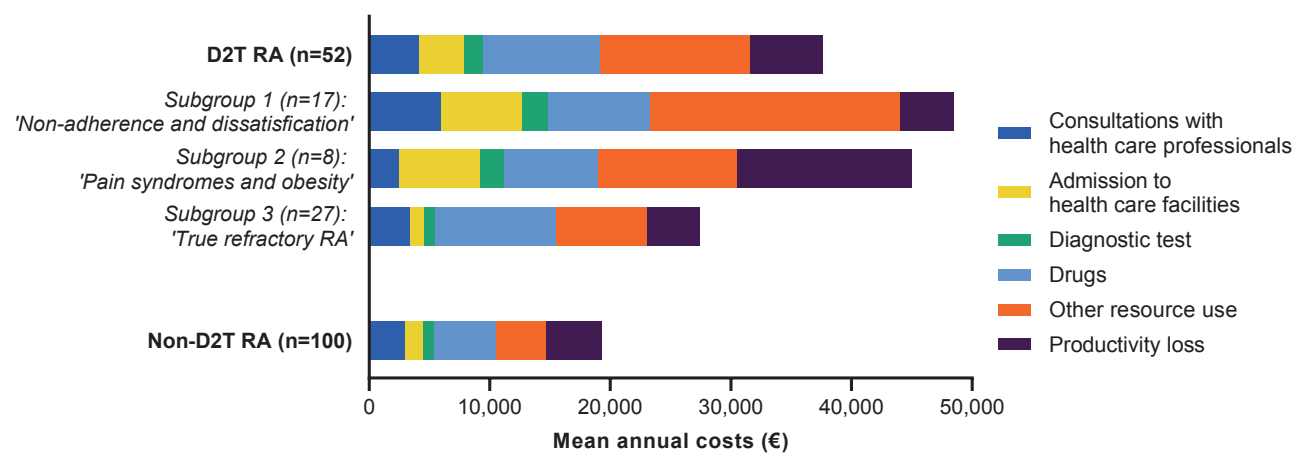

Figure 1. Estimations of annual mean costs in euros per patient, for D2T RA, its subgroups, and non-D2T RA patients D2T: difficult-to-treat; n: number; RA: rheumatoid arthritis.

subgroup 2 patients with 'pain syndromes and obesity' €48,689 (€36,378-60,710), subgroup 3 patients with RA closest to the concept of 'true refractory RA' $€ 27,438$ (€20,830-34,659), $p=0.07$ (Figure 1).

\section{Determinants of higher total costs}

In univariable linear regression analysis, having D2T RA was associated with higher total costs (Table 4). After adjustment for age, gender, disease duration and number of comorbidities, having D2T RA remained statistically significantly associated with higher total costs ( $B(95 \% \mathrm{Cl}, \mathrm{p})$ : 0.63 (0.17-1.09, <0.01), Supplementary Table 7).

No association between D2T RA and higher total costs was found after adjustment for disease activity score, physical functioning, quality of life, presence of concomitant fibromyalgia, depression and anxiety. Here, only functional disability (according to Health Assessment Questionnaire (HAQ)) remained independently associated with higher total costs ( $B(95 \% \mathrm{Cl}, \mathrm{p})$ : 0.99 (0.61-1.38, <0.001), Table 4). The association between HAQ and higher total costs did not differ statistically significantly between D2T and non-D2T RA patients, as indicated by the non-significant interaction between D2T and HAQ in the model $(p=0.91)$.

\section{Discussion}

In this cost-of-illness study, D2T RA patients were found to visit their rheumatologist more frequently, were more often admitted to day care facilities, underwent more laboratory tests and used more drugs, especially tsDMARDs. Consequently, cost-of-illness was found to be statistically significantly higher for D2T RA.

For D2T RA, mean annual total costs were estimated at €37,605 (equivalent to \$42,189), 
Table 4. Association between having D2T RA, higher total costs and previously identified determinants of higher costs

\begin{tabular}{lcc}
\hline & $\begin{array}{c}\text { Univariable analysis } \\
\beta(95 \% \mathrm{Cl}, \mathrm{p} \text {-value })\end{array}$ & $\begin{array}{c}\text { Multivariable analysis } \\
\beta(95 \% \mathrm{Cl}, \mathrm{p}-\mathrm{value})\end{array}$ \\
\hline D2T & $0.96(0.52-1.39,<0.001)$ & $0.23(-0.24-0.70,0.33)$ \\
DAS28-ESR, higher level & $0.31(0.18-0.43,<0.001)$ & $-0.13(-0.30-0.04,0.13)$ \\
Physical functioning (HAQ), a higher level of functional & $1.14(0.88-1.40,<0.001)$ & $0.99(0.61-1.38,<0.001)$ \\
disability & & \\
Quality of Life (EQ-5D-5L) & & \\
Index (Dutch tariff), lower level & $2.55(1.79-3.31,<0.001)$ & $0.73(-0.38-1.84,0.19)$ \\
Concomitant fibromyalgia & $1.04(0.50-1.57,<0.001)$ & $0.42(-0.10-0.93,0.11)$ \\
Depression & $1.09(0.31-1.87,<0.01)$ & $-0.02(-0.75-0.71,0.96)$ \\
Anxiety & $1.00(0.32-1.69,<0.01)$ & $0.26(-0.43-0.95,0.45)$ \\
\hline
\end{tabular}

ß: regression coefficient; Cl: confidence interval; D2T: difficult-to-treat; DAS28-ESR: disease activity score assessing 28 joints based on erythrocyte sedimentation rate; EQ-5D: Euro Quality of Life using 5 Dimensions (0-1, higher score reflects higher quality of life); ${ }^{28} \mathrm{HAQ}$ : health assessment questionnaire $(0-3$, higher score reflects higher level of functional disability):;9 RA: rheumatoid arthritis.

(Concomitant) fibromyalgia according to ACR 2010 criteria. ${ }^{30}$ Depression and anxiety according to hospital anxiety and depression scale (0-21, score $>10$ reflects probable depression or anxiety). ${ }^{32}$

As costs were highly skewed, natural log transformation was performed. B (multiplied by 100) should be interpreted as the percentage difference: e.g. costs for D2T RA patients are 96\% higher than for non-D2T RA patients in univariable analysis. ${ }^{33}$

almost twice the costs of non-D2T RA (€19,217, equivalent to $\$ 21,560)$. Although these estimates are in line with those of a previous SLR, with total costs ranging from $\$ 2,407$ to $\$ 83,845$, our costs estimates for D2T RA might have been expected to be among the higher part of this range. ${ }^{9}$ However, absolute costs reported in different studies are difficult to compare because of divergent cost items and health care systems among countries and differences in methodology used. ${ }^{34}$ Of note, the majority of costs in the SLR ranged between $\$ 10,000$ and $\$ 30,000$ (i.e. lower than our costs estimates for D2T RA), and only one outlier study reported costs above \$40,000 (i.e. \$83,845).9,35 In this Norwegian study, $68 \%$ of these costs were related to productivity loss, which may be explained by relatively high wages in Norway ${ }^{35}$ Thus, in accordance with results of previously studied RA populations, D2T RA patients can be considered as a subgroup with relatively high costs. Other cost-of-illness studies specifically in D2T RA have not been performed before, also hampering direct comparisons of our results.

Interestingly, informal help of family and friends was the main contributor to total costs in D2T RA patients (28\%) and only accounted for $19 \%$ of total costs in non-D2T RA patients. According to the Methods and Guidelines for Economic Evaluations in Health Care, costs of informal help of family and friends should be taken into account, using the price of formal home help as a surrogate price. ${ }^{20}$ These costs are frequently not incorporated when assessing cost-of-illness, ${ }^{9}$ although they have been shown to be a major contributor to total $\operatorname{costs}^{36}$ similarly as in our study. As informal help of family 
and friends might also have resulted in productivity loss of these individuals, cost-ofillness may be even higher than we identified. In our study, only the minority of partners of D2T and non-D2T RA patients ( $0 \%$ vs $2 \%$ ) reported to work less hours a week due to the patient's disease. However, it was not assessed whether other relatives, e.g. their grownup children, reduced their working hours. Therefore, additional productivity loss as well as related costs may have occurred.

In this study, as well as in cost-of-illness studies in general, it is difficult to fully distinguish costs directly related to (D2T) RA from costs related to comorbidities. RA patients are known to have an increased risk and prevalence of particular comorbidities (e.g. cardiovascular diseases, ${ }^{37}$ osteoporosis ${ }^{38}$ and psychiatric disorders ${ }^{39}$ ). Moreover, a higher number of comorbidities has been found in D2T RA patients. ${ }^{14}$ In our study, after adjustment for the number of comorbidities, a significant association between having D2T RA and higher total costs remained. Nevertheless, (costs related to) RA and comorbidities should probably not be seen as distinct and, therefore, fully distinguishing these costs may not be possible and appropriate. ${ }^{40}$

Among D2T RA subgroups, surprisingly, numerically the lowest total costs were found in the subgroup of patients closest to the concept of 'true refractory RA'. Here, as expected, costs related to drugs were the main contributor to total costs and these were numerically highest compared with those of the other two subgroups. Of the higher total costs in the D2T RA subgroups 'non-adherent dissatisfied' patients and patients with 'pain syndromes and obesity', costs related to informal help of family and friends and productivity loss, respectively, were the main contributors. Therefore, the relatively higher total costs in these two subgroups of D2T RA might be related to the suboptimal identification of the factors contributing to D2T RA in routine daily practice (e.g. treatment non-adherence, fibromyalgia). These results should be interpreted with care as they were not statistically significant, probably due to the relatively small samples. Nevertheless, the tendencies above suggest the need for more individualised management strategies. If factors contributing to D2T RA will be identified and adequately addressed, ${ }^{14}$ costs may decrease. However, cost-effectiveness of such a strategy should be established in future studies.

After adjustment for functional disability, having D2T RA was no longer statistically significantly associated with higher total costs. In multivariable analysis, functional disability was the only independent determinant of higher total costs and could therefore be considered to be a key determinant. This corresponds to findings in prior studies. ${ }^{16,17,41}$ In our study, higher disease activity, reduced quality of life, presence of concomitant fibromyalgia, depression or anxiety were also determinants of higher costs in univariable analyses, as also described previously. ${ }^{16-18,42}$ All these factors have previously been associated with D2T RA. ${ }^{14}$ This suggests that preferably prevention and, secondary, adequate management of functional disability and the other determinants 
is important to eventually decrease the economic burden of D2T RA. Our results should be confirmed in larger D2T RA cohorts. However, particularly the D2T RA 'non-adherent dissatisfied' patients and patients with 'pain syndromes and obesity' who also have functional disability are probably the patient groups wherein the most benefit could be gained.

This study has several limitations. Due to the cross-sectional study design, data on resource use (apart from drug use and laboratory tests) have been collected by a questionnaire over the past three months to minimise recall bias. Subsequently, these data were extrapolated over a year. As patients were consecutively enrolled over a considerable period, and not specifically at the moment when they first fulfilled the D2T RA definition, changes in resource use and treatment could have occurred at any time during the assessed three months. Therefore, we expect that these extrapolated data at the group level generally would reflect the past year. Nevertheless, a long-term follow-up study would be preferrable to more precisely assess all costs associated with D2T RA over a longer time period, as well as the changes therein. Another limitation is that official drug prices were used to estimate drug costs, while in the Netherlands, also for outpatients, b/tsDMARDs are paid for by the hospital. Discounts on the b/tsDMARD purchase prices are negotiated by each individual hospital (or collaborative hospital group) with the pharmaceutical companies, often resulting in lower prices. We could not use negotiated prices however, since they are not available publicly. Therefore, real-life drug costs might be lower than those estimated. Furthermore, presenteeism, i.e. work performance negatively impacted by RA, was not assessed as a contributor to productivity loss. No major contribution of presenteeism to total costs is to be expected, as the percentage of patients with paid work was relatively low in our study. Additionally, no consensus has been reached on the assessment methodology of presenteeism. ${ }^{43-45}$ Moreover, this study was conducted in one country, which limits extrapolation to other countries due to different health care systems and costs. Nevertheless, as this study is the first study on the cost-of-illness of D2T RA, its results, and especially those regarding health care utilisation, give a first and clinically relevant insight into the significantly higher economic burden of D2T RA. Hopefully, this will result in more research accompanied by more funding opportunities in this important area.

In conclusion, significantly higher health care utilisation as well as costs were found in D2T compared with in non-D2T RA patients. Main contributors to total costs were costs related to informal help of family and friends, drugs and productivity loss. Functional disability was identified as a key determinant of higher costs in RA. The high economic burden of D2T RA further substantiates the need to optimise management strategies. 


\section{References}

1. Kalkan A, Hallert E, Bernfort L, et al. Costs of rheumatoid arthritis during the period 19902010: a register-based cost-of-illness study in Sweden. Rheumatology (Oxford) 2014;53:15360.

2. Smolen JS, Landewé RBM, Bijlsma JWJ, et al. EULAR recommendations for the management of rheumatoid arthritis with synthetic and biological disease-modifying antirheumatic drugs: 2019 update. Ann Rheum Dis 2020;79:685-99.

3. Singh JA, Saag KG, Bridges SL, et al. 2015 American College of Rheumatology Guideline for the Treatment of Rheumatoid Arthritis. Arthritis Rheumatol 2016;68:1-26.

4. Smolen JS, Breedveld FC, Burmester GR, et al. Treating rheumatoid arthritis to target: 2014 update of the recommendations of an international task force. Ann Rheum Dis 2016;75:3-15.

5. Colebatch AN, Edwards CJ, Østergaard $M$, et al. EULAR recommendations for the use of imaging of the joints in the clinical management of rheumatoid arthritis. Ann Rheum Dis 2013;72:804-14.

6. Smolen JS, Aletaha D, Mclnnes IB. Rheumatoid arthritis. Lancet 2016;388:2023-38.

7. Kievit W, Fransen J, de Waal Malefijt MC, et al. Treatment changes and improved outcomes in RA: an overview of a large inception cohort from 1989 to 2009. Rheumatology 2013;52:1500-8.

8. Hresko A, Lin TC, Solomon DH. Medical Care Costs Associated With Rheumatoid Arthritis in the US: A Systematic Literature Review and Meta-Analysis. Arthritis Care Res 2018;70:14318.

9. Hsieh P-H, Wu O, Geue $\mathrm{C}$, et al. Economic burden of rheumatoid arthritis: a systematic review of literature in biologic era. Ann Rheum Dis 2020;79:771-7.

10. Buch $\mathrm{MH}$. Defining refractory rheumatoid arthritis. Ann Rheum Dis 2018;77:966-9.

11. de Hair MJH, Jacobs JWG, Schoneveld JLM, et al. Difficult-to-treat rheumatoid arthritis: an area of unmet clinical need. Rheumatology (Oxford) 2017;57:1135-44.

12. Kearsley-Fleet L, Davies R, De Cock D, et al. Biologic refractory disease in rheumatoid arthritis: Results from the British Society for Rheumatology Biologics Register for Rheumatoid Arthritis. Ann Rheum Dis 2018;77:1405-12.
13. Nagy G, Roodenrijs NMT, Welsing PMJ, et al. EULAR definition of difficult-to-treat rheumatoid arthritis. Ann Rheum Dis 2021;80:31-5.

14. Roodenrijs NMT, van der Goes MC, Welsing PMJ, et al. Difficult-to-treat rheumatoid arthritis: contributing factors and burden of disease. Rheumatology 2020:keaa860.

15. Roodenrijs NMT, de Hair MJH, van der Goes $\mathrm{MC}$, et al. Characteristics of difficult-to-treat rheumatoid arthritis: results of an international survey. Ann Rheum Dis 2018;77:1705-9.

16. Verstappen SMM, Verkleij H, Bijlsma JWJ, et al. Determinants of direct costs in Dutch rheumatoid arthritis patients. Ann Rheum Dis 2004;63:817-24.

17. Jacobsson LTH, Lindroth $Y$, Marsal L, et al. Rheumatoid arthritis: what does it cost and what factors are driving those costs? Results of a survey in a community-derived population in Malmö, Sweden. Scand J Rheumatol 2007;36:179-83.

18. Kim S-K, Kim S-H, Lee $\mathrm{C}-\mathrm{K}$, et al. Effect of fibromyalgia syndrome on the health-related quality of life and economic burden in Korea. Rheumatology (Oxford) 2013;52:311-20.

19. Aletaha D, Neogi T, Silman AJ, et al. 2010 Rheumatoid arthritis classification criteria: An American College of Rheumatology/ European League Against Rheumatism collaborative initiative. Ann Rheum Dis 2010;69:1580-8.

20. Zorginstituut Nederland. Guideline for economic evaluations in healthcare. 2016:145.https://english.zorginstituutnederland.nl/ publications/reports/2016/06/16/guidelinefor-economic-evaluations-in-healthcare

21. de Jong PHP, Hazes JM, Buisman LR, et al. Best cost-effectiveness and worker productivity with initial triple DMARD therapy compared with methotrexate monotherapy in early rheumatoid arthritis: cost-utility analysis of the tREACH trial. Rheumatology (Oxford) 2016;55:2138-47.

22. van den Hout WB, Goekoop-Ruiterman YPM, Allaart CF, et al. Cost-utility analysis of treatment strategies in patients with recentonset rheumatoid arthritis. Arthritis Rheum 2009;61:291-9.

23. Verhoeven MMA, Tekstra J, van Laar JM, et al. Effect on Costs and Quality-adjusted Lifeyears of Treat-to-target Treatment Strategies Initiating Methotrexate, or Tocilizumab, or Their Combination in Early Rheumatoid Arthritis. J Rheumatol 2020:jrheum.200067. 
24. Rothman KJ. No adjustments are needed for multiple comparisons. Epidemiology 1990;1:43-6.

25. Feise RJ. Do multiple outcome measures require $\mathrm{p}$-value adjustment? BMC Med. Res. Methodol. 2002;2:1-4.

26. Althouse AD. Adjust for Multiple Comparisons? It's Not That Simple. Ann Thorac surg 2016;101:1644-5.

27. Radner $\mathrm{H}$, Chatzidionysiou $\mathrm{K}$, Nikiphorou $\mathrm{E}$, et al. 2017 EULAR recommendations for a core data set to support observational research and clinical care in rheumatoid arthritis. Ann Rheum Dis 2018;77:476-9.

28. EuroQol Group. EuroQol--a new facility for the measurement of health-related quality of life. Health Policy 1990;16:199-208.

29. Maska L, Anderson J, Michaud K. Measures of functional status and quality of life in rheumatoid arthritis: Health Assessment Questionnaire Disability Index (HAQ), Modified Health Assessment Questionnaire (MHAQ), Multidimensional Health Assessment Questionnaire (MDHAQ), Health Assessment. Arthritis Care Res (Hoboken) 2011;63:S4-13.

30. Wolfe F, Clauw DJ, Fitzcharles $M-A A$, et al. Fibromyalgia criteria and severity scales for clinical and epidemiological studies: A modification of the ACR preliminary diagnostic criteria for fibromyalgia. J Rheumatol 2011;38:1113-22.

31. Spinhoven P, Ormel J, Sloekers PP, et al. A validation study of the Hospital Anxiety and Depression Scale (HADS) in different groups of Dutch subjects. Psychol Med 1997;27:36370.

32. Zigmond AS, Snaith RP. The hospital anxiety and depression scale. Acta Psychiatr Scand 1983;67:361-70.

33. Cole TJ, Altman DG. Statistics Notes: Percentage differences, symmetry, and natural logarithms. BMJ 2017;358:j3683.

34. Knies S, Ament AJHA, Evers SMAA, et al. The transferability of economic evaluations: Testing the model of welte. Value Heal 2009;12:730-8.
35. Kvamme MK, Lie E, Kvien TK, et al. Two-year direct and indirect costs for patients with inflammatory rheumatic joint diseases: Data from real-life follow-up of patients in the NOR-DMARD registry. Rheumatol (United Kingdom) 2012;51:1618-27.

36. Westhovens R, Boonen A, Verbruggen L, et al. Healthcare consumption and direct costs of rheumatoid arthritis in Belgium. Clin Rheumatol 2005;24:615-9.

37. Avina-Zubieta JA, Thomas J, Sadatsafavi M, et al. Risk of incident cardiovascular events in patients with rheumatoid arthritis: A metaanalysis of observational studies. Ann Rheum Dis 2012;71:1524-9.

38. Xue AL, Wu SY, Jiang $L$, et al. Bone fracture risk in patients with rheumatoid arthritis. Med (United States) 2017;96.

39. Marrie RA, Hitchon CA, Walld R, et al. Increased Burden of Psychiatric Disorders in Rheumatoid Arthritis. Arthritis Care Res 2018;70:970-8.

40. Radner $H$, Yoshida K, Smolen JS, et al. Multimorbidity and rheumatic conditions Enhancing the concept of comorbidity. Nat Rev Rheumatol 2014;10:252-6.

41. Wallman JK, Eriksson JK, Nilsson JA, et al. Costs in Relation to Disability, Disease Activity, and Health-related Quality of Life in Rheumatoid Arthritis: Observational Data from Southern Sweden. J Rheumatol 2016;43:1292-9.

42. Silverman S, Dukes EM, Johnston SS, et al. The economic burden of fibromyalgia: Comparative analysis with rheumatoid arthritis. Curr Med Res Opin 2009;25:829-40.

43. Kigozi J, Jowett $\mathrm{S}$, Lewis $\mathrm{M}$, et al. The Estimation and Inclusion of Presenteeism Costs in Applied Economic Evaluation: A Systematic Review. Value Heal 2017;20:496-506.

44. Zhang W, Gignac MAM, Beaton D, et al. Productivity loss due to presenteeism among patients with arthritis: Estimates from 4 instruments. J Rheumatol 2010;37:1805-14.

45. Zhang W, Chiu JA, Bansback N, et al. An update on the measurement of productivity losses due to rheumatoid diseases. Best Pract Res Clin Rheumatol 2012;26:585-97. 


\section{Supplementary}

Supplementary Table 1. Costs of health care utilisation

Costs $2019(€)^{1}$

\section{Costs directly related to health care utilisation and other resource use}

Admission to health care facilities, per day

General ward of academic hospital

685.40

General ward of general hospital

472.95

Intensive care

2151.21

Day care

324.12

Rehabilitation center

491.10

Nursing home

179.36

Consultations with health care professionals, per consultation

Rheumatologist or other medical specialist

90.75

Rheumatology trainee or nurse

38.43

General practitioner

35.23

Exercise therapist

Hydrotherapist

35.23

Occupational therapist

Physiotherapist

35.23

Podiatrist

35.23

Social worker

69.39

Psychologist

68.33

Psychotherapist

100.82

Psychiatrist

100.82

District nurse (per hour)

Formal house help (per hour)

21.35

Diagnostic tests, per test

Laboratory tests

Supplementary Table 2

Imaging measures

- x-ray

38.80

- Ultrasonography

89.13

- CT

152.13

$-\mathrm{MRI}$

237.01

Drugs

Informal help from family and friends, per hour

\section{Costs related to productivity loss}

Productivity loss, per hour

Disability pension, per month 1615.80

CT: computed tomography; MRl: magnetic resonance imaging.

1. Costs were estimated according to Methods and Guidelines for Economic Evaluations in Health Care. ${ }^{20}$ 


\section{Blood, per measurement}

Activated partial thromboplastin time

$\begin{array}{lr}\text { Alanine transaminase } & 2.23\end{array}$

$\begin{array}{ll}\text { Albumin } & 1.72\end{array}$

$\begin{array}{lr}\text { Alkaline phosphatase } & 2.08\end{array}$

$\begin{array}{ll}\text { Amylase } & 2.58\end{array}$

$\begin{array}{ll}\text { Anti-citrullinated protein antibodies } & 12.12\end{array}$

$\begin{array}{ll}\text { Anti-HBsAg } & 13.72\end{array}$

$\begin{array}{ll}\text { Antineutrophil cytoplasmic antibodies } & 8.97\end{array}$

$\begin{array}{ll}\text { Antinuclear antibodies } & 8.97\end{array}$

$\begin{array}{lr}\text { Arterial blood gas analysis } & 5.90\end{array}$

$\begin{array}{lr}\text { Aspartate transaminase } & 2.06\end{array}$

$\begin{array}{ll}\text { Bicarbonate } & 5.90\end{array}$

$\begin{array}{ll}\text { Bilirubin } & 1.72\end{array}$

$\begin{array}{lr}\text { Brain natriuretic peptide } & 19.79\end{array}$

$\begin{array}{ll}\text { C-reactive protein } & 4.35\end{array}$

$\begin{array}{lr}\text { Calcium } & 2.06\end{array}$

$\begin{array}{lr}\text { Calprotectin } & 50.46\end{array}$

$\begin{array}{ll}\text { Chloride } & 1.72\end{array}$

$\begin{array}{ll}\text { Cholesterol } & 2.12\end{array}$

$\begin{array}{ll}\text { Coombs test } & 4.95\end{array}$

$\begin{array}{lr}\text { Creatinine (including eGFR) } & 6.98\end{array}$

$\begin{array}{ll}\text { Creatinine kinase } & 8.51\end{array}$

$\begin{array}{ll}\text { Erythrocyte sedimentation rate } & 1.81\end{array}$

$\begin{array}{ll}\text { Ferritin } & 9.74\end{array}$

$\begin{array}{ll}\text { Free light chains } & 17.69\end{array}$

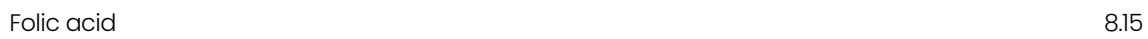

$\begin{array}{ll}\text { Gamma-glutamyltransferase } & 2.06\end{array}$

$\begin{array}{lr}\text { Glucose } & 1.89\end{array}$

$\begin{array}{lr}\text { HbAlc } & 8.43\end{array}$

$\begin{array}{ll}\text { Hemoglobin } & 1.83\end{array}$

$\begin{array}{ll}\text { High-density lipoprotein cholesterol } & 3.39\end{array}$

$\begin{array}{ll}\text { Immunoglobulin } G & 12.12\end{array}$

$\begin{array}{ll}\text { Iron }(\mathrm{Fe}) & 3.25\end{array}$

$\begin{array}{lr}\text { Lactate dehydrogenase } & 2.06\end{array}$

$\begin{array}{ll}\text { Leukocyte differentiation (complete count) } & 1.81\end{array}$

$\begin{array}{ll}\text { Leukocytes } & 1.85\end{array}$

$\begin{array}{ll}\text { Lipase } & 2.31\end{array}$

$\begin{array}{ll}\text { Magnesium } & 3.69\end{array}$

$\begin{array}{lr}\text { Parathyroid hormone } & 9.76\end{array}$ 
Supplementary Table 2. Continued

\begin{tabular}{|c|c|}
\hline & Costs $2019(€)^{\prime}$ \\
\hline Phosphate & 1.89 \\
\hline Potassium & 1.89 \\
\hline Prostate specific antigen & 9.63 \\
\hline Prothrombin time & 3.56 \\
\hline Prothrombin time and international normalised ratio & 9.17 \\
\hline Reticulocytes & 3.06 \\
\hline Rheumatoid factor & 12.12 \\
\hline Sodium & 1.89 \\
\hline T4 & 8.17 \\
\hline Thrombocytes & 1.83 \\
\hline Thyroid-stimulating Hormone & 7.14 \\
\hline Total protein & 1.78 \\
\hline Transferrin (including saturation) & 6.69 \\
\hline Triglycerides & 3.07 \\
\hline Troponin & 8.25 \\
\hline Urea & 1.72 \\
\hline Uric acid & 1.80 \\
\hline Vitamin $\mathrm{Bl}$ & 10.17 \\
\hline Vitamin B6 & 10.13 \\
\hline Vitamin $\mathrm{Bl} 2$ & 9.75 \\
\hline Vitamin D (25-hydroxy) & 10.85 \\
\hline \multicolumn{2}{|l|}{ Urine, per measurement } \\
\hline Urine micro-albumin & 4.95 \\
\hline Urine sediment (microscopic examination) & 1.92 \\
\hline Urine screening & 2.25 \\
\hline
\end{tabular}

eGFR: estimated glomerular filtration rate; HbAlc: Hemoglobin Alc; HBsAg: Hepatitis B surface antigen; T4: Free thyroxine.

1. Costs were estimated according to Methods and Guidelines for Economic Evaluations in Health Care. ${ }^{20}$ 
Chapter 11

Supplementary Table 3. Costs of drugs

Costs $2019(€)$

\section{csDMARDs, per dose}

Azathioprine

$\begin{array}{lr}100 \mathrm{mg} & 0.35 \\ 125 \mathrm{mg} & 0.48 \\ 150 \mathrm{mg} & 0.48\end{array}$

Hydroxychloroquine

200mg

0.14

Leflunomide

$10 \mathrm{mg}$

0.99

20mg

1.17

Methotrexate

Orally

$2.5 \mathrm{mg}$

2.76

$5.0 \mathrm{mg}$

5.52

$7.5 \mathrm{mg}$

8.28

$10.0 \mathrm{mg}$

3.00

$12.5 \mathrm{mg}$

5.76

$15.0 \mathrm{mg}$

8.52

$17.5 \mathrm{mg}$

11.28

$20.0 \mathrm{mg}$

6.00

$22.5 \mathrm{mg}$

8.76

$25.0 \mathrm{mg}$

11.52

$27.5 \mathrm{mg}$

14.28

$30.0 \mathrm{mg}$

9.00

Subcutaneously

$2.5 \mathrm{mg}$

22.89

$5.0 \mathrm{mg}$

17.99

$7.5 \mathrm{mg}$

10.28

$10.0 \mathrm{mg}$

8.23

$12.5 \mathrm{mg}$

10.29

$15.0 \mathrm{mg}$

12.35

$17.5 \mathrm{mg}$

14.41

$20.0 \mathrm{mg}$

16.47

$22.5 \mathrm{mg}$

18.61

$25.0 \mathrm{mg}$

20.59

$27.5 \mathrm{mg}$

22.62

$30.0 \mathrm{mg}$

24.66

Sulfalazine

$1000 \mathrm{mg}$

0.22

$1500 \mathrm{mg}$

0.33 
Supplementary Table 3. Continued

Costs $2019(€)^{\prime}$

Sulfalazine

2000mg

0.44

bDMARDs, per dose

Abatacept

Subcutaneously

$125 \mathrm{mg}$

277.95

Intravenously

$500 \mathrm{mg}$

783.26

$750 \mathrm{mg}$

1174.89

Adalimumab

$40 \mathrm{mg}$

538.44

Anakinra

$100 \mathrm{mg}$

34.30

Certolizumab pegol

200mg

505.76

Golimumab

$50 \mathrm{mg}$

1125.83

Etanercept

$25 \mathrm{mg}$

105.63

$50 \mathrm{mg}$

211.25

Infliximab

100mg

519.54

Tocilizumab

Subcutaneously

$162 \mathrm{mg}$

Intravenously

400mg

840.29

$600 \mathrm{mg}$

1260.42

$800 \mathrm{mg}$

1680.58

Rituximab

500mg

1267.28

$1000 \mathrm{mg}$

2534.56

tsDMARDs, per dose

Baricitinib

$2 \mathrm{mg}$

$4 \mathrm{mg}$

34.38

Tofacitinib

$5 \mathrm{mg}$ 
Supplementary Table 3. Continued

Costs $2019(€)$

Tofacitinib

$10 \mathrm{mg}$

\section{Glucocorticoids, per dose}

Prednisone

$2.5 \mathrm{mg}$

$5.0 \mathrm{mg}$

0.03

$7.5 \mathrm{mg}$

0.32

$10.0 \mathrm{mg}$

0.17

$12.5 \mathrm{mg}$

0.46

$15.0 \mathrm{mg}$

$17.5 \mathrm{mg}$

0.51

$20.0 \mathrm{mg}$

0.14

$22.5 \mathrm{mg}$

0.43

$25.0 \mathrm{mg}$

0.33

$27.5 \mathrm{mg}$

0.62

$30.0 \mathrm{mg}$

0.16

Pain killers, regular use per day ${ }^{2}$

Paracetamol

NSAIDS

Unselective

cox-2

0.45

Opioids

0.80

b: biological; cs: conventional synthetic; COX: cyclooxygenase; CT: computed tomography; DMARD: diseasemodifying antirheumatic drug; mg: milligram; NSAIDs: non-steroidal anti-inflammatory drugs; ts: targeted synthetic. 1. Costs were estimated according to www.medicijnkosten.nl (accessed in December 2019).

2. Costs were estimated based on the costs of regular use of the cheapest drug per group of pain killer. 
Supplementary Table 4. Work productivity

\begin{tabular}{lccc}
\hline & D2T RA (n=52) & Non-D2T RA (n=99) & p-value \\
\hline Paid job & 20 & 25 & $0.54^{2}$ \\
Paid job and not (partially) work disabled, $\%$ & 14 & 23 & $0.20^{2}$ \\
hours/week & $30(12) ; 24(20-40)$ & $27(11.3) ; 30(20-38)$ & $0.66^{1}$ \\
Partially a paid job and partially work disa-bled, $\%$ & 6 & 2 & $0.34^{2}$ \\
$\quad$ hours/week & $17(2) ; 16(16-16)$ & $16(0.7) ; 16(16-16)$ & $0.20^{1}$ \\
Absenteeism, \% & 20 & 34 & $0.69^{2}$ \\
$\quad$ days/3 months & $1.5(0.7) ; 2(1-2)$ & $17.7(28.3) ; 4(3-21)$ & $0.044^{1}$ \\
Fully work disabled, \% & 26 & 17 & $0.06^{2}$ \\
Duration of being work disabled, years & $15(11) ; 13(5-22)$ & $14(11) ; 11(4-25)$ & $0.81^{1}$ \\
Became fully work disabled in past year, \% & 0 & 3 & $0.55^{2}$ \\
Retired, \% & 33 & 44 & $0.12^{2}$ \\
Other, \% & 20 & 14 & $0.48^{2}$ \\
\hline
\end{tabular}

D2T: difficult-to-treat; n: number; RA: rheumatoid arthritis.

Numbers are mean (standard deviation); median (interquartile range). Differences between groups were analysed using: 1. Mann-Whitney U Test; 2. Fisher's exact test. 
Supplementary Table 5. Health care utilisation during the past three months, within the three subgroups of D2T RA

\begin{tabular}{|c|c|c|}
\hline & \multicolumn{2}{|c|}{$\begin{array}{c}\text { Subgroup } 1(\mathrm{n}=17) \\
\text { 'Non-adherence and dissatisfaction' }\end{array}$} \\
\hline & Mean $(95 \% \mathrm{Cl})$ & Median (IQR) \\
\hline \multicolumn{3}{|c|}{ Consultations with health care professionals } \\
\hline Rheumatologist, number & $1.8(1.4-2.2)$ & $2.0(1.0-2.0)$ \\
\hline Rheumatology nurse, number & $0.6(0.4-0.8)$ & $1.0(0.0-2.0)$ \\
\hline General practitioner, number & $0.9(0.4-1.4)$ & $0.0(0.0-2.0)$ \\
\hline Other medical specialist, number & $2.3(0.6-1.3)$ & $2.0(0.5-3.0)$ \\
\hline Paramedic, number & $6.7(2.4-11.6)$ & $3.0(0.0-10.0)$ \\
\hline Psychosocial support, number & $1.9(0.4-4.4)$ & $0.0(0.0-2.5)$ \\
\hline District nurse, hours/week & $5.7(0.4-13.7)$ & $0.0(0.0-0.0)$ \\
\hline Formal house help, hours/week & $11.1(3.4-20.6)$ & $0.0(0.0-22.8)$ \\
\hline
\end{tabular}

\section{Admission to health care facilities}

Hospital, days

General ward

$3.2(2.4-11.8)$

$0.0(0.0-0.0)$

Intensive care unit

$0.0(0.0-0.0)$

$0.0(0.0-0.0)$

Day care

$0.4(0.1-0.9)$

$0.0(0.0-1.0)$

Rehabilitation center or nursing home, days

$0.0(0.0-0.0)$

$0.0(0.0-0.0)$

Surgery, number

$0.0(0.0-0.0)$

$0.0(0.0-0.0)$

\section{Diagnostic tests}

Laboratory tests, number of administrations

$2.4(1.3-4.0)$

$2.0(1.0-2.0)$

Imaging measures, number

X-ray

Ultrasonography

CT

MRI
$1.0(0.0-2.0)$

$0.0(0.0-1.0)$

$0.0(0.0-0.5)$

$0.0(0.0-0.5)$

$1.9(1.5-2.3)$
$1.0(1.0-1.0)$

Drugs

DMARDs, number

$\operatorname{csDMARD}(\mathrm{s}), \%$

bDMARD, \%

tsDMARD, \%

59

Glucocorticoid therapy, \%

Pain killers, \%

Regular use of paracetamol, \% 88

Regular use of NSAIDs, \%

12

35

Regular use of opioids, \% 18

b: biological; Cl: confidence interval; cs: conventional synthetic; CT: computed tomography; D2T: difficult-to-treat; DMARD: disease-modifying antirheumatic drug; IQR: interquartile range; MRI: magnetic resonance imaging; n: number; NSAIDs: non-steroidal anti-inflammatory drugs; RA: rheumatoid arthritis; ts: targeted synthetic; ${ }^{\wedge}$ : unless 
Subgroup $2(n=8)$

'Pain syndromes and obesity'
Subgroup $3(n=27)$

'True refractory $R A^{\prime}$

Mean $(95 \% \mathrm{Cl})$

Median (IQR)^

$1.4(0.6-2.0)$

$1.0(1.0-2.0)$

$1.6(1.2-2.0)$

$1.5(0.3-2.0)$

$0.6(0.3-1.0)$

$0.0(0.0-1.0)$

$1.5(0.6-2.8)$

$0.0(0.0-1.0)$

$1.0(0.5-1.5)$

$1.0(0.0-1.0)$

4.1 (0.8-9.7)

$0.3(1.0-2.0)$

1.7 (1.0-2.6)

$1.0(0.0-3.0)$

6.4 (1.4-13.6)

$0.0(0.0-17.0)$

3.8 (2.1-5.8)

$1.0(0.0-9.0)$

0.3 (0.2-1.0)

$0.0(0.0-0.0)$

$0.4(0.1-0.9)$

$0.0(0.0-0.0)$

$0.0(0.0-0.0)$

$0.0(0.0-0.0)$

11.1 (3.4-20.6)

$0.0(0.0-0.0)$

9.8 (2.6-26.0)

$0.0(0.0-20.0)$

13.7 (5.8-24.4)

$0.0(0.0-26.0)$

$2.3(0.7-4.7)$

$0.0(0.0-5.5)$

$0.1(0.1-0.4)$

$0.0(0.0-0.0)$

$0.0(0.0-0.0)$

$0.0(0.0-0.0)$

$0.0(0.0-0.0)$

0.0 (0.0-1.0)

0.8 (0.1-1.6)

0.0 (0.0-1.8)

$0.7(0.2-1.2)$

$0.0(0.0-0.0)$

$0.0(0.0-0.0)$

$0.0(0.0-0.0)$

$0.0(0.0-0.0)$

$0.0(0.0-0.0)$

$0.0(0.0-0.0)$

$0.0(0.0-0.0)$

$0.0(0.0-0.0)$

$1.8(1.2-2.3)$

$1.5(1.0-2.8)$

$2.1(1.6-2.7)$

$2.0(1.0-2.0)$

$1.5(0.6-2.5)$

$1.0(0.3-2.8)$

$1.3(0.8-1.9)$

$1.0(0.0-3.0)$

0.5 (0.1-1.4)

$0.0(0.0-0.8)$

0.3 (0.1-0.5)

$0.0(0.0-0.0)$

$0.3(0.1-0.6)$

$0.0(0.0-0.8)$

0.1 (0.0-0.3)

$0.0(0.0-0.0)$

0.6 (0.1-1.5)

$0.0(0.0-1.0)$

$0.1(0.0-0.2)$

$0.0(0.0-0.0)$

$1.5(0.7-2.3)$

$1.5(0.3-2.8)$

$1.6(1.3-2.0)$

$2.0(1.0-2.0)$
50

38

38

38

100

25

75

25
70

52

22

44

96

33

33 
Supplementary Table 6. Estimations of annual mean costs in euros per patient, within the three subgroups of D2T RA

\begin{tabular}{|c|c|c|}
\hline & \multicolumn{2}{|c|}{$\begin{array}{c}\text { Subgroup } 1(\mathrm{n}=17) \\
\text { 'Non-adherence and dissatisfaction' }\end{array}$} \\
\hline & Mean $(95 \% \mathrm{Cl})$ & Median (IQR) \\
\hline \multicolumn{3}{|l|}{ Costs directly related to health care utilisation $(€)$} \\
\hline - Consultations with health care professionals & $5966(2055-10556)$ & $2221(0-6478)$ \\
\hline - Admission to health care facilities & $6654(216-21140)$ & $0(0-1296)$ \\
\hline - Diagnostic tests & $2214(887-4294)$ & $232(144-382)$ \\
\hline - Drugs & 8446 (5568-11091) & $10313(1115-13918)$ \\
\hline Total costs directly related to health care utilisation & $23280(13064-40200)$ & $17264(11287-23870)$ \\
\hline \multicolumn{3}{|l|}{ Costs related to other resource use $(€)$} \\
\hline $\begin{array}{l}\text { - Devices needed to perform daily activities and } \\
\text { adaptations in and around the house }\end{array}$ & $1867(29-4512)$ & $0(0-198)$ \\
\hline - Informal help from family and friends & $17602(3925-40608)$ & $2221(0-16655)$ \\
\hline $\begin{array}{l}\text { - Additional costs (e.g. alternative therapies, } \\
\text { additional energy and travel costs) }\end{array}$ & $1225(507-2052)$ & $120(0-2398)$ \\
\hline Total costs related to other resource use & 20694 (5941-442 21) & $18470(12841-48600)$ \\
\hline \multicolumn{3}{|l|}{ Costs related to productivity loss $(€)$} \\
\hline - Incidental absence due to illness & - & - \\
\hline - $\quad$ Structural absence due to illness ${ }^{\ddagger}$ & - & - \\
\hline - Disability pension & $4562(1212-8618)$ & $0(0-9695)$ \\
\hline Total costs related to productivity loss & $4562(1212-8618)$ & $0(0-9695)$ \\
\hline Total costs $(€)$ & $48536(22184-81772)$ & $18470(12841-63866)$ \\
\hline
\end{tabular}

Cl: confidence interval; D2T: difficult-to-treat; n: number; IQR: interquartile range; RA: rheumatoid arthritis; *: p<0.05;

ł: i.e. costs related to productivity loss using the friction cost method, only applicable to patients with structural

Supplementary Table 7. Association between having D2T RA, higher costs and other population characteristics

\begin{tabular}{lcc}
\hline & $\begin{array}{c}\text { Univariable analysis } \\
\beta(95 \% \mathrm{Cl}, \mathrm{p}-\mathrm{value})\end{array}$ & $\begin{array}{c}\text { Multivariable analysis } \\
\beta(95 \% \mathrm{Cl}, \mathrm{p}-\mathrm{value})\end{array}$ \\
\hline D2T & $0.96(0.52-1.39,<0.001)$ & $0.63(0.17-1.09,<0.01)$ \\
Age, higher & $-0.04(-0.06--0.02,<0.001)$ & $-0.04(-0.06--0.02,<0.001)$ \\
Female gender & $0.17(-0.32-0.66,0.49)$ & $-0.03(-0.49-0.43,0.90)$ \\
Disease duration, longer & $0.01(-0.01-0.03,0.18)$ & $0.02(-0.00-0.03,0.09)$ \\
$\begin{array}{l}\text { Comorbidities according to EULAR domains }{ }^{27}, \text {, higher } \\
\text { number }\end{array}$ & $0.19(-0.01-0.39,0.06)$ & $0.18(-0.03-0.39,0.09)$ \\
\hline
\end{tabular}

ß: regression coefficient; Cl: confidence interval; D2T: difficult-to-treat; EULAR: European League Against Rheumatism; RA: rheumatoid arthritis.

As costs were highly skewed, natural log transformation was performed. ß (multiplied by 100) should be interpreted as the percentage difference: e.g. costs for D2T RA patients are 96\% higher than for non-D2T RA patients in univariable analysis. ${ }^{33}$ 


\section{Subgroup $2(n=8)$}

'Pain syndromes and obesity'
Median (IQR)

$$
986(0-5979)
$$

$2593(0-13432)$

987 (374-3239)

12076 (10796-14554)

21166 (14828-31643)
$2459(740-4643)$

6716 (1502-12646)

$2013(572-3906)$

7748 (5667-11907)

22609 (16050-29100)
Subgroup $3(\mathrm{n}=27)$
'True refractory $R A^{\prime}$
Median (IQR)

$$
\begin{gathered}
705(0-4475) \\
0(0-1296)
\end{gathered}
$$$$
671 \text { (271-1109) }
$$

10570 (5958-13706)

14371 (9046-20284)
944 (634-1303)

10031 (7852-12151)

15465 (12481-18853)

$$
\begin{gathered}
10132(6497-13768) \\
1407(90-4237)
\end{gathered}
$$

11538 (7196-15570)

$$
\begin{gathered}
9993(6662-14434) \\
240(0-973)
\end{gathered}
$$

11309 (6952-17478)
-

14542 (7672-17627)

14542 (7672-17627)

48689 (36378-60710)
19390 (4847-19390)

19390 (4847-19390)

45149 (38108-65807)

$$
200(25-441)
$$

6127 (2303-11704)

1226 (484-2364)

7554 (3522-13397)

$110(20-306)$

4309 (1492-7756)

4419 (1522-7849)

27438 (20830-34659)

$$
0(0-0)
$$

2221 (0-6662)

396 (0-1052)

$3700(600-7772)$

$$
\begin{gathered}
0(0-0) \\
- \\
0(0-0) \\
0(0-2374)^{*}
\end{gathered}
$$

22830 (13564-43293)

absence (>85 consecutive days per year) due to illness or to those who became work disabled in the past year. Differences in total costs between groups were analysed using Kruskal-Wallis test. $95 \% \mathrm{Cl}$ is based on bootstrapping. 



\section{CHAPTER 12}

Non-adherence in difficult-to-treat rheumatoid arthritis from the perspectives of patients and rheumatologists: a concept mapping study

Rheumatology 2021. doi: 10.1093/rheumatology/keab130 [Epub ahead of print 9 Feb 2021]

Nadia M.T. Roodenrijs, Marlies C. van der Goes, Paco M.J. Welsing, Eline P.C. van Oorschot, Elena Nikiphorou, Nienke C. Nijhof, Janneke Tekstra, Floris P.J.G. Lafeber, Johannes W.G. Jacobs, Jacob M. van Laar, Rinie Geenen 


\section{Abstract}

\section{Objectives}

Treatment non-adherence is more frequent among difficult-to-treat (D2T) than among non-D2T rheumatoid arthritis (RA) patients. Perceptions of non-adherence may differ. We aimed to thematically structure and prioritise barriers (i.e. causes and reasons for non-adherence) and facilitators of optimal adherence from the patients' and rheumatologists' perspectives.

\section{Methods}

Patients' perceptions were identified in semi-structured in-depth interviews. Experts selected representative statements regarding 40 barriers and 40 facilitators. Twenty D2T and 20 non-D2T RA patients sorted these statements during two card-sorting tasks: first, by order of content similarity and, second, content applicability. Additionally, 20 rheumatologists sorted the statements by order of content applicability to the general RA population. The similarity sorting was used as input for hierarchical cluster analysis. The applicability sorting was analysed using descriptive statistics, prioritised and compared between D2T RA patients, non-D2T RA patients and rheumatologists.

\section{Results}

Nine clusters of barriers were identified, related to the health care system, treatment safety/efficacy, treatment regimen and patient behaviour. D2T RA patients prioritised adverse events and doubts about effectiveness as most important barriers. Doubts about effectiveness were more important to D2T than to non-D2T RA patients $(p=0.02)$. Seven clusters of facilitators were identified, related to the health care system and directly to the patient. All RA patients and rheumatologists prioritised a good relationship with the health care professional and treatment information as most helpful facilitators.

\section{Conclusions}

D2T RA patients, non-D2T RA patients and rheumatologists prioritised perceptions of nonadherence largely similarly. The structured overviews of barriers and facilitators, provided in this study, may guide improvement of adherence. 


\section{Introduction}

Clinical outcomes of rheumatoid arthritis (RA) patients have significantly improved over the past decades.' However, around $5 \%$ to $20 \%$ of patients remain symptomatic despite treatment according to European League Against Rheumatism (EULAR) recommendations. ${ }^{2-5}$ These patients can be classified as having difficult-to-treat (D2T) RA. ${ }^{6}$ D2T RA is often a multifactorial disease state, in which several factors may contribute to the persistence of signs and/or symptoms., ${ }^{2,78}$

Treatment non-adherence is one of the factors that could contribute to D2T RA. ${ }^{2,8}$ Our recent study confirmed the importance of non-adherence in D2T RA with significantly higher non-adherence rates in D2T than in non-D2T RA patients (40\% versus $22 \%$ ). ${ }^{8}$ An optimal drug response can only be achieved if a patient adheres to treatment instructions and, therefore, inflammation may persist in case of non-adherence. In D2T RA, this could eventually result in having used all available disease-modifying antirheumatic drugs (DMARDs) without (apparently) having any option left. Thus, non-adherence is highly relevant, specifically, as non-adherence is potentially modifiable: if non-adherence will be identified by the health care professional (HCP) and adequately addressed, the D2T RA state may ameliorate. ${ }^{9}$

To optimise non-adherence, insights into perceptions of non-adherence are needed. It is essential to identify both adherence barriers (i.e. any factor inducing suboptimal or non-adherence: causes and reasons for non-adherence) as well as facilitators (i.e. circumstances that could improve adherence) to allow their implementation in treatment strategies. ${ }^{10}$ Identification of perceptions of non-adherence has previously been based on patients' opinions, ${ }^{10-12}$ although few studies have thematically structured patient input. Concept mapping, for example using card-sorting tasks, has been shown to be a valid and reliable method to thematically structure and prioritise perceptions from the patients' perspective..$^{13-15}$ In this method, patients structure perceptions of nonadherence themselves.

Perceptions of non-adherence may differ between D2T and non-D2T RA patients. Due to, for instance, disease-related factors (e.g. a higher number of previously failed drugs and higher disease activity levels in D2T RA), ${ }^{8}$ different adherence barriers may apply to D2T than to non-D2T RA patients. ${ }^{16,17}$ Additionally, perceptions of non-adherence may differ between D2T RA patients and HCPS. This discordance could potentially aggravate the problem. As the role of the rheumatologist is crucial in the treatment of RA and in optimising adherence, it is important for the HCP to be vigilant to the issue of non-adherence in D2T RA and to understand causes and patient-related reasons as well as helpful facilitators. Therefore, identification of perceptions of both patients and rheumatologists is important.

The aim of this study was to thematically structure barriers and facilitators of optimal 
adherence using concept mapping, and to prioritise and compare these perceptions from the patients' and rheumatologists' perspectives.

\section{Methods}

\section{Study design and participants}

Consecutive RA outpatients, fulfilling the 2010 American College of Rheumatology (ACR)/ EULAR classification criteria for $\mathrm{RA}^{18}$ and treated according to the current standard of care (treat-to-target) for at least one year, had been recruited for a previous, cross-sectional study into factors potentially contributing to D2T RA. ${ }^{8}$ These patients had been enrolled from February 2019 to August 2020 at the Department of Rheumatology \& Clinical Immunology of the University Medical Center (UMC) Utrecht, the Netherlands. Patients had been classified as having D2T RA if they fulfilled all three criteria of the new EULAR definition, ${ }^{6}$ in short: 1. Previous failure of $\geq 2$ biological and/or targeted synthetic DMARDs with different mechanisms of action; 2. Symptoms and signs suggestive of active/ progressive disease (e.g. DAS28-ESR>3.2); 3. Management perceived as problematic by the rheumatologist and/or patient. RA patients who did not fulfil all three D2T RA criteria were allocated to the non-D2T RA group. Further details regarding participants and study procedures have been described previously. ${ }^{8}$

For the current study on treatment non-adherence, random samples of D2T and non-D2T RA patients were generated by a computer from the sample described above. Patients were invited by telephone for an additional study visit from February 2020 to August 2020. To be eligible, patients had to be fluent in Dutch language and have no hearing or visual impairments, or reading restrictions.

This study was approved by the medical ethics committee of the UMC Utrecht and was performed according to the Helsinki declaration. Written informed consent from all participants was obtained.

\section{Study procedures}

\section{Step I: Interviews}

Semi-structured in-depth interviews were conducted to collect as many statements as possible regarding (a) barriers and (b) facilitators of optimal adherence. Prior to these interviews, a pilot interview was undertaken in collaboration with a patient research partner ( $\mathrm{NCN}$ ) to limit the patient burden and to assess the relevance and comprehensiveness of the questions. Two female researchers (NMTR, EPCVO) conducted the interviews, in which a confidential environment was ensured and open-ended questions were asked in accordance with a predetermined interview guide (Supplementary File 1). The first ten interviews were carried out face-to-face at the outpatient clinic. Due to the COVID-19 
outbreak, the subsequent interviews were performed via a video call. All interviews were audio-recorded for verbatim transcription. Interviews were performed until saturation of information occurred (i.e. when no new information was gained during two consecutive interviews, Supplementary File 2). This study was conducted following the consolidated criteria for reporting qualitative research (COREQ) checklist (Supplementary File 3).19

\section{Step 2: Selection of statements}

Statements were extracted regarding (a) barriers and (b) facilitators of optimal adherence. The statements were reduced to 40 statements per category, which has been shown to be a manageable number in card-sorting tasks (step 3)..$^{5}$

First, unmistakably duplicate statements (in terms of content) were removed (in consensus by NMTR, EPCVO). Second, three researchers (NMTR, EPCVO, RG) independently made a selection of 40 statements per category. Statements were removed when they were too similar to another statement, too abstract, vague or inapplicable to all participants. Statements were then divided into three categories: chosen by at least two researchers, chosen by one researcher or not chosen by any of the researchers. Three other researchers (MCvdG, PMJW, JMvL) and one patient research partner ( $\mathrm{NCN}$ ) independently reviewed the categorisation of the statements using the same criteria and noted whether they agreed with the categorisation. Statements that were selected by at least four members were discussed and then selected for the final set of statements after consensus was reached. Lastly, wording and clarity of the statements were discussed and amended if needed.

\section{Step 3: Card-sorting tasks}

Twenty D2T and 20 non-D2T RA patients participated in the card-sorting tasks: they sorted the selected barriers and facilitators (randomly numbered and printed on separate cards) by order of content similarity and content applicability. A sample size of ten to 20 patients per group has been shown to be a working number for concept mapping and to ensure a variety of opinions, ${ }^{13}$ and 25 to 30 participants will likely yield similar results to those of several hundred participants. ${ }^{20}$

Due to the COVID-19 outbreak, the methodology was changed into patients sorting at home without supervision of one of the researchers. A detailed manual was created to describe all steps of the card-sorting tasks, a study call was performed including an extensive explanation and patients with remaining questions were contacted in an additional call.

In the first card-sorting task, patients sorted the individual cards with different barriers and facilitators printed on them, by order of content similarity. The following rules applied: all statements had to be placed in a group, a statement could only be placed in one group, at least two and a maximum of 20 statements per group were allowed, and at least 
four and a maximum of ten groups had to be formed. After the patients completed the sorting, they were asked to describe each group with an overarching word or sentence (i.e. an open card sorting).

In the second card-sorting task, patients sorted the individual cards with different barriers and facilitators printed on them, by order of content applicability (importance and helpfulness, respectively) into five predetermined groups. Group one had to contain statements that were least applicable to the individual and group five contained statements that were most applicable to the individual (i.e. forced closed card sorting). The following rules applied: all statements had to be placed in a group, a statement could only be placed in one group, and all five groups had to contain eight statements. Additionally, rheumatologists of the Utrecht RA Cohort Study group performed the second card-sorting task, now using an online questionnaire (OptimalSort, Optimal Workshop, Wellington, New Zealand). They sorted the barriers and facilitators by order of content applicability to the general population of RA patients.

\section{Statistical analyses}

The similarity of statements was classified using hierarchical cluster analysis, a statistical technique to classify similar objects into separate clusters. ${ }^{21}$ The cells of the input proximity matrix included the frequency of statements being not sorted in the same group. The number of statements that were not sorted in the same group was squared to get squared Euclidean distances. Then, Ward's method was used to cluster the most similar statements. At the end, a set of clusters was derived and presented as dendrogram and agglomeration schedule, which showed which statements were combined in each stage of the process. The project group (NMTR, MCVdG, PMJW, EPCVO, NCN, JMVL, RG) decided on the final number of clusters using the output of the hierarchical cluster analysis. The main criterion to decide on the final set of clusters was that clusters comprised statements with a consistent content that diverged from the content of other clusters.

Patient characteristics and applicability of statements and clusters were summarised descriptively. Results of D2T patients were compared with those of non-D2T RA patients and rheumatologists, and tested for statistically significant differences using independent T-, Mann-Whitney U, Fisher's exact or chi-square test for continuous (depending on distribution), binary and categorical variables, respectively. Two-sided tested $\mathrm{p}$-values $<0.05$ were considered statistically significant. Statistical analyses were performed using IBM SPSS Statistics v24 (IBM Corp, Armonk, NY, USA).

\section{Results}

\section{Interviews}


Saturation of information occurred after 14 RA patients (7 D2T and 7 non-D2T RA patients) participated in the in-depth interviews. All invited patients chose to participate. Patient characteristics were similar to those of the total previous study population, ${ }^{8}$ ensuring heterogeneity and representativeness of the sample (Supplementary Table 1). The median duration of interviews was 21 minutes (interquartile range 19-31 minutes).

\section{Selection of statements}

Three hundred ninety statements were collected: 206 barriers and 184 facilitators of optimal adherence (Figure 1). One hundred sixty-two duplicates were removed (72 barriers and 90 facilitators). From the remaining statements, a final set of 40 statements per category was derived (Supplementary Tables 2 and 3).

\section{Card-sorting tasks}

Patients

Fifty RA patients were invited and, of these, 40 patients participated (20 D2T and 20 nonD2T RA patients). Patient characteristics were similar to those of the total previous study population,, ensuring heterogeneity and representativeness of the sample (Table 1).

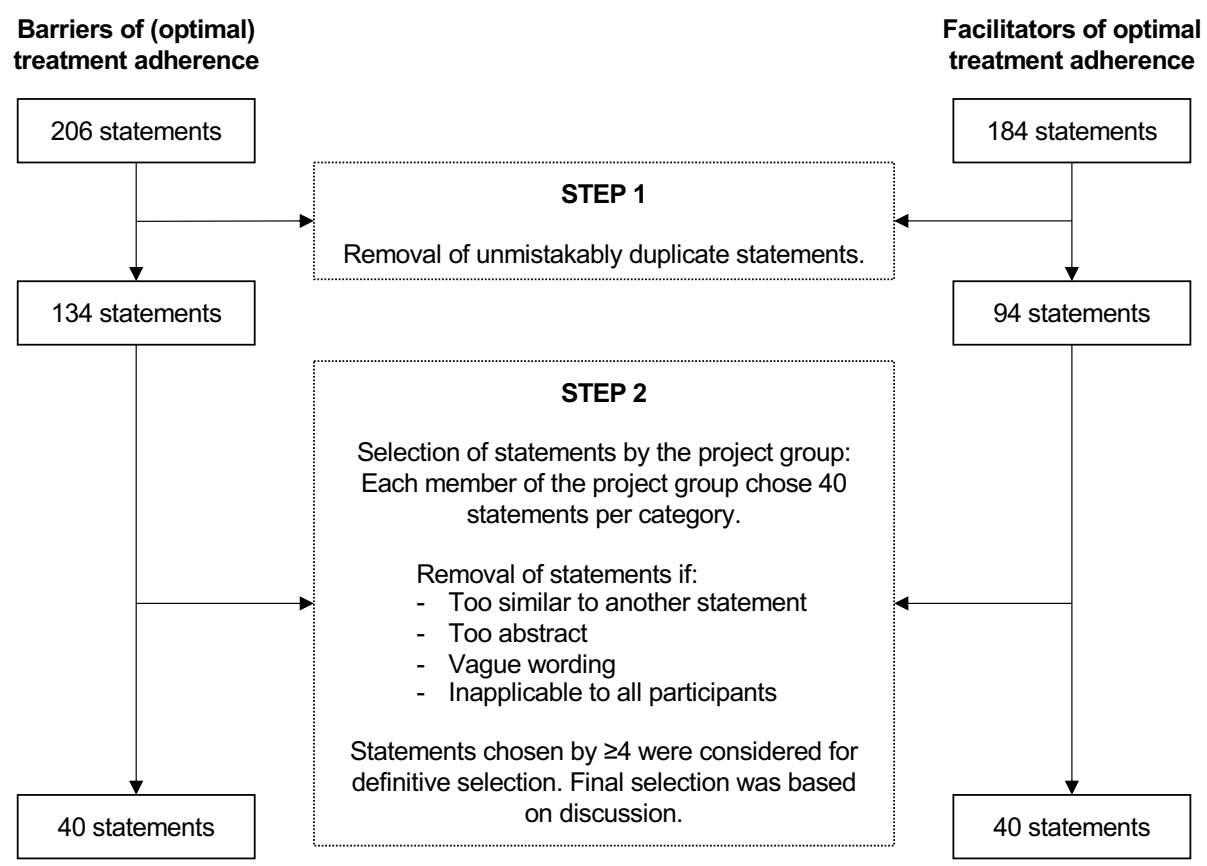

Figure 1. Flow chart of selection of statements

Project group: NMTR, MCvdG, PMJW, EPCVO, NCN, JMvL, RG. 
Table l. Characteristics of patients who participated in the card-sorting task in comparison with the total previous study population ${ }^{8}$

\begin{tabular}{|c|c|c|c|c|}
\hline & \multicolumn{2}{|c|}{$\begin{array}{l}\text { Patients who participated in } \\
\text { the card-sorting task }\end{array}$} & \multicolumn{2}{|c|}{$\begin{array}{l}\text { Total previous study } \\
\text { population }\end{array}$} \\
\hline & $\begin{array}{l}\text { D2T RA } \\
(n=20)\end{array}$ & $\begin{array}{l}\text { Non-D2T RA } \\
(n=20)\end{array}$ & $\begin{array}{l}\text { D2T RA } \\
(n=52)\end{array}$ & $\begin{array}{l}\text { Non-D2T RA } \\
(n=100)\end{array}$ \\
\hline \multicolumn{5}{|l|}{ (Socio-)demographics } \\
\hline Age, years, mean (SD) & $60.4(8.6)$ & $61.1(7.8)$ & $60.2(11.4)$ & $64.5(10.9) *$ \\
\hline Female, $\mathrm{n}(\%)$ & $15(75)$ & $14(70)$ & $38(73)$ & $72(72)$ \\
\hline \multicolumn{5}{|l|}{ Education, n (\%) } \\
\hline None & $0(0)$ & $0(0)$ & $1(2)$ & $0(0)^{\ddagger}$ \\
\hline Primary school & $1(5)$ & $0(0)$ & $3(6)$ & $2(2)$ \\
\hline $\begin{array}{l}\text { Secondary school/secondary vocational } \\
\text { education }\end{array}$ & $11(55)$ & $5(25)$ & $30(58)$ & $48(49)$ \\
\hline High vocational education/university & $8(40)$ & $15(75)$ & $18(34)$ & $49(49)$ \\
\hline \multicolumn{5}{|l|}{ Work participation, n (\%) } \\
\hline Paid work & $2(10)$ & $7(35)$ & $7(14)$ & $23(23) \ddagger$ \\
\hline Paid work and partly work disabled & $2(10)$ & $2(10)$ & $3(6)$ & $2(2)$ \\
\hline Fully work disabled & $7(35)$ & $3(15)$ & $16(31)$ & $17(17)$ \\
\hline Retired & $5(25)$ & $6(30)$ & $16(31)$ & $43(44)$ \\
\hline Other & $4(20)$ & $2(10)$ & $10(19)$ & $14(14)$ \\
\hline \multicolumn{5}{|l|}{ Disease characteristics } \\
\hline Disease duration, years, median (IQR) & $18.5(13.0-27.8)$ & $11.5(5.3-27.8)$ & $17.0(9.0-25.0)$ & $14.0(8.0-24.0)$ \\
\hline RF positivity, n (\%) & $15(75)$ & $13(65)$ & $39(75)$ & $65(65)$ \\
\hline ACPA positivity, n (\%) & $13(65)$ & $14(70)$ & $38(73)$ & $65(65)$ \\
\hline DAS28-ESR, median (IQR) & $3.7(3.0-4.5)$ & $2.0(1.4-2.8)$ & $4.1(3.5-6.1)$ & $2.5(1.8-3.3) *$ \\
\hline $\begin{array}{l}\text { Comorbidities, number according to EULAR } \\
\text { domains, }{ }^{22} \text { median (IQR) }\end{array}$ & $2(1-2)$ & $1(0-1)$ & $2(1-3)$ & $1(0-1) *$ \\
\hline \multicolumn{5}{|l|}{ Drugs } \\
\hline \multicolumn{5}{|l|}{ Failed DMARDs, number, median (IQR) } \\
\hline csDMARDs & $3(2-3)$ & $1(0-3)$ & $3(3-5)$ & $2(1-3) *$ \\
\hline $\mathrm{b} / \mathrm{tsDMARDs}$ & $4(3-5)$ & $0(0-0)$ & $4(3-6)$ & $0(0-1) *$ \\
\hline Current DMARDs, number, median (IQR) & $2(1-2)$ & $1(1-2)$ & $2(1-2)$ & $1(1-2)$ \\
\hline csDMARDs, n (\%) & $16(80)$ & $18(90)$ & $37(71)$ & $86(86) *$ \\
\hline bDMARDs, n (\%) & $11(55)$ & $8(40)$ & $27(52)$ & $39(39)$ \\
\hline tsDMARDs, n (\%) & $3(15)$ & $0(0)$ & $12(23)$ & $0(0) *$ \\
\hline \multicolumn{5}{|l|}{ Administration route of current DMARDs, $\mathrm{n}(\%)$} \\
\hline Oral & $17(85)$ & $18(90)$ & $45(87)$ & $86(86)$ \\
\hline Subcutaneous & $4(20)$ & $8(40)$ & $11(21)$ & $30(30)$ \\
\hline Intravenous & $7(35)$ & $0(0)$ & $16(31)$ & $9(9)$ \\
\hline Current glucocorticoids, n (\%) & $11(55)$ & $2(10)$ & $27(52)$ & $16(16)^{*}$ \\
\hline Current painkillers, n (\%) & $19(95)$ & $9(45)$ & $49(94)$ & $64(64)$ \\
\hline
\end{tabular}




\begin{tabular}{lcccc}
\hline & $\begin{array}{c}\text { Patients who participated } \\
\text { in the card-sorting task }\end{array}$ & $\begin{array}{c}\text { Total previous study } \\
\text { population }\end{array}$ \\
& $\begin{array}{c}\text { D2T RA } \\
(\mathrm{n}=20)\end{array}$ & $\begin{array}{c}\text { Non-D2T RA } \\
(\mathrm{n}=20)\end{array}$ & $\begin{array}{c}\text { D2T RA } \\
(\mathrm{n}=52)\end{array}$ & $\begin{array}{c}\text { Non-D2T RA } \\
(\mathrm{n}=100)\end{array}$ \\
\hline $\begin{array}{l}\text { Current other non-antirheumatic drugs, number, } \\
\text { median (IQR) }\end{array}$ & $6(3-8)$ & $2(1-4)$ & - & - \\
$\begin{array}{l}\text { Treatment non-adherence } \\
\text { Level of MARS-5, median (IQR) }\end{array}$ & $24(22-25)$ & $24(23-25)$ & $24(21-25)$ & $24(23-25) \ddagger$ \\
$\begin{array}{l}\text { Discrepancy in supplied and prescribed drugs, } \\
\mathrm{n}(\%)\end{array}$ & $8(40)$ & $4(20)$ & $21(40)$ & $22(22))^{\ddagger *}$ \\
\hline
\end{tabular}

ACPA: anti-citrullinated protein antibodies; b: biological; cs: conventional synthetic; D2T: difficult-to-treat; DAS28ESR: disease activity score assessing 28 joints using erythrocyte sedimentation rate; DMARD: disease-modifying antirheumatic drug; EULAR: European League Against Rheumatism; IQR: interquartile range; MARS-5: medication adherence reporting scale (5-25, higher score reflects higher level of adherence); ${ }^{23} \mathrm{n}$ : number; RA: rheumatoid arthritis; RF: rheumatoid factor; SD: standard deviation; ts: targeted synthetic; t: $n=99 ;$ *: $p<0.05$.

In the total previous study population, D2T and non-D2T RA patients were compared. Differences were analysed using independent T-test, Fisher's exact test, chi-square test or Mann-Whitney U test, as appropriate. Statistical significance was not tested in the participants of the card-sorting task because of the smaller sample size.

DMARDs of different administration routes were prescribed as well as different numbers of drugs.

Non-adherence according to a discrepancy between supplied and prescribed drugs was more frequent among D2T than among non-D2T RA patients (this difference was statistically significant in the total previous study population; statistical significance was not tested in the participants of the card-sorting task because of the smaller sample size, Table 1). The self-reported level of adherence (according to a questionnaire) did not differ between D2T and non-D2T RA patients, as described previously. ${ }^{8}$

\section{Rheumatologists}

Of 52 invited rheumatologists, 20 participated in the card-sorting task. Four of them were still in training. The participating rheumatologists each treated an estimated mean of 189 unique RA patients per year.

\section{Barriers of (optimal) treatment adherence: structured overview}

Patients sorted the barriers into a mean of 6.3 groups (range 4-10). In total, 246 groups were created and 245 groups were named. Twenty-nine labels were used by more than one patient, e.g. relationship with the physician, discipline, adverse events, usability issues, distrust.

After discussing the results of the hierarchical cluster analysis, the project group chose the 9-cluster option with four overarching categories (Figure 2a). Options of 5, 7, 8 and 10 clusters were also considered. Decreasing the number of clusters below 9 resulted in 
the combination of clusters 'adverse events' and 'doubts about safety', the combination of clusters 'doubts about effectiveness', 'low disease activity' and 'cost-utility evaluation', and the combination of clusters 'administration route' and 'complexity of drug regimen'. These clusters were considered to be too distinct to combine. Increasing the number of clusters above 9 separated the cluster 'health behaviour', which did not result in new, clearly distinguishable clusters. Each cluster represented two to eight barriers (Supplementary Table 2).

Barriers of (optimal) treatment adherence: importance

The prioritisation of the importance of the barriers is shown in Figure $2 b$ (details in Supplementary Table 2). The cluster 'adverse events' was the most important barrier in D2T and non-D2T RA patients and rheumatologists. D2T RA patients ranked 'doubts about effectiveness' as second most important and ranked this cluster as more important than non-D2T RA patients (mean (standard deviation (SD)): 3.7 (0.7) vs 3.2 (0.7), p=0.02). Additionally, D2T RA patients ranked 'complexity of drug regimen' as more important than rheumatologists (mean (SD): 2.7 (0.9) vs 2.3 (0.5), p=0.03). Conversely, D2T RA patients ranked 'health behaviour' as less important than rheumatologists (mean (SD): 2.4 (0.7) vs 3.0 (0.5), p<0.01).

\section{Facilitators of optimal treatment adherence: structured overview}

Patients sorted the facilitators into a mean of 6.2 groups (range 4-10). In total, 252 groups were created and 250 groups were named. Twenty-three labels were used by more than one patient, e.g. pharmacy, help from others, routine, information.

After discussing the results of the hierarchical cluster analysis, the project group chose the 7-cluster option with two overarching categories (Figure 3a). Decreasing the number of clusters below 7 resulted in the combination of clusters 'good relationship with the HCP' and 'treatment information'. These clusters were considered to be too distinct to combine. Increasing the number of clusters above 7 separated the cluster 'aids', which did not result in new, clearly distinguishable clusters. Each cluster represented three to eleven facilitators (Supplementary Table 3).

Three facilitators were combined in the cluster 'miscellaneous': 'the absence of major life events', 'low personal costs related to drug use' and 'a reward after drug use'. Patients' names for this group were, for example, 'other' or 'external'.

\section{Facilitators of optimal treatment adherence: helpfulness}

The prioritisation of the helpfulness of the facilitators was largely similar to D2T RA patients, non-D2T RA patients and rheumatologists (Figure 3b, Supplementary Table 3). 'Good relationship with the $\mathrm{HCP}^{\prime}$ was ranked as the most helpful cluster, followed by 'treatment information' and 'routine and reminders'. 'Help from the pharmacy' was more helpful to 
A. Structured overview of barriers of (optimal) treatment adherence

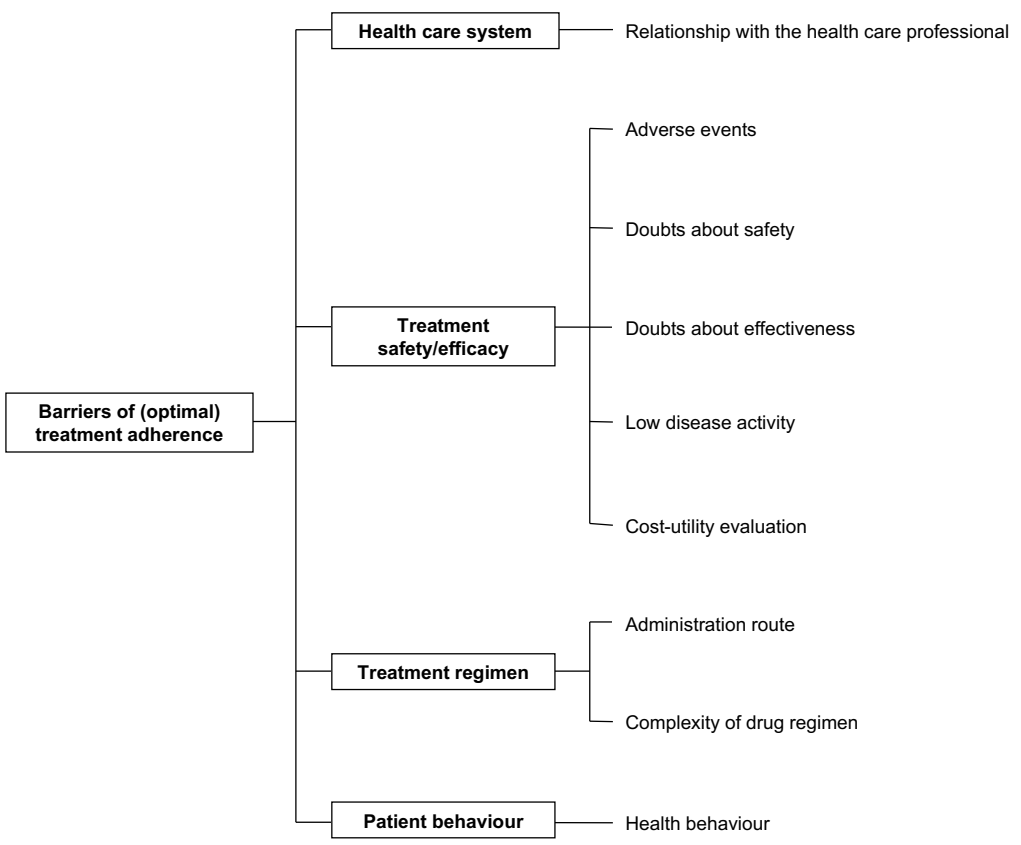

B. The helpfulness of clusters with barriers of (optimal) treatment adherence

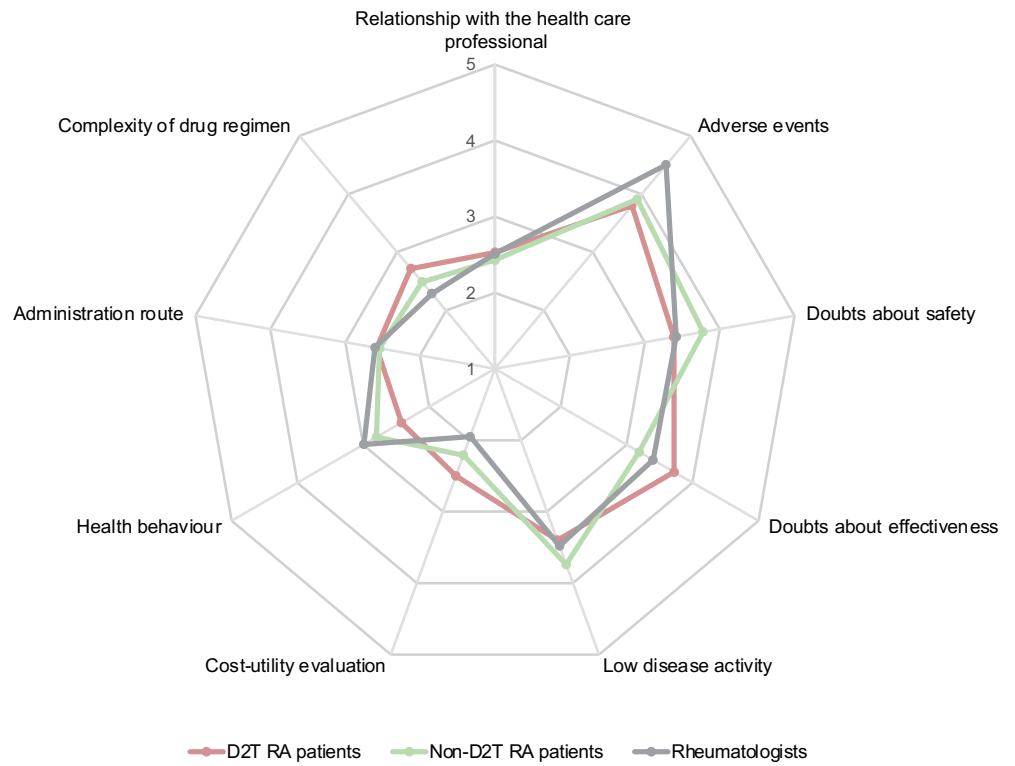

Figure 2. Barriers of (optimal) treatment adherence

A. The nine clusters are organised in four overarching categories (shown in the contoured boxes). B. Mean scores of importance of clusters. Importance was scored from 1 (least important) to 5 (most important).

D2T: difficult-to-treat; RA: rheumatoid arthritis. 
D2T RA patients than rheumatologists indicated (mean (SD): $3.2(0.8)$ vs $2.7(0.5), p=0.04$ ).

A. Structured overview of facilitators of optimal treatment adherence

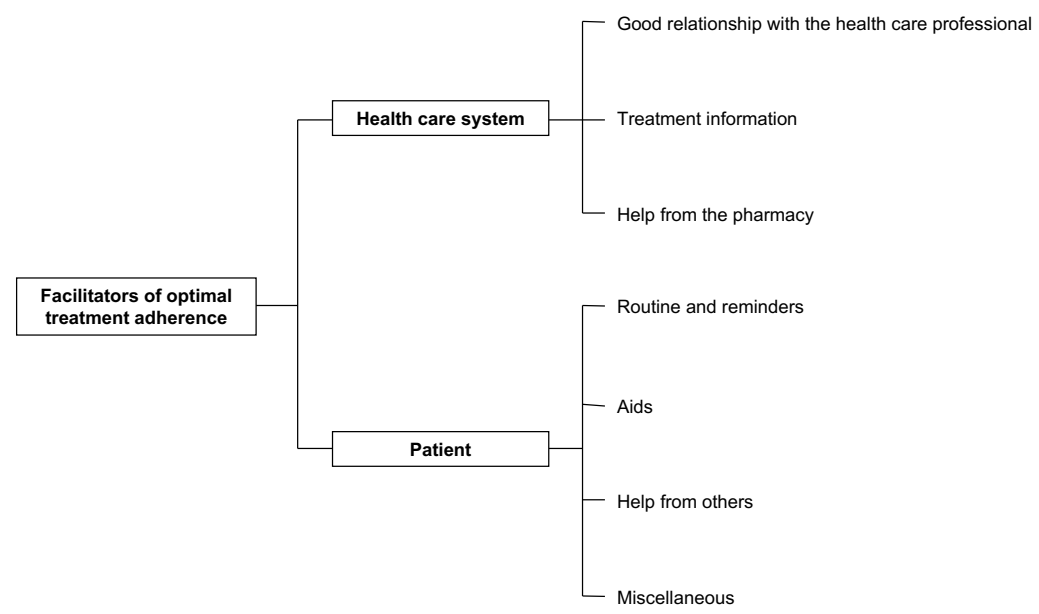

B. The helpfulness of clusters with facilitators of optimal treatment adherence

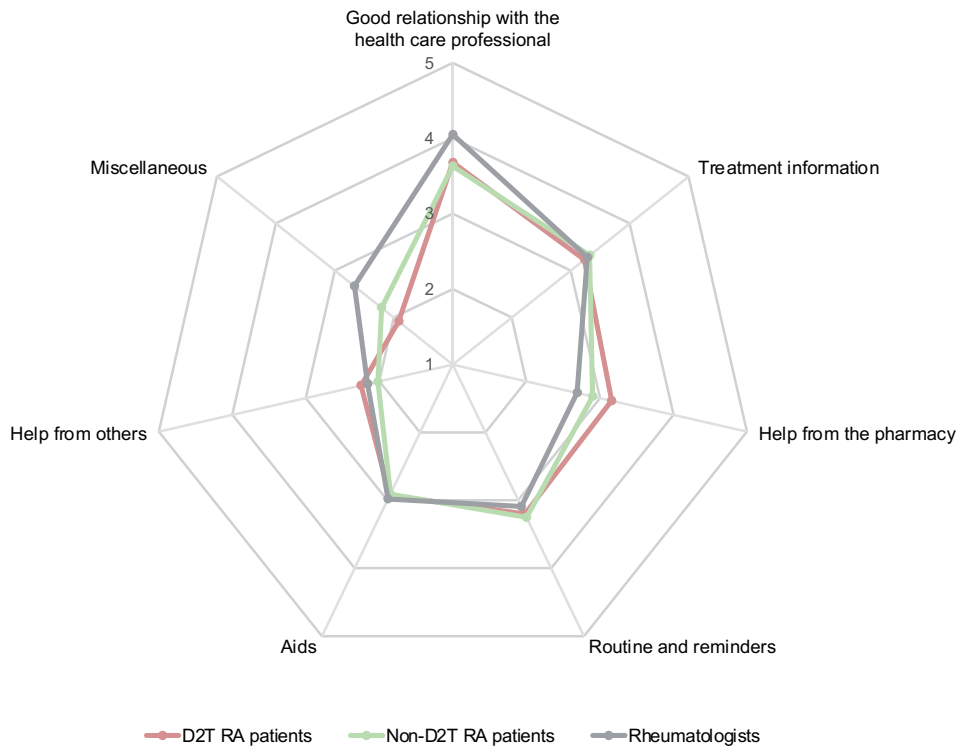

Figure 3. Facilitators of optimal treatment adherence

A. The seven clusters are organised in two overarching categories (shown in the contoured boxes). B. Mean scores of helpfulness of clusters. Helpfulness was scored from 1 (least helpful) to 5 (most helpful).

D2T: difficult-to-treat; RA: rheumatoid arthritis 


\section{Discussion}

This study delineates the hierarchical structures of barriers and facilitators of optimal treatment adherence. Nine clusters of adherence barriers were identified in four overarching categories: health care system, treatment safety/efficacy, treatment regimen and patient behaviour. Additionally, seven clusters of facilitators of optimal adherence were identified in two overarching categories: health care system-related and patient-related. D2T and non-D2T RA patients and rheumatologists prioritised the presence of adverse events as the most important adherence barrier. For D2T RA patients as a group, adverse events were followed by doubts about effectiveness, which were a less important barrier for non-D2T RA patients. The facilitators of optimal adherence were prioritised largely similarly by all RA patients and rheumatologists: a good relationship with the HCP, treatment information, and routine and reminders were considered most helpful. Help from the pharmacy was considered significantly more helpful to D2T RA patients than rheumatologists indicated.

Although our study focussed on treatment non-adherence in general, most statements provided by the patients were in fact related to medication adherence, for instance, those in the barrier clusters 'adverse events' and 'administration route'. This suggests that patients, and perhaps also their rheumatologists guided by EULAR recommendations, ${ }^{5}$ thought less of adherence to non-pharmacological treatments, for instance lifestyle advises, of which adherence improvement would also be beneficial. Our study among RA patients differs from the recently published EULAR points to consider on treatment non-adherence that included patients with musculoskeletal diseases in general. ${ }^{24}$

The World Health Organisation described five different dimensions of adherence barriers, which resemble the clusters we identified as well as barriers identified in previous studies: condition-related ('low disease activity'), treatment-related ('adverse events', 'administration route', 'complexity of drug regimen'), patient-related ('doubts about safety', 'doubts about effectiveness', 'health behaviour'), health system-related (cluster 'relationship with the $\mathrm{HCP}^{\prime}$ ) and socioeconomic-related factors ('cost-utility evaluation'). ${ }^{10-12,16,25-29}$ Although all the clusters we identified can be placed in these five domains, patients in our study sorted the barriers somewhat differently resulting in a hierarchical structure with other overarching categories. As our overviews were structured by patients themselves, they may be preferred in daily practice to screen barriers and facilitators that individual patients consider important and helpful.

Despite the variety in the socioeconomic status of participants, few adherence barriers in the socioeconomic domain were mentioned during the interviews. Costs were mentioned, but other socioeconomic-related barriers that have previously been associated with non-adherence were not brought up in our study (e.g. lack of support from relatives, cultural aspects and religion). ${ }^{25}$ As a hypothesis, this may be related to the 
Dutch (and Western) culture with a more prominent focus on individualism instead of collectivism. ${ }^{30-32}$ Nevertheless, these socioeconomic-related barriers have previously not been confirmed to play a role in non-adherence in RA specifically. ${ }^{27}$

The identified facilitators of optimal adherence largely resemble the facilitators in previous studies. ${ }^{1116}$ However, some facilitators were not specifically mentioned during the interviews in our study: e.g. experience from others and maintaining autonomy. This may be a result of the relatively long disease duration of the participants (median 14 years in D2T and 17 years in non-D2T RA patients) and their extended experience with drug use. Hence, these other facilitators should be considered in patients with early RA.

Perceptions of barriers and facilitators of optimal treatment adherence were largely similar between all RA patients and rheumatologists, although some differences were identified in the prioritisation of adherence barriers. Particularly, D2T RA patients prioritised doubts about effectiveness as an important barrier, which may be explained by their higher number of previously failed drugs and higher disease activity levels. ${ }^{8}$ Additionally, the prioritisation of adherence barriers among rheumatologists seemed to be more aligned with the prioritisation of non-D2T than with those of D2T RA patients. As only $5 \%$ to $20 \%$ of RA patients can be classified as having D2T RA, $3,4,7$ the perceptions of rheumatologists may predominantly be based on non-D2T RA patients. Therefore, our results suggest that D2T RA patients, as a group, should be addressed somewhat differently than non-D2T RA patients, for example, by more explicitly discussing their (possible) doubts about effectiveness and the importance of treatment adherence, to achieve an optimal treatment response. Additionally, perceptions of non-adherence between D2T RA patients and rheumatologists could be further aligned.

However, to the extent that the perceptions of non-adherence may differ between individual patients, we suggest that HCPs should be open to the occurrence of all possible barriers and facilitators. Therefore, a discussion between patients and HCPs remains essential. This is emphasised by the most helpful facilitators we identified: a good relationship with the HCP and treatment information. This discussion can be guided by the structured overviews of barriers and facilitators and should be conducted in all phases of the treatment process (i.e. before treatment initiation, in the treatment initiation phase and in the treatment persistence phase; Table 2 presents an overview of a structured approach to address treatment (non-)adherence). For D2T RA patients specifically, this discussion should include doubts about effectiveness among other potential barriers. The discussion should also focus on patient information and education. Not only did patients identify treatment information as a helpful facilitator, educational interventions have also been shown to be able to improve adherence. .,33,34 $^{2}$

Furthermore, other facilitators could be implemented to optimise adherence. Patients, and D2T RA patients specifically, may benefit from help from the pharmacist (e.g. reminders when drugs should be ordered, a consultation with the pharmacist about drug use). 
Table 2. A structured approach to address treatment (non-)adherence in clinical practice

\begin{tabular}{|c|c|c|c|}
\hline & \multirow{2}{*}{$\begin{array}{l}\text { Before treatment } \\
\text { initiation }\end{array}$} & \multicolumn{2}{|c|}{ During treatment } \\
\hline & & $\begin{array}{l}\text { Initiation } \\
\text { phase }\end{array}$ & $\begin{array}{l}\text { Persistence } \\
\text { phase }\end{array}$ \\
\hline Discuss the importance of treatment adherence & $\mathrm{X}$ & $\mathrm{x}$ & $\mathrm{x}$ \\
\hline Discuss barriers of treatment adherence: & $\mathrm{x}$ & $\mathrm{x}$ & $\mathrm{x}$ \\
\hline \multicolumn{4}{|l|}{ Adverse events* } \\
\hline Experience with previous adverse events & $x$ & & \\
\hline Presence of adverse events & & $x$ & $x$ \\
\hline Doubts about safety & $x$ & $x$ & $x$ \\
\hline Doubts about effectiveness & $x$ & $x$ & $x$ \\
\hline Low disease activity\# & & & $x$ \\
\hline Cost-utility evaluation^ & $x$ & $x$ & $x$ \\
\hline \multicolumn{4}{|l|}{ Administration route* } \\
\hline Thoughts about administration route & $x$ & & \\
\hline Experience with the administration route & & $x$ & $x$ \\
\hline Complexity of drug regimen* & $x$ & $x$ & $x$ \\
\hline Health behaviour ${ }^{ \pm}$ & $x$ & $x$ & $x$ \\
\hline Discuss facilitators of optimal adherence: & $\mathrm{x}$ & $\mathrm{x}$ & $\mathrm{x}$ \\
\hline \multicolumn{4}{|l|}{ Treatment information } \\
\hline Provide information & $x$ & & \\
\hline Check whether additional information is needed & & $x$ & $x$ \\
\hline Help from the pharmacy*s & $x$ & $x$ & $x$ \\
\hline Routine and reminders & $x$ & $x$ & $x$ \\
\hline Aids & $x$ & $x$ & $x$ \\
\hline Help from others & $x$ & $x$ & $x$ \\
\hline Maintain a good relationship between patient and HCP & $\mathrm{x}$ & $\mathrm{x}$ & $\mathrm{x}$ \\
\hline \multicolumn{4}{|c|}{ 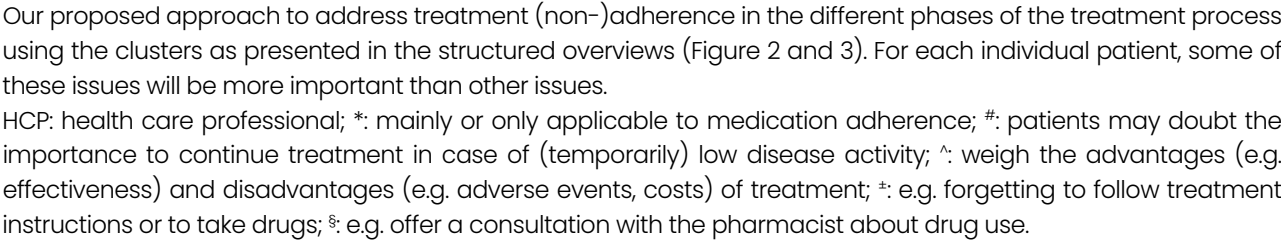 } \\
\hline
\end{tabular}

Additionally, the complexity of the drug regimen could be reduced by implementing aids (e.g. a patient-friendly drug strip or pill box). Also, the role of routine and reminders (e.g. linking the moment of drug use to a fixed moment of the day, using apps and e-health) ${ }^{35}$ and help from others (e.g. relatives and psychologists) ${ }^{9}$ could be discussed. Nevertheless, also helpfulness of these facilitators will vary between patients and, therefore, optimising adherence should be tailored to the individual patient. Additional guidance can be found 
in the recently published EULAR points to consider on treatment non-adherence. ${ }^{24}$ Future studies should address whether the use of these structured overviews in discussions together with the implementation of these facilitators will ultimately improve adherence. A major strength of this study is the combination of qualitative and quantitative methods to thematically structure perceptions of non-adherence. Additionally, a patient research partner was involved in the whole study as a researcher and co-author, providing a patient's opinion on, for example, the selection of statements and the number of clusters. Hence, the influence of the researchers has been minimised. As a limitation, this study was conducted in one country, which may limit generalisability of the results, particularly to non-Western countries. The relatively low level of non-adherence (especially the selfreported values) of participants may be another limitation. However, self-reported nonadherence is known to often underreport non-adherence assessed by other methods, such as a discrepancy between supplied and prescribed drugs. ${ }^{36}$ We also found a discrepancy (Table 1 and Supplementary Table 1), in line with previously reported rates. ${ }^{36-39}$ Still, the results might have been even more relevant if only non-adherent patients would have participated, although the feasibility of such studies is probably low. The relatively low response rate among rheumatologists (38\%) should be considered another limitation. This might be due to the large workload during the COVID-19 outbreak. We do not know whether this could have influenced the results. Furthermore, some patients expressed difficulties in performing the similarity sorting and categorised statements as 'not applicable' or 'other'. Nevertheless, the influence on the results may be limited as the face validity of the structured overviews is high.

In conclusion, this study provides structured overviews of perceptions of treatment non-adherence. Adherence barriers were structured into health care system-related (relationship with the HCP), treatment safety/efficacy-related (adverse events, doubts about safety, doubts about effectiveness, low disease activity, cost-utility evaluation), treatment regimen-related (administration route, and complexity of drug regimen) and patient behaviour-related barriers (health behaviour). Facilitators of optimal adherence were structured into health care system-related (a good relationship with the HCP, treatment information, help from the pharmacy) and patient-related facilitators (routine and reminders, aids, and help from others). On average, the perceived importance of adherence barriers was only slightly different in D2T RA patients compared with non-D2T RA patients and rheumatologists. The helpfulness of facilitators of optimal adherence was prioritised largely similarly by all RA patients and rheumatologists: good relationship with the HCP and treatment information were most helpful. These findings further indicate the importance of a discussion about non-adherence between patients and rheumatologists to improve adherence. 


\section{References}

1. Smolen JS, Aletaha D, Mclnnes IB. Rheumatoid arthritis. Lancet 2016;388:2023-38.

2. de Hair MJH, Jacobs JWG, Schoneveld JLM, et al. Difficult-to-treat rheumatoid arthritis: an area of unmet clinical need. Rheumatology (Oxford) 2017;57:1135-44.

3. Buch $\mathrm{MH}$. Defining refractory rheumatoid arthritis. Ann Rheum Dis 2018;77:966-9.

4. Kearsley-Fleet L, Davies R, De Cock D, et al. Biologic refractory disease in rheumatoid arthritis: Results from the British Society for Rheumatology Biologics Register for Rheumatoid Arthritis. Ann Rheum Dis 2018;77:1405-12.

5. Smolen JS, Landewé RBM, Bijlsma JWJ, et al. EULAR recommendations for the management of rheumatoid arthritis with synthetic and biological disease-modifying antirheumatic drugs: 2019 update. Ann Rheum Dis 2020;79:685-99.

6. Nagy G, Roodenrijs NMT, Welsing PMJ, et al. EULAR definition of difficult-to-treat rheumatoid arthritis. Ann Rheum Dis 2021;80:31-5.

7. Roodenrijs NMT, de Hair MJH, van der Goes $\mathrm{MC}$, et al. Characteristics of difficult-to-treat rheumatoid arthritis: results of an international survey. Ann Rheum Dis 2018;77:1705-9.

8. Roodenrijs NMT, van der Goes MC, Welsing PMJ, et al. Difficult-to-treat rheumatoid arthritis: contributing factors and burden of disease. Rheumatology 2020:keaa860.

9. Gossec L, Molto A, Romand X, et al. Recommendations for the assessment and optimization of adherence to diseasemodifying drugs in chronic inflammatory rheumatic diseases: A process based on literature reviews and expert consensus. Jt Bone Spine 2019;86:13-9.

10. Hugtenburg JG, Timmers L, Elders PJ, et al. Definitions, variants, and causes of nonadherence with medication: a challenge for tailored interventions. Patient Prefer Adherence 2013;7:675-82.

11. Voshaar $\mathrm{M}$, Vriezekolk J, van Dulmen $\mathrm{S}$, et al. Barriers and facilitators to diseasemodifying antirheumatic drug use in patients with inflammatory rheumatic diseases: a qualitative theory-based study. BMC Musculoskelet Disord 2016;17:442.

12. Ritschl V, Lackner A, Boström C, et al. I do not want to suppress the natural process of inflammation: New insights on factors associated with non-adherence in rheumatoid arthritis. Arthritis Res Ther 2018;20:234.

13. Trochim KM. An introduction to concept mapping for planning and evaluation. Eval Prog Plan 1989:1-16.

14. Rosas SR, Kane M. Quality and rigor of the concept mapping methodology: A pooled study analysis. Eval Program Plann 2012;35:236-45.

15. Kool MB, van Middendorp $H$, Boeije HR, et al. Understanding the lack of understanding: invalidation from the perspective of the patient with fibromyalgia. Arthritis Rheum 2009;61:1650-6.

16. Pasma A, van't Spijker A, Hazes JMWW, et al. Factors associated with adherence to pharmaceutical treatment for rheumatoid arthritis patients: a systematic review. Semin Arthritis Rheum 2013;43:18-28.

17. Li L, Cui Y, Yin R, et al. Medication adherence has an impact on disease activity in rheumatoid arthritis: a systematic review and meta-analysis. Patient Prefer Adherence 2017;11:1343-56.

18. Aletaha D, Neogi T, Silman AJ, et al. 2010 Rheumatoid arthritis classification criteria: An American College of Rheumatology/ European League Against Rheumatism collaborative initiative. Ann Rheum Dis 2010;69:1580-8.

19. Tong A, Sainsbury P, Craig J. Consolidated criteria for reporting qualitative research (COREQ): A 32-item checklist for interviews and focus groups. Int J Qual Heal Care 2007;19:349-57.

20. Wood JR, Wood LE. Card Sorting: Current Practices and Beyond. J Usability Stud 2008;4:1-6.

21. Romesburg H. Cluster Analysis for Researchers. Belmont, CA: : Lifetime learning Publications 1984.

22. Radner H, Chatzidionysiou K, Nikiphorou E, et al. 2017 EULAR recommendations for a core data set to support observational research and clinical care in rheumatoid arthritis. Ann Rheum Dis 2018;77:476-9.

23. Thompson K, Kulkarni J, Sergejew AA. Reliability and validity of a new Medication Adherence Rating Scale (MARS) for the psychoses. Schizophr Res 2000;42:241-7.

24. Ritschl V, Stamm TA, Aletaha D, et al. 2020 EULAR points to consider for the prevention, screening, assessment and management of non-adherence to treatment in people with 
rheumatic and musculoskeletal diseases for use in clinical practice. Ann Rheum Dis 2020:annrheumdis-2020-218986.

25. Sabaté E. WHO | Adherence to long-term therapies: evidence for action. World Health Organization 2015.

26. Achaval S de, Suarez-Almazor ME. Treatment adherence to disease-modifying antirheumatic drugs in patients with rheumatoid arthritis and systemic lupus erythematosus. Int J Clin Rheumtol 2010;5:31326.

27. Van Den Bemt BJF, Zwikker HE, Van Den Ende CHM. Medication adherence in patients with rheumatoid arthritis: A critical appraisal of the existing literature. Expert Rev Clin Immunol 2012;8:337-51.

28. Lin J, Sklar GE, Oh VM Sen, et al. Factors affecting therapeutic compliance: A review from the patient's perspective. Ther Clin Risk Manag 2008;4:269-86.

29. Salt E, Frazier SK. Adherence to diseasemodifying antirheumatic drugs in patients with rheumatoid arthritis: A narrative review of the literature. Orthop Nurs 2010;29:260-75.

30. Tafarodi RW, Swann WB. Individualismcollectivism and global self-esteem: Evidence for a cultural trade-off. J Cross Cult Psychol 1996;27:651-72.

31. Eckersley R. Is modern Western culture a health hazard? Int J Epidemiol 2006;35:252-8.

32. Santos HC, Varnum MEW, Grossmann I. Global Increases in Individualism. Psychol Sci 2017;28:1228-39.

33. Lavielle $M$, Puyraimond-Zemmour $D$, Romand $X$, et al. Methods to improve medication adherence in patients with chronic inflammatory rheumatic diseases: A systematic literature review. RMD Open 2018;4:e000684.

34. Ritschl V, Stamm TA, Aletaha D, et al. Prevention, screening, assessing and managing of nonadherent behaviour in people with rheumatic and musculoskeletal diseases: systematic reviews informing the 2020 EULAR points to consider. RMD Open 2020;6:e001432.

35. Bosworth HB, Zullig LL, Mendys $\mathrm{P}$, et al. Health Information Technology: Meaningful Use and Next Steps to Improving Electronic Facilitation of Medication Adherence. JMIR Med Informatics 2016;4:e9.

36. Lam WY, Fresco P. Medication Adherence Measures: An Overview. Biomed Res Int 2015;2015:1-12.

37. Murage $\mathrm{M}$, Tongbram $\mathrm{V}$, Feldman $\mathrm{S}$, et al. Medication adherence and persistence in patients with rheumatoid arthritis, psoriasis, and psoriatic arthritis: a systematic literature review. Patient Prefer Adherence 2018;12:1483503.

38. López-Medina C, Moltó A, Gérald F, et al. Assessment of the adherence to diseasemodifying drugs in patients with chronic inflammatory rheumatic diseases: Results of a survey of 1594 patients. Jt Bone Spine 2019;86:610-4.

39. Hope HF, Hyrich KL, Anderson J, et al. The predictors of and reasons for non-adherence in an observational cohort of patients with rheumatoid arthritis commencing methotrexate. Rheumatology 2020;59:213-23. 


\section{Supplementary}

\section{Supplementary File 1. Interview guide}

\section{Introduction}

- Previous studies have shown that (almost) nobody uses their medication perfectly and is fully adherent all the time. This is very understandable.

- To be able to improve management of treatment adherence, we would like to collect as much information as possible about this topic. This is the aim of the interview.

- You, the patient, are the expert. All answers you provide us with are correct and valuable. There are no wrong answers. The more information you give, the better we will be able to help you and other patients.

\section{Questions to ask during the interviews}

1. Could you think of reasons for treatment non-adherence?

- Can you give as many reasons as possible?

- Why is this a reason whether to take medication or not?

- Can you think of more reasons?

2. What would be reasons for you to be non-adherent?

- Why is this a reason for you?

- Can you think of more reasons?

3. What would be reasons for you to be adherent?

- Why is this a reason?

- Can you think of more reasons?

4. Which (external) factors can hamper optimal treatment adherence?

- Why will this hamper you to take your medication?

- Do you think this could also hamper optimal treatment adherence in other patients?

- Can you think of more factors that could hamper optimal treatment adherence?

5. Which (external) factors can facilitate optimal treatment adherence?

- Why will this help you to take your medication?

- Do you think this will also be helpful for other patients?

- Can you think of more factors to facilitate treatment adherence?

\section{Closure}

- Do you think that all important topics have been discussed?

- Do you have anything else to tell us that was not yet discussed in the interview? 


\section{Supplementary File 2. Interviews: extraction of statements and saturation of information}

Verbatim transcription (by EPCVO) was performed after each interview had been conducted. Statements were extracted regarding (a) barriers and (b) facilitators of optimal adherence (by EPCVO and confirmed by NMTR). The extracted statements were collected in one Excel document and sorted by content similarity. In case of unmistakably duplicate statements in terms of content, these statements were added to the same row (after achieving agreement between NMTR and EPCVO). After each additional interview, newly extracted statements were added in order of content similarity. At the time no new, i.e. unmistakably not duplicate statements in terms of content, could be distilled from the two latest, consecutive interviews (according to NMTR, EPCVO and RG), it was decided that saturation of information had occurred and that no new interviews need to be conducted.

Supplementary File 3. COREQ (COnsolidated criteria for REporting Qualitative research) checklist $^{19}$

Supplementary Table 3 can be accessed via: doi: 10.1093/rheumatology/keabl30. 
Supplementary Table 1. Characteristics of patients who participated in the interview study in comparison with the total previous study population ${ }^{8}$

\begin{tabular}{|c|c|c|c|c|}
\hline & \multicolumn{2}{|c|}{$\begin{array}{l}\text { Patients who participated in } \\
\text { the interview study }\end{array}$} & \multicolumn{2}{|c|}{$\begin{array}{l}\text { Total previous study } \\
\text { population }\end{array}$} \\
\hline & $\begin{array}{l}\text { D2T RA } \\
(n=7)\end{array}$ & $\begin{array}{c}\text { Non-D2T } \\
(n=7)\end{array}$ & $\begin{array}{l}\text { D2T RA } \\
(n=52)\end{array}$ & $\begin{array}{l}\text { Non-D2T RA } \\
(n=100)\end{array}$ \\
\hline \multicolumn{5}{|l|}{ (Socio-)demographics } \\
\hline Age, years, mean (SD) & $59.4(11.3)$ & $64.4(10.0)$ & $60.2(11.4)$ & $64.5(10.9)^{*}$ \\
\hline Female, $\mathrm{n}(\%)$ & $5(71)$ & 5 (71) & $38(73)$ & $72(72)$ \\
\hline \multicolumn{5}{|l|}{ Education, n (\%) } \\
\hline None & $0(0)$ & $0(0)$ & 1 (2) & $0(0)^{\ddagger}$ \\
\hline Primary school & $1(14)$ & $0(0)$ & $3(6)$ & $2(2)$ \\
\hline $\begin{array}{l}\text { Secondary school/secondary vocational } \\
\text { education }\end{array}$ & $4(57)$ & $4(57)$ & $30(58)$ & $48(49)$ \\
\hline High vocational education/university & $3(29)$ & $3(43)$ & $18(34)$ & $49(49)$ \\
\hline \multicolumn{5}{|l|}{ Work participation, n (\%) } \\
\hline Paid work & $2(43)$ & $2(29)$ & $7(14)$ & $23(23)^{\ddagger}$ \\
\hline Paid work and partly work disabled & $0(0)$ & $0(0)$ & $3(6)$ & $2(2)$ \\
\hline Fully work disabled & $1(14)$ & $1(14)$ & $16(31)$ & $17(17)$ \\
\hline Retired & $2(29)$ & $3(43)$ & $16(31)$ & $43(44)$ \\
\hline Other & $1(14)$ & $1(14)$ & $10(19)$ & $14(14)$ \\
\hline \multicolumn{5}{|l|}{ Disease characteristics } \\
\hline Disease duration, years, median (IQR) & $19.0(13.0-25.0)$ & $18.0(12.0-29.0)$ & $17.0(9.0-25.0)$ & $14.0(8.0-24.0)$ \\
\hline RF positivity, n (\%) & $4(57)$ & $3(43)$ & $39(75)$ & $65(65)$ \\
\hline ACPA positivity, $\mathrm{n}(\%)$ & $5(71)$ & $4(57)$ & $38(73)$ & $65(65)$ \\
\hline DAS28-ESR, median (IQR) & $4.0(3.5-6.2)$ & $2.1(1.7-3.3)$ & $4.1(3.5-6.1)$ & $2.5(1.8-3.3) *$ \\
\hline $\begin{array}{l}\text { Comorbidities, number according to EULAR } \\
\text { domains, }{ }^{22} \text { median (IQR) }\end{array}$ & $2(1-3)$ & $2(1-2)$ & $2(1-3)$ & $1(0-1) *$ \\
\hline \multicolumn{5}{|l|}{ Drugs } \\
\hline \multicolumn{5}{|l|}{ Failed DMARDs, number, median (IQR) } \\
\hline csDMARDs & $6(5-6)$ & $2(1-2)$ & $3(3-5)$ & $2(1-3)^{*}$ \\
\hline $\mathrm{b} / \mathrm{tsDMARDs}$ & $4(4-5)$ & $0(0-1)$ & $4(3-6)$ & $0(0-1)^{*}$ \\
\hline Current DMARDs, number, median (IQR) & $2(1-2)$ & $1(1-1)$ & $2(1-2)$ & $1(1-2)$ \\
\hline csDMARDs, $n(\%)$ & $4(57)$ & $7(100)$ & $37(71)$ & $86(86) *$ \\
\hline bDMARDs, n (\%) & $3(43)$ & $1(14)$ & $27(52)$ & $39(39)$ \\
\hline tsDMARDs, n (\%) & $4(57)$ & $0(0)$ & $12(23)$ & $0(0)^{*}$ \\
\hline \multicolumn{5}{|l|}{ Administration route of current DMARDs, $n(\%)$} \\
\hline Oral & $6(86)$ & $7(100)$ & $45(87)$ & $86(86)$ \\
\hline Subcutaneous & $0(0)$ & $1(14)$ & $11(21)$ & $30(30)$ \\
\hline Intravenous & $3(43)$ & $0(0)$ & $16(31)$ & $9(9)$ \\
\hline Current glucocorticoids, n (\%) & $5(71)$ & $1(14)$ & $27(52)$ & $16(16)^{*}$ \\
\hline Current painkillers, $\mathrm{n}(\%)$ & $7(100)$ & $4(57)$ & $49(94)$ & $64(64)$ \\
\hline
\end{tabular}




\begin{tabular}{|c|c|c|c|c|}
\hline & \multicolumn{2}{|c|}{$\begin{array}{l}\text { Patients who participated } \\
\text { in the interview study }\end{array}$} & \multicolumn{2}{|c|}{$\begin{array}{l}\text { Total previous study } \\
\text { population }\end{array}$} \\
\hline & $\begin{array}{l}\text { D2T RA } \\
(n=7)\end{array}$ & $\begin{array}{l}\text { Non-D2T } \\
(n=7)\end{array}$ & $\begin{array}{l}\text { D2T RA } \\
(n=52) \\
\end{array}$ & $\begin{array}{l}\text { Non-D2T RA } \\
(n=100)\end{array}$ \\
\hline \multicolumn{5}{|l|}{ Treatment non-adherence } \\
\hline Level of MARS-5, median (IQR) & $23(22-25)$ & $24(20-25)$ & $24(21-25)$ & $24(23-25) \ddagger$ \\
\hline $\begin{array}{l}\text { Discrepancy in supplied and prescribed drugs, } n \\
(\%)\end{array}$ & $4(57)$ & $0(0)$ & $21(40)$ & $22(22) \neq *$ \\
\hline \multicolumn{5}{|c|}{$\begin{array}{l}\text { ACPA: anti-citrullinated protein antibodies; b: biological; cs: conventional synthetic; D2T: difficult-to-treat; DAS28- } \\
\text { ESR: disease activity score assessing } 28 \text { joints using erythrocyte sedimentation rate; DMARD: disease-modifying } \\
\text { antirheumatic drug; EULAR: European League Against Rheumatism; MARS- } 5 \text { : medication adherence reporting } \\
\text { scale (5-25, higher score reflects higher level of adherence); }{ }^{23} \text { : number; RA: rheumatoid arthritis; RF: rheumatoid } \\
\text { factor; ts: targeted synthetic; }{ }^{\ddagger}: \mathrm{n}=99 ;{ }^{*}: \mathrm{p}<0.05 \text {. } \\
\text { In the total previous study population, D2T and non-D2T RA patients were compared. Differences were analysed } \\
\text { using independent T-test, Fisher's exact test, chi-square test or Mann-Whitney U test, as appropriate. Statistical } \\
\text { significance was not tested in the participants of the interviews because of the smaller sample size. }\end{array}$} \\
\hline
\end{tabular}


Supplementary Table 2. The importance of clusters of barriers of (optimal) treatment non-adherence including its individual statements

\begin{tabular}{|c|c|c|c|c|}
\hline Cluster & Statement & $\begin{array}{c}\text { D2T } \\
\text { RA } \\
(n=20)\end{array}$ & $\begin{array}{l}\text { Non-D2T } \\
\text { RA } \\
(n=19)\end{array}$ & $\begin{array}{l}\text { Rheuma- } \\
\text { tologist } \\
(n=20)\end{array}$ \\
\hline \multirow[t]{6}{*}{1} & Relationship with the health care professional & $2.5(1.1)$ & $2.4(1.1)$ & $2.5(0.8)$ \\
\hline & the health care professional does not listen and think along & $2.7(1.4)$ & $2.1(1.4)$ & $2.7(1.3)$ \\
\hline & a lack of confidence in the health care professional & $2.0(1.4)$ & $1.8(1.5)$ & $2.2(1.1)$ \\
\hline & no trust that the most optimal treatment is given & $2.3(1.2)$ & $2.8(1.6)$ & $2.5(1.2)$ \\
\hline & $\begin{array}{l}\text { the benefits and adverse effects of the drugs cannot be discussed } \\
\text { properly with the health care professional }\end{array}$ & $3.0(1.3)$ & $2.3(1.4)$ & $2.6(1.4)$ \\
\hline & $\begin{array}{l}\text { insufficient information is given about the importance of } \\
\text { consistent medication use, such as the need to achieve a certain } \\
\text { blood drug level }\end{array}$ & $2.8(1.6)$ & $3.1(1.1)$ & $2.8(1.1)$ \\
\hline \multirow[t]{4}{*}{2} & Adverse events & $3.8(0.9)$ & $3.9(1.0)$ & $4.5(0.4) *$ \\
\hline & $\begin{array}{l}\text { drug use provokes resistance, for example, due to nausea or the } \\
\text { taste of tablets }\end{array}$ & $3.7(1.1)$ & $3.9(1.2)$ & $4.5(0.8)^{*}$ \\
\hline & $\begin{array}{l}\text { drug use results in annoying adverse events, such as nausea, } \\
\text { fatigue or bruises }\end{array}$ & $4.2(1.0)$ & $4.1(1.0)$ & $4.7(0.6) *$ \\
\hline & $\begin{array}{l}\text { the drugs and adverse events are worse than the symptoms of } \\
\text { rheumatoid arthritis }\end{array}$ & $3.6(1.3)$ & $3.8(1.2)$ & $4.4(0.6)^{*}$ \\
\hline \multirow[t]{6}{*}{3} & Doubts about safety & $3.4(0.8)$ & $3.8(0.7)$ & $3.4(0.6)$ \\
\hline & the adverse events in the information leaflet are terrifying & $3.3(1.4)$ & $3.8(1.0)$ & $4.3(1.1) *$ \\
\hline & the drugs can damage the body & $3.9(0.9)$ & $4.2(1.0)$ & $3.6(1.2)$ \\
\hline & a high number of prescribed drugs results in doubts about safety & $3.8(1.3)$ & $4.4(0.8)$ & $4.1(1.2)$ \\
\hline & the dosage of the drugs is very high & $3.2(0.9)$ & $3.3(1.2)$ & $3.2(1.3)$ \\
\hline & $\begin{array}{l}\text { a homeopathic drug is preferred, for instance, a drug of herbal } \\
\text { origin }\end{array}$ & $2.8(1.6)$ & $3.2(1.5)$ & $2.1(1.1)$ \\
\hline \multirow[t]{6}{*}{4} & Doubts about effectiveness & $3.7(0.7)$ & $3.2(0.7)^{*}$ & $3.4(0.6)$ \\
\hline & drugs are not sufficiently effective & $4.0(1.1)$ & $3.3(1.2)$ & $4.1(1.2)$ \\
\hline & $\begin{array}{l}\text { drugs are only beneficial for a few symptoms of the disease, but } \\
\text { not for all of them }\end{array}$ & $4.2(0.9)$ & $3.5(1.2)$ & $3.1(1.3) *$ \\
\hline & drugs were insufficiently beneficial in the past & $3.8(1.3)$ & $2.9(1.2)$ & $3.2(1.4)$ \\
\hline & $\begin{array}{l}\text { there are doubts about the mechanism of action and effects of } \\
\text { the drugs }\end{array}$ & $3.8(1.0)$ & $3.6(1.3)$ & $3.8(1.2)$ \\
\hline & the purpose of the drug is unclear & $2.9(1.4)$ & $2.6(1.7)$ & $2.8(1.5)$ \\
\hline \multirow[t]{4}{*}{5} & Low disease activity & $3.4(1.0)$ & $3.7(0.9)$ & $3.5(0.8)$ \\
\hline & $\begin{array}{l}\text { the disease has been stable for a long time, resulting in the } \\
\text { perception that less drugs are needed }\end{array}$ & $3.6(1.4)$ & $3.9(1.0)$ & $3.9(1.0)$ \\
\hline & $\begin{array}{l}\text { the disease is temporarily stable, resulting in the perception that } \\
\text { drugs are not needed anymore }\end{array}$ & $3.5(1.3)$ & $3.6(1.1)$ & $3.2(1.3)$ \\
\hline & the symptoms of RA did not return after drug discontinuation & $3.1(1.1)$ & $3.7(1.2)$ & $3.4(1.4)$ \\
\hline \multirow[t]{2}{*}{6} & Cost-utility evaluation & $2.5(1.0)$ & $2.2(1.0)$ & $2.0(1.0)$ \\
\hline & health care and drugs are expensive & $2.8(1.5)$ & $2.2(1.5)$ & $1.6(0.9) *$ \\
\hline
\end{tabular}




\begin{tabular}{|c|c|c|c|c|}
\hline Cluster & Statement & $\begin{array}{c}\text { D2T } \\
\text { RA } \\
(n=20)\end{array}$ & $\begin{array}{c}\text { Non-D2T } \\
\text { RA } \\
(n=19)\end{array}$ & $\begin{array}{c}\text { Rheuma- } \\
\text { tologist } \\
(n=20)\end{array}$ \\
\hline & $\begin{array}{l}\text { rheumatoid arthritis, as a disease, is not as important compared } \\
\text { to other diseases, such as cancer }\end{array}$ & $2.3(1.4)$ & $2.2(1.2)$ & $2.3(1.4)$ \\
\hline \multirow[t]{9}{*}{7} & Health behaviour & $2.4(0.7)$ & $2.8(0.7)$ & $3.0(0.5) *$ \\
\hline & $\begin{array}{l}\text { a feeling of shame exists while using the drugs, for example, while } \\
\text { being in public places }\end{array}$ & $1.7(0.8)$ & $1.9(1.1)$ & $1.9(0.7)$ \\
\hline & it is a burden to use drugs on a daily basis & $2.9(1.3)$ & $3.3(1.2)$ & $3.7(1.4)$ \\
\hline & drugs are being forgotten & $2.7(1.7)$ & $3.4(1.6)$ & $3.8(1.4)^{*}$ \\
\hline & it is not (yet) a habit to use the drugs & $2.2(1.4)$ & $2.7(1.4)$ & $3.0(1.3)$ \\
\hline & a lack of discipline and accuracy exists to use the drugs properly & $2.3(1.3)$ & $2.4(1.1)$ & $3.2(1.3)^{*}$ \\
\hline & $\begin{array}{l}\text { distraction, due to external circumstances that currently require } \\
\text { attention }\end{array}$ & $3.2(1.7)$ & $3.2(1.6)$ & $3.4(1.4)$ \\
\hline & drug use is not supervised by others & $1.9(1.0)$ & $2.2(1.5)$ & $2.1(1.0)$ \\
\hline & it does not matter to skip the drugs sometimes & $2.6(1.5)$ & $3.4(1.5)$ & $3.0(1.2)$ \\
\hline \multirow[t]{4}{*}{8} & Administration route & $2.6(0.9)$ & $2.5(0.8)$ & $2.6(0.8)$ \\
\hline & drug use evokes fear, for example, injecting drugs with a syringe & $2.8(1.4)$ & $3.1(1.3)$ & $3.6(1.5)$ \\
\hline & drugs in syringe form are less preferred than those in tablets & $3.0(1.3)$ & $2.8(1.4)$ & $2.4(1.0)$ \\
\hline & drugs in tablets are less important than those in syringes & $2.0(0.9)$ & $1.6(0.9)$ & $1.9(0.9)$ \\
\hline \multirow[t]{7}{*}{9} & Complexity of drug regimen & $2.7(0.9)$ & $2.5(0.8)$ & $2.3(0.5) *$ \\
\hline & $\begin{array}{l}\text { the tools that should facilitate drug use are user unfriendly, such } \\
\text { as a pill box that is too small }\end{array}$ & 2.3 (1.3) & $2.3(1.4)$ & $1.7(0.9)$ \\
\hline & $\begin{array}{l}\text { drugs are user unfriendly, for example, because the tablets are so } \\
\text { small that they are difficult to see }\end{array}$ & $2.9(1.4)$ & $2.3(1.1)$ & $1.9(0.9)^{*}$ \\
\hline & $\begin{array}{l}\text { swallowing of drugs is difficult, for instance, because the tablets } \\
\text { are too big }\end{array}$ & $2.8(1.3)$ & $2.7(1.2)$ & $2.4(1.2)$ \\
\hline & $\begin{array}{l}\text { too many actions have to be performed before drug use, for } \\
\text { instance, drugs that have to be dissolved in water before intake }\end{array}$ & $2.8(1.4)$ & $2.1(1.2)$ & $2.1(1.1)$ \\
\hline & $\begin{array}{l}\text { drugs do not have to be used daily, therefore it becomes hard to } \\
\text { remember when they have to be used }\end{array}$ & $3.0(1.4)$ & $3.1(1.1)$ & $2.5(1.1)$ \\
\hline & $\begin{array}{l}\text { a high number of drugs results in confusion, for example, at what } \\
\text { moment which drug has to be taken }\end{array}$ & 2.7 (1.3) & 2.5 (1.3) & 3.3 (1.3) \\
\hline
\end{tabular}

Importance was scored from 1 (least important) to 5 (most important).

Numbers are mean (standard deviation). Differences between D2T and non-D2T RA patients as well as between D2T RA patients and rheumatologists were analysed using independent T-tests. *: $p<0.05$ compared with D2T RA patients. 
Supplementary Table 3. The helpfulness of clusters of facilitators of optimal treatment adherence including its individual statements

\begin{tabular}{|c|c|c|c|c|}
\hline Cluster & Statement & $\begin{array}{c}\text { D2T } \\
\text { RA } \\
(n=20)\end{array}$ & $\begin{array}{c}\text { Non-D2T } \\
\text { RA } \\
(n=19)\end{array}$ & $\begin{array}{c}\text { Rheuma- } \\
\text { tologist } \\
(n=19)\end{array}$ \\
\hline \multirow[t]{4}{*}{1} & Relationship with the health care professional & $3.7(0.9)$ & $3.6(1.1)$ & $4.1(0.9)$ \\
\hline & $\begin{array}{l}\text { easy accessibility of the health care professional, meaning it } \\
\text { possible to get in touch at any time when having a question }\end{array}$ & $3.8(1.1)$ & $3.6(1.2)$ & $3.9(1.4)$ \\
\hline & $\begin{array}{l}\text { having a good relationship with the health care professional } \\
\text { facilitating discussion of questions and problems }\end{array}$ & $3.7(1.3)$ & $3.8(1.2)$ & $4.1(1.2)$ \\
\hline & sufficient guidance from the health care professional & $3.7(0.8)$ & $3.5(1.1)$ & $4.2(0.8)$ \\
\hline \multirow[t]{6}{*}{2} & Treatment information & $3.2(0.7)$ & $3.3(0.9)$ & $3.3(0.7)$ \\
\hline & $\begin{array}{l}\text { clear information about drug storage, for example about drugs } \\
\text { that should be stored in a refrigerator }\end{array}$ & $2.9(1.2)$ & $2.5(1.3)$ & $2.5(1.0)$ \\
\hline & $\begin{array}{l}\text { clear information on the drug package about drug use, such as } \\
\text { when drugs have to be used }\end{array}$ & $3.4(1.1)$ & $3.1(1.0)$ & $2.9(1.4)$ \\
\hline & $\begin{array}{l}\text { receiving information about why drugs have to be used and what } \\
\text { kind of effects they have }\end{array}$ & $3.0(1.3)$ & $3.5(1.4)$ & $4.3(1.2) *$ \\
\hline & receiving clear information on how drugs should be used & $3.6(0.9)$ & $3.7(1.1)$ & $3.8(1.3)$ \\
\hline & $\begin{array}{l}\text { knowing that, according to current scientific knowledge, the most } \\
\text { optimal drug treatment is given }\end{array}$ & $3.3(1.6)$ & $3.7(1.5)$ & $2.9(1.4)$ \\
\hline \multirow[t]{7}{*}{3} & Help from the pharmacy & $3.2(0.8)$ & $2.9(0.8)$ & $2.7(0.5)^{*}$ \\
\hline & a consultation with the pharmacist about drugs use & $2.5(1.1)$ & $2.2(1.1)$ & $2.4(1.1)$ \\
\hline & getting a reminder from the pharmacy to order the drugs & $3.4(1.4)$ & $3.1(1.3)$ & $2.8(1.2)$ \\
\hline & standard delivery of the drugs by the pharmacy & $3.5(1.2)$ & $3.1(1.2)$ & $2.9(1.4)$ \\
\hline & sufficient stock of drugs in the pharmacy & $3.3(1.2)$ & $3.2(1.3)$ & $2.7(1.3)$ \\
\hline & the possibility of collecting the drugs at another pharmacy & $2.6(1.5)$ & $2.8(1.5)$ & $2.2(1.2)$ \\
\hline & getting the same brand of drugs with every prescription & $3.7(1.4)$ & $3.1(1.5)$ & $3.2(1.3)$ \\
\hline \multirow[t]{12}{*}{4} & Routine and reminders & $3.2(0.6)$ & $3.3(0.6)$ & $3.1(0.4)$ \\
\hline & having a fixed rhythm in drug use & $4.5(0.8)$ & $4.5(0.8)$ & $4.3(1.1)$ \\
\hline & $\begin{array}{l}\text { linking the moment of drug use to a fixed moment of the day, for } \\
\text { instance, during breakfast }\end{array}$ & $4.4(0.9)$ & $4.2(1.0)$ & $4.2(1.1)$ \\
\hline & adjusting times of drug use based on daily rhythm & $3.8(1.1)$ & $3.8(1.0)$ & $2.9(1.6)$ \\
\hline & $\begin{array}{l}\text { adjusting times of drug use based on their side effects, for } \\
\text { example, using the drugs in the evening to experience less nausea }\end{array}$ & $3.5(1.2)$ & $4.0(1.2)$ & $3.8(1.0)$ \\
\hline & checking off drugs on a list if they have been used & $2.3(1.3)$ & $2.4(1.3)$ & $3.1(1.5)$ \\
\hline & counting the number of drugs before intake & $2.6(1.4)$ & $1.7(0.8) *$ & $1.4(0.8) *$ \\
\hline & $\begin{array}{l}\text { preparing drugs for the next moment of use, immediately after } \\
\text { drug use }\end{array}$ & $2.5(1.3)$ & $3.0(1.2)$ & $2.3(1.2)$ \\
\hline & putting drugs on a visible spot & $3.5(1.5)$ & $3.7(1.4)$ & $2.5(1.3)^{*}$ \\
\hline & hanging a reminder about drug use on a visible spot & $2.7(1.5)$ & $2.6(1.3)$ & $2.9(1.3)$ \\
\hline & $\begin{array}{l}\text { setting a reminder for drug use, for instance, by using an alarm } \\
\text { clock or a special app }\end{array}$ & $3.2(1.4)$ & $3.2(1.6)$ & $4.1(1.2)^{*}$ \\
\hline & writing down when drugs should be used & $2.6(1.4)$ & $2.7(1.1)$ & $2.6(1.3)$ \\
\hline
\end{tabular}


Supplementary Table 3. Continued

\begin{tabular}{|c|c|c|c|c|}
\hline Cluster & Statement & $\begin{array}{c}\text { D2T } \\
\text { RA } \\
(n=20)\end{array}$ & $\begin{array}{c}\text { Non-D2T } \\
\text { RA } \\
(n=19)\end{array}$ & $\begin{array}{c}\text { Rheuma- } \\
\text { tologist } \\
(n=19)\end{array}$ \\
\hline \multirow[t]{10}{*}{5} & Aids & $2.9(0.4)$ & $2.9(0.6)$ & $3.0(0.4)$ \\
\hline & $\begin{array}{l}\text { user-friendly packaging of drugs, for example, a package that is } \\
\text { easy to open }\end{array}$ & $3.6(1.4)$ & $3.5(1.0)$ & $3.3(1.0)$ \\
\hline & $\begin{array}{l}\text { user-friendly drugs, such as tablets that can be easily split into } \\
\text { halves or the type of syringe pen which has to be used }\end{array}$ & $3.6(1.0)$ & $2.9(1.3)$ & $3.8(1.3)$ \\
\hline & $\begin{array}{l}\text { differences in appearance of the drugs facilitating easy } \\
\text { recognition, such as different colours and tablet forms }\end{array}$ & $3.2(1.3)$ & $2.9(1.1)$ & $2.3(1.2) *$ \\
\hline & using a pill box & $3.2(1.5)$ & $3.4(1.4)$ & $3.2(1.2)$ \\
\hline & user-friendly tools, for example a big pill box & $3.3(1.4)$ & $2.7(1.2)$ & $2.5(1.3)$ \\
\hline & using a medication strip that shows the days of the week & $2.7(1.5)$ & $2.9(1.5)$ & $2.9(1.6)$ \\
\hline & $\begin{array}{l}\text { using sachet packing, combining all drugs that should be used at } \\
\text { the same time in one sachet }\end{array}$ & $2.1(1.0)$ & $2.5(1.5)$ & $4.4(0.7) *$ \\
\hline & $\begin{array}{l}\text { the possibility of storing drugs while being outside the house, for } \\
\text { instance, by using a cool bag }\end{array}$ & $2.3(1.1)$ & $2.6(1.4)$ & $2.3(0.9)$ \\
\hline & taking the drugs everywhere & $2.5(1.1)$ & $2.8(1.4)$ & $2.1(1.1)$ \\
\hline \multirow[t]{4}{*}{6} & Help from others & $2.3(0.9)$ & $2.0(0.7)$ & $2.2(0.7)$ \\
\hline & reminders about drug use by others & $1.9(1.1)$ & $2.0(1.2)$ & $2.5(1.2)$ \\
\hline & help from others by using the drugs, such as help with injecting & $1.9(1.0)$ & $2.5(1.5)$ & $2.8(1.1)$ \\
\hline & supervision on drug use by others & $2.0(1.3)$ & $2.1(1.2)$ & $2.6(1.4)$ \\
\hline \multirow[t]{4}{*}{7} & Miscellaneous & $1.9(0.8)$ & $2.2(1.1)$ & $2.7(1.0) *$ \\
\hline & the absence of major life events & $2.4(1.4)$ & $2.6(1.5)$ & $2.2(1.1)$ \\
\hline & low personal costs related to drug use & $2.8(1.5)$ & $1.2(1.4)$ & $2.2(1.2)$ \\
\hline & a self-chosen reward after drug use & $1.6(1.1)$ & $1.3(0.7)$ & $2.2(1.5)$ \\
\hline
\end{tabular}

Helpfulness was scored from 1 (least helpful) to 5 (most helpful).

Numbers are mean (standard deviation). Differences between D2T and non-D2T RA patients as well as between D2T RA patients and rheumatologists were analysed using independent T-tests. *: p<0.05 compared with D2T RA patients. 
Non-adherence in D2T RA: a concept mapping study 



\section{CHAPTER 13}

General discussion 
In line with the scope of this thesis - to define and improve our understanding of difficult-to-treat rheumatoid arthritis (D2T RA) - studies to (further) characterise D2T RA and (systematic) literature reviews have been performed. Additionally, management recommendations have been developed. In this chapter, the results of these studies will be put in a broader perspective, including discussions on their strengths, limitations and clinical implications. Furthermore, future perspectives and research opportunities will be discussed.

\section{Terminology and definition of the concept of D2T RA}

The first step in improving the management and outcomes of rheumatoid arthritis (RA) patients who are difficult to treat was to create uniform terminology and a clear definition for this patient group, ${ }^{1-3}$ which is complicated by the heterogeneity in this disease state (Chapter 4 and 10) ${ }^{4,5} \mathrm{~A}$ myriad of factors can contribute to the persistence of signs and/or symptoms in these patients: factors resulting in persistent inflammatory disease activity (e.g. DMARDs being truly ineffective ('true' refractory RA) or treatment non-adherence) and non-inflammatory factors mimicking inflammatory RA activity (e.g. concomitant fibromyalgia).,2,5-7 The term 'difficult-to-treat (D2T)' RA covers all these potentially contributing factors and has therefore been chosen to describe this patient population in the new European League Against Rheumatism (EULAR) definition for this disease state (Chapter 5). ${ }^{8}$ RA patient are classified as having D2T RA if they fulfil all three criteria of the definition, in short: 1. previous failure of $\geq 2$ biological and/or targeted synthetic disease-modifying antirheumatic drugs (b/tsDMARDs) with different mechanisms of action; 2 . symptoms and signs suggestive of active/progressive disease; 3. management perceived as problematic by the rheumatologist and/or patient.

The D2T RA definition can be used in daily clinical practice and research to form a basis to improve the care for these patients. However, the definition should be validated before large-scale implementation. Validation of the definition in retrospective cohorts will be difficult because the definition contains some subjective criteria and items (e.g. 'the management is perceived as problematic'), which have probably not been structurally collected in retrospective cohorts. Therefore, as a first step, rheumatologists' and patients' acceptance in clinical practice can be used to validate the definition, as a sign of (face) validity. Additionally, it could be tried to validate the definition in a crosssectional or prospective cohort of D2T RA patients in which classification as D2T RA by an expert panel of rheumatologists or disease progression over time may be used as appropriate reference standards.

\section{Factors contributing to D2T RA}

The heterogeneity in D2T RA also complicates the management of these patients 
(Chapter 4). ${ }^{4}$ Unravelling the heterogeneity of D2T RA, including the occurrence and importance of specific factors contributing to the disease state, is therefore crucial in order to facilitate more appropriate, tailored management strategies. In our clinical study into D2T RA (according to the new EULAR definition (Chapter 5)), m multiple factors that were previously suggested to be associated with worse disease outcomes (e.g. treatment irresponsiveness, higher levels of disease activity, functional disability) were found to occur more frequently in D2T RA compared with in non-D2T RA: limited drug options because of adverse events or comorbidities, a mismatch in patient's and rheumatologist's wish to intensify treatment, concomitant fibromyalgia and poorer coping were independently associated with D2T RA; additionally, treatment non-adherence, depression and higher levels of anxiety were more commonly found among D2T RA patients (Chapter 10).,5,17,9-16 In an exploratory analysis, we could also identify three subgroups of D2T RA patients. Although these results should be replicated in another cohort of D2T RA patients due to the relatively small sample size, the various contributing factors that can be present as well as the identified subgroups further emphasise the heterogeneity of D2T RA and show the need to individualise treatment strategies.

For some other previously suggested factors (i.e. smoking, obesity, concomitant crystal arthritis and concomitant osteoarthritis and subluxation), no differences could be found between D2T and non-D2T RA patients.1.18-21 The relatively small sample size of the clinical study may be an explanation and these associations should be reassessed in, preferably, a larger cohort of patients. Additionally, smoking and obesity have been suggested to influence the immunology underlying DMARD inefficacy (Chapter 6), 18,22,3133,23-30 and, therefore, their role in D2T RA may be much more complex. Moreover, obesity may also hamper proper grading of inflammatory disease activity (Chapter 7 ), ${ }^{1,34}$ which further complicates this complex interplay. Until the role of these factors is clarified, we still recommend to consider these factors as potentially contributing to D2T RA.

Unfortunately, the role of 'true' refractory RA has not been addressed in our clinical study (Chapter 6 and 10).5,35 Unravelling immunological mechanisms by means of in-depth research on blood markers and (synovial) tissue characteristics has not (yet) been performed and may help to improve our understanding of 'true' refractory RA. In case such markers would be present, this may also facilitate the identification of (subgroups of) patients as well as the development of personalised and/or new pharmacological strategies.

\section{Scarcity of evidence}

The management of D2T RA is further hampered by the scarcity of evidence on this topic. In the systematic literature reviews (SLRS) that have been performed on diagnostic issues and therapeutic strategies in D2T RA, no studies were identified including D2T RA 
patients specifically (Chapter 7 and 8).34,36 This is not surprising, considering the recent establishment of the EULAR definition of D2T RA (Chapter 5). ${ }^{8}$ However, few studies were also identified on management strategies in RA patients who fulfilled the first part of the definition, i.e. failure of at least two b/tsDMARDs with different mechanisms of action. ${ }^{37-44}$ Furthermore, the majority of the identified evidence had a moderate to high risk of bias. Due to the indirectness and low-quality of the current evidence, no firm conclusions can be drawn about the most optimal management strategies for D2T RA.

The lack of current knowledge of some aspects of RA in general also contributes to the suboptimal management of D2T RA patients. First, a gold standard to assess inflammatory disease activity does not exist (Chapter 3 and 7), ${ }^{34,45}$ despite the ultimate aim of RA treatment is reaching clinical remission, which is defined as 'the absence of signs and symptoms of significant inflammatory disease activity'. ${ }^{4}$ Validated composite indices are frequently used to assess the presence or absence of inflammatory disease activity, but doubt about inflammation may remain after using these traditional measures, specifically in D2T RA (Chapter 7).34,47 This may result in incorrect assessment and therewith inappropriate treatment adjustments.' Furthermore, prediction of response to DMARDs for individual RA patients, i.e. 'personalised medicine', is still not possible. ${ }^{48}$ As the pathogenesis of (D2T) RA is likely heterogeneous (chapter 6), individual patients may benefit from different (b/ts)DMARDs making a personalised approach necessary. Despite many efforts to identify factors that could be used to predict treatment response, no reliable predictors have yet been found to identify optimal DMARD therapy for individual patients (Chapter 2). ${ }^{49-51}$ Therefore, treatment choices are still based on trial-and-error resulting in ineffective DMARD treatment and, with that, valuable time loss in the management of RA, which results in worse disease outcomes and may increase the likelihood of developing D2T RA. 52,53

\section{Management of D2T RA}

Despite the scarcity of (high-quality) evidence regarding the management of D2T RA, indirect evidence has been identified that, together with expert opinion, has resulted in the development of EULAR recommendations for the management of D2T RA (Chapter 9). ${ }^{54}$ The first step in managing the heterogeneous population of D2T RA patients should be to ascertain the diagnosis of RA and to exclude alternative mimicking diseases (e.g. polymyalgia rheumatica, Chapter 7). ${ }^{34}$ If the diagnosis of RA is ascertained, an evaluation of the presence or absence of inflammatory disease activity is advised (Chapter 7) as well as a thorough assessment of factors potentially contributing to the D2T RA state (including coexistent mimicking diseases (e.g. concomitant fibromyalgia) as described in Chapter 10) to identify the factor(s) that underlie the patient's current signs and symptoms., ${ }^{5,34}$ Instead of further adjusting DMARD treatment based on trialand-error, the factors contributing to the disease state, including the presence or 
absence of inflammation (and, although not yet possible, preferably also the underlying immunological mechanisms) should drive appropriate pharmacological and nonpharmacological therapeutic strategies (Chapter 7, 8 and 9). ${ }^{34,36,54}$ Noteworthy, in D2T RA patients in whom inflammation is absent or in whom persistent inflammation is not directly related to RA disease activity (e.g. treatment non-adherence), DMARD therapy should not be intensified and non-pharmacological interventions should be considered (Chapter 8 and 12).36,55

Some of the clinically identified contributing factors could be modifiable (i.e. treatment non-adherence, a mismatch between patient's and rheumatologist's wish to intensify treatment, depression, anxiety and poorer coping, Chapter 10). ${ }^{5}$ These contributing factors could be most interesting to address in management strategies because adequate management of these may ameliorate the D2T RA state. Although it remains debatable to what extent these factors are truly modifiable, addressing them adequately could at least reduce associated signs and symptoms. Nevertheless, the identification of all potentially contributing factors in an individual D2T RA patient will be essential in the management of D2T RA, as all these factors - modifiable or not - require different pharmacological and/or non-pharmacological management strategies.

Adequate management of modifiable contributing factors may not only ameliorate the D2T RA state, but may also prevent the development of D2T RA if they are addressed in an earlier phase of the disease. To this end, the identification of risk factors at RA onset for developing D2T RA may be helpful to identify D2T RA patients earlier in the disease course to enable early intervention. In our clinical study, we identified lower socioeconomic status as an independent risk factor at RA onset for developing D2T RA (Chapter 10). ${ }^{5}$ Although no previous studies have been performed on risk factors for developing D2T RA, lower socioeconomic status has previously been associated with higher burden of disease, higher risk of irresponsiveness to bDMARDs and higher risk of mortality.,56-59 Therefore, we recommend that RA patients with a lower socioeconomic status (e.g. using education level or income as surrogates) are monitored more intensively for the presence or development of factors contributing to D2T RA (e.g. treatment non-adherence or depression). When these contributing factors develop in patients at risk and are adequately addressed, the risk of acquiring D2T RA can potentially be diminished.

\section{Burden of D2T RA}

The burden of D2T RA has been clearly indicated in this thesis. First, the patients' burden of disease was indicated by worse levels of functional disability, quality of life, physical activity, fatigue and pain in D2T RA patients compared with in non-D2T RA patients (Chapter 10). ${ }^{5}$ Second, rheumatologists expressed their unmet clinical needs regarding D2T RA in the international survey (Chapter 4).4 They indicated items that 
are not fully covered by current EULAR management recommendations, e.g. interfering comorbidities and pain syndromes. ${ }^{50,60,61}$ Third, the high economic burden has been shown by assessing the cost-of-illness (Chapter 11). ${ }^{62}$ Mean annual total costs related to D2T RA were almost twice as high as costs related to non-D2T RA. Although this costof-illness study was only performed in one country, which limits broad extrapolation, the results give a first insight into the significantly higher health care utilisation and economic burden of D2T RA.

The high patients', rheumatologists' and economic burden of D2T RA indicate that management strategies should be improved and substantiate the need for a swift implementation of the abovementioned clinical implications and developed recommendations in daily practice to decrease the burden of D2T RA.

\section{Future perspectives and research opportunities}

The studies in this thesis have contributed to define and improve our understanding of D2T RA by characterising the concept of D2T RA and summarising the current knowledge on this topic, which has eventually resulted in the development of management recommendations for D2T RA. Multiple gaps of knowledge have been exposed, which warrant further research.

\section{Further understanding of D2T RA}

Despite the heterogeneity that has already been shown to be present in D2T RA, additional insights into the D2T RA state are needed.

First, the findings of our clinical study should be validated in another, and preferably larger, cohort of D2T RA patients. Preferably, this should be a cohort study with longterm follow-up in which the earlier results cannot only be replicated, but also the development of D2T RA can be assessed in more detail as well as its natural course. Long-term follow-up studies, preferably starting at RA onset, can help to resolve whether the clinically identified contributing factors (e.g. depression) are underlying the disease state, have developed as a result of having D2T RA, or both. These studies can also give insights into additional risk factors for developing D2T RA, such as the change in disease activity over time and the duration of (b/ts)DMARD prescription in relation to (b/ts)DMARD failure. Additionally, long-term follow-up studies would enable to study the fluidity of the D2T RA state, which is currently unknown. If only a modifiable factor, such as treatment non-adherence, contributes to the D2T RA state and is adequately addressed, it may be expected that patients can again be classified as not having D2T RA. More knowledge on the course of D2T RA may also gain valuable insights into the contributing factors that are most promising to address in management strategies and potentially promising management approaches when D2T RA is present.

Furthermore, some contributing factors should be assessed in more detail. As one of 
these factors, treatment non-adherence should be studied more thoroughly. In our clinical study, non-adherence was identified as a contributing factor according to a discrepancy between prescribed and supplied drugs (an indirect measure, chapter 10). ${ }^{5}$ However, the self-reported level of non-adherence was relatively low according to a questionnaire (another indirect measure). Conflicting results of various assessment measures of non-adherence have previously been described and especially selfreported questionnaires are known to overreport adherence rates. ${ }^{63}$ Additionally, incorrect administration of the hospital and pharmacy may influence adherence rates according to a discrepancy between prescribed and supplied drugs. Therefore, a direct measure, i.e. serum drug levels, may more accurately assess adherence, although individual's specific drug metabolism and 'white coat' adherence (i.e. improved treatment adherence around clinic visit appointments) could complicate their interpretation. ${ }^{63}$ Thus, a perfect measure of adherence does not yet exist and, therefore, a multi-measure approach combining these measures is currently advised. ${ }^{63}$ A study using serum drug levels to assess adherence in D2T RA will follow shortly, which will enable this multi-measure approach.

Additionally, mechanisms underlying DMARDs being truly ineffective are still largely unknown in D2T RA and should be further studied (Chapter 6). ${ }^{2,35}$ The importance of antidrug antibodies has not yet been assessed in patients fulfilling the D2T RA definition, although these have been found to play a role in bDMARD inefficacy and, thus, are likely to contribute (Chapter 6). ${ }^{35,64}$ Some hints were found for a role of Thl7 cells, activated memory B cells (CD95+ Ki67, which do not circulate in the peripheral blood, but resident in sites, such as lymph nodes) and synovial lymphoid aggregates (Chapter 6), ${ }^{35}$ although other immunological mechanisms (and the influence of genomics) should also be compared between D2T and non-D2T RA patients to assess which mechanisms are active in D2T RA. This may also strengthen our understanding of the influence of clinical characteristics (e.g. smoking, obesity) on these mechanisms. To this end, analyses of synovial tissue, biomarker studies, whole genome sequencing and epigenetic analyses may be useful, ${ }^{65-67}$ although a more systemic approach incorporating data on immunological pathways, signalling networks, proteins and genes may be preferred. Ultimately, a better understanding of these underlying mechanisms may facilitate the development of personalised and/or new pharmacological strategies and may also contribute to the ongoing classification of (relevant subgroups of) D2T RA.

By comparing immunological mechanisms that are active between D2T and nonD2T RA patients, the heterogeneity of D2T RA may complicate the interpretation of the results, in case some factors only play a role in part of the D2T RA population. Therefore, it may be preferred to make an additional comparison between patients with 'true' refractory RA (without other potentially contributing factors) and other D2T RA patients. This may also help to assess whether 'true' refractory RA really exists, which is currently 
unknown (Chapter 6).35

\section{Management of D2T RA}

With the development of the EULAR definition of D2T RA and the EULAR recommendations for the management of D2T RA, it was aimed to create uniform terminology, a uniform definition and more explicit and comprehensive management recommendations for this subpopulation of RA patients (Chapter 5 and 9) 8,54 Thereby, the ultimate aims were to improve outcomes of D2T RA patients and to provide further guidance to health care professionals. Whether the recommendations will be implemented successfully and whether this will resulted in the achievement of these aims should be addressed in future studies. Additionally, the recommendations should be updated in 3 to 5 years because, hopefully, new evidence on the management of D2T RA will emerge.

One of the main conclusions of the SLRs that have been performed to inform the management recommendations is the scarcity of high-quality evidence on diagnostic issues and therapeutic strategies in D2T RA (Chapter 7 and 8). ${ }^{34,36}$ Although studies in RA in general could provide valuable insights, it should be noted that the patient population is different and, therefore, additional studies in D2T RA patients specifically will be needed. Herewith, the heterogeneity of D2T RA should be taken into account, since it may be expected that not all patients benefit from the same management strategies.

Regarding diagnostic issues, the most interesting topic would be to assess which diagnostic measure should be used to assess the presence or absence of inflammatory disease activity in D2T RA patients in whom there is explicit doubt about the presence of inflammation. Currently, the recommendation that ultrasonography may be preferred is extrapolated from studies reporting a good correlation between ultrasonography and composite indices in the general population of RA patients, while this correlation is lacking in RA patients in whom there is explicit doubt (Chapter 7). ${ }^{34,68-76} \mathrm{~A}$ study assessing inflammatory disease activity at patient and joint level in D2T RA patients in whom there is explicit doubt, will be needed to increase the level of evidence. Additional potentially promising diagnostic measures could also be assessed, e.g. the multi-biomarker disease activity (MBDA) score, magnetic resonance imaging (MRI) and optical spectral transmission (OST) measures (Chapter 3 and 7). ${ }^{34,45,77-81}$ Hitherto, the lack of a true gold standard remains a challenge. As traditional measures are not trustworthy in D2T RA patients in whom there is doubt about the presence of inflammation (i.e. validated composite indices at patient level and clinical assessment of a joint being swollen at joint level), another reference standard should be used. In these patients, histology of synovial tissue (obtained by synovial biopsies) in combination with treatment response over time (e.g. response to glucocorticoid therapy) may be used as a more appropriate reference standard in such studies. 
Regarding pharmacological therapeutic strategies, randomised controlled trials (RCTs) in D2T RA patients (i.e. who failed at $\geq 2 \mathrm{~b} /$ tsDMARDs with different mechanisms of action) comparing different b/tsDMARDs are currently lacking (Chapter 8). ${ }^{36}$ These RCTs are needed to identify the most optimal pharmacological therapy. Before patients are enrolled, it should be tried to address all factors contributing to D2T RA (besides DMARDs being truly ineffective) as optimal as possible. Additionally, the presence of inflammatory disease activity should be ascertained and, preferably, ineffective DMARDs should be stopped first. Therewith, a homogeneous patient population will be selected of D2T RA patients having inflammatory RA activity. However, inflammation in these D2T RA patients may result from differences in the underlying immunology and, thus, the population is likely still somewhat heterogeneous. Therefore, these RCTs should preferably be combined with the assessment of the underlying immunology (e.g. in synovial tissue) to be able to understand why DMARDs are effective or not. This approach may eventually enable treatment stratification and personalised medicine. Regarding non-pharmacological therapeutic strategies, trials in D2T RA patients specifically have not yet been performed (Chapter 8). ${ }^{36}$ The additional benefit of non-pharmacological interventions might be even higher in D2T than in non-D2T RA patients because of the substantial higher patients' burden of D2T RA (Chapter 10). ${ }^{5}$ Due to this substantial higher burden, non-pharmacological interventions might not only be beneficial for D2T RA patients with predominantly non-inflammatory complaints (i.e. not (directly) related to inflammation), but also for the total population of D2T RA patients. However, it may be suggested to enrol only D2T RA patients without inflammation to study the effectiveness of non-pharmacological interventions on non-inflammatory complaints. Herewith, the potential beneficial effect of the therapeutic strategy is not confounded by additional inflammatory symptoms.

Although studies in different subgroups of D2T RA would result in more homogeneous patient populations, it will probably be difficult to enrol patients in these studies as multiple contributing factors frequently seem to coexist in individual patients. Therefore, in an ideal world were resources are not restricted, a holistic, multifaceted intervention trial may be another possibility. This trial should start with a 'time-out' in which the diagnosis of RA will be ascertained, the presence or absence of inflammation should be evaluated and potentially contributing factors should be identified. Then, a holistic management protocol should be applied, in which appropriate combinations of pharmacological and non-pharmacological strategies will be tailored to the disease state of the individual patient. At every follow-up visit, the contributing factors should be reassessed and the presence or absence of inflammatory disease activity should drive appropriate treatment changes. Such strategy will be a reflection of the ideal management of D2T RA patients and will hopefully show if and how outcomes of these patients can be improved. 


\section{Concluding remarks}

In conclusion, the concept of D2T RA has now been formalised with the EULAR definition of D2T RA. RA patients can be classified as having D2T RA when fulfilling all three of the following criteria: 1. previous failure of $\geq 2 \mathrm{~b} /$ tsDMARDs with different mechanisms of action; 2. symptoms and signs suggestive of active/progressive disease; 3 . the management is being perceived as problematic by the rheumatologist and/or patient. D2T RA is a heterogeneous disease state in which one or multiple factors may contribute to the persistence of signs and/or symptoms suggestive of active disease (e.g. DMARDs being truly ineffective, treatment non-adherence, concomitant fibromyalgia). The heterogeneity of D2T RA should be acknowledged in daily clinical practice and warrants a holistic approach.

Despite the scarcity of (high-quality) evidence, the management of D2T RA patients can now be guided by the consensus-based EULAR recommendations. As first step in the management of D2T RA, the diagnosis of RA should be ascertained. Then, the presence or absence of inflammatory disease activity should be evaluated as well as all factors potentially contributing to the D2T RA state to identify the origin of the patient's current signs and symptoms. These outcomes should guide individualised pharmacological and non-pharmacological therapeutic strategies.

The patients', rheumatologists' and economic burden of D2T RA are significant, which substantiates the need for a swift implementation of the abovementioned recommendations in daily practice to decrease the burden of D2T RA. Additionally, more research into D2T RA is needed to increase our understanding of the underlying immunology, to further optimise diagnostics and therapeutic strategies, and to determine factors that could facilitate early identification of (relevant subgroups of) patients to enable preventive interventions as well as treatment stratification and, with that, to improve outcomes of D2T RA patients. 


\section{References}

1. de Hair MJH, Jacobs JWG, Schoneveld JLM, et al. Difficult-to-treat rheumatoid arthritis: an area of unmet clinical need. Rheumatology (Oxford) 2017;57:1135-44.

2. Buch $\mathrm{MH}$. Defining refractory rheumatoid arthritis. Ann Rheum Dis 2018;77:966-9.

3. Kearsley-Fleet L, Davies R, De Cock D, et al. Biologic refractory disease in rheumatoid arthritis: Results from the British Society for Rheumatology Biologics Register for Rheumatoid Arthritis. Ann Rheum Dis 2018;77:1405-12.

4. Roodenrijs NMT, de Hair MJH, van der Goes $\mathrm{MC}$, et al. Characteristics of difficult-to-treat rheumatoid arthritis: results of an international survey. Ann Rheum Dis 2018;77:1705-9.

5. Roodenrijs NMT, van der Goes MC, Welsing PMJ, et al. Difficult-to-treat rheumatoid arthritis: contributing factors and burden of disease. Rheumatology 2020:keaa860.

6. Roodenrijs NM, de Hair MJ, van der Goes MC, et al. Correspondence to viewpoint 'Defining refractory rheumatoid arthritis' by Buch. Ann Rheum Dis 2019;78:e105.

7. Buch MH. Response to 'Correspondence to viewpoint 'Defining refractory rheumatoid arthritis' by Buch' by Roodenrijs et al. Ann Rheum Dis 2019;78:el06.

8. Nagy G, Roodenrijs NMT, Welsing PMJ, et al. EULAR definition of difficult-to-treat rheumatoid arthritis. Ann Rheum Dis 2021;80:31-5.

9. Maxwell JR, Gowers IR, Moore DJ, et al. Alcohol consumption is inversely associated with risk and severity of rheumatoid arthritis. Rheumatology 2010;49:2140-6.

10. Imhof $\mathrm{A}$, Froehlich $\mathrm{M}$, Brenner $\mathrm{H}$, et al. Effect of alcohol consumption on systemic markers of inflammation. Lancet 2001;357:763-7.

11. Xue AL, Wu SY, Jiang $L$, et al. Bone fracture risk in patients with rheumatoid arthritis. Med (United States) 2017;96.

12. Harrold LR, Andrade SE. Medication Adherence of Patients with Selected Rheumatic Conditions: A Systematic Review of the Literature. Semin Arthritis Rheum 2009;38:396402.

13. Naranjo A, Ojeda S, Francisco $F$, et al. Fibromyalgia in patients with rheumatoid arthritis is associated with higher scores of disability. Ann Rheum Dis 2002;61:660-1.

14. Ranzolin A, Brenol JCT, Bredemeier $M$, et al. Association of concomitant fibromyalgia with worse disease activity score in 28 joints, health assessment questionnaire, and short form 36 scores in patients with rheumatoid arthritis. Arthritis Care Res 2009;61:794-800.

15. Zhang L, Cai P, Zhu W. Depression has an impact on disease activity and healthrelated quality of life in rheumatoid arthritis: A systematic review and meta-analysis. Int J Rheum Dis 2020;23:285-93.

16. Machin AR, Babatunde $O$, Haththotuwa R, et al. The association between anxiety and disease activity and quality of life in rheumatoid arthritis: a systematic review and metaanalysis. Clin Rheumatol 2020;39:1471-82.

17. Englbrecht $M$, Kruckow $M$, Araujo $E$, et al. The Interaction of Physical Function and Emotional Well-being in Rheumatoid ArthritisWhat is the Impact on Disease Activity and Coping? Semin Arthritis Rheum 2013;42:48291.

18. Chang $\mathrm{K}$, Yang $\mathrm{SM}$, Kim $\mathrm{SH}$, et al. Smoking and rheumatoid arthritis. Int $\mathrm{J}$ Mol Sci 2014;15:22279-95.

19. Westhoff G, Rau R, Zink A. Rheumatoid arthritis patients who smoke have a higher need for DMARDs and feel worse, but they do not have more joint damage than non-smokers of the same serological group. Rheumatology 2008;47:849-54.

20. Ruiz-Medrano E, Espinosa-Ortega HF, ArceSalinas CA. The effect of concomitant hand osteoarthritis on pain and disease activity in patients with rheumatoid arthritis. Clin Rheumatol 2019;38:2709-16.

21. George MD, Baker JF. The Obesity Epidemic and Consequences for Rheumatoid Arthritis Care. Curr Rheumatol Rep 2016;18:1-9.

22. Westhoff $G$, Rau R, Zink A. Rheumatoid arthritis patients who smoke have a higher need for DMARDs and feel worse, but they do not have more joint damage than non-smokers of the same serological group. Rheumatology (Oxford) 2008;47:849-54.

23. Kuzuya K, Saeki Y, Hashimoto J, et al. Smoking reduces efficacy of biologics differently by target cytokines in rheumatoid arthritis. Arthritis Rheumatol 2017;69:(suppl 10).

24. Abhishek A, Butt S, Gadsby K, et al. Anti-TNF-a agents are less effective for the treatment of rheumatoid arthritis in current smokers. J Clin Rheumatol 2010;16:15-8.

25. Hyrich $\mathrm{KL}$, Watson $\mathrm{KD}$, Silman $\mathrm{AJ}$, et al. Predictors of response to anti-TNF-alpha therapy among patients with rheumatoid arthritis: results from the British Society 
for Rheumatology Biologics Register. Rheumatology (Oxford) 2006;45:1558-65.

26. Mattey DL, Brownfield A, Dawes PT. Relationship between pack-year history of smoking and response to tumor necrosis factor antagonists in patients with rheumatoid arthritis. J Rheumatol 2009;36:1180-7.

27. Söderlin MK, Petersson IF, Geborek P. The effect of smoking on response and drug survival in rheumatoid arthritis patients treated with their first anti-TNF drug. Scand J Rheumatol 2012;41:1-9.

28. Heimans L, Van Den Broek M, Le Cessie S, et al. Association of high body mass index with decreased treatment response to combination therapy in recent-onset rheumatoid arthritis patients. Arthritis Care Res 2013;65:1235-42.

29. Toussirot É, Michel F, Binda D, et al. The role of leptin in the pathophysiology of rheumatoid arthritis. Life Sci 2015;140:29-36.

30. Palming J, Gabrielsson BG, Jennische E, et al. Plasma cells and Fc receptors in human adipose tissue-lipogenic and antiinflammatory effects of immunoglobulins on adipocytes. Biochem Biophys Res Commun 2006;343:43-8.

31. Wojtal KA, Rogler G, Scharl M, et al. Fc Gamma Receptor CD64 Modulates the Inhibitory Activity of Infliximab. PLoS One 2012;7:e43361.

32. Tolusso B, Alivernini S, Gigante MR, et al. Biomolecular features of inflammation in obese rheumatoid arthritis patients: management considerations. Expert Rev Clin Immunol 2016;12:751-62.

33. Alivernini $\mathrm{S}$, Tolusso $\mathrm{B}$, Gigante $\mathrm{MR}$, et al. Overweight/obesity affects histological features and inflammatory gene signature of synovial membrane of Rheumatoid Arthritis. Sci Rep 2019;9:1-12.

34. Roodenrijs NMT, Kedves $M$, Hamar A, et al. Diagnostic issues in difficult-totreat rheumatoid arthritis: a systematic literature review informing the 2020 EULAR recommendations for the management of difficult-to-treat rheumatoid arthritis. RMD open 2021;7:e001511.

35. Roodenrijs NMT, Welsing PMJ, Schoneveld L, et al. Immunological mechanisms underlying DMARD inefficacy in difficult-to-treat rheumatoid arthritis: a narrative review with systematic literature search. Manuscript in preparation.

36. Roodenrijs NMT, Hamar A, Kedves $M$, et al. Pharmacological and non-pharmacological therapeutic strategies in difficult-to-treat rheumatoid arthritis: a systematic literature review informing the EULAR recommendations for the management of difficult-to-treat rheumatoid arthritis. RMD open 2021;7:e001512.

37. Emery $\mathrm{P}$, Keystone $\mathrm{E}$, Tony $\mathrm{HP}$, et al. IL-6 receptor inhibition with tocilizumab improves treatment outcomes in patients with rheumatoid arthritis refractory to anti-tumour necrosis factor biologicals: Results from a 24-week multicentre randomised placebocontrolled trial. Ann Rheum Dis 2008;67:151623.

38. Burmester GR, Blanco R, Charles-Schoeman $C$, et al. Tofacitinib (CP-690,550) in combination with methotrexate in patients with active rheumatoid arthritis with an inadequate response to tumour necrosis factor inhibitors: A randomised phase 3 trial. Lancet 2013;381:451-60.

39. Genovese MC, Fleischmann RM, Greenwald M, et al. Tabalumab, an anti-BAFF monoclonal antibody, in patients with active rheumatoid arthritis with an inadequate response to TNF inhibitors. Ann Rheum Dis 2013;72:1461-8.

40. Genovese MC, Kalunian K, Gottenberg JE, et al. Effect of Filgotinib vs Placebo on Clinical Response in Patients With Moderate to Severe Rheumatoid Arthritis Refractory to DiseaseModifying Antirheumatic Drug Therapy: The FINCH 2 Randomized Clinical Trial. JAMA 2019;322:315-25.

41. Kremer JM, Emery P, Camp HS, et al. A Phase llb Study of ABT-494, a Selective JAK-1 Inhibitor, in Patients With Rheumatoid Arthritis and an Inadequate Response to Anti-Tumor Necrosis Factor Therapy. Arthritis Rheumatol 2016;68:2867-77.

42. Weinblatt M, Thomson G, Chen K, et al. FRI0171 Clinical responses in patients with inadequate response to bdmards upon treatment with upadacitinib. Ann Rheum Dis 2019;78:759.

43. Blom M, Kievit W, Donders ART, et al. Effectiveness of a third tumor necrosis factor-a-blocking agent compared with rituximab after failure of 2 TNF-blocking agents in rheumatoid arthritis. J Rheumatol 2011;38:2355-61.

44. Finckh A, Ciurea A, Brulhart L, et al. Which subgroup of patients with rheumatoid arthritis benefits from switching to rituximab versus alternative anti-tumour necrosis factor (TNF) agents after previous failure of an anti-TNF agent? Ann Rheum Dis 2010;69:387-93.

45. Roodenrijs NMT, de Hair MJH, Wheater G, et al. 
The multi-biomarker disease activity score tracks response to rituximab treatment in rheumatoid arthritis patients: a post-hoc analysis of three cohort studies. Arthritis Res Ther 2018;20:256.

46. Smolen JS, Breedveld FC, Burmester GR, et al. Treating rheumatoid arthritis to target: 2014 update of the recommendations of an international task force. Ann Rheum Dis 2016;75:3-15.

47. Collignon $\mathrm{O}$. Methodological issues in the design of a rheumatoid arthritis activity score and its cut-offs. Clin Epidemiol 2014;6:221-6.

48. Isaacs JD, Ferraccioli $G$. The need for personalised medicine for rheumatoid arthritis. Ann Rheum Dis 2011;70:4-7.

49. Roodenrijs NMT, van der Goes MC, Welsing $\mathrm{PMJ}$, et al. Is prediction of clinical response to methotrexate in individual rheumatoid arthritis patients possible? A systematic literature review. Jt Bone Spine 2020;87:13-23.

50. Smolen JS, Landewé RBM, Bijlsma JWJ, et al. EULAR recommendations for the management of rheumatoid arthritis with synthetic and biological disease-modifying antirheumatic drugs: 2019 update. Ann Rheum Dis 2020;79:685-99.

51. Sutcliffe M, Radley G, Barton A. Personalized medicine in rheumatic diseases: how close are we to being able to use genetic biomarkers to predict response to TNF inhibitors? Expert Rev Clin Immunol 2020;16:389-96.

52. Aletaha D, Smolen J, Ward MM. Measuring function in rheumatoid arthritis: Identifying reversible and irreversible components. Arthritis Rheum 2006;54:2784-92.

53. Smolen JS, Aletaha D, McInnes IB. Rheumatoid arthritis. Lancet 2016;388:2023-38.

54. Nagy G, Roodenrijs NMT, Welsing PMJ, et al. EULAR recommendations for the management of difficult-to-treat rheumatoid arthritis. Manuscript in preparation.

55. Roodenrijs NMT, Goes MC van der, Welsing $\mathrm{PMJ}$, et al. Non-adherence in difficult-to-treat rheumatoid arthritis from the perspectives of patients and rheumatologists: a concept mapping study. Rheumatology 2021. Accepted for publication.

56. Group ES. Socioeconomic deprivation and rheumatoid disease: What lessons for the health service? Ann Rheum Dis 2000;59:7949.

57. Jacobi CE, Mol GD, Boshuizen $\mathrm{HC}$, et al. Impact of socioeconomic status on the course of rheumatoid arthritis and on related use of health care services. Arthritis Rheum 2003;49:567-73.

58. Marra CA, Lynd LD, Esdaile JM, et al. The impact of low family income on self-reported health outcomes in patients with rheumatoid arthritis within a publicly funded health-care environment. Rheumatology 2004;43:1390-7.

59. Verstappen SMM. The impact of socioeconomic status in rheumatoid arthritis. Rheumatology 2016;56:1051-2.

60. Smolen JS, Landewé R, Bijlsma J, et al. EULAR recommendations for the management of rheumatoid arthritis with synthetic and biological disease-modifying antirheumatic drugs: 2016 update. Ann Rheum Dis 2017;76:960-77.

61. Singh JA, Saag KG, Bridges SL, et al. 2015 American College of Rheumatology Guideline for the Treatment of Rheumatoid Arthritis. Arthritis Rheumatol 2016;68:1-26.

62. Roodenrijs NMT, Welsing PMJ, Goes MC van der, et al. Health care utilisation and economic burden of difficult-to-treat rheumatoid arthritis: a cost-of-illness study. Rheumatology 2021:keab078.

63. Lam WY, Fresco P. Medication Adherence Measures: An Overview. Biomed Res Int 2015;2015:1-12.

64. Van Schouwenburg PA, Rispens T, Wolbink GJ. Immunogenicity of anti-TNF biologic therapies for rheumatoid arthritis. Nat Rev Rheumatol 2013;9:164-72.

65. Orr C, Sousa E, Boyle DL, et al. Synovial tissue research: A state-of-the-art review. Nat Rev Rheumatol 2017;13:463-75.

66. Donlin LT, Park SH, Giannopoulou E, et al. Insights into rheumatic diseases from nextgeneration sequencing. Nat Rev Rheumatol 2019;15:327-39.

67. Winthrop $\mathrm{KL}$, Weinblatt $\mathrm{ME}$, Bathon $\mathrm{J}$, et al. Unmet need in rheumatology: reports from the Targeted Therapies meeting 2019. Ann Rheum Dis 2020;79:88-93.

68. Ceponis A, Onishi M, Bluestein HG, et al. Utility of the ultrasound examination of the hand and wrist joints in the management of established rheumatoid arthritis. Arthritis Care Res 2014;66:236-44.

69. Filer A, Mandelin Al, DiCarlo E, et al. Histological and Clinical Correlates of Ultrasound Measures of Joint Inflammation: Analysis of RA Tissue Obtained By Ultrasound Guided Biopsy in Phase 1 of the Accelerating Medicines Partnership RA Network - ACR Meeting Abstracts. Arthritis Rheumatol 2017;69. 
70. Kawashiri SY, Kawakami A, Iwamoto N, et al. The power Doppler ultrasonography score from 24 synovial sites or 6 simplified synovial sites, including the metacarpophalangeal joints, reflects the clinical disease activity and level of serum biomarkers in patients with rheumatoid arthritis. Rheumatology (Oxford) 2011;50:962-5.

71. Mandl P, Naredo E, Wakefield RJ, et al. A systematic literature review analysis of ultrasound joint count and scoring systems to assess synovitis in rheumatoid arthritis according to the OMERACT filter. J Rheumatol 2011;38:2055-62.

72. Yokota K, Tsuzuki Wada T, Akiyama Y, et al. Detection of synovial inflammation in rheumatic diseases using superb microvascular imaging: Comparison with conventional power Doppler imaging. Mod Rheumatol 2018;28:327-33.

73. Vlad V, Berghea F, Libianu S, et al. Ultrasound in rheumatoid arthritis - Volar versus dorsal synovitis evaluation and scoring. BMC Musculoskelet Disord 2011;12:124.

74. Ngai $\mathrm{Ng} \mathrm{S}$, Bjørndal Axelsen M, Ostergaard $M$, et al. OP0135 How well does whole body magnetic resonance imaging agree with whole body ultrasound in the assessment of joint inflammation in rheumatoid arthritis patients. Ann Rheum Dis 2019;78:143.

75. Ciurtin C, Brown G, Cotton A, et al. THU0138 Das 28 correlated poorly with the objective evidence of inflammation as detected by ultrasound (US) examination of hands and feet in patients with established rheumatoid arthritis (RA). Ann Rheum Dis 2017;76:253.1-253.

76. Takase-Minegishi K, Horita N, Kobayashi K, et al. Diagnostic test accuracy of ultrasound for synovitis in rheumatoid arthritis: systematic review and meta-analysis. Rheumatology (Oxford) 2018;57:49-58.

77. Krabbe $\mathrm{S}$, Ammitzbøll-Danielsen $\mathrm{M}$, Østergaard $M$, et al. Sensitivity and specificity of optical spectral transmission imaging in detecting joint inflammation in rheumatoid arthritis. Ann Rheum Dis 2016;75:632-3.

78. van Onna $\mathrm{M}$, Ten Cate $\mathrm{DF}$, Tsoi $\mathrm{KL}$, et al. Assessment of disease activity in patients with rheumatoid arthritis using optical spectral transmission measurements, a noninvasive imaging technique. Ann Rheum Dis 2016;75:511-8.

79. Besselink NJ, van der Meijde P, Rensen WHJJ, et al. Optical spectral transmission to assess inflammation in hand and wrist joints of rheumatoid arthritis patients. Rheumatology (Oxford) 2018;57:865-72.

80. Colebatch AN, Edwards CJ, Østergaard $M$, et al. EULAR recommendations for the use of imaging of the joints in the clinical management of rheumatoid arthritis. Ann Rheum Dis 2013;72:804-14.

81. Johnson TM, Register KA, Schmidt CM, et al. Correlation of the Multi-Biomarker Disease Activity Score with Rheumatoid Arthritis Disease Activity Measures: A Systematic Review and Meta-Analysis. Arthritis Care Res (Hoboken) 2018;71:1459-72. 



\section{ADDENDUM}

Summary

Nederlandse samenvatting

Acknowledgements

List of publications

Contributing authors

Curriculum Vitae 



\section{Summary}

In Chapter 1 of this thesis, we provided an introduction on difficult-to-treat rheumatoid arthritis (D2T RA). RA is the most common chronic inflammatory joint disease, affecting approximately $1 \%$ of the adult population. It is characterised by synovitis resulting in pain and stiffness in the joints and potentially leading to structural joint damage. Additionally, extra-articular manifestations can occur and a higher risk of certain comorbidities (e.g. cardiovascular disease) may be present. All these manifestations result in a significant patients' burden of disease: functional disability, quality of life and premature mortality. Treatment with disease-modifying antirheumatic drugs (DMARDs) is the cornerstone of the therapeutic strategy as these drugs can suppress inflammation and may prevent irreversible joint damage, therewith reducing the patients' burden of disease. In RA management recommendations, tight control and treat-to-target strategies require regular assessment of disease activity and already allow adaptation of treatment after 3 months if remission or low disease activity is not reached. Composite indices, such as the disease activity score assessing 28 joints (DAS28), are most frequently used for the assessment of disease activity.

Despite significant improvement in the management of RA, a substantial proportion of patients remains symptomatic after treatment with multiple biological and/or targeted synthetic (b/ts)DMARDs according to European League Against Rheumatism (EULAR) RA management recommendations. These patients can be referred to as having 'difficultto-treat' RA. Current management recommendations mainly focus on the early phase of the disease and on pharmacological management. Less guidance is available for the management of D2T RA, which is also hampered by the lack of uniform terminology and a definition, although its management often is a true clinical challenge. Therefore, the aims of this thesis were to define and improve our understanding of D2T RA, to contribute to the optimisation of the management of D2T RA patients and therewith to improve outcomes of this patient population.

In Chapter 2, we aimed to identify predictors of clinical response to methotrexate - part of the initial management of RA - after 3 to 6 months by a systematic literature review $(\mathrm{SLR})$. Reliable predictors are needed, as ineffective treatment may increase the risk of joint damage and the patients' burden of disease. Out of 30 selected papers, 102 different predictors and 11 predictive models were found. Female gender, smoking and rheumatoid factor positivity appeared to be weak predictors of non-response in multiple studies, although this could not be replicated in all studies. Additionally, a promising validated epigenetic model was found that needs further validation. However, for the individual patient, no reliable predictors of clinical response to methotrexate at 3 to 6 months after treatment initiation were identified. 
In Chapter 3, we studied the correlation between the multi-biomarker disease activity (MBDA) score and DAS28 during rituximab treatment. The MBDA score has been developed as a more objective and sensitive disease activity measure than the DAS28, and may specifically be helpful in patients in whom there is explicit doubt about the presence of inflammation. The MBDA score has been shown to track response to multiple (b/ts)DMARDs, although not yet for rituximab. In a post-hoc analysis of three cohort studies containing data from 57 RA patients, the MBDA score was determined in serum samples obtained at baseline and 6 months. The MBDA score correlated statistically significantly with DAS28 at baseline and 6 months. Additionally, change in MBDA score correlated significantly with change in DAS28 upon rituximab treatment.

In Chapter 4, we aimed to get more insight into the concept of D2T RA and the unmet needs that are not covered by the current EULAR RA recommendations. An online survey was distributed among rheumatologists and over 400 responses were received from 33 different (mainly European) countries. The results of this survey showed that there is a wide variety in characteristics of the concept of D2T RA: failure of $\geq 2 \mathrm{~b} / \mathrm{tsDMARDs}$ with different mechanisms of action, at least moderate disease activity or symptoms suggestive of active disease and inability to taper glucocorticoid treatment were considered as most important characteristics. D2T RA was seen as a heterogeneous condition, which underscores the difficulty in establishing an unambiguous definition of D2T RA. Current RA EULAR management recommendations were found not to address many aspects of this disease state, such as extra-articular manifestations, comorbidities and pain syndromes, indicating the rheumatologist's burden.

In Chapter 5, the EULAR definition of D2T RA is presented. This definition is based on the identified characteristics on the concept of D2T RA from the international survey (Chapter 4), a scoping literature review and extensive discussions among experts of the EULAR Task Force on D2T RA (patients, rheumatologists and other health care professionals). The following three criteria were agreed by all Task Force members as mandatory elements of the definition of D2T RA: 1. treatment according to EULAR recommendations and failure of $\geq 2 \mathrm{~b} / \mathrm{tsDMARDs}$ with different mechanisms of action after failing conventional synthetic (cs)DMARD therapy (unless contraindicated); 2. signs suggestive of active/progressive disease; 3. the management of signs and/or symptoms is perceived as problematic by the rheumatologist and/or the patient.

InChapter6, we providedanoverview of possibleimmunologicalmechanisms underlying DMARD inefficacy and (unacceptable) side effects in D2T RA by a narrative review based on a systematic literature search. Examples of immunological mechanisms are discussed, including the influence of different levels of gene expression regulation and 
clinical characteristics (i.e. smoking and obesity). The exact mechanisms underlying DMARD inefficacy in D2T RA patients in whom all currently available (b/ts)DMARDs are truly ineffective (i.e. 'true' refractory RA) remain largely unknown as well as whether 'true' refractory RA really exists, as studies in these patients are lacking. However, the identified heterogeneity between individuals supports the assumption that (D2T) RA is a syndrome, in which the pathogenesis differs between individuals.

In Chapter 7, we summarised the evidence on diagnostic issues in D2T RA by a SLR to inform the Task Force in charge of the development of the EULAR recommendations for the management of D2T RA (Chapter 9). These issues concern the optimal confirmation of the diagnosis of RA and of mimicking diseases that may underlie current RA complaints, as well as the optimal assessment of inflammatory disease activity when in doubt based on traditional measures. In this SLR, the scarcity of highquality studies regarding these diagnostic issues is highlighted. No diagnostic tests with sufficient validity and accuracy were found to confirm nor exclude the diagnosis of RA nor its mimicking diseases. Ultrasonography may have an additional value to assess the presence of inflammatory activity in D2T RA patients, including those with concomitant obesity or fibromyalgia.

In Chapter 8, we summarised the evidence on pharmacological and nonpharmacological therapeutic strategies in D2T RA patients by a SLR to inform the Task Force in charge of the development of the EULAR recommendations for the management of D2T RA (Chapter 9). This SLR underscores the scarcity of highquality evidence on the pharmacological and non-pharmacological treatment of D2T RA patients including those with comorbidities. We found that the effectiveness of $b /$ tsDMARDs decreased gradually with the number of previously failed bDMARDs. Additionally, the evidence indicated a tendency of non-TNFi bDMARDs to be more effective than TNFis in RA patients who failed at least one TNFi. Furthermore, a beneficial effect of several non-pharmacological interventions was found for the improvement of non-inflammatory complaints (e.g. pain, fatigue), goal setting and self-management.

In Chapter 9, the EULAR recommendations for the management of D2T RA are presented. These recommendations are based on the evidence from the SLRS on diagnostic issues and therapeutic strategies in D2T RA (Chapter 7 and 8), together with expert opinion of the members of the EULAR Task Force on D2T RA (patients, rheumatologists and other health care professionals). Two overarching principles and ten recommendations were formulated regarding the confirmation of the diagnosis of RA, the assessment of the presence or absence of inflammatory disease activity, pharmacological interventions to reduce inflammatory disease activity (also in patients with comorbidities) and non- 
pharmacological interventions to improve treatment adherence, functional disability, pain, fatigue, goal setting and self-efficacy. They promote a holistic management approach and individually tailored therapeutic strategies. The evidence supporting the recommendations was relatively weak. Nevertheless, the level of agreement among the Task Force members was generally high.

In Chapter 10, we further characterised D2T RA, by identifying risk factors and factors contributing to the D2T RA state, and determined the patients' burden of disease. To this end, 52 consecutive RA patients visiting the outpatient clinic have been enrolled who fulfilled the new D2T RA definition (Chapter 5), as well as 100 non-D2T RA patients. Lower socioeconomic status at RA onset wa s found to be an independent risk factor for developing D2T RA (odds ratio 1.97 (95\% confidence interval 1.08-3.61, p=0.03)). In D2T RA patients, higher occurrences of multiple contributing factors were found compared with in non-D2T RA patients: limited drug options because of adverse events ( $94 \% \mathrm{vs}$ $57 \%$ ) or comorbidities (69\% vs $37 \%$ ), a mismatch in patient's and rheumatologist's wish to intensify treatment (patient's wish to intensify treatment: $37 \%$ vs 6\%), concomitant fibromyalgia (38\% vs 9\%) and poorer coping (higher levels in D2T RA) were independently associated with D2T RA. Also, treatment non-adherence (discrepancy in prescribed and supplied drugs: $40 \%$ vs $22 \%$ ), depression ( $15 \%$ vs $4 \%$ ) and higher levels of anxiety were more commonly found among D2T than among non-D2T RA patients. In an exploratory analysis, three subgroups of D2T RA patients could be identified, reflecting the heterogeneity of the D2T disease state: 1. 'non-adherent dissatisfied patients'; 2. patients with 'pain syndromes and obesity'; 3. patients with RA closest to the concept of 'true refractory RA'. Furthermore, the patients' burden of disease was significantly higher in D2T than in non-D2T RA patients.

In Chapter Il, we determined the impact of D2T RA on (costs related to) health care utilisation, other resource use and work productivity. To this end, data of the same patients as in Chapter 10 (52 D2T and 100 non-D2T RA patients) were collected via a questionnaire and an electronic patient record review during the study visit. D2T RA patients were found to visit their rheumatologist more frequently, were more often admitted to day care facilities, underwent more laboratory tests and used more drugs. Consequently, annual mean total costs were statistically significantly higher in D2T RA patients: €37,605, versus €19,217 for non-D2T RA. Functional disability (according to health assessment questionnaire ( $\mathrm{HAQ})$ ) was identified as a key determinant of higher total costs in RA.

In Chapter 12, we further assessed treatment non-adherence in D2T RA by thematically structuring and prioritising barriers and facilitators of optimal adherence from the 
perspectives of patients and rheumatologists. First, interviews were conducted to identify perceptions of D2T and non-D2T RA patients. Second, a project group selected representative statements regarding 40 barriers and 40 facilitators. Third, 20 D2T and 20 non-D2T patients sorted the statements in two card-sorting tasks: first, by order of content similarity and, second, by content applicability. Independently, twenty rheumatologists sorted the statements by order of content applicability to the general RA population. The similarity sorting was used to create structured overviews from the patient's perspective: nine clusters of adherence barriers were identified related to the health care system, treatment safety/efficacy, treatment regimen and patient behaviour; additionally, seven clusters of facilitators of optimal adherence were identified related to the health care system and directly to the patient. The applicability sorting was used to prioritise the statements and to compare the priority order of D2T RA patients with those of non-D2T RA patients and rheumatologists. D2T RA patients, non-D2T RA patients and rheumatologists prioritised perceptions on non-adherence largely the same and only some differences were identified in adherence barriers. D2T RA patients prioritised adverse events and efficacy doubts as most important barriers. D2T and non-D2T RA patients and rheumatologists prioritised a good relationship with the health care professional and treatment information as most helpful facilitators.

In Chapter 13, we put the results of the studies in this thesis in a broader perspective, discussed strengths, limitations and clinical implications and provided future perspectives. In conclusion, the concept of D2T RA has now been formalised with the EULAR definition. D2T RA is a heterogeneous disease state in which one or multiple factors may contribute to the persistence of signs and/or symptoms. The heterogeneity of D2T RA should be acknowledged in daily practice and warrants a holistic approach. Despite the scarcity of (high-quality) evidence, the management of D2T RA patients can now be guided by the consensus-based EULAR recommendations. As first step, the diagnosis of RA should be ascertained. Then, the presence or absence of inflammatory disease activity should be evaluated as well as all factors potentially contributing to D2T to identify the origin of the patient's current signs and symptoms. These outcomes should guide individualised pharmacological and/or non-pharmacological therapeutic strategies. The patients', rheumatologists' and economic burden of D2T RA are significant, which substantiates the need for a swift implementation of the abovementioned recommendations in daily practice to decrease the burden of D2T RA. Additionally, more research into D2T RA is needed to increase our understanding of the underlying immunology, to further optimise diagnostics and therapeutic strategies, and to determine factors that could facilitate early identification and treatment stratification and, with that, to improve outcomes of D2T RA patients. 



\section{Nederlandse samenvatting}

In Hoofdstuk 1 van dit proefschrift introduceerden we het onderwerp 'moeilijk te behandelen reumatoïde artritis (RA)' en de doelen van dit proefschrift.

RA is de meest voorkomende chronische gewrichtsziekte; de ziekte komt voor bij bijna $1 \%$ van de volwassen bevolking. Het is een auto-immuun ziekte, wat betekent dat het afweersysteem ontregeld is. Hierdoor keert het zich tegen het eigen lichaam. Bij RA leidt dit tot ontstekingen van het gewrichtskapsel, wat zich bij de patiënt uit in pijn en stijfheid. Uiteindelijk kan dit leiden tot permanente schade en vervormingen van de gewrichten. Daarnaast kan RA ook voorkomen buiten de gewrichten (zoals in de longen en ogen) en geeft het een hoger risico op bepaalde andere ziekten (ook wel comorbiditeiten genoemd, bijvoorbeeld hart- en vaatziekten). Dit alles leidt tot een grote ziektelast voor de patiënt: er kan sprake zijn van verminderd functioneren, een verminderde kwaliteit van leven en vroegtijdig overlijden.

In de behandeling van RA spelen 'disease-modifying antirheumatic drugs' (DMARDs) de belangrijkste rol. DMARDs zijn medicijnen die de ontstekingen kunnen onderdrukken en daardoor kunnen voorkomen dat er gewrichtsschade optreedt. Om de arts en patiënt te begeleiden in de behandeling van RA zijn er door verschillende verenigingen behandelrichtlijnen opgesteld, bijvoorbeeld door de Europese vereniging voor reumatologie, de 'EULAR'. In de huidige behandelrichtlijn wordt aangeraden om de ziekteactiviteit regelmatig te controleren. Ook wordt aangeraden om de behandeling aan te passen indien de ziekte na drie maanden behandeling nog niet voldoende onder controle is. Om de ziekteactiviteit te bepalen, worden er voornamelijk samengestelde ziekteactiviteitscores gebruikt. De DAS28, een ziekteactiviteitscore die 28 gewrichten bekijkt, is hier een voorbeeld van. Deze score kijkt naar het aantal gezwollen en het aantal pijnlijke gewrichten, de ontstekingswaarde in het bloed en de mate waarin de patiënt op dat moment klachten ervaart van de ziekte.

De behandeling van RA is de afgelopen jaren enorm verbeterd. Het aantal DMARDs dat gebruikt kan worden om RA te behandelen is uitgebreid: er zijn nu ook biologische DMARDs (bDMARDs) die zich specifiek richten op bepaalde cellen of eiwitten in het afweersysteem en 'targeted synthetic DMARDs' (tsDMARDs) die zich richten op het doorgeven van boodschappen in cellen van het afweersysteem. Doordat deze medicijnen op de markt zijn gekomen en ingezet worden wanneer de klassieke DMARDs niet voldoende werken, is de ziektelast voor de meeste RA-patiënten verminderd. Voor een deel van deze patiënten lukt het echter niet om de ziekte goed onder controle te krijgen, hoewel deze patiënten reeds behandeld zijn met diverse nieuwe medicijnen (b/ tsDMARDs). Dit zijn de RA-patiënten die we beschouwen als 'moeilijk te behandelen'. In de huidige behandelrichtlijnen is er weinig aandacht voor deze patiëntengroep, terwijl de behandeling juist voor deze patiënten lastig is. 
De doelen van dit proefschrift waren ten eerste om vast te stellen wanneer er sprake is van moeilijk te behandelen RA en daarnaast om meer inzicht te krijgen in dit ziektebeeld. Beide zijn nodig om de behandeling van moeilijk te behandelen RA te verbeteren en om uiteindelijk de ziektelast van deze patiënten te verminderen.

In Hoofdstuk 2 was ons doel om factoren te vinden die kunnen voorspellen of de behandeling met het medicijn methotrexaat (een klassieke DMARD die wordt gegeven als onderdeel van de eerste behandeling van RA) succesvol zal zijn, drie tot zes maanden na de start van deze behandeling. Betrouwbare voorspellers zijn nodig omdat een onsuccesvolle behandeling ervoor zorgt dat de ziekte actief blijft. Dit kan het risico op gewrichtsschade verhogen, evenals de ziektelast. Om deze voorspellers te vinden, hebben we eerdere onderzoeken over dit onderwerp systematisch doorzocht en samengevat. In 30 artikelen vonden we 102 verschillende voorspellers en elf voorspelmodellen. In verschillende onderzoeken bleken vrouwelijk geslacht, roken en de aanwezigheid van reumafactor (een antistof in het bloed) zwakke voorspellers voor onsuccesvolle behandeling. Deze resultaten werden echter niet in alle onderzoeken bevestigd. Daarnaast vonden we een veelbelovend voorspelmodel, alhoewel de voorspellende waarde nog in meer onderzoeken bevestigd moet worden. Al met al vonden wevoor de individuele patiënt geen voorspellers die het succes van behandeling met methotrexaat, drie tot zes maanden na de start, betrouwbaar kunnen voorspellen.

In Hoofdstuk 3 bestudeerden we het verband tussen de MDBA-score (een score op basis van twaalf verschillende eiwitten die een rol hebben binnen het afweersysteem) en de DAS28 tijdens behandeling met het medicijn rituximab (een bDMARD). De MBDAscore is ontwikkeld als een alternatief voor de DAS28 om de ziekteactiviteit objectiever en gevoeliger te bepalen. Dit is met name relevant voor patiënten bij wie er twijfel is over de aanwezigheid van ziekteactiviteit op basis van de DAS28 (inclusief patiënten met moeilijk te behandelen RA). Eerder onderzoek heeft laten zien dat er een verband is tussen de MBDA-score en de DAS28 tijdens behandeling met verschillende (b/ts) DMARDs. Er is echter nog geen onderzoek gedaan tijdens behandeling met rituximab. Voor dit onderzoek hebben we gebruik gemaakt van het bloed van 57 RA-patiënten dat in drie eerdere onderzoeken is verzameld. De MBDA-score is bepaald in bloed dat werd afgenomen vóór behandeling met rituximab en in bloed dat zes maanden na behandeling werd afgenomen. We vonden een significant verband tussen de MBDAscore en de DAS28, zowel vóór de behandeling met rituximab als zes maanden na de behandeling. Ook vonden we een significant verband tussen de verandering in de MBDA-score en de verandering in de DAS28 gedurende deze periode.

In Hoofdstuk 4 wilden we meer inzicht krijgen in het concept moeilijk te behandelen RA 
en in de informatie die mist in de huidige EULAR-behandelrichtlijnen. We hebben een vragenlijst verspreid onder reumatologen, waarop we meer dan 400 reacties ontvingen vanuit 33 verschillende (voornamelijk Europese) landen. Deze reacties toonden grote verschillen in de karakteristieken van het concept moeilijk te behandelen RA. De reumatologen beschouwden de volgende karakteristieken als het meest belangrijk: behandeling met minimaal twee b/tsDMARDs met verschillendewerkingsmechanismen zonder succes, het hebben van ten minste matige ziekteactiviteit of andere klachten die de suggestie wekken dat RA actief is en het onvermogen om prednison-achtige middelen te kunnen afbouwen. Moeilijk te behandelen RA werd gezien als een heterogeen ziektebeeld, wat het lastig maakt om een éénduidige definitie op te stellen. De vragenlijst heeft ook inzicht gegeven in onderwerpen waarvoor onvoldoende hulp wordt geboden in de huidige EULAR-behandelrichtlijnen, zoals RA-activiteit buiten de gewrichten (in andere organen), de behandeling van comorbiditeiten en bijkomende pijnsyndromen, wat de moeilijkheden in de behandeling voor de reumatoloog aantoont.

In Hoofdstuk 5 presenteerden we de EULAR-definitie van moeilijk te behandelen RA. Deze definitie is gebaseerd op de karakteristieken van moeilijk te behandelen RA zoals beschreven in Hoofdstuk 4, een globaal literatuuronderzoek en de meningen van verschillende experts van de EULAR-werkgroep die zich richt op moeilijk te behandelen RA (patiënten, reumatologen en andere zorgverleners). De volgende drie criteria werden beschouwd als essentiële onderdelen van de definitie: 1. behandeling volgens de huidige EULAR-behandelrichtlijnen waarbij er ten minste twee b/tsDMARDs met verschillende werkingsmechanismen zijn gebruikt zonder succes; 2. de aanwezigheid van klachten en/of symptomen die de suggestie wekken van RA-activiteit; 3 . de behandeling van de klachten en/of symptomen wordt beschouwd als een probleem door de reumatoloog en/of de patiënt.

In Hoofdstuk 6 gaven we een overzicht van mechanismen in het afweersysteem die er mogelijk voor kunnen zorgen dat DMARDs niet succesvol zijn bij patiënten met moeilijk te behandelen RA ('echt refractaire RA') of ernstige bijwerkingen geven. Om deze mechanismen te vinden, hebben we eerdere onderzoeken over dit onderwerp systematisch doorzocht. We hebben de verschillende mechanismen in het afweersysteem beschreven die we hebben gevonden, inclusief de invloed van genen en andere factoren (zoals roken en overgewicht) hierop. De mechanismen in het afweersysteem die ervoor zorgen dat DMARDs ernstige bijwerkingen geven, hebben waarschijnlijk vaak direct te maken met het werkingsmechanisme van het medicijn. De mechanismen die ervoor zorgen dat DMARDs niet succesvol zijn, blijven grotendeels onbekend, omdat hier nooit eerder onderzoek naar is gedaan bij patiënten met moeilijk te behandelen RA. We vonden echter wel grote verschillen in het afweersysteem tussen 
verschillende patiënten met RA. Dit ondersteunt de veronderstelling dat de oorzaak van (moeilijk te behandelen) RA verschilt van persoon tot persoon.

In Hoofdstuk 7 hebben we eerdere onderzoeken over diagnostische problemen bij patiënten met moeilijk te behandelen RA systematisch doorzocht en samengevat. Dit overzicht werd gemaakt om de EULAR-werkgroep, gericht op het ontwikkelen van behandelrichtlijnen voor moeilijk te behandelen RA (Hoofdstuk 9), te informeren. De diagnostische problemen waar we in geïnteresseerd waren, betroffen het optimaal vaststellen van de diagnose RA, het uitsluiten van andere ziekten die hierop lijken en het vaststellen van RA-ziekteactiviteit wanneer er twijfel is op basis van ziekteactiviteitscores, zoals de DAS28, en klinische inschatting. Het beschreven overzicht toont hoe schaars onderzoek van hoge kwaliteit naar deze diagnostische problemen is. We vonden geen betrouwbare of accurate testen die de diagnose RA of ziekten die hierop lijken kunnen vaststellen of uitsluiten. Daarnaast vonden we dat echografie misschien van toegevoegde waarde zou kunnen zijn om de aanwezigheid van ziekteactiviteit bij patiënten met moeilijk te behandelen RA vast te stellen, evenals bij patiënten die daarnaast ook overgewicht of een pijnsyndroom hebben.

In Hoofdstuk 8 hebben we eerdere onderzoeken over medicamenteuze en nietmedicamenteuze behandelingen voor patiënten met moeilijk te behandelen RA systematisch doorzocht en samengevat. Ook dit overzicht werd gemaakt om de EULAR-werkgroep, gericht op het ontwikkelen van behandelrichtlijnen voor moeilijk te behandelen RA (Hoofdstuk 9), te informeren. Het beschreven overzicht onderstreept hoe schaars onderzoek van hoge kwaliteit naar deze behandelingen is bij deze patiëntengroep, inclusief de patiënten met comorbiditeiten. We vonden dat de effectiviteit van b/tsDMARDs geleidelijk afnam wanneer een patiënt eerder een hoger aantal bDMARDs had gebruikt zonder succes. Tevens toonden de gevonden onderzoeken aan dat er een tendens is dat medicijnen die tumornecrosefactor-alfa remmen (TNFa-remmers, een bDMARD) minder effectief zijn dan andere bDMARDs voor patiënten die eerder ten minste één TNFa-remmer hebben gebruikt zonder succes. Daarnaast vonden we een voordelig effect van verschillende niet-medicamenteuze behandelingen om klachten te verbeteren die niet direct gerelateerd zijn aan ontsteking (bijvoorbeeld pijn en vermoeidheid) evenals het verbeteren van het stellen van behandeldoelen en zelfeffectiviteit (het vertrouwen van een persoon in de eigen bekwaamheid).

In Hoofdstuk 9 presenteerden we de EULAR-behandelrichtlijnen voor moeilijk te behandelen RA. Deze richtlijnen zijn gebaseerd op de bevindingen van eerdere onderzoeken over diagnostische problemen en behandelingen (Hoofdstuk 7 en 8) en 
de meningen van de verschillende experts van de EULAR-werkgroep die zich richt op moeilijk te behandelen RA (patiënten, reumatologen en andere zorgverleners). Er zijn twee overkoepelende principes en tien aanbevelingen opgesteld over het bevestigen van de RA-diagnose, het vaststellen van de aan- of afwezigheid van RA-ziekteactiviteit, de medicamenteuze behandeling om ontstekingen te verminderen (ook in patiënten met comorbiditeiten) en de niet-medicamenteuze behandeling om therapietrouw, verminderd functioneren, pijn, vermoeidheid, het stellen van behandeldoelen en zelfeffectiviteit te verbeteren. De aanbevelingen promoten een holistische aanpak (met een brede benadering) en een behandeling die wordt afgestemd op de individuele patiënt. Het bewijs waarop de aanbevelingen gebaseerd zijn, was relatief zwak. Desondanks was de mate waarin de leden van de EULAR-werkgroep het eens waren met de opgestelde principes en aanbevelingen over het algemeen hoog.

In Hoofdstuk 10 hebben we moeilijk te behandelen RA verder gekarakteriseerd door risicofactoren te identificeren evenals factoren die bijdragen aan moeilijk te behandelen RA. Ook hebben we de ziektelast van de patiënten bepaald. Aan dit onderzoek hebben 52 patiënten deelgenomen die voldeden aan de EULAR-definitie van moeilijk te behandelen RA (Hoofdstuk 5) en 100 RA-patiënten die niet moeilijk te behandelen zijn. We vonden dat een lagere sociaaleconomische status (op basis van onderwijsniveau) op het moment van het stellen van de RA-diagnose een onafhankelijke risicofactor was voor het ontwikkelen van moeilijk te behandelen RA. Ook vonden we dat verschillende factoren die mogelijk bijdragen aan moeilijk te behandelen RA, vaker voorkwamen bij deze patiënten dan bij RA-patiënten die niet moeilijk te behandelen zijn. Verschillende factoren waren onafhankelijk geassocieerd met moeilijk te behandelen RA: verminderde behandelopties door bijwerkingen ( $94 \%$ en $57 \%$ ) of comorbiditeiten (69\% en $37 \%$ ), het niet overeenkomen van de wens om de behandeling te intensiveren (de patiënt wil de behandeling intensiveren, de reumatoloog niet: $37 \%$ en $6 \%$ ), een bijkomend pijnsyndroom (38\% en 9\%) en verminderde coping (verminderde vaardigheid om met problemen om te gaan). Daarnaast kwamen therapieontrouw (40\% en $22 \%$ ), depressie ( $15 \%$ en $4 \%$ ) en angstklachten vaker voor bij patiënten met moeilijk te behandelen RA. In een verkennend onderzoek vonden we ook drie subgroepen binnen de patiënten met moeilijk te behandelen RA, wat de heterogeniteit van moeilijk te behandelen RA onderstreept. Deze groepen konden als volgt omschreven worden: 1. 'therapieontrouwe patiënten die ontevreden zijn met de behandeling'; 2. patiënten met 'pijnsyndromen en overgewicht'; 3. patiënten met RA het dichtstbij het concept van 'echt refractaire RA'. Verder bleek ook de ziektelast van patiënten met moeilijk te behandelen RA significant hoger dan die van patiënten die niet moeilijk te behandelen zijn.

In Hoofdstuk 11 hebben we de impact bepaald van moeilijk te behandelen RA op (kosten 
gerelateerd aan) zorggebruik, ander middelengebruik en werkproductiviteit. Hiervoor hebben we de gegevens gebruikt van dezelfde patiënten als beschreven in Hoofdstuk 10 (52 patiënten met moeilijk te behandelen RA en 100 RA-patiënten die niet moeilijk te behandelen zijn), vanuit het patiëntendossier tijdens een onderzoeksbezoek en via een vragenlijst. Patiënten met moeilijk te behandelen RA bezochten vaker hun reumatoloog, werden vaker opgenomen op de dagbehandeling, hadden vaker bloedafnames en gebruikten meer medicijnen dan RA-patiënten die niet moeilijk te behandelen zijn. Daarmee waren ook de jaarlijkse gemiddelde totale kosten voor patiënten met moeilijk te behandelen RA significant hoger: €37.605, tegen €19.217 voor RA-patiënten die niet moeilijk te behandelen zijn, wat de economische ziektelast aantoont. Daarnaast vonden we dat verminderd functioneren de belangrijkste bijdrager is aan hogere kosten in RA.

In Hoofdstuk 12 hebben we therapieontrouw bij patiënten met moeilijk te behandelen RA verder onderzocht door belemmerende en bevorderende factoren voor therapieontrouw thematisch te structureren en te prioriteren vanuit het perspectief van de patiënt en de reumatoloog. Eerst hebben we interviews over therapieontrouw gehouden met patiënten met moeilijk te behandelen RA en RA-patiënten die niet moeilijk te behandelen zijn. Vanuit deze interviews zijn er door een projectgroep representatieve stellingen geselecteerd: 40 belemmerende en 40 bevorderende factoren. Vervolgens hebben 20 patiënten met moeilijk te behandelen RA en 20 RA-patiënten die niet moeilijk te behandelen zijn de stellingen gesorteerd in twee kaartsorteertaken: eerst op de mate van gelijkheid en vervolgens op de mate waarin de stellingen op hen van toepassing waren. Onafhankelijk daarvan hebben ook 20 reumatologen de stellingen gesorteerd op de mate waarin de stellingen volgens hen van toepassing waren op algemene RA-patiënten. De sortering op gelijkheid werd gebruikt om overzichten te maken vanuit het perspectief van de patiënt: binnen de belemmerende factoren waren er negen groepen te onderscheiden, gerelateerd aan het zorgsysteem, de veiligheid en de effectiviteit van de behandeling, de regels rondom de behandeling en het patiëntgedrag; daarnaast waren er binnen de bevorderende factoren zeven groepen te onderscheiden, gerelateerd aan het zorgsysteem en direct aan de patiënt. De sortering op de toepasselijkheid van de stellingen werd gebruikt om de stellingen te prioriteren en om de volgordes die zijn toegekend door patiënten met moeilijk te behandelen RA te vergelijken met de volgordes van RA-patiënten die niet moeilijk te behandelen zijn en die van reumatologen. Patiënten met moeilijk te behandelen RA, RA-patiënten die niet moeilijk te behandelen zijn en reumatologen prioriteerden de belemmerende en bevorderende factoren grotendeels hetzelfde. Patiënten met moeilijk te behandelen RA beschouwden 'bijwerkingen' en 'twijfel over de effectiviteit van medicijnen' als belangrijkste belemmerende factoren voor therapietrouw. Patiënten met moeilijk te behandelen RA, RA-patiënten die niet moeilijk te behandelen zijn en 
reumatologen beschouwden 'een goede band met de zorgverlener' en 'informatie over de behandeling' als meest behulpzame bevorderende factoren voor therapietrouw.

In Hoofdstuk 13 hebben we de resultaten van de onderzoeken in dit proefschrift in een breder perspectief geplaatst, de sterktes en zwaktes bediscussieerd evenals implicaties voor de klinische praktijk en aanbevelingen gedaan voor toekomstig onderzoek.

Concluderend kunnen we stellen dat het concept moeilijk te behandelen RA nu formeel is vastgesteld met de EULAR-definitie. Moeilijk te behandelen RA moet worden beschouwd als een heterogeen ziektebeeld waarbij er één of meerdere factoren kunnen bijdragen aan het blijven bestaan van klachten. Deze heterogeniteit moet worden onderkend in de dagelijkse praktijk en vraagt om een holistische benadering. Ondanks het gebrek aan onderzoek (zeker van hoge kwaliteit) wordt de behandeling van deze patiëntengroep nu ondersteund door de EULAR-behandelrichtlijnen die gebaseerd zijn op consensus van internationale experts. Als eerste stap in de behandeling is het van belang om vast te stellen dat RA nog steeds de juiste diagnose is. Om te ontdekken waar de huidige klachten van de patiënt vandaan komen moet vervolgens de aan- of afwezigheid van ontstekingen worden vastgesteld evenals de factoren die mogelijk bijdragen aan het ziektebeeld. Afhankelijk van deze bevindingen moeten zowel medicamenteuze als niet-medicamenteuze behandelingen worden overwogen, toegespitst op de individuele patiënt.

De ziektelast voor een patiënt met moeilijk te behandelen RA is hoog, maar ook de belasting voor de reumatoloog en de kosten gerelateerd aan dit ziektebeeld zijn hoog. Dit vraagt om een snelle implementatie van bovenstaande aanbevelingen in de dagelijkse praktijk, zodat de ziektelast kan worden verminderd. Daarnaast is er meer onderzoek nodig naar moeilijk te behandelen RA: ten eerste om onze kennis over de onderliggende mechanismen in het afweersysteem te vergroten, ten tweede om de diagnostische testen en behandeling te verbeteren, en ten derde om factoren te vinden die helpen bij het tijdig herkennen van deze patiënten en het voorspellen van het succes van behandelingen. Hierbij is het ultieme doel om de uitkomsten van patiënten met moeilijk te behandelen RA te verbeteren. 



\section{Acknowledgements}

Allereerst veel dank aan alle patiënten die bereid waren om deel te nemen aan de verschillende studies beschreven in dit proefschrift. Zonder jullie zou dit proefschrift er niet zijn geweest en bovenal waren jullie de belangrijkste motivator voor dit werk.

Ook ben ik veel dank verschuldigd aan mijn promotoren, copromotoren en andere begeleiders. Ik heb enorm veel van jullie geleerd en kijk ernaar uit om onze samenwerking zowel in de kliniek als in de onderzoekswereld voort te zetten.

Prof. dr. Van Laar, beste Jaap, tijdens ons kennismakingsgesprek was het binnen tien minuten beklonken: ik zou promotieonderzoek gaan doen en ik werd reumatoloog. Ondanks dat het promotietraject niet geheel verliep zoals gepland, bedacht je steeds weer nieuwe projecten waar ik me op kon storten en gaf je me ook de vrijheid om mijn eigen promotietraject vorm te geven. Bedankt voor alle kansen die je me de afgelopen jaren gegeven hebt.

Prof. dr. Lafeber, beste Floris, afspraken met jou leverden altijd nieuwe inzichten op. Ik heb veel gehad aan jouw focus en realisme en deel jouw behoefte aan een langetermijnvisie. Ik waardeer het zeer dat je onderdeel van mijn promotieteam wilde zijn en wil je hier hartelijk voor bedanken.

Dr. Van der Goes, beste Marlies, jouw plaats binnen mijn promotieteam veranderde al snel naar één van de belangrijkste. Ik heb bewondering voor de manier waarop je zo veel dingen tegelijkertijd doet. Je hebt hetzelfde pad als ik bewandeld en je gaf me daardoor altijd het vertrouwen dat het uiteindelijk allemaal goed zou komen. Je pragmatische klinische blik, nuchterheid en de mogelijkheid om over van alles en nog wat te kunnen sparren zijn van essentiële waarde geweest voor dit proefschrift. Veel dank hiervoor.

Dr. Welsing, beste Paco, je engelengeduld is bewonderenswaardig. Het duurde even voordat je een officiële rol kreeg binnen mijn promotieteam, maar desondanks kon ik altijd bij je terecht en was ik altijd zeker van een snelle reactie. Ik waardeer dit zeer, bedankt voor je begeleiding.

Dr. Jacobs, beste Hans, ondanks het veelal uitblijven van een relevante auteursplek ben je enorm betrokken geweest bij mijn promotietraject. Initieel was de manier van feedback even wennen, maar uiteindelijk had je toch (bijna) altijd gelijk. Je hebt je sporen in de wetenschap absoluut verdiend en ik wil je bedanken voor alles wat je me hebt geleerd.

Dr. De Hair, beste Marjolein, geen officiële taak meer, maar toch kan ik je zeker niet overslaan in dit dankwoord. Tijdens mijn eerste stappen in het onderzoek nam je me bijna letterlijk aan de hand, met veel geduld probeerde je me uit de klinische modus te halen. Dank voor al je hulp.

Dr. Tekstra, beste Janneke, gedurende mijn promotietraject ben je altijd zijdelings betrokken geweest. Ik bewonder het hoe de patiënt bij jou altijd op één staat. 
Alle andere coauteurs, ook zonder jullie bijdragen was dit proefschrift er niet geweest. My special gratitude goes out to prof. dr. Désirée van der Heijde and prof. dr. György Nagy for your efforts and supervision in the EULAR projects regarding difficult-to-treat rheumatoid arthritis. Additionally, a special thanks to dr. Elena Nikiphorou for your help in the project regarding treatment non-adherence. I am very much looking forward to the work visit to King's College in London, which hopefully will not be delayed much longer. Tot slot een speciaal woord van dank aan prof. dr. Rinie Geenen, ik kijk met veel plezier terug op onze prettige samenwerking, vooral in het project over therapieontrouw.

Leden van de beoordelingscommissie, prof. dr. Emmelot-Vonk, prof. dr. Huizinga, prof. dr. Van Schaardenburg, prof. dr. Visseren, prof. Van de Wijgert, dank voor uw tijd om mijn proefschrift te beoordelen. Ik kijk ernaar uit om met u over de inhoud van gedachten te wisselen.

(Ex-)collega-onderzoekers, postdoc's, laboranten, researchverpleegkundigen, studenten en secretaresses van de afdeling Reumatologie \& Klinische Immunologie, ik heb veel gehad aan jullie nuttige feedback, hulp bij alle projecten en bovenal aan de plezierige werksfeer. In het bijzonder Maxime en Mylène, doordat onze promotietrajecten gelijk opliepen hebben we veel waardevolle inzichten kunnen delen. Daarnaast Anne Karien voor alle hulp bij het METC-werk, Marion voor hulp bij het uitvoeren en verwerken van de echogeleide synoviumbiopten, Arno voor het verwerken van alle samples, Karin en Anneloes voor hulp bij de inclusie van patiënten en Diana voor hulp bij de praktische zaken.

Reumatologen, AIOS en reumaconsultenten, zowel in het UMC Utrecht, het Meander MC, als verder in de regio, bedankt voor jullie inzet bij het verwijzen van patiënten en verdere bijdragen aan de beschreven studies. Ik kijk er naar uit om met jullie samen te werken in de kliniek.

Internisten en AIOS uit het UMC Utrecht, bedankt voor het warme welkom terug in de kliniek. Ik kijk uit naar onze (nog korte maar vast krachtige!) samenwerking.

Lieve vrienden, clubgenoten en (schoon-)familie, bedankt voor alle interesse in mijn onderzoek en welkome afleiding in de vorm van ontelbare etentjes, borrels, wandelingen, reizen, etc. Ook dit laatste is enorm belangrijk geweest voor dit proefschrift. Ik ben heel blij met jullie in mijn leven.

In het bijzonder Anita en Jessica, wat een voorrecht dat jullie mijn paranimfen zijn! Anita, heerlijk dat onze smaak altijd precies overeenkomt. Ik waardeer het zeer dat ik altijd bij je terecht kan voor advies en een luisterend oor. Jessica, wat fijn dat jij destijds mijn mentor 
werd in het Meander en dat we sindsdien al zo veel (onderzoeksgerelateerde) hoogteen dieptepunten hebben kunnen delen. Dank voor deze dierbare vriendschappen!

Lieve papa en mama, altijd kan ik bij jullie terecht om uit te razen en kan ik er daarna weer tegenaan met goede adviezen. Jullie opvoeding heeft me gemaakt tot wie ik nu ben en daarmee is jullie aandeel aan dit proefschrift ook absoluut essentieel. Bedankt voor jullie betrokkenheid en onvoorwaardelijke steun, niet alleen bij het vieren van de pieken, maar ook bij de onvermijdelijke dalen.

Lieve Frank, jouw vechtlust heeft me ontelbare keren geholpen tijdens de totstandkoming van dit proefschrift. Je vond mijn medische kennis erg interessant maar tegelijkertijd ook bijzonder irritant, helemaal wanneer ik me weer eens te veel met jou bemoeide. Ik weet zeker dat je enorm trots zou zijn, maar wat had ik dit moment ook graag met jou gevierd.

Lieve Wouter, voordat ik begon met promoveren was je al gewaarschuwd, dit zou voor jou ook nog wel eens zwaar kunnen worden. Zelf vond je dit reuze meevallen en denk je dat je aan geen letter van dit proefschrift hebt bijgedragen. Hier ben ik het zeker niet mee eens, zonder deze fijne thuisbasis en oneindige steun was dit proefschrift er nooit gekomen. Bedankt voor alles, ik kijk uit naar onze toekomst samen. 



\section{List of publications}

Roodenrijs NMT, de Hair MJH, van der Goes MC, Jacobs JWG, Welsing PMJ, van der Heijde D, Aletaha D, Dougados M, Hyrich KL, McInnes IB, Müller-Ladner U, Šenolt L, Szekanecz Z, van Laar JM, Nagy G. Characteristics of difficult-to-treat rheumatoid arthritis: results of an international survey. Ann Rheum Dis 2018;77:1705-1709.

Roodenrijs NMT, de Hair MJH, Wheater G, Elshahaly M, Tekstra J, Teng YKO, Lafeber FPJG, Hwang CC, Liu X, Sasso EH, van Laar JM. The multi-biomarker disease activity score tracks response to rituximab treatment in rheumatoid arthritis patients: a post hoc analysis of three cohort studies. Arthritis Res Ther 2018;20:256.

Roodenrijs NMT, van der Goes MC, Welsing PMJ, Tekstra J, van Laar JM, Lafeber FPJG, Bijlsma JWJ, Jacobs JWG. Is prediction of clinical response to methotrexate in individual rheumatoid arthritis patients possible? A systematic literature review. Joint Bone Spine 2020;87:13-23.

Roodenrijs NMT, de Hair MJH, van der Goes MC, Jacobs JWG. Correspondence to viewpoint 'Defining refractory rheumatoid arthritis' by Buch. Ann Rheum Dis 2019;78:e105.

Nagy G, Roodenrijs NMT, Welsing PMJ, Kedves M, Hamar A, van der Goes MC, Kent A, Bakkers M, Blaas E, Šenolt L, Szekanecz Z, Choy E, Dougados M, Jacobs JWG, Geenen R, Bijlsma JWJ, Zink A, Aletaha D, Schoneveld JL, van Riel P, Gutermann L, Prior Y, Nikiphorou E, Ferraccioli G, Schett G, Hyrich KL, Müller-Ladner U, Buch MH, Mclnnes IB, van der Heijde $D^{*}$, van Laar JM*. EULAR definition of difficult-to-treat rheumatoid arthritis. Ann Rheum Dis 2021;80:31-35.

Roodenrijs NMT, Welsing PMJ, van der Goes MC, Jacobs JWG, van der Heijde D, van Laar JM, Nagy G. Response to: 'Correspondence on 'EULAR definition of difficult-totreat rheumatoid arthritis' by Novella-Navarro et al. Ann Rheum Dis 2020. doi: 10.1136/ annrheumdis-2020-219535 [Epub ahead of print 4 Dec 2020]

Roodenrijs NMT, van der Goes MC, Welsing PMJ, Tekstra J, Lafeber FPJG, Jacobs JWG, van Laar JM. Difficult-to-treat rheumatoid arthritis: contributing factors and burden of disease. Rheumatology 2020. doi: 10.1093/rheumatology/keaa860 [Epub ahead of print 17 Dec 2020]

Roodenrijs NMT, Welsing PMJ, van der Goes MC, Tekstra J, Lafeber FPJG, Jacobs JWG, van Laar JM. Health care utilisation and economic burden of difficult-to-treat rheumatoid 
arthritis: a cost-of-illness study. Rheumatology 2021. doi: 10.1093/rheumatology/keab078 [Epub ahead of print 27 Jan 2021]

Roodenrijs NMT, Kedves M, Hamar A, Nagy G, van Laar JM, van der Heijde D, Welsing PMJ. Diagnostic issues in difficult-to-treat rheumatoid arthritis: a systematic literature review informing the EULAR recommendations for the management of difficult-to-treat rheumatoid arthritis. RMD Open 2021;7:e001511.

Roodenrijs NMT, Kedves M, Hamar A, Nagy G, van Laar JM, van der Heijde D, Welsing PMJ. Pharmacological and non-pharmacological therapeutic strategies in difficult-to-treat rheumatoid arthritis: a systematic literature review informing the EULAR recommendations for the management of difficult-to-treat rheumatoid arthritis. RMD Open 2021;7:e001511.

Roodenrijs NMT, van der Goes MC, Welsing PMJ, E.P.C. van Oorschot, E. Nikiphorou, N.C. Nijhof, Tekstra J, Lafeber FPJG, Jacobs JWG, van Laar JM. Non-adherence in difficult-totreat rheumatoid arthritis from the perspectives of patients and rheumatologists: a concept mapping study. Rheumatology 2021. doi: 10.1093/rheumatology/keabl30 [Epub ahead of print 9 Feb 2021]

Nagy G*, Roodenrijs NMT*, Welsing PMJ, Kedves M, Hamar A, van der Goes MC, Kent A, Bakkers M, Pchelnikova P, Blaas E, Šenolt L, Szekanecz Z, Choy E, Dougados M, Jacobs JWG, Geenen R, Bijlsma JWJ, Zink A, Aletaha D, Schoneveld JL, van Riel P, Dumas S, Prior Y, Nikiphorou E, Ferraccioli G, Schett G, Hyrich KL, Müller-Ladner U, Buch MH, McInnes IB, van der Heijde D*, van Laar JM*. EULAR recommendations for the management of difficult-totreat rheumatoid arthritis. Manuscript in preparation.

Roodenrijs NMT, Welsing PMJ, van Roon J, Schoneveld Jan LM, van der Goes M, Nagy G, Townsend MJ, van Laar JM. Immunological mechanisms underlying DMARD inefficacy in difficult-to-treat rheumatoid arthritis: a narrative review with systematic literature search. Manuscript in preparation.

Messelink MA*, Roodenrijs NMT*, van Es B, Hulsbergen-Veelken CAR, Jong S, Overmars LM, Reteig LC, Tan SC, Tauber T, van Laar JM, Welsing PMJ, Haitjema S. Identification and prediction of difficult-to-treat rheumatoid arthritis patients in structured and unstructured routine care data: results from a hackathon. Submitted for publication.

* Authors contributed equally 



\section{Contributing authors}

\section{Daniel Aletaha}

Department of Internal Medicine III, Division of Rheumatology, Medical University of Vienna, Vienna, Austria

\section{Margot Bakkers}

EULAR Standing Committee of People with Arthritis/Rheumatism in Europe (PARE), Zurich, Switzerland

\section{Johannes W.J. Bijlsma}

Department of Rheumatology \& Clinical Immunology, University Medical Center Utrecht, Utrecht, the Netherlands

\section{Etiënne Blaas}

Department of Rheumatology \& Clinical Immunology, University Medical Center Utrecht, Utrecht, the Netherlands

\section{Maya H. Buch}

NIHR Manchester Musculoskeletal Biomedical Research Unit, Central Manchester NHS Foundation Trust, Manchester Academic Health Science Centre, Manchester, United Kingdom

Centre for Musculoskeletal Research, School of Biological Sciences, Faculty of Biology, Medicine \& Health, University of Manchester, United Kingdom

Leeds Institute of Rheumatic \& Musculoskeletal Medicine, University of Leeds, United Kingdom

\section{Ernest Choy}

CREATE Centre, Section of Rheumatology, School of Medicine, Division of Infection and Immunity, Cardiff University, Cardiff, United Kingdom

\section{Maxime Dougados}

Université de Paris Department of Rheumatology - Hôpital Cochin. Assistance Publique Hôpitaux de Paris INSERM (U1153) Clinical epidemiology and biostatistics, PRES Sorbonne Paris-Cité, Paris, France

\section{Sophie Dumas}

Department of Pharmacy, Marin Hospital, Assistance Publique-Hopitaux de Paris, Hendaye, France 


\section{Mohsen Elshahaly}

Department of Rheumatology \& Rehabilitation, Suez Canal University, Ismailia, Egypt

\section{Gianfranco Ferraccioli}

School of Medicine, Catholic University of the Sacred Heart, Rome, Italy

\section{Rinie Geenen}

Department of Psychology, Utrecht University, Utrecht, the Netherlands

\section{Marlies C. van der Goes}

Department of Rheumatology, Meander Medical Center, Amersfoort, the Netherlands Department of Rheumatology \& Clinical Immunology, University Medical Center Utrecht, Utrecht, the Netherlands

\section{Loriane Guttermann}

Department of Pharmacy, Paris Descartes University, Hôpital Cochin, Assistance Publique Hôpitaux de Paris, Paris, France

\section{Maria J.H. de Hair}

Department of Rheumatology \& Clinical Immunology, University Medical Center Utrecht, Utrecht, the Netherlands

\section{Attila Hamar}

Department of Rheumatology, Faculty of Medicine, University of Debrecen, Debrecen, Hungary

\section{Désirée van der Heijde}

Department of Rheumatology, Leiden University Medical Center, Leiden, the Netherlands

\section{Ching Chang Hwang}

Crescendo Bioscience, San Francisco, CA, USA

\section{Kimme L. Hyrich}

NIHR Manchester Musculoskeletal Biomedical Research Unit, Central Manchester NHS Foundation Trust, Manchester Academic Health Science Centre, Manchester, United Kingdom

Centre for Musculoskeletal Research, School of Biological Sciences, Faculty of Biology, Medicine \& Health, University of Manchester, Manchester, United Kingdom 


\section{Johannes W.G. Jacobs}

Department of Rheumatology \& Clinical Immunology, University Medical Center Utrecht, Utrecht, the Netherlands

\section{Melinda Kedves}

Department of Rheumatology, Bács-Kiskun County Hospital, Kecskemét, Hungary

\section{Alison Kent}

Salisbury Foundation Trust NHS Hospital, Wiltshire, United Kingdom

\section{Jacob M. van Laar}

Department of Rheumatology \& Clinical Immunology, University Medical Center Utrecht, Utrecht, the Netherlands

\section{Floris P.J.G. Lafeber}

Department of Rheumatology \& Clinical Immunology, University Medical Center Utrecht, Utrecht, the Netherlands

\section{Xinyu Liu}

Crescendo Bioscience, San Francisco, CA, USA

\section{Ian B. Mclnnes}

Institute of Infection, Immunity and Inflammation, University of Glasgow, Glasgow, United Kingdom

\section{Ulf Müller-Ladner}

Department of Rheumatology and Clinical Immunology, Justus-Liebig University Giessen, Kerckhoff Clinic Bad Nauheim, Bad Nauheim, Germany

\section{György Nagy}

Department of Rheumatology \& Clinical Immunology, Semmelweis University, Budapest, Hungary

Department of Genetics, Cell and Immunobiology, Semmelweis University, Budapest, Hungary

\section{Nienke Nijhof}

Department of Rheumatology \& Clinical Immunology, University Medical Center Utrecht, Utrecht, the Netherlands 


\section{Elena Nikiphorou}

Centre for Rheumatic Diseases, King's College London, London, United Kingdom

\section{Eline P.C. van Oorschot}

Department of Rheumatology \& Clinical Immunology, University Medical Center Utrecht, Utrecht, the Netherlands

\section{Polina Pchelnikova}

EULAR Standing Committee of People with Arthritis/Rheumatism in Europe (PARE), Zurich, Switzerland

\section{Yeliz Prior}

School of Health and Society, Centre for Health Sciences Research, University of Salford, Salford, United Kingdom

\section{Piet van Riel}

Department of Rheumatic Diseases, Radboud University Nijmegen Medical Centre, Nijmegen, the Netherlands

\section{Joel van Roon}

Department of Rheumatology \& Clinical Immunology, University Medical Center Utrecht, Utrecht, the Netherlands

\section{Eric H. Sasso}

Crescendo Bioscience, San Francisco, CA, USA

\section{Georg Schett}

Department of Internal Medicine 3, Rheumatology and Immunology, Friedrich-Alexander University of Erlangen-Nuremberg and Universitatsklinikum Erlangen, Erlangen, Germany

\section{Jan L.M. Schoneveld}

Department of Rheumatology Bravis Hospital, Roosendaal, the Netherlands

\section{Ladislav Šenolt}

Department of Rheumatology, lst Faculty of Medicine, Charles University and Rheumatology Institute, Prague, Czech Republic

\section{Zoltán Szekanecz}

Department of Rheumatology, Faculty of Medicine, University of Debrecen, Debrecen, 
Hungary

\section{Janneke Tekstra}

Department of Rheumatology \& Clinical Immunology, University Medical Center Utrecht, Utrecht, the Netherlands

\section{Y.K. Onno Teng}

Department of Nephrology, Leiden University Medical Center, Leiden, the Netherlands

\section{Michael J Townsend}

Biomarker Discovery OMNI, Genentech Research \& Early Development, South San Francisco, CA, USA

\section{Paco M.J. Welsing}

Department of Rheumatology \& Clinical Immunology, University Medical Center Utrecht, Utrecht, the Netherlands

\section{Gill Wheater}

Department of Biochemistry, The James Cook University Hospital, Middlesbrough, United Kingdom

\section{Angela Zink}

Epidemiology Unit, German Rheumatism Research Centre, Berlin, Germany 



\section{Curriculum Vitae}

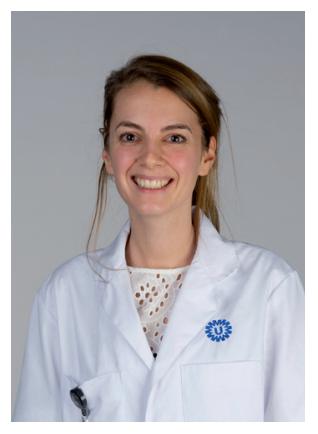

Nadia Roodenrijs was born on the $3^{\text {rd }}$ of May 1990 in Wateringen, the Netherlands. In 2008, she finished high school (gymnasium) at the 'Christelijk Lyceum' in Delft. After graduation, she moved to Utrecht and started as a medical student at the Utrecht University. In 2014, she obtained her medical degree. During her medical training, she had a special interest in Rheumatology, Dermatology and Tropical Medicine and conducted several research projects and internships in these fields. An internship in Kuala Lumpur, Malaysia, was among the highlights of her medical training.

Subsequently, she started her residency training in Rheumatology in 2015. As first part of her residency training, she worked at the department of Internal Medicine at the Meander Medical Center in Amersfoort under supervision of dr R. Fijnheer and dr R.J. Bosma.

At the end of 2017, she interrupted her residency training to start as a PhD candidate at the department of Rheumatology \& Clinical Immunology at the University Medical Center (UMC) Utrecht under supervision of prof dr J.M. van Laar, prof dr F.P.J.G. Lafeber, dr M.C. van der Goes and dr P.M.J. Welsing. During her PhD, she participated as fellow in the European League Against Rheumatism (EULAR) Task Force on the development of recommendations for the management of difficult-to-treat rheumatoid arthritis (D2T RA). This work formed the basis of her thesis and was performed under supervision of prof dr J.M. van Laar (UMC Utrecht), prof dr G. Nagy (Semmelweis University, Budapest, Hungary), prof dr D. van der Heijde (Leiden UMC) and dr P.M.J. Welsing (UMC Utrecht). Additionally, she set up a clinical study into factors contributing to D2T RA, the results of which are presented in this thesis. She received the Rheumatology Grant 2019 awarded by the 'Nederlandse Vereniging voor Reumatologie' for a research proposal on a sub study regarding treatment non-adherence in D2T RA, in collaboration with dr E. Nikiphorou (King's College, London, United Kingdom). Furthermore, she participates in the Emerging EULAR Network (EMEUNET) committee of EULAR as member of the Visibility \& Global Affairs subcommittee.

In January 2021, she continued her residency training at the department of Internal Medicine at UMC Utrecht under supervision of prof dr H.A.H. Kaasjager and dr J.J. Oosterheert. Starting from January 2022, this will be followed by residency training at the department of Rheumatology \& Clinical Immunology at UMC Utrecht under supervision of dr E. Ton and prof dr J.M. van Laar. Simultaneously, she will perform a fellowship in Clinical Pharmacology under supervision of dr W. Knol.

Nadia lives together with Wouter Moll in Utrecht. They are expecting their first child in September 2021. 


